\section{Oral Session}

\section{Stem Cell Biology and Stem Cell Therapy}

S. Seeber, Essen; W. Hiddemann, München

\section{1}

\section{Stem cell plasticity and biology}

Lemischka I.

Princeton, USA

2

\section{Stem cell therapy in myocardial infarction - experimental and} clinical data

Dimmeler $S$.

Molecular Cardiology, University of Frankfurt

Increasing evidence suggest that hematopoietic stem and progenitor cell can contribute to the formation of new blood vessels after critical ischemia Neovascularization thereby is believed to be stimulated by endothelial progenitor cells, which can be generated out of peripheral blood mononuclear cells or isolated CD34- or CD133-positive cells. Furthermore, experimental studies have demonstrated that transplantation of bone marrow-derived or circulating blood-derived progenitor cells is capable of regenerating cardiac contractile function after myocardial infarction.

Therefore, we tested whether contractile function after acute myocardial infarction might be improved by local progenitor cell therapy using either bone marrow-derived monocytic cells (BMC) or circulating blood-derived progenitor cells (EPCs). Currently, data are available from 19 patients with acute myocardial infarction, which have been transplanted with either BMC (9 patients, $245+71$ Mio cells) or EPCs (10 patients, 10.2+7.1 Mio cells). Cells have been selectively infused into the infarct artery $4.5+0.9$ days after acute myocardial infarction. At baseline and after 4 months, cardiac function and coronary flow reserve was monitored

The presentation will summarize current experimental data supporting the concept that progenitor cells may be used for possible therapeutic neovascularization and cardiac regeneration. First clinical data will be presented.

Can stem cells be used for grafting in Parkinson's disease?

\section{Brundin P.}

Section for Neuronal Survival, Wallenberg Neuroscience Center, Lund University, Sweden

Parkinson's disease is characterized primarily by motor symptoms that in clude tremor, rigidity and bradykinesia. Neuropathologically, there is a dramatic loss of dopaminergic neurons in the substantia nigra and related areas in the midbrain.

Grafts of human embryonic dopamine neurons placed in the target of the substantia nigra, i.e. the striatum, have been found to ameliorate several of the motor symptoms. Using positron emission tomography, it has been possible to demonstrate that grafted dopamine neurons survive and reinstate dopaminergic neurotransmission in the brains of Parkinson's disease patients.

Several lines of evidence suggest that a crucial threshold of surviving dopaminergic neurons must be exceeded for the grafts to become functional and relieve symptoms. In about one third of the 18 patients operated in our center in Lund,Sweden, the beneficial effects of neural grafts are very dramatic.

Although the beneficial functional effects have been dramatic in some grafted Parkinson's disease patients, a number of reasons explain why only a relatively small number of operations have been performed so far. Firstly, tissue from large numbers of donor embryos is needed for each patient, and this imposes major logistical problems. It is due to a low survival rate of grafted dopamine neurons (estimated at less than 10\%). Secondly, all pa- tients do not improve equally and it is difficult to predict which patients will show the best response. Thirdly, a study from the US reported in 2001 that around $15 \%$ of patients grafted with cultured mesencephalic tissue develop disabling involuntary movements, as an unwanted side-effect of the transplants. This study suggested that there was an excess of dopamine produced in the grafts, but a recent reevaluation of patients operated in Lund do not support this conclusion. Despite the issue of involuntary movements being of less concern than initially believed, the lack of large numbers of cells suitable for transplantation has inhibited progress. Thus, there is definitely a need for alternative sources of donor tissue that can be used for transplantation in Parkinson's disease in the future, and will make the procedure independent of a continuous supply of embryonic brain tissue. Clearly various forms of stem cells, possibly also those residing in bone marrow, are interesting future options.

\section{Hematopoietic Stem Cell Transplantation and Gene Transfer}

\section{v. Kalle $C$.}

Program in Molecular and Gene Therapy, Department of Experimental Hematology, Children's Research Foundation, Children's Hospital Medical Center, University of Cincinnati, USA

New and exciting developments have transformed the character of hematopoietic stem cell transplantation. The harvest, ex vivo handling and engraftment process are changing towards a more simple and well-tolerated therapeutic application. Successful engraftment of allogeneic stem cells begins to be understood as a process of establishing immunotolerance for the graft, enabling to reduce and eventually altogether eliminate the destruction of innate stem cells by toxic conditioning therapy. These ongoing developments are decisive prerequisites for the development and clinical application of genetic and other molecular strategies aimed at the therapeutic manipulation of stem cells. Genetic correction of stem cells with integrating vector systems is a promising long-term goal. The observation of novel bone marrow derived stem cell activities in other organ systems have renewed and intensified this interest. Like more conventional pharmaceutical new drug development, the genetic manipulation of stem cells has taken roughly 15 years from first conceptual success to the successful completion of initia clinical trials. In 2000, Fischer and colleagues reported the first successful genetic therapy of X-linked immunodeficiency. The retrovirus-mediated stem cell gene transfer used in this and other trials is still hampered by low efficiency, requiring a selective advantage of the modified stem cell population or toxic bone marrow conditioning to allow a clinically significant engraftment of such cells. More recent preclinical experimentation has allowed to define new retrovirus and lentivirus vector pseudotypes that achieve the integration of therapeutic vectors at much higher percentages in small and large animal hematopoiesis. Vector systems have been introduced that can selectively expand or protect genetically modified cells. These advances will transfer into the clinical field, defining new opportunities for the treatment of genetic disease and cancer with autologous and allogeneic stem cells. Similar to pharmaceutical dose increments, increasing the potency and amount of vector as well as the number of gene modified cells will, however, also increase the likelihood of encountering side effects of genetic modification. The genetic manipulation introduces unique genetic markers that differ in each affected stem cell. Newly developed technology has allowed us to count the number of manipulated stem cells active in several interesting preclinical and clinical studies, to track their biological fate in vivo over time and to begin assessing potential genetic toxicity. 


\section{Lung Cancer}

W. Eberhardt, Essen; M. Garbrecht, München

\section{Histology and molecular pathology of malignant lung tumours}

Junker $K$

Institut für Pathologie, BG-Kliniken Bergmannsheil, Klinikum der RuhrUniversität Bochum

Malignant lung tumours are classified according to the 1999 WHO-classification of lung and pleural tumours, which comprises about 100 tumours or preinvasive and tomour-like lesions of the lung. Under the heading of preinvasive lesions among others squamous dysplasia, carcinoma in situ, and atypical adenomatous hyperplasia are listed.

Carcinogenesis of malignant lung tumours can be subdivided into early (normal mucosa, hyperplastic and metaplastic lesions), intermediate (different grades of dysplasia) and late phases (carcinoma in situ, invasive carcinoma). Analysing normal, hyperplastic, and dysplastic epithelium as well as carcinoma in situ and invasive tumour from the same patient it is possible to show a sequence of allelic losses: $3 p-9 p-8 p-17 p-5 q$.

$3 p$ losses are observed in over $90 \%$ of small cell lung cancers and squamous cell carcinomas and in about $50 \%$ of adenocarcinomas. 3p14.2 deletions affect the fhit tumour suppressor gene. p16INK4A is located on chromosome 9p (9p21) and regulates the activation of RB by inhibition of the CDK4-cyclin D1-kinase activity. Aberrant p16INK4A methylation is seen as an early event in the carcinogenesis of lung cancer. As further tumour suppressor gene, $\mathrm{p} 53$ is located on chromosome $17 \mathrm{p}$ (17p13.1). Inactivation of p53 by mutation is observed in over $75 \%$ in small cell lung cancer and in about $50 \%$ in non-small cell lung cancer. The occurrence of p53 alterations characterises an intermediate phase of carcinogenesis in lung cancer. $35 \%$ of small cell lung cancers and $22 \%$ of non-small cell lung cancers show at least single microsatellite alterations, which may occur in the early stage of carcinogenesis. High telomerase activity can be established in most malignant lung tumours as well as in metaplastic changes of the bronchial mucosa and may so represent an early event in the carcinogenesis of lung cancer. 7 closely related genes form the myc-oncogene family. Their activation is seen as an essential mechanism and an early event in the pathogenesis of human lung cancer. Especially in pulmonary adenocarcinomas k-ras-codon 12-mutations can be observed. They are also found in late preinvasive lesions of the bronchial mucosa as well as in atypical adenomatous hyperplasia

Finally a - possibly incomplete - sequence of genetic alterations can be named in the carcinogenesis of malignant lung tumours. Their careful analysis may lead to progress in the molecular diagnosis and in the treatment of lung cancer.

Chemotherapy in lung cancer - state of the art and future perspectives.

Laack $E$.

Medizinische Klinik II (Onkologie und Hämatologie), Universitätsklinikum Hamburg-Eppendorf

Treatment of patients with lung cancer is a particular challenge in oncology, because lung cancer is the most frequent cause of death in Western Europe and in the United States. More than two-third of patients with non-small cell lung cancer (NSCLC) have distant metastases at diagnosis. Since the 1990's the therapy of patients with NSCLC has been changing, and some progress has been achieved. New and better tolerated cytotoxic agents have altered the therapeutic approach towards patients with distant metastases. A standard chemotherapy does not yet exist for this stage of disease. The results of randomized trials indicate the superiority of combination chemotherapy in comparison to single agent chemotherapy. One of the most controversially discussed questions is the role of cisplatin. Up to now there are only few data available concerning the comparison of cisplatin-based with cisplatinfree combinations. Current data of randomized trials indicate that cisplatin does not play an important role in the era of new cytotoxic agents for the palliative care of patients with NSCLC.

In small cell lung cancer (SCLC) combination chemotherapy is the basis of each treatment strategy. Platin-based as well as anthracykline-based chemotherapy could both be regarded as standard therapy. Dose intensification of chemotherapy did not significantly prolong survival. At present a plateau has been reached in gaining further benefits from chemotherapy in NSCLC as well SCLC. First results of new treatment approaches, like tyro- sine kinase inhibitors, are encouraging. These „biologicals“ will extend the therapeutic possibilities - alone or in combination with chemotherapy - in an adjuvant or palliative setting.

Current developments of combined modality treatment and modern options of radiotherapy of lung cancer

Stuschke M

Essen

8

New treatment strategies in lung cancer

Stahel $R$.

Zürich

\section{Acute Myeloid Leukemia}

W.D. Ludwig, Berlin; U. Jäger, Wien

9

The Genetic Basis of AML and MDS

Look T.

Boston, USA

10

The leukemic stem cell and stem cell regulatory genes

Buske C. (1), Feuring-Buske M. (1), Humphries R.K. (2), Hiddemann W. (1)

(1) GSF-National Research Center for Environment and Health und Medizinische Klinik III Klinikum Großhadern, LMU, München,

(2) The Terry Fox Laboratory, BCCA, Vancouver, Canada

Acute myeloid leukemia (AML) arises from the clonal expansion of a malignant transformed hematopoietic stem cell or early progenitor cell. In the recent years there has been considerable progress in characterizing leukemic stem cells and in dissecting the molecular mechanisms which determine their perturbed proliferation and differentiation behaviour. One intriguing characteristic of leukemic stem cells is their ability to efflux the Hoechst 33342 DNA binding dye, the so called ,Side Population“ (SP). Recently, the $\mathrm{ABC}$ transporter $\mathrm{ABCG} 2$ was identified as the molecular determinant of the SP phenotype. Importantly, SP-positivity in the CD34-positive/CD38negative stem cell compartment seems to differentiate between normal and leukemic stem cells in patients with AML. Another hallmark of leukemic stem cells is their perturbed expression of transcription factors, which are known to be key regulators of normal stem cell development. One example is the family of homeobox (Hox) genes, highly conserved transcription factors which are normally selectively expressed at the early progenitor stage and then silenced at later stages of hematopoietic differentiation. The Hox gene regulatory network is implicated in human leukemogenesis at the level of the Hox genes themselves (e.g. NUP98-HOXD13, NUP98-HOXA9, ETV6-CDX2), at the level of the Hox-cofactor PBX1 in the t(1;19) E2APBX1 fusion gene and at the level of the homeobox gene upstream regulator MLL, one of the most frequently altered genes in human leukemias. The relevance of perturbed homeobox gene expression was highlighted in functional assays inducing dysregulated expression of HOX genes or MLL-fusion genes in hematopoietic stem cells by retroviral gene transfer: transplantation of these stem cell populations resulted in a myeloproliferative syndrome or frank AML in lethally irradiated recipient mice. The detailed understanding of the pathobiology of the leukemic stem cell will allow a deeper insight into the process of malignant transformation and will finally help to differentiate between normal and leukemic stem cells and to develop strategies to counteract the oncogenic potential of dysregulated transcription factor expression. 
Proteomics of transcription factors C/EBPalpha and PU.1 in myeloid differentiation and leukemia

Behre G.

Department of Internal Medicine III, University Hospital Grosshadern, LMU Munich

The transcription factors C/EBPalpha and PU.1 are responsible for normal myeloid differentiation from stem cells to monocytes and granulocytes, a process which is blocked in acute myeloid leukemia (AML). Our recent results indicate that interactions of transcription factors with other nuclear proteins play not only an important role in normal differentiation in the hematopoietic system, but also in the pathogenesis of myeloid leukemias. Thus, the transcription factor PU.1 (which drives monocytic differentiation and can be mutated in AML) is activated by the co-activator c-Jun, a protein-protein interaction which can be disturbed by GATA-1 (leading to erythrocytic differentiation), by C/EBPalpha (resulting in granulocytic differentiation) or the leukemic fusion protein AML1-ETO (contributing to AML). In contrast to PU.1, the transcription factor C/EBPalpha is crucial for the differentiation of granulocytes. Our data indicate that C/EBPalpha is activated by the Ras signaling pathway via phosphorylation on serine 248 Dominant negative mutations of C/EBPalpha have been found in some patients with AML, but not in AML patients with the $t(8 ; 21)$ translocation. However, AML1-ETO-positive AML blasts had undetectable C/EBPalpha protein levels. In summary, transcription factor protein-protein interactions are important for normal myeloid development as well as for leukemogenesis. High throughput proteomics can identify multiprotein complexes and expression changes of multiple proteins at the same time by mass spectrometric analysis of gel separated proteins. We describe an application of proteomic methods (2D gel electrophoresis and mass spectrometry) towards identifying further regulated protein targets (interacting proteins and proteins altered in expression) of transcription factors C/EBPalpha and PU.1 and demonstrate the utility of this discovery-based global strategy in elucidating novel pathways. This global high throughput functional proteomics approach will lead to new insights into the network of protein-protein interactions and target proteins involved in myeloid development and leukemia, and provide new targets for rational pathogenesis-based therapies of leukemia and cancer.

\section{2}

\section{Oncogenic activation of signal transduction in AML}

Serve H. (1), Müller-Tidow C. (1), Steffen B. (1), Schwäble J. (1), Mizuki M. (2), Puccetti E. (3), Ruthardt M. (3), Berdel W.E. (1)

(1) Department of Medicine, Hematology/Oncology, University of Münster, Germany, (2) Osaka University, Osaka, Japan, (3) Department of Medicine, Hematology , J.W. Goethe-Universität Frankfurt, Germany

The fully malignant phenotype of AML is the result of several molecular events that induce increased proliferation, survival, and the characteristic differentiation blockade. Activating mutations in proximal signal transduction pathways like receptors or their signalling intermediates have been held responsible for enhanced proliferation and survival. On the other hand, the fusion proteins resulting from chromosomal translocations have been thought to contribute to the leukemic phenotype by disrupting the transcriptional control of differentiation. However, our data show that this view of the molecular events leading to AML might be oversimplified, since we could define considerable functional crosstalk between the two mutation classes. Mutations of the receptor tyrosine kinase Flt3 occur in $25-30 \%$ of AML cases. Previously, we have shown that they activate the receptor and induce leukemic transformation. Genome-wide expression analyses of myeloid progenitor cells revealed the mutation-specific repression of several genes known to be involved in myeloid gene regulation. Pu.1 and $\mathrm{C} / \mathrm{EBP} \alpha$, both induced by ligand-activation of wildtype Flt3, were suppressed in their expression and function by the Flt3-mutations, which thereby inhibited myeloid differentiation. On the other hand, analyses of the common target genes of the leukemia-associated fusion proteins AML1ETO, PML-RAR $\alpha$ and PLZF-RAR $\alpha$ revealed that all 3 fusion proteins induced the expression of plakoglobin, an important read-out of the Wnt-signalling pathway. The fusion proteins induced signalling downstream of plakoglobin and led to transactivation of TCF/LEF dependent promoters Several known Wnt target genes like cyclin D1 and c-myc were induced by the fusion proteins and high cyclin D1 levels were found in $\mathrm{t}(8 ; 21)$ positive patient samples. In myeloid cell lines and primary mouse bone marrow, stable transfection with plakoglobin stimulated cellular proliferation and colony growth of undifferentiated progenitor cells. Our data suggest that oncogenic activation of signal transduction pathways might directly im- pinge upon myeloid differentiation while leukemogenic fusion proteins activate unexpected signal transduction pathways that are known to control proliferation and cell fate decision. The results underline the potential of molecular target definition in AML that will provide the rationale for therapeutic intervention with small molecules designed to inhibit signal transduction.

\section{High Grade Lymphoma}

M. Pfreundschuh, Homburg; M. Kneba, Kiel

\section{3}

Classification and biology of high grade non Hodgkin's lymphoma

Stein $H$.

Berlin

14

Chemotherapy of aggressive lymphomas

Hartmann F., Pfreundschuh M. für die Deutsche Studiengruppe hoch-maligne Non-Hodgkin-Lymphome (DSHNHL)

Medizinische Klinik I, Universitätskliniken des Saarlandes

The demonstration that the 25-year old CHOP regimen is as efficient, but less toxic than the intensified 2nd and 3rd generation regimens together with the recognition that the prognosis of a given patient is more dependen on pretherapeutically identifiable prognostic factors rather than on the type of chemotherapy employed, have led many cooperative groups to distinguish three populations of patients with aggressive lymphomas with respect to therapy: 1st young low-risk, 2nd young high-risk and 3rd elderly patients ( $>60$ years). For the first time since 25 years, the DSHNHL succeeded in improving treatment results (CR rates and survival after 4 years by $14 \%$ each) in elderly patients by shortening time intervals between chemotherapy cycles from 3 to 2 weeks (CHOP-14). Similar improvements after a median follow up of only 2 years were achieved by the French GELA by adding the monoclonal anti-CD20 antibody rituximab to the classical 3-weekly CHOP (R-CHOP-21). The high-priority question in elderly patients with aggressive lymphomas is the question if the combination of CHOP-14 and rituximab can further improve treatment results and will be answered by the ongoing DSHNHL 1999-1 (Ricover 60) trial. In young low-risk (normal LDH) patients, the DSHNHL achieved a significant improvement of treatment results by the addition of etoposide to the classical CHOP (CHOEP). Therefore, CHOEP-21 can be regarded as the new standard treatment for this patient population. The ongoing MINT trial with participants from 20 countries will show whether the addition of rituximab to CHOEP will results in further improvement of results. Alternatively, cytostatic drug doses of CHOEP can be escalated moderately by use of G-CSF (,HiCHOEP“), which will be compared to CHOEP-21 in the trial DSHNHL 1999-2. In young high-risk patients, the situation is problematic: because high-dose strategies failed to prove their superiority so far and the strategies which resulted in improvement of treatment results in the other patient subpopulations have not been tested in this group, it is hardly justifiable to treat these patients outside the setting of a prospective trial. While CHOP-21 is formally still the standard regimen in this group, CHOEP-14 could be justified for the rare young patient who does not qualify for such a trial due to the excellent tolerability of CHOEP-14 in young low-risk patients and the superiority of the 2-weekly CHOP in the elderly.

\section{5}

\section{Relapse after high dose therapy: Rescue or resignation?}

Schmitz N. (1), Glass B. (2)

\section{(1) AK St. Georg Hamburg, (2) Universitätsklinikum Göttingen}

High-dose therapy (HDT) followed by transplantation of autologous peripheral blood progenitor cells (PBPC) is curative for $20-80 \%$ of patients with relapsed aggressive Non-Hodgkin lymphoma (NHL) largely depending on the status of disease prior to transplantation. Patients who relapse after HDT have limited options. We were able to show that second transplants in patients with lymphoma can be successful. Five years after the 
second transplant $29 \%$ of patients who had received a second autograft and $34 \%$ of patients who had received an allograft for relapse after autografting were alive. The major causes of death for these patients were relapse after autografting and transplant-related toxicity after allografting. These data refer to patients who were treated between 1983 and 1999. Therefore, the role of rituximab which has been shown to be effective also in patients with relapse after autotransplantation and the role of allogeneic transplantion after reduced-intensity conditioning are not fully mirrored by these data. Because both modalities are effective and may reduce mortality in patients with relapsed aggressive NHL further improvement of survival rates after secondary transplants are expected. Further aggressive treatment of younger patients who relapse after autologous transplantation is warranted.

\section{6}

\section{GELA strategies for the treatment of aggressive lymphomas in} adult patients

Coiffier B.

Hospices Civils de Lyon, Pierre-Bénite, France

Aggressive lymphomas encompass diffuse large B-cell lymphomas (DLCL), systemic anaplastic large cell lymphomas (ALCL), and peripheral T-cell lymphomas (PTCL). Patients with Burkitt's lymphoma or lymphoblastic lymphoma are usually not included in this group of patients but treated with regimen used in pediatric patients (COP-COPADEM) or for acute lymphoblastic leukemia in adults. During the last 20 years, GELA has firstly included all these patients in the same protocol (LNH-84), and then divided them according to internal prognostic factors (LNH-87), then according to the International Prognostic Index (LNH-93), and recently according to diagnosis and prognostic factors (LNH-98). Currently the GELA is thinking about the next generation of studies (LNH-03), not yet completely defined. However, the conclusions of these previous studies will lead us for the design of this study. These conclusions and how they affect the design of the future LNH- 03 will be presented to the meeting, as well as the most primordial questions regarding these patients that should be answered The first line of conclusions regards the diagnosis and the necessity to divide patients into these 3 subgroups (DLCL, ALCL and PTCL) because of the efficacy of rituximab in B-cell lymphomas. This drug has recently demonstrated in broad activity for these patients, the fact that it decreases the importance of standard prognostic parameters leading us to simplify the decision tree for these patients. No monoclonal antibody is available for Tcell lymphomas but they may be divided between ALCL patients having a good outcome and PTCL patients having the worst outcome. We did not succeed to find a specific regimen with greater activity in PTCL patients but we will certainly continue to search. The second line of conclusions regards the greater efficacy of ACVB (our standard regimen since 1984) over CHOP in several settings. The next generation will combine ACVB with rituximab. The third line of conclusions was the inefficacy of radiation therapy to prolong the disease-free survival or decrease the relapse rate in these patients. Radiotherapy will have no place in the design of our future studies.

\section{Chronic Myeloid Leukemia}

\section{Bergmann, Frankfurt/M.; J. Drach, Wien}

\section{7}

\section{Molecular and chromosomal mechanisms of resistance to imatinib (STI571, Glivec ${ }^{\circledR}$ ) therapy}

Kreil S. (1), Müller M.C. (1), Hanfstein B. (1), La Rosée P. (2), Corbin A.S. (2), Lahaye T. (1), Schoch C. (3), Berger U. (1), Gschaidmeier H. (4), Hehlmann R. (1), Hochhaus A. (1)

(1) III. Medizinische Universitätsklinik, Fakultät für Klinische Medizin Mannheim der Universität Heidelberg, Mannheim, Germany, (2) Oregon Health \& Sciences University, Portland, OR, USA, (3) Medizinische Klinik III, Universität München, Germany, (4) Novartis Pharma Deutschland $\mathrm{GmbH}$

Selective inhibition of the BCR-ABL tyrosine kinase by imatinib is a promising therapeutic strategy in patients (pts) with chronic myelogenous leukemia (CML). Despite significant hematologic and cytogenetic responses, resistance occurs, particularly in pts with advanced disease. We sought to determine the underlying mechanisms. Sixty-six pts with CML in chronic phase $(n=13)$, accelerated phase $(n=16)$, myeloid blast crisis $(n=33)$, lymphoid blast crisis $(n=2)$ and ALL $(n=2)$, resistant to imatinib were investi- gated. Median treatment interval was 148 days (6-882). Pts were evaluated for genomic amplification of BCR-ABL, overexpression of BCR-ABL transcripts, clonal karyotypic evolution, and mutations of the ATP binding site of the BCR-ABL tyrosine kinase domain. Results: (i) Median levels of BCR-ABL transcripts were not significantly changed at the time of resistance but $6 / 55$ pts showed a $>10$-fold increase; (ii) genomic amplification of BCR-ABL was found in 2/32 pts evaluated by FISH; (iii) additional chromosomal aberrations were observed in 19/36 (52\%) pts, 13 being aneuploidy; (iv) heterogeneous point mutations of the tyrosine kinase domain or the activation loop resulting in reactivation of BCR-ABL were detected in 22/66 pts (33\%). Mutations comprised M244V (n=1), Q252H (n=1), Y253H $(n=3)$, Y253F $(n=1)$, E255K $(n=3)$, E255V $(n=1)$, T315I $(n=6)$, M351T $(n=4)$, E355G $(n=1)$, H396R $(n=1)$. ABL autophosphorylation assays demonstrated an increase of the IC50 for imatinib from $0.025 \mu \mathrm{M}$ for wildtype ABL to $1.8 \mu \mathrm{M}$ for $\mathrm{Y} 253 \mathrm{~F}, 3.7 \mu \mathrm{M}$ for $\mathrm{Y} 253 \mathrm{H}$, and $>5.0 \mu \mathrm{M}$ for E255K or V and T315I. Restriction analysis was used to confirm the mutation and to track it during the course of treatment. In three pts, the proportion of the mutated clone decreased after withdrawal of imatinib. Reactivation of BCR-ABL was confirmed by CRKL immunoblotting in nine pts with point mutations. Median proportion of phosphorylated CRKL was significantly higher in pts with mutations than in pts lacking mutations. Conclusions: Although the development of imatinib resistance is challenging, the fact that BCR-ABL is active in many resistant pts suggests that it remains a good drug target. However, pts with clonal evolution are more likely to have BCR-ABL independent mechanisms of resistance. Therapeutic decisions should be based on the individual cause of resistance. Early combination therapy is suggested in advanced disease to prevent clonal selection of resistant cells.

\section{8}

Interim analysis of treatment with imatinib in $\mathbf{3 0 0}$ patients with BCR-ABL positive leukemias: Evaluation of response and resistance

Lahaye T. (1), Riehm B. (1), Kreil S. (1), Berger U. (1), Müller M.C. (1), Merx K. (1), Paschka P. (1), König H. (1), Sellhorst A. (1), Schoch C. (2), Gschaidmeier H. (3), Hehlmann R. (1), Hochhaus A. (1)

(1) III. Medizinische Universitätsklinik, Fakultät für Klinische Medizin Mannheim der Universität Heidelberg, Mannheim; (2) Medizinische Klinik III, Klinikum Großhadern, Universität München; (3) Novartis Pharma GmbH, Nürnberg, Germany

Between 7/99 and 3/02, 300 patients (pts.) with BCR-ABL+ leukemias (171 $\mathrm{m}, 129 \mathrm{f}$, median age 60 , range $16-82$ years; late chronic phase, $\mathrm{CP}, \mathrm{n}=139$; accelerated phase, AP, $n=80$; myeloid blast crisis, $B C, n=76$, lymphoid $B C$ or BCR-ABL+ acute lymphoblastic leukemia, $n=5$ ) were treated with imatinib at a single center in cooperation with 118 referring institutions. Initial dosage of imatinib was either $400 \mathrm{mg}(\mathrm{n}=153)$ or $600 \mathrm{mg} /$ day $(\mathrm{n}=147)$. 134 pts. (96\%) in CP, $69(88 \%)$ in AP, and $35(49 \%)$ in BK and $2(40 \%)$ in $\mathrm{BCR}-\mathrm{ABL}+\mathrm{ALL}$ reached a hematologic response. Major cytogenetic response $(\mathrm{Ph}+\leq 35 \%)$ was observed in $63 \%$ of pts. in $\mathrm{CP}, 35 \%$ in $\mathrm{AP}$, and $13 \%$ in myeloid $\mathrm{BC}$; being complete in $48 \%$ of $\mathrm{CP}, 24 \%$ of $\mathrm{AP}$, and $8 \%$ of myeloid BC pts. Survival at 18 months was $98.5 \%$ in CP, $73.7 \%$ in AP, and $18.8 \%$ in myeloid BC. In 175 sample pairs from CP pts., cytogenetic response was compared to molecular response determined by quantitative RTPCR using the real time LightCycler technology. BCR-ABL transcript levels of complete, partial, and minor responders were significantly different. None of the patients showed a complete molecular remission considering an acceptable sample quality and analysis of 10-20 ml of peripheral blood. Residual disease in complete cytogenetic responders spans a range over three orders of magnitude. In myeloid BC, median survival after start of imatinib therapy was 5.9 months. Considering the interval between progress to blast crisis and start of treatment, median survival was 9.3 months and better than data published with conventional chemotherapy. Negative prognostic factors for survival in myeloid BC pts. were platelets $<100 / \mathrm{nl}$, a 2 nd $\mathrm{Ph}$-chromosome, or aberrations of $17 \mathrm{p} .17$ of 76 pts. $(22 \%)$ in myeloid BC were primarily resistant, $16(21 \%)$ relapsed under therapy with imatinib. In 16 of $80(20 \%)$ pts. in AP imatinib lost its efficacy. In CP 10 of $139(7.2 \%)$ pts. progressed. Clonal evolution with development of novel cytogenetic aberrations was seen in 19/36 (53\%)pts. at the time of resistance. A mutation of the tyrosine kinase domain was detected in 20/61 (33\%, 6 in CP, 7 in $\mathrm{AP}$ and 7 in $\mathrm{BC}$ ) relapsed pts. In conclusion, the efficacy of imatinib to treat CML pts. with interferon resistant $\mathrm{CP}$ and advanced disease was confirmed in this single center interim analysis. Despite high rates of cytogenetic responses, residual disease was never eradicated. Therapeutic decisions in relapsing patients should be based on the individual mechanism of resistance. 
Randomized comparison of Interferon/Hydroxyurea vs Hydroxyurea monotherapy in chronic myeloid leukemia (CML-Study II)

Metzgeroth G., Berger U., Maywald O., Hochhaus A., Reiter A., Engelich G., Pfirrmann M., Hasford J., Heinze B., Heimpel H., Hossfeld D.K., Kolb H.-J., Löffler H., Pralle H., Queißer W., Hehlmann R. and the German CML Study Group.

III. Medizinische Universitätsklinik, Fakultät für Klinische Medizin Mannheim der Universität Heidelberg, Mannheim

In contrast to CML-study I, which compared strictly interferon alpha (IFN) monotherapy with hydroxyurea (HU) or busulfan, several international studies allowed IFN to be given in combination with various chemotherapies and demonstrated a significant survival advantage for IFN compared to HU suggesting therapeutic superiority of the combination IFN plus HU. Therefore a randomized trial was initiated to compare the combination of IFN and HU vs HU monotherapy (CML-study II). From February 1991 through December 1994426 patients with newly diagnosed CML in chronic phase were recruited, 376 patients were randomized and 340 patients were $\mathrm{Ph} / \mathrm{BCR}-\mathrm{ABL}$ positive and evaluable. Randomization was unbalanced 1:2 in favor of the combination therapy since study conditions were identical to CML-study I and HU patients of study I $(n=194)$ could be used for the HU control group. In total, 534 patients were evaluable (226 patients with IFN/HU and 308 patients with HU monotherapy). Median observation time of living patients still at risk is 7.6 years $(7.9$ years for IFN/HU and 7.3 years for HU monotherapy). The risk profile (new score) is available for 532 patients: 200 patients (38\%) are low, 239 patients (45\%) intermediate and 93 patients $(17 \%)$ high risk. Most patients' criteria at diagnosis were distributed evenly. HU monotherapy patients revealed more weight $(\mathrm{p}=0.0353)$ and splenomegaly $(\mathrm{p}=0.0097) .104(19 \%)$ of the 534 patients underwent allogeneic stem cell transplantation in chronic phase. 65 patients $(64 \%)$ were low, $30(28 \%)$ intermediate and $9(8 \%)$ high risk. Median survival time is 5.3 years for IFN/HU and 4.4 years for HU monotherapy patients $(p=0.0063)$. Duration of chronic phase is 4.6 years for IFN/HU and 3.4 years for $\mathrm{HU}(\mathrm{p}<0.0001)$. Analysis of prognostic subgroups revealed a survival advantage of IFN/HU patients in the intermediate but not in the high risk group. Analysis of the low risk group is inconclusive due to the high percentage (total 33\%; 37\% of IFN/HU patients) of allogeneic transplantations in this group. We conclude that IFN in combination with $\mathrm{HU}$ achieves a significant long-term survival advantage over HU monotherapy.

\section{0}

\section{Establishment of an in vivo model of imatinib resistance}

Miething C., Mugler C., v. Bubnoff N., Hoepfl J., Grundler R., Peschel C., Duyster J.

\section{Med. Dept., Klinikum Rechts der Isar, Munich, Germany}

The development of imatinib (STI-571), a tyrosine kinase inhibitor specifically blocking the enzymatic activity of the causative oncogene Bcr-Abl, has a profound impact on treatment of CML and Bcr-Abl expressing ALL $(\mathrm{Ph}+\mathrm{ALL})$. Unfortunately, patients with blast crisis or with Bcr-Abl positive ALL relapse at a high rate in spite of initial good response to STI571 treatment. Point mutations found in the Abl kinase domain in patients resistant to imatinib have been shown to account for the treatment failure in some, but not all cases. We employed a murine retroviral infection/transplantation model of CML to investigate mechanisms of STI571 resistance in vivo. Bone marrow cells were retrovirally infected with Bcr-Abl and transplanted into lethally irradiated mice, which developed a CML-like syndrome with short latency. Subsequently, we have serially transplanted spleen cells from a mouse with a CML-like syndrome to nine sublethally irradiated mice, which also developed a similar CML like disease within 4-6 weeks. With rising leukocyte counts, STI571 treatment was initiated with an oral application of $0.5 \mathrm{mg}$ STI571 twice a day. The mice responded initially well to the treatment, but all of them relapsed within 6-10 weeks. As determined by FACS and cytologic examination, 7 out of 9 mice relapsed with ALL, whereas two mice progressed with rising neutrophil counts in the peripheral blood. Automatic sequencing of the Bcr-Abl kinase domain from 6 of the 9 mice on a genomic level did not show specific mutations. SouthernBlot analysis gave evidence of clonal evolution. Interestingly, at least in three mice with B-ALL the same clone had contributed to development of STI571 resistant disease. Analysis of resistant leukemic cells in-vitro by anti-phosphotyrosine western-blot demonstrated that Bcr-Abl is still inhibited by STI571. Thus, further genetic events may have contributed to resistance, and we are currently performing cDNA arrays to pinpoint those alterations. In addition we are sequencing the DNA flanking the retroviral insertion sites, as random integration of the retrovirus and subsequent genetic changes may have contributed to the resistant phenotype. In conclusion, the mouse model of CML represents an valuable tool in the investigation of molecular mechanisms of STI571 resistance and will help to identify new treatment combinations for STI571 resistant CML or Ph+ ALL.

\section{1}

\section{Imatinib (STI571) pharmacokinetics in patients with CML}

Schleyer E. (1), Ottmann O.-G. (2), Leopold T. (1), Pursche S. (1), Berndt A. (1), Ehninger G. (1), Kreuzer K.-A. (3), le Coutre P. (3)

(1) Universitätsklinik Carl Gustav Carus Dresden, (2) Klinikum der Wolfgang Goethe Universität Frankfurt, (3) Humboldt Universität Berlin, Charite

The 2-phenylaminopyrimidine derivative STI571 (imatinib) is a selective inhibitor of the tyrosine kinase domain of the oncogenic bcr/abl fusion protein and has prooven efficacy in the treatment of chronic myeloid leukemia (CML) and bcr/abl acute lymphatic leukemia (ALL). Despite its broad clinical use, pharmacokinetic data are limited. We here report the pharmacokinetics of STI571 investigated in 32 patients with CML. From 32 patients analysed, 16 received $400 \mathrm{mg}$ STI571 o.d. (once daily) and another 16 patients $600 \mathrm{mg}$ o.d., respectively. In 6 patients with 400 and 6 patients with $600 \mathrm{mg}$ STI571 o.d. serum samples were drawn over a period of at least 24 hours up to 120 hours after the initial intake of STI571, while in another 20 patients samples were available from the first treatment day. Moreover, we analysed STI571 concentrations in the cerebrospinal fluid (CSF) parallel to serum in 3 patients and could approximate the liquor/serum ratio of STI571, as well as the $24 \mathrm{~h}$ urine elimination of STI571 and its main metabolite, the $\mathrm{N}$-desmethyl derivative, in another 3 patients.

\begin{tabular}{llllllll}
\hline dose & $\begin{array}{l}\mathrm{t} 1 / 2 \text { terminal } \\
\mathrm{h}\end{array}$ & $\begin{array}{l}\mathrm{t} 1 / 2 \text { absorption } \\
\mathrm{h}\end{array}$ & $\begin{array}{l}\mathrm{AUC} \\
\mu \mathrm{g} * \mathrm{ml} / \mathrm{h}\end{array}$ & $\begin{array}{l}\mathrm{Cmax} \\
\mu \mathrm{g} / \mathrm{ml}\end{array}$ & $\begin{array}{l}\mathrm{tmax} \\
\mathrm{h}\end{array}$ & $\mathrm{Vss} / \mathrm{s} \mathrm{l}$ & $\begin{array}{l}\text { Clear.total } \\
\mathrm{ml} / \mathrm{min}\end{array}$ \\
\hline $\begin{array}{l}600 \mathrm{mg} \\
(\mathrm{vc})\end{array}$ & $\begin{array}{l}16.9 \\
(35 \%)\end{array}$ & $\begin{array}{l}1.58 \\
(90 \%)\end{array}$ & $\begin{array}{l}85.9 \\
(66 \%)\end{array}$ & $\begin{array}{l}6.76 \\
(69 \%)\end{array}$ & $\begin{array}{l}3.84 \\
(55 \%)\end{array}$ & $\begin{array}{l}133 \\
(78 \%)\end{array}$ & $\begin{array}{l}147 \\
(53 \%)\end{array}$ \\
$\begin{array}{l}400 \mathrm{mg} \\
(\mathrm{vc})\end{array}$ & $\begin{array}{l}16.6 \\
(19 \%)\end{array}$ & $\begin{array}{l}2.45 \\
(47 \%)\end{array}$ & $\begin{array}{l}37.5 \\
(25 \%)\end{array}$ & $\begin{array}{l}2.02 \\
(32 \%)\end{array}$ & $\begin{array}{l}4.07 \\
(37 \%)\end{array}$ & $\begin{array}{l}160 \\
(51 \%)\end{array}$ & $\begin{array}{l}187 \\
(25 \%)\end{array}$ \\
\hline
\end{tabular}

Table 1 depicts the pharmacokinetic parameters calculated from the serum concentrations using a 2 compartment model fitting algorithm. A significant variability was found for the AUC that ranged from 34.7 to $197.0 \mu \mathrm{g} \cdot \mathrm{h} / \mathrm{ml}$ for the 600 and from 25.8 to $51.8 \mu \mathrm{g} \cdot \mathrm{h} / \mathrm{ml}$ for the $400 \mathrm{mg}$ STI571/day group, while the Cmax was distributed from 2.1 to $15.6 \mu \mathrm{g} / \mathrm{ml}$ and 1.2 to $2.8 \mu \mathrm{g} / \mathrm{ml}$, respectively. The over-proportional AUC and Cmax for the $600 \mathrm{mg}$ group resulted from one patient, in whom the individual STI571 concentration far exceeded the mean STI571 serum concentrations with a Cmax of $15.6 \mu \mathrm{g} / \mathrm{m}$ and an AUC of $197 \mu \mathrm{g} \cdot \mathrm{h} / \mathrm{ml}$. In 3 patients with bcr/abl positive ALL we found an average of $3.8 \%$ CSF concentration $(27.6-79.8 \mathrm{ng} / \mathrm{ml})$ related to the serum STI571 level. The $24 \mathrm{~h}$ urine elimination of STI571 and its main metabolite was $7.6 \%(4.1 \%$ to $12 \%)$ of the administered dose, while the $\mathrm{N}$ desmethyl derivative/STI571 ratio shows a value of $32 \%$ in urine (14\% to $50 \%$ ) and of $16 \%$ (10 to $21 \%$ ) in patient serum. In conclusion, our data demonstrates considerable interindividual variability of STI571 pharmacokinetics.

Further pharmacokinetic studies are ongoing and will investigate the impact of STI571 levels on outcome and toxicity, the interindividual variabillity of metabolism, the influence of cytochrom P450 heterogeneity, the cellular concentrations and uptake as well as a possible activity of the $\mathrm{N}$-desmethyl metabolite.

\section{2}

Dynamics of lineage-restricted mixed chimerism following sexmismatched allogeneic bone marrow transplantation in CML

Thiele J. (1), Wickenhauser C. (1), Kvasnicka H.M. (1), Varus E. (1), Beelen D.W. (2), Schaefer U.W. (2)

(1) Institute of Pathology, University of Cologne, Cologne, (2) Department of Bone Marrow Transplantation, University of Essen, Essen, Germany

Objectives: Scant knowledge is available about the quantity and dynamics of lineage-specific mixed chimerism $(\mathrm{mCh})$ of hematopoietic and endothelial cells following bone marrow transplantation (BMT).

Material and Methods: Bone marrow (BM) trephine biopsies were studied in 12 patients with $\mathrm{Ph}^{1+}$ chronic myeloid leukemia (CML) after BMT including a sex-mismatched host/donor constellation. Methods of processing involved dual color fluorescence in situ hybridization (FISH) with $\mathrm{x}$ - and $\mathrm{y}$ chromosome-specific DNA-probes and proper detection systems for a si- 
multaneous determination of the bcr/abl locus. These techniques were applied after previous immunophenotyping by monoclonal antibodies (CD34 CD61, anti-glycophorin C, CD68) to identify the various cell lineages.

Results: In the $\mathrm{CD} 34^{+}$progenitor population a significant degree of $\mathrm{mCh}$ ranging between 22 and $26 \%$ remaining host cells was found in the early and late (up to 200 days after BMT) posttransplant period. However, only $10 \%$ of these cells harbored the bcr/abl translocation gene. This result fits well with corresponding molecularbiological findings (minimal residual disease). Conversion of $\mathrm{mCh}$ occurred during leukemic relapse with more than $90 \%$ host-derived precursors of which $50 \%$ revealed the bcr/abl locus. This feature implies that a considerable amount of $\mathrm{CD}_{3} 4^{+}$cells are nonneoplastic in nature. A mCh was expressed to a significantly lower degree than in the progenitor cell pool in the compartment of nucleated erythroid percursors $(5 \%)$ and $\mathrm{CD}^{+} 8^{+}$macrophages $(8 \%)$. Compared to these data the slightly increased frequency $(16 \%)$ found in $\mathrm{CD} 61^{+}$megakaryopoiesis was probably due to the polyploid state of these cells. Abrupt changes from donor to host type cells was significantly associated with a transformation into recurrent leukemia. The $\mathrm{CD} 34^{+}$endothelial cells showed a minor degree of $\mathrm{mCh}$, because donor-derived elements ranged from only $18 \%$ to 25 $\%$ during the early and late posttransplant period. In keeping with the othe hematopoietic cell lineages leukemic relapse was characterized by an almost complete conversion of the endothelial cells to a host type.

Conclusions: In conclusion, our findings support the assumption of a CD34 progenitor cell origin of the endothelial cell layer and that conversion of $\mathrm{mCh}$ is indicating a leukemic relapse.

\section{3}

\section{Differential sensitivity of bcr-abl gene mutations responsible for clinical resistance towards glivec (STI571) and alternate kinase inhibitors}

von Bubnoff N. (1), Gschaidmeier H. (2), Schneller F. (1), Peschel Ch. (1), Duyster J. (1)

(1) III. Medizinische Klinik und Poliklinik, Klinikum rechts der Isar der Technischen Universität München, Germany, (2) Clinical Research and Development, Novartis Pharma GmbH, Nürnberg, Germany

STI571 (Glivec, Novartis, Basel, Switzerland), a competitive inhibitor at the ATP-binding site of Bcr-Abl has been shown to be capable of very rapidly reversing the clinical and hematological abnormalities of Philadelphia chromosome-positive $(\mathrm{Ph}+)$ leukemia. While chronic phase $\mathrm{CML}$ patient durably respond, CML blast crisis and $\mathrm{Ph}+\mathrm{ALL}$ patients relapse within weeks to months of therapy. As we have shown previously, different mutations of Bcr-Abl can be responsible for molecular-based resistance of $\mathrm{Ph}+$ leukaemia to glivec. To date, we have analysed 12 consecutive patients with $\mathrm{Ph}+$ leukaemia and acquired resistance towards glivec. We were able to detect mutated Bcr-Abl in ten out of twelve patients only in samples derived at the time of resistant disease. Looking at the frequency of mutations in cases of $\mathrm{Ph}+$ leukaemia resistant to glivec, mutated Bcr-Abl seems to be the causative abnormality for the majority of patients with lymphoid disease, whereas in myeloid disease, mutations can be found two to four times less frequently. We found $\mathrm{Y} 253 \mathrm{H}$ in three cases, $\mathrm{E} 255 \mathrm{~K}$ in two, $\mathrm{E} 255 \mathrm{~V}$ in one, $\mathrm{T} 315 \mathrm{I}$ in three, and $\mathrm{H} 396 \mathrm{P}$ in one case(s). All point mutations conferred resistance to glivec in vitro, resulting in IC50 values that were spanning a range of $2 \mu \mathrm{M}(\mathrm{H} 396 \mathrm{P})$ to $>10 \mu \mathrm{M}$ (Y253H and T315I; IC50 of wild-type: $0,2 \mu \mathrm{M})$. Positions Y253, E255 and T315 are located within the ATP-binding site of Bcr-Abl, where a conformational change at critical positions results in steric clash with the drug. H396 is positioned within the activation loop, distant from regions directly interacting with the compound. This region of $\mathrm{Abl}$ has to be in a closed (inactive) conformation to allow binding of glivec to occur. Therefore, we propose a model where H396P leads to a stabilization of the activation loop in the open (active) conformation. To overcome resistance due to mutated $\mathrm{Bcr}-\mathrm{Abl}$, we are currently testing alternate compounds for inhibition of mutated Bcr-Abl, assuming that: 1. positions critical for binding may not be the same for different compounds, 2. inhibition may be achieved using compounds that bind at distinct sites (e.g. tyrphostin-type inhibitors) and, 3. unlike imatinib, an alternate compound may be capable of binding Bcr-Abl (especially H396P) regardless of the conformation of the activation loop.

\section{Case Reports I}

R. Fuchs; Eschweiler; F. Schmalzl, Hall in Tirol

\section{4}

Paralytic ileus due to generalized varicellosis showing dermal, hepatic and bone marrow alterations in multiple myeloma after autotransplantation

Henrich D., Dingeldein G., Weiss B., Hoffmann M., Uppenkamp M.

Klinikum Ludwigshafen/Rhein $\mathrm{gGmbH}$

We report on a 52-year-old lady with multiple myeloma type IgG kappa stadium IIIa (Salmon and Durie), which was diagnosed in May 2001, and who was treated with double highdose chemotherapy in September 2001 and January 2002. She was conferred to our hospital because of heavy abdominal pain mainly in the epigastric region persisting for seven days in February 2002.

Peptic ulcers could be excluded by gastroscopy. In the proximal duodenal parts a relevant amount of greenish fluid could be seen, a possible sign for distal intestinal obstruction. To secure the diagnosis of an ileus we performed abdominal radiographs. After 3 days significant radiologic signs of an ileus arose. Apart from a massive colonic dilatation we could neither find any intestinal obstruction in small intestinal and colonic radiography as sign of a mechanical ileus nor we could detect mesenterial ischemia of the superior or inferior mesenteric artery in an angiography. Finally, we diagnosed a paralytic ileus of unknown origin. As the patient developed temperatures up to $39.7^{\circ} \mathrm{Celsius}$ and a typical exanthema of a varicella infection a few days later, generalized varicellosis was the probable cause of the patient's condition. Serological tests revealed an elevated titer of specific IgM, which proved our hypothesis. In our laboratory workup we saw elevated levels of alcaline phosphatase, $\gamma$-guadenosine triphosphate, total bilirubine and the transaminases tantamount to hepatic coreaction in generalized varicellosis. The total blood count showed transient pancytopenia due to impaired haematopoesis by the viral infection. The patient was started on intravenously aciclovir and immunoglobulin replacement therapy, which proved successful quickly.

In the literature there are only few reports on paralytic ileus as leading symptom of a generalized varicellosis in immunocompromized patients. As far as we know, this is one of the first reports on generalized varicellosis in a patient pretreated with highdose chemotherapy and autologous stem cell transplantation.

\section{5}

Successful treatment of a patient with hairy cell leukemia and autoimmune thrombocytopenia with rituximab

Hensel M., Ho A.D.

Medizinische Klinik V, Universität Heidelberg

In this report, we describe the first successful treatment with rituximab in a patient with autoimmune thrombocytopenia associated with HCL and pentostatin treatment. This association is extremely rare, and therapeutic options in this heavily pretreated patients have been very poor. Both in patients with refractory hairy cell leukemia and patients with autoimmune thrombocytopenia, there is first evidence of promising activity of the anti-CD20 monoclonal antibody rituximab.

In 1974, a 45-year old man was diagnosed with HCL. A long lasting complete remission was achieved with initial splenectomy. A first relapse occurred in 1996, which was successfully treated with 6 cycles of pentostatin. He was in CR until August 2000, as he developed a second relapse which was again successfully treated with pentostatin. One month after the fifth administration of pentostatin, the diagnosis of immune mediated thrombocytopenia was established and the patient was treated with corticosteroids. Even with high doses of prednisone and later with intravenous immunoglobulin, a sustained elevation of platelets could not be reached. With a combined therapy of corticosteroids, vincristin and oral cyclophosphamide, platelets stabilized for some months. Cyclophosphamide and prednisone were slowly tapered and finally discontinued in September 2001. At that time, the patient had a normal peripheral blood count. Five months later, platelets decreased again to $61 / \mathrm{nl}$ with normal leukocytecount, hemoglobin-level and without atypical lymphocytes in the peripheral blood. We decided to treat him with rituximab in the conventional dose of $375 \mathrm{mg} / \mathrm{m}^{2}$ i.v. weekly for 4 cycles. One week after the first administration of rituximab, the peripheral blood count has recovered with a platelet count 
well into the normal range. 4 months after the fourth rituximab infusion, the patient is still in complete hematologic remission without further medication.

In conclusion, rituximab seems to be a new, promising drug in the treatment of refractory autoimmune thrombocytopenia even in patients with underlying lymphoproliferative disorders.

\section{6}

Successful salvage high dose chemotherapy (HDCT) with autologous stem cell transplantation (ASCT) in a patient with five relapses of Hodgkin's disease

Hentrich M., Brack N., Pohlmann H., Schmid P., Lutz. L., Hartenstein R.

Städtisches Krankenhaus München-Harlaching, IV. Medizinische Abteilung

Objectives: HDCT with ASCT has been frequently used in patients with refractory or relapsed Hodgkin's disease (HD). However, data on HDCT in pts with more than 3 relapses prior to transplantation are scarce.

Methods: We report on a patient with a 5th relapse of HD successfully salvaged by HDCT and ASCT. The available literature on this topic will be reviewed.

Case Report: In 1982 a stage IIIB mixed cellularity (MC) type HD had been diagnosed in a 27 years-old man. After administering 6 courses of CVPP CR was achieved. A 1st relapse involving left axillary lymph nodes occured in 1984 and was treated by mantle- and inverted Y-field radiotherapy which resulted in a 2nd CR. However, in 10/88 a 2nd relapse with enlarged hilar lymph nodes was diagnosed. 2 courses of COPP-ABV-IMEP were applied leading to a 3rd CR. 17 months later a 3rd relapse involving cervival lymph nodes and the right tonsil was histologically confirmed. The patient received 2 courses Dexa-BEAM and achieved a 4th CR which lasted for 7 months. At that time he presented with enlarged cervical lymph nodes and recurren involvement of the right tonsil. Tonsilectomy and a surgical excixion of the lymph nodes were performed. Submandibular and cervical lymph nodes increased in size again and were excised in 11/91 revealing both, a lymphocyte predominant HD (submandibular region) and a nodular sclerosis type HD located cervically. Involved field radiotherapy to the cervical and supraclavicular region $(40 \mathrm{~Gy})$ was performed. The patient achieved a 5 th $\mathrm{CR}$ lasting for 58 months. In 11/96 CT-scans revealed enlarged mediastinal lymph nodes but a mediastinoscopy failed to demonstrate malignancy. However, in 2/97 an extensive 5th relapse of MC-type HD was confirmed by excision of lymph nodes located at the left upper tigh. Lymphadenopathy at multiple sites, pulmonary infiltrates, bone marrow infiltration and destruction of 2 ribs were found on further examination. The patient was included in a HDCT program. 4 courses of IEV (ifosfamide/epirubicin/etoposide) followed by G-CSF and PBSC collections resulted in a PR. HDCT consisted of busulfan $16 \mathrm{mg} / \mathrm{kg}$ and cyclophosphamide $120 \mathrm{mg} / \mathrm{kg}$ followed by ASCT $(6.8 \times 106 / \mathrm{kg} \mathrm{CD} 34+$ cells $)$. Engraftment occured on day +9 . The patient achieved a complete remission and has remained well without evidence of recurrent disease 57 months after PBSCT.

Conclusions: This rare case underlines that even in multiple relapsed HD durable remissions may be obtained after HDCT and ASCT.

27

\section{Acute myelofibrosis in a patient with diffuse large cell lym-} phoma and renal cancer

Mohren M. (1), Essbach U. (2), Franke A. (1), Klink A. (1), Maas C. (1), Markmann I. (1), Scheinpflug K. (1), Jentsch-Ullrich K. (1)

(1) Klinik für Hämatologie/Onkologie und (2) Institut für Pathologie, Universität Magdeburg, Germany

Introduction: Aggressive Non Hodgkin's Lymphoma (NHL) can be successfully treated with standard chemotherapy including alkylating agents and anthracyclines. Relapse is frequent whereas complications such as secondary malignancies rarely occur. Acute myelofibrosis is a rare form of AML that may arise de novo or after treatment with alkylating agents or topoisomerase II inhibitors usually 2-5 years after exposure. We report on a patient with NHL and concurrent renal cancer who developed acute myelofibrosis (AMF) 2 months after completion of chemotherapy.

Patient: A 60 year old female patient presented with pancytopenia and a right sided 4,5 cm diameter renal mass. Diagnostic work up revealed diffuse large cell lymphoma with severe bone marrow infiltration and renal cancer T1N0M0G2. There was no evidence of further lymphoma manifestations and/or solid tumor metastasis. Cytogenetic and molecular bone marrow evaluation showed metaphases with a normal karyotype as well as a second clone with a ringed chromosome 7 and a third clone with an enlarged chromosome 2 with additional material not resulting from reciprocal translocation and 1-2 markers.

Clinical course: The patient initially underwent nephrectomy and then received 6 courses of CHOP 14. Anemia persisted, although there was no further need for transfusions. Bone marrow biopsy after $3 \times \mathrm{CHOP}$ showed complete remission. 2 months after completion of therapy the patient again presented with pancytopenia. Repeat bone marrow biopsies showed abscence of lymphoma or solid tumor, but massive fibrosis with an increased number of atypical megakaryocytes consistent with acute myelofibrosis Because of the dismal prognosis of this disease entity therapy was limited to palliative supportive care. The patient died 3 months later due to pulmonary infection.

Conclusion: After resolution of lymphoma in response to multiagen chemotherapy and in the abscence of renal cancer following nephrectomy our patient developed acute myelofibrosis. Considering the early manifestation of AMF, the complex cytogenetic aberrations found in our patient's bone marrow cells at initial diagnosis and the coincidence of lymphoma and renal cancer, AMF in this patient rather accounts for a third malignancy and reflects her individual increased susceptibility to malignant disease.

\section{8}

Transfusion-associated graft-versus-host disease (TA-GVHD) after fludarabine treatment in a 72-year-old patient with follicular lymphoma

Schabath R., Busemann C., Schuler E., Jost D., Ludwig W.D.

HELIOS Klinikum Berlin, Robert-Rössle-Klinik, Charité, CAMPUS Berlin-Buch, Medizinische Klinik für Hämatologie, Onkologie und Tumorimmunologie

TA-GVHD is a rare immunological complication resulting from engraftment of donor lymphocytes in the recipient after transfusion of not irradiated blood products. Patients at highest risk include premature infants and patients who are immunosuppressed as an effect of either immunodeficiency disease or immunosuppressive therapy. An additional high risk group represent immunocompetent recipients who are heterozygous at a particular HLA-locus and who received blood from a donor who is homozygous at the same locus. We report on a 72-year-old female patient with follicular nonHodgkin's lymphoma (NHL), first diagnosed in 1984. During the last 12 years, multiple chemotherapeutic protocols were administered. The lates included 5 cycles of rituximab from 10-11/00, 2 cycles of fludarabine $\left(25 \mathrm{mg} / \mathrm{m}^{2}\right)$ given $3-4 / 01$ without response. In May 2001, we started with a regimen consisting of chlorambucil (15mg, day $1-4)$, mitoxantron $\left(8 \mathrm{mg} / \mathrm{m}^{2}\right.$, day 1) and prednisolon $\left(25 \mathrm{mg} / \mathrm{m}^{2}\right.$, day $\left.1-5\right)$. The patient received a blood transfusion with two leukocyte-depleted, but not irradiated, units of packed red cells. A second cycle of chemotherapy was administered in 6/01. Four weeks after transfusion, the patient developed an erythematous maculopapular rash originating on the trunk and a conjunctivitis. Laboratory features showed a liver involvement. A skin biopsy showed morphological features of acute GVHD (degree 3). The chimerism analysis in the $\mathrm{pB}$ demonstrated a donor CD4+ T-cell population in a relation of 7:1 to the CD4+ Tcell population of the host. At the same time we could observe a partial remission of the intestinal manifestations of the lymphoma, possibly not only induced by chemotherapy but as a consequence of a graft-versus-lymphoma effect. The GVHD was treated with prednisolon beginning with $100 \mathrm{mg}$ p.o.. The dosis was reduced stepwise over a period of 6 months. One year after occurrence of TA-GVHD, the patient is alive and withou symptoms of GVHD. However follow-up investigations revealed progressive follicular lymphoma. Discussion: TA-GVHD ist prevented by inactivating immunologically competent cells using gamma irradiation. Guidelines exist for the use of irradiated blood products for specific indications, but so far there are no strong recommodationes for NHL-patients or patients treated with purine analogs. We propose that NHL-patients treated with purine analogs shall be considered as being at high risk of developing TA-GVHD.

\section{9}

Hepatits C virus-associated mixed cryoglobulinemia in a $47 \mathrm{y} / \mathrm{o}$ patient, initially treated with chemotherapy for non-hodgkin's lymphoma - report of a case and review of the literature

Staak J.O., Diehl V., Josting A.

\section{UNI-Klinik Köln, Klinik I für Innere Medizin}

Objectives: Chronic hepatitis $\mathrm{C}$ virus (HCV)-infection is frequently associated with a variety of autoimmune phenomenons. Mixed cryoglobulinemia (EMC) appears in up to $50 \%$ of chronic HCV-infected patients. Cryoglobu- 
lins consist of immunoglobulin complexes precipitating in vitro when cooled to low temperatures. Cryoprecipitates are deposited in small vessels. Resulting vasculitis is responsible for clinical symptoms such as weakness, arthralgia and purpura. Complications are glomerulonephritis and neuropathic lesions. Several mechanisms for HCV-induced clonal lymphoproliferation evolving into B-cell non-Hodgkin's lymphoma (NHL) are discussed. Treatment of $\mathrm{HCV}$-associated EMC aimes at reducing circulating immunocomplexes and causal therapy with interferon (IFN) and ribavirin.

Results: We report on a 47-year-old male patient (pt) who was initally apparent in 12/00 with small spotted exanthema of the lower legs with paresthesia rising in phases. Histology revealed leukocytoclastic vasculitis. Other findings were paraproteinemia (IgM kappa) and small cell lymphoid infiltration of bone marrow (10-20\%). Pt was then treated with six cycles CHOP chemotherapy for stage IV immunocytoma. After a short period of recovery symptoms worsened again and the pt was admitted to the University hospital Cologne for relapse therapy. Clinical findings: weakness, bilateral vasculitis of lower legs, peripheral neuropathy, arthralgia, bilateral pleural effusions, ascites and splenomegaly; laboratory: mildly elevated liver function tests and CRP; virology: chronic active hepatitis C; liver biopsy: grade 3 fibrosis with mild inflammatory activity; bone marrow: reactive changes; immunology: high EMC titer with monoclonal IgM and polyclonal IgG. The pt received plasmapheresis and treatment with PEG-IFN and ribavirin for HCV-associated EMC. After 4 months of therapy clinical symptoms almost resolved with decreasing paraproteinemia and ongoing clearance of HCVRNA.

Conclusions: EMC is a frequent finding in HCV-infected pts. It is associated with clonal B-cell proliferation and may evolve into NHL since there prevalence of frank NHL in 5-10\% of HCV positive EMC pts. Mechanisms of malignant transformation are still unknown. B-cells from pts with EMC and HCV strongly express the bcl-2 oncogene product. Despite initial evidence of lymphoproliferation in the pts' bone marrow NHL was not confirmed, without evidence of bcl-2. If overt NHL might still develop in this $p$ remains to be evaluated.

\section{0}

\section{Successful treatment of systemic lupus erythematosus with rituximab}

Weide R., Heymanns J., Pandorf A., Köppler H.

\section{Oncology group practice, Koblenz}

Background: SLE is a chronic, inflammatory autoimmune disease which may involve multiple organ systems. Treatment consists of immunosuppression, cytotoxic treatment, plasmapheresis and immunoglobuline therapy. Treatment of patients refractory to standard treatment approaches is difficult and results are poor.

Methods: We describe a 39-year old patient with SLE suffering from grand mal epilepsia due to cerebral vasculopathy with positive lupus anticoagulant, who was refractory to standard treatment modalities. The patient was treated with the anti-CD20 monoclonal antibody rituximab $\left(375 \mathrm{mg} / \mathrm{m}^{2} \times 4\right.$, repeated in weekly intervalls). Rituximab-application were delivered in $10 / 00,03 / 01$ and 10/01. Since $03 / 02$ she receives maintenance therapy with rituximab $375 \mathrm{mg} / \mathrm{m}^{2}$ every 3 months.

Results: The patient showed a major improvement of her clinical condition, 20 months after the beginning of the rituximab therapy she is free of any symptoms. Inflammation parameters, ANA and lupus anticougulant declined significantly after the treatment.

Conclusion: Rituximab may be a new effective therapy in SLE.

\section{Oral Session}

\section{Stem Cell Transplantation}

J. Finke, Freiburg; D. Beelen, Essen

31

Impact of high resolution HLA-A,B,C donor-recipient matching on clinical outcome of allogeneic hematopoietic stem cell transplantation (hsct) from unrelated donors

Ferencik S. (1), Ottinger H.D. (2), Beelen DW. (2), Kremens B. (3), Shaefer U.W. (2), Grosse-Wilde H. (1)

(1) Institute of Immunology, (2) Department of Bone Marrow Transplantation, (3) Department of Pediatrics, University Hospital Of Essen, Germany

At our institution unrelated donors for HSCT are routinely matched by DNA-based PCR-SSP typing at low resolution (,2 digit level') for HLAA,B,C and high resolution (,4 digit level') for HLA-DRB1,DQB1 with the respective patients. To answer the question, whether HSCT outcome migh be improved by high resolution HLA-A,B,C, i.e. sequence based typing (SBT), a retrospective single center study was performed. Enrolled were 227 adult unrelated transplants for early $(\mathrm{N}=118)$ and advanced $(\mathrm{N}=109)$ hematological malignancies. In all study patients the conditioning regimen used and the protocol for prophylaxis of graft-versus-host disease (GvHD) were identical and all enrolled donor/recipient pairs were fully HLAmatched according to the routine outlined above. For the purpose of this study, HLA-A,B,C SBT was performed in all donor/recipient pairs and revealed only $73(32.2 \%)$ of the 227 transplants to be identical at the allelic level. In contrast, $36.6 \%, 20.7 \%, 6.6 \%$, and $3.1 \%, 0.4 \%$ and $0.4 \%$ of the transplants exhibited $1,2,3,4,5$ or even 6 HLA-A,B,C allele mismatches in $\mathrm{GvH}$-direction respectively. Uni- and multivariate statistical analysis showed overall survival after HSCT to be comparable from HLA-A,B,Cidentical $(\mathrm{N}=73)$ and partially HLA-mismatched donors exhibiting 1 through 6 HLA-class I allele disparities $(\mathrm{N}=154)$. The cumulative risk of clinically relevant GvHD was $50+/-8 \%$ in the HLA-identical and $56+/-5$ $\%$ in the HLA class I allele-mismatched subgroup ( $p>0,05)$. The cumulative risk of graft lost after transplants from fully HLA-matched and HLA class I allele mismatched donors was $3.2 \%(2 / 73) \%$ and $4.1 \%(6 / 154)$, respectively $(p>0,05)$. Thus, if present at all, the beneficial effect of prospective HLA-A,B,C SBT typing for donor selection seems to be modest, since it could not be demonstrated in our retrospective approach enrolling 227 well selected transplants.

This study was supported in part by a grant of the Deutsche José Carreras Leukämie-Stiftung e.V., Munich, project no. R12.

\section{2}

Secondary hematopoietic failure is a frequent complication of allogeneic stem cell transplantation

Holler E. (1), Jobst K. (1), Hahn J. (1), Erdmann A. (1), Dada A. (2), Ullrich $H$. (2), Poetscher Ch. (1), Wittnebel S. (1), Meidenbauer N. (1), Klouche M. (2), Reichle A. (1), Schmitz G. (2), Andreesen R. (1)

(1) Abteilung Hämatologie/Intern.Onkologie und (2) Institut f.Klin.Chemie und Laboratoriumsmedizin des Klinikums der Universität, 93042 Regensburg

Objectives: Studies analyzing the effects of different stem cell sources in allogeneic transplantation frequently address parameters such as primary engraftment and GvHD. Little information, however, is available, on the incidence and prognostic signifance of secondary hematopoietic failure (SHF). Material and Methods: All consecutive patients (pts) receiving allogeneic stem cell grafts and surviving at least 3 months (=76 out of $106 \mathrm{pts}$ ) were analyzed for occurrence of SHF defined as a decrease of neutrophils (ANC) to $<0.5$ or $<1.0 / \mathrm{nl}$ or platelets (plts) to $<20 / \mathrm{nl}$ in the first 6 months following transplantation. Stem cell source, CD34 dose, donor type, CMV status, myelo-suppressive viral infections or drugs such as virostatics or MMF and acute or chronic GvHD as well as severe microangiopathy were evaluated as possible risk factors.

Results: Overall, SHF defined as ANC $<1.0$ or plts $<20$ was observed in $13 / 26(50 \%)$ of pts receiving bone marrow, in $21 / 37(57 \%)$ of pts receiving unselected peripheral blood stem cells (PBSC) and in 6/13 (46\%) of pts receiving CD34 selected stem cell grafts. Median time to secondary neutropenia was 125 d's after transplant, and median time to secondary drops in plt counts was 60 d's. Severe secondary neutropenia defined as ANC $<0.5$ was not observed in marrow grafted pts but occured in $22 \%$ of pts transplanted 
with either source of PBSC. When expected risk factors were analyzed, use of virostatic drugs was of marginal significance (64\% vs $40 \%$ SHF, p 0.06 ). The strongest associations, however, were observed for presence of acute and chronic GvHD as well as severe microangiopathy: Severe SHF occured in a low number of pts in the absence of GvHD (33\% in marrow, $17 \%$ in PBSC and 20\% in CD34selected grafts) but increased dramatically in those with GvHD grade III or IV (67\% after SCT using marrow, 100\% in pts receiving either source of PBSC). In multivariate analyis, severe acute and chronic GvHD remained the only significant risk factors. Transplant related mortality was significantly increased in pts with SHF.

Conclusions: Our data show a high incidence of SHF as a complication following allogeneic SCT. Severe acute and chronic GvHD were the main risk factors, especially in pts receiving peripheral blood stem cell grafts. Our data suggest direct myelosuppressive effects of GvHD, and better understanding of these interactions may help to improve outcome by avoiding consecutive infectious complications in GvHD pts

\section{3}

Outcome of allogeneic hematopoietic stem cell transplantation in patients with ALL - a retrospective multicenter analysis

Kiehl M.G. (1), Kraut L. (1), Remberger M. (2), Schwerdtfeger R. (3), Kroeger N. (4), Bornhaeuser M. (5), Martin H. (6), Scheid C. (7), Fauser A.A. (1), Zander A. (4), Ehninger G. (5), Hoelzer D. (6), Diehl V. (7), Ringden $O$. (2)

(1) KMT Zentrum Idar-Oberstein, (2) Uni-Klinik Huddinge, (3) DKD Wiesbaden, (4) Uni-Hamburg, (5) Uni-Dresden, (6) Uni-Frankfurt, (7) Uni-Koeln

We retrospectively analysed 220 patients (pts.) receiving a myeloablative allogeneic stem cell transplant for ALL between 1994 and 2002 at seven BMT centers in Germany (6) and Sweden (1). Study population consists of 21 pts with unspecified ALL, 5 biphenotypic ALL, 15 B-ALL, 54 pre-B ALL, 12 pro-B-ALL (2 pre-pre-B-ALL), 68 c-ALL, 32 T-ALL, 12 pre-TALL and 1 NK leukemia (53 of all $\mathrm{Ph}+$ ). 88 of these pts were female and 132 male. Mean age was 25.6 years (range: $1-57$ ). 148 pts were transplanted

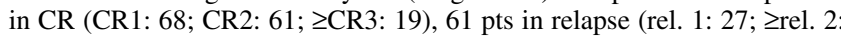
34 ) and 11 pts were refractory to chemotherapy prior to transplant. $64 \%$ of pts received BM and 36\% PBSC from an HLA-identical sibling (29\%), HLA-mismatched sibling (6\%), MUD (52\%), or an HLA-mismatched unrelated donor $(13 \%)$. A TBI based conditioning was used in $78 \%$ of pts. All pts received CsA for GvHD prophylaxis. Overall median EFS was 269 days. 82 pts are still alive (55-2326 days post Tx) and 138 deceased (cause of death: 60 relapse, $13 \mathrm{GvHD}, 40$ infection, 16 multi organ failure and 9 other reasons; TRM 57\%). We observed an advantage regarding EFS in favor of pts transplanted in 1 . CR (median $1313 \mathrm{~d}$, mean $1293 \mathrm{~d}$ ) in comparison to pts transplanted in $\geq 2$. CR (median $247 \mathrm{~d}$, mean $770 \mathrm{~d} ; \mathrm{p}=0.025$ ) or relapsed disease (median $145 \mathrm{~d}$, mean $436 \mathrm{~d} ; \mathrm{p}<0.0001$ ). In addition, $\mathrm{Ph}+$ pts. had no poorer outcome than Ph- pts. Overall incidence of acute GvHD (aGvHD) was $60 \%$ (III-IV ${ }^{\circ}: 12 \%$ ). We observed no differences with regard to incidence and severity of aGvHD comparing pts receiving BM or PBSC AGvHD incidence was significantly increased in pts receiving an HLA mismatched related graft $(\mathrm{p}=0.035)$ in comparison to pts receiving a matched related graft. GvHD was not correlated with GvL as incidence and severity of aGvHD were equally distributed in relapsed and non-relapsed pts. Effects of phenotype on EFS seems to demonstrate a better outcome for B-lineage ALL pts in comparison to T-lineage ALL ( $\mathrm{p}=0.067$; cumulative long-term survival: B-lineage ALL: 40\%; T-lineage ALL: 20\%) but not in comparison to c-ALL pts ( $\mathrm{p}=0.224$; cumulative long-term survival: $28 \%$ ). Myeloablative allogeneic SCT in ALL pts should be performed in first CR. Transplantation in 2. or 3. CR as well as in relapsed or refractory disease is associated with a significantly higher relapse rate and TRM. New strategies including reduced conditioning might be less suitable since GvHD did not correlate with GvL.

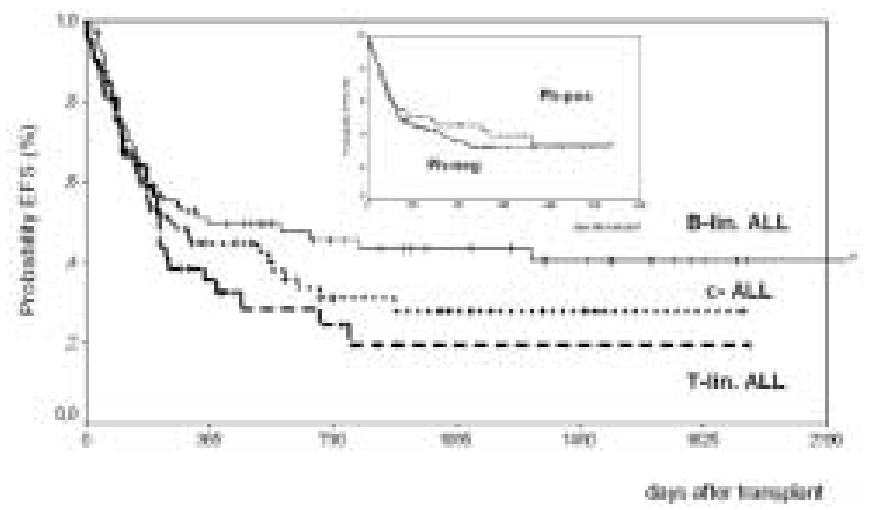


Transplantation of highly purified peripheral blood CD34+ cells from sibling donors in first chronic phase chronic myeloid leukemia

Peceny R., Elmaagacli A.H., Kummer G., Ottinger H., Trenschel R., Schaefer U.W., Beelen D.W.

Dept. for Bone Marrow Transplantation, University of Essen Medical School, Germany

Since September 1998, we are studying prospectively the feasibility of transplantation using G-CSF-mobilized highly purified peripheral blood CD34+ cells from HLA-identical sibling donors.

Forty adult patients with chronic myeloid leukemia in first chronic phase were transplanted in this program up to date. The median Gratwohl score was 2 (1-4). No prophylactic immunosuppression was given with the exception of one patient who received an unmanipulated graft due to poor donor mobilization. All patients engrafted rapidly. The median follow up is 17 months (2-42 months). All except one patient who died of metastatic prostate cancer 27 months after transplant are surviving in at least complete hematologic remission. 27 of 40 patients are in complete molecular remission. At 3 years, the cumulative probability of OS is $90 \%+/-9.5 \%$ and of EFS $81.5 \%+/-10.5 \%$. Two patients developed an early secondary graft failure and were successfully retransplanted with a unmanipulated graft from their original donor. One patient relapsed hematologically despite full Tcell chimerism, but could be rescued with donor lymphocyte infusions and a short course of alpha-Interferon. This patient is in molecular remission again with a well controlled GvHD of the liver and hemolytic anemia, a ful chimera and in good health. Only in 2 patients developed acute skin GvHD with a maximum stage 1 after transplantation of highly purified CD34+ cells. After donor lymphocyte infusions ( 28 pat.) or transplant of unmanipulated grafts ( 3 pat.), 5 patients developed acute GvHD with a maximum grade III, 3 of these 5 patients suffering from extensive chronic GvHD. All of these patients received a starting T-cell dose of at least $1 \times 10^{\wedge} 7 \mathrm{CD} 3+$ $/ \mathrm{kg}$ body weight. With escalating T-cell doses starting with $3 \times 10^{\wedge} 5 \mathrm{CD} 3+$ $/ \mathrm{kg}$ body weight and escalating by $1 / 2 \log$, we have seen only one patient developing an acute skin GvHD stage 2, easily managed with prednisone. In conclusion, transplantation of highly purified peripheral blood CD34+ cells from sibling donors appears to be safe, is effectively preventing acute GvHD and is capable to induce complete hematologic and molecular remissions. This transplant approach with very low mortality and markedly reduced transplant related morbidity will be further evaluated in a multicenter phase III trial. Also in the light of new developments in conventional treatment, allogeneic transplantation is still the only curative therapy for chronic myeloid leukemia.

35

Early allogeneic transplantation in patients with high-risk acute myeloid leukemia defined by karyotype at diagnosis and response to first induction therapy: Results of the ongoing AML HD98A trial of the AML SG ULM

Schlenk R.F. (1), Bunjes D. (1), Döhner K. (1), Fischer J.T. (2), Fröhling S. (1), Füllgraf B. (1), Glasmacher A. (3), Götze K. (4), Grimminger W. (5), Germing U. (6), Hartmann F. (7), Kneba M. (8), Koller E. (9), Mergenthaler H.G. (10), Salwender H. (11), Waterhouse C. (12), Döhner H. (1) for the AML SG ULM

(1) UNI-Klinik Ulm, (2) Städtisches Klinikum Karlsruhe, (3) UNI-Klinik Bonn, (4) TU München, (5) Bürgerhospital Stuttgart, (6) UNI-Klinik Düsseldorf, (7) UNI-Klinik Homburg, (8) UNI-Klinik Kiel, (9) Hanusch

Krankenhaus Wien, (10) Katharinenhospital Stuttgart, (11) Krankenhaus

Altona Hamburg, (12) Krankenhaus München-Schwabing

Karyotype and response to induction therapy are the most important prognostic factors in acute myeloid leukemia (AML). In our ongoing treatmen trial induction and consolidation therapy are stratified according to the karyotype and response to first induction therapy. High-risk patients are defined either by karyotype $(\operatorname{abn}(3 q),-5 / 5 q-,-7 / 7 q-, a b n(12 p)$, abn $(17 p)$, complex) or persistent AML after first induction therapy with idarubicin, cytarabine and etoposide (ICE). Patients with persistent AML after ICE are assigned to second induction therapy with A-HAM (high-dose cytarabine, mitoxantrone, ATRA orally $45 \mathrm{mg} / \mathrm{sqm} /$ day d. $3-5$ and $15 \mathrm{mg} / \mathrm{sqm} /$ day d. 6-28). Patients with partial (PR) or complete remission (CR) after ICE are assigned to a second cycle of ICE followed by one cycle of HAM. All highrisk patients are assigned to allogeneic transplantation (Allo-Tpl) either from matched related donors (MRD), matched unrelated donors (MUD) or haploidentical related donors (HRD). At the interim analysis from March 2002, 116 of 462 patients were high-risk patients. 30 high-risk patients were defined by karyotype and 86 high-risk patients by persistent leukemia after ICE. So far 67 patients received an Allo-Tpl: $n=32$ PBSC MRD, $n=1$ bone marrow MRD, $n=29$ PBSC MUD, $n=5$ PBSC HRD. The conditioning regimens were as follows: $n=33 \mathrm{TBI} /$ cyclophosphamid(Cy) and $n=12$ busul $\operatorname{fan}(\mathrm{Bu}) / \mathrm{Cy}$ (some patients with additional thiotepa or etoposide), $\mathrm{n}=14$ antibody-targeted radionuclide therapy (188Re-Anti-CD66) and $n=3$ bisphosphonate-targeted radionuclide therapy (186Re HEDP) in combination with $\mathrm{Bu} / \mathrm{Cy}(\mathrm{n}=14)$ or TBI/Cy $(\mathrm{n}=2)$ as well as Fludarabine(Flud)/melphalan/ thiotepa $(\mathrm{n}=1), \mathrm{n}=3 \mathrm{Bu} /$ Flud, $\mathrm{n}=1$ Flud/Cy/idarubicin/etoposide, $\mathrm{n}=1$ TBI/Flud. For rejection prophylaxis patients receiving grafts from MUD or HRD were treated with anti-thymocyte globulin. The median follow-up after Allo-Tpl is 18 months and treatment related mortality, relapse probability and survival after Allo-Tpl at this time point are $40 \%, 43 \%$ and $33 \%$ respectively. In univariate analysis complete remission at Allo-Tpl $(\mathrm{p}=0.04)$ and conditioning regimen including antibody-targeted radionuclide therapy with 188 Re-Anti-CD66 ( $\mathrm{p}=0.03$ ) were associated with a statistically significant better survival. In conclusion in our ongoing treatment trial early AlloTpl was feasible in $57 \%$ of our high-risk AML patients.

36

Simultaneous quantification of BCR-ABL Transcripts and T-Cell Receptor excision circles after allogeneic bone marrow transplantation

Przybylski G.K. (1), Kreuzer K.A. (1), Laurisch A. (1), Siegert W. (2), + Schmidt C.A. (3)

(1) Charite, Campus Virchow Klinikum, Humboldt Universität Berlin, (2) Charite, Campus Mitte, (3) EMA Universität Greifswald

Reconstitution of the donor T-cell repertoire after bone marrow transplantation (BMT) is poorly understood. Quantification of T-cell recptor excision circles (TREC) is a new tool for detecting recent thymic emigrants. In order to measure the thymic output and the minimal residual disease after allogeneic BMT, TREC count and bcr-abl transcript numbers were quantified in 14 CML patients by real time PCR. To more accurately determine the TREC counts we prepared a duplex vector containing both the target (TREC) and the reference gene (RAG2). Since the same vector dilutions were used to obtain the target and the reference standard curve the absolute TREC-numbers were not influenced by the errors in DNA quantification, preparing of dilution series and variation of vector stability. Prior to BMT all patients showed markedly lowered TREC counts compared to healthy individuals, most likely due to chemotherapy. After BMT TRECs became undetectable in 12/14 patients and reappeared within 4 month after BMT in 10 patients. Four patients remained TREC-negative. All these four patients ahowed an acute graft versus host disease (GvhD). We next compared TREC numbers and bcr-abl transcript levels. Three patients who remained bcr-abl positive after BMT were TREC negative. There seems to be an inverse correlation between TREC and bcr-abl transcript numbers, however, number of patients analyzed is to small to draw a definite conclusion. We herein describe for the first time a more accurate, duplex vector based, rea time PCR for TREC quantification. Our data suggest, that patients developing GvhD as well as patients with persisting bcr-abl transcripts show an impaired reconstitution of naive T-cells.

\section{7}

Anti-CD45 mediated cytoreduction for allogeneic stem cell transplantation

Wulf G., Luo K., Goodell M., Brenner M.

Universitätsklinikum Göttingen

Monoclonal antibodies have evolved as a valuable therapeutic modality in the treatment of some hematological neoplasias. In a murine model, we here addressed the in vivo effects of an antibody against the common leukocyte antigen CD45 (clone 30F11) on the lymphohematopoietic system, and its role in conditioning regimens for bone marrow transplantation. Following systemic application in vivo, all cells expressing the CD45 antigen were accessible to the antibody. Hematopoietic progenitor cells were efficiently labeled, but spared from cytolysis. In the peripheral blood all leukocytes were transiently diminished, with a prolonged effect on lymphatic cells. Accordingly, T-cells were depleted from the lymphatic organs, and their architecture severely distorted for more than 30 days. When applied in conditioning regimens for bone marrow transplantation, anti-CD45 treatment had no impact on syngeneic engraftment. However, in the allogeneic situation, addition of anti-CD45 treatment to total body irradiation as low as $8 \mathrm{~Gy}$ allowed engraftment across a full MHC mismatch. In conclusion, anti-CD45 treat- 
ment targets all lymphohematopoietic cells, spares progenitor cells, efficiently deplets T-cells, and may have a role as an adjunct to conditioning regimens in allogeneic stem cell transplantation.

\section{Euthanasia \\ J. Beyer, Marburg; H. Samonigg, Graz}

38

\section{Debates on euthanasia}

Frewer A.

Ethik und Geschichte der Medizin, Universitaet Goettingen

At the moment in Germany and in many other countries an intensive debate on ethical and legal aspects of euthanasia is going on. Cases like ,Diane P. at the European Court for Human Rights in Strasbourg or current opinion polls mirror the high resonance in this area. Not least because of the changes of the laws in the Netherlands and in Belgium in Germany is a series of professional and emotional statements in the discussion. The introductory contribution ,debates on euthanasia ' will present definitions of the basic terms which differentiate the field between ,active" and ,passive' euthanasia. Furthermore, main arguments pro and con killing on demand or physician-assisted-suicide are discussed. The problems of oncology in particular raise the ethical questions of a ,good " perimortal medicine. One of the key tasks of medical ethics should be the critical analysis of the discourse and to underline the fruitful perspectives of palliative care and hospice services. Finally, some aspects of the „Grundsätze der Bundesärztekammer zur ärztlichen Sterbebegleitung" and the up to now not very much debated European Convention „Protection of the human rights and dignity of the terminally ill and the dying" will be presented.

\section{9}

\section{Euthanasia: moral obligation or criminal act}

\section{Birnbacher D.}

\section{Philosophisches Institut, Universität Düsseldorf}

Der Beitrag geht von zwei Voraussetzungen aus: 1. dass Sterbehilfe eine aus dem Gebot der Leidensminderung folgende moralische Verpflichtung des Arztes ist, und 2. dass diese Verpflichtung - wie alle Verpflichtungen - begrenzt ist, einerseits durch das Selbstbestimmungsrecht des Patienten, andererseits durch die Gewissensfreiheit des Arztes und durch das gesellschaftliche Interesse an der Vermeidung von Missbräuchen und an der Aufrechterhaltung von Vertrauen. Erörtert wird die Frage: Ist die in Deutschland gegenwärtig vorherrschende Tabuierung der aktiven Sterbehilfe unter diesen Voraussetzungen zweckdienlich und sinnvoll? Eine Reihe von Gründen sprechen dafür: Eine wie immer begrenzte Praxis der aktiven Sterbehilfe könnte Missbräuchen Tür und Tor öffnen, ärztliche Gewissenskonflikte verschärfen oder das internationale Ansehen Deutschlands beschädigen. Auf der anderen Seite ist aber auch eine Tabuierung nicht ohne Gefahren: Da der Begriff ,aktiv“ von vielen Ärzten auf alle Verhaltensweisen bezogen wird, bei denen der Arzt handelnd den Tod eines Patienten (mit-)verursacht, werden irrigerweise auch solche leidensmindernde Maßnahmen für unzulässig gehalten, die weithin als ethisch und rechtlich unbedenklich gelten, etwa der aktive Behandlungsabbruch, die den Tod mit Sicherheit beschleunigende Palliation und die ärztliche Beihilfe zum Patientensuizid bei terminaler Krankheit. Eine Abwägung von Pro und Contra führt aus Sicht des Referenten auf die Unabweisbarkeit einer gedanklichen, praktischen und rechtlichen Neuorientierung, die die Grenze zwischen dem Erlaubten und Unerlaubten weniger bei den Mitteln als bei den Zwecken zieht und - im Anschluss an den „Alternativentwurf Sterbehilfe“ von 1986 - eine Entkriminalisierung der aktiven Sterbehilfe zumindest für Extremfälle vorsieht.

\section{Euthanasia in the Netherlands: New Developments}

Gordijn B.

\section{UNI-Klinik Nijmegen}

In den Niederlanden wurde lange Zeit eine sogenannte Politik der Duldung von Euthanasie praktiziert. Obwohl offiziell strafbar wurde die lebensbeendende Handlung unter bestimmten, vorab bekannten Bedingungen geduldet Das bedeutete, dass ein Arzt, der an Euthanasie beteiligt war, nicht strafverfolgt wurde, wenn er diesen Bedingungen genügt hatte. Vor kurzem wurde diese Duldungspraxis gegen eine neue gesetzliche Regelung eingetauscht. In ihr ist festgelegt, dass bestimmte Formen von Euthanasie straffrei sind. Die Präsentation soll dem besseren Verständnis der jüngsten Entwicklungen in den Niederlanden dienen sowie zur Förderung einer konstruktiven Debatte beitragen. Zunächst erfolgt eine Skizze der Duldungspolitik in Sachen Euthanasie, wie sie bis vor kurzem gehandhabt wurde. Im Anschluss daran wird die neue gesetzliche Regelung skizziert. Schließlich folgen einige Reflexionen über die neue Gesetzgebung.

\section{1}

\section{Euthanasia - are there solutions?}

Meran J.G.

\section{Wilhelminenspital der Stadt Wien, A-1171 Vienna}

Euthanasia and the discussion of autonomy are important issues in the care of heamato-oncologic patients. Besides the pressing philosophical question whether it is always wrong for a doctor intentionally to kill a patient, even if this patient is suffering and asking for death, there are so many matters of degree - grey zones - in clinical practice that have to be be adressed. For the huge majority of patients euthanasia is not the main question, as they want to life and not to die. However, sometimes there appears the question whether life is worth living, whereas the most relevant issue in this context seems to find out the value of a certain therapy for the situation of the dying patient. The search to find the kairos - the right moment - to stop or modify treatment appears to be a real practical problem in oncology. The need of improving professional skills to sensitively find out the needs of our patients may be one of the ways to solve problems. Patients fear to become dependent and to loose control in a situation where only futile treatment is applied and the life is prolonged without any benefit. Advance directives have received considerable attention. Problems and chances of these devices will be discussed. Too much can be too little - in the treatment and care of dying patients. Modern palliative medicine has to enhance the awareness for the needs of these patients and provide a broad spectrum of options as well as training-opportunities. New concepts like the artificial coma to treat pain will be evaluated and discussed. Menschenwürde is a key concept, but not sufficiant to solve practical problems. Clinical practice needs professiona skills and loving care more than sophisticated philosophical dispute.

\section{Providing Health Care for Patients with Malignancies in Practice or Hospital: Competition or Cooperation?}

\section{Böning, München; C. Peschel, München}

\section{2}

The point of view of the clinician

Trümper L.

Göttingen
The point of view of resident doctor

Heymanns $J$. 
Integrative care for tumor patients in regional tumor networks

Schriever $F$

Praxis für Innere Medizin, Hämatologie und Internistische Onkologie, Wartenberg

Building functional regional networks that care for tumor patients and their family members and friends will be a major task for the German health care system. These networks have been evolving since in recent years therapy of tumor patients has been extended from hospital wards to ambulatory of fices. Thereby, need for cooperation between in-hospital and ambulatory treatment has significantly increased. This trend will further increase once hospital costs will be financed according to diagnosis related groups (DRGs) making it necessary to cut down on the number of inpatient beds and to reduce the length of stay of inpatients. In addition, professionals caring for tumor patients are confronted with a series of tasks that have not been addressed sufficiently. Among these are professional help for friends and family members of tumor patients, integration of self-help, volontary groups, and spiritual help and building inpatient and ambulatory palliative care centers and inpatient hospices. Although bias towards these issues is steadily decreasing in academic medicine, the financial problems of the German health care system are dramatically increasing. In contrast, integrative regional networks offer the chance of focussing the potential of already existing health care institutions. Members of these regional tumor networks are among others: oncology and palliative care wards, outpatient departments and offices, self-help groups, volunteers, psycho-oncology, spiritual help and in-patient hospices. Important issues that also need to be addressed by these regional networks are improving documentation and data transfer and providing elements for definition of standards of care. It will a major feat in haematology and oncology to guide the upcoming changes of the German health care system into regional tumor networks that will significantly improve the care for tumor patients, their family members and friends.

\section{5}

\section{The point of view of the health insurance companies}

Baumgartner $R$

AOK Bayern Die Gesundheitskasse, München

Derzeitiger Stand der Diagnostik und Therapie aus Sicht der Krankenkassen

Chancen und Risiken durch Integrierte Versorgung

- was ist Integrierte Versorgung

- Module der Integrierten Versorgung

- bestehende Versorgungsmodelle

- Ist die Integrierte Versorgung das Instrument der Zukunft

Zusammenfassung

Ausblick

\section{6}

Medical care of hematologic/oncologic patients - the point of view of the Resident Doctors' Association

Munte A.

Kassenärztliche Vereinigung Bayern, München

Niedergelassene Fachärzte spielen in der Onkologie eine immer größere Rolle.

Dies betrifft zum Teil die chirurgische Primärtherapie durch ambulante Operateure und Belegärzte. Die Diskussion über vergleichbare Qualitätsstandards in hauptamtlichen Abteilungen, belegärztlichen Abteilungen und bei ambulanten Operationen ist zu begrüßen. Niedergelassene Ärzte bringen ein hohes Leistungsniveau ein und können dies beweisen.

In der Strahlentherapie ist der Trend zur ambulanten Durchführung ungebrochen. Für den Patienten bedeutet dies einen Zuwachs an Lebensqualität. Für das System der kassenärztlichen Versorgung resultiert daraus ein erheblicher finanzieller Mehraufwand. Das Leistungsvolumen im Rahmen der Ermächtigungen steigt, teilweise kommt es auch zu Niederlassungen von Strahlentherapeuten im Krankenhaus. Gemeinsam mit den Regionalkassen arbeiten wir an einer Lösung des finanziellen Problems. Die Situation der niedergelassenen Hämatologen/Onkologen ist durch drei Faktoren gekennzeichnet. a) Strukturell bedingte Mengenentwicklung: Inwieweit unsere Bevölkerung einer zunehmenden Krebsgefährdung unterliegt, können wir wegen des Fehlens von Krebsregistern nicht zuverlässig beurteilen. Aber allein schon die Verschiebung der Alterspyramide führt zu einem starken Anstieg des Behandlungsbedarfs. Durch therapeutische Innovationen wird dieser Anstieg in dreierlei Hinsicht vergrößert: Mehr Krankheiten werden behandelbar, die ambulante Durchführung wird erleichtert und durch bessere Überlebensraten ergibt sich wachsender Bedarf für Folgetherapien.

All diese Faktoren sind zusammen unter -Verlagerung von stationär nach ambulant- zu diskutieren.

b) Distribution von Arzneimitteln: Mit der wachsenden Bedeutung und den wachsenden wirtschaftlichen Anteilen hochpreisiger Arzneimittel in der ambulanten Versorgung verschärft sich das Problem der unterschiedlichen Kostenstrukturen für stationären und ambulanten Bereich bezüglich der Apothekenversorgung. Hier besteht Handlungsbedarf. In der einseitigen Öffnung der Krankenhausapotheken für ambulante Krankenhausbehandlungen kann die Lösung nicht liegen!

c) Off-Label-Use: Niedergelassene Hämatologen/Onkologen sind hier in eine Klemme geraten, aus der sie befreit werden müssen. Bezüglich der forensischen Problematik fehlt dem niedergelassenen Arzt der ,breite Rücken der Universität“. Ein Verzicht auf Off-Label-Anwendungen kann humanitäre und ärztliche Probleme aufwerfen. Und last but not least sehen niedergelassene Internisten sich zunehmend der Regressdrohung ausgesetzt, wenn sie in diesem Bereich tätig - auch erfolgreich tätig - sind.

\section{Poster Presentation}

\section{Acute Lymphoblastic Leukemia}

\section{7}

\section{Mechanisms of acute leukemic cell lysis by the clonal natural killer cell line NK-92}

Bug G. (1), Romanski A. (1), Tonn T. (2), Seifried E. (2), Hoelzer D. (1), Ottmann O.G. (1)

(1) III. Medizinische Klinik, Klinikum der J.W. Goethe-Universität, Frankfurt, Germany, (2) Red Cross Blood Donor Service Hessia, Frankfurt, Germany

Objectives: The human natural killer (NK) cell line NK-92 has previously been shown to effectively kill malignant target cells of various origin without affecting normal tissues and hematopoiesis. As an attractive candidate for use in adoptive immunotherapy, it is the only NK cell line that has entered clinical trials to date. Acute lymphoblastic leukemias (ALL) show variable sensitivity towards NK-cell- including NK-92-lysis, but this has not be investigated in detail. Aim of this study was to evaluate the responsiveness of ALL blasts to NK-92 cells and to analyse possible mechanisms of resistance.

Methods: Primary leukemic blasts (T-lineage ALL and Philadelphia chromosome positive $[\mathrm{Ph}+]$ or negative $[\mathrm{Ph}-]) \mathrm{B}$-precursor-ALLs were examined in addition to a panel of leukemic cell lines. Cytotoxic activity of NK92 cells against leukemic target cells was determined in vitro using a flow cytometry based cytotoxicity assay.

Results: Of 14 patient-derived samples examined, 6 proved to be sensitive (>=20\% lysis) to NK-92-mediated in vitro cytotoxicity (4/4 T-ALLs, 1/5 [Ph-]-ALLs, 0/6 [Ph+]ALL). NK-92 displayed pronounced cytotoxic activity against T-ALL cell lines. Except for the EBV infected SD-1 cell line, Blineage ALL cells were resistant against NK-92 treatment. No difference in NK-92-mediated cytotoxixity was observed between p185(BCR-ABL) expressing or wild type U937 cells. Effector-to-target cell contact mediated by adhesion molecules (ICAMs) seemed to be prerequisite for cytolysis of some (K562, MOLT-4), but not all (SD-1, MOLT-3) cell lines. Up-regulation of ICAM-1 or ICAM-3 by IFNgamma or TNFalpha in patient derived ALLs did not alter the cytolytic activity of NK-92 effector cells. Tumor escape from cytotoxic effector cell lysis has been suggested to be mediated through stimulation of the inhibitory KIR2DL4 receptor by HLA-G expressed on the tumor cells. However, cells genetically engineered to express HLA-G (721.221.G1) and wild type cells lacking HLA-G (721.221) did not differ in their susceptibility to lysis by NK-92 cells.

Conclusions: Our in vitro analysis of the antileukemic activity of NK-92 human leukemic cells suggests that the majority T-ALLs are susceptible to lysis. In contrast, B-precursor ALL are heterogeneous in their susceptibilty to lysis. The presence of the Philadelphia chromosome as well as the expression of HLA-G is not associated with resistance against lysis by NK-92. 
sibling the hematological recovery was uneventful with $\mathrm{WBC}>1 / \mathrm{nl}$ on day

Anti leukemia-specific humoral immune response in children with T-lineage acute lymphoblastic leukemia

Dohnal A., Panzer-Grümayer R.

Children's Cancer Research Institute Vienna

Objectives: Although recent studies suggest that acute lymphoblastic leukemia induces $\mathrm{T}$ cell anergy, there exist data that provide evidence for a cellular and thus also humoral immune response against leukemia-specific antigens. We have recently demonstrated the presence of a persistent humoral immune response in children with T-lineage acute lymphoblastic leukemia (T-ALL) and speculated that this immune response has an effect on the leukemic T cells. Consequently, the aim of the current study is to test whether the detected humoral immune response is directed against the leukemic T cells.

Methods: Mononuclear cells from bone marrow (BM-MNCs) obtained from children with T-ALL at the time of diagnosis are separated into leukemic T cells (positive selection by magnetic cell sorting (MACS) using anti-CD7 magnetic beads) and normal B cells. The latter are used for EBV transformation in order to establish IgG-producing lymphoblastoid B cell lines (LCLs). LCLs are screened by clonotypic PCR using the specific sequence of those IgH rearrangements that were initially identified at diagnosis. By Western blotting supernatant-IgG of primary LCLs and plasma-IgC from bone marrow of T-ALL patients are tested for their reactivity against leukemic T cells (positive selected fraction by MACS) and leukemic cell lines (Jurkat, Molt-3, Peer, HSB-2).

Results: In one patient we successfully EBV-transformed normal B cells. In these primary LCLs one of the three IgH rearrangements that had been identified at diagnosis in BM-MNCs could be detected. Supernatant-IgG from these LCLs recognized a $\sim 100 \mathrm{kD}$ protein in the autologous leukemic cells (ALCs) as well as in all leukemic T cell lines. Three additional smaller proteins were exclusively detected in ALCs. By using plasma-IgG from two other patients for Western blotting, several proteins were detected in ALCs as well as in leukemic $\mathrm{T}$ cells from three additional patients and in all leukemic $\mathrm{T}$ cell lines, whereas no reactivity was observed with plasma-IgG from a healthy donor. Non of these proteins was detectable in normal $\mathrm{T}$ cells from the same donor.

Conclusion: A leukemia-specific humoral immune response is evident in children with T-ALL which is based on the reactivity of plasma-IgG with leukemic $\mathrm{T}$ cells from several children and on the presence of leukemia-specific IgG in the supernatant of primary LCLs from one patient.

49

Imatinib can be safely coadministered with synergistic high-dose chemotherapy and subsequent TBI-containing conditioning therapy in refractory $\mathbf{P h}+$ leukemia to allow successful allogeneic transplantation in a state of minimal residual disease

Fruehauf S. (1), Topaly J. (1), Schad M. (2), Goerner M. (1), Zeller W.J. (2), Ho A.D. (1)

(1) Med. Klinik u. Poliklinik V, Univ. Heidelberg, (2) D0200, Deutsches Krebsforschungszentrum (DKFZ) Heidelberg

Patients with BCR-ABL+ ALL have a dismal prognosis. Even tyrosine kinase inhibitor imatinib (formerly termed STI571) can only induce short lasting remissions. This prompted us to investigate whether combinations of imatinib and different cytostatic agents would display synergistic activity in the BCR-ABL+ lymphatic blast crisis cell line BV173, in primary cells in vitro and in the clinic. Among a range of combinations with imatinib, significant synergism was observed for imatinib +irradiation, +cytarabine, + mitoxantrone, +mafosfamide. A potentiation of toxicity in BCR-ABLleukemic cell lines or normal CD34+ progenitors by addition of imatinib to cytostatic drugs was not observed.

Based on our favorable preclinical data we treated a 19 -year-old patient with $\mathrm{Ph}+\mathrm{ALL}$ who was refractory to standard induction therapy and only partially responded to imatinib alone with imatinib-based combination therapy. Following imatinib +MitoFLAG (mitoxantrone, fludarabine, cytarabine, G-CSF) the patient became severely aplastic for the first time and no leukemic cells were detectable in the PB by flow cytometry (FCM) during deep marrow aplasia (WBC 0,05/nl, 800 events counted). However, in the recovery phase the patient showed a leukemic cell population of $2 \%$ in the $\mathrm{PB}$ and $15 \%$ in the BM. Subsequently, a conditioning therapy (TBI/cyclophosphamide) supplemented with imatinib was given. Again, the leukemic cell population in the PB dropped below the detection limit during conditioning therapy and in the BM only $1.5 \%-6 \%$ leukemic cells were noted at this time. Following PBSC transplantation from an HLA-identical
16 and thrombocytes $>50 / \mathrm{nl}$ on day 21 after transplantation. In a follow-up examination 2 months after transplant a leukemic cell population was for the first time not detectable any more in BM by FCM. A nested RT-PCR was also negative. This case shows that combination therapy of imatinib and synergistically active cytostatic drugs or irradiation can be safely administered and can induce a leukemic cell depletion that was not possible by either high-dose induction chemotherapy or imatinib alone. Although the combined modality treatment was not curative by itself it helped to achieve a state of minimal residual disease which allowed successful allogeneic stem cell transplantation. This treatment approach should be systematically explored in refractory or relapsing high-risk $\mathrm{Ph}+$ leukemias.

\section{0}

\section{4-year-old gravida at 21 weeks with Philadelphia-chromo- some positive acute lymphoblastic leukemia}

\author{
Hähling D. (1), Subert R. (1), Freund M. (2)
}

(1) Klinikum Schwerin, (2) Universität Rostock

We report on a 24-year-old gravida1 para1 at 21 weeks (07/2001) diagnosed with Philadelphia-chromosome positive acute lymphoblastic leukemia ( $\mathrm{Ph}+$ ALL). The woman was admitted to our hospital with a leucocytosis of 123 $\mathrm{Gpt} / 1$, a thrombocytopenia of $23 \mathrm{Gpt} / 1$ and anemia (hemoglobin of 7,0 $\mathrm{mmol} / \mathrm{l})$. Her bone marrow was hypercellular with an excess of blast $(90$ $\%$,peroxidase stain(-), PAS stain (-)), that displayed a positive immunphenotype for CD 10, CD 19, CD 34, HLA DR, TdT. Cytogenetics showed a Philadelphia-chromosome (m-bcr).

The woman received induction-therapy according to the GMALL protocol $5 / 93$ with daunorubicin, vincristin, prednisolone and asparaginase followed by cyclophosphamide, cytarabine and mercaptopurine. She also underwent prophylactic intrathekal methotrexate. After 4 weeks she achieved complete remission. She delivered a healthy male infant $(49 \mathrm{~cm}, 2880 \mathrm{~g})$ at 35 week by cesarean section. After delivery she underwent prophylactic brain radiation and consolidation with cyclophosphamide and cytarabine. 2 weeks later she got her first relapse. She was treated with Imatinib(Glivec), vincristine, daunorubicin and rituximab (Mabthera) and achieved again complete remission. At the university of Rostock she received an allogenic bone marrow transplantation (12/2001)with HLA-identical unrelated donor. For conditioning she was given cyclophosphamide $120 \mathrm{mg} / \mathrm{kg}$,ATG $3 \times 520 \mathrm{mg}$ and TBI with 12 Gy in 6 doses with lung shielding. The patient developed acute graft-versus-host disease of skin, liver und lung Grade II-III.

She is alive in complete hematological, cytogenetical and molecular remission.

\section{1}

\section{Functional Ig heavy chain gene rearrangements in children with T-cell acute lymphoblastic leukemia are derived from normal B cell clones}

Hübner S., Panzer-Grümayer R.E.

Children's Cancer Research Institute, St.Anna Kinderspital, Vienna

Objectives: We have recently identified oligo/clonal complete Ig heavy chain $(\operatorname{IgH})$ gene rearrangements in children with T-cell acute lymphoblastic leukemia (T-ALL). Sequence analysis indicates that these rearrangements are potentially functional and thus cannot be leukemia clone-specific. We therefore hypothesize that these $\mathrm{IgH}$ rearrangements originate from normal clonally expanded B cells. The aim of this study was to confirm this hypothesis by characterization of these B cell populations and to investigate whether they persist during therapy.

Methods: Twenty-three children with T-ALL were included in the study. They were selected on the availability of bone marrow samples from diagnosis and follow-up time points. The leukemias were of immature $(n=10)$, intermediate $(n=5)$ and mature $(n=8)$ T cell phenotype. For the detection of clonal IgH rearrangements at diagnosis a two-round VH family PCR was performed. Rearrangements were analyzed for their open reading frame, VH gene usage and somatic hypermutation. In order to confirm their B cell origin, mononuclear cells (MNC) from diagnosis were sorted into normal B cells and leukemic $\mathrm{T}$ cells by flow cytometry and were subjected to a threeround PCR using specific primers for the respective rearrangements. The same PCR approach was used to analyze total bone marrow MNCs from follow-up time points for the persistence of these rearrangements.

Results: We detected oligo/clonal IgH rearrangements in 13/23 (56\%) patients with an immature $(n=8)$, intermediate $(n=2)$, and mature $(n=3)$ T-ALL. A median of 2 (range 1-5) potentially functional rearrangements were identified per patient. They preferentially used VH4 gene segments $(56 \%)$ and 
displayed antigen-driven somatic hypermutations in $37 \%$. In three of the patients we confirmed that the $\mathrm{IgH}$ rearrangements were derived from normal B cells and showed that the respective B cell clones accounted for 1-8\% of total bone marrow B cells. Furthermore, we demonstrated in two patients that these B cell clones persisted in the bone marrow during the course of therapy and even thereafter.

Conclusion: Our findings demonstrate the presence of a persistent humora immune response in children with T-ALL. Since these patients did not have clinical signs of infections at diagnosis or during the weeks before, we speculate that this immune response is directed against the leukemia itself.

\section{2}

\section{Detection of the BCR-ABL mutation E255K prior to STI571} treatment in patients with $\mathrm{Ph}+$ acute lymphoblastic leukemia

Komor M. (1), Güller S. (1), Waßmann B. (1), Wagner S. (1), Gschaidmeier H. (2), Hoelzer D. (1), Koeffler H.P. (3), Ottmann O.G. (1), Hofmann W.K. (1)

(1) Department of Hematology, University Hospital, Frankfurt/Main, Germany (2) Novartis Pharma AG, Nürnberg, Germany, (3) Division of Hematology and Oncology, Cedars Sinai Medical Center, UCLA School of Medicine, Los Angeles, CA, USA

Objectives: The tyrosin kinase inhibitor STI571 (Imatinib ${ }^{\mathrm{TM}}$ ) binds competitively to the ATP binding site of the ABL kinase, thereby inhibiting autophosphorylation of the oncogenic protein BCR-ABL and preventing the activation of downstream signaling pathways. Development of resistance is common in Ph+ ALL and advanced CML and is associated with the occurance of at least 8 different point mutations in the ATP binding site. Comparative studies on leukemic cells obtained from CML and Ph+ ALL patients before and after treatment with STI571 reported the mutations in resistent samples after a short time of therapy, but never prior to STI571 therapy. It was the aim of the study to determine whether or not patients with $\mathrm{Ph}+$ ALL in whom resistance developed as a consequence of the E255K mutation already harboured this subclone prior to STI571 treatment.

Material and Methods: Genomic DNA (gDNA) and RNA were extracted from 30 bone marrow samples of 21 patients with Ph+ ALL. First, the migration pattern of 30 cDNAs was analyzed by PCR-SSCP. Thereafter, detailed mutational analysis was performed on initial, STI571 naive bone marrow samples of 4 individuals with Ph+ALL, for whom the mutation E255K in association with STI571-treatment has been shown. A 166 bp PCR-fragment spanning from nt 862 to nt 1027 was cloned and a total of 440 clones were analyzed by direct sequencing.

Results: PCR-SSCP analysis detected aberrant DNA-migration patterns in 4 of 6 STI571-resistant samples, but no mutation was detected in any initial sample. At least 100 clones of every initial sample were analyzed by direct sequencing. This more sensitive technique revealed presence of the E255K mutation in 2 initial samples, one clone each. For comparison, 25 clones of resistent samples were analyzed as well. In resistent samples, E255K was identified in $10-30 \%$ of the analyzed clones.

Conclusions: We identified for the first time the mutation E255K in STInaive leukemic samples of $\mathrm{Ph}+$-ALL-patients. The findings suggest, that the mutation exists in a very small sub-population of leukemic cells at the beginning of the disease. The presence of E255K in cells prior to STI571 may result in different directions of further research to identify mechanisms of resistance to STI571.

\section{3}

The role of $\mathrm{V}(\mathrm{D}) \mathrm{J}$-recombination in recurrent chromosomal translocations associated with lymphoid malignancies

Marculescu R. (1), Le T. (2), Simon P. (3), Jäger U. (2), Nadel B. (2)

(1) Inst. f. Med. u. Chem. Labordiagnostik, (2) Univ. Klinik f. Innere Med I, Abt. f. Hämatologie u. Hämostaseologie, (3) Uni. Klinik f. Chirurgie, Universität Wien, Allgemeines Krankenhaus

Lymphoid malignancies are often initiated by specific chromosomal translocations between Ig/TCR gene segments and cellular proto-oncogenes. In many cases, illegitimate $\mathrm{V}(\mathrm{D}) \mathrm{J}$ recombination has been proposed to be in volved in the translocation process, but this has never been established. Using an extra-chromosomal V(D)J recombination assay we directly and functionally tested which of several proto-oncogene sequences are able to target $\mathrm{V}(\mathrm{D}) \mathrm{J}$ recombination. Our results support the presence of at least two distinct mechanisms by which $\mathrm{V}(\mathrm{D}) \mathrm{J}$ recombination leads to chromosomal translocations. In some cases, illegitimate $\mathrm{V}(\mathrm{D}) \mathrm{J}$ recombination occurs between an Ig or TCR locus and a proto-oncogene locus bearing a fortuitous, but functional recombination signal sequence (RSS). T(7;9)(q34;q32)
(TCRß/TAL2) and $\mathrm{t}(11 ; 14)(\mathrm{p} 13 ; \mathrm{q} 11)(\mathrm{LMO} 2 / \mathrm{TCR} \delta)$, both recurrently found in pediatric T-ALL, are of this type (type I). In a second category (type II), however, only the breaks at the immune locus are V(D)J mediated. Breaks at the proto-oncogene locus must be initiated by other (yet unknown) mechanisms, and subsequently invade the $\mathrm{V}(\mathrm{D}) \mathrm{J}$ synaptic complex during the rearrangement process. $\mathrm{t}(14 ; 18)(\mathrm{q} 32 ; \mathrm{q} 21)(\mathrm{BCL} 2 / \mathrm{IgH})$ and $\mathrm{t}(11 ; 14)(\mathrm{q} 13 ; \mathrm{q} 32)(\mathrm{BCL} 1 / \mathrm{IgH})$ belong to this category. In order to further define the factors governing the occurrence of type I translocations, we precisely quantified the recombinogenic potentials of the TAL2 and LMO2 proto-oncogene breakpoints. We found them to be comparable to those of physiologic RSS. We then analyzed thymic tissue from healthy individuals for the presence of this translocations, and, in agreement with our in vitro data, we detected them at a high frequency. In addition, comparison of the rearrangements amplified from healthy individuals and from leukemia samples suggests a more complex, multi-step involvement of the V(D)J-recombinase in some type I translocations, and provides novel insights into the molecular mechanisms of proto-oncogene deregulation leading to leukemia.

54

Allogeneic transplantation in bcr-abl-positive acute lymphoblastic leukemia: intensification of conditioning using ${ }^{188}$ rhenium-labelled anti-cd66 monoclonal antibodies

Martin H. (1), Klein S.A. (1), Bug G. (1), Buchmann I. (3), Zenz T. (2), Wiesneth M. (4), Dohr D. (5), Reske S.N. (3), Döhner H. (2), Hoelzer D. (1), Bunjes D. (2)

(1) UNI-Klinik Frankfurt Hämatologie, (2) UNI-Klinik Ulm Hämatologie, (3) Nuklearmedizin Ulm, (4) Tranfusionsmedizin Ulm, (5) Strahlentherapie Ulm

Objectives: In patients with $\mathrm{BCR}-\mathrm{ABL}^{+} \mathrm{ALL}$, allogeneic transplantation is regarded as treatment of choice if a HLA-matched donor is available. Despite impressive results from a few single centers, the long-term outcome after allotransplants in prospective studies remains to be improved.

Material and Methods: Over a period of 43 months a total of 19 allografted patients with BCR-ABL+ ALL and a median age of 45 (range 19-59) years received radioimmunotherapy (RIT) intensified conditioning using ${ }^{188} \mathrm{Re}$ labelled anti-CD66 MoAbs as described for AML-patients elsewhere (Bunjes et al, Blood 2001, 98:565)

Status at Tx: CR1 $(n=11)$, advanced leukemia $(n=8)$

Conditioning: RIT+TBI 12Gy+CTX $120(n=14)$

RIT+fludarabine 150+melphalan 140 (Flu-Mel) $(\mathrm{n}=5)$

Donors: HLA-id sib. $(\mathrm{n}=10)$, MUD $(\mathrm{n}=9)$

T-cell-depletion: RIT+TBI ( $\mathrm{n}=13$ of 14$)$; Flu-Mel $(\mathrm{n}=0 / 5)$

Two TBI/TCD-pts. with incomplete engraftment received an non-TCD 2nd graft.

Results: The median ${ }^{188}$ Re bone marrow dose was 12,5 (range 6,7-21,9) Gy. TRD occurred in 5 of 19 pts. ( 3 in CR1, 2 in advanced leukemia). Four of 5 TRD were in patients receiving non-TCD grafts. Eight patients $(3 \mathrm{CR} 1,5$ advanced) relapsed after a median of 117 (102-251) days. No patient receiving RIT+Flu-Mel in advanced disease survived. Six patients (5/11 CR1) are in CCR after a median follow-up of 790 (range 176-1169) days. EFS in patients receiving TBI + RIT significantly correlated with the ${ }^{188} \mathrm{Re}$ bone marrow dose $(n=14, p=0.02)^{*}$. Actuarial EFS, TRM and relapse rate for patients transplanted in CR1 versus advanced disease are $42 \pm 16 \%$ vs $19 \pm 16 \%, 32 \pm 15 \%$ vs. $25 \pm 15 \%$, and $33 \pm 16 \%$ vs. $83 \pm 15 \%$ ( $=0.07$ ), respectively.

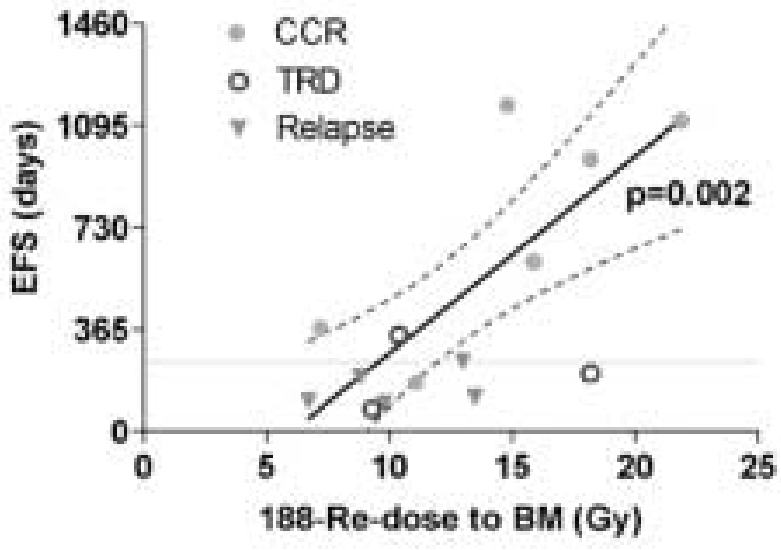

Conclusions: This interim analysis of an ongoing study shows an encouraging low relapse rate for BCR-ABL+ ALL patients receiving RIT + TBI + CTX in CR1. We conclude that leukemia-free correlates with total marrow TBI + RIT irradiation dose. 
Non-disjunction of chromosomes leading to hyperdiploid B cell precursor acute lymphoblastic leukemia in childhood is an early event in leukemogenesis

Panzer-Grümayer E.R. (1), Fasching K. (1), Panzer S. (2), Schmitt K. (3), Haas OA. (1), Gadner H. (1)

(1) CCRI and St. Anna Kinderspital,Vienna, (2) UNI-Wien, (3) LKH Linz, Austria.

Objectives: A hyperdiploid karyotype is found in 30\% of B cell precursor (BCP) acute lymphoblastic leukemias (ALL) in childhood. Its implication for the development of leukemia as well as the time point of its occurrence during leukemogenesis is unknown. We therefore aimed at identifying the time of non-disjunction in relation to the immunoglobulin heavy chain (IgH) rearrangements in the pre/leukemic cell and to the pre- or postnatal period of the patient. As the IgH gene is located on chromosome 14 that is trisomic in the majority of hyperdiploid BCP ALL, a clonal marker is available for each of these chromosomes provided all three $\mathrm{IgH}$ genes are rearranged individually.

Material and Methods: In one informative cytoplasmic mu negative BCP ALL from a 2.6 years-old boy three IGH rearrangements were identified by VH family-specific PCRs. ASO primers were designed for their detection in single cells and neonatal blood spots.

Results: We confirmed that all three rearrangements are contained within a single leukemia clone. Two of these rearrangements - the incomplete DJH and one of the complete VDJH rearrangements - were found to have homologous DJH joinings. Thus, the chromosome harboring the original DJH rearrangement must have duplicated before the subsequent joining of a $\mathrm{VH}$ segment to one of these incomplete rearrangements. These findings suggest that an incomplete DJH rearrangement on one allele and presumably a nonfunctional VDJH rearrangement on the second allele were present in the diploid cell before mitosis.Using neonatal blood spots as a source of the earliest available hematopoietic cells in this patient for the retrospective analysis of clonotypic IgH rearrangements, we demonstrate that all three rearrangements were present already at birth, indicating that doubling of chromosome 14 as well as the ongoing rearrangement processes occur early during leukemogenesis.The in utero initiation of hyperdiploid BCP ALL was confirmed in two other children by the presence of leukemia clone-specific IgH rearrangements in their neonatal blood spots.

Conclusions: Since it was shown previously that hyperdiploidy results from one single abnormal mitosis, non-disjunction of chromosome 14 is likely to concur with that of the other multiplied chromosomes present in a hyperdiploid leukemia. Thus, we provide the first evidence that non-disjunction leading to hyperdiploid BCP ALL is an early event in leukemogenesis.

\section{6}

Clinical Resistance to the ABL Kinase Inhibitor Imatinib is not associated with increased Expression of Multidrug Resistance activity in Ph+/Bcr-Abl+ Acute Lymphoblastic Leukemia

Pfeifer H. (1), Hansen M. (1), Evers R. (2), Wassmann B. (1), Gambacorti C. (3), Scheuring U. (1), Hoelzer D. (1), Ottmann O.G. (1)

(1) Department of Hematology, University Hospital, Frankfurt/Main, Germany, (2) Georg-Speyer-Haus, Frankfurt Main, Germany, (3) Instituti di Tumori, Mailand, Italy

Background: Imatinib showed in clinical trials anti-leukemic activity against bcr-abl expressing leukemias, including $\mathrm{Ph}+\mathrm{ALL}$. Hematologic response rates of more than $60 \%$ in $\mathrm{Ph}+\mathrm{ALL}$ were achieved, but secondary resistance to STI571 developed in the overwhelming majority. In some Imatinib-resistant cell lines an increased P-gp expression has been demonstrated. P-GP is a membrane protein encoded by MDR-1 gene.

Patients and Methods: To test whether expression of MDR1 and MRP1 in leukemic cells is upregulated in Imatinib-refractory $\mathrm{Ph}+\mathrm{ALL}$ blasts, we used a flow cytometric based calcein assay. The direct Imatinib transport was examined by MDR1, MRP1 and MRP2 transfected cell lines, imatinib concentrations were measured by HPLC. To evalute MDR1 expression in patients receiving Imatinib therapy, samples from 7 primary resistant, 6 responding and 8 secondary resistant adult $\mathrm{Ph}+\mathrm{ALL}$ patients were analyzed, too. Total RNA was extracted, reverse transcribed and MDR 1 mRNA expression was analyzed by quantitative Real-Time-PCR (Taqman-technology). To standardize results expression of GAPDH was quantitated. Additionally the multidrug resistance activity factor (MAF), a calculated factor which corresponds to the function of MDR was measured in 3 primary resistant and 7 secondary resistant patients by flow cytometry. (MAF $<10$ reflecting no MRD-activityn).
Results: The Calcein AM transport of the P-gp substrat was efficiently inhibited by Imatinib (IC50 1 $\mu \mathrm{M}$ ), suggesting a competetive effect. Direct drug transport measured by HPLC showed that only P-gp has the ability to transport Imatinib effectively. The median level of MDR1 (ratio of copy number of MDR1 and GAPDH) expression was 1,58x10-1 for patients prior to therapy and 4,39 x 10-2 after developing resistance. The median level prior imatinib was 4,94 × $10-2$ for responders, and 1,3 x 10-2 for non-responders. There was no significant difference between bone marrow and peripheral blood samples. The multidrug activity factor was low. No patient had an actvity higher than 8 .

Conclusion: Our data show that STI is a substrate of P-GP but not of MRP1 or 2. The comparison of drug resistance activity in patients with secondary resistant $\mathrm{Ph}+\mathrm{ALL}$ does not contribute significantly to upregulation of the membrane transporters P-gp or MRP-1.

\section{7}

Central nervous system relapse in patients with relapsed or refractory Philadelphia chromosome positive $(\mathrm{ph}+)$ acute leukemia during treatment with the ABL kinase inhibitor imatinib mesylate (glivec)

Pfeifer H. (1), Wassmann B. (1), Scheuring U. (1), Brueck P. (1), Binckebank A. (1), Schleyer E. (2), Gschaidmeier H. (3), Hoelzer D. (1), Ottmann O.G. (1)

(1) Department of Hematology, University Hospital, Frankfurt/Main, (2) Carl Gustav Carus University, Dresden, (3) Novartis Pharma AG, Nürnberg, Germany

Imatinib mesylate is a selective ABL kinase inhibitor displaying promising single agent activity in advanced $\mathrm{Ph}+$ leukemias, although the response duration in relapsed or refractory $\mathrm{Ph}+\mathrm{ALL}$ and CML blast crisis is usually short. Due to lack of information regarding possible interactions, the clinical trials excluded the simultaneous use of Imatinib and intrathecal prophylaxis. Preclinical data suggest that penetration of the blood brain barrier by imatinib is limited, which could conceivably lead to an elevated risk of CNS relapse.

We examined the clinical effects of Imatinib to determine the risk of CNS relapse in 107 consecutive patients with relapsed or primary refractory acute lymphoblastic leukemia $(\mathrm{Ph}+\mathrm{ALL} ; \mathrm{n}=65)$ or chronic myeloid leukemia in blast crisis $(B C ; n=42)$ who were enrolled into multicenter clinical phase II trials. Leukemic involvement of the CNS was defined by the presence of at least $5 / \mathrm{ml}$ leukemic blasts in the CSF, obtained by lumbar puncture performed at the time of neurological symptoms or clinical suspicion of CNS involvement. During imatinib therapy, CNS relapse occurred in 13 of 107 patients $(11 \%) .5$ of these cases occurred in the 32 patients who were refractory to Glivec $(16 \%)$. Of the 75 responding patients, $4(5 \%)$ presented with an isolated CNS relapse and 4 pts. $(5 \%)$ experienced simultaneous $\mathrm{CNS}$ and systemic relapse. Median time from start of imatinib to diagnosis of CNS relapse was 87 days (14-424 d). Patients with a lymphoid phenotype were at particular risk, as 8 of 65 pts. with Ph+ALL, 1 of 10 with LyBC and 3 pts. with bilineage BC developed CNS relapse. Among 29 pts. with $\mathrm{MyBC}$, only one developed possible CNS leukemia as shown by CCT (chloroma), without cytologic verification. None of the pts. with CNS relapse had a history of leukemic involvement of the CNS. Simultaneous measurements of imatinib concentrations in plasma and CSF in 2 pts. revealed 17 -fold and 65 -fold lower mean concentrations in CSF $(1363 \mathrm{ng} / \mathrm{ml}$ and $1790 \mathrm{ng} / \mathrm{ml}$ versus $79.8 \mathrm{ng} / \mathrm{ml}$ and $27.6 \mathrm{ng} / \mathrm{ml}) .5 \mathrm{pts}$. received prophylactic i.th. triple therapy (AraC, Dexa, MTX) parallel to imatinib. With short term follow up, no non-hematologic side effects were observed.

Imatinib is a promising treatment option for advanced $\mathrm{Ph}+$ leukemias. However risk of CNS relapse relapse during single agent imatinib therapy is high.As simultaneous i.th. therapy and administration of Glivec appear to be feasible, currently data suggest that CNS prophylaxis should be adopted as routine 
Significance of early minimal residual disease (MRD) analysis by Bcr-Abl quantification during imatinib (Glivec) therapy in $\mathrm{Ph}+$ acute lymphoblastic leukemia patients

Scheuring U.J., Pfeifer H., Wassmann B., Brück P., Gschaidmeier H., Hoelzer D., Ottmann O.G.

Department of Hematology and Oncology, J. W. Goethe University Hospital, Frankfurt, Germany

Objectives: Abl tyrosine kinase inhibitor imatinib mesylate (Glivec, formerly STI571) induces remissions in $60-70 \%$ of patients with Philadelphia chromosome-positive $(\mathrm{Ph}+)$ acute lymphoblastic leukemia (ALL), but responses are short-termed. Therefore, it is important to discern patients with pending relapse from those with longer remissions by MRD analysis to guide timing and intensity of additional therapeuties. The aim of the present study was to test whether early Bcr-Abl levels and changes in bone marrow $(\mathrm{BM})$ or peripheral blood (PB) are predictive of response duration and survival in $\mathrm{Ph}+\mathrm{ALL}$ patients treated with imatinib.

Methods: We followed a cohort of 56 patients treated with imatinib in phase II trials (CSTI109, CSTI114) by quantitative PCR of Bcr-Abl levels in bone marrow (BM) and peripheral blood (PB). All patients had received chemotherapy (at start: 1patient in CR, 2 in PR, 22 in 1st, 10 in 2nd , 1 in 4th relapse and 20 refractory) 18 patients allogeneic stem cell transplantation (SCT) and 4 patients autologous SCT prior to imatinib. Serial BM and PB samples were analyzed by RNA extraction, reverse transcription and quantitative Taqman PCR for Bcr-Abl using plasmid titrations and normalized by GAPDH houskeeping gene levels.

Results: Imatinib induced a complete hematologic response (CHR) or marrow response (marrow-CR) in 40 patients (good responders) and a partia $(n=2)$ or no $(n=14)$ remission in the remaining patients (poor responders). Compared to baseline, the median Bcr-Abl/GAPDH ratios decreased significantly in PB by $2.65,2.64$ and $3.11 \log$ steps after 2,4 weeks and at the time of best response, respectively. In BM, the decline of median Bcr$\mathrm{Abl} / \mathrm{GAPDH}$ was $0.75,1.37$ and $2.78 \mathrm{logs}$, respectively. Thus, Bcr-Abl levels decreased more rapidly in PB than in BM (median time to best level 31 versus 39 days). Low Bcr-Abl/GAPDH ratios $<10-4$ in PB and $<10-2$ in $\mathrm{BM}$ after 2 weeks were significantly associated with good responses after 4 weeks. Moreover, Bcr-Abl levels $(<10-2)$ in BM of good responders after 4 weeks discriminated between two groups of patients with significantly different median time to progression (139 versus 22 days).

Conclusions: The data show that Bcr-Abl levels in PB and BM after 2 weeks of STI571 treatment and in BM after 4 weeks have predictive relevance and may guide the application of additional therapies, including stem cell transplantation, donor lymphocyte infusions, interferon-alpha or chemotherapy.

Preservation of HLA and CD86, but loss of CD40 and CD80 on blasts of patients with acute myeloid leukemia (AML)

Vollmer M. (1), Schmitt A. (2), Greiner J. (1), Reinhardt P. (2), Ringhoffer M. (1), Wiesneth M. (2), Döhner H. (1), Schmitt M. (1)

(1) Third Department of Internal Medicine, University of Ulm, Robert-Koch-Str. 8, 89081 Ulm, Germany, (2) Department of Transfusion Medicine, German Red Cross Blood Center, Helmholtzstr. 10, 89081 Ulm, Germany

The expression of human leukocyte antigen (HLA) molecules on the targeted leukaemic blasts (LB) is pivotal for immunotherapies employing antigen peptides alone or pulsed on dendritic cells (DC). Leukemia associated antigens (LAA) recognized by T lymphocytes like WT-1, PR-1, PRAME and RHAMM have been identified. CD80 and CD86 as costimulatory molecules give a second signal to $\mathrm{T}$ lymphocytes mandatory for the lysis of leukaemia cells, CD40 was shown to enhance the efficacy of antigen presentation. In the present study, the expression of HLA-ABC, HLA-A2, HLADR, CD40, CD80 and CD86 was examined by FACS analysis of MNC from 15 healthy volunteers (HV), 15 patients with AML at the time of diagnosis and 5 AML patients at the time of relapse. Expression of HLA-ABC and HLA-A2 was detected at the same level on LB and on lymphocytes of HV. Suprisingly, CD86 and HLA-DR expression was two to five times higher on LB than on lymphocytes of HV. In AML patients, the expression of HLA and CD86 molecules was five to ten times higher on LB than on normal lymphocytes. The costimulatory molecules CD40 and CD80 molecules on AML blasts were downregulated. This might at least partially explain the immune escape of blasts in AML patients. The preservation of
HLA molecules and CD86 on LB at the time of diagnosis and even at relapse of AML are sine qua non prerequisites for the feasibility of LAA targeted immunotherapies of the disease.

\section{0}

Expression profiles of tumor-associated antigens (TAA) in patients with acute myeloid leukemia (AML)

Greiner J., Ringhoffer M., Taniguchi M., Szmaragowska A., Döhner H., Schmitt $M$.

\section{3rd Department of Medicine, University of Ulm, Germany}

For a specific immunotherapy of tumors, the identification of tumor-associated antigens (TAA) eliciting an immune response in the tumor-bearing host is mandatory. Significant mRNA expression of such TAA is a prerequisite for immunotherapy in leukemias. Simultaneous expression of different antigens is a basis for therapies with polyvalent vaccines. In this study, TAA previously characterized in solid tumors and antigens newly characterized in myeloid leukemias, so-called leukemia associated antigens (LAA) were evaluated for their expression in up to $60 \mathrm{AML}$ patients and in leukemia cell lines by RT-PCR and real-time PCR. The differential expression of the TAA/LAA was investigated in PBMN and CD34 positive cells of healthy volunteers and in a panel of normal tissues. Three groups of antigens with different expression pattern could be distinguished:

1) Antigens with no mRNA expression in leukemia patients and healthy volunteers like the antigens MAGE1, MAGE3, SSX2, SCP1, GAGE, HER2/neu, RAGE1 and NY-CO-38,

2) antigens with mRNA expression in leukemias but also in PBMN of healthy volunteers like proteinase 3, PINCH, HSJ2, Syntaxin, MAZ, RBPJkappa, and

3) antigens with tumor-restricted mRNA expression: PRAME was detected in $32 / 50(64 \%)$, hTERT in $7 / 25(28 \%)$, MPP11 in 43/50 (86\%), RHAMM in $35 / 50(70 \%)$, NewRen60 in 25/35 (71\%), BAGE in 7/12 $(58 \%)$ and WT1 in $40 / 60(66 \%)$ of AML patients.

These antigens were evaluated for their differential mRNA expression in normal tissues using a normized tissue panel. The antigens BAGE and hTERT showed tumor-specific, MPP11, RHAMM, PRAME and WT1 highly tumor-restricted expression in leukemic cells. Real-time RT-PCR for PBMN of untreated AML patients and healthy volunteers revealed a tumorspecific expression of the antigens RHAMM, PRAME and WT1, the antigen MPP11 is overexpressed in leukemic patients.

Taken together, the antigens BAGE, hTERT, MPP11, PRAME, RHAMM and WT1 might be candidate target structures for immunotherapies of leukemia patients. Because of their simultaneous expression in AML, the design of a polyvalent vaccine is possible.

\section{1}

\section{Expression patterns of NK-cell markers in acute leukemias}

Weiss B. (1), Hoffmann M. (1), Anders C. (2), Uppenkamp M. (1)

(1) Medizinische Klinik A, (2) Institut für Hämostaseologie und Immunhämatologie, Klinikum der Stadt Ludwigshafen am Rhein

Immunophenotyping is part of the initial routine work up in acute leukemias, detecting lineage infidelity and maturaty of the neoplastic clone. These factors have great influence on therapy and prognosis, especiallly in acute lymphoblastic leukemia. Immunophenotyping can also render additional information with regard to special biological circumstances in cases of acute myeloid leukemia. Lineage infidelity is one of the most important factors. A frequent finding is in this respect the expression of markers that are specific for NK-cells, like CD16, CD56, CD57, and CD94. We report on the different patterns of expression of NK- cell markers in routinely detected acute leukemias with special emphasis on CD56.

in common acute myeloid leukemia, especially with myelomonocytic or monoblastic differentiation.

in acute lymphoblastic leukemia and lymphoblastic lymphoma, especially with T-cell phenotype.

in myeloid/natural killer cell acute leukemia, first reported by Scott and coworkers (1994), with morphological and immunophenotypic features of acute promyelocytic leukemia.

in myeloid/NK cell precursor acute leukemiawith aggressive clinical behaviour, lymphoblastoid morphology, often extramedullar manifestations and response to acute myeloid leukemia induction therapy. And

in blastic NK-cell lymphoma/leukemia with skin involvement, blastic morphology, lacking coexpression of myeloid markers and response to lymphoma-type chemotherapy. 


\section{AML-Clinical Research}

62

Early allografting during aplasia using modified conditioning therapy to induce and sustain remission in patients with high-risk acute myeloid leukemia

Bornhäuser M., Schaich M., Illmer T., Haack M., Schaekel U., Nettesheim O., Helwig A., Geissler G., Freiberg-Richter J., Babatz.J., Röllig C., Schuler U., Thiede C., Ehninger G.

Med. Klinik und Poliklinik I, Universitätsklinikum der TU Dresden

Objectives: The purpose of this study was to show whether conditioning therapy using fludarabine and reduced doses of alkylating agents can be performed during the aplastic phase after initial induction chemotherapy in patients with AML and high-risk cytogenetics or insufficient reduction of blast counts. The aim was to establish fast lymph-hematopoietic engraftment from related and unrelated donors in order to reduce the risk of relapse and induce graft-versus-leukemia effects.

Material and Methods: Seven patients with a median age of 45 years (3654) were included so far. Five patients had a high-risk karyotype (complex, inv 3 or $\mathrm{t}(3 ; 3)$ ). In 2 patients with normal karyotype no reduction of blast counts was achieved by induction therapy. Two to three weeks after induction with mitoxantrone/cytarabine/etoposide (MAV, n=2), MAV + amsacrine/cytarabine (MAMAC, $n=4$ ) or one additional cycle of Ida-FLAG $(\mathrm{n}=1) 5 \times 30 \mathrm{mg} / \mathrm{m}^{2}$ fludarabine were combined with either $8 \mathrm{mg} / \mathrm{kg}$ busulfan $(n=5)$ or $150 \mathrm{mg} / \mathrm{m}^{2}$ melphalan. Patients with unrelated donors received antithymocyte globulin (10 mg/kg ATG Fresenius). All patients received GCSF mobilized peripheral blood stem cells (PBSC). GvHD prophylaxis was performed with cyclosporine A (CSP). No growth factors were given. Four patients with suspected aspergillosis during induction treatment received 35 granulocyte transfusions from sibling donors.

Results: Grade 3 mucositis was documented in 5 patients. One patient experienced grade 3 liver toxicity. Rapid engraftment for neutrophils $(>0.5 \mathrm{Gpt} / 1$ on day 12 , range 10-16) and platelets (> $50 \mathrm{Gpt} / \mathrm{l}$ day 14 , range 11-28) was achieved. Acute GvHD grade I-III occurred in all patients. Chronic GvHD was documented in 4 patients with a follow-up of $>100$ days. One patient experienced pulmonary aspergillosis that resolved after treatment with amphotericine B. With a median follow-up of 20 months (range 2- 36) all patients are alive in remission. Complete donor chimerism was achieved after 2-3 weeks and is sustained in the myeloid and lymphatic compartment. Conclusions: We could demonstrate the feasibility of fludarabine-based conditioning therapy started before hematopoietic recovery after induction chemotherapy. Early allogeneic HSCT seems to be an effective strategy to achieve long-term remission in patients with high-risk acute myeloid leukemia. Without a potential sibling donor the search for an alternative donor should be initiated as soon as the results of cytogenetics are available.

\section{3}

\section{Treatment of acute myeloid leukemia (AML) in patients older than 60 years: report of the AML97-\#38 study of the east german hematology and oncology study group (OSHO)}

Hegenbart U., Krahl R., Kliem C., Rudolph M., Grommisch L., Pönisch W., Wedding U., Herold M., Subert S., Freund M., Fiedler F., Pasold R., Peter N., Meran J., Fasshauer E., Dachselt K., Grobe N., Assmann M., Kettner E., Steglich J., Huhn R., Schulze M., Schultze W., Ittel T., Klinkenstein C., Doepper J., Winkelmann C., Knauf W., Niederwieser D.

\section{Ostdeutsche Studiengruppe für Hämatologie und Onkologie (OSHO)}

Objectives: Patient selection plays a major role in the treatment of leukemia in elderly patients over 60a. In order to avoid biases, a study was designed to register all patients with AML at diagnosis. Subsequently, patients were treated following one of three protocols: a curative for patients eligible for chemotherapy, a palliative for patients with contraindications for intensive chemotherapy and a supportive for patients failing palliative treatment or contraindications for treatment with chemotherapy.

Methods: Curative treatment protocol consisted of one cycle of AraC 2 g/m iv on days $1,3,5,7$ and mitoxantrone $10 \mathrm{mg} / \mathrm{m}^{2}$ iv day $1-3$. Patients entering complete remission were treated with 2 consolidation courses $\left(240 \mathrm{mg} / \mathrm{m}^{2}\right.$ AraC day $1-5,10 \mathrm{mg} / \mathrm{m}^{2}$ mitoxantrone day $\left.1+2\right)$. Since $6 / 99$, patients with HLA-identical siblings were eligible for treatment with allogeneic hematopoietic stem cell transplantation (HSCT) after minimal conditioning as second consolidation. Palliative treatment consisted of idarubicin $10 \mathrm{mg}$ po day 1 in combination with thioguanin $40 \mathrm{mg}$ po day 1-5, AraC $80 \mathrm{mg} \mathrm{sc}$ day $1-5$ or etoposide $100 \mathrm{mg}$ po day 1-5. As supportive treatment transfusions were applied.

Results: Since 03/98 312 patients were enrolled in these protocols. Of those, 187 entered the curative, 96 the palliative and 29 the supportive arm. 117 patients (median age 65a; 60-80) with de novo AML, 70 patients (median age $68 \mathrm{a} ; 60-79$ ) with secondary AML receiving intensive chemotherapy and 78 patients (median age $75 \mathrm{a} ; 62-90$ ) receiving palliative treatment are evaluable to date. CR was reached in $86(73,5 \%, 95 \%$ CI:64,4-80,9) patients with de novo and in $41(58,6 \%, 95 \% \mathrm{CI}: 46,2-70,2)$ patients with secondary AML with an early death rate of $12 \%$ and $18,6 \%$ (de novo AML: $n=117$, median OS 13 months, OS (2 years) $0,33 \pm 0,05$; secondary AML: $\mathrm{n}=70$, median OS 12 months, OS (2 years) $0,22 \pm 0,08$ ). Median overall survival (OS) of the 78 patients treated in the palliative arm was 52 days. Finally, the median OS of patients in the supportive arm $(n=22)$ was 20 days.

Conclusion: A high proportion of elderly patients with AML can be treated in the curative arm and high CR rate with acceptable early death rate can be achieved. To improve results, a phase II study for AML patients $>60$ years to compare consolidation with chemotherapy or allogeneic related HSCT after minimal conditioning was initiated.

64

High correlation of the proteome-set in bone marrow aspirate and peripheral blood samples in AML patients with over 80 percent blast cells in both compartments

Hütter G. (1), Letsch A. (1), Poland J. (2), Schnölzer M. (3), Sinha P. (2), Thiel E. (1)

(1) Medizinische Klinik III, Hämatologie, Onkologie und Transfusionsmedizin, Universitätsklinikum Benjamin Franklin, Berlin, (2) Institut für Laboratoriumsmedizin und Pathobiochemie, Universitätsklinikum Charité, Berlin, (3) Zentrale Proteinanalytik, Deutsches Krebsforschungszentrum, Heidelberg

Objectives: Proteins, rather than genes or mRNAs, represent the key players in the cell. The proteome determines the cellular phenotype and its plasticity in response to external signals. To investigate proteomic alterations during the treatment of human acute myeloid leukemia, peripheral blood samples are much more easier to collect than bone marrow aspirate. But it is unknown, whether the protein expression of blasts in the bone marrow are comparable to peripheral blood.

Material and Methods: Three patients with newly diagnosed AML and the presence of over $80 \%$ blast in bone marrow aspirate and peripheral blood were examined. After purification with Ficoll-gradient an IEF two-dimensional electrophoresis was performed. Protein identification was related to the data of 130 proteins analyzed previously in a human gastric cancer cell line.

Results: For each patient 80 proteins were evaluable in the sample from peripheral blood and bone marrow. Overall, most protein spots showed no change between blood and bone marrow. Only in three molecules namely: phosoglycerolmutase, thioredoxin reductase, and annexin 6 , changes of the protein expression were observed. These changes were not connected with the origin of the sample.

Conclusions: We can therefore conclude, that the proteome-set of leukemic blast cells from marrow and blood, does not differ substantially when drawn from AML patients with over 80 percent blast cells in both compartments.

\section{5}

No prognostic role of CD82 expression in acute myeloid leukemia

Jänike M. (1), Illmer T. (2), Schenkluhn A. (1), Ehninger G. (2), Neubauer A. (1), Burchert A. (1)

(1) Klinik für Hämatologie/Onkologie/Immunologie der Philipps Universität Marburg, (2) Medizinische Klinik und Poliklinik I, Uniklinikum Carl Gustav Carus, TU Dresden

CD82 (KAI1) is a member of the tetraspanin family. Loss or down-regulation of CD82 expression was shown to be a poor prognostic marker in patients suffering from breast-, prostate-, pancreatic-, bladder- or colon cancer. In contrast, we have previously shown that CD82 is overexpressed in the leukemic fraction of the vast majority of acute myeloid leukemia (AML) patients. Based on these findings, we asked whether the level of CD82 expression is of prognostic value also in AML. CD82 expression was quantitated by real time polymerase chain reaction in 77 AML patients treated within the large multicenter AML-SHG 96 trial. The study population was divided according to their CD82 expression status into high and low ex- 
pressors, both of which displayed significantly higher CD82 expression levels than healthy donors $(n=14)$. The level of CD82 expression correlated with the percentage of blasts at diagnosis. However, there was no statistically significant correlation between the level of CD82 expression and either of the following parameters: age, white blood count at diagnosis, CD34 expression. There was also no correlation between CD82 expression and remission rate $(\mathrm{p}=0.821)$ regardless of age. Finally, CD82 expression level had no impact on overall survival $(\mathrm{p}=0.18)$ or disease free survival (DFS) $(\mathrm{p}=0,08)$. However, in the group of patients below 60 years ( 14 low expressor, 16 high expressor) there was a statistically significant better DFS in the low expressor group $(\mathrm{p}=0.028)$.

We show here that CD82 does not, in contrast to solid tumors, play a prognostic role in AML. Consequently, there appears to be a significantly different biological role for the expression of the tetraspanin CD82 in malignant hematopoiesis as opposed to an expression in solid malignancies.

\section{6}

\section{Do patients with acute promyelocytic leukemia (APL) really have a good prognosis? Risk factors for early death and complications in the era of all-trans retinoic acid (ATRA) Results of the AMLCG}

Lengfelder E. (1), Saussele S. (1), Haferlach T. (2), Schoch C. (2), Weisser A. (1), Ludwig W.D. (3), Staib P. (4), Heinecke A. (5), Sauerland M. (5), Berdel W. (6), Wörmann B. (7), Hehlmann R. (1), Hiddemann W. (2), Büchner Th. (6) for the AML Cooperative Group (AMLCG)

(1) III. Medizinische Klinik, Klinikum Mannheim, Universität Heidelberg, (2) Medizinische Klinik III, Klinikum Großhadern, Universität München,

(3) Charité Campus Berlin-Buch, Robert-Rössle-Klinik, Universität Berlin,

(4) Klinik I für Innere Medizin, Universität zu Köln, (5) Institut für

Medizinische Informatik und Biomathematik und (6) Medizinische Universitätsklinik A, Universität Münster, (7) Städtisches Klinikum Braunschweig.

Objectives: On average, $10 \%$ of patients with APL included in prospective therapy studies still die in the early phase of treatment. In order to identify risk factors for early death (ED) and to reduce the early mortality, we analyzed rate and type of complications and time and cause of ED in newly diagnosed APL between the start of therapy and the recovery of blood cell counts after induction.

Methods: The patients were treated by the German AMLCG with ATRA and an intensified double induction (TAD/HAM) including $\mathrm{HD}$ ara-C, followed by consolidation and maintenance therapy. Patients $\geq 60$ years (y) received the second cycle (HAM), if complete remission (CR) was not reached after the first cycle (HAM necessary in $13 \%$ of the patients $\geq 60 \mathrm{y}$ ). Results: Since November 1994, the results of induction therapy are evaluable in 106 patients (median age $44 \mathrm{y}$, range 16 to 82 ). The rate of CR of all patients was $89 \%$, of partial remision (PR) $1 \%$ and of ED $10 \%$. The ED rate and the complications differed significantly dependent on age and on the initial white blood cell (WBC) count:

\begin{tabular}{|c|c|c|c|c|}
\hline & $<60 \mathrm{y}$ & $\geq 60 y$ & $\begin{array}{l}\text { WBC } \\
<5000 / \mu 1\end{array}$ & $\begin{array}{l}\text { WBC } \\
\geq 5000 / \mu 1\end{array}$ \\
\hline $\mathrm{n}$ & 82 & 24 & 70 & 36 \\
\hline CR/PR (\%) & 94 & 75 & 97 & 75 \\
\hline $\mathrm{ED}(\%)$ & $6^{*}$ & $25^{*}$ & $3 * *$ & $25^{* *}$ \\
\hline Bleeding $(\%)^{+}$ & 15 & 19 & $9 * * *$ & $30 * * *$ \\
\hline Infection $(\%)^{+}$ & 39 & 38 & 39 & 39 \\
\hline Cardiac dysfunction $(\%)^{+}$ & $1^{\#}$ & $26^{\#}$ & $2^{\# \#}$ & $18^{\# \#}$ \\
\hline ATRA syndrome $(\%)$ & 25 & 35 & 25 & 32 \\
\hline
\end{tabular}

+ WHO grade $\geq 3, * \mathrm{p}=0.02, * * \mathrm{p}=0.0008, * * * \mathrm{p}=0.009, \# \mathrm{p}=0.001, \# \# \mathrm{p}=0.009$

WHO grade $\geq 3$ hepatotoxicity, diarrhea, neurotoxicity and nephrotoxicity were seen in $8 \%, 16 \%, 1 \%$ and $2 \%$ of all patients, respectively. Most frequent symptoms of the ATRA syndrome were fever (41\%), pulmonary infiltrates $(59 \%)$, pleural/pericardial effusions $(49 \%)$ and weight gain $(51 \%)$ The median duration of ATRA therapy until ATRA syndrome was 14 (3 to 36) days. Thromboembolic complications were observed in 3 patients on days 3,22 and 24 of the ATRA therapy, respectively (one sinus cavernosus thrombosis, two myocardial infarctions). 7 of 11 (64\%) ED occurred within the first week of therapy. Causes of death were bleeding $(n=5)$, multiorgan failure $(n=2)$, cardiac failure $(n=2)$, myocardial infarction $(n=1)$, infection $(n=1)$. The median initial WBC count of the patients with ED was $26000 / \mu 1$ (range 1200 to 95000 ).

Conclusions: In APL patients with initially higher WBC count and of older age, the chance of cure is reduced by a high risk of ED, mainly due to bleeding and cardiovascular problems. As ATRA may play a role in the pathogenesis of both complications, the possible benefit of prophylaxis with corticosteroids should be investigated.

\section{Treatment of relapsed acute myeloid leukemia with imatinib}

Mohr M., Kratz-Albers K., Oenaldi-Mohr D., Buechner Th., Berdel W.E., Serve $H$.

UK-Münster; Innere Medizin A; Hämatologie, Onkologie und Pneumologie

Imatinib is a selective inhibitor of tyrosine kinases, like c-abl, bcr-abl, c-ki and PDGFR. Clinical application of imantinib in bcr-abl positive CML as well as in bcr-abl positive ALL represents the most promising improvement in the treatment of hematologic malignancies in the last years. Since most AML blasts express considerable amounts of c-kit, targeting of this receptor tyrosine kinase with imantinib seems feasible. Here, we report about the treatment of relapsed AML patients with this substance. Eight patients who were diagnosed with AML and who were refractory or relapsed to prior chemotherapy were treated with a dose of $400 \mathrm{mg}$ Imatinib daily. Prior to the first imatinib application total blast count in bone marrow and in periphera blood count was measured. During the ongoing study the patients differential blood count was monitored. A follow-up bone marrow aspiration was performed when either peripheral blast clearance was reached or the treatment had to be stopped.

A reduction of the bone marrow blast count appeared in four of eight patients $(50 \%)$. In one of these patients we could observe a complete blas clearance $(12.5 \%)$. This Patient also showed a decrease of the peripheral blast count from max. $70 \%$ (d7) to $0 \%$ and an increase in relative and absolute neutrophile count from $8 \%(9001 \mathrm{luk} / \mu \mathrm{l})$ to $85 \%(3500 \mathrm{leuk} / \mu \mathrm{l}) \mathrm{max}$ on day 48 . However during this period the patient remained transfusion dependend for erythrocytes and platelets. Two patients developed CTC $3-4^{\circ}$ neutropenia, in one patient the therapy was stopped whereas in the other case dose reduction was performed.

In conclusion, response to monotherapy with imatinib could be seen in $4 / 8$ patients. Although the clinical benefit for most of these heavily pretreated patients was marginal, these results justify the conduction of larger clinical trials to evaluate a possible role for drugs targeting tyrosine kinases in AML.

\section{8}

\section{Unusual extramedullary presentations of acute myelogenous leukemia}

Pelz H.F., Wirths S., Hebart H., Kanz L., Denzlinger C.

\section{Uni-Klinik Tübingen}

In most cases, extramedullary manifestations of AML are associated with advanced systemic disease or relapse. Several reports are available on single cases of leukemic infiltrates strictly confined to the skin (,leukemia cutis"), but manifestations at other single extramedullary sites have only very rarely been observed. We report two such unusual cases of extramedullary AML, one confined to a group of cervical lymph nodes (pat. 1) and one confined to the testis (pat. 2)

Pat. 1, a 67 years old female patient presented with a swollen cervica lymph node. She felt well, without any signs of infection, bleeding tendency or other symptoms. Laboratory tests and bone marrow examination were normal. CT showed enlarged lymph nodes on the left side of the neck but no other abnormalities. A lymph node was removed and revealed AML M4 PET disclosed high metabolic activity on the left side of the neck without any further sites involved. The patient was treated with idarubicin and cytarabine $\left(, 3+7^{*}\right)$. CT and PET showed complete remission. She was subjected to another course of idarubicin and cytarabine $\left(,, 3+4^{*}\right)$ and consolidation therapy with high-dose cytarabine. Due to diarrhea following chemotherapy and prolonged recovery the patient did not receive further treatment. She remains in complete remission 12 months after initial diagnosis. Pat. 2, a 42 years old male patient presented with painful swelling of one testicle. Histological examination after semicastratio revealed AML M1. Laboratory tests and bone marrow examination were normal. He was treated with idarubicin and cytarabine $\left(, 3+7^{*}\right)$. During neutropenia the patient developed hepatic candidiasis and chemotherapy had to be discontinued. There is no evidence of leukaemia 18 months after initial diagnosis.

Prognosis of aleukemic leukemia is generally regarded as poor: in most cases systemic disease develops rapidly and response rate and response duration appear to be less favourable. CR durations of $12+$ and $18+$ months observed in our patients with primary lymph node or testicular AML are remarkable. Possibly with these sites might be less dismal than with aleukemic leukemia cutis. 


\section{Poor response to therapy with STI 571 in two patients with recurrent or refractory c-kit positive acute myelogenous} leukemia (AML)

Perz. J., Krasniqi F., Steiger S., Krämer A., Ho A.D.

Medizinische Klinik und Poliklinik V, Abteilung für Hämatologie, Heidelberg

Aims: STI-571 is a selective tyrosine kinase inhibitor targeting bcr-abl, PDGFR and c-kit. Recently succesful treatment of a patient with c-kit positive AML refractory to standard chemotherapy has been reported. We administered STI 571 to two patients with recurrent (Patient 1) or refractory (Patient 2) AML in order to assess the efficacy of the drug. Patients: Patient 1 (61 years, female) presented with second relaps of secondary AML after mylodysplasia. C-kit was expressed on $90 \%$ of blasts and blasts karyotype was normal. With low-dose cytarabine first remission was achieved, with oral idarubicine, followed by etopiside/ mitoxantrone patient came to second remission. In second relaps she did not responde to cytarybine. Patient 2 (61, male) suffered from AML secondary to chronic myelomonocytic leukemia. C-kit was expressed on $90 \%$ of blasts and cytogenetic revealed trisomy 8. Patient 2 did not respond to induction chemotherapy with cytarabine/doxorubicine and to mercaptopurine.

Methods: STI 571 was given orally at daily dose of $600 \mathrm{mg}$ in Patient 1 for 28 days and in Patient 2 for 9 days. No severe side effects were observed. Results: Patient 1 presented leukocytosis of $60 / \mathrm{nl}$ with $90 \%$ blasts in peripheral blood at the beginning of therapy. To reduce the blast count she got additionally $10 \mathrm{mg}$ mitoxantrone at day 1 . At day 14 leukocytes decreased to $15,2 / \mathrm{nl}$ but still $87 \%$ peripheral blasts were found. At day 24 leukocytes rose to $114 / \mathrm{nl}$ with $97 \%$ peripheral blasts and she got additional $10 \mathrm{mg} \mathrm{mi}-$ toxantrone again. STI 571 was given until day 29 , when she died after cerebral hemorrhagy. In patient $280 \%$ bone marrow blasts were found at the beginning of STI 571 therapy and peripheral leukocyte count was $24 / \mathrm{nl}$ without peripheral blasts. At day 10 leukocyte count rose to $50,8 / \mathrm{nl}$ with $14 \%$ peripheral blasts, so STI 571 therapy has been stopped. Patient 2 did not respond to further therapies with idarubicine and mitoxantrone and succumbed 112 days later to disease progress with peripheral bleedings and renal failure.

Conclusions: In two AML patients no reduction of blast count was seen after STI 571 therapy. We postulate that c-kit receptor and the depending signal pathway could not be inhibited by the drug. This finding suggests that c-kit receptor pathway is deregulated in AML blasts. Further investigations with AML cell lines are necessary to prove this hypothesis.

\section{0}

\section{Successful combination of gemtuzumab ozogamicin and minimal conditioning before allografting in patients with relapsed myelogenous leukemia}

Prange-Krex G., Jenke A., Babatz.J., Helwig A., Haack M., Thiede Ch., Ehninger G., Bornhäuser $M$.

\section{Universitätsklinikum Dresden, Medizinische Klink und Poliklinik I}

Treatment with gemtuzumab ozogamicin (mylotarg ${ }^{\circledR}$ ) is an emerging therapeutic option for older patients with relapsed acute myelogenous leukemia (AML). New strategies of minimal conditioning might allow to introduce this compound into the conditioning therapy of patients with high-risk AML.

Patient 1: A 40 year-old woman with acute myeloid leukemia (FAB M2, normal karyotype, aberrant CD 7 coexpression) experienced early relapse after induction chemotherapy and also failed a subsequent autologous stem cell transplantation after conditioning with Busulfan $16 \mathrm{mg} / \mathrm{kg}$. She had suffered from chronic renal insufficiency since the start of induction chemotherapy.

Patient 2: A 19 year old patient with AML (FAB M4, FLT3 activating mutation) relapsed 3 months after allogeneic bone marrow transplantation from a matched sibling donor.

Since the preceding high-dose chemotherapy did not allow for a second conventional conditioning therapy in both patients, they received $6 \mathrm{mg} / \mathrm{m}^{2}$ and $3 \mathrm{mg} / \mathrm{m}^{2}$ mylotarg three and two weeks before transplantation, respectively. Minimal conditioning was performed with 3 x $30 \mathrm{mg} / \mathrm{m}^{2}$ fludarabine (day -3 to -1 ) and 2 Gy total body irradiation (day 0). G-CSF mobilized peripheral blood stem cells (PBSC) were infused on day 0. Patient 1 received PBSC from a matched unrelated volunteer donor. PBSC from the original sibling donor were infused in patient 2. Patient 1 received CsA and mycophenolate mofetil (day 0-35) as GvHD prophylaxis. No prophylactic immunosuppression was administered in patient 2 .
Rapid donor engraftment could be documented and both patients remain in complete remission after a follow-up of 10 and 9 months with limited chronic GvHD. The major toxicity observed was acute GvHD grade II. We observed no liver toxicity $>$ grade 2 . Although the patient number is still limited, we conclude that mylotarg seems to be a feasible adjunct to minimal conditioning before allogeneic transplantation of hemopoietic stem cells from related and unrelated donors. This strategy might help to improve the efficacy of allografting after minimal conditioning in patients with advanced AML.

71

\section{Risk adapted therapy in acute promyelocytic leukemia: AIDA} 2000 - a AML SHG study group treatment protocol

Schäkel U. (3), Schuler U. (3), Wandt H. (8), Aulitzky W.E. (11), Bodenstein H. (7), Fetscher S. (9), Schalk K. (6), Fiedler F. (2), Gramatzki M. (4), Knigge O. (1), Schmitz N. (5), Wagner T. (10), Schaich M. (3), Thiede C. (3), Ehninger G. (3)

(1) Universitätsklinikum Charite Berlin, (2) Krankenhaus Küchwald Chemnitz, (3) Universitätsklinikum Dresden, (4) Universitätsklinikum Erlangen, (5) Allgemeines Krankenhaus St. Georg Hamburg, (6) St. Vincent-Krankenhaus Limburg /Lahn, (7) Klinikum Minden, (8) Städtisches Klinium Nürnberg, (9) Städtisches Krankenhaus Süd Lübeck, (10) Universitätsklinikum Lübeck, (11) Robert-Bosch-Krankenhaus Stuttgart

Since November 2000 we registered 27 patients ( 14 pat. $\leq 60$ ys, 13 pat. > $60 \mathrm{ys}$ ) with newly diagnosed acute promyelocytic leukemia. Based on different international studies we established a risk adapted treatment protocol according to age ( $\leq 60 \mathrm{ys} />60 \mathrm{ys})$ and initial leucocyte count $(<=/>10$ GPT/l). In the induction therapy ATRA $45 \mathrm{mg} / \mathrm{m}^{2} / \mathrm{d}$ until CR but not more than 90 days and idarubicin $12 \mathrm{mg} / \mathrm{m}^{2} / \mathrm{d}$ for 4 days were administerd. The 1 . consolidation therapy consists of daunorubicin $60 \mathrm{mg} / \mathrm{m} / 2 \mathrm{~d}$ for 3 days in patients $\leq 60 \mathrm{ys}, 45 \mathrm{mg} / \mathrm{m}^{2} / \mathrm{d}$ for 3 days in patients $>60 \mathrm{ys}$ and additionally Ara-C in patients with initially leucocytes $>20 \mathrm{GPT} / 1200 \mathrm{mg} / \mathrm{m}^{2} / \mathrm{d}$ as continuous infusion for 7 days in patients $\leq 60 \mathrm{ys}$ and $100 \mathrm{mg} / \mathrm{m}^{2} / \mathrm{d}$ in patients > $60 \mathrm{ys}$ ). As 2. consolidation course mitoxantrone $10 \mathrm{mg} / \mathrm{m}^{2} / \mathrm{d}$ for 3 days and Ara-C in patients with initially leucocytes $>20 \mathrm{GPT} / \mathrm{l}$ twice daily $3 \mathrm{~g} / \mathrm{m}^{2} / \mathrm{d}$ for 4 days in patients $\leq 60 \mathrm{ys}$ and $1 \mathrm{~g} / \mathrm{m}^{2} / \mathrm{d}$ in patients $>60 \mathrm{ys}$ were administered. As maintenance therapy over 2 years patients are treated with 6-mercaptopurine daily $90 \mathrm{mg} / \mathrm{m}^{2} / \mathrm{d}$, methotrexate weekly $15 \mathrm{mg} / \mathrm{m}^{2} / \mathrm{d}$ and intermittend ATRA $45 \mathrm{mg} / \mathrm{m}^{2} / \mathrm{d}$ over 15 days every 3 months. The main objectives of the treatment protocol are to investigate 1. the efficiency and toxicity of the treatment plan, 2. the patients outcome with the prognosis dependent reduction of the intensity of consolidation therapy and whether the high risk group of patients seems to benefit from more intensive therapy, 3. the detection of kinetics of the PML-RAR $\alpha$ transcript for further therapeutical decisions and 4. correlating other pretreatment factors with the risk of relapse.

At time of evaluation $92 \%(24 / 26)$ of all patients and $100 \%$ of patients $\leq$ 60 ys achieved complete remission. 3 patients $>60$ ys died: 1 patient during induction therapy due to tumor lysis syndrome and haemorrhagic disorder, 1 patient after induction therapy and another during consolidation therapy due to sepsis. In $19 \%$ of the patients the initial white blood count was $>10$ GPT/I. None of the other patients relapsed and all are still in continued complete remission.

Comparable to other trials these first results indicate a high effectiveness in the treatment of acute promyelocytic leukemia.

\section{2}

\section{AML-patients with complex karyotype aberrations have a significant decreased chemosensitivity in-vitro}

Schinköthe T. (1), Schoch C. (2), Diehl V. (1), Staib P. (1)

(1) Klinik I für Innere Medizin, Uniklinik Köln, Köln, (2) Labor für spezielle Leukämiediagnostik, Universität München, München

Patients with acute myeloid leukaemia (AML) and complex karyotype aberrations show a poor prognosis. Only $45 \%$ of these patients reach complete remission $(\mathrm{CR})$ compared to an overall $\mathrm{CR}$ rate of $68 \%$. After a TAD-TAD treatment patients with a complex karyotyp showed a CR rate of $23 \%$ vs. $63 \%$. Changes in chemosenstivity in patients with complex karyotype aberrations might be the reason for these differences.

Material and Methods: For the in-vitro chemosensitivity testing we used the differential staining cyto-toxicity (DiSC) assay. Freshly isolated leukemic cells were incubated in duplicate with different amounts of ara-C, daunorubicin, idarubicin, mitoxantrone for four days $\left(37^{\circ} \mathrm{C}, 5 \% \mathrm{CO} 2\right)$. Complex chromosome aberrations were detected by fluorescence in-situ hybridisation 
(FISH) and defined as three or more numerical and/or structural chromosome aberrations excluding translocations $\mathrm{t}(8 ; 21)(\mathrm{q} 22 ; \mathrm{q} 22), \mathrm{t}(15 ; 17)(\mathrm{q} 22$ q11-q12) and inv(16)(p13q22).

Results: Samples from 129 patients with AML were tested. 26 patients showed a complex (20\%) and 46 a non-complex (36\%) karyotype aberration whereas 57 patients had a normal karyotyp (44\%). Comparing the invitro chemosensitivity, the patients with a complex karyo-typ aberration showed a significantly decrease in sensitivity against ara- $\mathrm{C}(\mathrm{p}=0.046)$, daunorubicin $(\mathrm{p}=0.019)$ and idarubicine $(\mathrm{p}=0.019)$. No significant changes were observed in case of mitoxantrone $(\mathrm{p}=0.162)$.

Conclusions: Our data demonstrate a close correlation between complex karyotyp aberra-tions and a decreased in-vitro chemosensitivity against ara-c and daunorubicin, that are in-cluded in treatments like TAD. In contrast, chemosensitivity-testings are able to reveal drugs like mitoxantrone which showed no significant differences in chemosensitivity in cells with or without complex karyotyp aberrations. Chemosensitivity-tests might be usefull to improve future clinical treatments of patients with a high risk of failure of standard treatment.

\section{3}

\section{Complete remission of third recurrence of acute myleoid leukemia after treatment with imatinib (STI-571)}

Schittenhelm M., Aichele O., Brümmendorf T., Kanz L., Denzlinger C.

Medizinische Klinik, Abteilung für Haematologie / Onkologie / Rheumatologie und Immunologie, Eberhard-Karls-Universität Tübingen

Novel well tolerated treatment strategies are urgently needed for elderly patients with acute myeloid leukemia (AML). Imatinib (STI-571) is a possible candidate drug for a subgroup of these patients who have c-kit positive disease.

We report the case of a 76-year old female patient with third relapse of AML who was successfully treated with imatinib. First presentation was in October 1998 with AML M5. First and second complete remission (CR1 and 2) were obtained with conventional induction and consolidation treat ment (induction and consolidation treatment for CR1 included multidrug resistance modulator PSC 833); CR3 was obtained with gemtuzumab ozogamicin followed by maintenance with low-dose interleukin-2. Durations of CR1, 2 and 3 were 14, 6, and 12 months. The decision to try imatinib in third relapse was guided by bright expression of c-kit on the patient's blasts. Treatment with imatinib, $600 \mathrm{mg} /$ day, was well tolerated. On day 33 dose was reduced to $400 \mathrm{mg} /$ day for pancytopenia and three days later treatment with imatinib was stopped completely for pneumonia. The patient recovered with i.v. antibiotics and antimycotics and s.c. G-CSF Reevaluation of bone marrow on day 24 and day 51 (15 after end of treatment) demonstrated reduction of blasts to $19 \%$ and $0 \%$ and absence of c-ki positive cells. Absence of malignant blasts from bone marrow was again confirmed on day 45 after the end of treatment when peripheral blood cells had recovered, indicating that a third complete remission has been achieved in this patient. Ten and fourteen weeks after end of treatment the patient had normal blood counts and treatment with imatinib $400 \mathrm{mg} /$ day was started again with the intention to prolonged remission duration.

This is the first report demonstrating that imatinib can induce complete remission in relapsed c-kit positive AML in an elderly patient. Prolonged cytopenia remains a considerable problem indicating that norma hematopoiesis might not independent of the signalling cascades inhibited by imatinib. Nevertheless our report supports further study of this drug in c-kit positive AML.

Extramedullary initial manifestations of acute myeloid leukemia (AML)

\section{Schmitt-Graeff A., Kvasnicka H.M., Thiele J.}

(1) Pathologisches Institut, Albert-Ludwigs-Universität Freiburg, Germany, (2) Institut für Pathologie, University of Cologne, Germany

Extramedullary myeloblastic tumors, so-called myelosarcomas (granulocytic sarcomas, chloromas) have been reported only sporadically in the pertinent literature which reflects their rather infrequent occurrence. These lesions may accompany or signal relapse of acute myeloid leukemia (AML) or coincide with blastic transformation of a chronic myeloproliferative disorder. However, even more rarely primary myelosarcomas may preceede AML by months or years or may be associated with myelodysplastic syndromes (MDS) that never progress to full-blown leukemia. In a retrospective evaluation a clinicopathological study on these latter two variants of isolated extramedullary manifestations of AML was performed to elucidate certain aspects of site involvement and histopathology by application of enzyme- and immunohistochemistry. For this reason, we selected 11 patients presenting with a myelosarcoma in combination with MDS and 6 patients revealing only uncharacteristic reactive changes of the bone marrow. Of these patients 14 developed AML following an observation time of 3 years. Focal leukemic infiltrates were most often localized in the oral mucosa, skin, retroperitoel soft tissue, small intestinal mucosa, testis, pleura-lungs These lesions were usually regarded by the clinicians as putative malignan lymphomas unless immunohistological evaluation in particular lysozyme immunoperoxidase and chloroacetate esterase reaction as well as CD68, CD43 and CD34, CD56 monoclonal antibodies proved their true nature. Although at that time bone marrow findings were inconclusive a straightforward diagnosis was reached by considering the possibility of a (primary) myelosarcoma in these patients.

\section{5}

Resistance to treatment in primary acute myeloid leukemia is not due to a dysfunctional apoptotic apparatus per se

Schneiderat P., Zimmermann I., Fiegl M., Schoch C., Hiddemann W., Braess $J$.

\section{Medizinische Klinik III, Klinikum Grosshadern der LMU München}

Objectives: Deficiencies or structural defects of the apoptotic machinery have been postulated as a potential mechanism for a broad resistance of acute myeloid leukemia (AML) blasts towards cytotoxic therapy comprizing chemotherapeutic agents with diverse pharmacodynamic principles but also cell-mediated cytotoxicity of the graft-versus-leukemia effect e.g. in the setting of allogeneic transplantation. This hypothesis was systematically tested in the present investigation by functionally analysing early, intermediate and late events of the apoptotic process in primary AML blasts following activation of the intrinsic and extrinsic pathway of apoptosis (VP16 and AraC as DNA damaging agents, FAS-ligand as an activator of the death receptor pathway).

Material and Methods: Early events such as the apoptotic volume decrease (AVD) were measured by the micro-culture kinetic (MicK) assay, downstream effects were assessed by measurement of caspase 3 activation and final loss of viability (LOV) was measured by a tetrazolium based assay (WST).

Results: Samples of 31 patients with diagnosis of AML were investigated (karyotypes - favourable 4, normal 16, unfavourable 6, unknown significance 4, not done 1). Activation of the extrinsic pathway by FAS-ligand was unable to induce apoptosis in primary AML - instead the proapoptotic signal was shown to "fade" already in the early phase of the apoptotic sequence. However, activation of the intrinsic pathway induced severe cytotoxicty in all tested samples that showed the characteristic features of typical apoptosis with a prominent apoptotic volume decrease (blebbing) in the early phase, significant increases in caspase 3 activity (intermediate or effector phase) and breakdown of cellular energy production in the late phase of apoptosis. These characteristics did not differ between AML with prognostically favourable versus unfavourable karyotypes or between clinically responding versus refractory AML - indicating that a functional apoptotic apparatus is present even in the unfavourable subgroup of AML.

Conclusions: Hence, our data indicate that the mechanism for a broad clinical resistance is not so much a dysfunctional apparatus per se but rather the consequence of an antiapoptotic regulation that impedes an otherwise functional apoptotic machinery.

\section{6}

WHO-Category: AML with recurring chromosome abnormalities: FAB subtype, age distribution and incidence of additional genetic abnormalities in an unselected series of 1897 cytogenetically and moleculargenetically analysed AML

Schoch C. (1), Haferlach T. (1), Schnittger S. (1), Kern W. (1), Dugas M. (2), Hiddemann W. (1)

(1) Laboratory for Leukemia Diagnosis, Department of Internal Medicine III, Ludwig-Maximilians-University, Munich, (2) Department of Medical Informatics, Biometrics and Epidemiology, Ludwig-Maximilians-University, Munich

The WHO category AML with recurring chromosomal abnormalities in cludes 1 . AML with $\mathrm{t}(8 ; 21)(\mathrm{q} 22 ; \mathrm{q} 22) / \mathrm{AML} 1-\mathrm{ETO}, 2$. acute promyelocytic leukemia with $\mathrm{t}(15 ; 17)(\mathrm{q} 22 ; \mathrm{q} 12) /$ PML-RARA and variants, 3 . AML with $\operatorname{inv}(16)(\mathrm{p} 13 \mathrm{q} 22) / \mathrm{t}(16 ; 16)(\mathrm{p} 13 ; \mathrm{q} 22) / \mathrm{CBFb}-\mathrm{MYH} 11$ and 4 . AML with 11q23/MLL abnormalities. In an unselected series of 1897 AML (1632 de novo AML, 148 AML after an antecedent hematological disorder and 117 
therapy-related AML) 87 cases with $\mathrm{t}(8 ; 21), 99$ with $\mathrm{t}(15 ; 17), 87$ cases with $\operatorname{inv}(16) / t(16 ; 16)$ and 55 cases with $11 \mathrm{q} 23$ /MLL rearrangement were observed. Thirteen different partners of the MLL gene were identified. Al cases with inv(16)/t(16;16) showed a FAB subtype AML M4eo, in 70 cases with $\mathrm{t}(15 ; 17)$ an AML M3 and in 29 patients an AML M3v was diagnosed. In patients with $\mathrm{t}(8 ; 21) 67$ cases of AML M2, 5 of M1 and 1 of M4 were observed (no data on FAB subtype: 14). In AML with 11q23/MLL abnormalities AML M5a, M5b and M4 were the most common morphologies occurring in $82 \%$ but also M0, M1 and M2 cases were observed in $18 \%$ of cases. Additional cytogenetic abnormalities occurred in 69 cases $(79.3 \%)$ with $\mathrm{t}(8 ; 21), 44$ (44.4\%) with $\mathrm{t}(15 ; 17), 38(43.7 \%)$ with inv(16)/t $(16 ; 16)$ and in 20 patients $(36.3 \%)$ with $11 \mathrm{q} 23 /$ MLL rearrangement. The incidence of $\mathrm{t}(8 ; 21), \mathrm{t}(15 ; 17)$, inv(16)/t(16;16) and 11q23/MLL-abnormalities was sig nificantly higher in patients younger than 60 years in comparison to patients $>60$ years, $(7.1 \%$ vs. $2.3 \%, 7.4 \%$ vs $3.1 \%, 6.8 \%$ vs. $2.5 \%$ and $5.3 \%$ vs $0.8 \%$, respectively, for all $\mathrm{p}<0.0001)$. For MLL abnormalities, and inv(16) the incidence was significantly higher in t-AML than in de novo AML $(8.5 \%$ vs. $2.6 \%, p=0.0005 ; 11.1 \%$ vs. $4.5 \%, p=0.0016)$. For $t(8 ; 21)$ and $\mathrm{t}(15 ; 17)$ no significant differences in incidence were observed between de novo and t-AML $(6.8 \%$ vs. $4.8 \%, 2.6 \%$ vs. $5.9 \%)$. In conclusion, AML with recurring abnormalities as defined by the WHO classification account for $26.7 \%$ of AML under the age of 60 , but for only $8.7 \%$ of AML 60 years or older. In t-AML these abnormalities occur in $29 \%$ of cases. In future furthe cytogenetic categories such as trisomies, monosomies, deletions and other unbalanced recurring karyotype abnormalities should be considered for the classification of AML. Furthermore, molecular genetic defects including partial tandem duplications of the MLL gene, FLT3 length mutations and point mutations of genes (i.e.: AML1, CEBPalpha, FLT3) could be included to categorize a larger proportion of AML on a genetic basis.

\section{7}

Dramatic alterations in refractory and relapsed acute myeloid leukaemia (AML) in comparison to AML prior to treatment detected by combining SSH and CDNA microarrays

\author{
Staber P. (1), Hoefler G. (2), Schauer S. (2), Linkesch W. (1)
}

(1) Klinische Abteilung für Hämatologie, Med. Univ. Klinik Graz, (2) Institut für Pathologie, Karl-Franzens-Universität Graz

Acute myeloid leukaemia (AML) is a clonal proliferating disease of myeloid blasts with an unsatisfying prognosis. The poor survival is related to the high frequency of relapse or refractory disease. Comparing newly diagnosed AML prior to high dose chemotherapy (HDC) with refractory and relapsed AML after induction therapy (Cytarabin and Daunorubicin) and consolidation therapy (Cytarabin and Mitoxantron), we aimed to detect common mechanisms in the development of resistance to HDC in vivo.

Methods: Bone marrow biopsies with an at least $80 \%$ blast infiltration were fixed immediately. To enrich differentially expressed sequences we created an SSH (subtractive suppressive hybridisation)- library comparing a pool $(n=9)$ of newly diagnosed AML-samples prior to therapy to a pool $(n=9)$ of relapsed AML-samples. The resulting PCR products were printed on glass slides together with cDNAs of IMAGE clones of putative relevant genes described in the literature gaining highly specific microarrays covering 4100 genes. Validation of the hybridisation experiments was done by quantitative RT-PCR and immunohistochemistry.

Results: Microarray analysis of 28 samples identified 70 commonly, at leas twofold deregulated genes in the 2 investigated groups. The results firstly suggest a crucial role of the microenvironment for developing a relapsed or refractory disease. This is indicated for example by upregulation of factors affecting platelet aggregation, inflammation, iron-metabolism, cell-cell and cell-matrix interaction. In addition relapsed and refractory AML showed a highly significant activation of the MAP-Kinase cascade downstream to ras, leading to an increase of the proliferating fraction, as seen by immunohistochemistry using MIB-1 (Ki67 antigen). Apoptotic markers appeared markedly downregulated.

The combination of an SSH- library and cDNA microarrays provides a broad view on the relevant mechanisms in a certain disease and could serve as a proper alternative to full genome chip analysis.
The infusion rate of Ara-C has no impact on remission rate, toxicity and event free survival in patients with de novo or secondary acute myeloid leukemia (AML): Results of a phase III-trial

Uharek L., Mantovani L., Krahl R., Hasenclever D., Pönisch W., Herold M., Pasold R., Franke A., Freund M., Fiedler N., Peter N., Subert R., Meran J., Wedding U., Kämpfe E., Kettner E., Grobe N., Dölken G., Florschütz A., Schultze W., Ittel T.H., Dachselt K., Huhn R., Schmoll H.J., Opitz B., Schulze M., Aßmann M., Schott G., Steglich J., Schirmer V. Helbig W., Niederwieser D. for the East German Hematology and Oncology Group (OSHO)

Medizinische Klinik und Poliklinik II, Hämatologie-Onkologie, Universitätsklinik Leipzig

Based on a pharmacological model of intracellular ARA-C uptake, conversion to ARA-CTP and resulting drug kinetics, we have calculated the optimal infusion-rate (IR) for intermediate ARA-C. In 1997, a phase III-study was initiated to investigate whether the application of optimized ARA-C IR can lead to reduced toxicity at similar or superior response rates.

Study Design: Pts were randomized to receive Ara-C at two different IRs during induction and consolidation treatment: (1) Arm A / experimental: $1 \mathrm{x}$ $2 \mathrm{~g} / \mathrm{m}^{2} / \mathrm{d}$ Ara-C over 8 hours (IR $250 \mathrm{mg} / \mathrm{m}^{2} / \mathrm{h}$ ), (2) Arm B / standard: 2 x $1 \mathrm{~g}$ Ara-C over 3 hours (IR $333 \mathrm{mg} / \mathrm{m}^{2} / \mathrm{h}$ ). Induction and first consolidation consisted of Ara-C (day 1,3,5,7) in combination with an anthracycline (IDA $12 \mathrm{mg} / \mathrm{m}^{2}$ or MITOX $10 \mathrm{mg} / \mathrm{m}^{2} ; \mathrm{d} 1-3$ ). The dose was reduced by ever a day for the second consolidation. The third consolidation consisted either of allogeneic or autologous stem cell transplantation or of chemotherapy identically to second consolidation.

Results: From 02/97 to 04/02 434 pts entered the study. In $05 / 02$, the study was closed and unblinded. The present calculation is based on 310 pts who have completely ended the induction therapy. The median follow up is 22 (2-58) months. Overall, CR was reached in 169/232 pts with de novo AML (73\%;95\%CI:66\%-78\%) and 51/78 pts with secondary AML $(51 \% ; 95 \%$ CI:40\%-63\%). Early death rate was $8 \%$ and $15 \%$ in both groups No statistical significant differences were detected between arm A and B with regard to CR (66\% vs. $68 \%)$, early death rate ( $11 \%$ vs. $8 \%)$ and OS at 2 years $(0.40$ vs. 0.44$)$. A detailed analysis of toxicity data, hematological recovery and transfusion requirements also revealed no significant differences between both study arms. 30 (26\% of the 116 pts, who have completed the intended therapy) pts were allografted, 33 (28\%) were autografted, and $53(46 \%)$ have received a third chemotherapy consolidation. As expected, the underlying cytogenetic aberration turned out to be the most important prognostic factor.

Conclusion: The calculated optimized ARA-C IR is not superior to the standard (IR $333 \mathrm{mg} / \mathrm{m}^{2} / \mathrm{h}$ ) application mode of ARA-C with regard to effectiveness and toxicity. Overall results of both treatment arms are similar to those of other current AML trials and are clearly superior to previous trials of the OSHO.

79

Duration and nadir of leukopenia may be prognostic factors for the success of induction chemotherapy in AML

v. Lilienfeld-Toal M., Tiedge K., Hahn C., Schmidt-Wolf I.G.H., Glasmacher A.

Medizinische Klinik und Poliklinik I, Uniklinikum Bonn, Sigmund Freud Str. 25, 53105 Bonn

Objectives: It is an old but perhaps not well known clinical experience that patients with acute myeloid leukemia who maintain higher leukocyte counts after myelosuppressive chemotherapy are less likely to achieve remission. We now examined this phenomenon systematically.

Methods: 75 consecutive patients with newly diagnosed AML who received first line myelosuppressive induction chemotherapy in our hospital from October 1999 until December 2001 were evaluated retrospectively. Six patients had to be excluded ( 5 incomplete documentation, 1 no myelosuppressive chemotherapy). Of these 69 patients 32 were female and 37 were male with a median age of 60 (interqu. range 48-68) years. They received a standard induction therapy with idarubicin and cytarabine, some also received etoposide and all-trans-retinoic-acid.

Results: 46/69 (67\%) patients had less than 5\% blasts in the bone marrow after standard induction therapy (group 1) whereas 23/69 (33\%) had more than $5 \%$ blasts (group 2). Both groups had a similar duration of leukopenia below $1.0 \mathrm{G} / 1$ with a median duration of $16 \pm 7$ days for group 1 and $17 \pm$ 10 days for group 2 . However, there was a significant difference in the lowest number of leukocytes (nadir). Group 2 with residual blasts after 
chemotherapy had a median of $0.5 \pm 0.3 \mathrm{G} / 1$ for the lowest number of leukocytes, while leukocytes in group 1 fell to a median of $0.3 \pm 0.2 \mathrm{G} /$ $(\mathrm{p}=0.006)$. This resulted in a positive predictive value for achieving a reduction of blasts below $5 \%$ of 0.78 if the nadir fell below $0.5 \mathrm{G} / 1$ with a sensitivity of 0.83 and a specificity of 0.52 (table 1 ).

\begin{tabular}{llll}
\hline & Nadir & Nadir & \\
& $<0.5 \mathrm{G} / 1$ & $\geq 0.5 \mathrm{G} / 1$ & \\
\hline Blasts $<5 \%$ (group 1) & 38 & 8 & 46 \\
Blasts $>5 \%($ group 2) & 11 & 12 & 23 \\
\hline & 49 & 20 & \\
\hline
\end{tabular}

There was also a significant difference in the duration of leukocytes below $0.5 \mathrm{G} / 1$ (group 1: $8 \pm 6$ days, group 2: $5 \pm 7$ days, $\mathrm{p}=0.025$ ). A duration of neutropenia below $0.5 \mathrm{G} / 1$ of at least 2 days yielded a positive predictive value of 0.81 with a sensitivity of 0.76 and a specificity of 0.65 for achieving a reduction of blasts below $5 \%$ (table 2 ).

\begin{tabular}{llll}
\hline & $\begin{array}{l}\text { Nadir }<0.5 \mathrm{G} / 1 \\
\geq 2 \mathrm{~d}\end{array}$ & $\begin{array}{l}\text { Nadir }<0.5 \mathrm{G} / 1 \\
<2 \mathrm{~d}\end{array}$ & \\
\hline Blasts $<5 \%$ (group 1) & 35 & 11 & 46 \\
Blasts $>5 \%($ group 2) & 8 & 15 & 23 \\
\hline & 43 & 26 & \\
\hline
\end{tabular}

Conclusion: These results show that patients with AML who had only 1 day of leukopenia below $0.5 \mathrm{G} / 1$ or whose leukocyte count did not fall below 0.5 $\mathrm{G} / 1$ at all had a lower remission rate. It will be of great interest to characterise the remaining leukocytes in leukopenia and to examine their relevance in further prospective studies.

This study was supported by Leukämie Initiative Bonn.

\section{0}

\section{Acute myeloid leukaemia (AML) after hypereosinophilic syndrome with response to imatinib mesylate (glivec): possible molecular mechanism}

von Bubnoff N. (1), Sandherr M. (1), Schwarz K. (1), Schneller F. (1), Gschaidmeier H. (2), Peschel Ch. (1), Duyster J. (1)

(1) III. Medizinische Klinik und Poliklinik, Klinikum rechts der Isar der Technischen Universität München, Germany, (2) Clinical Research and Development, Novartis Pharma GmbH, Nürnberg, Germany

We present a case of a 49-year old male patient with AML and extramedullary disease. Four years earlier, a diagnosis of hypereosinophilic syndrome was made. Histologic examination of a pathological fracture of the left humerus revealed AML with eosinophilic differentiation. Blastoid cells stained positive for cKit. At that time, the white blood cell count was $154 \mathrm{G} / 1$ with $83 \%$ eosinophils and $1 \%$ blastoid cells. The patient received two courses of ICE without response. Salvage treatment with HAM was administered, and a search for a matched unrelated stem cell donor (MUD) was initiated. A bone marrow specimen again showed refractory disease. The patient experienced nausea and vertigo. Magnetic resonance imaging and examination of cerebrospinal fluid confirmed CNS-involvement. After identification of a MUD the patient was scheduled for allogenic stem cell transplantation. Due to progressive disease receiving hydroxyurea, we decided to give glivec $400 \mathrm{mg}$ daily, based on the finding of strong positivity of blastoid cells for cKit. Hydroxyurea was discontinued. The white blood cell count decreased from 5G/1 with 55\% eosinophils before imatinib to 4.4G/1 with no eosinophils within ten days of treatment with glivec. The platelet count rose to normal values. Bone marrow examination showed disappearance of both eosinophils and myeloid blasts. Although peripheral blood and bone marrow fulfilled the criteria of complete hematologic remission, symptomatic CNS-involvement persisted. Therefore, radiation treatment of the brain and spinal cord was performed prior to allogenic stem cell transplantation. Point mutations causing constitutive activation of cKit have been demonstrated to be causative for a number of different neoplastic conditions, among them human gastrointestinal stromal tumors (GIST), and mastocytosis. Therefore, we sequenced the cKit receptor tyrosine kinase in bone marrow samples. We were not able to detect activating point mutations in exon 9-12. However, most importantly, we found a deletion in exon 9 of cKit as sole abnormality. This deletion in cKit has been shown to transform NIH3T3 cells. Therefore, we assume that this patients disease was driven by mutant cKit and that the remission observed while receiving glivec was caused by inhibition of cKit by imatinib. Further clinical and molecular studies will show, if the identified deletion indeed plays a pathophysiological role in cases of eosinophilic AML which can be reversed by treatment with imatinib.
Palliative outpatient treatment in patients with acute myeloid leukaemia

Steegmanns R. (1), Köppler H. (1), Heymanns J. (1), Jacobs G. (2), Schimke J. (2), Steinmetz T. (3), Schmitz S. (3), Weide R. (1)

(1) Oncology group practice Koblenz, (2) Saarbrücken, (3) Köln

Objective: Evaluation of feasability and outcome in patients with acute myeloid leukaemia (AML) who were diagnosed and received palliative treatment on an outpatient basis.

Methods: Retrospective analysis of 47 consecutive, unselected AML-patients who were diagnosed and received palliative outpatient treatment between 06/95-08/01 in three community based oncology group practices Treatment consisted of blood and platelet transfusion, fresh frozen plasma when necessary, antimicrobial treatment and cytoreductive chemotherapy to control the blast cell count.

Results: The median age of the patients was 70 years (57-86), 28 were male and 19 female. 20 patients had de novo AML (43\%) and 27 patients were transformed from MDS into AML (57\%). At first presentation 11 patients (23\%) had granulocytes below 500/ul. Red cell transfusions (RBC): During the study period 871 units were transfused to 40 patients. A median of 18,5 units of RBC were transfused per patient (2-55).

Platelet transfusions (PLT): 279 PLT-units (cell separator) were transfused to 28 patients. A median of 6 PLT-units were transfused per patient (1-34). Fresh frozen plasma (FFP): 35 FFP-units were transfused to 2 patients. Febrile neutropenia occurred in 12 patients $(26 \%)$. A total of 21 febrile episodes were treated with antibiotic therapy $(21$ orally $(100 \%), 9$ intravenous $(43 \%))$. Oral therapy was successful in 8 episodes $(38 \%)$. Intravenous antibiotic therapy was successful in 6 episodes $(67 \%)$. During the treatment period 55 hospitalisations occurred in 27 patients. The median frequency of hospitalisation was 2/patient (1-8). The median duration of hospitalisation was 13 days (1-67). The reasons for hospitalisation were as follows: 49 disease related (89\%), 6 comorbidity (11\%). Median overall survival was 251 days (32-1331) with a median observation time of 236 days (32-1331). During the study period 38 patients have died $(81 \%) .17$ patients died at home (45\%) and 19 patients died in hospital $(50 \%)$

Conclusion: Palliative and supportive therapy in AML-patients can be performed in the ambulant setting. The observed median survival is long compared to published results of patients treated in hospitals. A considerable percentage $(45 \%)$ of patients can die at home.

\section{Angiogenesis}

\section{2}

Basic fibroblast growth factor is overexpressed in the bone marrow of patients with acute myeloid leukemia

Bieker R., Padró T., Kramer J., Kessler T., Retzlaff S., Hintelmann H., Büchner T., Berdel W.E., Mesters R.M.

Dept. of Medicine / Hematology and Oncology, Univ. of Münster, Germany.

Increased angiogenesis is a common feature in bone marrow of patient with untreated acute myeloid leukemia (AML) (Padró et al. Blood. 2000;95:2637). Basic fibroblast growth factor (bFGF) is known to play a critical role in physiologic and pathologic angiogenesis, including tumor neovascularization. Furthermore, bFGF is suggested to promote growth and dissemination of tumors. The present study investigates the expression of bFGF in patients with newly diagnosed AML.

bFGF levels were determined by immunohistochemical analysis (APAAP method) in the bone marrow of 74 AML patients. Control specimens $(n=18)$ were obtained from patients with various diseases without bone marrow involvement. Immunostaining scores (arbitrary units: AU) were calculated by multiplying the percentage of labeled cells and the intensity of cellular staining in bone marrow specimens (Padró et al. Leukemia, in press).

The expression of bFGF in the bone marrow was significantly increased in AML patients (median: 3,1 [interquartile range:1,83-3,9] AU) compared with controls $(1,95[1,5-2,3] \mathrm{AU})$. There were higher staining levels in the younger population $(<60$ years $3,4 \mathrm{AU},>60$ years $2,3 \mathrm{AU} ; \mathrm{p}=0.09)$. Concerning the FAB-subtypes, the M4 subtype showed the highest immunostaining scores (M4 3,7 AU vs M1 2,7 AU: P= 0.046; M4 3,7 AU vs M5 2,1 AU: $\mathrm{p}=0.005$; M4 3,7 AU vs M6 1,9 AU: $\mathrm{P}=0,004)$. The degree of bFGF expression in the bone marrow correlated with the staining level for vascular endothelial growth factor (VEGF) $(\mathrm{rs}=0.506, \mathrm{P}=0.01$ ), but not with the expression of the VEGF receptors VEGFR-1 and VEGFR-2. Furthermore, 
there was no correlation found between bFGF and microvessel density. We were able to demonstrate that the bFGF stimulated HL-60 cell line showed a significant higher colony formation than unstimulated cells $(\mathrm{P}<0.0001)$ Moreover, bFGF induced an increased proliferation of this cell line $(\mathrm{P}=$ 0.01 ) These effects of bFGF were significantly reduced in the presence of the polyclonal antibody against bFGF (R\&D, AB-33-NA, $\mathrm{P}<0.001)$.). In conclusion, there is evidence of increased expression of bFGF in the bone marrow of AML patients, which supports the hypothesis of an important role for bFGF in the patho-physiology of AML.

\section{Use of AC133-derived endothelial progenitor cells for coating} of intracoronary stents

Gehling U.M. (1), Guckenbiehl M. (2), Loges S. (1), Koester R. (2), Ito W. (2), Muenzel T. (2), Hossfeld D.K. (1), Fiedler W. (1)

(1) Abteilung für Hämatologie/Onkologie, (2) Abteilung für Kardiologie, Universitätsklinikum Eppendorf, Hamburg

Objectives: Coronaray stenting is complicated by a high rate of restenosis and thrombosis. Recent studies have shown that rapid regeneration of endothelium at sites of stenting can reduce reocclusion. In this context, AC133-derived endothelial progenitor cells (EPC) may have the capacity to enhance endothelialization. The aim of this study was to identify optimal stent surfaces for adherence and growth of EPC.

Material and Methods: AC133+ cells were isolated from granulocyte colony-stimulating factor (G-CSF)-mobilized peripheral blood by using an immunomagnetic device and cultured in IMDM that was supplemented with $10 \%$ fetal bovine serum, $10 \%$ horse serum, Flt3 Ligand $(50 \mathrm{ng} / \mathrm{ml})$, SCGF (100 ng/ml), and VEGF (50 ng/ml). After 14 days, 316L steel stents coated with either heparin, aluminium oxide, fibronectin, vitronectin, osteonectin, collagen type I, collagen Type IV, or laminin, and uncoated 316L stents were placed into cultures containing expanded AC133-derived EPC. Following two weeks of culture in the presence of SCGF and VEGF, layers of EPC on the stents were evaluated by microscopy. Cell density was scored in four categories according to visual assessment of mean cell density within three representative microscopic fields (categories: 1: 0-10 cells/field, 2 11-20, 3: 21-30, 4: 31-40)

Results: Within one week of culture, EPC became adherent on heparin-, aluminium oxide-, fibronectin-, vitronectin-, osteonectin-, and collagencoated stents, whereas there was no visible attachment to uncoated 316L steel. The highest numbers of cells were counted on stents coated with heparin, aluminium oxide, and collagen type IV ( $>31$ cells/field). Cell numbers were lower on collgen type I- and vitronectin-coated stents $(>21$ cells/field). Lowest cell numbers were detected on osteonectin- and laminincoated stents ( 0 - 10 cells/field)

Conclusions: AC133-derived EPC are suitable for coating coronary stents Determination of the optimal stent surface will be important to enhance their attachment and growth. Stent coating with AC133-derived EPC may offer therapeutical potential to reduce restenosis and thrombosis after intracoronary stent implantation.

\section{4}

\section{7-S immunoglobulins (Venimmun $\mathrm{N}$ ) in the treatment of idiopathic thrombocytopenia}

Guenzelmann S., Link D., Basara N., Kiehl M.G.

\section{Department of Haematology/Oncology, Idar-Oberstein, Germany}

Background: 7S immunoglobulins have an established place in the treatment of autoimmune thrombocytopenia. Intravenous (IV) administration leads to rapid increases in platelet count in $>90 \%$ of paediatric patients and $70-80 \%$ of adult patients with autoimmune thrombocytopenia. In acute forms of the disease, platelet levels generally remain within the normal range while in chronic cases platelet levels may fall again after 2-4 weeks We report here the results from 31 patients with acute immunocytopenia treated with Venimmun $\mathrm{N}$.

Methods: 31 patients ( 23 female, 8 male) with acute thrombocytopenia and detectable antibodies to GPIIb/IIIa were treated with Venimmun N. Additional inclusion criteria were a platelet count $\leq 30000 / \mu \mathrm{L}$ and/or haemmorrhagic signs and elevated megakaryocytopoiesis in bone marrow cytology. Patients were treated on an out-patient basis and received IV Venimmum N $(0.4 \mathrm{~g} / \mathrm{kg}$ body weight $)$ on 5 consecutive days. Assessments were made 2, 3, $4,5,10,17,24,31,38,50$ and 100 days post-infusion.

Results: A rapid increase in blood platelet counts was seen in all patients. To date the median observation time is 18 months, range 4-46 months. The platelet count remained stable and above $50000 / \mu \mathrm{L}$ in 18 patients and sta- ble between 50000 and $30000 / \mu \mathrm{L}$ for 7 patients. Platelet counts declined below $20000 / \mu \mathrm{L}$ in 6 patients and splenectomy was planned. A further dose of Venimmun $\mathrm{N}(1 \mathrm{~g} / \mathrm{kg}$ body weight over 2 consecutive days) was given prior to splenectomy and the procedures were carried out without any complications.

Conclusions: Our data confirm the good response in terms of increased platelet counts with Venimmun N. Consistent with previous observations a small number of patients $(20 \%)$ did not experience persisting remission but responded well to a renewed administration.

85

\section{Trofosfamide equally inhibits endothelial and tumor cell growth in vitro}

Klink T., Marxsen J.H., Wagner T.

Universität zu Lübeck

Objectives: Angiogenesis is an indisputable prerequisite for progressing tumor growth and metastasis. Thus, the inhibition of angiogenesis is considered an important target of cancer treatment. Intentional inhibition of the genomically stable endothelial cell would lead to tumor regression, despite possible development of resistances of the neoplastic cell. Experimental studies on mice showed reduced sprouting of blood vessel into tumor tissue while being continously treated with low-dose cyclophosphamide. That angiostatic effect also substantially reduced the murine tumor mass. The aim of this study was to compare the sensitivity of human endothelial cells and tumor cells to trofosfamide.

Material and Methods: We used the human soft-tissue sarcoma cell-line S117. Endothelial cells were isolated from human umbilical cord veins (HUVEC). For determination of the $\mathrm{IC}_{50}$, we cultured both cell-lines in 96well microtiter plates and treated them with the active metabolite $4 \mathrm{OH}$-trofosfamide in increasing concentrations from $2,5 \mathrm{nmol} / \mathrm{ml}$ to $40 \mathrm{nmol} / \mathrm{ml}$. Then, we stained the cells in each well with crystal-violet and measured the optical density of the solubilized dye with a microtiter plate-reader. The results were compared to untreated cells. Finally we analysed the cell-cycle of both cell-types, because alcylating agents preferentially affect cells in the Sphase of the cell cycle and are therefore most potent on rapid proliferating tissue. We labelled cells with the thymidine-analog bromodeoxyuridine and detected its incorporation into newly synthesized DNA by means of flow cytometry.

Results: The inhibitory effect of trofosfamide on proliferating S117 cells was similar to its effect on cultured HUVECs $\left(\mathrm{IC}_{50}: 7,1 \mathrm{nmol} / \mathrm{ml}\right.$ vs $5,2 \mathrm{nmol} / \mathrm{ml})$. The analysis of cell-cycle showed almost identical proportions of cells in S-phase. From these small differences in both cell-lines we draw the conclusion that non-malignant HUVECs were as sensitive as the tumor cells S117.

Conclusions: The cytostatic effect of trofosfamide seemed to be similar on the two cell lines studied in vitro. With long-term treatment the genomically instable tumor cells tend to become resistant whereas endothelial cells remain sensitive. In our opinion, with a similar initial sensitivity of both celllines, the antiangiogenic effect would predominate, when tumor cells have become resistant to treatment.

86

Comparison of endothelial cells grown from CD34+ cells from mobilized peripheral blood, cord blood or bone marrow

Keller U., Ott I., Götze K.S., von Bubnoff N., Wimmer M., Peschel C., Oostendorp R.A.J.

III. Medizinische Klinik, Techniscvhe Universität München, München

Endothelial precursor cells (EPC) circulate and vascularize damaged tissues in vivo. To characterize the ability of EPC to form functional endothelial cell layers, we isolated CD34+ cells from different sources (mobilized peripheral blood (MPB), cord blood (CB), and bone marrow (BM)) and cultured these cells under conditions that promote outgrowth of endothelial cells. Endothelial cell layers could be readily established from all sources. In BM preparations, fibroblastoid cells were depleted after one week by reselection for expression of CD31 and binding of UEA-1. Immunocytochemistry and FACS analyses show that endothelial cells cultured from CD34+ EPC express vWF, VEGF-R2 (KDR), CD31, Tie2, CD105, and variable expression of CD34 and VEGF-R3, but do not express CD45 or CD14. At late passages, expression of CD34 and CD105 diminishes. EPCderived endothelial cells could be expanded to clinically relevant preparations of about $10 \mathrm{e} 14$ to $10 \mathrm{e} 15$ total cells. DNA Microarray comparisons from endothelial cells of different EPC sources show a high degree of similarity between CB-derived endothelium and human umbilical vein-derived 
endothelial cells (HuvEC). On the other hand, MPB-derived endothelium shows higher expression of HDL-binding-protein, CD9 and MMP1 as compared to HuvEC. In addition, CB-derived endothelium shows high expression of Flt4 (VEGF-R3), suggesting that CB CD34+ cells have a greater potential to differentiate towards lymphatic endothelium than HuVEC or MPB-derived CD34+ cells. Currently, we investigate whether the human CD34+-derived endothelium cells can be used to revascularize damaged blood vessels in an in vivo model of ischemic heart damage. In conclusion, we show generation of clinically relevant numbers of endothelial cells from CD34+ EPC. These cells can be used for revascularization as well as serve as vehicle for gene-therapeutic targeting of blood vessels.

\section{7}

\section{Pioglitazone and rofecoxib combined with angiostatic} scheduling of chemotherapy in far advanced malignancies

Reichle A. (1), Bross K. (1), Vogt Th. (2), Bataille F. (3), Wild P. (3), Wodzynski A. (1), Zaiss M. (1), Wagner H. (1), Klebl F. (1), Messmann H. (4), Krause S.W. (1), Dengler R. (1)

(1) Department of Hematology and Oncology University of Regensburg, (2) Department of Dermatology University of Regensburg, (3) Department of Pathology University of Regensburg, Department of Medicine University of Regensburg, Germany

Objectives: Angiostatic, stromal and tumor cell-targeted therapies might control chemorefractory malignancies.

Material and Methods: A phase II trial was started to analyze the activity of a continuously applicated molecular-targeted therapy (daily $45 \mathrm{mg}$ pioglitazone $(\mathrm{P})$ po and $25 \mathrm{mg}$ rofecoxib $(\mathrm{R})$ po) combined with sequentially added angiostatic scheduled chemotherapy, indication group A (67 cases), capecitabine $2 \times 1 \mathrm{~g} / \mathrm{m}^{2}$ po from day 14 to 28 , every 3 weeks, indication group B (37 cases), trofosfamide $3 \times 50 \mathrm{mg}$ po daily, day $14+$ ) in heavily pretreated metastatic tumors (sarcomas, carcinomas, hematologic neoplasias) with acquired drug resistance and tumors with poor response rates to standard firstline treatment (intrinsic drug resistance).

Results: Up to now 104 patients with 19 different tumor entities are evaluable (mean 1.6, range 0 to 8 preceding systemic tumor therapies). Major side effects (WHO grade 3 and 4) were due to capecitabine treatment (therapy was stopped in 7 cases due to hand-foot-syndrome). Overall tumor responses were observed in 11 patients $(10 \%)$, including CR in 3 patients, PR in 8 patients. Additional 16 patients $(15 \%)$ achieved disease stabilization lasting $>6$ months. Clinical respone (CR, PR, SD >6 months) occured in 13 pts $(20 \%)$ in group A and 14 patients $(32 \%)$ in group B. Clinical response occured in 14 of 19 histologically diverse tumor types represented in the study. Among the 27 patients achieving clinical and objective responses (26\%), 17 had acquired chemoresistance and 10 patients were intrinsically chemoresistant. In 22 patients (20\%) first signs of clinical and/or histologic responses to biomodulation with pioglitazone and rofecoxib occured after a 14 day pretreatment period. Retrospective analysis showed that median PFS was not significant different within the two indication groups (group A and B, respectively, 4.0 months)

Conclusions: The new combination therapy is feasible with a manageable toxicity profile in patients with heavily pretreated advanced cancer. This is the first study of a combined modality treatment to show that novel therapeutic approaches are effective in a range of chemorefractory diseases.

\section{8}

\section{Thalidomide for the treatment of osteomyelofibrosis}

Strupp C., Germing U., Misgeld E., Kündgen A., Haas R., Gattermann N.

Klinik für Hämatologie/Onkologie, Heinrich-Heine-Universität Düsseldorf

Conventional treatment of myelofibrosis is only palliative and based on cytotoxic treatment (hydroxyurea, anagrelide), androgen therapy, steroids and splenectomy. Thalidomide is an immunmodulatory drug with a wide spectrum of activity that is not clearly understood. Current data suggest that the action of thalidomide is related to several different mechanisms, including suppression of tumor necrosis factor (TNF-alpha), effects on bFGF, VEGF, interleukins and interferons, down-regulation of selected cell surface adhesion molecules and changes in the lymphocyte-subsets.

We administered thalidomide to 16 patients ( $15 \mathrm{men} / 1$ women) with myelofibrosis, who suffered from transfusion-dependent anemia, thrombocytopenia or symptomatic splenomegaly. Median age was 59 years (range 52-78). Patients received thalidomide at an escalating dose from 100 to 400 $\mathrm{mg} / \mathrm{d}$ (median $300 \mathrm{mg}$ ). Administration of the drug was discontinued because of progressive disease (2) and polyneuropathy (2). Other adverse effects were obstipation (10), fatigue (8) and edema (2). Clinical response is now observed for a median duration of 9 months (3-20). Of the 15 evaluable patients, anemia improved in 7 out of 10 , thrombocytopenia improved in 5 out of 7 , splenomegaly was reduced in 3 out of 13 patients. In 7 out of 12 patients LDH decreased, as well as LDH increased in 4 patients during the course. LDH courses were not correlated with clinical response.

Thalidomide may be useful in the treatment of patients with osteomyelofibrosis

89

\section{Expression and activity of the VEGF receptor-2 in breast cancer cell lines}

Weigand M. (1), Kreienberg R. (1), Waltenberger J. (2)

(1) Universitätsfrauenklinik Ulm, (2) Innere Medizin II der Universität Ulm

Objectives: The vascular endothelial growth factor (VEGF) is a potent stimulator of tumor angiogenesis. VEGF and the VEGF receptor-2 are overexpressed in breast cancer compared to corresponding normal tissue. Expression of VEGFR-2 is found not only on endothelial cells of tumor vessels but also on epithelial breast cancer cells. We wanted to know if breast cancer cell lines express an active VEGFR-2 and therefore support the hypothesis of autocrine stimulation of tumor cells with VEGF.

Material and Methods: The expression of VEGFR-2 was analyzed on tumor cell lines and primary cultures derived from pleural effusions from patients with metastasized breast cancer using flow cytometry and immunohistochemistry. The breast cancer cell lines were stimulated with VEGF to show the activation of VEGFR-2. The cells were lysated, separated by SDS page and a phosphotyrosine blot was performed. To detect the phosphorylated VEGFR-2 we used an monoclonal antiphosphotyrosine antibody. We also investigated the stimulation of VEGFR-2 by VEGF with an in vitro kinase assay after radioactive labeling of the phosphotyrosine.

Results: Some of the breast cancer cell lines revealed a weak but distinct expression of VEGFR-2. Performing the phosphotyrosine blot, minor changes in the phosphorylation pattern of some cell lines were found. Using the in vitro kinase assay we were able to show an activation of VEGFR-2 in one of the breast cancer cell lines. Immunostaining showed expression of VEGFR2 by some of the primary cultures. Only two of the primary cultures could be analyzed by flow cytometry and phosphotyrosine blotting. These cultures did not show expression nor activity of VEGFR-2.

Conclusions: We could show expression of VEGFR-2 on some breast cancer cell lines supporting the hypothesis of VEGF as an autocrine activator of breast cancer cell lines. Activation of VEGFR-2 could only be shown in a few cell lines which could be due to low quantity of VEGFR-2, expression of a mutated kinase defect receptor or expression of a related receptor that cannot be activated by VEGF

\section{0}

\section{Suppression of vascular endothelial growth factor by the tuberous sclerosis complex gene 2}

Vu P.-A. (1), Licht T. (2), Essler M. (3), Kretzler M. (4), Ghashghaeinia M. (1), DeClue J.E. (5), Volkenandt M. (1), Wienecke R. (1)

(1) Department of Dermatology, LUM Munich, (2) Department of Medicine III, TU Munich, (3) Institute for Prevention of Cardiovascular Disease, LMUMunich, (4) Medical Policlinic Center, LMU Munich, (5) National Cancer Institute, Bethesda, USA

Germ-line inactivation of TSC2 causes tuberous sclerosis, an inherited predisposition leading to multiple angiofibromas. Since induction of angiogenesis has been linked to inactivation of other tumor suppressor genes, we studied whether TSC2 regulates angiogenesis. To this end, human TSC2 was reintroduced into TSC2(-/-) rat embryonic fibroblasts (REF). TSC2(-/-) REF overexpressed VEGF mRNA, secreted high levels of VEGF, and thereby stimulated actin-reorganization of endothelial cells. Reintroduction of TSC2 reverted VEGF secretion, and growth stimulation and stress fiberformation of endothelial cells. In conclusion, we show that VEGF is suppressed by TSC2. Our study may help understand the pathogenesis of tuberous sclerosis-associated angiofibromas and sporadic tumors, in which expression of TSC2 is lost. 


\section{Lung Cancer}

91

Efficacy and safety of docetaxel/ gemcitabine compared to sequential cisplatin/ gemcitabine/ docetaxel in the treatment of patients with metastatic non-small cell lung cancer (NSCLC)

Binder D. (1,4), Sailer V. (1), Schweisfurth H. (2), Temmesfeld-Wollbrück B. (1), Schüler G. (2), Grah C. (3), Suttorp N. (1), Beinert T. $(1,4)$

(1) Medizinische Klinik m. S. Infektiologie, Universitätsklinikum Charité, Berlin, (2) III. Medizinische Klinik, Carl-Thiem-Klinikum, Cottbus, (3) Gemeinschaftskrankenhaus Havelhöhe, Berlin, (4) formerly: Medizinische Klinik m. S. Onkologie und Hämatologie, Universitätsklinikum Charité, Berlin

Background: Over the past years chemotherapy has become increasingly accepted as treatment for UICC stage IV non small-cell lung cancer (NSCLC). Depending on age and performance of the patients, mono- to three-drug schedules have been applied, while two-drug regimens are most often used. Triple-agent chemotherapy may improve response and survival, but is associated with significant toxicity. We conducted a multicentric prospective, randomized study to evaluate a sequential three-drug schedule compared to a two-drug combination chemotherapy.

Patients: 44 patients (140 planned) with UICC stage IV NSCLC have been randomized to one of the following schedules. Arm A: gemcitabine $\left(900 \mathrm{mg} / \mathrm{m}^{2}, 30 \mathrm{~min}\right.$. infusion, $\left.\mathrm{d} 1+8\right)$ and docetaxel $\left(75 \mathrm{mg} / \mathrm{m}^{2}, 1\right.$ hour infusion, d1), repeated every three weeks for up to six cycles. Arm B: gemcitabine $\left(900 \mathrm{mg} / \mathrm{m}^{2}, \mathrm{~d} 1+8\right)$ and cisplatin $\left(70 \mathrm{mg} / \mathrm{m}^{2}, 1\right.$ hour infusion, $\left.\mathrm{d} 1\right)$ were given for three cycles, followed by three cycles of docetaxel (100 $\left.\mathrm{mg} / \mathrm{m}^{2}, \mathrm{~d} 1 \mathrm{q} 21 \mathrm{~d}\right)$.

Results: In arm A (23 pts.) and arm B (21 pts.), 8 of 19 pts. (42\%) and 8 of 18 pts. $(44 \%)$ responded to the treatment (WHO criteria). One arm B patien had a complete response. $4 / 3$ pts. (A/B) are currently under treatment or are not evaluable for response. 18/18 pts. (A/B) are evaluable for toxicity. Mild or moderate leukopenia was common in both arms. 5/4 pts. (A/B) developed grade III-IV leukopenia (WHO). One patient (A) had fever and grade 4 leukopenia, another patient (A) severe gastrointestinal bleeding. Other adverse effects have been (A/B): ${ }^{\circ}$ III/IV thrombocytopenia $(11 \% / 11 \%)$, ${ }^{\circ} \mathrm{II}-\mathrm{III}$ alopecia $(50 \% / 44 \%),{ }^{\circ} \mathrm{I}-\mathrm{II}$ fatigue $(28 \% / 28 \%),{ }^{\circ} \mathrm{II}-\mathrm{III}$ diarrhoea $(6 \% / 17 \%)$, ${ }^{\circ}$ I-II nausea/vomiting $(28 \% / 33 \%),{ }^{\circ}$ I-II mucositis $(17 \% / 22 \%)$.

Conclusion: Both schedules demonstrate good activity and acceptable toxicity. Further details including preliminary survival data will be reported at the meeting.

\section{2}

\section{Molecular and immunological screening of peripheral blood samples from patients with lung cancer}

Lahm H., Rieger N., Schindel M., Wildenberg U., Muley T., Hoffmann H., Manegold C., Neuner A., Fischer J. R.

Immunology-Molecular Biology Laboratory, Thoraxklinik-Heidelberg gGmbH, D-69126 Heidelberg, Germany

Objectives: Soluble mediators which are secreted by malignant cells can significantly influence the capacity of peripheral lymphocytes to secrete immunomodulatory cytokines. Many tumors have been found to secrete immunosuppressive molecules such as transforming growth factor $\beta 1$ (TGF $\beta 1$ ), interleukin-10 (IL-10) and Fas ligand. In addition, genetic and epigenetic alterations of neoplastic cells can directly influence the biological behavior of the tumor.

Material and Methods: We assess the capacity of peripheral lymphocytes to secrete cytokines upon stimulation with PHA. Molecular changes can be monitored in the periphery after purification of genomic DNA from serum or plasma of lung cancer patients. Upon chemical treatment with sodium bisulfite the DNA was subjected to a methylation-specific PCR.

Results: TGF $\beta 1$ is secreted by SCLC and we have demonstrated that suppression of IL-2 in SCLC-patients is correlated with reduced survival. Currently, the influence of cytokine secretion on the efficiency of a vaccination therapy is being investigated. In NSCLC, increased production of immunosuppressive IL-10 by the tumor and increased plasma IL-10 concentrations have recently been correlated to reduced survival. In NSCLC-patients we could indeed demonstrate an impairment of IL-2 secretion by these immunocompetent cells. More important, this reduction correlated with reduced survival. Thus different types of lung cancer may use distinct molecules to escape immune defense with clinically relevant success. Hypermethylation analyses of promoter regions encompass DNA repair enzyme
(MGMT), apoptosis-related (DAP kinase) and tumor suppressor genes (p14, p16, RAR $\beta$, RASSF1A). Furthermore, point mutations in codon 12 of k-ras and the $\beta$-tubulin gene (codons 147, 245 and 246) were investigated.

Conclusions: The concomitant assessment of immunological and molecular data provides the basis to collect a whole set of different biological data from a single patient. The combination of such markers may be of much greater prognostic and predictive value than each of them alone. A thorough analysis of these parameters may lead to a better prognosis and may potentially provide the basis of an improved customized therapy for an individual patient. Finally this may lead to a novel approach which may allow a tumor treatment which is based on its biological properties rather than its histogenetic origin.

\section{3}

Reversibility of growth inhibition of the non-small cell lung cancer cell line A549 by fenretinide (4-HPR) and generation of the 4-HPR resistant cell line A549/HPR

Ohlmann C.-H., Jung C., Jaques G.

Klinikum der Philipps-Universität Marburg, Klinik für Hämatologie, Onkologie, Immunologie

Objectives: Growth inhibition and induction of apoptosis by Fenretinide [N (4-hydroxyphenyl)retinamide, 4-HPR] has already been shown for a variety of cell lines including lung cancer cell lines. Furthermore, 4-HPR inhibits growth of cell lines unresponsive to all-trans-retinoic acid (ATRA), the mos important natural retinoid. Clinical application of ATRA in patients is limited by the reversibility of growth inhibition which requires long-term treatment with the retinoid causing mild to severe side effects. Because of its higher potency in inhibiting cell growth and the lower toxicity compared to ATRA, 4-HPR has been postulated as a putative agent for chemoprevention and therapy of solid tumors. The aim of our study was to have a close look at the effect of 4-HPR on the growth of the non-small cell lung cancer cell line (NSCLC) A549 for its reversibility and observed the effects of continuous exposure to 4-HPR on cell growth, cell morphology and resistance to the retinoid.

Material and Methods: The resistant cell line A549/HPR was developed by continuous cultivation of A549 in increasing concentrations of 4-HPR in $\mathrm{R} 10$ up to $10 \mu \mathrm{M}$. Proliferation rate was measured by counting the cell number in a Coulter Counter.

Results: Growth inhibition of A549 by 4-HPR is reversible as evidenced by analysing growth kinetics of A549. After treatment of the cells with $10 \mu \mathrm{M}$ 4-HPR for six days, removal of the retinoid resulted in a decrease of the population doubling time (pdt) comparable to untreated cells. Therefore, long-term application of 4-HPR will be needed in lung cancer patients. Under continuous exposure of A549 to $10 \mu \mathrm{M} 4$-HPR we were able to develop a 4-HPR resistant cell line, which, after an initial decrease, increased in cell growth again after a few weeks of incubation. Along with incubation, morphological changes became visible. The cells changed their shape from round/oval to a more spindle like shape. Within the cytoplasm, small vacuoles became visible. The morphologic changes appeared after $48 \mathrm{~h}$ of incubation and are characteristic for the 4-HPR resistant cell line A549/HPR. Conclusions: Reversibility of growth inhibition and development of resistance to 4-HPR may limit clinical application of 4-HPR in lung cancer patients. Therefore, clinical trials are needed to prove successful treatment of lung cancer patients with 4-HPR.

\section{4}

\section{Bendamustin for treatment of relapsed small cell lung cancer}

Schmittel A. (1), Hütter G. (1), Schulze K. (2), Thiel E. (1), Keilholz U. (1)

(1) Medizinische Klinik III (Hämatologie, Onkologie und Transfusionsmedizin, (2) Medizinische Klinik II (Kardiologie und Pulmologie), Universitätsklinikum Benjamin Franklin, Hindenburgdamm 30, 12200 Berlin

Objectives: The single agent activity of bendamustin in small cell lung cancer (SCLC) is high with a response rate of $46 \%$ in previously untreated patients (Heider et al. 1999). However, a small phase 2 trial in patients refractory to primary chemotherapy failed to show activity of second line bendamustin treatment.

Material and Methods: We investigated the activity of bendamustin in patients with relapsing SCLC after initial response to first line treatment. We treated patients with relapsed SCLC extensive disease with bendamustin $120 \mathrm{mg} / \mathrm{m}^{2}$, days $1+2$, every three weeks. In the absence of tumor progression a maximum of six cycles was given. Inclusion criteria were absence of 
brain metastases and time to relapse $\geq 2$ months after completion of first line chemotherapy. Patients refractory to first line therapy were excluded from this study.

Results: Until now 12 patients have been included into the trial, of which ten are evaluable for toxicity and response to treatment. Toxicity was mild One patient experienced a grade 3 leucopenia, the other patients only had grade 2 hematologic toxicity. One patient had a poststenotic pneumonia during therapy. No chemotherapy induced alopecia was observed. Four out of ten evaluable patients had a partial response after two and four cycles of therapy. Four patients had disease stabilization and two patients progressed. Mean time to progression was 12 weeks (4 -37 weeks)

Conclusions: This ongoing study demonstrates already a substantial activity of bendamustin in patients with relapsed SCLC and confirms the low toxicity found in other trials.

\section{5}

\section{Carboplatin and irinotecan as first line treatment for extensive} disease small cell lung cancer: a pilot study

\author{
Schmittel A., Hütter U., Ullrich A., Thiel E., Keilholz U.
}

Universitätsklinikum Benjamin Franklin, Medizische Klinik III (Hämatologie, Onkologie und Transfusionsmedizin), Hindenburgdamm 30, 12200 Berlin

Objectives: Recently, a Japanese randomised phase III trial demonstrated a significant increase in survival and response rates for cisplatin plus irinotecan therapy over the combination of cisplatin plus etoposide. Carboplatin is more commonly employed in Germany because of its favourable toxicity profile and equal effectivity.

Material and Methods: Ten patients with histologically or cytologically confirmed extensive disease small cell lung cancer were treated. Chemotherapy consisted of carboplatin AUC 5 on day 1 and irinotecan $50 \mathrm{mg} / \mathrm{m}^{2}$ on day 1 , 8 and 15 , cycles were repeated on day 28 , as recommended in an earlier phase I trial in a Japanese population. CT based response evaluation was performed after cycle 2, 4 and 6 and every 2 months during the follow up period. Patients regularly received 5-HT3 antagonists.

Results: Myelotoxicity was mild with four grade 2 anemias and three grade 2 thrombopenias. Grade 1 and 2 diarrhea was observed in 2 patients. One patient with diabetes mellitus developed a grade 3 neuropathy after the third treatment cycle. No complete response was observed, 8 of 10 patients had a partial response to treatment. Two patients progressed during therapy.

Conclusions: The combination of carboplatin (AUC 5) and irinotecan (50 $\mathrm{mg} / \mathrm{m}^{2}$ ) is effective with a very mild toxicity in SCLC. Because of the very low hematologic and non-hematologic toxicity we are currently evaluating an intensified regimen of carboplatin (AUC 6) and irinotecan $50 \mathrm{mg} / \mathrm{m}^{2}$ and initiate a subsequent randomised trial comparing carboplatin plus irinotecan with the combination of carboplatin and etoposide for the first time in the Caucasian population. Data will be presented at the meeting.

\section{6}

The loss of pro-apoptotic bak expression is associated with poor prognosis in patients with small-cell lung cancer*

Milosevic J. (1), Micke P. (1), Faldum A. (2), Sturm I. (3), Güner D. (3) Dörken B. (3), Buhl R. (1), Huber C. (1), Schuler M.(1), Daniel P.T. (3)

(1) Department of Medicine III, (2) Department of Statistics, Epidemiology and Informatics, Johannes Gutenberg University, Mainz, Germany; (3) Department of Medicine, Division of Hematology, Oncology and Tumor Immunology, Charité Campus Berlin-Buch, Berlin, Germany

Objectives: In experimental models the stress-induced apoptosis pathway plays a central role in tumor suppression and sensitivity towards anticance therapy. A key step in this death pathway is the release of mitochondrial cytochrome c into the cytoplasm, which is regulated by the BCL-2 family of proteins. In the present study we assessed the influence of the expression of pro-apoptotic BCL-2 family proteins on the outcome of a cohort of uniformly treated Small-Cell Lung Cancer (SCLC) patients from a single center.

Methods: Paraffin-embedded tumor biopsies from 94 patients with SCLC were retrospectively analyzed for the expression of pro-apoptotic BCL-2 family proteins by standard immunohistochemical techniques. Expression levels were scored in a blinded fashion as absent, low, intermediate or high based on specific cytoplasmic staining of the tumor cells. Complete clinica data of 85 patients were available for Cox regression analysis with forward and backward variable selection (inclusion criterion $\mathrm{p} \leq 0.05$, exclusion criterion $\mathrm{p}>0.1$ ).
Results: BAK expression was absent in 34 of 85 patients (40 \%). Low BAK expression was found in 19 patients $(22 \%)$, intermediate BAK expression in 18 patients $(21 \%)$, and high BAK expression in 14 of 85 patients $(16 \%)$. A regression analysis, patients with absent BAK expression exhibited a reduced survival as compared to patients with low BAK expression. Comparing patients with absent BAK expression and patients with intermediate or high BAK expression, a statistically satisfactory impact on survival could not be established in this cohort. BAX expression was absent in 62 of 94 patients $(66 \%)$, but failed to correlate with survival. Additional parameters associated with adverse prognosis were age, extensive disease, and elevated LDH levels.

Conclusions: Absent expression of the pro-apoptotic multidomain BCL-2 family protein BAK appears to be associated with adverse prognosis in SCLC patients. These results warrant confirmation in the context of prospective studies.

* Supported by the Max-Eder-Programm of the Deutsche Krebshilfe (project no. 70-2952).

\section{7}

\section{Growth hormone producing non small cell lung cancer - a rare cause of Pierre Marie Bamberger syndrome}

Trenn G. (1), Petersen S. (2)

(1) KKH Bottrop, (2) Universitätsklinikum Essen

Various paraneoplastic syndromes are associated with primary lung cancer. The syndrome of hypertrophic pulmonary osteoarthropathy is a consequence of elevated growth hormone $(\mathrm{GH})$ releasing hormone levels produced by both small (SCLC) and non small cell lung cancer(NSCLC).Here we present the case of a 53 year old patient who was admitted to our hospital because of severe pain in his limbs. Due to a swelling of his fingers the diagnosis of chronic polyarthritis was made 6 months prior to admission and MTX was started without clinical improvement.Work up of the patient revealed the presence of lung carcinoma. The changes in nuclear bone scan were compatible with the diagnosis of hypertrophic pulmonary osteoarthropathy (Pierre Marie Bamberger syndrome. The GH-level was markedly elevated. An adenoma of the pituitary gland could be excluded. Histologic analysis of a tumour specimen showed a neuroendocrine NSCLC. An additional immunophenotyping was performed using a polyclonal antibody against GH. $60 \%$ of tumour cells stained positive for $\mathrm{GH}$ expression. Since mediastinoscopy showed infiltration of mediastinal lymph nodes a neoadjuvant therapy was initiated. After three cycles of paclitaxel/cisplatin and a hyperfractionated radiation of the tumour a partial remission was achieved and a Ro-resection of the tumour was performed. Postoperatively the patient's GH-level normalized and the bone pain disappeared. The patient is well and free of tumour nine months after completion of therapy.

While many neurocrine lung cancers were shown to produce GH-releasing hormone the release of growth hormone itself by the tumour is extremely rare.

\section{8}

\section{Local expression patterns of BMP5 in lung cancer}

Wölfl S. (1), Odyvanova L. (1), Kroll T. (1), Treiber K. (1,2), Sänger J. (2), Waldau A. (1), Höffken K. (1), Clement J.H. (1)

(1) Klinik für Innere Medizin II, Friedrich-Schiller-Universität Jena, (2) Institut für Pathologie Bad Berka

Using cDNA array analysis we identified a number of genes that showed a clear tumor specific regulation. Our results included upregulation of the well established tumor marker PCNA and cancer specific regulation of other genes previously described as lung cancer associated like LUCA1 and LUCA2, which are downregulated in the tumor samples. Interestingly, our results included a very clear difference for BMP5 expression between tumor and tumor free resection margins. BMP5 was expressed at a lower level in all lung cancer samples analyzed, except for a leiomyom, which is found very rarely in lung. Immunohistochemistry confirmed this observation and additionally showed a regional specific expression pattern. In cancers where BMP5 expression is reduced, the BMP5 free zone correlates with the area that shows enhanced PCNA expression. In samples of benign tumors (e.g. leiomyom) a clear expression of BMP5 in the tumor is seen which correlates with a lower expression of PCNA in this tumor. 
In conclusion, BMP5 is expressed in a region specific manner in lung cancer, showing clear BMP5 expression in the tumor free areas of the histolog ical section and no BMP5 immunoreactivity within the tumor area. In benign tumors BMP5 is still expressed. This finding indicates that BMP5 may be a marker for less agressive tumors in lung cancer.

This work was supported by the Deutsche Krebshilfe e.V and the BMBF.

\section{CML - Basic Research}

\section{9}

Interferon $\alpha$, but not imatinib (STI571) causes expression of myeloblastin in vivo: A potential mechanism how Interferon $\alpha$ elicits a myeloblastin specific cytotoxic T-cell response in chronic myeloid leukemia

Burchert A. (1), Wölfl S. (2), Schmidt M. (3), Brendel C. (1), Denecke B. (1), Cai C. (1), Odyvanova L. (2), Lahaye T. (4), Müller M.C. (4), Berg T. (5), Gschaidmeier H. (6), Wittig B. (3), Hehlmann R. (4), Hochhaus A. (4), Neubauer A. (1)

(1) Klinik für Hämatologie/Onkologie/Immunologie der Philipps Universität Marburg, (2) Friedrich-Schiller-Universität Jena, Klinik für Innere Medizin, (3) Mologen GmbH, (4) Fakultät für Klinische Medizin Mannheim der Universitaet Heidelberg, III. Medizinische Klinik, Mannheim, (5) Charité - Virchow Klinikum, Medizinische Klinik, Gastroenterologie - Hepatologie, Humboldt Universität, Berlin, (6) Novartis Pharma GmbH

Chronic myeloid leukemia (CML) is a clonal disease of hematopoietic stem cells caused by a reciprocal translocation of the long arms of chromosome 9 and 22. In HLA-A*0201 positive individuals response after interferon-alpha (IFN $\alpha$ ) was shown to be associated with the emergence of CML-specific cytotoxic T-cells that recognize PR-1, a myeloblastin- (MBN) derived nonapeptide. In contrast, imatinib potently induces remissions from CML by specific inhibition of the ABL-tyrosine-kinase. Here, we explored molecular regulations associated with CML-responses under different treatment forms using cDNA-array. Expression of MBN was found to be downregulated in remission under imatinib therapy ( 0 of $7 \mathrm{MBN}$-positive patients). In contrast, $\mathrm{MBN}$ transcription was readily detectable in the peripheral blood in 8 of 8 tested IFN $\alpha$-patients in complete remission ( $\mathrm{p}=0.0002)$. Further analysis of the granulocytic and mononuclear fraction but also sorted CD3, CD14 and CD20 positive white blood compartments revealed that MBN was induced in all white blood compartments including the CD14 positive (monocytic) fraction, but not in CD20 positive lymphocytes. IFN $\alpha$-dependent MBN transcription was confirmed in vitro by IFN $\alpha$ stimulation of peripheral blood mononuclaer cells (PBMC) and sorted monocytic and lymphocytic sub-fractions, and by IFN $\alpha$-mediated activation of the MBN promoter in reporter gene assays. We then speculated that IFN $\alpha$ mediated upregulation of MBN expression on antigen presenting monocytes may augment an autologous cytotoxic T-cell response. Indeed, using tetrameric compexes MBN specific cytotoxic T-cells were found 4 of 4 tested IFN $\alpha$-treated HLA$\mathrm{A}^{*} 0201$ positive individuals, whereas these cells could only be detected in 2 of 11 HLA-A*0201 positive imatinib treated patients (19\%) in complete hematological or cytogenetic remission $(\mathrm{p}=0.011)$. Thus, we postulate a novel mechanistic pathway of IFN $\alpha$ response, whereby IFN $\alpha$ induced MBN expression contributes to the specific ability of IFN $\alpha$, but not imatinib to elicit an autoimmune MBN-specific T cell response. This hypothesis is substantiated by the fact that identical effects of IFN $\alpha$ on MBN expression and MBN-CTL response are seen in hematologically healthy, HLA-A*0201 positive hepatitis $\mathrm{C}$ patients.
BCR/ABL D-FISH - a mandatory examination for primary diagnosis of CML

Duba H.-Ch. (1), Kühr T. (2), Petzer A. (3), Mehringer A. (1), Erdel M. (1), Utermann G. (1), Thaler J. (2)

(1) Institut für Medizinische Biologie und Humangenetik der Universität Innsbruck, Schöpfstrasse 41, A-6020 Innsbruck, Austria, (2) IV. Interne Abteilung, A.ö. Krankenhaus der Kreuzschwestern, Grieskirchner Str. 42, A-4600 Wels, Austria, (3) Universitätsklink Innsbruck, Klinische Abteilung für Hämatologie und Onkologie, Anichstr. 35, A-6020 Innsbruck, Austria

Objectives: Imatinib and Interferon-alpha alone or in combination with cytostatic drugs can induce major and durable cytogenetic responses in chronic myelogenous leukaemia (CML) patients. Since these patients have a significant survival benefit, frequent follow up investigations have become clinically important. Although conventional cytogenetics is still considered the ,gold standard" for evaluating CML during treatment, recently developed methods such as hypermetaphase-FISH on bone marrow cells and in terphase-FISH on bone marrow and peripheral blood cells, performed with probes suitable for detection of the BCR/ABL rearrengement, have shown some advatages in comparison to conventional cytogenetics. It was demonstrated recently [Huntly et al: Blood, 98, 1732-1738, 2001] that a highly sensitive BCR/ABL D-FISH probe detects deletions of ABL and/or BCR and neighbouring sequences on the derivative chromosome 9. These deletions provide a powerful and independent prognostic indicator in CML.

Material and Methods: We have performed FISH with the highly sensitive LSI $^{\circledR}$ BCR/ABL Dual Color, Dual Fusion Translocation Probe probe from Vysis in CML patients treated with Interferon-alpha and YNK-01 (oral prodrug of Ara-C) $(n=147)$, Imatinib $(n=22)$ and on archived cell suspensions from CML patients at first diagnosis $(\mathrm{n}=22)$.

Results: We have detected deletions in $11,52 \%(22 / 191)$ of patients $[8,84 \%$ $=13 / 147$ in IFN + YNK-01 group; $18,18 \%=4 / 22$ in Imatinib group; $22,73 \%=5 / 22$ in first diagnosis group], the majority of them showing poor response to Interferon and/or poor outcome.

Conclusions: Deletions on the derivative chromosome 9 result in rapid disease progression due to loss of one or more genes within the deleted region [Huntly et al.: Blood 99, 4547-4553, 2002]. Therefore BCR/ABL sensitive D-FISH, for detection of the Philadelphia chromosome and deletions of the derivative chromosome 9 , should be incorporated into future diagnostic strategies as well as management decisions of CML.

101

In BCR-ABL positive cells, STAT-5 tyrosine-phosphorylation integrates signals induced by imatinib mesylate and Ara-C

Kindler T. (1), Breitenbücher F. (1), Kasper S. (1), Gschaidmeier H. (2), Huber C. (1), Fischer T. (1)

(1) III. Medical Department, Johannes Gutenberg-University, (2) Novartis Pharma, Nürnberg, Germany

Imatinib mesylate, a newly developed kinase inhibitor with selectivity for $\mathrm{Bcr}-\mathrm{Abl}$, demonstrated potent antileukemic activity in clinical trials. However, reponse rates and duration of remission in transformed phases of CML are limited and molecular resistance is frequently observed.

Objectives: Combination therapy with chemotherapeutic agents may exhibit synergistic effects and prevent development of resistance. To develop effective therapeutic strategies, understanding of molecular mechanisms of therapeutic agents is essential.

Methods: Here, we investigated the effects of cytosine-arabinoside (Ara-C) and imatinib mesylate on STAT-5 tyrosine-phosphorylation, DNA-binding, cellular proliferation and induction of apoptosis in cell lines and primary hematopoietic cells.

Results: In BCR-ABL positive cells, the transcription factor STAT-5 is constitutively activated by tyrosine phosphorylation. STAT-5-activation results in upregulation of bcl-XL and increased resistance to induction of apoptosis. Imatinib mesylate treatment strongly suppressed STAT-5 tyrosine-phosphorylation in K562 and primary CML blasts. In contrast to JAK-2 and PI3-kinase inhibition, exposure of K562 cells to imatinib mesylate resulted in obvious suppression of proliferation. Reduced cell growth was due to specific induction of caspase activation followed by apoptotic cell death. Interestingly, exposure of BCR-ABL positive cell lines and primary CML blasts to Ara-C also resulted in specific downregulation of tyrosine phosphorylated STAT- 5 levels. As a consequence, DNA-binding of STAT-5 was strongly suppressed. Treatment of K562 cells with Ara-C in combination with imatinib mesylate revealed synergistic effects at the level of STAT-5 tyrosinephosphorylation and DNA-binding, cell growth and induction of apoptosis. 
Conclusions: Overall, in this report we demonstrate that STAT-5 tyrosinephosphorylation is a specific target of imatinib mesylate and of Ara-C. Our results suggest that, in combination therapy, inhibition of STAT-5 activation may be responsible for synergistic or additive effects on BCR-ABL positive cells.

\section{2}

C-kit (CD117) and PDGFR expression on leukemic blasts from bone marrow biopsies of CML patients in accelerated phase (AP) and blast crisis (BC) and response to therapy with imatinib mesylate

Marx A. (1), Beck J. (1), Hansen T. (1), Gschaidmeier H. (2), Huber C. (1), Kirkpatrick C.J. (1), Fischer T. (1)

(1) Johannes Gutenberg-University, Department of Pathology and Hematology/Oncology, Mainz, Germany; (2) Novartis Pharma, Nürnberg, Germany

In CML, clinical trials using imatinib mesylate showed a high rate of hematologic and cytogenetic remissions. In $\mathrm{AP}$ and $\mathrm{BC}$, remissions are usually shortlived. However, there is a considerable heterogeneity in these patients regarding degree and duration of responses achieved.

Objectives: Since in addition to bcr-abl, imatinib also targets c-kit and PDGFR we reasoned whether expression of these receptors on leukemic blasts influences clinical results.

Methods: By immunohistochemistry (IH), we examined expression of ckit, PDGF a and $B$ receptor on leukemic blasts in a series of bone marrow trephine biopsies from 19 imatinib treated patients in AP and BC.To obtain reproducible and comparable results an immunoreactivity score (IRS) based on the percentage of immunoreactive cells and their staining intensitiy (ranging from 0 to 12 ) was employed.

Results: On myeloid leukemic blasts, strong expression of c-kit was observed (mean IRS 8.9), whereas lymphoid blasts were completely negative for c-kit (IRS 0). All cases showed PDGFR a and $\beta$ expression with varying immunoreactivity score (PDGF receptor a: mean IRS 5.8; PDGF receptor $\beta$ : mean IRS 8.2). IRS classes (low, intermediate, high) were compared to response to imatinib therapy. 6 patients did not show any response, demonstrating mainly low or intermediate IRS for c-kit with a mean IRS of 6.0 (SD 1.9). 13 patients with partial response or return to chronic phase showed low, intermediate or high IRS for c-kit (mean IRS 6.7; SD 4.3). With regard to PDGFR expression, we found a mean IRS of 6.2 (SD 2.0) for PDGFR a and 8.8 (SD 2.9) for PDGFR $\beta$ in the responders compared to 4.3 (SD 1.2) and 5.8 (SD 2.2), respectively in the non-responders.

Conclusions: In this cohort, we did not find an apparent difference in expression of c-kit and PDGF receptors on leukemic blasts from responding patients compared to non-responders. Further analysis will include duration of responses and cytogenetic parameters

\section{3}

\section{Wilms'-tumor-antigen (WT1-) derived peptides elicit a T- cell response in patients with chronic myeloid leukemia (CML)}

Hilbers U. (1), Hartung G. (1), Geppert T. (1), Lange T. (1), Hegenbart U. (1), Deininger M. (1), Schmitt M. (2), Niederwieser N. (1), Uharek L. (1)

(1) Medizinische Klinik und Poliklinik II, Hämatologie-Onkologie, Universitätsklinik Leipzig, (2) Medizinische Klinik und Poliklinik III, Hämatologie-Onkologie, Universitätsklinik Ulm

WT1 is overexpressed ubiquitously in various types of leukemia cells, but not in normal cells, indicating WT1 to be an attractive target antigen for anti-leukemia cytotoxic T- lymphocytes (CTLs). Therefore, we studied the frequency of HLA-A0201-restricted CTLs specific for WT1 in patients with CML before and following allogeneic stem cell transplantation.

Methods: Unselected PBMCs were stimulated with the peptides RMFPNAPYL (aa 126-134) and SLGEQQYSV (aa 187-195), and the frequency of WT1-specific CTLs was determined by Elispot-analysis of IFN-secreting responder cells. An HIV-specific peptide served as negative control. In patients with high numbers of WT1 specific cells, the responding cells were purified by the IFN-secretion assay (Miltenyi Biotec) and further characterized with regard to their immunologic phenotype. In order to detect the expression of the WT1 gene in tumor cells, a quantitative RT-PCR was carried out.

Results: In contrast to 17 conventionally treated patients with CML who showed no WT-1-specific reactivity, WT1-specific CTLs were present in high frequency (up to 211:100.000) in 4 out of 10 pts up to 780 days after allogeneic transplantation. The CTL-response against both WT-1 peptides found in all positive patients is a clear indication of multiple epitopes to be involved. The response was either permanent or temporary with loss of reactivity after the patients became bcr/able negative. Phenotyping (CD27, CD45RA, HLA-DR, CCR7) of separated responding cells showed the CD8+ effector cell type to dominate. Despite high numbers of WT-1-specific CTL's, all patients showed regular engraftment.

Conclusion:The competence of peptide- reactive CTLs to specifically lyse WT1- expressing tumor cells remains to be demonstrated. Nevertheless, our data strongly suggest that the WT1 gene product is a promising target protein in the immunotherapy of leukemia and certain other WT1-expressing tumors.

\section{4}

Clonal evolution of $\mathrm{Ph}$-negative hematopoiesis in CML patients treated with imatinib

Hochhaus A. (1), Schoch C. (2), Haferlach T. (2), Lahaye T. (1), Kreil S.

(1), Berger U. (1), Gschaidmeier H. (3), Hehlmann R. (1)

(1) III. Medizinische Universitätsklinik, Fakultät für Klinische Medizin Mannheim der Universität Heidelberg, Mannheim, (2) Medizinische Klinik III, Klinikum Großhadern, Universität München, (3) Novartis Pharma, Nürnberg, Germany

Trisomy 8 was observed in Philadelphia $(\mathrm{Ph})$ negative hematopoiesis in three chronic myeloid leukemia (CML) patients treated with the selective tyrosine kinase inhibitor imatinib: Patient \#1: A 50 year old male patien was diagnosed with $\mathrm{Ph}+\mathrm{CML}$ in 1996 and treated with hydroxyurea (HU) and interferon $\alpha$ (IFN). In 7/01, acceleration with basophilia was reported. Therapy was changed to imatinib $(600 \mathrm{mg} /$ day $)$ within the expanded access protocol 0114 . In $4 / 02$, whilst in complete hematologic remission, cytogenetic evaluation of bone marrow (BM) metaphases revealed four differen clones: 46, XY, Ph [15]; 47, XY, Ph, +8 [3]; 46, XY [2]; 47, XY, +8 [3]. Fluorescence in situ hybridisation (FISH) confirmed trisomy 8 in 46/100 interphases. Patient \#2: A 51 year old female patient was diagnosed with $\mathrm{Ph}+$ CML in 1998 and treated with HU. Due to acceleration of the disease, imatinib therapy was commenced at a daily dosage of $600 \mathrm{mg}$ in $11 / 00$ (protocol 0114). In 5/01, 10/13 BM metaphases showed the typical Ph translocation without additional aberrations. In 3/02, complete cytogenetic remission was reached, the ratio BCR-ABL/ABL determined by real time RT-PCR was $0.3 \%$ consistent with complete cytogenetic remission. However, in 7/10 metaphases and 8/105 interphases trisomy 8 was detected. Patient \#3: A 36 year old female patient was diagnosed with $\mathrm{Ph}+\mathrm{CML}$ in $1 / 01$ and treated with HU and IFN. In 9/01, a combination therapy with imatinib and pegylated IFN $\alpha 2 \mathrm{a}$ (Pegasys) was started, and continued as imatinib monotherapy at a dosage of 300-400 mg daily since $10 / 01$. In 5/02, cytogenetic evaluation and FISH revealed trisomy 8 in 4/15 Ph-negative metaphases and 5/30 interphases. None of the patients reported showed any cytogenetic aberrations apart from the $\mathrm{Ph}$ translocation prior to the imatinib therapy. When trisomy 8 was observed in $\mathrm{Ph}$ negative metaphases, BM morphology did not show any signs of dysplasia. The emergence of cytogenetically unrelated $\mathrm{Ph}$ negative clones with additional aberrations in CML may support the multistep model of leukemic transformation and the concept of genetic instability inherent in CML. However, the detection of two independent clones harbouring trisomy 8 ( $\mathrm{Ph}$ pos. and $\mathrm{Ph}$ neg.) in patient \#1 may be related to imatinib long term toxicity. Therefore, even in good responders to imatinib, $\mathrm{BM}$ cytologic and cytogenetic analyses should be continued in regular intervals.

\section{5}

Global gene expression analysis by oligonucleotide microarrays can detect aberrant signaling pathways in CD34+ cells from patients with myelofibrosis with myeloid metaplasia

Hofmann W.K. (1), Jones L.C. (2), Tefferi A. (3), Wachsman W. (4), Hoelzer D. (1), Koeffler H.P. (2)

(1) University Hospital, Frankfurt/Main, (2) UCLA School of Medicine, Los Angeles, (3) Mayo Clinic and Mayo Foundation, Rochester, (4) UCSD School of Medicine, La Jolla

Objectives: Myelofibrosis with myeloid metaplasia (MMM) is a rare clona stem-cell disorder that leads to ineffective hematopoiesis, bone marrow fibrosis, and extramedullary hematopoiesis. To understand possibly altered cellular pathways, we used oligonucleotide microarrays to analyze global gene expression in CD34+ cells from patients with MMM.

Material and Methods: The gene pattern of expression in purified CD34+ cells from 6 patients with MMM was compared to the expression data from CD34+ cells from 4 healthy individuals by high-density oligonucleotide microarray analysis (HG-U95Av2, Affymetrix, Santa Clara, CA). Genes that 
were classified as up- and down-regulated had raw data values of at least 1,500 in the MMM and control samples, respectively, and demonstrated >5fold changes in expression. For confirmation, the expression of selected genes was analyzed by real-time polymerase chain reaction.

Results: Forty-three up-regulated and 26 down-regulated genes in MMM were selected for further study. Our data reveal enhanced expression of several transcription factors: AP-1 proteins JUNB and JUND, the hematopoietic transcription factor GATA2, and ATF-3 which stimulates the transcription of genes important for fibroblast growth in response to stress signals. Furthermore, we find that regulators of G-protein signaling including the guanine nucleotide exchange factor NET1, $\beta$-arrestin 2 , the G0/G1 switch regulatory gene G0S8, and a G-protein receptor induced phoshoprotein C8FW are all overexpressed in MMM. Among the down-regulated genes in MMM are two proteins whose activities are important for cell cycle mechanisms: BUB3, a mitotic checkpoint kinase, and MAD2, a monitor for spindle kinetochore attachment. Other cell cycle-related proteins whose expression is decreased in MMM include BCL1, CDC2 and CDC20. We also find that the antiproliferative cytokine TGF $\beta$ is down-regulated $>10$-fold in MMM. Conclusions: We hypothesize that dysregulation of these intracellular signaling pathways and/or decreased expression of key cell cycle proteins may play a role in the pathogenesis of MMM.

\section{6}

\section{Genome-wide expression profiling of highly enriched CD34+ hematopoietic progenitor cells in CML}

Kronenwett R. (1), Steidl U. (1), Roes N. (1), Neumann F. (1), Martin S (1), Junge B. (1), Kliszewski S. (1), Schroeder T. (1), Maercker C. (2), Hermann S. (2), Gattermann N. (1), Haas R. (1)

(1) Klinik für Hämatologie, Onkologie und klinische Immunologie, Heinrich-Heine-Universität Düsseldorf, Germany, (2) Deutsches Ressourcenzentrum für Genomforschung, Heidelberg/Berlin, Germany

In this study, we examined gene expression profiles of immunomagnetically enriched CD34+ hematopoietic progenitor cells from 7 patients with untreated CML in chronic phase in comparison to CD34+ cells from 12 healthy donors using cDNA array technology. For a genome-wide analysis nylon arrays spotted with 75,000 cDNA clones were hybridized with radioactively labelled cDNA from CD34+ cells of 2 CML patients or of 2 healthy volunteers. Comparing CML with normal CD34+ cells, 1352 sequences were at least 2-fold differentially expressed. 993 sequences had a lower and 359 a higher expression in CML suggesting a broader repertoire of active genes in normal cells in comparison to the malignant counterpart To corroborate the expression data, we used a cDNA array covering 1185 defined genes and examined CD34+ cells from bone marrow (BM) of 5 patients and 10 healthy donors. Comparing both groups we found 154 genes which were significantly differentially expressed in CML $(\mathrm{p}<0.01)$. The expression profiles reflected the functional properties of the leukemic cells as several genes were upregulated in CML which are involved in cell cycle progression and apoptosis inhibition such as cyclins, CDK4 or defender against cell death as well as genes involved in the bcr-abl signal transduction pathway such as members of the ras family, Jak2 or Akt. Besides, we found several genes for which differential expression was not described so far, as for example overexpression of the proto-oncogen ski and reduced expression of the tumor suppressor PRL-1. Downregulation of DNA repair enzymes such as mutL or ERCC5 might explain the occurrence of secondary genetic aberrations in CML. Interestingly, the opioid receptor $\mu 1$ was upregulated in CML suggesting a pathophysiological role for this molecule The lack of expression of zyxin, a focal contact protein at sites of adhesion, might play a role for the adhesion defect in CML. The intraindividual comparison of CML CD34+ cells from BM and peripheral blood (PB) showed no difference between both compartments. This was surprising since normal CD34+ cells had distinct molecular phenotypes in BM and PB. Hierarchical clustering revealed a closer relationship of CML CD34+ cells to normal $\mathrm{BM}$ cells than to normal circulating CD34+ cells.

In conclusion, we provide novel molecular insight into the biology of CML. We identified new candidate genes which may be pathophysiologically relevant and which might become targets for new therapeutic approaches.

\section{Cerivastatin significantly inhibits bone marrow colony growth} from patients with CML chronic phase

Kuhn C., Metzgeroth G., LaRosée P., Hastka J., Hochhaus A., Hehlmann R., Willer A.

III. Medizinische Klinik, Universitätsklinikum Mannheim

Objectives: Cerivastatin is an inhibitor of the enzyme 3-hydroxy-3-methyglutaryl coenzym A ( HMG-CoA) reductase, the major regulatory enzyme of the mevalonate pathway. Antiproliferative effects of cerivastatin have been described in solid tumors and hematological malignancies such as AML. However, the exact mechanism is not completely understood until now. Induction of growth arrest, apoptosis or functional suppression of RAS and Rho are discussed. Therefore, we wanted to study if cerivastatin has antiproliferative activity on BCR-ABL positive CML in chronic phase and if the effect is mediated by apoptosis.

Methods: Inhibition of colony growth was determined using a methylcellulose based colony forming assay with bone marrow mononuclear cells. In total, we studied four patients with BCR-ABL positive CML in early chronic phase prior to treatment and four healthy individuals as controls. Using the BCR-ABL positive cell line BV173, we analyzed growth inhibition by viability counts and apoptosis using the AnnexinV/ Propioniumiodit method.

Results: A selective and dose dependent effect on proliferation of colonies from patients with CML was observed after 14 days of culture: at 0.16 $\mathrm{ng} / \mathrm{ml}$, a median $40 \%$ inhibition of the colony numbers both from patients with CML and from healthy individuals ( n.s.); in contrast, at $0.016 \mathrm{ng} / \mathrm{ml}$, a significant inhibition of colony numbers from all patients with CML, when compared to healthy individuals (median $28 \%$, range $35-24 \%$ and $2 \%$, range $14-26 \%$, respectivly; $\mathrm{p}=0.03$ ). Furthermore, in the 48 hours culture with BV173, a growth inhibitory effect of cerivastatin was observed: $29 \%$ $(3 \mathrm{ng} / \mathrm{ml}), 30 \%(10 \mathrm{ng} / \mathrm{ml})$ and $33 \%(20 \mathrm{ng} / \mathrm{ml})$. However, $24 \mathrm{hr}$ exposure to increasing doses of cerivastatin revealed merely slight pro-apoptotic activity: $3.6 \%(0 \mathrm{ng} / \mathrm{ml}), 5 \%(3 \mathrm{ng} / \mathrm{ml}), 7 \%(10 \mathrm{ng} / \mathrm{ml})$, and $11.6 \%(20 \mathrm{ng} / \mathrm{ml})$ apoptotic cells

Conclusion: We have shown that cerivastatin i)has antiproliferative activity in the CML cell line BV173, ii) induces selective growth inhibition of BCRABL positive primary cells as detected in colony forming assays and iii) has only a moderate pro-apoptotic activity in the BCR-ABL positive cell line BV173, suggesting rather low cytocidal activity. Therefore, cerivastatin could be useful for therapy of hematologic disorders like CML in combination with other drugs.

\section{8}

Dynamics of bone marrow features improve prognostic classification in $\mathrm{Ph}^{1+} \mathrm{CML}$

Kvasnicka H.M. (1), Thiele J. (1),Schmitt-Graeff A. (2), Diehl V. (3)

(1) Institute of Pathology, University of Cologne, Germany, (2) Institute of Pathology, University of Freiburg, Germany, (3) First Clinic of Medicine, University of Cologne, Germany

Objectives: Risk classification of stable phase $\mathrm{Ph}^{1+}$ chronic myelogenous leukemia (CML) is generally focused on hematological variables. However, recent data provide confirming evidence that bone marrow characteristics at time of admission are significantly correlated with disease outcome and present prognostic factors independent of therapy.

Material and Methods: A multicenter observational study on 159 consecutively recruited patients in first chronic phase of $\mathrm{Ph}^{1+} \mathrm{CML}$ including representative sequential bone marrow biopsies at standardized intervals during therapy was performed to evaluate the prognostic impact of changing bone marrow patterns. In particular, the degree of argyrophilic fibers and the extend of erythropoietic precursors were quantified following immunohistochemical and morphometric techniques.

Results: According to the Sokal risk score $45 \%$ of our patients could be classified as low risk on admission, $34 \%$ were intermediate and $21 \%$ were high risk cases, respectively.With regard to pretreatment bone marrow histological patterns 46 cases presented with a granulocytic subtype, in 67 patients a predominant proliferation of atypical micromegakaryocytes was observed and in about $30 \%$ of our patients already a mild to overt increase in reticulin fibers was detected. Evaluation of the sequential bone marrow trephines showed a significant increase in fibers in about half of our patients independently of therapeutic regimens. An excessive reduction of erythropoiesis was observed in 70 patients before therapy. However, in $47 \%$ of the repeated bone marrow examinations a significant regeneration of the erythroid cell lineage was evident during therapy. Regarding survival, a manifest myelofibrosis in the first year of follow-up exhibited a worse prognostic 
impact independently of therapy. Furthermore, patients without relevant regeneration of the erythroid islets or even a further reduction of this cell lineage had significantly shorter survival times. These findings were independently of clinical risk classification by the Sokal and the Hasford score.

Conclusions: Our data strongly implicate that prognostic classification of chronic phase CML can be significantly improved by inclusion of morphological parameters and that in particular dynamics of myelofibrosis and regeneration of erythropoiesis during therapy are strongly associated with survival. Therefore, representative bone marrow biopsies should be taken during the course of the disease.

\section{9}

\section{High levels of BAX, low levels of MRP and high platelets are independent predictors of response to imatinib in myeloid blast crisis of CML}

Lange T. (1), Günther C. (1), Köhler T. (2), Leiblein S. (1), Musiol S. (1), Krahl R. (1), Al-Ali H.K. (1), v. Hoomissen I. (3), Niederwieser D. (1), Deininger M.W.N. $(1,4)$

(1) Department of Hematology, University of Leipzig, Germany;

(2) Roboscreen Gesellschaft für molekulare Biotechnologie mbH, Leipzig, Germany; (3) Novartis Pharma, Basel, Switzerland; (4) BMT/Leukemia Center, OHSU, Portland, OR, USA

Objectives: The results of allogeneic stem cell transplantation for chronic myeloid leukemia (CML) in myeloid blast crisis (M-BC) are better if patients are transplanted in remission. Imatinib (STI571, Gleevec ${ }^{\circledR}$ ) induces remissions in a subset of patients with little toxicity compared to conventional chemotherapy. Thus, it is important to identify factors predictive of response to Imatinib alone.

Material and Methods: CD34+ cells were separated from forty-six patient with CML in myeloid blast crisis [defined as $\geq 30 \%$ of blasts in the peripheral blood (PB) or bone marrow (BM)] and studied for expression of BCR $\mathrm{ABL}$ and a panel of drug-resistance and apoptosis-related genes (MRP, MDR-1, BAD, BAX, BCL-2, BCL-XL, MDM-2, Survivin) by TaqmanPCR. In addition in vitro sensitivity to graded concentrations of Imatinib was determined in CFU-GM assays. Together with other baseline parameters, the results were analysed for their impact upon response to Imatinib.

Results: In univariate analysis, only low PB blasts, basophils, low levels of MRP, BCL-2 and MDM-2 and high platelets, high levels of BAX and MDM-2 were associated with responsive disease. In multivariate analysis, only high platelets, high BAX and low MRP retained significance and predicted a favourable response to Imatinib. Combining the three parameters into a score accurately discriminated responders and non-responders $(\mathrm{p}=$ $0.00001)$.

Conclusions: We show that high levels of BAX mRNA, low levels of MRP mRNA and a high platelet count are independently predictive of a favourable response to Imatinib. If confirmed in a larger series, the predictive score may help to allow restricting more toxic combination therapies to patients who are unlikely to respond to Imatinib alone. This may be particularly relevant in the context of a subsequent allograft.

\section{0}

Regular screening by dual colour FISH detects submicroscopic aberrations beside primary chromosomal rearrangements in CML

Locher M. (1), Greulich-Bode K. (2), Grießhammer M. (1), Berger U. (3), Heinze B. (1)

(1) Innere Medizin III Uniklinik, Ulm (2) DKFZ, Heidelberg; (3) III Med. Klinik Universität Heidelberg, Mannheim

Objectives: $\mathrm{CML}$ is characterized by a reziprocal $\mathrm{Ph}$-translocation between chromosome 9 and 22, in which the genes bcr and abl merge to the bcr/abl oncogene. Recently submicroscopic deletions of the derivative chromosome 9 that span the translocation breakpoint, have been associated to a poor prognosis. The question is, how frequent are submicroscopic aberrations in patients with normal or variant $\mathrm{Ph}-\mathrm{Tl}$ and in patients with or without additional cytogenetic aberrations. We used dual colour FISH to screen for aberrations of genes, known to be involved in blast crisis specific aberrations and disease progression (on the chromosomes 8, 9, 17 and 22). Moreover the region of a supposed tumour suppressor gene on chromosome 1 was included in our study.

Material and Methods: 51 bone marrow or blood samples of 47 patients with the diagnosis of CML were analysed. The samples were sent from 24 cities of the German CML study group. 39/51 samples were taken from patients in chronic phase, $4 / 51$ in accelerated phase and 6/51 in blast phase.
One patient suffered from relapse after BMT. 20-25 mitoses have been analysed by GTG-banging. Item dual colour FISH analyses with 5 single locus probes: bcr/abl DF, c-myc, 19q21, 1p36 and 17p13.3/q21.1(Lis1), were applied on each sample. 4 probes were commercially available(Vysis, Qbiogene) and 19p produced by our own. Usually 200 interphase nuclei and 15 metaphases were scored by FISH analysis, using an automatic image analysis system.

Results: Two of 47 patients were $\mathrm{Ph}-$ and $45 \mathrm{Ph}+$. 13/47 exhibit additional aberrations of the Ph-translocation. First results of FISH analysis: 5/26 samples, until now analysed, showed an aberrant pattern of bcr/abl rearrangement due to variant or complex $\mathrm{Ph}-\mathrm{Tl}$ or loss of fusion signals. $2 / 22$ analysed samples indicated an aberrant pattern of c-myc and $1 / 10$ samples had an aberrant pattern of 19q 21. Genetic defects were not not found until now in the regions of $1 \mathrm{p} 36$ and $17 \mathrm{p} 13.3 / \mathrm{q} 21.1$. Cytogenetic results were usually confirmed. However, FISH analyses detected in some samples new aberrations, not found by conventional cytogenetics.

Conclusions: FISH analysis indicated aberrations in addition to conventional cytogenetics. However, the loss of fusion signals from bcr/abl rearrangement are less often than published. Also it seems, that single gene aberrations are not necessarily accompanied by the other aberrations tested. Further analyses will be done and the complete data set will be presented.

\section{1}

\section{BCR/ABL induced VEGF expression: role of PI3-kinase and mTOR}

Mayerhofer M. (1), Valent P. (1), Griffin J.D. (2), Sillaber C. (1)

(1) Department of Internal Medicine I, Division of Hematology \& Hemostaseology, Vienna, Austria, (2) Department of Adult Oncology, Dana Farber Cancer Institute, Harvard Medical School, Boston, USA

Objectives: In CML, VEGF is overexpressed in the bone marrow and peripheral blood. Aim of the present study was to examine whether the $\mathrm{BCR} / \mathrm{ABL}$ oncogene induces VEGF gene expression, and to characterize respective signal transduction pathways.

Methods: To study BCR/ABL-dependent VEGF-expression, IL-3-dependent $\mathrm{Ba} / \mathrm{F} 3$ cells inducibly expressing $\mathrm{BCR} / \mathrm{ABL}$ and primary $\mathrm{BCR} / \mathrm{ABL}$ positive CML cells were employed. BCR/ABL-dependent VEGF expression was investigated by analyzing VEGF promoter activities, VEGF mRNA and VEGF protein. Proliferation and viability of cells were evaluated by $3 \mathrm{H}$-incorporation assay, cell cycle analysis and annexin- $\mathrm{V}$ staining. Results: $\mathrm{Ba} / \mathrm{F} 3$ cells induced to express $\mathrm{BCR} / \mathrm{ABL}$ were found to exhibit substantial amounts of VEGF mRNA whereas cells starved from growth factor exhibited only baseline levels of VEGF mRNA. In the same cells, expression of BCR/ABL led to an approximately three-fold increase in VEGF promoter activity and substantially increased VEGF protein levels in supernatants. Moreover, BCR/ABL was found to promote expression of hypoxia inducible factor 1 (HIF-1), a major transcriptional regulator of VEGF gene expression. BCR/ABL-induced VEGF gene expression was counteracted by the PI3-kinase inhibitor LY294002 and rapamycin, an antagonist of mTOR, but not by inhibition of MEK through PD98059. Similarly, BCR/ABL-dependent HIF-1alpha activity was abolished by addition of LY294002 and rapamycin. As for $\mathrm{Ba} / \mathrm{F} 3$ cells, expression of VEGF mRNA was inhibited by LY294002 and rapamycin in primary CML cells, but was not affected by PD98059. We next analyzed the effects of rapamycin on growth and viability of various BCR/ABL-positive cell lines. As expected, rapamycin induced cell cycle arrest and inhibited proliferation of BCR/ABL-positive cell lines as well as in primary CML cells, but did not induce apoptosis as determined by annexin-V staining. Interestingly, proliferation of BCR/ABL-positive cell lines resistant to STI571 (STI571-resistant K562 cells and STI-resistant $\mathrm{Ba} / \mathrm{F} 3 \mathrm{p} 210$ cells) was also inhibited by rapamycin.

Conclusion: Together, our data show that BCR/ABL induces VEGF gene expression through a pathway involving PI3-kinase, mTOR, and HIF1alpha. Moreover, the mTOR-inhibitor rapamycin was found to inhibit growth of BCR/ABL positive cells by induction of cell cycle arrest. These observations may have implications for the pathogenesis of CML and for the development of new treatment strategies. 
Early reduction of BCR-ABL mRNA transcript levels predicts cytogenetic response to imatinib in chronic phase $\mathrm{CML}$ patients after failure of interferon alpha

Merx K. (1), Müller M.C. (1), Kreil S. (1), Lahaye T. (1), Paschka P. (1), Schoch C. (2), Weißer A. (1), Kuhn C. (1), Gschaidmeier H. (3), Berger U. (1), Hehlmann R. (1), Hochhaus A. (1)

(1) III. Medizinische Klinik, Universitätsklinikum Mannheim, (2) Medizinische Klinik III, Klinikum Großhadern, München, (3) Novartis Pharma GmbH, Nürnberg, Germany

Objectives: The degree of tumor load reduction as measured by cytogenetic response is an important prognostic factor for chronic myelogenous leukemia (CML) patients (pts.) on therapy. We sought to determine whether BCR-ABL transcript levels can predict chromosomal response.

Patients and Methods: Residual disease was evaluated in 120 CML pts. in chronic phase $(\mathrm{CP})$ treated with the selective tyrosine kinase inhibitor imatinib after resistance or intolerance to interferon alpha (IFN). Median time of therapy was 401 days (range 111-704). BCR-ABL and total ABL transcripts were measured in 486 peripheral blood $(\mathrm{PB})$ specimens with a real time RT-PCR approach using fluorescent labeled hybridization probes (LightCycler technology) and results were expressed as the ratio BCRABL/ABL. Cytogenetic response was determined in three-monthly intervals.

Results: From 101 evaluable pts., 42 achieved a complete (CR, 0\% Philadelphia chromosome [Ph] positive metaphases), 18 a partial (PR, 1$34 \% \mathrm{Ph}+$ ), 13 a minor (MR, 35-94\% $\mathrm{Ph}+$ ), and 26 no response (NR, $>94 \%$ $\mathrm{Ph}+)$. All PB samples were RT-PCR positive. Proportion of $\mathrm{Ph}+$ metaphases and simultaneous ratios BCR-ABL/ABL correlated with $\mathrm{r}=0.74, \mathrm{p}<0.0001$. In order to investigate whether early molecular analysis may predict cytogenetic response, quantitative RT-PCR data obtained after one and two months of therapy were compared with cytogenetic response at six months. Ratios BCR-ABL/ABL after one month were not predictive, but results after two months correlated with the consecutive cytogenetic response $(p=0.0008)$ The probability for a major cytogenetic response was significantly higher in patients with a ratio BCR-ABL/ABL $<20 \%$ after two months of imatinib therapy.

Conclusions: We conclude, that (1) quantitative determination of residual disease with real time RT-PCR is a reliable and sensitive method to monitor CML pts. on imatinib therapy; (2) ratios BCR-ABL/ABL correlate well with cytogenetic response; (3) in IFN pretreated pts. all complete responders to imatinib have evidence of residual disease with the limited follow-up available; and (4) cytogenetic response at six months of therapy in CP pts. is predictable with real time RT-PCR at two months.

\section{3}

\section{Dynamics of BCR-ABL mRNA expression in newly diagnosed CML patients treated with imatinib}

Müller M.C. (1), Gattermann N. (2), Lahaye T. (1), Deininger M.W.N. (3), Berndt A. (4), Frühauf S. (5), Reckzeh B. (6), Fischer T. (7), Kreil S. (1), Merx K. (1), Gschaidmeier H. (8), Hehlmann R. (1), Hochhaus A. (1), for the German STI Study Group

(1) III. Medizinische Universitätsklinik, Fakultät für Klinische Medizin Mannheim der Universität Heidelberg, (2) Med. Klinik, Universität Düsseldorf, (3) Abt. Hämatologie, Universität Leipzig, (4) Klinik f. Innere Medizin, TU Dresden, (5) Med. Klinik und Poliklinik V, Heidelberg, (6) Abt. Hämatologie/Onkologie, Universität Marburg, (7) Med. Klinik III, Universität Mainz, (8) Novartis Pharma GmbH, Nürnberg, Germany

Imatinib (STI571), a selective inhibitor of the BCR-ABL tyrosine kinase represents a promising new approach in the treatment of chronic myeloid leukemia (CML). An interim analysis of a phase III study in newly diagnosed CML patients (pts) revealed 68\% complete cytogenetic responders (CCR) within a median treatment interval of 14 months. We sought to determine the dynamics of BCR-ABL mRNA expression in $139 \mathrm{CML}$ pts (79 $\mathrm{m}, 60 \mathrm{f}$, median age 54, range 20-71 years) in first chronic phase from 17 participating German centers.

Pts were randomized to receive imatinib $(n=70)$, or interferon alpha $($ IFN $)+$ Ara-C $(n=69)$. The distribution of pts according to the new CML score was not different. Response to therapy was sequentially monitored by conventional cytogenetics from bone marrow metaphases $(n=586)$. In parallel, 922 peripheral blood samples were analyzed by qualitative nested and quantitative RT-PCR for BCR-ABL transcripts using the LightCycler technology. After a median observation time of 12 months 46 of 68 evaluable pts $(66 \%)$ on imatinib therapy vs. 4 of 67 pts $(6 \%)$ on IFN based therapy achieved CCR. Due to lack of response or intolerance 26 IFN treated pts crossed over to imatinib of whom ten consecutively achieved CCR. Levels of residual disease at first CCR were not different between imatinib treated pts (median ratio BCR-ABL/ABL 0.55, range $0.024-47 \%$ ), pts on IFN based therapy $(1.8$, range $0.79-5.1 \%)$, and imatinib after IFN (1.2, range 0.028-47) However, best molecular response in CCR was significantly different between imatinib treated pts $(0.12$, range $0.0019-7.7 \%)$ vs IFN based therapy (0.83, range $0.24-1.1 \%), p=0.0033$, but not between primary imatinib therapy and imatinib after IFN $(0.14$, range $0-2.5 \%)$, or between primary IFN therapy and imatinib after IFN. All pts on primary imatinib or IFN therapy remain PCR positive as yet. One of ten pts reached a sustained molecular remission after crossover from IFN to imatinib. BCR-ABL levels before and after one month of therapy were not predictive for cytogenetic response in any treatment group.

Considering the limited observation time we conclude, that (i) quantitative RT-PCR is an appropriate method to monitor treatment response to imatinib, (ii) levels of BCR-ABL parallel cytogenetic response, (iii) in complete cytogenetic responders, the level of residual disease is lower in pts after imatinib therapy as compared to IFN, but residual disease is rarely eliminated.

\section{4}

Strong correlation between bone marrow cytogenetics and FISH on peripheral blood neutrophils but not total leukocytes in CML patients on Imatinib

Reinhold U. (1), Hennig E. (1), Al-Ali H.-K. (1), Leiblein S. (1), Niederwieser D. (1), Deininger M. $(1,2)$

(1) Abteilung Hämatologie/Onkologie, Universität Leipzig,

(2) BMT/Leukemia, OHSU, Portland, USA

Objectives: The standard technique of monitoring patients with $\mathrm{Ph}$-positive CML is cytogenetics of bone marrow (BM) metaphases. However, it is not always possible to obtain sufficient numbers of metaphases for analysis, and $\mathrm{BM}$ aspiration is an unpleasant invasive procedure. Interphase FISH on peripheral blood (PB) leukocytes has therefore been advocated as a substitute to monitor patients on Interferon-alpha. In order to determine if this approach might also be useful to follow patients treated with Imatinib (STI571), we compared (a) BM cytogenetics and FISH for BCR-ABL performed on PB leukocytes in CML patients on various therapies (except Imatinib) and (b) BM cytogenetics with FISH on total as well as lineage-selected PB leukocytes in patients on Imatinib.

Material and Methods: Paired BM and PB specimens were studied in 14 CML patients (not treated with Imatinib) and 16 patients on Imatinib. In the latter patients, PB leukocytes were also FACS-sorted into neutrophils (CD33+), monocytes (CD14+), B-cells (CD19+) and T-cells (CD3+). The LSI BCR-ABL-DNA ES probe (Vysis) was used to do hypermetaphase FISH on BM samples and interphase FISH on PB specimens. Twenty-five metaphases or 500 interphase nuclei were analyzed in each sample.

Results: A strong correlation between BM metaphases and PB interphases $(r=0.9137, p<0.001)$ was found in patients not on Imatinib. By contrast, in the patients treated with Imatinib, this correlation was much weaker $(\mathrm{r}=$ $0.6428, p=0.013$ ). However, in the latter patients, there was a good correlation between BM cytogenetics and PB neutrophils $(r=0.89, \mathrm{p}<0.001)$, and reasonably good correlation between $\mathrm{BM}$ cytogenetics and $\mathrm{PB}$ monocytes $(r=0.76, p=0.001)$. No correlation was found in the case of $B$ cells $(r=0.3772, p=0.15)$ or $T$ cells $(r=0.1635, p=0.56)$.

Conclusions: In agreement with previous studies our results show a strong correlation between BM cytogenetics and PB FISH in CML patients on various therapies other than Imatinib. By contrast, this correlation is much weaker in patients on Imatinib, where a strong correlation is seen only in $\mathrm{CD} 33+$ cells. This is likely to reflect the fact that $\mathrm{Ph}$-negative lymphocytes constitute a significant proportion of the total PB leukocytes in Imatinibtreated patients. FISH analysis of unselected WBC is therefore not useful to monitor patients on Imatinib.

\section{5}

Deregulated tyrosine kinases in chronic myeloproliferative disorders and therapeutic implications.

Reiter A. (1), Hochhaus A. (1), Hehlmann R. (1), Cross N.C.P. (2)

(1) III. Medizinische Klinik, Universitätsklinikum Mannheim, (2) Wessex Regional Genetics Laboratory, Salisbury, U.K. \& Human Genetics Division, University of Southampton, U.K.

With the exception of chronic myeloid leukemia (CML), chronic myeloproliferative disorders (CMPDs) are a heterogeneous spectrum of conditions for which the molecular pathogenesis is only poorly understood. Most 
cases have a normal or aneuploid karyotype, but a minority present with an acquired reciprocal translocation that is amenable to molecular analysis Molecular cloning of the most common rearrangements at 5q31-33 and 8 p11 revealed that they each disrupt genes encoding tyrosine kinases, specifically the platelet-derived growth factor receptor B (PDGFRB) on chromosome 5 and the fibroblast growth factor receptor 1 (FGFR1) on chromosome 8 (8p11 myeloproliferative syndrome; EMS). As a consequence, constitutively active fusion proteins are produced that are functionally and structurally analogous to BCR-ABL. To date, four partner genes for both PDGFRB (ETV6/TEL, HIP1, H4/D10S170 and RAB5) and FGFR1 (ZNF198, CEP110, FOP and BCR) have been described, but FISH analysis has indicated that several other partners remain to be identified for both genes. Some of the cytogenetic abnormalities seen in CMPD may appear as a simple reciprocal translocation between either 22q11 or, less commonly, $9 \mathrm{q} 34$ and a third chromosomal region regarded to as variants of the Philadelphia chromosome. Occasional patients, however, are BCR-ABL negative and instead have distinct fusion genes involving encoding tyrosine kinases; for example, the $\mathrm{t}(9 ; 12)(\mathrm{q} 34 ; \mathrm{p} 13)$ fuses ETV6 to ABL and the $\mathrm{t}(9 ; 22)(\mathrm{p} 24 ; \mathrm{q} 11)$ fuses BCR to JAK2. In addition, we have identified a BCR-FGFR1 fusion in two patients with a $t(8 ; 22)(\mathrm{p} 11 ; \mathrm{q} 11)$ and a BCR PDGFRA fusion in a patient with a $t(4 ; 22)(\mathrm{q} 12 ; \mathrm{q} 11)$, highlighting the importance of deregulated tyrosine kinases in the pathogenesis of CMPD's. In the great majority of cases tyrosine kinase fusion genes are associated with a relatively aggressive disease. With the advent of targeted signal transduction therapy, an accurate clinical and molecular diagnosis of CMPDs has become increasingly important. Currently, patients with fusion genes involving ABL, PDGFRB or PDGFRA are candidates for treatment with imatinib (STI571). However, imatinib is inactive against FGFR1 and so it is unlikely that patients with EMS would benefit. In contrast, other compounds have been developed, eg. SU5404, SU6668 and PD173074 that also function as ATP binding site inhibitors and which possess anti-FGFR activity. FGFR inhibitors are therefore good candidates for treatment of EMS

\section{6}

Combination of heat shock protein 90 inhibition and imatinib shows synergistic activity in imatinib-resistant chronic myelogenous leukemia

\section{Schad M. (1), Topaly J. (2), Laufs S. (1), Zeller W.J. (1), Fruehauf S. (2)}

(1) Deutsches Krebsforschungszentrum (DKFZ) Heidelberg,

(2) Med. Klinik und Poliklinik V, UNI Heidelberg

The tyrosine kinase inhibitor imatinib (STI571, Glivec ${ }^{\circledR}$ ) has clearly proven its efficacy for treatment of chronic myelogenous leukemia (CML). However, drug resistance occurs. Overexpression of the BCR-ABL protein (p210), a supposed mechanism of resistance, is frequently observed in imatinib-resistant cells. The heat shock protein 90 blocker 17-allylamino-17demethoxy-geldanamycin (17AAG) is known to induce degradation of p210 by the proteasome with subsequent apoptosis in CML cells. As combination therapy is expected to overcome drug resistance to imatinib, we examined the effect of its combination with 17AAG on CML cell lines and primary CML cells.

BCR-ABL+ CML cell lines BV173, EM3, K562, LAMA84 (imatinib-sensitive), K562R (imatinib-resistant) and primary CML cells were used. Examination of EM3 cells by annexin V/propidium iodide assay revealed that the combination of imatinib and 17AAG induces apoptosis substantially more effectively than each of these drugs alone after 24 and 48 hrs of incubation. However, analysing inhibition of proliferation using the MTT assay in imatinib-sensitive CML cell lines, only combination index (CI; medianeffect method of Chou and Talalay) values indicating additive $(\mathrm{CI}=1)$ or slightly antagonistic $(\mathrm{CI}>1)$ effects were achieved at a $75 \%$ growth inhibition level by simultaneous treatment with both drugs. In contrast, the agents acted synergistically $(\mathrm{CI}=0.6, \mathrm{n}=3,75 \%$ growth inhibition level) in imatinib-resistant $\mathrm{K} 562 \mathrm{R}$ cells. In primary CML cells, combination of both drugs showed noticeable higher reduction in CFU-GM colony formation than each of them alone. BCR-ABL mRNA levels were more than 10-fold increased in $\mathrm{K} 562 \mathrm{R}$ cells compared to their sensitive counterparts using quantitative real-time PCR. A dose-dependent upregulation of BCR-ABL mRNA expression was found for both compounds as well as their combination in surviving CML cells. In contrast, on protein level (Western Blot) only treatment with imatinib alone caused an increase of p210, which could be counteracted by co-treatment with $17 \mathrm{AAG}$, even despite a further increase of BCR-ABL mRNA expression in cells treated by the drug combination.

Inducing degradation of p210, application of 17AAG is able to lower its level when increased by overexpression, a known mechanism of resistance to imatinib. Combination of imatinib plus 17AAG shows synergistic activity on imatinib-resistant K562R cells and may thus be useful to overcome imatinib resistance caused by $\mathrm{p} 210$ upregulation.
Clonal cytogenetic evolution in chronic myeloid leukemia patients treated with imatinib mesylate

Schoch C. (1), Haferlach T. (1), Kern W. (1), Schnittger S. (1), Berger U. (2), Hehlmann R. (2), Hiddemann W. (1), Hochhaus A. (2)

(1) Laboratory for Leukemia Diagnosis, Medical Department III, LudwigMaximilians-University, Munich, (2) III. Medizinische Klinik, Klinikum Mannheim der Universität Heidelberg

Recently, the selective competitive inhibitor of the BCR-ABL protein tyrosine kinase imatinib was introduced into the therapy of chronic myeloid leukemia (CML) in accelerated phase and blast crisis. In these advanced phases of CML secondary chromosomal aberrations are frequently observed in addition to the Philadelphia- $(\mathrm{Ph})$ translocation. We evaluated the prognostic impact of additional karyotypic changes on response to imatinib and the occurrence of new chromosome aberrations during therapy. We investigated 140 patients with BCR-ABL positive CML. Overall, 1555 months of imatinib treatment were monitored. Twenty patients were in blast crisis, 34 patients in accelerated phase, and 86 in chronic phase at start of therapy with imatinib. In 109 patients the Ph translocation was the sole karyotype abnormality, while in 31 patients additional karyotypic changes were observed prior to imatinib therapy. Patients with additional chromosome abnormalities were more frequent in blast crisis than those without (41.9\% vs. $6.4 \%, \mathrm{p}<0.0001)$ and were less frequent in chronic phase $(35.5 \%$ vs. $68.8 \%$, $\mathrm{p}=0.0008)$. Of the 31 patients showing chromosome abnormalities in addition to the $\mathrm{Ph}$-translocation prior to imatinib no cytogenetic response was observed in 16 cases $(51.6 \%)$ compared to 33 of 109 patients $(30.3 \%)$ without additional karyotypic changes $(\mathrm{p}=0.028)$. With respect to the stage of the disease 46 of 86 patients in chronic phase $(53.5 \%)$ but only 6 of 20 cases in blast crisis $(30 \%)$ showed a complete cytogenetic response $(\mathrm{p}=0.058)$. Only 20 of 86 patients in chronic phase $(23.3 \%)$ but 11 out of 20 cases in blast crisis were cytogenetic non-responders $(55 \%)(\mathrm{p}=0.005)$ Therefore, cytogenetic response to imatinib therapy was related to the presence of additional karyotype changes as well as to stage of the disease, but response did not differ according to additional cytogenetic abnormalities within the respective disease stage. During imatinib treatment $12.9 \%$ of patients acquired additional karyotype changes. Patients without cytogenetic response to imatinib were more likely to develop additional karyotype changes during the course of the disease. The incidence and the pattern of abnormalities occurring during treatment with imatinib is comparable to the clonal evolution during the natural course of the disease or during treatment with hydroxyurea or interferon alpha and does not show a specific pattern for imatinib treatment.

\section{8}

Structuro-genetic selection and validation of the dual-specific Src/Abl kinase inhibitor CGP76030: A potential new treatment modality for advanced Bcr-Abl-positive, STI571 resistant leukemia

Simon N. (1,2), Mitina O. (1,2), Hallek M. (1,2), Warmuth M. (1,2)

(1) Med. Klinik III, Klinikum Grosshadern, München,

(2) GSF - Forschungszentrum für Umwelt und Gesundheit,

Hämatologikum, KKG Gentherapie, München

Philadelphia-( $\mathrm{Ph})$-positive leukemias are characterized by the expression of Bcr-Abl, a fusion protein with deregulated tyrosine kinase activity. Recently, STI571, an Abl tyrosine kinase inhibitor, was shown to be of value for the treatment of Ph1+CML. However, STI571 is relatively inefficient in CML blast crisis and Ph1+ B-ALL. Lack of response or development of resistance to STI571 is caused by a direct resistance of leukemic cells due to different molecular mechanisms including Bcr-Abl gene amplification and point mutations disrupting STI571 binding. We could show that Bcr-Abl associates with and activates Src family tyrosine kinases by an Abl-kinase independent mechanism. Using a murine in-vivo model system we found that Bcr-Abl transduced bone marrow from Src kinase knockout mice (lyn/-hck-/-fgr-/-) is deficient in inducing Bcr-Abl-dependent B-ALL. In difference to wild type controls, mice transplanted with marrow from this triple Src kinase knockout strain showed no signs for disease development after more the 6 month of follow up. This suggested a role for Src kinases in induction of leukemia in vivo. Interestingly, genetic characterization of the interaction of STI571 and Abl established a structural rationale for the identification of dual-specific Src/Abl kinase inhibitors: both Src kinases an Abl share high homology as to a potential inhibitor binding pocket located adjacent to the ATP binding site. Using mutants of Abl and Src kinases which blocked the access to this proposed inhibitor binding site, a compound was identified, CGP76030, which inhibited Src kinases and Abl with equal po- 
tencies and overlapping binding modes. Biologically, CGP76030 blocked growth and survival of several Bcr-Abl-positive cell lines with potencies equal to STI571. However, in marked contrast to STI571, CGP76030 blocked cell growth and survival even in cells expressing various STI571/CGP76030-resistant Abl mutants. This suggested that a Src-related action of CGP76030 might contribute to the biological effects of the compound. Studies on potential signaling mechanisms demonstrated that CGP76030 inhibited the activity of Src family tyrosine kinases and Akt in dependently of $\mathrm{Abl}$ inhibition and was able to induce apoptosis in the presence of active Bcr-Abl, STAT5 and Jnk. In summary, the use of CGP76030 allowed to overcome a molecular resistance of Bcr-Abl to STI571, pointing to a potential role of these findings for the treatment of STI571 resistant $\mathrm{Ph} 1+$ leukemia

\section{9}

Altered molecular phenotype of CD105+ mesenchymal stem and progenitor cells in CML

Steidl U., Roes N., Fenk R., Rohr U.-P., Neumann F., Kliszewski S., Martin S., Gattermann N., Haas R., Kronenwett $R$.

Heinrich-Heine-Universität Düsseldorf; Klinik für Hämatologie, Onkologie und klinische Immunologie

In CML it has been shown that changes in the stromal cell compartment within the bone marrow have growth-stimulatory effects on the malignant cells. The underlying molecular mechanisms are largely unknown. In this study, we examined gene expression profiles (1,185 genes) of CD105+ stromal stem and progenitor cells of patients with newly diagnosed CML in chronic phase $(n=3)$ in comparison to healthy controls $(n=5)$. Using Clontech's Atlas Human 1.2 I cDNA arrays we found 63 genes which were consistently at least 2-fold differentially expressed in CML-CD105+ cells CML-CD105+ cells expressed the caspases 2 and 3 significantly 3.3 -fold and 2.1-fold less suggesting a decreased apoptotic activity in comparison to healthy controls. Looking at proliferation-mediating cell cycle control and DNA synthesis genes we found expression of 16 genes to be 2.1- to 3.8-fold lower than in normal controls (RCL, PLK1, CDC20, CDC28, MCM3, MCM5, RFC38, RFC40, helicase II, E2F1, B-MYB, NDKB, RAD23A RAD23B, LIG1, HMGI). In contrast, the proliferation-inhibiting p68 kinase, CDK inhibitor 2B, and hDMP1 were 2.6- to 3.2-fold higher expressed in CML-CD105+ cells. Taken together, those data suggest a reduced proliferative activity of CD105+ stromal stem and progenitor cells in patients with CML. Expression of the heat shock proteins HSP40, HSP70, and HSP73, as well as glutathion S-transferase A1 was also diminished in CMLCD105+ cells indicating a weaker stress defense system. Endothelial-monocyte-activating polypeptide II (EMAPII) which is known to induce tumor regression was 3.4-fold higher expressed in CML-CD105+ cells. This might be interpreted as an unavailing attempt to control growth of the malignant cells in CML. Two G protein-coupled receptors, opioid receptor mu1 and neuromedin B receptor, were 5.2-fold and 3.0-fold higher expressed in CML-CD105+ cells. On the other hand, the receptor tyrosine kinase EphA1 was 2.6-fold less expressed when compared to healthy controls. In summary, our data characterize the molecular phenotype of bone marrow CD105+ stromal stem and progenitor cells in CML. Gene expression patterns reflect a lower apoptotic rate and proliferation activity of stromal stem and progenitor cells. Further, we could demonstrate alterations in the expression of surface receptors suggesting changed signal perception in CML-CD105+ cells Our data may provide a molecular basis for an improved understanding of the pathogenesis of CML.

120

Farnesyltransferase inhibitors enhance imatinib action in chronic myelogenous leukemia cells

Topaly J. (1), Zeller W.J. (2), Ho A.D. (1), Fruehauf S. (1)

(1) Med. Klinik u. Poliklinik V, Univ. Heidelberg, (2) D0200, Deutsches Krebsforschungszentrum (DKFZ) Heidelberg

Farnsyltransferase inhibitors (FTI) were reported to inhibit growth of chronic myelogenous leukemia (CML) cells. We investigated the activity of FTI L-744,832 and LB42918 alone and in combination with imatinib (formerly termed STI571), a tyrosine kinase inhibitor known for its efficacy in treatment of CML, in CML cell lines and primary CML cells.

LB42918 was more potent with ED50 $=3.5 \pm 0.8 \mu \mathrm{M}($ mean \pm S.D., $n=3)$ in the BCR-ABL+ cell line EM-3 vs. ED50 $=25.4 \pm 1.9 \mu \mathrm{M}$ for $\mathrm{L}-744,832$ after $48 \mathrm{hrs}$ of treatment and assessment by MTT assay. The growth of BCRABL+ K562 and LAMA-84 cells was not measurably inhibited by L744,832 doses up to $25 \mu \mathrm{M}$. Similarly, the growth of K562 was not affected by $25 \mu \mathrm{M}$ of LB42918. However, for LB42918 in LAMA-84 cells an ED50 value of $29.7 \pm 10.5 \mu \mathrm{M}$ could be determined. In the FTI-sensitive cell line EM-3 combined effects of both FTI with imatinib were assessed using the median-effect method of Chou and Talalay. Both combinations were strongly synergistic with combination index (CI) values of $0.47 \pm 0.18$ and $0.67 \pm 0.15$ at ED75 for imatinib+L-744,832 and imatinib+LB42918 respectively. Using the Annexin V/propidium iodide assay we observed a strong increase of the apoptotic cell fraction in EM-3 cells treated for 24 hrs by the combination imatinib+L-744,832 as compared to treatment with each drug alone. The influence of imatinib and L-744,832 on CFU-GM colony formation of primary CML cells obtained from 4 patients was investigated and a mutual enhancement of inhibitory effects was observed again. Interestingly, in the FTI-resistant cell lines K562 and LAMA-84 a trend to lower ED50 values of imatinib was determined when up to $5 \mu \mathrm{M}$ of $\mathrm{L}-744,832$ or LB42918 were added, signifying potentiation of imatinib (see Table for imatinib+L-744,832, all doses are given in $\mu \mathrm{M}$ ).

\begin{tabular}{llll}
\hline & $\begin{array}{l}\text { ED50 of } \\
\text { imatinib alone }\end{array}$ & $\begin{array}{l}+1 \mu \mathrm{M} \\
\mathrm{L}-744,832\end{array}$ & $\begin{array}{l}+5 \mu \mathrm{M} \\
\mathrm{L}-744,832\end{array}$ \\
\hline K562 & $0.65 \pm 0.15$ & $0.55 \pm 0.21$ & $0.39 \pm 0.08$ \\
LAMA-84 & $0.42 \pm 0.16$ & $0.23 \pm 0.12$ & $0.18 \pm 0.09$ \\
\hline
\end{tabular}

The strong variation of FTI activity in different CML cells derived from patients in CML blast crisis would probably preclude the use of FTI as monotherapy in advanced phases of the disease. However, due to observed potentiation of imatinib FTI may find their place as supplement for CML patients on imatinib treatment.

\section{1}

STI571 decreases the mutation frequencies in cells expressing the active Bcr-Abl tyrosine kinase

van der Kuip H., Wohlbold L., Miething C., Duyster J., Aulitzky W.E.

Dr. Margarete Fischer Bosch-Institut für Kinische Pharmakologie Stuttgart, Robert Bosch Krankenhaus, Stuttgart, TU-München

CML progresses through clinical phases, becoming more and more resistant to treatment. The fact that this transition is accompanied by accumulating genetic alterations led to the suggestion that Bcr-Abl itself causes the genomic instability of chronic-phase disease. We measured the frequency of HPRT mutations in cells transfected with wild type and mutant Bcr-Ab forms. Cells expressing wtBcr-Abl showed an increase of spontaneous and gamma irradiation-induced HPRT mutations when compared with their BcrAbl negative counterparts. Importantly, the Bcr-Abl kinase activity is essential for this mutator phenotype: STI571-treatment of cells expressing wtBcrAbl significantly decreased the mutation frequency to a value comparable to that observed in Bcr-Abl negative cells with the same treatment. An amino acid substitution within the kinase domain (Y793F) in Bcr-Abl also resulted in diminished mutation frequencies. In contrast, mutation of the Grb2 binding site $(\mathrm{Y} 177 \mathrm{~F})$ had no effect on the mutator phenotype of Bcr-Abl. In summary, the present study shows that the Bcr-Abl kinase activity is responsible for the mutator phenotype observed in Bcr-Abl positive cells and that STI571 reverses this mutator phenotype in hematopoietic cell lines.

122

\section{Decrease of bone marrow angiogenesis in CML patients} treated with the tyrosine kinase inhibitor imatinib

Willer A. (1), Lahaye T. (1), Hastka J. (1), Saussele S. (1), Gschaidmeier H. (2), Hehlmann R. (1), Hochhaus A. (1)

(1) III. Medizinische Universitätsklinik, Fakultät für Klinische Medizin Mannheim der Universität Heidelberg, Germany, (2) Novartis Pharma, Nürnberg, Germany

Background: An increase of angiogenesis in the bone marrow (BM) has been observed in a variety of hematological malignancies including chronic myeloid leukemia (CML). However, the pathogenetic role of angiogenesis in leukemia is still conflicting. Imatinib (STI571) decreases tyrosine phosphorylation by competitive inhibition of ABL, c-kit, and PDGF-R. Hematologic and cytogenetic responses have been observed in BCR-ABL positive CML in chronic phase (CP) and in advanced disease.

Objectives: To elucidate a possible role of angiogenesis for pathogenesis of CML, we aimed to investigate the association between angiogenesis and disease activity. 
Methods: BM biopsies were taken from patients before and during treatment with imatinib. In total, 16 patients with CML were monitored. Median treatment duration was 11 months (range 1-21). Eight patients were in $\mathrm{CP}$ four in accelerated phase (AP) and four in myeloid blast crisis (BC). BM angiogenesis in thin sections was determined after immunostaining of endothelial cells by counting the microvessels; hematologic response was evaluated in peripheral blood and BM, and molecular response was determined by quantitative RT-PCR.

Results: Treatment with imatinib resulted in a significant decrease, i.e. normalization of BM microvessel density (median 10, range 3-50 vs. median 4 , range $1-6, p=0.0006$ ) in all patients with $\mathrm{CML}$ who were sensitive to imatinib. In contrast, in patients who were resistant to imatinib (one patien with $\mathrm{CP}$ and one with $\mathrm{BC}$ ), an increase of BM microvessel density was observed (range 8.7-9 vs. 9.5-11.3). Furthermore, a good correlation of BM angiogenesis to hematologic and molecular remission was observed in most patients (11/12) with CP and AP, but only in one of four patients with BC who was found to be imatinib resistant $(\mathrm{p}=0.018)$; in the three remaining $\mathrm{pa}$ tients with $\mathrm{BC}$, a decrease of BM microvessel density was detected despite lack of hematologic remission.

Conclusions: We conclude that (i) BM microvessel density correlates well with disease activity and clinical course in CP and AP CML, (ii) lack of response in $\mathrm{BC}$ despite reduction of microvessels suggests a direct inhibitory effect of imatinib on angiogenesis, (iii) progression of leukemia does not depend on BM angiogenesis.

\section{Case Reports}

\section{3}

\section{Sweet's syndrome associated with acute myeloid leukemia: A case report.}

Adorf D., Börner F., Lipp T., Nerl C.

Krankenhaus München-Schwabing

Introduction: Sweet's syndrome is an acute febrile neutrophilic dermatosis in which approximately $20 \%$ of the reported patients have an associated cancer. In literature the most common associated malignancy seems to be acute myeloid leukemia or myelodysplastic syndrome. Sweet's syndrome is characterized by pyrexia, neutrophilia and the abrupt appearance of erythematous, painful, cutaneous plaques primarily on the upper extremities, head and neck. Extracutaneous manifestations may occur and most often involve the musculoskeletal system. Histologically, the salient feature is a dense dermal infiltrate of neutrophils.

We report a case of a 56 year old woman, who was admitted to hospital with fever of unknown origin and severe and painful cutaneous lesions on the right tigh and the left forearm. A biopsy of the lesions showed no specific histological features, only few of the microbiological tests showed grampositive cocci without humoral signs of inflammation. Antibiotic therapy and application of immunoglobulins were unsuccessful. Cytomorphology and histology of the bone marrow showed myelodysplastic features but reactive bone marrow alterations could not be excluded. In the following weeks painful cutaneous erythematous, well-demarcated papules and plaques in changing locations appeared. Sonography and MR-tomography of M. deltoideus, biceps and trizeps of both sides showed partially myolytic and myositic features. In combination with humoral signs of inflammation and an elevated serum-creatinkinase a myositis was diagnosed. Vasculitis or other rheumatologic diseases could be ruled out. After corticosteroid therapy the cutaneous lesions and the humoral signs of inflammation disappeared, the serum-creatinkinase was normalized.

$2 \frac{1}{2}$ months later the patient was admitted to our hospital with a leucocytosis of $27,7 / \mathrm{nl}$, mild anemia and thrombocyto-penia. The differential count showed $23 \%$ of blasts. Cytomorphologic and immunocytologic analysis of the bone marrow confirmed acute myeloid leukaemia, subtype FAB M4 The karyotype was normal. The patient received 2 cycles of polychemotherapy with idarubicin, cytarabine, etoposid and 2 cycles of high dose cytarabine and mitoxantrone with a complete remission after the first course. 6 months after the first treatment she is still in complete remission without any cutaneous lesions. Retrospectivally the cutaneous and musculoskeleta lesions could be interpretated as an malignancy (AML)-associated sweet's syndrome.

\section{Gastrointestinal stroma cell tumour (GIST) of the esophagus}

Clauss M. (1), Berkovic D. (2), Kania U. (3), Rösler E. (4), Reis H.E. (5)

\section{Kliniken Maria Hilf GmbH Mönchengladbach}

A 62 year old women with a chronic hepatitis $C$ virus infection was routinely examinated for esophageal varices by gastroscopy. $4 \mathrm{~cm}$ proximal of the cardia a nodular structure below the mucosal surface was detected. An endosonography was performed which showed an inhomogenous tumour of the muscularis propria with a diameter of $4 \mathrm{~cm}$ and no signs of infiltrative growth into other parts of the esophageal wall. Staging procedures showed no other manifestations of the tumour. A leiomyoma of the esophagus was suspected and a resection was proposed to the patient.

The tumour was enucleated successfully without touching the mucosa. The first pathohistologic review seemd to confirm the clinical diagnosis of a leiomyosarcoma with good differentiation $(\mathrm{G} 1)$. However, a reference pathologist examination revealed positivity of the tumour cells for CD117 antigen changing the primary diagnosis into that of a gastrointestinal stroma cell tumour (GIST)

GIST are a rare entitiy of mesenchymal neoplasia of the gastrointestinal system. Usually they are found in the stomach or the duodenum. A GIST of the esophagus has to our knowledge not yet been described. Probably incidence of GIST may be much higher than expected because very often they are difficult to discriminate from leiomyoma or tumours of neurogen differentiation.

Recently the CD 117 membrane antigen has been discovered as very specific for GIST which may help to correctly diagnose this Tumour more accurately in the future.

This seems of high importance for the clinician because GIST show a different biological behaviour than other mesenchymal neoplasmas. Most striking is the proliferative dependence of these tumours on a certain tyrosine kinase of the stem cell factor receptor. This is clearly demonstrated by the antitumour effects of the tyrosine kinase inhibitor STI 571 in GIST.

Today primary resection of the tumour is considered as the treatment of choice as long as there is no evidence of widespresd disease. There is no recommendation for any adjuvant therapy. For palliative treatment only STI 571 seems to bring some benefit for the patient as conventional chemotherapy or radiation have only shown very marginal effects.

Painful gynecomastia as a leading symptom of gastric cancer

Fischer R., Südhoff T., Graeven U., Hollerbach S., Stefanou A., Teschendorf C., Reinacher-Schick A., Schmiegel W.

Medizinische Klinik der Ruhr-Universität, Knappschaftskrankenhaus Bochum

A 71-year-old patient presented at his urologist with chronic fatigue and newly recognized painful gynecomastia. In the laboratory investigation a high level of B-HCG $(950 \mathrm{mIE} / \mathrm{ml})$ attracted attention. The testicles were unsuspicious. A CT-Scan revealed a retroperitoneal mass, close to the pancreas. With the suspect of an extragonadal germ cell tumor the patient was admitted to hospital for resection of the tumor. The histological examination showed a mixed tumor with parts of a choriocarcinoma and embryonal carcinoma. To perform further diagnostic and therapeutic procedures the patient was transferred to our hospital.

We saw the patient 10 days after operation with jaundice, the B-HCG level was $(797 \mathrm{mIE} / \mathrm{ml})$. Sonographically signs of intrahepatic cholestasis were present. ERCP revealed a complete stenosis of the bile duct, probably caused by a clip placed during the operation. The endoscopy revealed a massive gastric infiltration by a tumor suspicious mass. Histological samples from initial lymph node resection and endoscopy were studied by three experienced pathologists. Two favored germ cell tumor (GCC) whereas for one pathologist samples were compatible with gastric cancer.

Thus diagnosis of GCC was established and PEB chemotherapy were started. After 4 courses we performed a restaging. In the endoscopic examination the gastric tumor was completely vanished. However the B-HCG levels were not normalized $(376 \mathrm{mIE} / \mathrm{ml})$. On endosonographic examination several enlarged perigastric lymph nodes were seen $(10-15 \mathrm{~mm})$. The cytological samples (FNP) from the lymph nodes revealed poorly differentiated vital tumor cells, which were suggestive for gastric cancer according the cytopathologist. Since staging including cytology revealed residual cancer only in perigastric lymph nodes gastrectomy with lymph node dissection and biliodigestive anastomosis was performed.

Conclusions: 1.) Painful gynecomastia can be the leading symptom in BHCG producing gastric cancer. 
2.) The differential diagnosis of poorly differentiated gastric cancer and retroperitoneal germ cell cancer may be difficult.

3.) PEB showed considerable activity in poorly differentiated gastric cancer.

126

\section{Primary intraocular lymphoma (PIOL) as uveitis masquerade syndrome}

Fricke H.-J. (1), Königsdörffer E. (2), Augsten R. (2), Voigt U. (2), Janz A. (2), Kosmehl H. (3), Höffken K. (1)

(1) Department of Internal Medicine II, (2) Department of Ophthalmology, (3) Institute of Pathology, Friedrich-Schiller-University Jena, Germany

Correct diagnosis in uveitis masquerade syndromes is often difficult. In patients with PIOL, clinical features of uveitis are common. They vary depending on the site of tumor cell infiltration (vitreous, retina, subretinal pigment epithelium). On first presentation, decreased vision and floaters are typical. In such cases, vitreous cellular infiltration is often the only evidence of the disease. This is the reason why autoimmune or infectious etiology is presumed. When lymphoma involves vitreous and retina, diagnosis may be more apparent but it is, nevertheless, difficult to prove it. Moreover, because of steroid therapy is effective both in uveitis and PIOL, the correct diagnosis of PIOL is often delayed until PIOL becomes resistant to steroid therapy (or the patient develops CNS disease).

We report a 67 year old woman who suffered from a decreased vision of the left eye since $8 / 2001$ and the right eye since $1 / 2002$. At presentation on our departments in 2/2002 we found keratic precipitates, a mild cellular reaction of the anterior chamber, an infiltration of the vitreous and yellowish subretinal infiltrates. The findings were found resistant to steroid therapy. Therefore, a pars plana vitrectomy was performed. The cytologic examination was negative (due to the small cell count after prior steroid therapy). Only when analyzing the vitreous liquid for immunoglobulin heavy chain rearrangement monoclonality as evidence for malignant lymphoma was detected.

Further diagnostic procedures did not show any evidence for an involvement of the CNS of other organs. High dose therapy with Ara-C improved the symptoms. Consolidation therapy by local radiation is planned.

Acute severe adverse events in advanced metastatic cancer following treatment with vinorelbine

Gengenbacher D., v. Hornstein W., Unger C., Weber R.

Klinik für Tumorbiologie an der Albert-Ludwigs-Universität Freiburg im Breisgau, Klinik für Internistische Onkologie, Breisacherstr. 117, 79106 Freiburg

The vinca-alkaloid Vinorelbine is an effective antineoplastic drug that is widely used for the treatment of cancer patients. This drug has been approved in the treatment of advanced breast cancer as well as advanced NSCLC patients. Herein we report a single center experience of severe side effects in 6 of 47 patients (12.8\%) with a median age of 55 years who had been treated with vinorelbine infusions between $07 / 01$ and $02 / 02$ ( 1 chordoma, 1 cervix carcinoma, 4 breast cancer) in our center. All but one patient suffered from unbearable pain during the time of vinorelbine infusion requiring a significant increase in the dosing of opioid medication. In 5 of the 6 patients pain lead to the discontinuation of the actual vinorelbine infusion. All of these patients were on high doses of analgetics prior to vinorelbine therapy for reasons of cutaneous or local inflammation at their tumor sites One patient developed life threatening bronchospasm and a fluid lung after vinorelbine infusion. This patient presented with lymphangiosis carcinomatosa and recieved antiobstructive therapy for chronic pulmonary obstruction. Before vinorelbine treatment, all of the patients reported here had prior cycles of either taxane, anthracycline or platin based chemotherapy. All but one patient had been treated with prior radiotherapy. For the patient who was radio naive, vinorelbine infusions could be continued for further ten cycles. In this patient dexamethasone was used as premedication. These clinical observations may implicate that the therapeutic use of vinorelbine in cancer patients should be carefully used in selected cancer patients. Patients presenting with either i) any tumor inflammation of the skin ii) extensive pain caused by the tumor iii) with prior bronchospasms or with iiii) prior radiotherapy are recommended an intensified monitoring under vinorelbine infusion. The use of dexamethasone in premedication prior to vinorelbine infusion may help to overcome side effects of vinorelbine chemotherapy in heavily pretreated and advanced metastatic cancer patients.
A rare case of extramedullary acute myeloid leukemia (chloroma) with arm paresis, maculopapular exanthema, stomach and orbital involvement

Glossmann J.-P., Diehl V., Josting A.

First Department of Internal Medicine, University Hospital Cologne, Joseph-Stelzmann-Str. 9, 50931 Koeln, Germany

The diagnosis of chloroma can be difficult in lack of demonstrable abnormality in the peripheral blood or bone marrow. Chloroma, also called granulocytic sarcoma or extramedullary myeloblastoma, is an extramedullary tumor mass composed of immature cells of the myeloid series. They occur in association with acute myeloid leukemia, as a sign of leukemic transformation in myeloproliferative or myelodysplastic disorders or rarely as an isolated finding unaccompanied by other hematological malignancies. Chloroma occur at a variety of sites, most commonly involving the bone, periosteum, soft tissue, lymph nodes and skin. If untreated, leukemia usually develops within 2 years.

Here we report a rare case of a 71 year old patient with chloroma initially presenting with incomplete paresis of the left arm, periorbital swelling and a maculopapular exanthema. Peripheral blood and bone marrow including cytogenetic analysis were normal. Physical examination additionally revealed an enlargement of the left testicle. Perioperative evaluation of cryocut sections of the testicle suggested a malignant lymphoma. MRI findings were those of a malignant brachial plexus lesion. Abdominal CT scans showed a thickening of the stomach walls. Biopsies of stomach an skin showed a infiltration of CD45- and chloracetatesterase-positive blast cells. Myeloperoxidase revealed intracytoplasmatic granules. The patient was treated with two cycles of chemotherapy including high-dose cytarabine, thioguanin daunorubicin and mitoxantrone (TAD, HAM). Six weeks after start of treatment all skin and organ lesions disappeared. The paretic arm totally recovered after four months.

Based on our experience and literature review we conclude treatment that patients with chloroma should be treated with intensive chemotherapy similar to that used to treat acute myeloid leukemia (AML) as soon as possible This case confirms the importance of including chloroma in the differential diagnoses of a variety of diseases.

\section{9}

\section{Spontaneous splenic rupture followed by the development of OPSY - a fatal course of patients with lymphoproliferative syndroms: a case report on two patients and review of the literature}

Heistinger M., Smetanig W., Rupacher E., Jukic Z., Klocker J., Geissler D.

\section{LKH Klagenfurt}

Spontaneous splenic rupture is a known complication in patients with enlarged spleen, but also occurs in patients with lymphoproliferative syndroms with envolvement of this organ even without splenomegaly. We report on two patients with lymphoproliferative disease ( pat.1: multiple myeloma, pat.2: high grade NHL), who presented with abdominal pain, decreasing haemoglobin-values and hypotension. Ultrasound examination and prompt paracentesis was diagnostic in both patients revealing intraperitoneal haemorrhage. In both cases immediate splenectomy was successfully performed, but OPSY occured in the later course and both patients died because of septic shock. For a better outcome of such patients several considerations should be taken in account:

1. Anaemia is common in lymphoproliferative syndromes, so progressive decrease of haemoglobin values can easily be interpreted as a sign of progression of the underlying disease. Immediate splenectomy could therefore be delayed, this contributes to a poorer prognosis of such patients.

2. Lymphoproliferative disease often is associated with secondary immunodeficiency. Recurrent pneumococcal bacteremia is regarded as a warning sign of immunodeficiency and associated with high mortality.

3. OPSY is a known and serious event in patients after splenectomy. In most cases this clinical syndrome is caused by streptococcus pneumoniae and may occur at any time after removal of the spleen. This is why vaccination with antipneumococcal vaccine is generally recommended for these patients. Another strategy is longterm antibiotic prophylaxis. But as described in our cases, antipneumococcal vaccination could not work when OPSY occured immediately after splenectomy. Although adequate antibiotic therapy was administered immediately this did not influence the fatal outcome. Although there are limited data in the literature, we discuss the possible benefit of a general antipneumococcal vaccination of patients with lymphoproliferative disease. 
Successful treatment of a gastrointestinal bleeding in refractory CLL induced very severe thrombocytopenia by application of Fibrogammin

\section{Hütter G., Blau I.W., Knauf W., Thiel E.}

Medizinische Klinik III, Hämatologie, Onkologie und Transfusionsmedizin, Universitätsklinikum Benjamin Franklin, Berlin

A 53-year-old man with B-chronic lymphatic leukemia was admitted because of a gastrointestinal bleeding in severe thrombocytopenia. The diagnosis of CLL was made on Mai 1989, and up to April 1999 the patient responded to intermittent chlorambucil, Thereafter treatments showed no further response, several attempts with fludarabine, bendamustin, and modified CHOP-regimes were made. Best response was seen after treatment with rituximab, and since November 2001 the disease was refractory to any therapy.

For our patient no origin of bleeding was found in gastro- and colonoscopy. We administered a course of Campath-1H, with good response of leucocytes but no effect on thrombocytopenia and transfusion frequency. An aspergillus pneumonia forced us to stop Campath-1H therapy. At this time transfusion frequency was 7-17 (median 12) packed red cells and 5-8 (median 6) packed platelets per week.

Although plasmatic coagulation was only modestly impaired, after seven weeks of persistent bleeding in thrombocytopenia we administered factor XIII (Fibrogammin) initially at a dosage of $1250 \mathrm{E}$ twice a day and after 10 weeks the dosage was reduced to $1250 \mathrm{E}$ once a day. Gastrointestinal bleeding disappeared after a few days of treatment with Fibrogammin. Frequency of transfusion decreased to 0-10 (median 4) packed red cells and 2-4 (median 3) packed platelets per week.

After regeneration of the leukocytes and recovery from aspergillus pneumonia we made a second course with Campath- $1 \mathrm{H}$, but once more without any effect on thrombocytopenia.

In this situation, an allogenic stem cell transplantation of an unrelated donor was performed after conditioning with Fludarabine and Treosulfane.

Conclusions: Bleeding in thrombozytopenia that shows not effect on administration of packed platelets is a therapeutic obstacle. In our case, a chronic gastrointestinal bleeding was the main problem. From admission to the day of transplantation the patient had 81 days of a severe thrombocytopenia with 0-3 platelets /nl. Since substitution with FXIII, gastrointestinal bleeding was controllable. Efficacy of Fibrogammin was seen at a dosage of $1250 \mathrm{E}$ per day. Even in a situation with high dosage chemotherapy and allogeneic stem cell transplantation, thrombocytopenia was no serious problem during Fibrogammin therapy. FXIII substitution may helpful and lifesaving in patients with a bleeding in severe thrombocytopenia.

\section{1}

\section{An unusual course of a Klatskin's tumor: A case report}

Kortus-Götze B. (1), Nötzel, E. (2), Schnabel, S. (2), Holle, A. (2), Liebe, S. (2), Freund, M. (1).

(1) University of Rostock, Department of Internal Medicine, Division of Hematology and Oncology and (2) Gastroenterology, Ernst-HeydemannStraße 6, 18057 Rostock, Germany

Background: A benign stricture of the extrahepatic bile ducts may occur months and even many years after a cholecystectomy. Prolonged obstructions lead often to a secondary biliary cirrhosis, which occasionally gives an indication for a liver transplantation. Moreover, strictures of the extrahepatic bile ducts may also be a sign of a Klatskin's tumor, which is rare and has a poor prognosis.

Objectives: We report on a sixty seven-year-old female patient, who had undergone a laparoscopic cholecystectomy in 1993. Six years later, in 1999, this patient complained about nausea, vomiting and fatigue. A painless jaundice and a bilirubin value of $71,2 \mu \mathrm{mol} / 1$ were noticeable. A stricture of the extrahepatic bile duct caused by several metal clips was found intraoperatively. The metal clips were removed and a T-tube drainage inserted, but one month later jaundice developed again. A performed ERCP showed the known stricture, which was suspected to be caused by a malignant tumor The tumor serum marker CA 19-9 has been conspicuously increased from the beginning of our treatment, but repeated cytological and histological examinations, performed during several ERCPs, PTCs and cholangioscopies, were without any sign of malignancy. In the following months recurrent cholangitis due to insufficient biliary drainages were observed. Many ERCPs, PTCs and cholangioscopies with implantations and changes of stents and drainages were necessary for relieving jaundice. Despite these in- terventions and manipulations, a secondary biliary cholangitis with cirrhosis CHILD C established as a complication of a permanent obstruction of the bile duct and an insufficient drainage effect.

Results: In a multidisciplinary approach in July 2001 it was decided to put this patient on the liver transplantation waiting list with the diagnosis: secondary biliary cirrhosis. In May last year a liver for this patient was available and the liver transplantation was performed successfully. Surprisingly, the histological examination revealed a Klatskin's tumor; TNM: pT2 pM0 $(0 / 1) \mathrm{Mx}$ G1. One year after the liver transplantation the patient is alive and has a good quality of life.

Conclusion: In our opinion this is an unusual course of a Klatskin's tumor Furthermore, this case might also show how difficult it can be, despite of several examinations through very experienced and skillful endoscopists and examiners, to establish a diagnosis.

\section{2}

\section{IgM-paraprotein as a marker of tumor burden in a patient with Burkitt-lymphoma}

Maas C., Franke A., Jentsch-Ullrich K., Klink A., Markmann I., Mohren M.

Department of Hematology/ Oncology; Magdeburg University Hospital, Magdeburg, Germany

Introduction: $\mathrm{LDH}$ is a well established but rather nonspecific tumormarker in patients with Non Hodgkin's Lymphoma (NHL). IgM is a typical surface-marker on NHL B-cells and an IgM-paraprotein is seen in up to $50 \%$ of patients with Burkitt-lymphoma (BL), especially if presenting with extensive tumor. Disappearance of the paraprotein has been seen with $\mathrm{CR}$ and reappearance has been noted with relapse of lymphoma. We report on the clinical course of a patient with BL and an IgM-k-paraprotein, who was successfully treated with polychemotherapy according to the German-BALL-protocol.

Patient: A 24 year old male patient presented with diarrhea, abdominal pain, fever, weight loss and nocturnal sweats. Diagnostic work-up revealed gastric ulcer, ileitic inflammation, ascites, pleural effusion and mesenteric tumor. $\mathrm{LDH}$ was elevated (4x normal range) and immunoelectrophoresis (IE) showed the presence of an IgM-k-paraprotein. Ileum and gastric biopsies revealed BL. There was no evidence of further lymphoma manifestations.

Clinical course: The patient underwent polychemotherapy according to the German-B-ALL-protocol with rapid clinical improvement. After two of six cycles no further tumor was found in ct-scans and serum-LDH went back to normal. However, the IgM-k-paraprotein was still detectable until the start of cycle four. At present ( 6 months after completion of therapy) the patient is still in CR with normal serum-LDH and absence of tumor or paraprotein. Conclusion: In our patient an IgM-k-paraprotein was still detected after achieving clinical CR and disappeared in the further clinical course. This is in concordance with findings in a small number of earlier reported patients. We conclude that IgM-paraprotein may be a more sensitive marker of tumor burden for BL than serum-LDH.

\section{3}

\section{Thromboembolic complications after splenectomy for hematologic disorders}

Markmann I. (1), Dworschak U. (1), Franke A. (1), Jentsch-Ullrich K. (1), Maas C. (1), Mewes S. (1), Scheinpflug K. (1), Weiss G. (2), Mohren M. (1)

(1) Department of Hematology / Oncology and (2) Surgery, Magdeburg University Hospital, Magdeburg, Germany

Introduction: Thromboembolism (TE) is an infrequent, but potentially fatal complication after splenectomy for hematologic disorders occuring in up to $10 \%$ of patients within the first 30 postoperative days. Thrombocytosis as well as immobilization are thought to play an important etiologic role. However, there exist no clear recommendations concerning prophylactic anticoagulation. We report on 3 patients, who experienced pulmonary embolism, 2 of whom with concurrent visceral thrombosis 8 to 35 days following splenectomy.

Patient 1: A 25 year old female with autoimmune hemolytic anemia was diagnosed with pulmonary embolism as well as portal vein thrombosis with liver and kidney failure 8 days after laparoscopic splenectomy. The further course was complicated by extension of thrombosis into the mesenteric vein leading to bowel ischemia and eventually death from multiple organ failure despite anticoagulation with full dose heparin.

Patient 2: A 22 year old male with ITP presented with pulmonary embolism (PE) and thrombosis of the portal and inferior cava vein 10 days after laparoscopic splenectomy. 
Patient 3: A 63 year old male with marginal zone lymphoma with splenomegaly and bone marrow involvement developed PE 35 days after open splenectomy.

All patients had been on low dose molecular weight heparin until complete mobilization and discharge from hospital and received full dose heparin after diagnosis of TE. Patient 2 and 3 were then switched to oral anticoagulation for a total period of 6 months.

Conclusions: Although generally infrequent we saw 3 patients with TE following splenectomy within a short period of time. Currently there exist no recommendations as to the length of prophylactic anticoagulation, especially since further risk factors for TE such as Protein C or S deficiency or the factor V Leiden mutation are usually not known in this patient group. Because of our own experience we decided to continue anticoagulation with low molecular weight heparin beyond mobilzation for at least 30 days post splenectomy. However as patient 3 shows even this regimen may not be sufficient for prevention, thus patients and physicians should be alert to symptoms of TE.

\section{4}

\section{A case of systemic alveolar rhabdomyosarcoma mimicking haematological neoplasia}

Maywald O. (1), Metzgeroth G. (1), Schoch C. (2), Ludwig W.D. (3), Neff W. (4), Hehlmann R. (1), Hastka J. (1)

(1) III. Medizinische Universitätsklinik, Fakultät für Klinische Medizin Mannheim der Universität Heidelberg, Mannheim, (2) Medizinische Klinik und Poliklinik III, Ludwig-Maximilians-Universität, Klinikum Grosshadern, München, (3) Abteilung für Hämatologie, Onkologie und Tumorimmunologie, Robert-Rössle-Klinik, Charite Humboldt-Universität Berlin, (4) Institut für Klinische Radiologie, Klinikum Mannheim

Alveolar rhabdomyosarcoma (RMS) is a rare malignancy, which is usually diagnosed in older children or younger adults. Primary tumour is often located in the extremities but is sometimes small or not detectable.

We report a case of a 16 year old boy presented with a short history of pain of the lower spine, fatigue, night sweat and fever. T1-weighted magnetic resonance imaging of the spine demonstrated a homogeneous suppression of the bone marrow fat signal; T2-weighted imaging revealed compression fractures of Th12, L1, L5 and a heterogeneous enhancement of the vertebral spine. In light of these findings, a haematological malignancy was supposed and the patient was admitted to our department.

White blood cell count, haemoglobin and platelet count were within normal range, however, in the differential blood count some myeloblasts and promyelocytes but no erythroblasts were visible. Lactate dehydrogenase was elevated to 2529 U/l. May-Grünwald-Giemsa stained bone marrow smears showed an infiltration by primitive and sometimes multinucleated cells. Immunostaining of these cells was positive for desmin and vimentin whereas cytokeratine and CD61 were not detectable. Flow cytometry demonstrated an expression of CD56 but no other lymphatic or myeloid antigens. Cytogenetic studies revealed a tetraploid karyotype with a translocation $\mathrm{t}(2 ; 13)(\mathrm{q} 35 ; \mathrm{q} 14)$ in 10 out of 13 metaphases, confirming the supposed diagnosis of bone marrow occupation due to alveolar rhabdomyosarcoma. The primary tumour was established as a subcutaneous plantar mass of $2.5 \mathrm{~cm}$ in diameter on the left foot.

The patient was treated with chemotherapy according to the CWS 96 protocol and died 2 years after diagnosis due to progressive disease. Diffuse marrow metastasis is particularly common in alveolar RMS and may mimic a heamatological malignancy. Compared to embryonal or undifferentiated RMS prognosis is poor ( $53 \%$ 5-year survival) mostly due to early metastasis.

\section{5}

Myelodysplastic syndrome with trisomy 21 in recipient-derived cells after allogeneic stem cell transplantation for philadelphianegative, bcr-negative chronic myeloid leukemia: A report of two cases

Al-Ali H.K., Friedrich T., Müller C., Niederwieser D.

\section{Abt. Hämatologie und Onkologie, Universität Leipzig}

Objectives: Relapse after allogeneic stem cell transplantation (ASCT) is usually due to regrowth of the malignant clone present prior to transplantation. Secondary MDS (SMDS) is a known long-term complication after autologous transplantation. We describe two cases of SMDS in recipient-derived cells with trisomy 21 after ASCT for Ph-negative CML. The intriguing questions refer to the time when the abnormal clone arose and whether this pattern of evolution is a possible complication after ASCT for Ph-negative
CML. Two female patients presented with leukocytosis and splenomegaly and were diagnosed as Ph-negative CML. Karyotype at diagnosis was normal. Patient 1, 33 years old, received ASCT from her HLA identical brothe after eight months treatment with Busulphan. Patient 2, 51 years old, received ASCT from a matched unrelated male donor after seven months treatment with Hydroxyurea. Both were not treated with IFN-a. Conditioning consisted of Busulphan $16 \mathrm{mg} / \mathrm{kg} / \mathrm{d}$ for 4 days and Cyclophosphamide $60 \mathrm{mg} / \mathrm{kg} / \mathrm{d}$ for 2 days. Both developed grade III acute GvHD of the skin. Immunosuppression was discontinued 12 months later in patient 1 . In patient 2, tapering of Cyclosporin A was ongoing.

Results: Both patients developed pancytopenia (WBC $<2 \times 109 / \mathrm{L}$, platelets $<10109 /$ L) 84 (patient 1) and 11 (patient 2) months after ASCT. Bone marrow was hypercellular, with blasts $>10 \%$. A diagnosis of MDS, RAEB-II (WHO-classification) was made. A mixed donor chimerism of $80 \%$ was found by FISH. 2\% recipient T-cells and 20\% recipient CD34+ cells. The karyotype was 47,XX,+21 [4], 46,XY [8] in patient 1 and 47,XX,inv(11)(p13p15),der(15q+),+21[16] 46,XY [8] in patient 2.

Conclusion: Cytogenetic abnormalities involving chromosomes 11, 15, 21 as well as others are described in association with Ph-negative CML. It is possible that an active primary aberration existed either within the malig nant clone or in another distinct clone. This clonal diversity may not become apparent as long as one clone has a proliferative advantage and no selective pressure is applied. After ASCT, such proliferative advantage may have occurred in host cells surviving ASCT. Alternativly, genetic damage in remaining recipient cells caused by the preparative regimen, may have taken place. Studying unbalanced genetic changes would be important to identify disease-specific gene rearrangements relevant to diagnosis or prognosis and may identify patients with increased risk of developing MDS after ASCT.

\section{6}

Treatment of aggressive Non-Hodgkin's Lymphoma (NHL) in a patient with Ataxia telangiectasia (AT)

Saußele S. (1), Dencausse Y. (1), Weiss A. (1), Hehlmann R. (1)

III. Medizinische Klinik, Fakultät für Klinische Medizin Mannheim der Universität Heidelberg

Patients with AT, an autosomal recessive genetic neurological disorder, are known to have an increased risk for cancer due to the mutated ATM gene on chromosome 11q22-23 resulting in both neuronal degeneration and genetic instability. $10 \%$ of homozygote AT patients develop cancer, mostly of the lymphoid system. Treatment of AT patients requires extremely careful planning and caution with respect to the use and dose of chemotherapy, since the patients suffer from increased chemosensitivity, radiosensitivity and an increased risk for infectious diseases such as pulmonary infections and sinusitis. One study on pediatric patients with chromosomal breakage syndromes included five patients with AT; the therapy was curative with adjustment to individual risk factors and tolerance. Radiation, alkylating agents and epipodophyllotoxines were omitted, and methotrexate ( $\mathrm{mtx}$ ) was limited to $1 \mathrm{~g} / \mathrm{m}^{2}$. Based on this study, it was recommended for patients with AT, incomplete resection and $\mathrm{LDH}<500 \mathrm{U} / 1$ to give a four cycle chemotherapy, which includes blocks A1-B1-A2-B2 of the childrens B-NHL protocol of the German study group in a modified order.

We report on a 24 year old female patient with AT and aggressive B-NHL. The staging examinations after diagnosis in February 2002 revealed a stage III disease with involvement of the right tonsil, right cervical lymph nodes and an abdominal tumor of $8 \times 8 \mathrm{~cm}$. The patient was treated according to the above protocol with a reduced dose of $\mathrm{mtx}$, cytarabine (arac) and doxorubicin (dxr) (block A: $\mathrm{mtx} 250 \mathrm{mg} / \mathrm{m}^{2}$, arac $75 \mathrm{mg} / \mathrm{m}^{2}$, fortecortin 10 $\mathrm{mg} / \mathrm{m}^{2}$, vincristine $2 \mathrm{mg}$, block B: instead of arac dxr $20 \mathrm{mg}$ day 4 and 5). After administration of block A1 and B1, a WHO grade II to III mucositis with severe obstipation (grade III) due to colitis was observed. Therefore, the chemotherapy dose could not be increased. The restaging examinations after block B1 in April 2002 revealed a complete remission. After block B2, a grade IV mucositis, a grade III infection and a leukopenia and an anemia grade III occurred, and the neurological symptoms worsened transiently. Until now, there are only few reports on B-NHL in AT patients over 20 years of age. The reported survival time in these patients did not exceed six months. Although we reduced the recommended dose of chemotherapy, the patient is still in remission. Toxicity was considerable but reversible. Therefore, we suggest to cautiously reduce the dose of chemotherapy in patients with AT over 20 years. 
Tremendous cutaneous toxicity of Imatinib Mesylate and allogeneic hematopoetic stem cell transplantation in a patient with CML

Schaich M. (1), Schäkel K. (2), Illmer T. (1), Nettesheim O. (1), Meurer M. (2), Ehninger G. (1), Bornhäuser M. (1)

(1) Med. Klinik und Poliklinik I, Universitätsklinikum Dresden,

(2) Klinik und Poliklinik für Dermatologie, Universitätsklinikum Dresden

A 52 year old male patient was treated with a daily dose of $800 \mathrm{mg}$ of the tyrosine kinase inhibitor Imatinib Mesylate (IM) for lymphoid blast crisis of chronic myeloid leukemia. After two months he was scheduled for an allogeneic stem cell transplantation from an unrelated donor. IM was stopped 48 hours before the start of the preparative regimen. Conditioning was performed with Fludarabine $120 \mathrm{mg} / \mathrm{m}^{2}$ and Busulfan $16 \mathrm{mg} / \mathrm{kg}$. On day 12 after transplantation the patient suffered from severe mucositis CTC grade 4 and generalized edema associated with an increase in blood urea and creatinine. Two days later purpura and massive symmetrical bullous eruptions of the skin developed on both lower legs.

Two days later the primarily translucent bullae became hemorrhagic and up to $50 \mathrm{ml}$ of a viscous sterile fluid could be aspirated out of each bulla. No signs of skin GvHD could be ascertained. Treatment with steroids at $1 \mathrm{mg} / \mathrm{kg}$ was initiated. After hematological engraftment, the wounds at both legs started healing. Another ten days later the skin damage was almost completely resolved and the clinical status of the patient improved substantially.

Cutaneous adverse reactions can be seen in up to $25 \%$ of patients with CML receiving higher doses of IM. However, the here reported case illustrates that cumulative toxicity of IM and conditioning therapy can be tremendous. With more patients receiving allogeneic stem cell transplantation after treatment with IM, clinicians should be aware of a potential increase of extramedullary toxicity after conditioning therapy.

\section{8}

\section{Atrophic gastritis „cures“ hemochromatosis}

Schatz M., Willer A., Hehlmann R., Hastka J.

3. Medizinische Klinik, Universitätsklinikum Mannheim, Universität Heidelberg

We describe a 72-year old male patient who first presented with a ferritin concentration of $2550 \mu \mathrm{g} / \mathrm{l}$ in 1993. Serum iron concentration was 47,2 $\mu \mathrm{mol} / \mathrm{l}$ and transferrin saturation $78 \%$. Desferal test was highly pathological. Liver biopsy confirmed iron overload. Molecular examination identified C282Y homozygosity of the HFE-gene. No signs of organ dysfunction was found. Primary hemochromatosis was diagnosed. The patient was started on a twice weekly phlebotomy regimen, which was continued until 1995. Under phlebotomy serum ferritin concentration normalized. In 1995 phlebotomy was stopped, after ferritin concentration remained stable at low levels for months.

At this time significant macrocytosis developed (MCV $120 \mathrm{fl})$. Serum cobalamin showed very low levels $(<50 \mathrm{ng} / \mathrm{l})$. Folate levels were normal. Schilling test indicated cobalamin malabsorption and normalized after application of intrinsic factor (IF). Antiparietal antibodies and anti-IF antibodies were negative. Endoscopic examination of the upper gastrointestinal tract identified chronic gastritis of the fundus with severe atrophy and metaplasia of the mucosa. Furthermore one polyp of the fundus and one of the body were detected, without any sign of bleeding. Histologic examination of the resected fundus polyp revealed carcinoma in situ.

In light of these findings, cobalamin deficiency of pernicious type was diagnosed and continuously parenteral cobalamin-substitution was started. Under this therapy, MCV turned to normal, ferritin level, however, did not rise. Nine years after the diagnosis the patient remains in a good health, without signs of hemochromatosis typical damages and without need of phlebotomies within last seven years. A possible explanation of this case could be the achlorhydria due to atrophic gastritis with impaired iron absorption.

\section{Transient Pelger-Huet-anomaly in acute infection}

Schmalz O., Binder T., Raghavachar A.

Klinikum Wuppertal, Department of Haematology and Oncology, Heusnerstraße 40,42283 Wuppertal

Objectives: The association of abnormal granulocyte nuclear formation and tuberculosis was first reported by Pelger in 1928. Huet, however, concluded that this defect was hereditary, and the association of Pelger-Huet anomaly (PHA) of granulocytes and tuberculosis was obscured for many years. But furthermore PHA is regularly observed in myelodysplastic syndrome (MDS). We describe PHA-like hyposegmentation of granulocyte nuclei in an adult patient presenting with a severe acute illness of probable virus infection.

Material and Methods: A 30 years old female patient was admitted to our hospital with fever, illness, headache, vomitting, diarrhoea, rheumatic pain and a morbilliform rash. On physical examination the patient was alert, fully oriented, and able to converse. Lymphadenopathy was found at the neck. The lungs, heart, abdomen, arms and legs were normal. Neurological examination showed a stiff neck. The temperature was 41.2 centigrees, the pulse 130 , and the respiration was 18 . The blood pressure was $90 / 60$ $\mathrm{mmHg}$. The CRP was $35,3 \mathrm{mg} / \mathrm{dl}$, the white cell count $4.9 / \mathrm{nl}$ with 8 percent lymphocytes, $4 \%$ monocytes, $4 \%$ eosinophils, $66 \%$ neutrophils and $14 \%$ bands. Nearly all neutrophils showed hyposegmented nuclei as in PHA and hypogranular cytoplasm. The platelet count was $67 / \mathrm{nl}$. The cerebrospinal fluid and the MRT of the head were normal. All microbiological, immonological and serological examinations of blood, sputum, urine and faeces were not able to detect the agent of the infection. X-ray of the chest, abdominal sonography, gastroscopy and rectosigmoidoscopy showed no focus. The patient was treated symptomatically with antiphlogistics without antibiotic treatment. In 2 weeks down all pathological findings turned to normal, especially the blood film showed normal neutrophils without PHAlike morphology. Being alert from this case, we then observed 8 more patients with various severe viral or bacterial infections showing the same transient PHA phenomenon.

Conclusions: Investigations surrounding this case suggest a possible association of PHA with acute infection. Shetty et al. (Blood 2000; 96:1388ff) have shown that the death of Pseudo-Pelger-Huet-cells in MDS patients might be inflicted by high amounts of proapoptotic cytokines such as TNF alpha and INF gamma. We suggest that the transient PHA changes in our index patient and the other patients follow the same pathway. Further investigations on the cytokine network in these cases are in progress.

\section{0}

\section{Successful treatment of multiple relapses of astrocytoma grade III with oral temozolomide}

\section{Schroeder M., Aul C.}

Medizinische Klinik 2, St. Johannes-Hospital, An der Abtei 7-11, D-47166 Duisburg

We report on a 52-year-old who was diagnosed as having astrocytoma grade III in the right temporo-parietal hemisphere in Januar 1993. The patient underwent neurosurgery and subsequently received postoperative radiotherapy with a total dose of $60 \mathrm{~Gy}$.

In 11/1998, a relapse of the brain tumor was diagnosed by MRT. There was no possibility of a second surgical intervention. At time of clinical progression manifested by Jackson epilepsies, we started an oral chemotherapy with temozolomide at a dose of $150 \mathrm{mg} / \mathrm{m}^{2} / \mathrm{d} 1-5$. Between 05/1999 and 06/2000, the patient received 12 cycles of this chemotherapy. Repeat MRT brain showed a complete regression of the astrocytoma. Therapy was stopped and the patient was followed-up at monthly intervals. In 11/2000, a second relapse at multiple sites of the brain was diagnosed. On applying the same schedule of chemotherapy (temozolomide $150 \mathrm{mg} / \mathrm{m}^{2} \mathrm{~d} 1-5$ at monthly intervals), the patient entered a second complete remission which lasted until 12/2001. A third relapse of his brain tumor could also be successfully treated with temozolomide. No significant side effects of chemotherapy were observed during the entire treatment period.

In summary, this case report demonstrates that temozolomide is an effective cytostatic drug for selected patients with astrocytoma grade III. 


\section{Rapid tumor lysis and sustained cytopenia after treatment of} relapsed Burkitt leukemia with rituximab and GM-CSF

\section{Schuler E., Matylis M., Hemmati P., Bargou R., Ratei R., Ludwig W.D.}

HELIOS Klinikum Berlin, Robert-Rössle Klinik, Charité Campus BerlinBuch, Medizinische Klinik mit Schwerpunkt Hämatologie, Onkologie und Tumorimmunologie

Rituximab has been recently demonstrated to add therapeutic benefit to standard chemotherapy in aggressive B-cell lymphomas. Its role in Burkitt leukemia (BL) has not yet been established.

We report on a 20-year-old patient, being diagnosed with BL. Treatment was started according to the B-ALL-Protocol (GM-ALL 06/99) including CNS prophylaxis. Routine CSF tests revealed no pathology. Complete remission was achieved after 4 cycles of chemotherapy (CT). CNS radiation was scheduled after completion of 6 cycles CT. 2 weeks after completion of the 6th cycle the patient presented with a rapid deteriorating performance status and facial palsy. Early relapse of Burkitt leukemia was diagnosed in CSF, BM and suspected organ involvement.

Therapy: Systemic therapy was planned according to a protocol performed in children (personal communication: A. Reiter, Univ. of Giessen). After cytoreductive treatment with Rituximab (d1-2) in combination with GMCSF (d1-5) a HD-Ara-C (3 g/m $\mathrm{m}^{2}$ and VP-16-containing regimen (CYVE) is applied (Patte et al., Blood, 2001, Vol. 97, 3370f.). Here GM-CSF application $(5 \mu \mathrm{g} / \mathrm{kg} \mathrm{BW}$ s.c) was initiated 2 hours prior to start of rituximab $\left(375 \mu \mathrm{g} / \mathrm{m}^{2}\right)$. Supportive medication followed local protocol. Corticosteroids were withdrawn $36 \mathrm{hrs}$ before start. After infusion of $5 \mathrm{mg}$ rituximab, the patient presented with a clinical picture of flush, emesis and hypotension. $20 \mathrm{~min}$ after start of rituximab, a rapid and sustained decline of WBC was detected (12 Gpt/l to $0,9 \mathrm{Gpt} / \mathrm{l})$. Tumor lysis syndrome with coagulation disturbances and renal impairment developed. $2.5 \mathrm{hrs}$ after start of rituximab severe capillary leakage with a shock syndrome required treatment with prednisolon $(2 \mathrm{mg} / \mathrm{kg} \mathrm{BW})$. Subsequently hypotension resolved rapidly. The 2nd application of rituximab (d2) was well tolerated. Sustained pancytopenia (ANC $<0,5 \mathrm{Gpt} / 1,>14$ days) was observed. BM at $\mathrm{d} 8$ showed hypocellularity with no residual leukemia involvement. LDH normalised (initial $7000 \mathrm{U} / \mathrm{l}$; maximum $30000 \mathrm{U} / \mathrm{l}$ ) and CYVE was administered as scheduled.

Conclusion: Rituximab in combination with GM-CSF in a patient with relapsed Burkitt leukemia induced severe tumor lysis syndrome and resulted in rapid leukemia depletion and sustained cytopenia. Our data demonstrate strong anti-tumor acitivity of rituximab in combination with GM-CSF in relapsed BL. Future clinical studies have to define the optimal timing and sequencing in combination with conventional $\mathrm{CT}$ in BL patients.

\section{2}

\section{Vascular toxicity of 5-Fluorouracil (5-FU)}

Stoll C., Unger C., Arends J.

\section{Tumor Biology Center at Albert-Ludwigs-University, Freiburg}

Cases: During the last 5 years we have seen 12 patients $(5 \mathrm{M}, 7 \mathrm{~F}$; range 52 81 years) treated with 5 -fluorouracil $(\mathrm{N}=6$ bolus doses; $\mathrm{N}=5$ constant infusion) or capecitabine $(\mathrm{N}=1)$ who experienced drug-related vascular dysfunction in different organs. 3 patients received platinum compounds at the same time. Mean time to onset of symptoms was 3.8 (range 1-6) days. 6 patients reported angina pectoris, 5 had ECG changes, atrial fibrillation developed in 1, tinnitus in another, 1 had cerebral ischemia with hemiplegia and 1 suffered from thrombotic occlusion of a popliteal artery. Stopping or reducing 5-FU dose resulted in symptom resolution in 3 but not in 2 others. Calcium channel blockers improved 5-FU tolerance in 1 but not in another, sotalex resolved symtoms in 1 patient. Raltitrexed was given subsequently to 2 patients without recurrence of problems.

Literature search: During treatment with 5-FU the following problems have been reported: brain infarctions, coronary artery spasms, myocardial infarctions, left ventricular dysfunction and cardiogenic shock. The incidence of vascular symptoms is between 0.5 and $20 \%$ with higher risk in patients with preexisting cardiovascular problems. The onset of symptoms usually is 2 to 6 days after the beginning of treatment. Myocardial damage appears to occur without the ischemia type release of myocardial enzymes. One patien experienced angina $30 \mathrm{~min}$ after dermal application of a 5-FU creme; one group reported cardiac problems after a mean of 14 hours of treatment with resolution of the symptoms within $60 \mathrm{~min}$ after ending the 5-FU infusion. Despite the lack of a complete understanding of 5-FU-induced toxicities, experimental data support at least 2 different ways of action. One mode includes the acute induction of vasospasms, which may be relieved by nitrates, but not by alpha-, beta- or calcium channel blocking agents. Another mode appears to be the slow development of endothelial damage with subsequent thrombus formation, extravasation and tissue inflammation as well as the release of vasoconstrictor substances like endothelin-1. Vascular toxicity seems to be independent of thymidilate synthase; thus raltitrexed may be used as an alternative chemotherapy drug.

Conclusions: We want to draw attention to the possibility during the use of 5-FU of two different modes of vascular dysfunction that may result in severe and potentially lethal organ dysfunction.

\section{3}

\section{Mediastinal Hodgkin's disease in a patient treated for} seminoma

van Aaken K., Arends J., Gaßmann C., Weber R., Unger C.

Tumor Biology Center at Albert-Ludwigs-University, Freiburg

We report on a 34 year old man with stage III A seminoma. Upon treatment with four cycles of platinum based chemotherapy chemorefractory mediastinal lymph node enlargement was diagnosed as Hodgkin's disease in this patient.

History: A painless testicular mass was noticed by the patient. Orchiectomy was performed in July 2001 and a seminoma was diagnosed which was localized to the right testicle infiltrating the regional lymph vessels and the rete testis. The patient was first addmitted to our hospital in September 2001. At that time CT-scans demonstrated several small retroperitonea lymph nodes $(<13 \mathrm{~mm})$ as well as mediastinal lymph node enlargement to a size of $25 \mathrm{~mm}$. AFP and B-HCG levels in serum were in normal range but LDH was slightly elevated (255 U/l; normal range $<240 \mathrm{U} / \mathrm{l})$. Staging was documented as pT2 cN2 cM1a, i.e. seminoma IIIA according to AJCC. After the application of 3 courses of PEB in November 2001 CT displayed residual mediastinal lymph nodes up to $15 \mathrm{~mm}$; the patient then received a fourth course of PEB.

A follow-up CT in March 2002 showed persistence of the enlarged mediastinal and left sided retroclavicular lymphomas. A PET scan was performed that revealed the enlarged lymphnodes to be metabolically active The patient underwent a thoracotomy and partial resection of the mediastinal lymph nodes. Histologic assessment resulted in the diagnosis of nodular sclerosing Hodgkin's lymphoma. Subsequently, additional chemotherapy treatment with 4 courses of ABVD is offered to the patient which will be followed by involved field radiotherapy.

Discussion: We hereby demonstrate the rare case of a patient presenting with seminoma and Hodgkin's disease. Histological reexamination of the seminoma confirmed the initial diagnosis and CD30 expression was used to differentiate Hodgkin's disease and seminoma. We further present an example that PET diagnostics may be helpful in the „Disease Management Programs" of seminoma patients with remaining lesions after completion of standard chemotherapy.

\section{4}

\section{Severe lead poisoning due to ayurvedian plant medicine from} India

Weide R. (1), Färber H. (2), Kaufmann F. (2), Heymanns J. (1), Pandorf A. (1), Köppler H. (1)

(1) Haematology/Oncology group practice, Neversstrasse 5, 56068 Koblenz, Germany, (2) Department of Microbiology, University Bonn, Germany

Background: Patients with incurable diseases living in Western societies often use alternative treatment modalities to improve their physical and spiritual well being. Treatment approaches from homeopathy, traditional chinese and indian medicine are frequently applied.

Patient and methods: We describe a 39 year old woman with a history of slowly progressive muscle dystrophia which was diagnosed at the age of 15 . Since she was 31 she had to use a wheel-chair. Before she presented to our group practice, she could walk a few meters and with special technical equipment she could manage her daily life with a minimum of help from others. The patient worked as a psychologist in a careers advicing center She presented to us in 09/01 with a hypochromic, microcytic anaemia with a haemoglobin of $7.9 \mathrm{~g} / \mathrm{dl}$. Clinical examination revealed a tetraparesis without any detectable muscle reflexes and atrophical muscles of her extremities. A bilateral radialis paresis was found with a loss of power (1/6). Laboratory examination revealed basophilic stippling in the erythrocytes, reticulocytosis, slightly reduced vitamin B6, mildly elevated creatininphosphokinase but was otherwise unremarkable. Bone marrow biopsy showed a marked dyserythropoiesis with $50 \%$ ringsideroblasts. After the examination of the bone marrow, the serum lead level was measured and found to be 
grossly elevated up to $88 \mathrm{ug} / \mathrm{dl}$. Reevaluation of the patient's history revealed that in $01 / 01$ she had been to india for an aryuvedian treatment approach to improve her muscle dystrophia. Since then she had taken regularly different natural plant drugs which were sent to her from india. Toxicologic analysis of these drugs revealed one drug to be heavily lead contaminated with a lead concentration of $50447 \mathrm{mg} / \mathrm{kg}$. The drug was stopped immediately.

Treatment: The patient was treated with 16 infusions of sodium-EDTA and afterwards for a period of 4 weeks with dimercaptopropionic acid orally. Her neurological condition improved so that she could return to work and her haematological parameters normalized. She was last seen in 03/02 when the serum lead level had decreased down to $34 \mathrm{ug} / \mathrm{dl}$.

Conclusion: This case report shows that naturally produced medicine, manufactored by non-controlled institutions may be heavily contaminated with lead and may cause serious toxicity.

\section{5}

\section{Osteolytic granulocytic sarcoma (chloroma) before allogeneic PBSC transplantation}

Wilhelm S., Kahl C., Leithäuser M., Wolff D., Steiner B., Hartung G., Casper J., Freund M.

Hematology and Oncology, University of Rostock, PO 100888 , 18055 Rostock, Germany

Granulocytic sarcoma or chloroma is defined as an extramedullary solid tumor, composed of granulocytic precursor cells at various levels of differentiation. Chloroma is mostly associated with acute and chronic leukemia, rarely with polycythemia vera and myelofibrosis

A case of chloroma, which developed 4 months after diagnosis of chronic myelogenous leukemia (CML), is reported. A 47-year-old male developed unspecific elevated markers of inflammation during the workup for allogeneic BMT. All screening tests were negative. Inflammation szintigraphy with $99 \mathrm{mTc}$ antibody we could detect an enrichment in the left femur. U1 trasound of this area showed a large hypodensic mass. Infilrtation of the bone could not be excluded. The diagnosis of chloroma was confirmed from the examination of a fine needle aspiration (FNA) specimen. The smears revealed numerous large, granular promyelocytes, myelocytes, metamyelocytes and many blasts. The patients showed no markers of a blast crisis in the peripheral blood or in the bone marrow. Two days after sonography a subtrochanteric femoral fracture occured. The treatment included surgery with intramedullary nailing, chemotherapy with glivec $(3 \times 200 \mathrm{mg} / \mathrm{d})$ and Cytarabine ( 2 × $40 \mathrm{mg} / \mathrm{wk}$ s.c.) and lokal radiotherapy (24 Gy) of the left thigh Two months later, a an allogeneic PBSC transplantation from an unrelated HLA-matched donor was performed. A myeloablative conditioning regimen including TBI ( $12 \mathrm{~Gy})$, ara-C $\left(2 \times 3 \mathrm{~g} / \mathrm{m}^{2} \mathrm{~d}-5\right.$ to -2$)$ with G-CSF priming $(480 \mu \mathrm{g} / \mathrm{d} \mathrm{d}-7$ to $\mathrm{d}-2)$ was administered. The patients developed a acute GvHD II $^{\circ}$ of the skin. We achieved complete remission on day +546 after allogeneic transplantation and the patient is in a good general condition.

We will report the diagnostic findings of chloroma with a review of the literature. We describe the first case of sonography demonstration of bone involvement in this tumor entity.

\section{Gene Therapy}

\section{6}

\section{Resistance to cytarabin after retroviral gene transfer of} cytidine deaminase into human hematopoietic cells

Bardenheuer W., Lehmberg K., Moritz T., Seeber S., Flasshove M.

Department of Internal Medicine (Cancer Research), University of Essen Medical School

The expression of the cDNA for human cytidine deaminase (CDD) in human hematopoietic progenitor or stem cells could provide a tool for rendering the hematopoietic system of cancer patients resistant to myelotoxicity during chemotherapy. In order to investigate if the transfer of CDD would confer resistance of human hematopoietic cells to cytarabin (ara-C), we used a retroviral SFß1 vector expressing CDD to transduce cytokine prestimulated human hematopoietic cells. Retroviral supernatant was produced by polyclonal producer cells from the cell line PG13. In clonogenic assays, the percentage of surviving colony-forming units increased from $34.5 \pm$ $7.4 \%$ to $53.5 \pm 8.2 \%$ at a concentration of $10 \mathrm{nM}$ ara-C, and from $18.3 \pm$ $2.7 \%$ to $32.1 \pm 6.0 \%$ at $25 \mathrm{nM}$ ara-C after mock versus SFB1-CDD vecto transduction $(\mathrm{n}=7$; mean $\pm \mathrm{SEM})$. In order to improve on these data, we constructed a new retroviral vector based on the SF91 backbone expressing CDD upstream and EGFP downstream of an internal ribosomal entry site. A vector expressing EGFP served as a mock control. Stably transduced PG13 cells were sorted by FACS to select for EGFP producing single cell clones and retroviral supernatant was used to transduce CD34-selected umbilica cord blood cells. Preliminary clonogenic assays indicate protection of transduced human clonogenic progenitor cells to higher concentrations of ara-C as compared with experiments using the SFß1 retroviral backbone and retroviral supernatants from polyclonal producer cells.

\section{7} Optimization of adeno-associated virus (AAV) retargeting
vectors for gene therapy by assessment of the virus-cell
contact time by single virus tracing (SVT)

Büning H. (1), Enssle J. (1), Leike K. (1), Huttner N. (1), Ried, M.U. (1), Rhode S. (3), Grimm St. (3), Bräuchle Ch. (3), Hallek M. (1,2,4)

(1) Genzentrum, (2) Medizinische Klinik III, LMU, München, Germany; (3) CeNS Center for NanoScience, Institut für Physikalische Chemie LMU, München, Germany; (4) KKG Gentherapie, GSF-National Center for Research on Environment and Health, München, Germany

Recombinant adeno-associated virus type 2 (rAAV) is one of the most promising vectors for human somatic gene therapy. However, AAV mediated gene delivery is not specific with regard to the target tissue, because of the widespread expression of its cellular attachment receptor heparan-sulfate proteoglycan (HSPG). This is an apparent limitation for a tissue specific gene delivery in vivo. To control the viral tropism we identified capsid positions, which allowed the insertion of cell specific ligands and the generation of rAAV retargeting vectors transducing cells expressing specific receptors (Girod et al., Nat Med 1999,5:1052). However, these retargeting vectors leave room for improvement with regard to the elimination of the wild type (wt) AAV tropism and the infectious titer. To improve this, it is necessary to understand the mechanisms of virus uptake and intracellular process in full detail. For this purpose, we generated several mutations of capsid regions potentially involved in these processes, starting with the potential HSPG binding region. These mutants could be packaged with genomic titers comparable to wild type. All mutants showed a significant reduction of their infection efficiency. Competition and HSPG binding studies revealed that the residual infection was not dependent of HSPG. Using the Single Virus Tracing technology developed by our group (Seisenberger et al., Science 2001,294:1929), we analyzed the interaction of virus mutants with the cell surface. WtAAV repetitively touched the cell membrane, before the virus entered the cell or diffused away. The average contact time of wtAAV with the membrane lasted $56+/-7 \mathrm{~ms}$. This contact time decreased significantly to $37+/-5 \mathrm{~ms}$ if non-human cell lines were used, correlating with the reduced infection efficiency. A comparable decrease in contact time to $36+/-2$ ms was detected for mutants, in which the HSPG binding domain of the capsid was deleted. This is the first report on precise measurements of the contact time of a virus (or functionally relevant mutants) on different cell lines. The results illustrate that both deletions of the HSPG binding motif in the AAV capsid and the use of non-human cells significantly reduce the contact time of AAV with the cell membrane. AAV seems to recognize species specific differences of cell surface proteoglycans, which serve as primary virus attachment receptor. Development of rAAV retargeting vectors will be further optimized with regard to these parameters.

148

\section{Non-viral transfection of CD3+ CD56+ enriched immunologic effector cells}

Buttgereit P., Schakowski F, Nagaraj S., Märten A., Ziske C, Gorschlüter M., Schmidt-Wolf I.G.H.

Medizinische Klinik und Poliklinik I, Rheinische Friedrich-WilhelmsUniversität, Bonn, Germany

Objectives: Gene therapy offers new and promising treatment for patients with hematological malignancies. Cells of the immune system are possible targets for immune and gene therapy. In general, gene therapeutic approaches require efficient gene transfer into host cells and sufficient transgene expression. Although many methods of gene transfer into mammalian cells exist, most do not allow efficient DNA transfer into primary lymphocytes. In contrast to gene transfer into tumor cells and many other cell types, which can be successfully performed using a variety of methods, the efficient expression of foreign DNA into primary lymphocytes presents unique problems and challenges, requiring a careful selection of the mode of gene 
transfer. With regard to immune therapeutic approaches we tested a novel non-viral gene transfer for the transfection of CD3+ CD56+ enriched immunological effector cells.

Material and Methods: Cytokine induced killer (CIK) cells were generated by incubation of peripheral blood lymphocytes with an anti-CD3 monoclonal antibody, interleukin-2, interleukin-1 beta and interferon gamma. CIK cells depend on exogenous source of cytokines like interleukin-2 for proliferation. Fourteen days after expansion, cells were transfected with the reporter gene green fluorescent protein (GFP). For transfection we used the novel non-viral electroporation based technology, termed nucleofection. This technique combines special electrical parameters and cell type specific solutions to deliver the DNA directly to the cell nucleus. Cells were analysed by flowcytometric analysis. Expression of GFP and viability of the nucleofected cells were determined.

Results: The nucleofection technique for gene transfer into the stimulated CIK cells resulted in a transient expression of GFP in more than $41 \%$ of the cells after 24 hours. Further analysis of the subpopulations showed a fraction of 5\% CD56+ GFP double positive cells. PI staining of the nucleofected cells showed that there was a low mortality rate ranging from $10-24 \%$.

Conclusions: Here, we transfected CD3+ CD56+ enriched immunological effector cells. These cells have been shown to eradicate established human lymphoma cells and may be valuable for adoptive immuntherapy. Using nucleofector technology, up to $41 \%$ of CIK cells showed expression of GFP with low toxicity. This new approach promises applications in transfection of primary lymphocytes for immune therapy in the basic and clinical research.

\section{9}

\section{Comparison of the effects of recombinant adenovirus- mediated expression of IL-2 and IL-12 on co-cultured lympho- cytes in human $B$ lymphoma cells}

Ebert O. (1,3), Wilbert D. (1), Buttgereit P. (1), Ziske C. (1), Flieger D. (2), Sauerbruch T. (1), Schmidt-Wolf I.G.H. (1)

(1) Medizinische Klinik und Poliklinik I, Rheinische Friedrich-WilhelmsUniversität, Bonn, Germany, (2) Medizinische Klinik II, Klinikum Aschaffenburg, Germany, (3) Carl. C. Icahn Institute for Gene Therapy and Molecular Medicine, Mount Sinai School of Medicine, New York, NY, USA

Modulation of the immune system by genetically modified lymphoma cell vaccines is of potential therapeutic value in the treatment of B cell lymphoma. However, the anti-tumor effect of any single immunogene transfer has been limited, especially against low immunogenic tumors. Recent reports of intra-tumoral co-injection of adenoviral vectors expressing IL-2 and IL-12 demonstrated the regression of pre-established solid tumors with high frequency. However, the significance of IL-2 and IL-12 immunogene therapy of hematopoietic neoplasms such as B cell lymphoma has not been addressed yet. Recently, we reported an adenoviral protocol leading to highly efficient gene transfer into B lymphoma cells. In this study, we attempted to evaluate the relationship between the amount of cytokine production by the combination of adenoviral-mediated IL-2 and IL-12 gene expression in lymphoma cells and the effective anti-tumor activity in vitro. Using three different human B cell lymphoma cell lines and primary samples from patients with B cell neoplasms, we transduced both IL-2 and IL-12 genes by adenoviral vectors, and monitored cytokine production and effects on proliferation and cytolytic activity of co-cultured lymphocytes. We found that most B-cell lymphoma cell lines could be transduced with much higher efficiency than primary tumor samples dependent on the expression of the coxsackie B-adenovirus receptor (CAR) and alpha (v) integrins. Transgene expression in Raji cells measured $72 \mathrm{~h}$ after infection with IL-2 or IL-12 expressing adenovirus averaged $11 \mathrm{ng} / \mathrm{ml} / 106$ cells and $220 \mathrm{ng} / \mathrm{ml} / 106$ cells respectively. The amount of produced cytokines led to dose-dependent increase of proliferation rates of peripheral blood lymphocytes (PBL) from healthy donors. In addition, transduced Raji cells were co-cultured with PBL for $72 \mathrm{~h}$ and stimulated lymphocytes were assayed for their cytolytic activity against unmodified lymphoma cells. IL-2 transduced lymphoma cells produced a superior anti-tumor effect compared to the combined effect of IL-2/IL-12 or IL-12 alone. In conclusion, these data indicate that the generation of adenovector modified lymphoma cell vaccines expressing IL-2 and IL-12 is technically feasible. Further studies of immunologic and in vivo efficacy are required.
Oncolytic vesicular stomatitis virus (VSV) for hepatocellular carcinoma

Ebert O., Shinozaki K., Huang T.-G., Woo S.L.C.

Carl C. Icahn Institute for Gene Therapy and Molecular Medicine, Mount Sinai School of Medicine, New York, NY, USA

Hepatocellular carcinoma (HCC) is the third leading cause of cancer deaths in the world, accounting for over 1 million cases annually. In the United States, its incidence has increased from 1.4 (1976-80) to 2.4 (1991-95) per 100,000 , which may be related to an increase in chronic hepatitis C (HCV) infection, with an estimated 16,600 new cases and 14,100 deaths in 2002. The prognosis for untreated HCC is very poor, with a median survival of 7.8 months and a 3 -year survival rate of only $10 \%$. The current treatmen modalities for HCC are hepatic resection, intra-tumoral ablation and orthotopic liver transplantation, which are not very effective and patient's prognosis remains poor. Conditionally replicating viruses targeted to tumors are being developed as a novel class of oncolytic agents. Vesicular Stomatitis Virus (VSV) is a negative-strand RNA virus with inherent specificity for replication in tumor cells due to their attenuated anti-viral responses. VSV as an oncolytic virus is particularly appealing for its exceptionally rapid replication rate of 1-2 hours in tumor cells, such that the oncolytic effects could be maximally manifested before the onset of potentially neutralizing anti-viral immune responses in the host. We have successfully rescued a recombinant VSV vector expressing the green fluorescent protein (rVSVGFP) and demonstrate its cytopathic effects and replication in cultured Morris rat HCC cells and in solitary nodules of HCC established in the livers of syngeneic Buffalo rats. In order to assess the tumoricidal potential of VSV to treat multi-focal HCC through hepatic artery infusion in an immune-competent host, such a model was developed in the livers of syngeneic Buffalo rats through portal vein infusion of the Morris rat HCC cells. Three days after intra-arterial infusion of rVSV-GFP, extensive necrosis was apparent in all tumor foci that were also positive for GFP by fluorescence analyses and VSV by immuno-histochemical staining. Importantly, the surrounding normal liver tissues were negative for the virus and any signs of pathology. Further development of this system for the treatment of orthotopic multifocal HCC in immune-competent animals could provide the scientific foundation for future development of recombinant VSV as a novel therapeutic agent for multi-focal HCC in patients.

\section{1}

Nonviral gene transfer into primary human B-CLL cells: Comparison of ballistic gene transfer and Nucleofection

Gresch O., Wolf J.

Department for Internal Medicine I, University of Cologne

Objectives: Efficient and safe gene transfer into tumor cells is an essentiell prerequisite for clinical vaccination trials using genetically modified lymphoma cells. Compared to viral gene transfer, nonviral transfection techniques such as lipofection and electroporation commonly fail to transfect human B-lymphoma cells efficiently. However, nonviral techniques for introduction of therapeutic genes into tumor cells may circumvent some disadvantages of viral gene transfer like insertional mutagenesis, triggering of the immune system and complex safety procedures. With regard to a clinical vaccination study we tested the novel Nucleofection method and ballistic gene transfer in combination with expression vectors encoding for the reporter gene GFP and two potential therapeutic genes: CD154 (CD40L) and GM-CSF.

Material and Methods: Primary human B-CLL cells taken from peripheral blood of patients after informed consent were transfected using ballistic gene transfer (biolistic PDS-1000/He apparatus, Bio-Rad) or novel Nucleofection technique (amaxa biosystems). 3 different expression vectors carrying cDNAs encoding for GFP, CD154 or GM-CSF were tested.

Results: Ballistic gene transfer lead to average transfection efficiencies between $1 \%$ and $2 \%$. In contrast, the Nucleofection method allows transient transfection of primary human B-CLL cells with efficiencies of more than $40 \%$ using conventional pMOK plasmid or linear MIDGE (minimalistic immunogenically defined gene expression) vectors. The average percentage of CD154 expressing CLL cells 5 hours after nucleofection of CD154MIDGEs was 41,5\%. Gene transfer of GM-CSFMIDGE vectors into CLL cells lead to secretion of approximately $3500 \mathrm{pg} / \mathrm{ml} \mathrm{GM-CSF}$ by $10^{6}$ cells in $24 \mathrm{~h}$. Gene expression and function in human B-CLL cells after nucleofection of MIDGE vectors can be demonstrated for $24 \mathrm{~h}$ to $72 \mathrm{~h}$.

Conclusions: In the present work we show that high transfection efficiencies of more than $40 \%$ into human B-CLL cells can be reached by the novel $\mathrm{Nu}$ cleofection technology. This technology combines special electrical param- 
eters and cell specific solutions to deliver DNA directly into the cell's nucleus. In summary, we have shown, that using the Nucleofection technique in combination with either conventional plasmids or MIDGE expression vectors results in high transfection efficacies. This permits the initiation of clinical vaccination trials and circumvents the use of viral gene transfer methods.

\section{2}

\section{Compared to single-stranded adeno-associated Virus (AAV) self-complementary adeno-associated virus (scAAV) improves the efficiency of the gene transfer into solid tumor cells in vitro}

Hacker U. (1), Lutz S. (3), Büning H. (3), King S. (2), Hallek M. (1)

(1) Medizinische Klinik III, Klinikum der LMU München-Großhadern,

(2) Klinische Kooperationsgruppe Gentherapie am Hämatologikum der GSF, (3) Genzentrum der LMU München

Background: Adeno-associated virus (AAV) vectors package single stranded genomes and require host-cell synthesis of the complementary strand for transduction. However, when the genome is half wild-type size, AAV can package either two copies, or dimeric inverted repeat DNA molecules. These self-complementary molecules (scAAV) spontaneously reanneal, alleviating the requirement for host-cell DNA synthesis. We generated scAAV vectors in order to test the efficiency of gene transfer into solid tumor cells in comparison to single stranded AAV.

Material and methods: Single stranded AAV and scAAV were produced using standard protocols. Genomic (dot blot) and infectious titers were determined. A number of human solid tumor cell lines were transfected in vitro at different multiplicities of infection (moi). GFP was used as rAAV marker gene and quantification of positive cells was done by flow cytometry $48 \mathrm{~h}$ after transfection. Irradiation of HeLa cells (100Gy) was performed in one control experiment prior to transduction.

Results: At moi 5, irradiated HeLa cells were 96,1\% positive for GFP expression using AAV vs. $98.8 \%$ using scAAV. Without irradiation at moi 1 , $30,5 \pm 3,3 \%$ of Hela cells were GFP positive using AAV compared to $73,2 \pm 0,8 \%$ using scAAV. At moi 2 the difference was $48,5 \pm 6,5 \%$ (AAV) vs. $88,5 \pm 0,8 \%$ (scAAV), at moi 5 the difference was $77,8 \pm 1,3 \%$ (AAV) vs $96,7 \pm 0,7 \%$ (scAAV). Using the human coloncarcinoma cell line Co115 transduction with AAV at moi 5 resulted in $30,5 \pm 1,8 \%$ of GFP positive cells compared to $72,3 \pm 1,7$ (scAAV). At moi 10 the difference was $35,4 \pm 0,7 \%$ (AAV) vs. $81,1 \pm 1,0 \%$ (scAAV), at moi 20 the difference was $43,7 \pm 2,5 \%$ (AAV) vs. $84,2 \pm 0,9 \%$ (scAAV). The results were confirmed in anothe colon carcinoma cell line DLD-1: Transduction with AAV at moi 5 resulted in $25,6 \pm 1,8 \%$ of GFP positive DLD-1 cells compared to $70 \pm 1,5$ (scAAV). At moi 10 the difference was $34,4,4 \pm 3,5 \%$ (AAV) vs. $82,4 \pm 0,8 \%$ (scAAV) at moi 20 the difference was $46,4 \pm 2,3 \%$ (AAV) vs. 91,9 $\pm 1,4 \%$ (scAAV).

Discussion and conclusions: In earlier reports AAV has been used for gene transfer into cells derived from solid tumors. However, gene transfer could only be demonstrated at very high moi, which would be difficult to achieve under in vivo conditions. The use of AAV vectors for an in vivo gene transfer approach is an attractive strategy for the future. The results presented here suggest that scAAV might be a step forward towards in vivo of solid tumors.

\section{3}

T cell activation by recombinant immunoreceptors:

CD3zeta-chain receptors are expressed with less stability but activate with higher efficiency than FcepsilonRI gamma-chain receptors

\section{Hombach A., Heuser C., Lösch C., Manista K, Abken H.}

Klinik I für Innere Medizin der Universität zu Köln,

Josef-Stelzmann-Str. 9, D-50924 Köln, Germany

Recombinant $\mathrm{T}$ cell receptors for use in adoptive immunotherapy are modularily composed of extracellular antigen binding and intracellular signalling domains preferentially derived from the CD3zeta- or FcepsilonRI gammachain. The impact of the signalling domain on the stability of immuoreceptor expression and function is not completely understood. To address this issue we expressed a panel of scFv-Fc-zeta and $\mathrm{scFv}$-Fc-gamma immunoreceptors in mouse $\mathrm{T}$ cell lines with and without endogenous CD3/TCR complex and in human peripheral $\mathrm{T}$ cells, respectively. The stability of zetachain immunoreceptor expression is substantially impaired in the presence of an endogenous TCR whereas the expression of gamma-chain receptors is not affected. Recombinant zeta-chain immunoreceptors in turn stabilize expression and function of the endogenous CD3/TCR complex. Lower membrane expression of zeta-chain immunoreceptors in TCR+ cells is due to in- creased degradation. Accordingly, the expression level of zeta-chain immunoreceptors in human peripheral blood T cells is significantly lower than those of gamma-chain receptors but expression of both receptors decreased upon prolonged cultivation. In contrast, target cell lysis and induction of IFN-gamma secretion is mediated with similar efficiency by both zeta- and gamma-chain immunoreceptors shortly after receptor engraftment. Upon prolonged propagation, however, the anti-tumor response mediated by the zeta-chain receptor is more efficient indicating that the high expression level of gamma-chain immunoreceptors early after receptor engraftment compensates its lower cellular activation capacity. These findings have substantial consequences for the design of recombinant immunoreceptors.

\section{4}

\section{Synergism of cisplatin and rAAV-2 vectors in the treatment of} non small cell lung cancer (NSCLC)

Rohr U.P., Wulf M.A., Steidl U., Stahn S., Fenk R., Haas R., Kronenwett R.

Klinik für Hämatologie, Onkologie und Klinische Immunologie, Universität Düsseldorf, Germany

New strategies such as a combination of viral transfer of therapeutic genes in addition to conventional chemotherapy might improve outcome in NSCLC. One promising candidate for a viral vector is the recombinan adeno-associated virus type 2 (rAAV-2). For examination of tropism for rAAV-2 into bronchial carcinomas we used 10 lung tumor cell lines in an attempt to reflect the heterogenicity of this tumor entity according to differen histological subtypes. Cell lines of NSCLC were highly susceptible for rAAV-2 showing transduction rates between $60 \%$ and $98.9 \%$ using a MOI of 50 per cell. In contrast, cell lines of small cell carcinomas were resistent to rAAV-2. Two rAAV-2 vectors were constructed containing either the tumor suppressor gene p53 or the FHIT-gene, which are deleted in more than $50 \%$ of NSCLC. Successful viral gene transfer was demonstrated by Western blot choosing the NSCLC cell lines H1299 and H23 which are deficient of p53 and FHIT.

Using 7.5x103 genomic rAAV-p53 particles per cell (MOI of 6), the relative growth rate (GR) was $73.1 \%$ (SD+14\%) after 3 days compared to untreated control or mock vector transduction. Combination experiments with cisplatin (IC 50 concentration) and rAAV-2-p53 vector showed a realtive growth rate of $35 \%(\mathrm{SD}+10 \%)$ after 3 days using rAAV-p53 vector compared to cisplatin treated cells and mock vector transduction. Using 3x104 genomic rAAV-2-FHIT particles per cell (MOI of 120), GR was $26.9 \%$ (SD+2\%). Combination experiments with cisplatin (IC 50 concentration) and rAAV-FHIT showed a decrease down to $2.9 \%$ after 3 days. In conclusion, NSCLC are highly susceptible for rAAV-2 vectors. Further, viral transfer of recombinant p53 or FHIT tumor suppressor genes results in an inhibition of proliferation of NSCLC cells and indicate a synergistic effect with cisplatin. The results might be the basis for a therapeutic approach using rAAV-2.

\section{5}

\section{Adenoviral transduction of tumor cells induces apoptosis in} co-cultured T lymphocytes

Scholz C. (1), Stärck L. (1), Willimsky G. (2,3), Blankenstein T. (2,3), Dörken B. (1), Daniel P.T. (1)

(1) Department of Hematology, Oncology and Tumorimmunology, Charité, Campus Berlin-Buch, Robert-Rössle Klinik, Humboldt Universität, Berlin, (2) Max-Delbrück-Center for Molecular Medicine, Berlin, (3) Institute of Immunology, Free University, Berlin

Objectives: Adenoviral gene transfer of immunmodulatory molecules has been employed successfully in tumor vaccination studies to induce rejection of transplanted syngeneic tumors. In contrast, the response observed when treating chemically induced murine tumors is rather limited. The same applies for human malignancies. A number of reasons including poor transduction efficiency or insufficient T cell infiltration have been held accountable for this lack of efficacy. However, little attention has been given to effects of the adenoviral transduction itself on the T cell system.

Material and Methods: To investigate the effects of adenoviral transduction on non-transduced by-stander cells, PBMC or purified T cells were co-cultered with tumor cells either non-transduced or adenovirally infected with Ad-lacZ at increasing multiplicity of infection (MOI). Alternatively, tumor cells were adenovirally transduced to express IL-7 and CD80, both inhibitors of T cell apoptosis. After 6 days of co-incubation non-adherent cells were harvested, assessed for $\mathrm{T}$ cell purity and restimulated with uninfected tumor cells. Subsequently, T cell mediated $51 \mathrm{Cr}$ release from tumor cells 
and activation induced cell death (AICD) of restimulated $\mathrm{T}$ cells were measured. Furthermore, levels of TNF- $\alpha$ and CD95/Fas ligand, both mediators of AICD, were assessed in culture supernatants.

Results: Preactivated T lymphocytes displayed similar cytotoxicity towards non-transduced tumor cells regardless of prior stimulation with non-infected or Ad-lacZ-transduced tumor cells. In contrast, restimulation of T cells by use of uninfected tumor cells resulted in $40 \pm 11,2 \%$ activation induced $\mathrm{T}$ lymphocyte death when the primary activation was done with Ad-lacZtransduced tumor cells compared with $26 \pm 4,6 \%$ AICD when uninfected tumor cells were initially employed $(\mathrm{P}=0,018)$. The levels of soluble CD95/Fas ligand or TNF- $\alpha$ were not affected by the primary culture conditions and supernatant transfer experiments could not demonstrate that soluble factors are the cause of increased T cell AICD. Interestingly, AICD was only partially inhibited if $\mathrm{T}$ cells were initially co-cultured with tumor cells adenovirally transduced to express IL-7 and CD80.

Conclusions: Our data suggest that adenoviral infection of tumor cells is capable of inducing apoptosis of co-cultured T cells. This may contribute to phenomena such as insufficient tumor infiltration by tumor reactive $\mathrm{T}$ lymphocytes.

\section{6}

\section{New strategies for the therapy of sarcomas using recombinant} adeno-associated virus 2 vectors that contain a suicide gene

Veldwijk M.R. (1), Berlinghoff S. (1), Topaly J. (2), Laufs S. (1), Fruehauf S. (2), Zeller W.J. (1)

\section{(1) D0200, DKFZ-Heidelberg, (2) Internal Medicin V, Uni-Klinik} Heidelberg

Adeno-associated virus 2 (AAV-2) vectors are used for clinical gene therapy of hereditary diseases and at this stage for preclinical cancer gene therapy. In this work a high susceptibility of some solid tumors to an rAAV-2 vector containing the humanized green fluorescent protein (GFP) gene was shown Among a series of primary cells and eight solid tumor cell lines infected with these AAV-2 supernatants, the highest infection rates (functional titer: $200 \mathrm{IU} / \mathrm{cell}$ ) were observed in soft tissue sarcoma cells (HS1; mean: 96\% $\mathrm{GFP}^{+}$cells) and in breast cancer cells (T47D; mean: 83\%, MCF-7; mean $85 \%$ ); other cell lines ( 1 ovarian tumor, 1 germ cell tumor, 1 osteosarcoma and 2 small cell lung cancer) were less permissive $(3 \%-12 \%)$; CD $34^{+}$peripheral blood progenitor cells showed the lowest transduction rates (max $4 \%$ ). These data suggest that the sarcoma and breast cancer cells are the most suitable candidates for further development of AAV-2 tumor suicide gene therapy. The first requirement for using these vectors in cell kill experiments was the construction of new rAAV-2 vectors containing a suitable suicide gene. The thymidine kinase (TK) gene was chosen in combination with the prodrug ganciclovir. Three novel TK-containing vectors were cloned. Since some of the new vectors contained no marker gene (GFP) and therefore did not allow fluorescence-based titration, a highly optimized titration method was established based on the real-time quantitative polymerase chain reaction so that $\mathrm{AAAV}-\mathrm{TK}$ vectors which did not contain a fluorescent marker gene could be titrated accurately. Four sarcoma cell lines were chosen (HS-1, HT1080, RDES and SK-N-MC) and a complete eradication of all rAAV-TK/eGFP transduced tumor cells was shown following exposure to ganciclovir $(2.5 \mu \mathrm{g} / \mathrm{ml})$ in vitro while at this dose level $>95 \%$ of mock-transduced tumor cells survived. Xenotransplantation tumor models for these human sarcoma cell lines were established. In proof of principle experiments mice transplanted with rAAV-TK/eGFP-transduced and ganciclovir-exposed tumor cells survived $>5$ months while in the non-transduced group all mice had died 1 month after inoculation. These data hold promise for a future clinical application of AAV-2-based suicide gene therapy.

\section{7}

\section{Single aminoacid substitutions affect chain pairing of adoptively transferred $\mathrm{T}$ cell receptors in human $\mathrm{T}$ cells}

Voss R.-H. (1), Juelch M. (1), Romero P. (2), Kuball J. (1), Antunes E.F. (1), Huber C. (1), Theobald M. (1)

(1) Department of Hematology and Oncology, Johannes GutenbergUniversity, Langenbeckstr. 1, 55101 Mainz, Germany, (2) Ludwig Institute of Cancer Research, University of Lausanne, Hopital Orthopedique Niveau 5 Est, Avenue Pierre Decker 4, Epalinges, 1005 Lausanne, Switzerland

Objectives: T cell receptors (TCRs) derived from CD8 x A2 $\mathrm{K}^{\mathrm{b}}$ transgenic (Tg) mice bypass human (Hu) MDM2-oncoprotein-specific self-tolerance. After adoptive transfer of these high affinity $\alpha \beta$-heterodimeric TCRs into human $\mathrm{T}$ cells the antigen specificity will be redirected towards the human MDM2 oncoprotein, paving the way for immunotherapy of human malig- nancies. One major concern is the eventual inter-chain pairing of the endogenous human TCRs and the exogenous murine $(\mathrm{Mu})$ TCRs due to conserved primary and tertiary structure. This might lead to ambiguous functional specificities as autoimmune reactivities in human tissue. As a model MDM2-specific TCRs were mutated by rational design in order to favor the chain pairing of the adoptively transferred murine TCRs and to weaken the formation of hybrid TCRs.

Material and Methods: The related genes for the $\alpha / \beta$-heterodimeric $\mathrm{T}$ cell receptor (TCR) of a transgenic murine $(\mathrm{Mu}) \mathrm{T}$ cell clone with MDM2(8188) epitope-specificity were retrovirally transferred into $\mathrm{Hu} \mathrm{T}$ cells. The multiplasmid cotransfection system enabled the individual combination of wildtype and mutated TCR genes in order to array wildtype, double point mutated (ie one replaced residue in each chain) and single point mutated (ie one mutation in either chain) TCRs. As a conceptual prerequisite for mutagenesis the residues were chosen according to the „knob-hole“ model, implying the inversion of sterically related residue pairs at the interface of the heterodimeric $\alpha \beta T C R$. As readout for structural avidity of the transduced T cells tetramer staining was applied, for functional avidity cytolytic assays against peptide pulsed T2 cells and human malignant cell lines were applied.

Results: The double point mutated murine TCRs, harbouring sterically inverted residues on both sides at the inter-chain contact side proved to be cytolytically functional whereas the single point mutated TCRs in both orientations were significantly impaired in tetramer binding and cytolytic efficacy.

Conclusions: The rational design of single aminoacid substitutions in heterodimeric TCRs is ideally suited to manipulate the structural and functional features of transduced $\mathrm{T}$ cells on a molecular basis. This model yields a proof of concept for the equipment of human $\mathrm{T}$ cells with new antigen specificities by murine TCR gene transfer and the simultaneous elimination of ambiguous reactivities originating from potentially hybrid $\mathrm{Hu} / \mathrm{Mu}$ TCRs

\section{Gastrointestinal Cancer}

158

Combination therapy of the matrix-metalloproteinase inhibitor Ro28-2653 and the cytostatic drug gemcitabine markedly inhibited tumor progression of orthotopically transplanted human ductal pancreatic adenocarcinoma and prolonged survival of tumor bearing SCID mice

\section{Alves F. (1), Borchers U. (1,2), Contag S. (1), Scheuer W. (3), Tietze L.F. (2), Krell H.W. (3)}

(1) Uni-Klinik Göttingen, Abtl. Hämatologie und Onkologie, (2) Organische Chemie, Universität Göttingen, (3) Roche Diagnostics GmbH, Pharma Research Penzberg

The present study was aimed at evaluating the effect of the matrix metalloproteinase (MMP)-inhibitor Ro28-2653 on tumor progression and survival using an orthotopic pancreatic carcinoma model in severe combined immunodeficient mice (SCID). Ro 28-2653 belongs to pyrimidine-2,4,6-triones, a new class of MMP-inhibitors and exhibits high selectivity towards MMP2, MMP9 as well as MT1-MMP and MT3-MMP. In controls receiving the vehicle only, the poorly differentiated ductal pancreatic adenocarcinoma invaded into adjacent organs and metastasized to different sites in the abdomen and to the lungs. Treatment with Ro28-2653 ( $45 \mathrm{mg} / \mathrm{kg}$ bw), orally once a day for 21 days or treatment with gemcitabine $(2.2 \mathrm{mg} / \mathrm{kg}$ bw) i.p., every $48 \mathrm{~h}$ for 21 days, alone or in combination were started one week after tumor cell implantation of the human pancreatic adenocarcinoma cell line PancTu1 in SCID mice $(n=9-13)$ and were well tolerated. Compared to controls treatment of Ro28-2653 alone reduced tumor volume significantly by $64 \%$, treatment of gemcitabine alone by $84 \%$ and the combination therapy by $94 \%$. The latter group showed marked induction of necrosis in the pancreatic tumors. Invasion was not observed and metastasis were markedly reduced in mice receiving gemcitabine alone or in combination with the MMP-inhibitor. Treatment with Ro28-2653 or gemcitabine alone as well as the combination therapy prolonged survival of the tumor bearing mice to further 13 days (Ro28-2653 alone), 26 days (gemcitabine alone) and 33 days (combination therapy).

In summary, the present study showed that treatment with the novel MMPinhibitor Ro28-2653 during early neoplastic progression is highly effective in inhibiting pancreatic ductal adenocarcinoma growth and progression, prolongs survival of tumor bearing mice and enhances the anticancer efficacy of the cytostatic drug gemcitabine. 
Evaluation of the novel epothilone BMS-247550 in patients (pts) with metastatic gastric adenocarcinoma previously treated with a taxane: An international phase II study

Bokemeyer C. (1), Kollmannsberger C. (1), Shah M.A. (2), Lenz H.-J. (3), Burris H.A. (4), Van Cutsem E. (5), Lebwohl D. (6), Voi M. (7), Bochtler H. (8), Kanz L. (1), Safran H. (9), Ajani J.A.(10)

(1) Tübingen University Hospital, Tuebingen, Germany; (2) Memorial Sloan-Kettering Cancer Ctr, New York, NY; (3) USC Norris Cancer Center, Los Angeles, CA; (4) Sarah Cannon Cancer Center, Nashville, TN; (5) University Hospital Gasthuisberg, Leuven, Belgium; (6) Bristol-Myers Squibb, Wallingford, CT; (7) Bristol-Myers Squibb, Waterloo, Belgium; (8) Bristol-Myers Squibb Deutschland, (9) Rhode Island Hospital, Providence, RI., (10) UT MD Anderson Cancer Ctr, Houston TX

Epothilones are a novel class of non-taxane microtubule-stabilizing agents, which bind to tubulin differently from taxanes. BMS-247550 is a derivative of epothilone B with improved metabolic stability, which induces -similarly to paclitaxel- tubulin polymerization and G2M arrest leading to apoptosis in cancer cells. BMS-247550 has preclinical activity in taxane-sensitive and -resistant tumors.

Patients and Methods: The primary objective of this study was to determine the efficacy and safety of BMS-247550 in pts with metastatic gastric cancer previously treated with one taxane-based chemotherapy regimen. Pts were pretreated with one taxane/fluoropyrimidine \pm platinum-based chemotherapy regimen in the metastatic or adjuvant setting. BMS-247550 was administered at a dose of $50 \mathrm{mg} / \mathrm{m}^{2}$ as a 1-hour infusion every 21 days.

Preliminary Results: Between January 2001 and August 2001, 23 pts were enrolled. Data are available for $23 \mathrm{pts}$ ( $18 \mathrm{male} / 3 \mathrm{female})$ with a median age of 52 years (range 22-75 years). Baseline ECOG performance status was 0 in 2 pts, 1 in 13 pts, and 2 in 6 pts. Twelve pts had adenocarcinoma of the gastroesophageal (GE) junction. Forty-eight $\%$ of pts had previously received a taxane/platinum/5-FU-containing regimen and $26 \%$ a taxane/platinum-containing regimen. Five of the 21 pts remain on treatment. A total of 56 courses of BMS-247550 have been administered (median 2, range 1-5) Severe (Grade 3-4) toxicities included: fatigue ( $9 / 23 \mathrm{pts})$; anorexia (4/23 pts); nausea/vomiting (5/23 pts); sensory neuropathy/neuropathic pain ( $3 / 23$ pts); myalgia/arthralgia (2/23 pts); abdominal pain/cramping (2/23 pts); diarrhea (1/23 pts); febrile neutropenia (1/23 pts); neutropenia (9/23 pts). Response data in 23 evaluable pts indicate 2 PR (10\%), 12 SD (PR+SD: 61\%). In order to reduce cumulative neurotoxicity and improve the therapeutic index, the regimen has been amended to $6 \mathrm{mg} / \mathrm{m}^{2}$ of BMS 247550 administered daily x 5 over 1 hour every 21 , a schedule which has been associated with no Grade 3 neuropathy in phase I evaluation.

Conclusion: Initial activity results with BMS-247550 are encouraging in pts with metastatic gastric cancer previously treated with one taxane-based chemotherapy regimen. Accrual is ongoing and updated results will be presented.

\section{0}

\section{Gemcitabine plus high-dose 5-FU/FA: A phase I trial}

Geuenich S. (1), Wilkowski R. (2), Vehling-Kaiser U. (3), Schalhorn A. (1), Hiddemann W. (1), Heinemann V. (1)

(1) Dept. of Internal Medicine III, (2) Dept. of Radiotherapy and Radiooncology, University of Munich. (3) Onkologische Praxis Landshut, Germany.

Gemcitabine (GEM) and 5-fluoruracil (5-FU) plus folinic acid (FA) both have proven activity in the treatment of patients with advanced pancreatic cancer. We report a phase I study to determine the maximum-tolerated dose (MTD), the dose-limiting toxicity (G3/4 toxicity) and efficacy including time to disease progression of GEM in combination with 5-FU-FA. FA (500 $\mathrm{mg}$ ) was given as a 2-hr infusion followed by a 24-hrs infusion of 5-FU and GEM delivered as a 0.5 -h infusion in patients with advanced 5-FU sensitive tumor entities ( 22 pancreatic cancer, 5 cholangiocellular cancer, 2 stomach; 3 adenocarcinoma and 1 mucoepidermoidal cancer of unknown primary). 32 patients ( 12 female / 20 male) with a median age of 63 yrs (range 37 to $75 \mathrm{yrs}$ ) and an ECOG performance status of 0-2 were enrolled into the study and treated on dose level I-VI. Before therapy 14 patients suffered from other diseases (diabetes mellitus type II (7), arterial hypertension (4), coronary heart disease (2), atrial fibrillation (2), hepatitis A/B (2), occlusive arterial disease type IIa (1), myocardial infarction (1), thromboembolic complication (3)). Chemotherapy was applied on days $1 / 2$ and $15 / 16$ of each cycle and was repeated on day 29 . All except 4 patients have had prior therapy (radiochemotherapy or chemotherapy alone).

\begin{tabular}{lclllll}
\hline $\begin{array}{l}\text { Dose } \\
\text { level }\end{array}$ & $\begin{array}{l}\text { No of } \\
\text { pts. }\end{array}$ & $\begin{array}{l}\text { FA } \\
{\left[\mathrm{mg} / \mathrm{m}^{2}\right]}\end{array}$ & $\begin{array}{l}5-\mathrm{FU} \\
{\left[\mathrm{mg} / \mathrm{m}^{2}\right]}\end{array}$ & $\begin{array}{l}\text { Gem } \\
{\left[\mathrm{mg} / \mathrm{m}^{2}\right]}\end{array}$ & $\begin{array}{l}\text { No of } \\
\text { DLT }\end{array}$ & $\begin{array}{l}\text { TTP[months }] \\
\text { (available pat.) }\end{array}$ \\
\hline I & 3 & 500 & 1750 & 800 & 0 & $3.7(3$ pts.) \\
II & 10 & 500 & 2000 & 800 & 2 & $5.3(8$ pts.) \\
III & 4 & 500 & 2000 & 900 & 0 & $5.6(2$ pts.) \\
IV & 5 & 500 & 2000 & 1000 & 2 & $4.7(3$ pts.) \\
V & 6 & 500 & 2250 & 1000 & 2 & $2.7(5$ pts.) \\
VI & 4 & 500 & 2500 & 1000 & 1 & n.a. \\
\hline
\end{tabular}

At dose level I and III no DLTs were observed. At level II two patients showed a DLT (myocardial infarction, granulocytopenia G4), at level IV DLTs were diarrhea G4 and granulocytopenia G4, and at level V two cases of DLTs were observed (sepsis, leucocytopenia G3). Until now, one patient showed a DLT (sepsis) at level VI. 21 patients were evaluable for time to disease progression. The median time to progression was 4.4 months (range 2.7 to 5.6 months). Two patients had a progression-free interval of greater than 10 months (1 patient at dose level II, 1 patient at dose level IV). In conclusion, the data show that up to dose level VI biweekly application of highdose FU/FA and GEM is a well tolerated and effective regimen.

\section{1}

\section{Capecitabine and Mitomycin combination salvage therapy in patients with advanced colorectal cancer refractory to oxaliplatin and irinotecan based chemotherapy}

Harba A.E. (1), Jordan K. (1), Kegel T. (1), Kühne I. (1), Kellner O. (1), Behrens R. (2), Schmoll H.-J. (1), Grothey A. (1)

(1) Klinik für Hämatologie/Onkologie, Uni Halle-Wittenberg,

(2) Praxis für Hämatologie, Halle

Background: Mitomycin C (MMC) has been used in the treatment of colorectal cancer for over 30 years. The combination of 5-FU continuous infusion and MMC in advanced disease results in higher response rate when compared to infusional 5-FU alone (Ross 98). The actual value of this combination as salvage therapy in oxaliplatin and irinotecan refractory patients has not yet been assessed. Furthermore, it is unclear if capecitbine (CAP) can be a substitute for infusional 5-FU in this setting. Therefore, we evaluated the combination of CAP and MMC as last-line therapy in pts. with advanced colorectal cancer after failure of oxaliplatin and irinotecan based chemotherapy.

Dosage and Schedule: CAP $1000 \mathrm{mg} / \mathrm{m}^{2}$ BID p.o. d 1-14 qd 22 and MMC 7 $\mathrm{mg} / \mathrm{m}^{2}$ every 6 weeks were given in an outpatient setting.

Results: To date, 18 pts. (female:male 5:13) are evaluable for toxicity and efficacy. Median age was 64 (range 33-77). Toxicity (NCI-CTC) was: grade 3 leucopenia in 1 pt., grade 3 thrombocytopenia in 1 pt., 2 pts. with grade 2 3 hand-foot-syndrome and 2 pts. with grade 4 anemia. MMC treatment had to be terminated due to fragmentocyte positive hemolysis in $1 \mathrm{pt}$. and due to prolonged grade 3 thrombocytopenia in another pt.

Efficacy: Of 18 pts. PR resulted in 2 pts, SD in 7 pts. and PD in 9 pts. Best response was observed between 4-6 weeks after initiation of CAP/MMC chemotherapy. The median time to progression in 5 pts. with stable disease was 16 weeks (range 10-28 weeks). The other 2 pts. with stable disease are still on treatment.

Conclusion: In some patients with refractory advanced colorectal cancer $\mathrm{CAP} / \mathrm{MMC}$ seems to be an active salvage treatment with a favourable toxicity profile. In a certain subgroup we saw unforeseen partial remission in these irinotecan and oxaliplatin refractory pts.. Furthermore MMC/CAP is favourable priced and suitable for outpatient use. In such pts. this regimen should be regarded as long as further active salvage options are available.

\section{2}

Protracted infusional 5-fluorouracil (5-FU) plus high dose folinic acid (FA) combined with bolus mitomycin C (MMC) in patients (pts) with gastrointestinal cancer: A phase I/II study

Hartmann J.T. (1), Quietzsch D. (2), Wein A. (3), Hofheinz R.D. (4), Oechsle K. (1), Honecker F. (1), Nehls O. (1), Koehne H. (5), Kanz L. (1), Bokemeyer C. (1)

(1) Medizinische Universitätsklinik Tübingen, (2) Klinikum Chemnitz, (3) Medizinische Universitätklinik I Erlangen, (4) Medizinische Universitätsklinik III Mannheim, (5) Medizinische Universitätklinik Dresden, Germany

Objectives: To define the maximum tolerated dose (MTD) of bolus MMC in combination with $24 \mathrm{~h}$-continuous infusion of 5-FU/FA, and to assess the toxicity and activity of this regimen in pts with previously treated colorectal and gastric cancer. 
Material and Methods: Overall 61 pts, all pretreated with at least one previous line of chemotherapy were entered in this study. Escalating doses of MMC starting from $6 \mathrm{mg} / \mathrm{m}^{2}$ in $2 \mathrm{mg} / \mathrm{m}^{2}$-steps to a maximum of $10 \mathrm{mg} / \mathrm{m}^{2}$ were applied on days 1 and 22 , given to fixed doses of $5-\mathrm{FU}\left(2.600 \mathrm{mg} / \mathrm{m}^{2}\right)$ as $24 \mathrm{~h}$-infusion and folinic acid $500 \mathrm{mg} / \mathrm{m}^{2}$ as $2 \mathrm{~h}$ infusion prior to 5 -FU weekly for 6 weeks (q, d50). At least 3 pts were treated at each dose level Escalation proceeded to dose level 3 unless 2 out of 3 or 4 out of 6 patients experience a dose-limiting toxicity (DLT) after the first cycle. On the MTD level pts with pretreated colorectal as well as gastric cancer were planned to enter the phase II trial to assess the activity of the regimen.

Results: 16 pts were entered in the phase I study, and all 3 consecutive dose levels have been tested. At the last dose level (MMC $10 \mathrm{mg} / \mathrm{m}^{2}$ ) grade III thrombocytopenia, dyspnea, mucositis and diarrhea were observed in one $\mathrm{pt}$ each $(17 \%)$, and therefore this dose level was considered as the MTD. 45 pts, 32 colorectal cancer, 11 gastric cancer, 2 Adeno-CUP, 24 pts after failure of first- and 21 pts after second-line chemotherapy, have been so far included at this dose-level. 33 pts are currently available for response evaluation. 13 pts $(41 \%)$ achieved disease stabilisation, 11 colorectal $(95 \%$ CI, 27 $69 \%)$ and 2 gastric cancer pts $(25 \%$; $95 \% \mathrm{CI}, 3-60 \%)$ and 6 partial responses were registered $(19 \%), 1 \mathrm{PR}(4 \%$; 95\% CI, 0-22\%) in colorectal and $5 \mathrm{PRs}$ $(63 \% ; 95 \% \mathrm{CI}, 21-86 \%)$ in gastric cancer. The median progression-free survival is currently $3.3+$ mos (range, 1-8.5+) in colorectal cancer and 5.1+ mos (range, 1.5-10.9+) in gastric cancer pts.

Conclusions: MMC $10 \mathrm{mg} / \mathrm{m}^{2}$ as bolus injection on days 1 and 22 combined with weekly $24 \mathrm{~h}$ continuous infusion of 5-FU/FA for $6 \mathrm{wks}$ is a safe and well tolerated regimen in the second-line setting. Final assessment of the efficacy and the impact on survival obtained in pretreated pts with gastric and colorectal cancer is still ongoing.

\section{3}

\section{Multicenter phase II study of irinotecan (CPT 11) as 3rd-line treatment in metastatic colorectal cancer after prior exposure to bolus and infusional 5-FU-based chemotherapy}

Hartmann J.T. (1), Knuth A. (2), Reiss H.E. (3), Ehninger G. (4), Niederle N. (5), Wilke H.-J. (6), Pflüger K.-H. (7), Buechele T. (8), Kanz.L. (1), Bokemeyer C. (1)

(1) Medizinische Universitätsklinik Tübingen, (2) Nordwest-Klinik Frankfurt, (3) Klinikum Mönchengladbach, (4) Medizinische Universitätsklinik Dresden, (5) Klinikum Leverkusen, (6) Klinikum Essen Mitte, (7) Klinikum Bremen, (8) Aventis Pharma Bad Soden

Objectives: The present study investigated CPT 11 as 3rd-line chemotherapy in patients (pts) with prior exposure to a bolus schedule of 5-FU/folinic acid followed by $24 \mathrm{~h}$-infusion 5 -FU $2.0-2.6 \mathrm{~g} / \mathrm{m}^{2}$ plus folinic acid 500 $\mathrm{mg} / \mathrm{m}^{2}$ i.v. for metastatic disease. Pts with histologically confirmed adenocarinoma of the colon or the rectum, measurable metastatic progressive disease and adequate bone marrow, liver and renal function were entered into the study after informed consent. Previous 5-FU based adjuvant chemotherapy and/or radiotherapy were allowed.

Material and Methods: CPT 11 was applied at a dose of $350 \mathrm{mg} / \mathrm{m}^{2}(300$ $\mathrm{mg} / \mathrm{m}^{2}$ for pts with WHO-performance status of 2 and/or age $>70 \mathrm{yrs}$ ) i.v. over $30 \mathrm{~min}$ every $3 \mathrm{wks}$ until progression of disease or occurrence of an unacceptable toxicity.

Results: 50 pts entered the study: sex, 21 male, 29 female; median age 59 (range, 39-77) yrs; median perfomance status 1 (range, 0-2). 50 patients were assessable for safety and toxicity monitoring and $46 \mathrm{pts}$ for response evaluation. A total of 216 cycles of CPT 11 with a median number of 4 cycles (range, 1-11) have been administered. The objective response rate was $15 \%$ (95\% CI, 6-29) and additionally $48 \%$ of pts achieved disease stabilisation $(95 \% \mathrm{CI}, 33-63 \%)$. Median time to progression and median surviva were 13 (range, 8.6-16.0) and 34 (range, 26.3-47.1) wks. The median duration of response was 24 wks (range, 13-27) and the median duration of disease stabilisation was 14 wks (range, 13-21). 1-yr survival was $28 \%(\mathrm{Cl}$ $95 \%, 16-40 \%$ ). NCIC-CTC grade $3 / 4$ toxicities included leucopenia in 14 $\%$, bilirubin evaluation $16 \%$, vomiting $8 \%$, diarrhea $24 \%$ as well as acute cholinergic syndrome in $8 \%$ of pts. Dose reductions were performed in $5 \%$ of cycles.

Conclusions: In case of failure of both bolus and infusional modulated 5 FU, CPT 11 has considerable efficacy in metastatic colorectal cancer pts The impact in the present study is comparable with that obtained in phase III studies giving irinotecan as 2nd-line treatment after failure of 5-FU based regimen. These results indicate that the efficacy of irinotecan in a 3rd-line treatment is obviously independent of the previously used 5-FUbased regimens in association with an acceptable safety profile of the drug.
A phase-Il-study of dose-dense irinotecan and oxaliplatin as second-line treatment for advanced colorectal cancer

Hartmann J.T. (1), Kaechele V. (2), Bokemeyer C. (1), Knuth A. (3), Ohl U. (4), Geiner L. (5), Souchon R. (6), Köhne C.H. (7), Lutz M.P. (2)

(1) Medizinische Universitätsklinik Tübingen, (2) Medizinische Universitätsklinik Ulm, (3) Nordwestklinik Frankfurt, (4) Klinikum Pforzheim, (5) Klinikum Wuppertal, (6) Klinikum Hagen, (7) Medizinische Universität Dresden

Objectives: The present study investigates a combination of Irinotecan (CPT-11) and oxaliplatin (L-OHP) as second-line therapy in metastatic colorectal cancer patients (pts) with prior exposure to either a bolus- or a continuous infusion regimen of 5-FU/folinic acid.

Material and Methods: A dose-intensified regimen with L-OHP $\left(85 \mathrm{mg} / \mathrm{m}^{2}\right.$ over $2 \mathrm{~h}$ on day 1 and 15$)$ and CPT11 $\left(80 \mathrm{mg} / \mathrm{m}^{2}\right.$ over $30 \mathrm{~min}$ on day 1,8 , 15; qd 28) was used. Pts with histologically confirmed adenocarcinoma of the colon or the rectum, measurable metastases, progressive disease and adequate bone marrow, liver and renal function were entered on study after informed consent. Previous 5-FU based adjuvant chemotherapy was allowed. Results: 35 pts, 24 male, 11 female, median age 62 (range, 42-76) yrs, were assessable for safety and toxicity monitoring as well as response evaluation. A median number of 4 cycles (range, 1-10) have been administered. The objective confirmed response rate was $31 \%$ (CI 95\%, 17-49\%) and additionally $40 \%$ of pts achieved disease stabilisation (95\% CI, 24-58\%). The median progression free- and overall survival were 6 mos (range, 1-14) and 9.1+ mos (range, 2.0-20.0+). NCIC-CTC grade III/IV toxicities included diarrhea in $29 \%$, neutropenia in $14 \%$, peripheral neurotoxicity $6 \%$ and lethargy in $9 \%$. Grade IV toxicity was observed in 4 pts (neutropenia $n=1$, diarrhea $\mathrm{n}=3$ ). Dose reductions were performed in 4 pts due to L-OHP associated peripheral neurotoxicity (PNP)/allergic reaction and in 9 pts due to CPT11 associated diarrhea/neutropenia. Almost all dose adjustments have been done during the first cycle except for PNP.

Conclusions: In case of failure of 5-FU first-line treatment or occurrence of unacceptable side effects, the combination of CPT11 and oxaliplatin has substantial antitumor activity in pretreated pts with progressive colorectal cancer. The toxicity profile is manageable.

\section{5}

Impact of sequential chemotherapy on survival in patients with advanced pancreatic cancer following Phase III trial comparing Gemcitabine + R115777 ( Zarnestra) versus Gemcitabine + placebo

\section{Hempel C., Oettle H., Lück K., Riess H.}

Charité, Campus Virchow-Klinikum, Medizinische Fakultät der HumboldtUniversität zu Berlin

The farnesyltransferase inhibitor R115777 (Zarnestra ${ }^{\mathrm{TM}}$; Z), targeting neoplasms with ras mutations like pancreatic cancer (PC), showed antitumor activity in PC cell lines as a single agent and in combination with gemcitabine (GEM). As a consequence, a randomized, multinational double blind phase III trial comparing GEM + Z versus GEM + placebo in patients (pts.) with advanced PC (R115777-INT-11 trial, Proc. ASCO 2002, - 517) was initiated.

Aim of this study was to evaluate the impact of second and third line treatment in patients progressing within the phase III trial. Pts.' characteristics: $\mathrm{n}=23$, median age 61 years [49-78], ECOG PS 0/1/2 in 5/13/5 pts., stage III/IV in $3 / 20$ pts. by time of diagnosis and $1 / 22$ after documented progression.

Median time to progression (TTP) on 1st line therapy was 13 [1-46] weeks (95\% CI 7.1-23; data still double blinded). After documented progression, 9 pts. stopped treatment. 14 pts. $(61 \%)$ were treated with 2 nd line regimen: Either combination of GEM with Cisplatin, Folinic Acid (FA) and 5-FU (4 pts.) or Oxaliplatin combined with FA and 5-FU. Paclitaxel administrated weekly was used as $3 \mathrm{rd}$ line therapy in 7 out of the 14 pts. progressing on 2nd line therapy.

Results: Median TTP in 14 pts. on 2nd line was 14 [2-71] weeks, an additional progression free median interval of 11 [1-19] weeks was observed in 7 pts. on 3rd line therapy. Median overall survival (OS) in all pts. treated in our institution was 33 [2-93] weeks (95\% CI 28.3-37.7) with a 1-year survival rate of $39 \%$. Median OS in pts. with 2 nd- or 3rd-line treatment was 69 [31-93] weeks (95\% CI 48.8-89), 5 pts. are still alive.

Conclusions: In the 23 pts. from our institution receiving GEM $+/-\mathrm{Z}$ as first line therapy within the context of a phase III trial, no change in TTP was to observe compared to TTP for the entire cohort of 688 pts. (16 weeks and 16.5 weeks, respectively). The median OS of 33 weeks and 1-year sur- 
vival of $39 \%$ in our patients were increased when compared to OS of all pts. in R115777-INT-11 trial with 27.5 weeks for GEM $+\mathrm{Z}$ and 26 weeks for GEM and 1-year survival rates of $27 \%$ and $24 \%$, respectively. The increase of OS seems to be related to pts. receiving 2 nd and 3 rd line treatment with a median OS of 69 weeks for this subgroup. These findings do underline the need to identify sequential therapy strategies in pts. with PC.

\section{6} A phase I study of high-dose 5-fluorouracil, sodium folinic acid,
and mitomycin c in combination with escalating doses of Caelyx ${ }^{\circledR}$ in patients with pretreated gastrointestinal cancer

Hofheinz R.D., Weisser A., Willer A., Worst P., Emig M., Hehlmann R., Hochhaus A.

Onkologisches Zentrum, III. Medizinische Klinik, Universitätsklinikum Mannheim

Introduction: Infusional 5-fluorouracil (5-FU), folinic acid (FA) and mitomycin C (MMC) have demonstrated activity in patients (pts) with gastrointestinal cancer. Using 3-weekly bolus MMC $10 \mathrm{mg} / \mathrm{sqm}$ with high-dose (HD) 5-FU/FA (AIO-schedule) as first-line therapy in patients with metastatic gastric cancer we observed a response rate of $55 \%$ and overall survival of 10.6 months. In order to improve these results, we decided to add liposomal, pegylated adriamycin (Caelyx) into this regimen. We therefore have launched a phase I study in patients with pretreated gastrointestinal cancer adding increasing doses of Caelyx to HD 5-FU/FA and MMC.

Dose-escalation schedule: 5 -FU 2,000mg/sqm and sodium-FA (Oncofolic) $500 \mathrm{mg} / \mathrm{sqm}$ mixed in one pump weekly x 6 , MMC $7 \mathrm{mg} / \mathrm{sqm}$ days 8 and 36 , Caelyx 15 - 25 - 30 - $35 \mathrm{mg} / \mathrm{sqm}$ (level I, II, III, IV, respectively) days 1 and 29. The cycle is repeated on day 57.

Results: Until now 14 pts have been recruited. The patients suffered from pancreatic cancer $(n=11)$, gastric cancer $(n=1)$ and gallbladder cancer $(n=2)$ They had been pretreated with 5-FU/capecitabine $(n=7)$ and/or gemcitabine $(n=13)$. Two pts were withdrawn because of anaphylactoid reaction during the first Caelyx administration. No dose limiting toxicity (DLT) has been observed on level I $(n=3)$. On level II, three out of four patients experienced DLT between treatment days 14 and 16 (leukopenia grade III-IV $\mathrm{n}=3$, mucositis grade III $\mathrm{n}=3$ ). Therefore, Caelyx $25 \mathrm{mg} / \mathrm{sqm}$ was determined as maximum tolerated dose (MTD). Because of the large difference between the dose levels of 15 and $25 \mathrm{mg} / \mathrm{sqm}$ we decided to also test a $20 \mathrm{mg} / \mathrm{sqm}$ level. Five pts have been recruited to this dose level so far. 3 pts completed the first cycle without DLT, one is still under treatment, and one patient developed leukopenia grade IV and mucositis grade III. 19 complete cycles have been administered, and antitumor activity (minor remission, NC, clinical benefit response) has been observed on all three dose levels. Interestingly, palmar-plantar erythrodysesthesia, regarded as overlapping toxicity is not likely to be increased by the combination of infusional 5-FU and Caelyx at the investigated doses.

Conclusion: The study is ongoing on the $20 \mathrm{mg} / \mathrm{sqm}$ dose level, and mature results will be presented.

\section{7}

\section{A phase I study of capecitabine in combination with escalating doses of mitomycin $\mathrm{c}$ in patients with pretreated gastro-} intestinal cancer

Hofheinz R.D. (1), Hartmann J.T. (2), Hartung G. (3), Weisser A. (1), Willer A. (1), Bokemeyer C. (2), Hehlmann R. (1), Hochhaus A. (1)

(1) III. Medizinische Klinik, Universitätsklinikum Mannheim, (2) II. Medizinische Klinik, Universitätsklinikum Tübingen, (3) Medizinische Klinik, Hämatologie und Onkologie, Universitätsklinikum Rostock

Introduction: Both Capecitabine, an oral prodrug of 5-fluorouracil (5-FU), and Mitomycin C (MMC) have demonstrated activity as single agents in patients (pts) with gastrointestinal (GI) cancer. Furthermore, a combination of MMC with infusional 5-FU can induce tumor remission even in patients pretreated with 5-FU. In a human xenograft model it has been demonstrated recently that capecitabine and MMC act synergistically due to an upregulation of the thymidine phosphorylase activity by MMC. We sought to exploit these preclinically observed effects in a phase I study in pts with pretreated GI cancer.

The dose-escalation regimen included capecitabine administered at a dose of $1,000 \mathrm{mg} / \mathrm{sqm}$ twice daily day 1-14 and MMC given as bolus at day 1 at doses of 4, 6, 8, $10 \mathrm{mg} / \mathrm{sqm}$ (Levels I, II, III, IV, respectively) to cohorts of at least three pts per dose level. This regimen is repeated on day 22. Two treatment cycles are considered for the evaluation of dose limiting toxicities (DLT) according to study protocol.
Results: Since 12/2001 16 pts have been included at the first three dose levels: Level I $n=3$, level II $n=6$, level III $n=7$. Toxicity data of dose levels I and II is available. Dose level III is currently under evaluation. Pts at level I and II suffered from gastric cancer $(n=2)$, colorectal cancer $(n=6)$, and pancreatic cancer $(n=1)$. These pts had been pretreated with a median of two different regimen (range 0-4). High-dose 5-FU ( $n=8)$, CPT-11 $(n=5)$, and oxaliplatin $(n=5)$ were the most commonly used drugs for previous treatment. No DLT has been observed in the first two dose levels. Out of 26 evaluable cycles on levels I and II ( $\mathrm{n}=9 \mathrm{pts}$ ) the following toxicities (graded according NCI-CTC) have been reported: Leukopenia $\mathrm{I}^{\circ}(\mathrm{n}=1)$, thrombocytopenia $\mathrm{I}^{\circ}$ $(\mathrm{n}=1)$, nausea/vomitus $\mathrm{I}^{\mathrm{O}} / \mathrm{II}^{\circ} / \mathrm{III}^{\circ}(\mathrm{n}=4 / 0 / 1)$ diarrhea $\mathrm{I}^{\circ} / \mathrm{II}^{\circ}(\mathrm{n}=2 / 2)$.

Conclusion: Mitomycin in combination with capecitabine is well tolerated at the investigated does levels. Accrual is ongoing at the third dose level $(8 \mathrm{mg} / \mathrm{sqm})$, and mature results will be presented.

\section{High incidence of genomic amplifications in pancreatic carcinoma cell lines - a matrix-CGH analysis}

Holzmann K. (1), Schwänen C. (1), Kohlhammer H. (1), Wessendorf S. (1), Gress T. (2), Lichter P. (3), Bentz M. (1)

(1) Abt. Innere Medizin III und (2) Abt. Innere Medizin I, Universität Ulm; (3) Abt. Molekulare Genetik, Deutsches Krebsforschungszentrum, Heidelberg

Objectives: Comparative genomic hybridisation (CGH) was developed for a genome wide analysis of DNA sequence copy number. However, due to the use of metaphase chromosomes only aberrations $>10 \mathrm{Mbp}$ are detectable. The approach of genomic DNA hybridization to microarrays (,Matrix$\mathrm{CGH}^{\prime}$, $\mathrm{MCGH}$ ) allows the detection of much smaller genomic gains and losses. We used this novel technique to analyse 14 pancreatic carcinoma cell lines.

Methods: 14 widely used pancreatic carcinoma cell lines were examined by Matrix-CGH. For these analyses, a dedicated microarray containing 498 DNA clones was used. These target clones were selected based on their chromosomal mapping positions covering (i) regions, which are recurrently affected in tumorigenesis or (ii) containing genes involved in the pathogenesis of many solid tumors. In all cases, MCGH results were compared with chromosomal CGH data.

Results: In the 14 cell lines, 29 high level DNA amplifications were identified. In four cell lines, amplifications mapping to 8q24 and 20q13 were detected. In all cases with 8q24-amplifications, the MYC gene was amplified. In three cases each, the EGFR gene mapping to chromosome 7 p12.3 and the FGF3 gene (11q13) were amplified. The other amplifications affected some genes, for which a pathogenetic role in pancreatic carcinoma has not been described, like e.g. two members of the BCL-family. Using chromosomal $\mathrm{CGH}$, only 8 high level DNA amplifications were identified in a total of 6 cases.

Conclusions: Compared to previously published data, by MCGH a much higher frequency of gene amplifications was found in pancreatic carcinoma cell lines. As also amplifications of new candidate genes were found, this approach may lead to new insights into the pathogenesis of pancreatic carcinoma.

\section{9}

Adjuvant radiochemotherapy (RTx/CTx) using 5-FU/Folinic acid (FA)/cisplatin (CDDP)/Paclitaxel (P) and radiation in patients (pts) with completely (RO) resected high-risk gastric cancer (UICC stages II-IV (MO)

Kollmannsberger C. (1), Budach W. (2), Stahl M. (3), Vanhöfer U. (4), Hehr Th. (2), Wilke J. (3), Schleicher J. (5), Schleucher N. (4), Böhlke I. (1), Kanz L. (1), Bokemeyer C. (1)

(1) Dept. of Hematology/Oncology, Univ. of Tuebingen; (2) Dept. of Radiation Oncology, Univ. of Tuebingen, (3) Dept. of Hematology/ Oncology Kliniken Essen Mitte; (4) Dept. of Hematology/Oncology Westdeutsches Tumorzentrum Essen; (5) Dept. of Hematology/Oncology Katharinenhospital Stuttgart

The US-Intergroup study 0116 has demonstrated a significant survival benefit for pts receiving adjuvant Rtx/CTx following complete resection of high-risk gastric cancer. The current study evaluates the feasibility, toxicity and efficacy of an optimised treatment strategy containing a 4-drug CTx regimen plus 5-FU-based RTx/CTx.

Patients and methods: Between 12/2000 and 4/2002 33 pts were registered. Treatment consisted of 2 cycles of $5-\mathrm{FU} 2000 \mathrm{mg} / \mathrm{m}^{2} / 24 \mathrm{~h}$ preceded by FA $500 \mathrm{mg} / \mathrm{m}^{2} / 2 \mathrm{~h}$ once weekly for 6 consecutive weeks, P $175 \mathrm{mg} / \mathrm{m}^{2}$ added 
in weeks 1 and 4 and CDDP $50 \mathrm{mg} / \mathrm{m}^{2}$ in weeks 2 and 5 (Kollmannsberger et al. Br J Cancer 2000). After one cycle of CTx pts receive Rtx with 45 Gy plus 5 -FU $225 \mathrm{mg} / \mathrm{m}^{2} / 24 \mathrm{~h}$ applied on all radiation treatment days followed by the second cycle of CTx. Eligibility criteria included completely resected gastric adenocarcinoma including a systematic D1 or D2 lymph node dissection (LND). Pts were stratified according to the extent of the LND.

Results: Four of 33 pts were primarily excluded due to metastases found on pre-treatment work-up. Pts characteristics: median age: 49 years [37-66] total/subtotal gastrectomy $84 \% / 16 \%$; D1/D2 LND 33\%/67\%; stage distribution: UICC stage IB 8\%, II 32\%, IIIA 36\%, IIIB 8\%, IV(M0) 16\%; Toxicity ( $\mathrm{n}=19$ pts who completed therapy) CTC III/IV: neutropenic infection 2 pts (11\%); leucopenia 8 pts (42\%); anorexia 3 pts (16\%); diarrhea 1 p (5\%); nausea/vomiting 2 pts (11\%); weight loss $\geq 10 \%$ (CTC II/III) 12 pts (64\%); early death $1 \mathrm{pt}(5 \%)$ due to pneumocystis infection; study discontinuation 2 pts (1x toxicity, 1x personal reasons). Dose reduction/treatmen delays during the first and second cycle were necessary in 6 and 9 applications, respectively. Two pts had an allergic reaction to P. However, dose intensity for 5-FU/FS was $>90 \%$ and for P and CDDP $>80 \%$ in both CTxcycles. After a median follow-up of 9 months [3-18], 3 pts (10\%) have relapsed and 26 pts $(90 \%)$ are alive without tumor recurrence.

Conclusions: This novel RTx/CTx regimen appears feasible and safe with acceptable toxicity. A second study arm consisting of 5-FU/FS/CDDP without P plus RTx/CTx has been opened, in order to assess both a 3 and a 4 drugs regimen in this setting. The study will continue to recruit a total number of $100 \mathrm{pts}$ in order to fully evaluate the feasibility, toxicity and activity of this approach. The results of this study will form the basis for a randomised phase III trial. Updated results will be presented.

\section{0}

Toxicity-profile of simultaneous $24 \mathrm{~h}$-infusion of $5-\mathrm{FU}$ and sodium-folinic acid as compared to sequential application of calcium-folinic acid and prolonged 5-FU-infusion

Köppler H., Spychalski N., Heymanns J., Weide R.

\section{Haematology/Oncology group practice, Koblenz}

Introduction: Folinic acid modulated 5-FU regimen are a cornerstone in palliative treatment of colorectal carcinoma. For prolonged infusion of 5-FU calcium-folinat is given as $2 \mathrm{~h}$-infusion before 5 -FU due to incompatibility of the two drugs leading to cristalization of calcium-folinic acid when mixed with 5-FU. Recently sodium-folinic acid has been shown to be compatible in solution with 5-FU. We report the toxicity of this approach as compared to the standard sequential application.

Methods: 35 patients received simultaneous mixed infusions of sodiumfolinic acid and 5-FU over $24 \mathrm{~h}$ with a total of 607 applications. Toxicity was compared to 82 patients treated with calcium-folinic acid ( $2 \mathrm{~h}$ infusion) followed by 5-FU 24h infusion (1634 applications). Sodium-folinic acid and calcium-folinic acid were given with $500 \mathrm{mg} / \mathrm{m}^{2}$. 5 -FU dose varied between 2000 and $2600 \mathrm{mg} / \mathrm{m}^{2}$.

Results: No increase in 5-FU/folinic acid associated toxicity WHO grade IIV (mucositis, diarrhea, hand- and foot syndrome) was seen in patients receiving sodium-folinic acid. No unexpected other toxicity occurred. Preliminary survival data demonstrate no disadvantage for the group treated with sodium-folinic acid.

Conclusion: We conclude that sodium-folinic acid has a safe toxicity-profile. No adverse reactions were seen. Infusion time is reduced by 2 hours per application saving office time and adding to patient's quality of life.

\section{1}

Mitomycin $\mathbf{C}$ in combination with capecitabine or biweekly high dose gemcitabine in patients with advanced biliary tract cancer: preliminary results of a parallel phase II trial

Kornek G.V. (1), Schmid K. (1), Schuell B. (1), Kwasny W. (2), Haider K.

(2), Depisch D. (2), Lang F. (3), Raderer M. (1), Scheithauer W. (1)

(1) Vienna Univ.Med.School, (2) General Hospital Wr.Neustadt,

(3) General Hospital Neunkirchen

Introduction: Patients with advanced biliary tract carcinoma face a particularly dismal prognosis and no standard palliative chemotherapy has been defined until today. Among several different single agents, mitomycin C (MMC) and more recently, the oral fluoropyrimidine capecitabine (CAPE) and the nucleoside analog gemcitabine (GEM) have been reported to exert antitumor activity. In view of a possible drug synergism, the present paralle phase II trial was initiated. The aim was to investigate the therapeutic efficacy and tolerance of MMC in combination with CAPE or GEM in previously untreated patients with advanced biliary tract cancer.
Patients and methods: Seventeen patients were treated with MMC $8 \mathrm{mg} / \mathrm{m}^{2}$ given as an iv bolus injection on day 1 plus oral CAPE $2500 \mathrm{mg} / \mathrm{m}^{2}$ on days 1-14 every 3 weeks, and 18 patients received MMC $8 \mathrm{mg} / \mathrm{m}^{2}$ on day 1 in combination with GEM $2200 \mathrm{mg} / \mathrm{m}^{2}$ given as a $30 \mathrm{~min}$ iv infusion on days $1+15$ every 4 weeks. In both treatment arms, chemotherapy was administered for a duration of 6 months unless prior evidence of progressive disease.

Results: Pretreatment characteristics were well balanced between the two treatment arms. The overall response rate among patients treated with MMC + CAPE was $35 \%(6 / 17)$ compared to $27.8 \%(5 / 18)$ among those treated with MMC+GEM. There was no significant difference in terms of median progression free survival ( 4.5 vs. 5 mos), and median overall surviva ( $>8$ months) has not been reached yet with either regimen. Chemotherapy was well tolerated in both arms with a comparable low rate of only mild or moderate non-haematologic adverse reactions. Similarly, only $2(12 \%)$ versus $3(17 \%)$ patients experienced WHO grade 3 haematologic side effects. Conclusion: Early results of this parallel phase II trial suggest a fairly good therapeutic index for both the combination of MMC with CAPE or GEM and thus warrant further investigation.

\section{Low dose Capecitabine plus Oxaliplatin in Metastatic Colorectal Cancer}

Stengele K., Krieger G.

Hegau-Klinikum Singen

Capecitabine plus Oxaliplatin (CapOx) is an effective therapy in metastatic colorectal cancer (MCRC). The standard regimen (Cap $2.5 \mathrm{~g} / \mathrm{qm}$ d1-d14, Ox $130 \mathrm{mg} / \mathrm{qm} \mathrm{d} 1, \mathrm{qd} 22$ ) requires dose modifications in more than a third of patients and may be inconvenient for clinical practice.

Objectives: To assess the efficacy and toxicity of low dose (LD) CapOx in unselected patients with MCRC in the community hospital setting.

Material and Methods: Retrospective analysis of a cohort of patients attending the outpatient clinic of a community hospital for palliative therapy of MCRC from 1/00 until 4/02. Patients were treated with Capecitabine p.o. $1.5 \mathrm{~g} / \mathrm{qm} \mathrm{d} 1-\mathrm{d} 14$ and Oxaliplatin i.v. $50 \mathrm{mg} / \mathrm{qm} \mathrm{d} 1$ and d8 qd22. Responding patients received at least 6 cycles. Clinical status, CEA, blood chemistry and toxicity were evaluated before every treatment cycle. Radiologic tumor parameters were assessed every 3 cycles.

Results: 45 pts were treated with 228 cycles LD CapOx (median 6 cycles) after informed consent. Patient characteristics: median age $64 \mathrm{y}, \mathrm{f} / \mathrm{m} \mathrm{15/30,}$ liver metastases $82 \%$, lung $27 \%$, other $13 \%, \geq 220 \%$. The median follow up is 9 months. The efficacy in 28 pts with CapOx as first line therapy was CR 1, PR $11(\mathrm{CR}+\mathrm{PR} 43 \%), \mathrm{NC} 6$, PD 7, NA 3; in 17 pts with second line therapy: CR 0, PR 4 (CR + PR 24\%), NC 7, PD 5, NA 1. Median survival for first line therapy: not available yet; for second line therapy: 12 months Toxicity: 3 pts had gastrointestinal toxicity WHO Gr 3/4 (2 diarrhea, 1 liver enzymes), one had thrombocytopenia Gr 3 and 5 pts receiving more than 6 cycles had neuropathy Gr 3 . Frequent Grade $1 / 2$ toxicities were sensory neuropathy in 31 pts $(69 \%)$, nausea/vomiting $40 \%$, hematological toxicity $22 \%$ and diarrhea $20 \%$. Only 2 pts had hand-foot syndrome (Gr 1). 14 pts did not receive 6 cycles: 10 because of progression, 3 because of gastrointestinal toxicity $\mathrm{Gr} 3 / 4(7 \%)$ and one due to change of residence. 8 pts (18 $\%$ ) had minor dose reductions for Gr $1 / 2$ toxicities. Update of follow up will be presented at the meeting.

Conclusions: LD CapOx is an effective and convenient regimen for palliative therapy of MCRC. This regimen should be compared with other innovative chemotherapies for MCRC.

\section{3}

Chronomodulated chemotherapy in locally advanced and metastatic colorectal cancer combining 5-FU and sodium folinate with Oxaliplatin (SOFOX) or Irinotecan (SOFIRI). The Jena experience in 52 patients

\section{Kubsch K. (1), Kliche K.O. (1), Raida M. (1), Masri-Zada R. (2), Höffken K. (1)}

(1) Klinik und Poliklinik für Innere Medizin II - Onkologie, Hämatologie, Endokrinologie und Stoffwechselerkrankungen, Friedrich-Schiller-Universität Jena, (2) Klinik und Poliklinik für Innere Medizin I - Gastroenterologie, Friedrich-Schiller-Universität Jena

Purpose: To study efficacy and tolerability of chronomodulated (CM) chemotherapy in patients with metastatic or locally advanced colorectal cancer. Furthermore, calcium folinate has been replaced by sodium folinate due to better feasibility. 
Patients and Methods: We treated 52 patients with metastatic or locally advanced colorectal cancer (,CRC“) with a total of 311 courses of a CM-therapy using Sodium-Folinate-5-FU-Oxaliplatine-CM (,SOFOX-CM“). The protocol is a 4-day course of chronomodulated infusion 5-FU (750 $\left.\mathrm{mg} / \mathrm{m}^{2} / \mathrm{d}\right)$ and sodium folinate $\left(150 \mathrm{mg} / \mathrm{m}^{2} / \mathrm{d}\right)$, simultaneously infused from $22.00 \mathrm{~h}$ to $10.00 \mathrm{~h}$. Oxaliplatine $\left(25 \mathrm{mg} / \mathrm{m}^{2} / \mathrm{d}\right)$ was infused from $10.00 \mathrm{~h}$ to $22.00 \mathrm{~h}$ on $\mathrm{d} 1-4$. Therapy was reinitiated on day 15 . Our „SOFIRI-CM“" protocol lasts for 5 days and uses identical dosages for 5-FU and sodium folinate with slightly different infusion times $(24.00 \mathrm{~h}$ to $12.00 \mathrm{~h}$ on $\mathrm{d} 2-5)$. Irinotecan is given on day $1\left(350 \mathrm{mg} / \mathrm{m}^{2} / \mathrm{d}\right)$ from $2.00 \mathrm{~h}$ to $8.00 \mathrm{~h}$. Therapy was reinitiated on $\mathrm{d} 21$. A total of 135 cycles of this protocol was administered, mainly ( $75 \%$ of all cases) as 2 nd CM-therapy.

Results: Within the whole group $(\mathrm{n}=52)$ response data were as follows: $\mathrm{CR}$ 1 (1.9\%), PR 7 (13.5\%), SD 20 (38.5\%), PD 20 (38.5\%), lost-to-follow-up $2(3.9 \%)$, too-early $2(3.9 \%)$. PFS was 4 months (0-24) (median; range). The patients reported here clearly represent a poor prognosis group, since 37 pts $(71.2 \%)$ had received prior chemotherapy. 7 patients $(13.5 \%)$ had received prior oxaliplatine, which led to SD in 1 patient and left 6 patients with progressive disease. Within this study 5 patients were reexposed to SOFOX-CM, which achieved SD in 3 patients, while 2 patients remained progessive. Among the chemonaive patients $(n=15) 6$ achieved CR $(1 \mathrm{x})$ and PR $(5 \mathrm{x})(40 \%)$ and another three individuals $(20 \%)$ showed a stabilization of their disease.

We found a moderate overall toxicity with grade $3-4$ neuropathy in $9,6 \%$ of patients during a total of 311 courses of SOFOX-CM and grade 3-4-diarrhea in $9,4 \%$ after 135 courses of SOFIRI-CM.. Only in 1 patient SOFOX$\mathrm{CM}$ had to be stopped due to grade 3-4 sensory neuropathy.

Conclusions: We found CM-therapy to be effective and safe in the treatment of advanced poor-prognosis malignancies of the GI-tract. In 3 of 5 cases „CM“ oxaliplatine could induce another disease stabilization after ,flatrate" oxaliplatine had failed. Sodium folinate offers the advantage of chemical compatibility with 5-FU, thus a single chamber port is sufficient.

\section{4}

\section{Frequency of hereditary non-polyposis colorectal cancer (HNPCC) in the region of Bonn/Rhein-Sieg: A prospective population-based study}

Lamberti C. (1), Matthiak M. (2), Bollmann M. (4), Vogel J. (5), Kindermann D. (6), Propping P. (3), Schmidt-Wolf I.G.H. (1), Sauerbruch T. (1)

(1) Medizinische Klinik und Poliklinik I, (2) Pathologisches Institut, (3) Institut für Humangenetik Universitätsklinikum Bonn, (4) Institut für Pathologie Bonn-Duisdorf, (5) Institut für Pathologie Siegburg, (6) Praxisgemeinschaft für Pathologie Bonn

Introduction: The majority of colorectal cancers (CRC) develop sporadically, but the real proportion of inherited CRC remains to be uncertain, yet. Especially in Germany no epidemiological data exist about the frequency of the most common - hereditary non polyposis colorectal cancer (HNPCC). Thus, incidence of HNPCC should be estimated in a defined region such as the region of Bonn/Rhein-Sieg according to the clinical criteria of HNPCC (positive Amsterdam criteria or looser criteria including extracolonic cancers known to be associated with HNPCC) and immunhistochemistry (lack of expression of most commonly involved DNA mismatch repair enzymes MSH2 or MLH1)

Methods: Tumor stage and therapy of patients with newly diagnosed colorectal cancer in the region of Bonn/Rhein-Sieg were documented according to the medical records and family history was assessed by a standardised questionnaire. Expression of MSH2 and MLH1 was determined by immunhistochemistry of paraffin-embedded tumor tissue.

Results: Approximately 800.000 habitants live in this region, i.e. 470 new cases of colorectal cancer per year could be expected. From $9 / 99$ to $10 / 0$ 814 patients were registered. $346(42.5 \%)$ patients gave their written consent and were asked about their family history. Of these patients, $36(10 \%)$ had a family history fulfilling the HNPCC criteria, 15 patients were younger than 45 years and 24 patients had syn-or metachronous HNPCC associated cancers. Merely, $21 \%$ of patients with family history of HNPCC showed a lack of expression of MSH2 or MLH1 MLH2 by immunhistochemistry. As expected, the proportion of patients with sporadic colorectal cancer lacking MSH2 or MLH1 was significantly lower $4.6 \%(n=16 ; p<0.02) .12$ out of the 16 patients were older than 60 years at diagnosis.

Conclusion: 1/5 of newly diagnosed patients had a family history of colorectal or other HNPCC associated cancers. However, the majority of these patients expressed both mismatch repair proteins MSH2 and MLH1, respectively. Thus, only a small proportion of patients fulfilled unambiguously the clinical as well as immunhistochemical criteria for HNPCC. A larger proportion of patients seems to have a familiar predisposition different from HNPCC.

The study was supported by the Deutsche Krebshilfe
Implications of the human Histon-Deacetylase HDAC9 on the pathogenesis of gastrointestinal malignancies

Mahlknecht U. (1), Schnittger S. (2), Will J. (1), Cicek N. (1), Hoelzer D. (1)

(1) Klinikum der J.W. Goethe Universität Frankfurt, Medizinische Klinik III, Franfkurt (2) Klinikum der Ludwig-Maximilians-Universität Großhadern, Medizinische Klinik und Poliklinik III, München

Epigenetically mediated modulation of gene promoter function through histone acetylation modifying enzymes, which regulate the acetylation state of histone proteins and other promoter-bound transcription factors is increasingly appreciated as a key component in the regulation of reversible gene expression and in the pathogenesis of cancer. While histone acetyltransferases (HATs), which are frequently part of multisubunit coactivator complexes, lead to the relaxation of chromatin structure and transcriptional activation, histone deacetylases (HDACs) tend to associate with multisubunit corepressor complexes, which result in chromatin condensation and transcriptional repression of specific target genes. We report herein the identification and characterization of human Histone Deacetylase 9 and its potential implications on the pathogenesis of gastrointestinal malignancies. The human HDAC9 genomic sequence spans a region of $458 \mathrm{~kb}$ and has one single chromosomal locus. Determination of the exon-intron splice junctions established that HDAC9 is encoded by 23 exons ranging in size from $22 \mathrm{bp}$ (exon 1) to $264 \mathrm{bp}$ (exon 11). Characterization of the 5 ' flanking genomic region revealed that the human HDAC9 promoter lacks both the canonical TATA and CCAAT boxes; CpG elements are missing. The human HDAC9 open reading frame is 3,036 bp long and encodes a 1,011 aa protein with a predictive molecular weight of $111,3 \mathrm{kDa}$ and an isoelectric point of 6.41. Fluorescence in situ hybridization analysis localized the human HDAC9 gene to chromosome $7 \mathrm{p} 21$, a region which has been associated particularly with the pathogenesis of gastrointestinal malignancies.

\section{6} Acute toxicity of combined radiochemotherapy after surgery
for advanced gastric cancer - first experiences

Mai S.K. (1), Hofheinz R. (2), Lohr F. (1), Wenz F. (1)

(1) Universitätsklinikum Mannheim, Institut für Klinische Radiologie, Sektion Strahlentherapie, (2) Universitätsklinikum Mannheim, III. Medizinische Klinik

Objectives: To present our experiences with regard to acute toxicity of adjuvant Radiochemotherapy for gastric cancer.

Material and Methods: Since February 200115 patients with gastric cancer were treated with combined radiochemotherapy according to the protocol published by MacDonald et al. Median age was 61 years (39-69). Male/female-Ratio was 9:6. Karnofsky-performance-index was between 60 and $100 \%$. Initially patients received $425 \mathrm{mg} 5 \mathrm{FU} / 20 \mathrm{mg}$ FA for 5 days, followed then by radiochemotherapy with a total dose of 45 Gy with single daily factions of $1,8 \mathrm{~Gy}$, five days per week. During radiotherapy chemotherapy was given on day 1-4 and $23 ; \mathrm{V} 25$ at reduced dose of $400 \mathrm{mg} 5 \mathrm{FU} / 20 \mathrm{mg}$ FA. Two more 5-day cycles of full dose chemotherapy were given 4 and 8 weeks after radiochemotherapy.

Results: 12 of 15 patients received therapy per protocol. One patient showed progress of disease during therapy, one patient declined therapy, and one patient died due to peritoneal spread before the last chemotherapy-cycle. Mean weight-loss amounted to $4,5 \mathrm{~kg}(0-10 \mathrm{~kg})$. Nine patients suffered from gastrointestinal toxicity $\geq$ Grad 3 according to the CTC-Scale. Hematologic side effects $\geq$ Grad 3 were not observed.

Conclusions: Adjuvant combined Radiochemotherapy after resection of gastric cancer shows a high toxicity. Special supportive treatment is necessary. 
Tumorsuppression induced by macrophage activating lipopeptide in an ultrasound-guided syngeneic pancreatic carcinoma mouse model

Märten A. (1), Schneider C. (1), Schmidt T. (1), Ziske C. (1), Sauerbruch T. (1), Mühlradt P. (2), Schmidt-Wolf I. (1)

(1) Department of Internal Medicine I, University of Bonn, 53105 Bonn, Germany; (2) Department of Immunbiology, GBF, 38124 Braunschweig, Germany

Carcinoma of the pancreas has an especially poor prognosis. Therefore novel therapeutic forms are required. In this study, we investigated the immunomodulatory capacity of a lipopeptide (macrophage activating lipopeptide, MALP) in a new orthotopic, ultrasound-guided pancreatic cancer mouse model, which is close to the biological situation and avoids stress and immunostimulation caused by laparatomy. Pancreatic carcinoma cells from the syngeneic, highly aggressive and metastatic cell line Panc 02 were administered ultrasound-guided orthotopically or subcutaneously, respectively. In both models, we could show a tumorsuppressive effect induced by a single intraperitoneally or intratumorally administration of synthetic MALP which was originally derived from mycoplasma fermentans. Mice showed obvious no side effects. The median survival increased from 17 days to 25 days. The life-prolonging effect $(\mathrm{p}<0.003)$ was coupled to a significant increase of cytotoxic T cells, $\beta 2$-integrin expression on lymphocytes and high levels of CD45RB as determined in murine spleens. Immunohistochemicals stainings showed a strong CTL infiltration.

MALP binds to toll-like receptor- 2 and -6 and induces the release of early stage leucocyte attracting factors, such as MIP-1, MCP-1, and MIP-2. Furthermore, the activaton of NF- $\mathrm{KB}$ in monocytes and macropphages induces the release of TNF- $\alpha$. Another source of Th1 cytokines are CD45bright T cells, a population which was found to be suppressed in tumor-bearing mice and normalized after MALP treatment. Similar was seen with regard to CD18; leucocyte adhesion was suppressed in tumor-bearing mice but expression was normalized after MALP treatment $(\mathrm{p}<0.0001)$.

After optimization of schedule for MALP administration, immunotherapy with this lipopeptide could be an offer for patients even in adjuvant situation.

\section{8}

Distinct apoptosis and growth control of combination chemotherapy with 5-Fluorouracil (5-FU), oxaliplatin (OXA), and SN-38 in HT-29 and Colo-206 colon cancer cell lines

\section{Gerold M., Andreas M.}

Klinikum der Universität München, Medizinische Klinik - Innenstadt, Department of Hematology/Oncology, Munich, Germany

Despite the availability of new drugs such as oxaliplatin (OXA) and irinotecan (with its active metabolite SN-38), 5-FU remains the most commonly used chemotherapeutic agent in colorectal cancer. Since 2-drug combinations are clinically superior to single agents, we evaluated a possible synergistic effect of triple combinations in the colorectal cancer cell lines HT-29 and Colo-206. Furthermore, we evalutated the proapoptotic effect of two cyclooxygenase-2 inhibitors, NS-398 and celecoxib, in conjunction with 5FU. Sequence of drugs and intervals between administration in 2-drug combinations have greater influence on apoptosis induction and growth inhibition than does dose escalation. Neither double nor ternery combinations of $1 \mu \mathrm{g} / \mathrm{ml} 5-\mathrm{FU}, 1 \mu \mathrm{g} / \mathrm{ml}$ OXA and $3 \mathrm{nM} \mathrm{SN}-38$ significantly exceeded the pro-apoptotic and growth-inhibitory effects of 5-FU alone. A $24 \mathrm{~h}$ preincubation with OXA resulted in less apoptosis but showed similar rates of growth inhibition than 5-FU alone. OXA alone induced less apoptosis than 5-FU, but a similar reduction of cell growth. HT-29 cells were sensitive to NS-398 as well as to celecoxib, while Colo-206 only demonstrated apoptosis when exposed to celecoxib. These findings were confirmed by measuring mitochondrial transmembrane potential using DIOC6. Coincubation of continuously administered 5-FU with $50 \mu \mathrm{M}$ NS-398 or $25 \mu \mathrm{M}$ celecoxib did not result in more apoptotic cells or a higher growth inhibition than 5FU alone. (Apoptosis was measured after propidium iodide staining of fixed cells by flow cytometry after a 5 day incubation. Cell viability in $\%$ was determined with a MTT-assay relative to untreated control. Results are mean of at least three experiments).

Conclusion: 1) Under the conditions tested, 5-FU alone was not consistently inferior to double or ternery combinations in apoptosis or proliferation assays of two widely used colorectal cancer cell lines in vitro. This contrasts to published data from recent clinical trials using double combinations, thus indicating that in vitro chemotherapy studies do not precisely correlate with the clinical response rate in patients.
2) Within the limitations of the in vitro system used, pronounced differences in apoptosis induction and growth control were observed in ternery combinations, that should be considered for future trial design.

3) COX-2-inhibitors may work antagonistically in conjuction with continuous exposure to 5-FU. Additional studies involving more cell lines are warranted.

CPT-11, Oxaliplatin, and 5-FU/FA combination chemotherapy for metastatic colorectal cancer - preliminary data of a phase I/II trial

Meinhardt G., Schlesinger A., Emmerich B.

Klinikum der Universität München, Medizinische Klinik - Innenstadt, Department of Hematology/Oncology, Munich, Germany

Objectives: We evaluated the safety and efficacy of a triple-drug combination consisting of oxaliplatin, CPT-11, and FA-modulated 5-FU in the treatment of advanced colorectal cancer.

Material and Methods: Non-randomized phase I/II study approved by the local ethics committee. Primary endpoints were toxicity and response. $\mathrm{Pa}$ tients (pts) on dose level I received oxaliplatin $60 \mathrm{mg} / \mathrm{m}^{2}$ for 2 hours, followed by irinotecan $65 \mathrm{mg} / \mathrm{m}^{2}$ for $30 \mathrm{~min}$, folinic acid (FA) $500 \mathrm{mg} / \mathrm{m}^{2}$ for 2 $\mathrm{h}$ and 5 -FU $2000 \mathrm{mg} / \mathrm{m}^{2}$ for $24 \mathrm{~h}$ on day 1 . On dose level II, irinotecan was increased to $80 \mathrm{mg} / \mathrm{m}^{2}$. Therapy was repeated on day 8 (without oxaliplatin) and day 15 with one additional week rest. Response was evaluated with CT scan and abdominal ultrasound of liver metastasis.

Results: Four patients (pts) were treated on dose-leve I, five on level II. Mean age was 60 years (49-67), 7 male, 2 female. 6 pts were previously untreated for metastatic disease, 3 had received one prior 5-FU based chemotherapy regimen. No dose reduction was necessary on level I, wheras on level II, one patient had to be switched to level I due to dose-limiting diarrhea. A mean of 4.25 (range 2-6) and 3 (1-6) cycles were administered on levels I and II, respectively. The regimen was well tolerated with no grade III or IV hematologic toxicity and no infectious complications related to therapy. Several patients could be treated on an outpatient basis. No neurotoxicy grade III/IV and no other serious adverse events (except one grade IV diarrhea) were reported. 3 pts achieved a partial response, 5 hat stable disease, 1 patient progressed after 3 cycles. As of June 2002, 8/9 pts are alive with time from study inclusion being $1+; 6+; 6+; 8+; 9+; 12+; 16+;$ and $23+$ months. One patient died after 11 month from disease progression. One patients was succesfully retreated with the same regimen after disease progression, other patients received alternative double drug combination depending on physician preference.

Conclusions: This triple drug regimen can be safely administered to patients with advanced colorectal cancer and is able to induce partial responses. Further evaluation of this regimen in a randomized fashion against a double drug regimen is warranted.

\section{0}

Oxaliplatin (L-OHP) in combination with high dose 5-FU(24h)/ folinic acid (FA) as second line therapy in patients with gemcitabine-refractory advanced pancreatic cancer. Results of a phase II trial

Pelzer U., Hempel C., Stieler J., Arnold D., Makowski M., Köneke D., Riess H., Oettle H.

\section{Charité, Campus Virchow Klinikum, Berlin}

Objective: L-OHP-FU has demonstrated synergy in advanced pancreatic cancer (Rougier, ASCO 2000). We evaluated a combination (L-OHP/5$\mathrm{FU}(24 \mathrm{~h}) / \mathrm{FA})$ to find an active second line regimen for patients with gemcitabine (GEM) resistant pancreatic cancer.

Methods: Following failure with GEM first line therapy (with or without a farnesyl-transferase inhibitor) we treated patients (pts.) in second line with 5 -FU $2600 \mathrm{mg} / \mathrm{m}^{2}(24 \mathrm{~h}) / \mathrm{FA} 500 \mathrm{mg} / \mathrm{m}^{2}$ (30 min.) on d1,d8,d15,d22 in combination with L-OHP $85 \mathrm{mg} / \mathrm{m}^{2}(3-4 \mathrm{~h})$ on day 8 and 22 . No therapy was given on days 29 and 36. Eligibility criteria were KPS $>60 \%$, adequate hematological, renal and liver functions and controlled pain. Pts. remained on treatment until progression; all treatments were given on an outpatient basis.

Results: 23 pts. with UICC stage IVa (1) and IVb (22) have been enrolled in our center between 11/00 and 11/01. The median age was 61(38-82) years, sex ratio: 10 female / 13 male, median Karnofsky PS 80 (70-90)\%. The regimen was tolerated with moderate toxicities, no WHO $\mathrm{IV}^{\circ}$ was seen. WHO III $^{\circ}$ were as follow: hematological toxicities were documented for $\mathrm{RBC} / \mathrm{WBC} / \mathrm{PLT}$ in 4/3/1 pts, Nausea/emesis in 6 pts and diarrhea in 2 pts. 
Neurotoxicity WHO grade I-II was common with reversible WHO grade III in 2 pts. Overall median survival probability from start with OFF is now $28[4,63 ; 95 \%$ CI:7.5;48.5] weeks, overall survival probability since primary diagnosis is $17[3 ; 31 ; 95 \% \mathrm{CI}: 15.5 ; 18.5]$ months with $11 / 23$ pts. still alive. The one year survival of these selected patients is $61 \%$.

Conclusion: Our results indicate that this combination is feasible and of acceptable toxicity in advanced pancreatic cancer as salvage therapy after GEM indicating an emerging need for sequential regimens in this previously considered chemoresistant disease. Based on these results, a multicenter randomized phase III study has started to compare „BSC (best supportive care)“vs. „BSC + OFF“ in second line therapy for gemcitabine (mono) pretreated patients.

\section{1}

\section{Preliminary results of a multicenter phase II trial investigating two schedules of capecitabine plus oxaliplatin as first-line} treatment in advanced colorectal cancer

Scheithauer W. (1), Kornek G.V. (1), Schüll B. (1), Dworan N. (1), Raderer M. (1), Lenauer A. (2) Lang F. (3), Schneeweiss B. (4), Längle F. (1), Depisch D. (2)

(1) Vienna Univ.Med.School, General Hospitals of (2) Wiener Neustadt, (3) Neunkirchen, (4) Kirchdorf, Austria

Capecitabine and oxaliplatin are two new agents with potential synergistic activity that have demonstrated promising antitumor efficacy in advanced colorectal cancer (ACC). Based on preclinical and suggestive clinical evidence that dose-intensification of the oral 5-FU prodrug might result in improved therapeutic results, the present randomised phase II study has been initiated.

Patients with bidimensionally measurable ACC previously untreated for metastatic disease were randomly allocated to receive oxaliplatin $130 \mathrm{mg} / \mathrm{m}^{2}$ on day 1 + capecitabine $2000 \mathrm{mg} / \mathrm{m}^{2}$ on days $1-14$ ever 3 weeks ( $\operatorname{arm~A)~or~}$ to oxaliplatin $85 \mathrm{mg} / \mathrm{m}^{2}$ on days $1+14$ combined with capecitabine $3500 \mathrm{mg} / \mathrm{m}^{2}$ on days $1-7$ and $14-21$ every 4 weeks ( $\operatorname{arm~B}$ ). In both treatment arms, chemotherapy was continued for a total of 6 months unless prior evidence of PD.

At present, 50 patients with a median follow-up time of $\geq 6$ months are evaluable for response and toxicity analysis. Pretreatment characteristics (M/F: 33/17; median age: 67 years [range, 37 to 75 ]; primary tumor: colon/rectosigmoid 28/22; no. of metastatic sites: single/multiple 18/32; prior (neo)adjuvant chemotherapy: 15 pts) were well balanced between the two treatment arms. Patients allocated to the high dose capecitabine combination arm A had a higher radiological confirmed response rate $57.7 \%$ vs $37.5 \%$ ), though median progression-free and overall survival times have not been reached yet. The dose-intense capecitabine + oxaliplatin combination arm caused more hematological toxicities (neutropenia/thrombocytopenia: $68 \% / 52 \%$ vs. $48 \% / 26 \%$ ) and thus resulted in more treatment delays (no.o pts./courses: $9 / 20$ vs. $6 / 8$ ) than the conventional arm B. There was no difference, however, in terms of incidence and/or degree of non-hematological adverse events: the most commonly encountered symptoms (all grades, arm $\mathrm{A} / \mathrm{B})$ included nausea/emesis $(60 \% / 61 \%)$, diarrhea $(44 \% / 52 \%)$, peripheral sensory neuropathy $(84 \% / 85 \%)$, fatigue $(56 \% / 52 \%)$ and transient elevation of liver functional parameters $(60 \% / 61 \%)$.

Preliminary results of this study suggest/confirm that both combination reg imens are feasible, tolerable, and clinically active. Whether the higher response activity of the dose-intensified capecitabine arm will translate into an improved therapeutic index of this combination should be clarified with availability of more mature study results.

\section{2}

\section{Prolonged infusion of gemcitabine in advanced solid tumors: A phase-I-study}

Schmid P., Fritz J., Schweigert M., Beinert T., Fenner M., Dieing A., Salk K., Flath B., Sezer O., Possinger K.

Medizinische Klinik mit Schwerpunkt Onkologie und Hämatologie, Charité Campus Mitte, Humboldt Universität Berlin

Introduction: Gemcitabine is an active agent in the treatment advanced solid tumors. The phosphorylation of gemcitabine into the active gemcitabinetriphosphate (dFdCTP) is catalyzed by deoxycytidine kinase. This enzyme is saturated at plasma concentrations achieved after standard infusions over 30 minutes. Therefore accumulation of higher intracellular dFdCTP-concentrations, which could be associated with an enhanced antineoplastic activity, might be achieved by prolongation of the infusion time.
Purpose: The objectives of this phase I trial were to determine the dose-limiting toxicities (DLT) and the maximum-tolerated dose (MTD) of gemcitabine given as a 4-hour intravenous infusion.

Patients and Methods: Patients with advanced and pretreated solid tumors were treated with gemcitabine as 4-hour infusion on days 1,8 and 15 every 4 weeks. The starting dose was $350 \mathrm{mg} / \mathrm{m}^{2}$ with an interindividual escalation in $50 \mathrm{mg} / \mathrm{m}^{2}$ increments.

Results: 21 patients received a total of 202 doses through 3 dose levels. All patients were assessable for toxicity, 12 were also assessable for response. The maximum-tolerated dose was $400 \mathrm{mg} / \mathrm{m}^{2}$. Dose limiting toxicity was observed in 4 of 5 patients at $450 \mathrm{mg} / \mathrm{m}^{2}$ consisting of thrombocytopenia in 2 pts, leukopenia in $1 \mathrm{pt}$ and a reversible elevation of transaminases WHOgrade 3 in 1 pt. Dose reductions were necessary in $5 \%$ of pts at $350 \mathrm{mg} / \mathrm{m}^{2}$, $11 \%$ at $400 \mathrm{mg} / \mathrm{m}^{2}$ and $30 \%$ at $450 \mathrm{mg} / \mathrm{m}^{2} .2 \%$ and $26 \%$ of doses at 400 $\mathrm{mg} / \mathrm{m}^{2}$ and $450 \mathrm{mg} / \mathrm{m}^{2}$ respectively had to be omitted. Dose modifications were mainly due to hematologic toxicity which was generally mild to moderate. Most common nonhematologic toxicities were mild to moderate nausea and vomiting, mild alopecia and rapidly reversible elevation of liver enzymes. Two and four patients with NSCLC, HCC and colorectal cancers achieved a partial remission and stable disease, respectively.

Conclusion: Gemcitabine $400 \mathrm{mg} / \mathrm{m}^{2}$ days 1,8 and 15 every 4 weeks can be safely administered as 4-hour infusion. The toxicity profile is similar to the profile with short time infusion. Although not being the primary objective, activity has been observed in several entities.

\section{3}

The selective Cox-2 inhibitor Rofecoxib inhibits cell proliferation of gastric cancer cell lines in a dose-dependent manner

*Schneider R., Cramer D., Grossmann D., Ehninger G., Miehlke S.

\section{Medizinische Klinik und Poliklinik I, Universitätsklinikum Dresden}

Objectives: Cox-2 gene expression is upregulated in several gastrointestinal malignancies. With the advent of clinically available selective Cox-2 in hibitors, therapeutic downregulation of Cox-2 might become an important strategy for preventing cancer of the gastrointestinal tract. The aim of this in vitro study was to investigate the effect of the selective Cox-2 inhibitor rofecoxib (VIOXX ${ }^{\circledR}$, MSD) on cell proliferation and Cox-2 gene expression in the human gastric cancer cell lines NCI, AGS and MKN 45.

Methods: The cells were incubated in 96-Well Plates containing DMEM- or RPMI-medium, supplemented with $1 \%$ calfserum, $1 \%$ glucose, $100 \mathrm{U} / \mathrm{ml}$ penicillin and HEPES-buffer. Rofecoxib (VIOXX ${ }^{\circledR}$, MSD) were added in concentrationen from $1 \times 10-1$ to $1 \times 10-7 \mathrm{mM}$ for 12,24 and 48 hours. The cell proliferation were quantified by BrdU-ELISA. For COX-2 Expression aliquotes of mRNA obtained from AGS, MKN-45 and NCI cell lines were analyzed by RT-PCR.

Results: Baseline Cox-2 gene expression varied between the investigated cell lines and showed the highest levels in NCI, medium levels in MKN 45 and lowest levels in AGS cells. Rofecoxib significantly reduced the cell proliferation in a dose-dependent manner in all three gastric cancer cell lines. The maximum suppression of proliferation were observed in NCI cells, in cubated for 12 hours in $0,1 \mathrm{mM}$ rofecoxib, reaching $44,9 \%(\mathrm{p}=0,0003)$. In MKN 45 and AGS cells rofecoxib reduced the proliferation up to $23,1 \%$ $(p=0,0001)$ and $27,3 \%(p=0,013)$, respectively. Suppression of cell proliferation was maintained for 24 hours in NCI and MKN 45, and for 12 hours in AGS cells.

Conclusion: Our preliminary data suggest that depending on the baseline Cox-2 gene expression rofecoxib inhibits cell proliferation in gastric cancer cell lines in a time- and dose- dependent manner. Further studies are needed to investigate whether the selective Cox -2 rofecoxib might have a potentail role for chemoprevention of upper gastrointestinal malignancies.

\section{4}

Benefit or disadvantage of applying chemotherapy in patients (PTS) aged over 75 years with colorectal cancer

Schroeder M. (1), Schäfer U. (2), Aul C. (1)

(1) Medizinische Klinik 2, St. Johannes-Hospital, An der Abtei 7-11, D-47166 Duisburg, (2) Medizinische Klinik 1, St. Vincenz-Hospital, Papendelle 6, D-47051 Duisburg

Although there is a marked increase in CRC in the 7th and 8th life decade (more than $60 \%$ of patients with CRC are over 70 years at diagnosis), only a minority of patients receive adjuvant or palliative chemotherapy with 5FU based protocols. Age over 65 years is often used as exclusion criterion 
for recruitment of pts into clinical studies. We should like to report our experience in 41 pts ( 24 men, 17 women) aged $>75$ years who were treated in an adjuvant or palliative setting in our institution since $1 / 1999$.

Age distribution: median age 79 years (range $75-88$ years)

Type of cancer: colon $n=27$, rectosigmoid $n=3$, sigmoid $n=4$, rectum $n=7$

Type of treatment: adjuvant CTX (Group I): $\mathrm{n}=12$ (10x Mayo, 2x AIO) \pm RTX

palliative CTX (Group II): $\mathrm{n}=29$ (different schedules: Mayo,

$\mathrm{AIO} \pm$ Oxaliplat \pm Irinotecan $)$

Results: Adjuvant CTX

$8 / 12$ pts alive without evidence of disease after a median follow up of

$20+$ mos.

$3 / 12$ pts deceased (2/12 tumor-related)

$1 / 12 \mathrm{pt}$ alive with metastases

Palliative CTX

$20 / 29$ pts still alive after a median follow up of $17+$ mos.

9/29 pts succumbed to complications of tumor progression, but there were no drug-related deaths.

Toxicity:

4/41 pts leucopenia $\mathrm{III}^{\circ}$ and $2 / 41$ thrombopenia $\mathrm{III}^{\circ}$ no unusual GIT-toxicity

$4 / 9$ pts with polyneuropathy III $^{\circ}$ after 6 cycles of oxaliplatin/5-FU

$1 \mathrm{pt}$ ( 81 years) refused further CTX due to unacceptable side effects.

In summary, our retrospective analysis of 41 pts aged $>75$ years demonstrates that CTX is a feasible therapeutic approach which could be applied with comparable benefit as in younger pts. Before starting chemotherapy however a thorough geriatric assessment of pts is required for reducing the risks of chemotherapy and improving the treatment outcome in this advanced age group. In our patient population there was no relevant increase in toxicity or mortality due to chemotherapy.

\section{5}

\section{Biweekly docetaxel plus cisplatin \pm human granulocyte colony-stimulating factor and/or erythropoetin in patients with advanced gastroesophageal cancer}

Schüll B. (1), Kornek G.V. (1), Raderer M. (1), Dworan N. (1), Haider K. (2), Kwasny K. (2), Depisch D. (2), Lang F. (3), Hejna M. (1), Scheithauer W. (1)

(1) Vienna Univ.Med.School, (2) General Hospitals Wr.Neustadt, (3) Neunkirchen, Austria

Purpose: A phase II trial was performed to determine the antitumor efficacy and tolerance of biweekly combined docetaxel and cisplatin with or without hematopoetic growth factor support in patients with advanced gastroesophageal cancer.

Patients and methods: Thirty-six patients with histologically confirmed metastatic disease were entered in this trial. Treatment consisted of 4-weekly courses of docetaxel $50 \mathrm{mg} / \mathrm{m}^{2}$ and cisplatin $50 \mathrm{mg} / \mathrm{m}^{2}$ both given iv on days 1 and 15. Depending on absolute neutrophil counts on the days of scheduled chemotherapeutic drug administration (1000-2000/ $\mu 1)$, a 5-days course of human granulocyte colony-stimulating factor (G-CSF) $5 \mu \mathrm{g} / \mathrm{kg} / \mathrm{c}$ was given subcutaneously (s.c.); in addition, if hemoglobin was $<12$.0 $\mathrm{mg} / \mathrm{dl}$, erythropoietin $10.000 \mathrm{IU}$ was administered s.c. three times per week. Results: All patients were evaluable for response. Four (11\%) achieved a complete response and $13(36 \%)$ a partial response, for an overall response rate of $47 \%$. Eleven additional patients $(31 \%)$ showed stabilization, and 8 $(22 \%)$ progressed while on treatment. The median time to progression was 8 months and the median survival was 10.5 months with $18(50 \%)$ still beeing alive. Hematologic toxicity was common, though WHO grade 4 neutropenia occurred only in 3 patients. Non-hematologic adverse events were usually mild to moderate. Grade 3 toxicities included alopecia (14\%), infection $(3 \%)$, neurotoxicity $(6 \%)$ and anaphylaxis $(3 \%)$.

Conclusions: These results of this trial suggest that the combination of docetaxel and cisplatin with or without G-CSF and/or erythropoietin has moderate toxicity and promising therapeutic activity in patients with advanced gastroesophageal cancer.
Ethnic differences in thymidylate synthase (TS) promoter polymorphic tandem repeats

Steiner M. (1), Seule M. (1), Hartung G. (2), Köhne C.H. (3), SalazarSanchez L. (4), Herrmann F.H. (4), Schuff-Werner P. (1)

(1) Institute of Clinical Chemistry \& Pathobiochemistry, (2) Division of Hematology/Oncology, Department of Internal Medicine, University of Rostock; (3) Department of Internal Medicine, Technical University of Dresden; (4) Institute of Human Genetics, University of Greifswald, Germany

Aims: Variability in the gene expression of thymidylate synthase driven by a variable number of TS gene enhancer region tandem repeats might have therapeutic implication for fluoropyrimidine drug treatment of malignancies. Compared to double repeat alleles, tandem repeat alleles encoding for three copy numbers were shown to be associated with greater translation efficiency. In addition, preliminary evidence suggests a prognostic value of this polymorphism in cancer patients undergoing 5-fluorouracil drug treatment. The present study was performed to determine ethnic differences in TS genotype and allele frequencies between Caucasian, Amerindian, and Asian subjects.

Methods: Peripheral blood genomic DNA was isolated from subjects of Caucasian origin $(n=131)$, Amerindians from the Atlantic Talamanca area of Costa Rica (tribe Cabecar, $n=97$ ), and South Korean Asian subjects $(n=349)$. Standard PCR amplification of the TS promoter enhancer region containing the tandem repeats was performed and the amplified DNA fragments analyzed by agarose gel electrophoresis. Newly identified alleles were confirmed by sequencing studies.

Results: The predominant TS promoter genotype in Caucasian subjects was $2 \mathrm{R} / 3 \mathrm{R}(53 \%)$ followed by $3 \mathrm{R} / 3 \mathrm{R}(25 \%)$ and $2 \mathrm{R} / 2 \mathrm{R}(21 \%)$. A new allele coding for one copy of the tandem repeat was detected in one subject whose genotype was $1 \mathrm{R} / 3 \mathrm{R}$. In contrast, the majority of Asian subjects demonstrated the genotype $3 \mathrm{R} / 3 \mathrm{R}(66 \%)$. The genotype $2 \mathrm{R} / 3 \mathrm{R}$ accounted for $29 \%$ and $2 \mathrm{R} / 2 \mathrm{R}$ for $3 \%$ of Asian subjects, respectively. An additional new allele coding for 5 tandem repeat copies was found in three Asian patients $(0.9 \%)$ all being heterozygous for the genotype 3R/5R. In addition, one Asian subject displayed the rare allele 4R. TS genotype distribution in Amerindians was almost balanced between 3R/3R (48\%) and 2R/3R (42\%) with a $10 \%$ proportion of $2 \mathrm{R} / 2 \mathrm{R}$. TS enhancer region tandem repeat allele frequencies were dominated by the variant $3 \mathrm{R}$ in Asians $(81.2 \%)$ followed by Amerindians $(68.6 \%)$ and Caucasians $(52.3 \%)$.

Conclusions: The present study demonstrates significant ethnic variation in the TS enhancer region alleles between Caucasian, Amerindian, and Asian populations thus adding support to the concept that pharmacogenetically important polymorphic enzyme systems vary across populations. The clinical impact of TS enhancer region allele genotyping as one molecular tool for individualizing fluoropyrimidine chemotherapy needs to be addressed in future studies.

\section{7}

Gemcitabine in combination with cisplatin, 5-fluorouracil [24 h] and folinic acid in patients with metastatic pancreatic cancer pretreated with gemcitabine

Stieler J., Arnold D., Peters B., Pelzer U., Hempel C., Makowski M., Lück K., Roller M., Langrehr J., Riess H., Oettle H.

Charité, Medizinische Fakultät der Humboldt-Universität zu Berlin. Campus Virchow-Klinikum, Med. Klinik mit Schwerpunkt Hämatologie und Onkologie

We evaluated a combination of Cisplatin (CDDP) with Gemcitabine $\left(1000 \mathrm{mg} / \mathrm{m}^{2} ; 30 \mathrm{~min}\right), 5-\mathrm{FU}\left(750 \mathrm{mg} / \mathrm{m}^{2}\right)$ as $24 \mathrm{~h}$-infusion, and Folinic Acid $\left(200 \mathrm{mg} / \mathrm{m}^{2} ; 60 \mathrm{~min}\right)$, given day 1 and 8 of a 22 -day schedule, to find an active regimen for patients (pts.) with advanced pancreatic cancer. Despite a phase I of this schedule showed dose limiting toxicity with CDDP at 30 $\mathrm{mg} / \mathrm{m}^{2}$ in pts. with esophageal cancer (Proc. ASCO 2002, Abstr. 671), we decided to administer a reduced dose of CDDP $\left(25 \mathrm{mg} / \mathrm{m}^{2}\right)$ due to patients' pretreatment and palliative intent of this schedule. Pts. characteristics: 15 pts., $7 \mathrm{f} / 8 \mathrm{~m}$, all stage IV pancreatic cancer (mets.: 12 liver, 1 lung, 2 peritoneal carcinomatosis) have been enrolled at our institution. Median age was 62 years [range 48-79] and median Karnofsky PS 80\% [70-100]. Pretreatment consisted of GEM as adjuvant therapy (5 pts.) or GEM in combination with an experimental drug, either an inhibitor of farnesyltransferase ( 3 pts.) or an antiangiogenesis inhibitor ( 7 pts.). Median time on pretreatment was 20 [11-33] weeks until disease progression. 
Results: Therapy characteristics: With a median number of 3+ (1-6) cycles per patient, a total of 34 cycles were administered. Toxicity: All pts. were evaluable for toxicity. Therapy was generally well tolerated with occurence of anaemia $\mathrm{WHO} \mathrm{III}^{\circ}$ in 2 pts. $/ 3$ cycles as highest grade, leading to a transfusion of a total 6 RBC. 1 pt. had nausea/emesis WHO-grade III, another pt showed elevation of bilirubin to $\mathrm{WHO} \mathrm{III}^{\circ}$, probably related to choledocha stent dysfunction. Other haematologic and symptomatic toxicity did not ex ceed WHO-grade II. One patient developed mild haemolysis with increase of creatinine (WHO $\mathrm{II}^{\circ}$ ) which was regarded as haemolytic-uraemic syndrome. Response: response evaluation was performed via CT scan every 6 8 weeks. Out of 10 evaluable pts. (others: 3 too early, 1 lost to follow, stopped treatment after first course), 4 pts. presented progressive disease while one more pt. progressed rapidly and was not sent to CT. 3 pts. had stable disease for $>2$ months. Minor response was observed in 2 pts. after $4+$ and 9 weeks.

Conclusions: This combination appears to be active and generally well tolerated in an outpatient setting. The schedule should be evaluated as first line therapy. The study is ongoing and updated results will be presented.

\section{8}

\section{Dynamic expression profile of p21WAF1/CIP1 and Ki-67 predicts survival in rectal carcinoma treated by preoperative radiochemotherapy}

Sturm I. (1), Rau B. (2), Lage H. (3), Berger S. (3), Schneider U. (3), Hauptmann S. (3), Wust P. (4), Riess H. (5), Schlag P.M. (2), Dörken B. (1,5), Daniel P.T. (1)

(1) Campus Berlin-Buch, Robert-Roessle Klinik, Department of Hematology, Oncology and Tumor Immunology (2) Campus Berlin-Buch, Robert-Roessle Klinik, Department of Surgery and Surgical Oncology (3) Campus Mitte, Institute for Pathology (4) Campus Virchow-Klinikum, Department of Radiation Oncology (5) Campus Virchow-Klinikum, Department of Hematology and Oncology, Charité Medical School, Humboldt University of Berlin

Objectives: We investigated p53 and its downstream effectors p21WAF1/CIP1, BAX and hMSH2 as well as the proliferation marker Ki67 (MIB-1) in patients undergoing preoperative radiochemotherapy for rectal carcinoma to identify prognostic/predictive factors. The focus of this study was on the dynamics of these genetic markers in a longitudinal study, i.e. prior to and after radiochemotherapy.

Material and Methods: Expression of p53, BAX, p21WAF1/CIP1, Ki-67 and hMSH2 was investigated by immunohistochemistry in pre- and posttherapeutic tumor samples in 66 patients. Tumor DNA was screened for p53 mutations by SSCP-PCR. Paired tumor samples (pre-therapy and post-therapy) were collected prospectively.

Results: Patients with a decrease in p21 expression following radiochemotherapy $(\mathrm{p}=0.03)$ had a survival benefit. This went along with an increase in proliferative activity: better survival was seen for those patients with an increase of Ki-67 expression upon therapy ( $\mathrm{p}<0.005)$. In addition, we observed a significantly better prognosis for patients with high hMSH2 expression. In contrast, pretherapeutic levels of p53, BAX or p21 expression and p53 mutation had no prognostic value, indicating that the combination of radiotherapy and chemotherapy might override defects in these genes.

Conclusions: These findings are novel and support the clinical relevance of p21 in the supression of both, proliferation and apoptosis. Thus, the dynamic induction of p21 WAF1/CIP1 was associated with a lower proliferative activity but an ultimately worse treatment outcome following neoadjuvant radiochemotherapy and tumor resection. Induction of p21 therefore represents a novel resistance mechanism in rectal cancer undergoing preoperative radiochemotherapy.

\section{9}

\section{Quantification of multiple genes hypermethylated in hepato- cellular carcinoma by real-time methylation specific PCR}

Usadel H. (1), Steffens M. (1), Brabender J. (2), Nomoto S. (3), Blum H.E. (1)

(1) University Hospital of Freiburg, Freiburg i. Br., Germany; (2) University of Cologne, Cologne, Germany; (3) Johns Hopkins University, School of Medicine, Baltimore, MD, USA.

Objectives: Methylation of $\mathrm{CpG}$ islands within the promoter regions of tumor suppressor or tumor-associated genes is a common mechanism leading to silencing of gene expression. This epigenetic mechanism was shown to be an integral part of cancer development in many types of cancer. Hepatocellular carcinoma (HCC) is one of the most common cancers worldwide and little is known about the relevance of DNA methylation in the initiation and progression of this disease. We sought to investigate the meaning of hypermethylation of the genes genes p16, GSTP1, MGMT, DAP kinase and APC in the progression of HCC by quantification of methylated allels within HCCs, matching nonmalignant and normal liver tissues.

Material and Methods: A specific as well as sensitive technique to investigate the methylation status of genes is methylation specific PCR (MSP). An advancement of this technique is called real-time MSP, which is quantitative and more sensitive compared to traditional MSP. Real-time MSP has been shown to be useful in detection of neoplastic tissue (i.e. prostate cancer). We used real-time MSP for quantitative methylation analyses of the genes p16, GSTP1, MGMT, DAP kinase and APC in $34 \mathrm{HCCs,} 34$ matching non-malignant, cirrhotic tissues and 16 normal liver tissue samples from patients who underwent cholecystectomy.

Results: Detection frequencies of methylated DNA in HCCs and matching non-malignant tissues, respectively, were as follows: p16 (73\% vs. $26 \%$ ), GSTP1 (53\% vs. $32 \%)$, MGMT (15\% vs. 6\%), DAP kinase (67\% vs. $97 \%$ ) and APC (100\% vs. $100 \%) .73 \%$ of HCCs showed methylation in at least 3 of these genes. In normal liver tissue methylated DNA of p16, GSTP1 and MGMT was not detectable. Methylated DAP kinase DNA was present a low levels in $31 \%$ and of APC in $100 \%$ of normal liver samples, respectively. Relative levels of methylated DNA of the genes were significantly different in the various tissue types $(\mathrm{P}<0.001)$. The APC and DAP Kinase genes showed hypermethylation in normal liver as well as in HCCs.

Conclusions: Hypermethylation of tumor-associated genes is common in HCCs. Furthermore, significantly higher levels and frequency of methylated p16 and GSTP1 DNA in matching non-malignant tissues as compared to HCCs indicate that these epigenetic events occur early in hepatocellular carcinogenesis and that they could be useful for early diagnosis in this disease.

\section{0}

\section{A new transcatheter arterial chemoembolization protocol in refractory liver metastasis of colorectal cancer - a feasibility study}

Voigt W. (1), Behrmann C. (2), Schlüter A. (2), Kegel T. (1), Grothey A. (1), Schmoll H.-J. (1)

(1) Department of Hematology/Oncology, Martin-Luther-University, Halle-Wittenberg, Halle, (2) Department of Radiology, Martin-LutherUniversity, Halle-Wittenberg, Halle

In patients (pts) with advanced colorectal cancer (CRC) refractory to systemic chemotherapy including 5-Fluorouracil (5-FU)/Folinic acid (FA), Oxaliplatin and Irinotecan we assessed the feasibility, toxicity and response to hepatic transcatheter arterial chemoembolisation (TACE). Pts treated had exclusively or dominant liver metastasis of CRC. The following protoco was applied via a selective transfemoral hepatic arterial approach: Mitomycin C $5 \mathrm{mg} / \mathrm{m}^{2}$, Interferon- $\alpha 2 \mathrm{~b} 4.5 \mathrm{Mio}$ IU, Dexamethasone $20 \mathrm{mg}$ mixed with Amilomer DSM 45/25 (Spherex ${ }^{\circledR}$ ) day 1 and 2 i.a. (bolus), Oxaliplatin $50 \mathrm{mg} / \mathrm{m}^{2}$ (2h) day 1 i.a., FA $500 \mathrm{mg} / \mathrm{m}^{2}$ (2h) i.v. day 1 and 5-FU $1500 \mathrm{mg} / \mathrm{m}^{2}(24 \mathrm{~h})$ i.a. day 1 . Cycles have been repeated at day $15-22$. However, in two pts 1 or 2 cycles, respectively were applied with significant delay. Dose was adjusted according to the pre-treatment performance status and elevation of alkaline phosphatase, bilirubin and serum albumin. Treatment has been continued until progression or emergence of intolerable toxicity.

Eleven pts received a total number of 48 TACE, with a range of 2-7 per pt. There was no TACE related mortality. Eight pts died 5 (1pt), 8 (1pt), 9 (1pt), $10(1 \mathrm{pt}), 11$ (4pts) months after initiation of treatment. Three pts are alive at $+13(1 \mathrm{pt}),+15(1 \mathrm{pt})$ and $+18(1 \mathrm{pt})$ months after start of the treatment. Toxicity (CTC) was mild to moderate with ${ }^{\circ} \mathrm{I}-\mathrm{II}$ asthenia (10 pts), ${ }^{\circ} \mathrm{I}-\mathrm{II}$ neurotoxicity (5 pts), ${ }^{\circ}$ II nausea and/or vomiting ( $\left.2 \mathrm{pts}\right)$ and ${ }^{\circ} \mathrm{II}$ diarrhea (1 pt). Treatment had to be postponed due to ${ }^{\circ} \mathrm{I}$ thrombocytopenia in two pts and due to protracted ${ }^{\circ} \mathrm{II}$ asthenia in one pt. No bleeding episodes or obvious in fectious complications were observed. Adverse events developed in three pt: one allergic reactions to oxaliplatin and contrast agent, respectively as well as a chemically induced gastric ulcer in one pt. Arterial catheter dislocation occurred in three pts. In ten pts evaluable for response, we observed $3 \mathrm{PR}, 2$ MR, 4 SD. Only one pt had PD of disease under treatment.

Conclusion: TACE using a combination of Mitomycin C, Dexamethasone and Interferon- $\alpha 2 b$ mixed with Sphere ${ }^{\circledR}$ followed by Oxaliplatin, FA and 5-FU appears to be an effective and feasible treatment option in the case of liver metastasis of CRC refractory to standard systemic chemotherapy. This treatment is associated with tolerable toxicity, mainly asthenia, neurotoxicity and thrombocytopenia. These preliminary data warrant further evaluation in a phase II study. 
A high incidence of 5-FU associated angina pectoris - a prospective study with 102 patients

Wacker A. (1), Lersch C. (2), Scherpinski U. (3), Busch R. (4), Seyfarth M. (1)

(1) Deutsches Herzzentrum und I. Med. Klinik der TU Muenchen,

(2) II. Med. Klinik der TU Muenchen, (3) Neurologische Klinik der TU Muenchen, (4) Institut für Medizinische Statistik und Epidemiologie der TU Muenchen

Objectives: Angina pectoris, arrythmic sudden death and myocardial infarction, all these cardiac events have been occasionally reported during 5-Fluorouracil chemotherapy. Underlying mechanisms leading to these symptoms are unknown; damage to the myocytes or vasospasms have been discussed. Methods: 102 consecutive and unselected patients were monitored with 12 lead ECG, echocardiography and radionuclidventriculography prior to $5 \mathrm{FU}$ chemotherapy and three months later.

Results: $19 \%$ of the patients developed reversible symptoms of angina pectoris during treatment which were lasted ongoing up to 12 hours after cessation of the infusion. All 19 patients showed corresponding ECG changes. 6 out of 19 patients with severe angina pectoris had subsequent coronary angiography. None of these patients did show coronary artery disease, two patients had low ventricular function (EF $34 \%$ ). Arrhythmias were screened for with holter monitoring during 5-FU chemotherapy. Bradycardia and ventricular extrasystolies increased significantly $(\mathrm{p}<0.01)$ during treatment compared to baseline values. The Qtc time was significantly prolonged $(\mathrm{p}<$ $0.01)$.

Conclusions: The incidence of angina pectoris in patients during 5-FU treatment seems higher than previously suspected. As myocardial ischemia can have fatal outcome, awareness of these symptoms and immediate treatment is important.

\section{2}

Influence of 2nd line chemotherapy on survival of patients with advanced gastric carcinoma

Weisser A., Hofheinz R., Willer A., Sadikaj L., Hehlmann R., Hochhaus A.

Onkologisches Zentrum, III. Medizinische Universitätsklinik, Klinikum Mannheim der Universität Heidelberg

Introduction: For patients with advanced gastric carcinoma (AGC), chemotherapy has been shown to prolong survival and to improve quality of life in comparison with patients treated with best supportive care. In contrast to colorectal cancer no 2nd line chemotherapy can be recommended for patients with AGC, although several agents (eg taxanes, irinotecan) have shown activity. To assess the efficacy of 2 nd line therapy we retrospectively evaluated the records of 59 consecutive patients with AGC, who had been treated within the framework of two phase II studies for 1st line chemotherapy on a monocentric basis between 1997 and 2000 .

Patients: 59 pts with advanced or metastatic gastric cancer were treated at our institution in two phase II studies for 1st line chemotherapy of AGC. One study evaluated a bolus regimen with mitomycin C (MMC), 5-fluorouracil (5-FU) and folinic acid (FA), another study a 24-hour high dose (HD) 5-FU/FA infusion in combination with 3-weekly MMC.

Results: Seven pts were lost for evaluation. 37 of 52 pts did not receive any 2nd line treatment. Fifteen pts received various 2 nd line therapies: HD 5-FU after 1st line bolus 5-FU $(n=4)$, irinotecan $(n=4)$, taxanes $(n=2)$, BBR3438 $(n=1)$, anthracyclines $(n=3)$, and cisplatin/5-FU $(n=1)$. Calculated from the start of 1 st line treatment, pts who were not amenable to 2 nd line therapy had a median survival of 9.5 months (range 3.1-25.8), whereas pts who were treated with 2 nd line treatment had an overall survival of 14.1 months (range 2.7-33.1). Median survival from the time of progression under 1st line chemotherapy was 3.1 months (range 0.4-12.1) for pts without 2nd line therapy, and 5.1 months (range 1.8-25.2) for patients who received 2 nd line therapy. For patients with 2 nd line treatment median survival from the start of this treatment was 3.5 months (range 0.1-21.9).

Conclusion: About a third of pts with AGC were amenable to 2nd line therapy. This was in most cases dependent on an inadequate performance status at the end of 1 st line treatment. Therefore, patients who qualify for 2 nd line therapy represent a highly selected group. Even in this subgroup the survival gain is minimal. Prospectively randomized studies on 2nd line treatment versus best supportive care in AGC are required.
Unusual first presentation of Hepatocellular Carcinoma (HCC) as a frontal mass with extracranial and intracranial invasion and vertebral bone metastasis requiring multimodal therapy with neurosurgery, radiotherapy and systemic treatment.

Willenbacher W. (1), Lohmann E. (2), Oeckler R. (3), Vierling P. (4), Brugger W. (1)

(1) Hospital Villingen-Schwenningen (VS) Dep. Hematology-Oncology, (2) Hospital VS Dep. Radiotherapy, (3) Hospital VS Dep. Neurosurgery, (4) Hospital VS Dep. Pathology

Clinical presentation: A 50 year old man, presented himself in 09/01 with a fast growing frontal mass with spiculae and intra; as well as extracerebral tumor(CT: $6 \times 4 \times 7.5 \mathrm{~cm})$. Staging showed bone metastasis to the 6 th thoracic vertrebra and two ribs as well as a mass in the left liver lobe (CT: $5 \times 6 \mathrm{~cm}$ ) A broad tumor marker profile showed no pathologic parameters. Hepatitis test were negative. A diagnosis of CUP was made and a neurosurgical rescetion of the frontal mass was performed to achieve local tumor control. Pathologic findings: A trabecular, pseudoglandular tumor with eosinophilic cytoplasm was described. Mitosis and intratumoral bleeding was frequent. In GIEMSA and PAS stainings production of gall was evident. Immunohistology showed the tumor cells to be highly positive for AFP. A diagnosis of metastasized HCC was made.

Treatment: Besides neurosurgery the patient received radiotherapy to the vertebral tumor $(30 \mathrm{~Gy})$ and 8 courses of weekly epirubicin $\left(25 \mathrm{mg} / \mathrm{m}^{2}\right)$ as well as 4 weekly bisphosphonats. Since then the patient remains in stable disease (liver) with no further distant metastasis.

Conclusions: HCC should be included in the differential diagnosis of bone and brain metastasis. Although therapeutic options are palliative a multimodal approach to such pt. seems appopriate.

\section{Lymphoma - Clinical Research}

\section{4}

\section{Marked anti-tumor activity of rituximab plus thalidomide in} paients with relapsed/resistant mantle cell lymphoma

Drach J. (1), Kaufmann H. (1), Püspök A. (2), Bankier A. (3), Urbauer E. (1), Chott A. (4), Raderer M. (1)

(1) Univ. Klinik für Innere Medizin I, Klinische Abteilung für Onkologie, (2) Univ. Klinik für Innere Medizin IV, Klinische Abteilung für Gastroenterologie und Hepatologie, (3) Univ. Klinik für Radiodiagnostik, (4) Institut für Klinische Pathologie; AKH Wien, Österreich

Objectives: Mantle cell lymphoma (MCL) represents a distinct entity within the non-Hodgkin's lymphomas, which is difficult to treat by conventional treatment approaches. Since the microenvironment plays an important role for growth and survival of malignant B-cells, we hypothesized that a treatment strategy targeting both the tumor cell and the microenvironment could be active in MCL. We therefore initiated a phase 2 clinical trial to evluate the toxicity and efficacy of rituximab plus thalidomide (R+THAL) in patients (pts) with CD20 positive MCL who relapsed after or did not respond to standard CHOP (or CHOP-like) chemotherapy.

Methods: $\mathrm{R}$ was administered at $375 \mathrm{mg} / \mathrm{m}^{2}$ on days $1,8,15$, and 22 ; THAL was given orally, with a daily dose of $200 \mathrm{mg}$ starting on day 1 and a dose escalation to $400 \mathrm{mg}$ on day 15 . THAL was continued after completion of R until progression or relapse.

Results: Up to now, 10 pts are evaluable ( 6 at first relapse, 3 pts at second or greater relapse, and $1 \mathrm{pt}$ with primary CHOP-resistant MCL). Median age of pts was 69 yrs (range, 50-74 yrs), and time from initial diagnosis ranged between 6 and 53 (median, 21) months. According to the International Prognostic Index, 6 pts were at intermediate-high or high risk. In 4 pts, fever and chills (grade 1-2) associated with the administration of $\mathrm{R}$ were observed, but no unexpected side effects of $\mathrm{R}$ were encountered by the addition of THAL. Side effects of THAL included fatigue, constipation, and peripheral neuropathy. Thromboembolic events were observed in 2 pts, and one pt had severe neutropenia associated with THAL resulting in discontinuation of treatment. R+THAL induced an objective response in 9 of 10 pts (3 CR including the pt who failed to respond to CHOP, $6 \mathrm{PR}$ ), and one pt had stable disease. Tumor responses were observed both at nodular and extranodular manifestations of the disease (bone marrow, stomach, colon). Remissions were found to be durable, with CRs lasting 18+, 14+, and 10+ months, respectively; these remission durations were equal to or even longer 
than the time to progression after the last chemotherapy $(17,15$, and 3 months, respectively). Four of the 6 pts, who achieved a PR, are in continuous remission at $20,14,14$, and 6 months.

Conclusion: These results suggest that $\mathrm{R}+\mathrm{THAL}$ has marked anti-tumor activity and a favorable toxicity profile in pts with relapsed and CHOP resistant MCL. Recruitment of pts is therefore ongoing.

\section{5}

\section{Intrathecal application of Rituximab in combination with standard chemotherapy in case of relapsed mantle cell lymphoma. A case report}

Dresel I., Jakob A., Peuckert A., Messerschmidt D., Ziesemer A., Hirsch F.W.

\section{Klinikum Offenburg, Ebertplatz 12, D-77654 Offenburg}

Objectives: In malignant lymphomas, primary central nervous system(CNS) involvment is rare. Later on in disease about $10 \%$ of patients show CNS relapse. In mantle cell lymphoma only $4 \%$ show CNS involvement.However the clinical course and prognosis in patients with CNS re-lapse is poor. Most of CNS lymphomas are B-cell neoplasms,with expression of CD20 surface antigen. Rituximab,a human chimeric anti CD20 antibody has shown response in CNS lymphomas. However,only $1-1,7 \%$ of the corresponding serum concentration levels could be reached in cerebrospinal fluid(CSF), when Rituximab was given as intravenous infusion. Therefore, intrathecal application of Rituximab with higher anti-CD20 concentrations in CSF might be more effective.

Methods/Case: We report on a 68-year old man with mantle cell lymphoma, first diagnosed in 1998. Since 1998 he received various chemotherapy regimes consisting of fludarabine, mitoxantrone, dexametha-sone, bendamustin and Rituximab.7 months after the last intravenous application of Rituximab,CNS relapse occured with intracerebral and meningeal manifestation. After intrathecal therapy with MTX and irradiation of CNS he showed a complete remission. 3 months later again a relapse with menigeosis lymphomatosa was diagnosed in CFS. No further manifestations could be found.We decided to treat the patient with intrathecal Rituximab. From 8/01 until 9/01 the patient received 8 intrathecal applications of Rituximab, $25 \mathrm{mg}$ in $2,5 \mathrm{ml} 0,9 \%$ saline, MTX $15 \mathrm{mg}$, ARA-C $40 \mathrm{mg}$ and dexamethasone $4 \mathrm{mg}$.Again the patient reached a complete remission.In 12/01 inguinal and parasacral lymphomas occured without any CNS involvment. Again treatment with bendamustin ans Rituximab was started. Until 4/02 patient showed a good response of peripheral lymphoma. In 5/02 again CNS neurological symptoms with weakness, ataxie and pain occured.Again a CNS relaps was found in CSF.After intrathecal application of Rituximab,MTX, ARA-C and dexamethasone for two times, again a reduction of cell count from 5000/3 to $18 / 3$ in CSF was seen.No other systemic manifestations of lymphoma could be found.

Results: Intrathecal application of Rituximab in combination with standard chemotherapy was well tolerated. No toxicity or clinical side effects were observed. In case of relaps it is possible to repeat this therapy.

Conclusion: Intrathecal application of Rituximab is well tolerated and seems to be a therapeutic option for CD20 lymphomas with CNS involvement. Further experience is necessary.

\section{6}

\section{Topotecan for treatment of relapsed or refractory primary central nervous system lymphoma (PCNSL)}

\section{Fischer L., Korfel A., Thiel E.}

Universitätsklinikum Benjamin Franklin der FU Berlin

Despite a high remission rate to initial treatment in PCNSL relapse occurrs in more than $50 \%$ of patients. No standard salvage therapy of PCNSL could be established thus far. The topoisomerase I inhibitor topotecan with some known effectiveness in relapsed or refractory peripheral Non-Hodgkin's lymphomas is able to cross the intact blood-brain barrier. Activity of topotecan against brain metastases of small cell lung cancer and breast cancer has recently been demonstrated. We examined the effectivity of topotecan in the treatment of relapsed or refractory PCNSL. Six patients with refractory and 6 with relapsed (time to relapse 6-42 months, median 9.5 months) nonAIDS-related PCNSL were treated with $1.5 \mathrm{mg} / \mathrm{m}^{2} / \mathrm{d}$ topotecan i.v. d1-5 3weekly. Pretreatment comprised high-dose methotrexate (HD-MTX) alone or in combination with BCNU, procarbacine, and dexamethasone (BMPD) in 5 patients and HD-MTX followed by high-dose cytarabine (HD-AraC) in 5 patients. Moreover, 1 patient received HD-MTX followed by procarbacine, $\mathrm{CCNU}$, and vincristine (PCV) followed by a modified $\mathrm{CHOP}$ regi- men. Three patients were pretreated by whole brain irradiation therapy (WBRT). The disease was restricted to the CNS in all but one patient who showed systemic manifestations in testis and adrenal gland.

An average of 2.3 (1-5) cycles topotecan were administered. 4/12 patients responded for an overall response rate of $33 \%$. One complete response (CR) was observed after 3 cycles. Partial response (PR) was seen in 3 patient after 1, 4 and 4 cycles respectively and stable disease (SD) was seen in 2 patients after 2 and 3 cycles. 5 patients progressed after 1 to 3 cycles. One patient is not evaluable for response yet. Neurologic symptoms improved in 3 responding patients and worsened despite response in 1 patient due to progressive leukoencephalopathy.

Six patients are alive at up to 24 months after starting topotecan. Four patients died of PD at 3-7 months, 1 of late neurotoxicity at 2 months, and 1 of an unknown cause. Toxicity was mainly hematologic. 2 patients developed neutropenic fever and 1 severe hypokalemia with paralytic ileus. In summary, monotherapy with topotecan was effective in these extensively pretreated patients, producing tolerable toxicity. Further evaluation of the role of topotecan in treatment of refractory or relapsed PCNSL is warranted.

\section{8}

\section{Combined systemic and intraventricular chemotherapy in primary CNS lymphoma: High efficiency and low rate of neurotoxicity}

Glasmacher A. (1), Pels H. (2), Kleinschmidt R. (3), Helmstaedter C. (4), Deckert M. (5), Van Roost D. (6), Zellner A. (7), Kroschinsky F. (8), Schackert G. (9), Neben K. (10), Krämer A. (10), Ho A. (10), Schulz H. (11), Engert A. (11), Diehl V. (11), Bode U. (12), Klockgether T. (2), Schlegel U. (2), Schmidt-Wolf I.G.H. (1)

(1) Med. Klinik u. Poliklinik I, (2) Klinik für Neurologie, Univ. Bonn, (3) Markus-Krankenhaus, Frankfurt, (4) Abteilung für Neuropsychologie, Univ. Bonn, (5) Institut für Neuropathologie, Univ. Köln, (6) Abteilung für Neurochirurgie, Univ. Bonn, (7) Klinik für Neurologie, Univ. Regensburg (8) Abteilung für Innere Medizin, (9) Klinik für Neurochirurgie, Univ. Dresden, (10) Med. Poliklinik, Univ. Heidelberg, (11) Med. Klinik I, Univ. Köln, (12) Pädiatrische Hämatologie-Onkologie, Univ. Bonn

Objectives: Since the combination of radiation and chemotherapy causes severe neurotoxicity in a large proportion of patients with primary central nervous system lymphoma (PCNSL), studies with chemotherapy alone are warranted. We evaluated the response rate, response duration and toxicity of combination chemotherapy in patients with previously untreated PCNSL. Methods: 65 consecutive patients with histologically proven PCNSL (mean age 59 years, range 27-75) received a total of 6 courses of combination chemotherapy with high-dose methotrexate (MTX) $\left(5 \mathrm{~g} / \mathrm{m}^{2}\right.$, course $\left.1,2,4,5\right)$ and cytarabine (ara-C) $\left(3 \mathrm{~g} / \mathrm{m}^{2} / \mathrm{d}, \mathrm{d} 1-2\right.$, courses 3,6$)$ combined with dexamethasone (courses 3-6), vinca alkaloids and ifosfamide alternating with cyclophosphamide. In addition, MTX, prednisolone and ara-C were applied via an Ommaya or Rickham reservoir in each course.

Results: 62/65 patients were evaluable for response, from which $37(60 \%)$ achieved a complete response (CR) and $6(10 \%)$ a partial response (PR). In $3 / 65$ patients response could not be determined because of complete tumor resection $(n=2)$ or because of interruption of treatment by the patient $(n=1)$. $13 / 65(20 \%)$ had a progressive disease, $6 / 65(9 \%)$ patients died from treatment-related complications (mainly neutropenic infections). The median observation time is 12 months (range 0-75). In 30 patients younger than 60 years a CR rate of $22 / 29(76 \%)$ and a PR rate of $3 / 29(10 \%)$ was obtained. $76 \%$ of these patients are free from treatment failure at a medium follow-up of 18 months, $7 / 29(24 \%)$ had a treatment failure (including induction failure and relapse) and 6/29 (21\%) have died. The Kaplan Meier estimate for survival in patients of all ages is 50 months (95\%-confidence interval 29-70 months). Ommaya reservoir infections occurred in 12/64 (19\%) and acute transient MTX related encephalopathy in 2 (subacute in 1) patients. Permanent cognitive dysfunction due to treatment was seen in only one patient after 12 courses of chemotherapy ( 6 at relapse).

Conclusion: Combined systemic and intraventricular chemotherapy is highly efficient in PCNSL. The results reported here for patients younger than 60 years (estimated 5-year survival $72+/-11 \%$ ) could be superior to previously reported therapy regimes. The survival rates for patients above 60 years reported here are comparable to those reported for combined radiochemotherapy. However, in contrast to radiochemotherapy permanent treatment-associated neurotoxicity is infrequent. 


\section{Erythropoietin decreases transfusion requirements in patients with relapsed lymphoma treated with the Cologne high-dose sequential chemotherapy regimen}

Glossmann J.-P. (1), Engert A. (1), Wassmer G. (2), Flechtner H. (3), Ko Y. (4), Rudolph C. (5), Illiger H.J. (6), Dörken B. (7), Wiedenmann S. (1), Diehl V. (1), Josting A. (1)

(1) First Dept. of Int. Med., University Hospital Cologne, (2) IMSIE, University Hospital Cologne, (3) Klinik und Poliklinik für Psychiatrie und Psychotherapie des Kindes- und Jugendalters, University Hospital Cologne, (4) Poliklinik Bonn, (5) II. Med. Klinik, CTK Cottbus, (6) Städt. Kliniken Oldenburg, (7) Virchow-Hospital, Humboldt University Berlin

Objectives: Patients treated with aggressive chemotherapy and stem cell support usually develop severe anemia requiring multiple red blood cell (RBC) transfusions. Erythropoietin (EPO) has been shown to reduce the number of transfusions in chemotherapy-induced anemia. This study evaluated the effects of EPO on RBC transfusions, hemoglobin $(\mathrm{Hb})$ levels and quality of life in patients with relapsed lymphoma treated with an aggressive high-dose sequential chemotherapy (HDSCT) regimen.

Material and Methods: Fifty-seven patients with early or late relapsed Hodgkin's disease (HD) or first relapse of aggressive Non-Hodgkin's lymphoma (NHL) were treated in a randomized multicenter study involving 23 centers. Patients were stratified according to gender, age $(\leq 40$ vs. $>40$ years $[\mathrm{y}]$ ) and $\mathrm{Hb}$ level ( $\leq 10 \mathrm{vs.}>10 \mathrm{~g} / \mathrm{dL}$ ) before random assignment to therapy . Pts in both study arms received two cycles DHAP; patients with PR or CR then received cyclophosphamide $4 \mathrm{~g} / \mathrm{m}^{2}$, followed by PBSC harvest; methotrexate $8 \mathrm{~g} / \mathrm{m}^{2}$ plus vincristine $1,4 \mathrm{mg} / \mathrm{m}^{2}$; and etoposide $2 \mathrm{~g} / \mathrm{m}^{2}$. The final myeloblative course was BEAM followed by autologous stem cell support. Pts in the experimental arm additionally received 10,000 IE EPO (NeoRecormon ${ }^{\circledR} 3 \mathrm{x} /$ week) from the start of therapy until the end of VP-16. Primary end-point of the study was number of RBC units needed during HDSCT. In addition, $\mathrm{Hb}$ levels and quality of life were measured.

Results: Forty-four of the 57 patients received therapy until end of the etoposide cycle and qualified for the per-protocol analysis. The median age of the 23 male and 21 female patients was $35,5 y$ (19-65y). 32 patients (73\%) had HD and 12 patients (27\%) NHL. 13 patients had stage I/II disease and 31 patients had stage III/IV at relapse. There was no difference in terms of age, gender and $\mathrm{Hb}$ level between the two groups. The mean number of RBC units given in the EPO arm was 4.5 compared to 8.3 in the control arm $(\mathrm{p}=0.0134)$. Five patients $(25 \%)$ in the $\mathrm{EPO}$ arm and 2 patients (8\%) in the control arm did not require transfusions. The mean $\mathrm{Hb}$ levels during therapy were $10.4 \mathrm{~g} / \mathrm{dL}$ in the EPO arm and $9.7 \mathrm{~g} / \mathrm{dL}$ in the control $(\mathrm{p}=0.018)$. Quality of life (QLQC30) and fatigue (MFI) assessment, however, showed no significant difference.

Conclusions: This prospectively randomized study shows that EPO is effective in decreasing RBC transfusion requirements in patients with relapsed lymphoma undergoing aggressive chemotherapy and stem cell support.

\section{0}

Radioimmunotherapy with 131I-anti-CD20 rituximab in B-cell lymphoma: results of the Goettingen RAIT Study Group

Griesinger F. (1), Jung W. (1), Glass B. (1), Trümper L. (1), Meller J. (1), Behr T. (2),Wörmann B. (3), Kaufmann, C. (4), Binder C. (1), Brittinger G. (1), Becker W. (1)

(1) UNI-Klinik Göttingen, (2) Uni-Klinikum Marburg, (3) Klinikum Braunschweig, (4) Wyk a.F.

Objectives: Radioimmunotherapy has been shown to be effective in CD20 + B-cell lymphomas. Both non-myeloablative as well as myeloablative regimens have been employed for low grade and high grade lymphomas with impressive response rates and remission durations. Recently, Press et al (2000) published a non randomised study using myeloablative 131I-antiCD20 RAIT (murine antibody) in combination with high dose chemotherapy for relapsed CD20+ B-cell NHL with favourable outcome when compared to a historic control treated with high-dose chemotherapy and total body irradiation. In our institution, 131I-RAIT using the anti-CD20 antibody (rituximab, 19 pts.) was performed in 19 pts with poor prognosis relapsed CD20 B-NHL in order to investigate feasibility and efficacy of this approach.

Methods: 22 pts with CD20+ B-NHL (aggressive NHL 9, transformed NHL 4 , follicular NHL (FL) 6, mantle cell lymphoma (MCL) 3) were enrolled in the study. 20/22 pts received myeloablative RAIT (19/20 rituximab, 1/20 murine anti CD22 MoAb) , $2 / 22$ (1 transformed, 1 aggressive NHL) nonmyeloablative RAIT with anti-CD22 MoAb. In 3 pts. myeloablative RAIT was given just after myeloablative chemotherapy in a ,tandem" setting with a (median interval 4 months). 10/22 pts had refractory B-NHL, 10/22 had chemosensitive relapses, and in $2 / 22$ pts, chemosensitivity was not assessed prior to RAIT. 12/22 pts only received conventional chemotherapy (CTx) prior to RAIT, 10/22 had high-dose (HD) CTx (5 with TBI) prior to RAIT. All pts were regularly followed up until death, for surviving pts., the last visit was on March 8th 2002.

Results: 5/13 aggressive B-NHL (2/9 aggressive, 3/4 transformed) had a $\mathrm{CR}, 2 / 13$ a PR, and 6/13 SD or PD. 2/9 aggressive B-NHL are in continuous complete remission $(\mathrm{CCR})$ for $45+$ and $48+$ months, $2 / 4$ transformed $\mathrm{B}$ NHL are in CR for $2+$ and $41+$ months. Response rate of FL was $6 / 6$ with 4 CR and 2 PR. 4/6 are in CCR for $16+, 22+, 31+$ and 54+ months. 3/3 MCL (1 with prior conventional CTx, 2 post HD-CTx) had CR, 2/3 pts relapsed and died 11 and 9 months after RAIT, 1 pt is in CCR for $28+$ months. TRM (treatment related mortality) was $1 / 22$ pts (septic death after non-myeloablative RAIT)

Conclusion: Myeloablative RAIT is a feasible and effective treatment modality for relapsed poor prognosis CD20+ B-NHL offering long term relapse free survival for a significant number of patients. These results are the basis for a multicenter prospective study in poor prognosis CD20+-B-NHL using a tandem HD-CTx and RAIT a pproach.

\section{1}

TOPiCS-ML: Therapy optimization protocols in chemo- and radiotherapy for patients with malignant lymphoma

\section{Hoffmann W. (1), Heidelbach S. (1), Paulus U. (2), Löffler M. (3)}

(1) Bremen Institute for Prevention Research, Social Medicine and Epidemiology (BIPSE), Linzer Str. 8-10, D-28359 Bremen, Germany, (2) Universität Köln, Klinik I für Innere Medizin, Arbeitsgruppe Biometrie, Herderstr. 52-54, 50931 Köln; (3) Universität Leipzig, Liebigstr. 27, 04103 Leipzig

Objectives: Do patients with Hodgkin's disease (HD) and high grade malignant Non-Hodgkin's lymphoma (NHL-h) who participate in therapy optimising protocols (TOP) have, on average, better outcomes than non-partipicants?

Material and Methods: TOPiCS-ML is based on a population-based retrospective incidence study with active case ascertainment from multiple clinical data sources covering six counties with a total population of 1.1 mio between 1984-98 (Northern Germany Leukemia and Lymphoma Study (NLL)). Incident cases of HD (first diagnosis between 1988-98) will be compared to participants of the German Hodgkin's Study Group (DHSG) TOP HD 4 to HD 9 (recruitment 1988-1998, PI Prof. Diehl, Cologne), incident cases of NHL-h (1994-98) with the German High Grade NonHodgkin's Lymphoma Study Group (GHNHLSG) study B (both arms; recruitment 1994-1998, PI Prof. Pfreundschuh, Homburg, Prof. Löffler, Leipzig). Eligibility (disease stage, specific histology) and presence of exclusion criteria will be assessed for each patient. Risk factors known or suspected to influence therapy outcome will be abstracted from medical records and other primary data sources.

Results: In a pilot study, TOPiCS-ML methods and instruments were tested in two departments of hematology/oncology in the City of Hamburg. Our standardized abstraction form was completed for 178 patients. Of these, only six (5 NHL-h, 1 HD) had participated in TOP. For both lymphoma entities combined, 38 patients positively met one or more exclusion criteria for their respective TOP. Another 14 NHL-h and 6 HD patients had diagnoses of concomitant disease. The pilot study established that in two major hematology/oncology departments

all patients originally ascertained in the incidence study could be retrieved data availability and validity was sufficient to determine eligibility/exclusion criteria for each patient

$31 \%$ to $43 \%$ of NHL-h patients and $4 \%$ to $11 \%$ of HD patients met $\geq$ exclusion criteria for their respective TOP. Exact values will depend on decisions on patients with concomitant disease.

On-site abstraction is time consuming and demanding. The abstractor must have MD level medical knowledge and pertinent experience with documentation routines in clinical hematology/oncology.

Conclusions: The main study will include a total of 599 patients (294 NHLh, $305 \mathrm{HD}$ ). Results of the pilot study indicate that TOPiCS-ML will be able to detect systematic differences in mortality between $30 \%$ to $60 \%$. 
Impact of high-dose chemotherapy and autologous or allogeneic hematopoetic transplantation in the treatment of peripheral t-cell lymphomas (PTCL)

Kahl C., Leithäuser M., Wilhelm S., Wolff D., Steiner B., Hartung G., Casper J., Freund M.

Division of Hematology and Oncology, Department of Internal Medicine, University of Rostock, Germany

Peripheral T-cell lymphomas (PTCL) are a rare entity of non-Hodgkin'slymphomas (NHL). Despite the poor outcome after conventional chemotherapy the impact of high dose chemotherapy (HDCT) and autologous or allogeneic stem cell transplantation is not well defined in these patients (pts.). In a retrospective study we evaluated the outcome of 15 patients (9 male, 6 female) with PTCL after HDCT with autologous (10 pts.) and allogeneic (5 pts.) stem cell transplantation between 1996 and 2001 at our department. At the time of transplantation 3 pts. were in 2 nd remission, 7 pts. were in PR and 3 pts. had refractory disease. Two pts. were treated with sequential HDCT (m-CHOEP). The conditioning regimes were heterogeneous. After HDCT 10 pts. (67\%, autologous 7, allogeneic 3) achieved $\mathrm{CR}$, two pts. (13\%, autologous 2 , allogeneic 0$)$ had refractory disease and three pts. (20\%, autologous 1 , allogeneic 2 ) died because of toxic side effects before evaluation of response was performed. The median overall survival (OS) was 10 months. The one year probability of survival for the autologous and allogeneic group were $58 \%$ and $40 \%$ respectively. At time of evaluation 6 pts. are alive, 9 pts. have died ( 4 severe infection, 1 late toxicity, 2 disease progression, 2 relapse).

Despite the small number of pts. in this study, HDCT with autologous or allogeneic hematopoetic transplantation seems to be an effective treatmen option that can achieve CR for pts. with PTCL. Because of the poor outcome of this pts. after conventional chemotherapy, HDCT seems to be a rationale option. Whether it improves survival in these patients should be further investigated.

CLAOP (liposomal doxorubicin modified chop) in patients with high grade NHL: A phase II study with 21 day intervals and a phase I study with 14 day intervals

Keilholz U., Szelenyi H., Schmittel A., Siehl J., Knauf W., Thiel E.

\section{UKBF, FU-Berlin}

CHOP is the most efficient and least toxic treatment for the first line treatment of high-grade Non-Hodgkin's-lymphoma (NHL). However, because of the cardiotoxicity of doxorubicin the use of $\mathrm{CHOP}$ is restricted in patients being at risk for cardiotoxicity (e.g. age, pre-existing cardiac dysfunction). Caelyx is a liposomal preparation of doxorubicin with no apparent cardiotoxicity at doses of up to $2000 \mathrm{mg} / \mathrm{m}^{2}$ and a half-life of 43 hours.

We initiated a phase IIa study in patients with high grade NHL and risk factors for cardiac toxicity with the CLAOP regimen given every 3 weeks $(\mathrm{Cy}-$ clophosphamide $750 \mathrm{mg} / \mathrm{m}^{2}$ day 1 , Caelyx $20 \mathrm{mg} / \mathrm{m}^{2}$ day 1 Vincristine $1 \mathrm{mg}$ i.v. day 1, Prednison $100 \mathrm{mg}$ p.o. day 1-5) and a phase I study with CLAOP14 given every two weeks with G-CSF support and escalating doses of Caelyx.

Thirteen patients with a median age of 79 years (range 40-92) have entered the phase II study. 67 cycles (median 5, range 2 to 6 ) have been given. Main side effects were haematologic: leucopenia grade III/IV in 2 patients, thrombopenia grade III/IV 1 patient. No organ toxicity greater grade II and especially no clinical cardiotoxicity was noticed. 6 patients have been entered into the phase I study. $20 \mathrm{mg} / \mathrm{m}^{2}$ of Caelyx was not associated with significant hematologic toxicity, however 5 febrile infections developed in 3 of 4 patients at this dose level after 4 to 6 cycles. $25 \mathrm{mg} / \mathrm{m}^{2}$ of Caelyx was associated with hematoxicity and 4 febrile infections in 2 of 2 patients. Currently a third cohort is accrued with $25 \mathrm{mg} / \mathrm{m}^{2}$ of Caelyx during the first three cycles and $20 \mathrm{mg} / \mathrm{m}^{2}$ for cycles 4 to 6 . All patients from both studies are evaluable for efficacy. Remission rates were 10 CR (53\%), 4 PR (21\%), and 5 PD $(26 \%)$.

Our preliminary data prove CLAOP to be an effective treatment for high grade NHL, which may be an ideal alternative for elderly patients or patients at risk for cardiac toxicity. CLAOP 21 can safely be given with 20 $\mathrm{mg} / \mathrm{m}^{2}$ of Caelyx without growth factor support. The dose recommendation for Caelyx in the intensified CLAOP14 regimen will either be $20 \mathrm{mg} / \mathrm{m}^{2}$ or $25 \mathrm{mg} / \mathrm{m}^{2}$ for cycles 1 to 3 and $20 \mathrm{mg} / \mathrm{m}^{2}$ for cycles 4 to 6 .
Fludarabin and bendamustin for relapsed and refractory indolent lymphoma - update of a multicenter phase II trial by the East German study group for hematology and oncology (OSHO)

Koenigsmann M. (1), Knauf W.U. (2), Herold M. (3), Kahl C. (4), Müller G. (5), Uhle R. (5), Lackner V. (6), Assmann M. (7), Jentsch-Ullrich K. (1), Franke A. (1)

(1) Universitätsklinik für Hämatologie und Onkologie, Magdeburg, (2) Berlin (FU), (3) Klinik Erfurt, (4) Universitätsklinik für Hämatologie und Onkologie, Rostock, (5) Praxis für Hämatologie und Onkologie Magdeburg, (6) Praxis für Hämatologie und Onkologie Rostock (7) Klinik Riesa

Objectives: Bendamustin is an effective cytotoxic drug and is likely to act as a bifunctional alkylating substance with additional activity as purinanalogon. Clinical activity in lymphoma pts has been previously shown in single agent as well as in combination trials with steroids, anthracyclins and vinca alkaloids. Bendamustin was combined with Fludarabin for patients (pts) with relapsed or refractory indolent lymphoma. This phase II-study was preceeded by a dose-finding study in which the maximum tolerable dose of Bendamustin together with Fludarabin was defined.

Material and Methods: Bendamustin was now given at $30 \mathrm{mg} / \mathrm{m}^{2} / \mathrm{d}$; Fludarabin at $30 \mathrm{mg} / \mathrm{m}^{2} / \mathrm{d}$, both drugs as iv infusion over $30 \mathrm{~min}$ on days 1 to 3 . Six cycles were to be given every 4 weeks.

Results: So far, 16 pts have entered the protocol at the established dose level. Seven pts had mantle cell lymphoma, 8 pts follicular lymphoma grade I and II, 1 pt lymphoplasmocytic lymphoma. Median age was 63 years (range 38-73). Stage of disease at relapse was III in 1 pt and IV in 15 pts. Before study entry, all pts had prior chemotherapy with or without additional radio- or immunotherapy. Six pts completed the chemotherapy protocoll, while therapy was halted in 8 pts after cycles $1(n=3), 3(n=2), 4(n=2)$ and 5 $(n=1)$. Reasons for discontinuation were disease progression $(n=3)$, cytopenia $(n=2)$ and newly manifested hemolytic anemia $(n=2)$. Hemolysis in these pts occurred after 1 and after 5 cycles of therapy, respectively, and was in one pt associated with disease-progression and in the second pt most likely therapy-induced. 2 pts are still under therapy. CR was reached in 6/14 evaluable pts, namely in 2 pts after 2 cycles and in 4 pts after 6 cycles. PR as best response was reached in 4 pts, yielding an overal response rate of 10/14 evaluable pts on an intent-to-treat basis.

Conclusions: The leading toxicity of Fludarabin in combination with Bendamustin is hematotoxicity. Despite unfavorable prognostic features (histologic subtype, stage of disease, pretreatment) response rates appear to be satisfactory with this regimen. The protocol is especially appropriate for elderly pts. Efficacy and toxicity are being further studied.

\section{5}

Chemotherapy alone versus chemotherapy with adjuvant whole-brain irradiation (WBI) for primary CNS Iymphoma (PCNSL) - first experience with a multicenter randomized trial

Korfel A. (1), Nowrousian M.R. (2), Hossfeld D.K. (3), Weber W. (4), Brücher J. (5), Berdel W.E. (6), Birkmann J. (7), Peschel Ch. (8), Pasold R. (9), Fischer L. (1), Sternemann U. (1), Thiel E. (1)

(1) Klinikum Benjamin Franklin, Freie Universität Berlin; (2) Universitätsklinikum Essen; (3) Universitätskrankenhaus Eppendorf; (4) Brüderkrankenhaus Trier; (5) Klinikum Aschaffenburg; (6) Westfälische WilhelmsUniversität Münster; (7) Klinikum Nürnberg Nord; (8) Klinik rechts der Isar, Technische Universität München; (9) Klinikum Ernst von Bergmann, Potsdam

The purpose of this trial was to assess the role of adjuvant WBI in patients with PCNSL after high-dose methotrexate (MTX)-based primary chemotherapy (CHT) +/- salvage CHT.

56 patients from 28 institutions were treated with up to 3 courses of BCNU $80 \mathrm{mg} / \mathrm{m}^{2}$ iv d1, MTX $1.5 \mathrm{~g} / \mathrm{m}^{2} / 24 \mathrm{~h}$ iv with leucovorin rescue after $36 \mathrm{~h} \mathrm{~d} 2$, procarbacine $100 \mathrm{mg} / \mathrm{m}^{2}$ po d1-8 and dexamethasone $3 \times 8 \mathrm{mg}$ po in course 1 only (BMPD). Patients without CR received idarubicine $12 \mathrm{mg} / \mathrm{m}^{2}$ iv d1-2 and ifosfamide $3 \mathrm{~g} / \mathrm{m}^{2} / 24 \mathrm{~h}$ iv d1 (Ida/Ifo) or cytarabine $2 \times 3 \mathrm{~g} / \mathrm{m}^{2} / 3 \mathrm{~h}$ iv d1-3. Complete responders to CHT were randomly assigned to WBI with 45 Gy/1.8 Gy or one additional CHT course.

CR was achieved in 30/56 patients (54\%) after BMPD. In 15/56 patients salvage CHT was applied (10 Ida/Ifo, $5 \mathrm{AraC}$ ) producing CR in $4.11 / 56 \mathrm{pa}-$ tients did not receive salvage CHT due to death or dropping out. 9/15 patients received rescue WBI. One patient was treated with CHT intrathecally, one patient dropped out. In summary, 34 patients achieved CR to CHT. Due to drop out for toxicity ( 7 patients), refusal ( 2 patients), decision of the 
treating physician ( 1 patient), and death (1 patient) 23 patients were randomized (12 CHT, $11 \mathrm{WBI).} 4$ patients randomized to WBI refused the treatment. Among those not randomized, 3 patients received adjuvant WBI out of the protocol. After a median follow-up of 26 months the median overall survival (OAS) of all patients was 20.8 months (95\% CI 13.2-28,4), the median time to progression (TTP) was 9.4 months (95\% CI 3.2-15.5). There was a trend towards longer OAS and TTP in patients treated at institutions with $>4$ PCNSL patients as compared to patients treated at institutions with less PCNSL patients (33.6 vs. 12.2 months; $\mathrm{p}=0.13$, and 12.5 vs. 6.5 months; $\mathrm{p}=0.077$ ). The OAS and TTP of the 10 patients with adjuvant WBI were compared to the 23 patients without adjuvant WBI. There was no significant difference between these both patients groups $(\mathrm{p}=0.50$ resp. $\mathrm{p}=0.85)$. Late neurotoxicity occurred in $8 / 56(14 \%)$ of patients, 6 of whom were never treated with WBI.

This trial was not feasible due to the high refusal rate of patients randomized for WBI and the high drop out rate. Thus, results concerning efficacy of adjuvant WBI are of limited evidence for obvious differences between the intent to treat and the per protocol population. No difference of OAS and TTP was found thus far between patients with vs. without adjuvant WBI Updated results will be presented.

Fludarabine / mitoxantrone versus high-dose chemotherapy, each combined with CD20-antibody rituximab, as first-line treatment of advanced follicular lymphoma

Meckenstock G. (1), Martin S. (1), Kobbe G. (1), Möller P. (2), Bentz M. (2), Rhagavachar A. (3), Aul C. (4), Wehmeier A. (5), Braumann D. (6), Plauth M. (7), Losem C. (8), Nusch A. (9), Kronenwett R. (1), Döhner H. (2), Haas R. (1)

(1) Universitätsklinikum Düsseldorf, (2) Universitätsklinikum Ulm, (3) Klinikum Wuppertal, (4) St. Johannes-Hospital Duisburg, (5) SanaKlinikum Remscheid, (6) Allg. Krankenhaus Hamburg-Altona, (7) Städt. Klinikum Dessau, (8) Onkolog. Praxis Neuss, (9) Onkolog. Praxis Velbert

In this prospective randomized multicenter study, patients aged $<60$ years with stage III/IV follicular lymphoma were treated either with 6 courses of fludarabine $\left(25 \mathrm{mg} / \mathrm{m}^{2} \mathrm{~d} 1-3\right) /$ mitoxantrone $\left(10 \mathrm{mg} / \mathrm{m}^{2} \mathrm{~d} 1\right)$ or with 3 courses of conventional CHOP followed by two courses of HAM (AraC $2 \times 2 \mathrm{~g} / \mathrm{m}^{2}$ $\mathrm{d} 1+2$, mitoxantrone $10 \mathrm{mg} / \mathrm{m}^{2} \mathrm{~d} 2+3$ ) preceeding high-dose therapy with BEAM (BCNU $300 \mathrm{mg} / \mathrm{m}^{2} \mathrm{~d}-7$, etoposide $300 \mathrm{mg} / \mathrm{m}^{2} \mathrm{~d}-7$ to $\mathrm{d}-4$, AraC $400 \mathrm{mg} / \mathrm{m}^{2} \mathrm{~d}-7$ to $\mathrm{d}-4$, melphalan $140 \mathrm{mg} / \mathrm{m}^{2} \mathrm{~d}-3$ ) and autologous peripheral blood stem cell transplantation (APSCT). In both arms, CD20-antibody rituximab was added on day 0 except for the first course. For this interim analysis, 37 of 40 patients were evaluable. In the FM/R arm $(n=20)$, the rate of complete remission (CR) was $39 \%$ after 3 courses, $60 \%$ after 5 courses, and $85 \%$ after 6 courses of chemo-/immunotherapy. In the highdose arm $(n=17), C R$ rate was $33 \%$ after CHOP/R, $50 \%$ after HAM/R, and $75 \%$ after final BEAM/R chemotherapy and APSCT. Molecular monitoring was performed using $\mathrm{t}(14 ; 18)$ nested PCR. In the FM/R arm, molecular remissions (MR) of 6 patients initially PCR positive in bone marrow and peripheral blood were observed in $14 \%$ after 3 courses, $67 \%$ after 5 courses, and $75 \%$ after 6 courses. MR rates of 4 PCR positive patients in the highdose arm were $75 \%$ after CHOP/R and $100 \%$ after HAM/R courses. At a median observation time of 12 months, event free and overall survival were not different comparing both arms. Peripheral stem cell mobilization was successful in $47 \%$ of $\mathrm{FM} / \mathrm{R}$ treated patients as compared to $100 \%$ after $\mathrm{CHOP} / \mathrm{R}$ and HAM/R treatment. Stem cell harvest was better in patients treated in the high-dose arm as compared to the FM/R arm (means: 12.8 vs $5.5 \times 10 \mathrm{E} 6 \mathrm{CD} 34$-positive cells $/ \mathrm{kg}$ bw). Regarding toxicity, both treatment options were well tolerated, with $2 \%$ documented severe infections (CTC grade III/IV) in the FM/R arm and $6 \%$ in the high-dose arm. For patients treated with FM/R hospitalization was unnecessary. In conclusion, FM/R is an effective regimen potentially achieving molecular remission, with low toxicity.

\section{7}

\section{Waldenström macroglobulinemia associated with multilobated} nuclei

Metzgeroth G., Sick Ch., Maywald O., Schatz M., Hehlmann R., Hastka J.

III. Medizinische Universitätsklinik, Fakultät für Klinische Medizin Mannheim, Universität Heidelberg

A number of multiple myeloma cases with excessively convoluted or lobulated nuclei have been published. These cases were associated with aggressive clinical course, thus the extensive nuclear lobulation is regarded to be a sign of poor prognosis. Up to now it is not clear, whether the conspicuous nuclear structures are really multiple nuclei, or fragments of one nucleus only.

We report a case of a 53-year-old man with Waldenström macroglobulinemia, who presented with impaired general performance status, suffering from fatigue, night sweats, fever and weight loss. The laboratory finding revealed low hemoglobin of $87 \mathrm{~g} / \mathrm{l}$. The lactic dehydrogenase was within the normal range, beta-2-microglobuline was increased to $3.1 \mathrm{mg} / \mathrm{l}$. Immunofixation detected monoclonal IgM-kappa paraprotein in serum and kappa-light chains in urine. Total protein in serum was $113 \mathrm{~g} / \mathrm{l}$, the $\mathrm{IgM}$ part beared $66.1 \mathrm{~g} / \mathrm{l}$. The urine protein excretion was $0,8 \mathrm{~g} / \mathrm{day}$. Bone marrow analysis showed a nearly entire infiltration by atypical cells, about $20 \%$ of them were bi- or multinucleated or revealed extensively lobulated nuclei. Immunostaining verified IgM-kappa inside the pathological population and an expression of the antigens CD19 and CD38. FISH analysis with a chromosome 8 painting probe on interphase nuclei revealed only two signals in each cell - even in those with multiple nuclei. Our findings suggest that the multilobated nuclear structures originate from one single nucleus. It is, however, not clear, whether the multilobulation is a result of atypical mitosis or of fragmentation when preparing the smears. In contrast to the multiple myeloma cases published, our patient showed good response to $\mathrm{COP}$ chemotherapy. 5 years after diagnosis the patient is still alive and in good health.

\section{8}

Dexamethasone, high-dose cytarabine, and cisplatin (DHAP) in combination with rituximab as salvage treatment for patients with refractory or relapsed aggressive non-Hodgkin's lymphoma: preliminary results of a multicenter phase II trial

Mey U. (1), Flieger D. (2), Birkmann J. (3), Ho A.D. (4), Huhn D. (5), Kaiser U. (6), Ko Y. (7), Krämer A. (4), Neubauer A. (6), Wilhelm M. (8), Hahn C. (1), Haarmann G. (1), Glasmacher A. (1), Schmidt-Wolf I.G.H. (1)

(1) Medizinische Klinik und Poliklinik I Uni-Bonn, (2) Klinikum Aschaffenburg, (3) Klinikum Nürnberg, (4) Medizinische Klinik und Poliklinik V Uni-Heidelberg, (5) Giesebrechtstraße, Berlin, (6) Klinik für Hämatologie, Onkologie und Immunologie Uni-Marburg, (7) Medizinische Poliklinik

Uni-Bonn, (8) Medizinische Poliklinik Uni-Würzburg

Objectives: Patients with relapsed or resistant aggressive lymphoma have a poor prognosis. With commonly used salvage chemotherapy regimens like DHAP, response and overall survival rates are unsatisfactory. This is particularly true for patients, who are not eligible for high-dose chemotherapy Several studies suggest both an improvement in response and overall survival rate in patients with aggressive lymphoma with a combination of chimeric anti-CD20 antibody, rituximab and the $\mathrm{CHOP}$ regimen. Therefore, we designed a multicenter phase II trial to evaluate the safety and efficacy of the combination of rituximab with the DHAP regimen in patients who relapsed after or were resistant to a CHOP-like regimen.

Material and Methods: Twenty-eight patients with relapsed or resistant aggressive B-cell NHL were included in this ongoing trial so far. The median age was 63 years. Of 13 patients in first relapse 12 had recurrence during the first 12 months after treatment. Five patients had second or subsequent relapse, 6 had primary refractory disease. Treatment consisted of rituximab infusions $\left(375 \mathrm{mg} / \mathrm{m}^{2}\right.$ per dose) on day 1 followed by dexamethasone $40 \mathrm{mg}$ d3-6 (d 3-5 in first cycle), cytarabine $2 \times 2000 \mathrm{mg} / \mathrm{m}^{2} \mathrm{~d} 4\left(2 \times 1000 \mathrm{mg} / \mathrm{m}^{2}\right.$ in first cycle), $2 \times 1000 \mathrm{mg} / \mathrm{m}^{2}$ for patients $>60$ years of age $\left(2 \times 500 \mathrm{mg} / \mathrm{m}^{2}\right.$ in first cycle), and cisplatin $25 \mathrm{mg} / \mathrm{m}^{2} \mathrm{~d} 3-6$ (d3-5 in first cycle) for a maximum of 4 cycles. When hematological toxicity did not allow continuation of chemotherapy on day 22, rituximab was given as single agent on day 22 and the next cycle was postponed until day 29 .

Results: The overall response rate in evaluable patients was $46.6 \%$ (7 of 15 patients). Two patients experienced a complete response (13.3\%), 5 patients had partial response $(33.3 \%)$. Six patients showed progressive disease $(40 \%)$. Grade $3 / 4$ nausea and vomiting were the only severe toxicities attributed to rituximab in one patient. Main toxicities were neutropenia and thrombocytopenia leading to dose reductions in 12 of 28 patients, primarily after the first cycle. Grade 3/ 4 nephrotoxicity did occur in only one patient. Conclusions: Preliminary analysis suggests that the combination of rituximab with the DHAP regimen in the treatment of relapsed or refractory aggressive lymphoma is feasible and effective. However, due to the compromised bone marrow reserve in this intensively pretreated patient population, dose reductions were mandatory during the first cycle in order to avoid severe hematological toxicities. 
Primary CNS lymphoma treated with HD-methotrexate, HD-busulfan/thiotepa and autologous stem cell transplantation: preliminary results of the OSHO-53 study

Montemurro M., Kiefer T., Schwenke M., Stockschläder M., Dölken G.

Universitätsklinikum Greifswald, Innere Medizin C - Hämato-Onkologie, Sauerbruchstraße, 17489 Greifswald

Introduction: High-dose methotrexate ( $>4 \mathrm{~g} / \mathrm{m}^{2}, 4 \mathrm{~h}$; HD-MTX) is the single most effective agent in the therapy of primary CNS lymphoma (PCNSL). The optimal number of MTX-cycles remains to be determined, but even after multiple courses of MTX about $50 \%$ of the patients do not achieve a complete remission. This makes additional therapy necessary, i.e. in most studies whole brain radiotherapy (WBRT). But radiation grossly contributes to neurotoxicity, especially in the elderly. Therefore the application of WBRT has been questioned for patients in CR after chemotherapy. A therapeutic option that avoids radiation-induced neurotoxicity is high-dose chemotherapy with lipophilic substances, such as busulfan and thiotepa in combination with autologous peripheral blood stem cell transplantation (PBSCT).

Material and Methods: Our phase II study was designed accordingly. Once diagnosis is ascertained by histology HD-MTX $\left(8 \mathrm{~g} / \mathrm{m}^{2}, 4 \mathrm{~h}\right)$ is given on $\mathrm{d} 1$ and $\mathrm{d} 10$ followed by leucapheresis. Then patients were stratified: SD/PD receive WBRT (45Gy) reference therapy, those in CR/PR continue with HDbusulfan $(16 \mathrm{mg} / \mathrm{kg}$ BW) / thiotepa $(10 \mathrm{mg} / \mathrm{kg} \mathrm{BW})(\mathrm{HD}-\mathrm{Bu} / \mathrm{TT})$ and PBSCT. In the case of CR treatment is finished, patients in PR receive WBRT (45gy).

Results: We report on the treatment results of the first 10 patients at a median follow up of 9 months. Median age at time of diagnosis is 59 years (3967y), 3 females and 7 males. In two cases MTX related toxicity (hepatic, renal) changed the course of treatment. MTX yielded excellent leukapheresis results. HD-Bu/TT was well tolerated with low toxicity. CNS - RESULTS: $9 \mathrm{CR}$ and 1 PR (protocol violation, SD after MTX, HD-Bu/TT, WBRT). 3 patients died (2 CR - 1 pneumonia, 1 encephalopathy; 1 PR encephalopathy), 5 of 7 patients alive have a Karnofsky-Index of $100 \%$ and continue their prior living activities, two patients have an index of $70 \%$ and $60 \%$ (aphasia, right hemiparesis). 6 month post transplantation the latter patient developed a histologically proven relapse, with abdominal and very unusual testicular lymphoma. Treatment with $\mathrm{CHOP} /$ rituximab has just started (as of May).

Conclusions: We conclude that responders to HD-MTX and HD-Bu/TT show very good results despite omission of neurotoxic WBRT. These promising data yet have to be confirmed by more patients treated.

\section{0}

HELYX Study Part I \& II: Treatment of low-grade gastric nonHodgkin's lymphoma of mucosa-associated lymphoid tissue (MALT) type stages IE \& II1E

Morgner A. (1), Dragosics B. (2), Ruskoné-Formestraux A. (3), Stolte M. (4), Ehninger G. (1), Miehlke S. (1), Herrmann T. (5), Dawel M. (5), Bayerdörffer E. (1)

(1) Med. Klinik \& Poliklinik I, TU Dresden, (2) Gesundheitszentrum Süd, Wien, (3) Service de Gastroentérologie, Hôtel-Dieu, Paris, (4) Institut für Pathologie, Klinikum Bayreuth, (5) Klinik \& Poliklinik f. Strahlentherapie \& Radioonkologie, Technische Universität Dresden

Background:Today, more than 650 patients have been treated for Helicobacter pylori (H. pylori) positive, primary gastric MALT lymphoma with antibiotics world-wide. Complete remission occurs in about $80 \%$ of patients with stage I lymphomas. We also gained new insights into cytogenetics characterizing the translocation $\mathrm{t}(11 ; 18)$ Aim: To investigate in a prospective, randomized study if complete lymphoma remission occur in H. pyloripositive gastric low-grade MALT lymphoma in stages IE and II1E (HELYX Part I) after eradication therapy. Patients that do not respond to eradication therapy or patients that are $\mathrm{H}$. pylori- negative will receive radiotherapy either a reduced dose $(25,2 \mathrm{~Gy})$ or the standard dose (36Gy) (HELYX Part II). Patients \& Methods: Patients, 18 to 75 years-old, with a histologically confirmed H. pylori- positive primary gastric low-grade MALT lymphoma stage I to II1E will be included into HELYX I. After eradication therapy, control examination will be performed in 3-monthly intervals for the first 12 months. Patients with complete lymphoma remission (cr) will be followedup every 3 months during the first year. Afterwards the control intervals depend on the PCR results: persistent B-cell monoclonality at 6-monthly intervals, polyclonal PCR once yearly. Patients with partial remission (pr) or no chance (nc) 12 months after eradication therapy as well as patients with an H. pylori- negative MALT lymphoma will enter HELYX II: radiation therapy. After randomization, patients will either receive the standard dose $36 \mathrm{~Gy}$ fractionated or a reduced dose of 25,2Gy fractionated. Control examination after radiotherapy will be performed as described above. In addition, B-cell monoclonality will be analyzed for residual lymphoma. The aim is to proof aquivalence of the reduced dose versus the actual standard dose. $\mathrm{Pa}$ tients with lymphoma progression at any time point of the study (HELYX I\&II) reach an individual end point.

This study may help to develop a multimodal step-up treatment plan for patients with primary gastric MALT lymphoma stages IE and IIIE, and to establish radiation therapy as a salvage therapy after failed $\mathrm{H}$. pylori eradication as well as for $\mathrm{H}$. pylori- negative MALT lymphomas.

\section{1}

\section{ABVD chemotherapy without obligatory radiotherapy in patients with early and intermediate stage Hodgkin's lymphoma}

Naumann R. (1), Hänel A. (2), Kroschinsky F. (1), Dawel M. (3), Baaske D. (4), Beuthien-Baumann B. (5), Freund S. (1), Zschuppe E. (6), Mölle M. (7), Schmidt H. (8), Grundeis M. (9), Günther E. (10), Hardt D. (11), Fiedler F. (2), Ehninger G. (1), Hänel M. (1)

(1) Universitätsklinikum Dresden, Med. Klinik, (2) Klinikum Chemnitz, Hämatologie, (3) Universitätsklinikum Dresden, Strahlentherapie, (4) Klinikum Chemnitz, Strahlentherapie, (5) Universitätsklinikum Dresden, Nuklearmedizin, (6) KH DD-Friedrichstadt, (7) Onkologische Praxis DDAltstrehlen, (8) KKH Hameln, (9) Onkologische Praxis Chemnitz, (10) Klinikum Reutlingen, (11) Klinikum Konstanz

Objectives: Combined treatment modality using ABVD chemotherapy and involved field radiation is effective in patients with early and intermediate stage Hodgkin's lymphoma. The aim of our prospective study was to reduce the toxicity by omitting the subsequent radiotherapy in patients with complete remission (CR) after ABVD.

Material and Methods: Since May 2000 a total of 39 patients with early ( $\mathrm{n}=$ 13 ) or intermediate stage $(n=26)$ Hodgkin's lymphoma (HL) were treated either with 4 or 6 cycles of ABVD. A consolidating involved field radiation (30 Gy) was performed only in patients who achieved less than CR following chemotherapy. Until now 26 patients (median age 33 years, range 1771 ; 18 male, 8 female) were evaluated after completing therapy. Results: After finishing ABVD 18 patients (69\%) showed a CR. Seventeen patients with CR were investigated by PET scan, in $17 / 17$ cases PET showed a negative result. 7 patients with partial response (3 patients were PET negative) after chemotherapy went into CR after radiotherapy, another patient with PET negative PR refused radiation. The observed side effects were comparable to the well known moderate toxicity of ABVD. After a median follow-up of 16 months all patients are alive in continuous CR.

Conclusions: 4 or 6 cycles of ABVD chemotherapy induce high CR rates in patients with early and intermediate HL. This strategy could reduce combined toxicity in a high proportion of patients. However, the results are preliminary and should be confirmed in a larger number of patients and with a longer follow-up.

\section{2}

\section{BACOPP-D as treatment in patients with advanced Hodgkin's lymphoma}

Naumann R. (1), Hänel A. (2), Kroschinsky F. (1), Friedrichsen K. (2), Zschuppe E. (3), Mölle M. (4), Freund S. (1), Fiedler F. (2), Ehninger G. (1), Hänel M. (1)

(1) Universitätsklinikum Dresden, Medizinische Klinik I, (2) Klinikum Chemnitz, Hämatologie, (3) KH DD-Friedrichstadt, (4) Onkologische Praxis DD-Altstrehlen

Objectives: The development of the escalated BEACOPP regimen let to an improved outcome in patients with advanced Hodgkin's lymphoma (HD9 study of the GHSG). However, the application of high dose etoposide (cumulative $4,8 \mathrm{~g} / \mathrm{m}^{2}$ per 8 cycles) seems to be associated with an increased incidence of secondary MDS and AML, respectively. Therefore, the aim of our ongoing HL3 study is to evaluate the efficacy and toxicity of the etoposide free as well as dose intensified BACOPP-D protocol.

Material and Methods: Since May 2000 a total of 33 patients (median age 35 years, range 18-65; 21 male, 12 female) with advanced Hodgkin's lymphoma (HL) were treated with BACOPP-D which included cyclophosphamide $1250 \mathrm{mg} / \mathrm{m}^{2}(\mathrm{~d} \mathrm{1})$, adriamycin $25 \mathrm{mg} / \mathrm{m}^{2}(\mathrm{~d} 1+2)$, dacarbazine 250 $\mathrm{mg} / \mathrm{m}^{2}$ (d 1-3), procarbazine $100 \mathrm{mg} / \mathrm{m}^{2}$ (d 1-7), prednisolone $40 \mathrm{mg} / \mathrm{m}^{2}$ (d 1-14), bleomycin $10 \mathrm{mg} / \mathrm{m}^{2}$ (d 8) and vincristine $1,4 \mathrm{mg} / \mathrm{m}^{2}$ (maximum 2 $\mathrm{mg}, \mathrm{d} 8)$ at three-weekly intervals. A consolidating involved field radiation 
(30 Gy) was performed only in patients who achieved less than CR following chemotherapy. Initial staging and post-treatment control included PET monitoring.

Results: Until now 26 patients are evaluable for treatment response and survival. With an median follow-up of 15 months (6-25 months) 23 patients are alive in CR $(n=7), C R u(n=14)$ and PR $(n=2)$. Two non-responding patients (including one lymphocyte-depleted-HL) were successfully salvaged. One 65 year-old woman died in CR following myocardial infarction caused by coronary heart disease. No HL-specific or treatment related death occurred. We analyzed the acute toxicity for 166 cycles of BACOPP-D CTC/WHO grade III-IV hematological toxicities per patient were observed as follows: leukopenia $96 \%$, anemia $48 \%$, thrombocytopenia $30 \%$. Anemia and thrombocytopenia are comparable to the BEACOPP-14 regimen but less than for escalated BEACOPP. CTC grade III-IV non-hematological side effects included alopecia (13\%), FUO (8\%), cardial toxicity $(4 \%)$ and pain (4\%).

Conclusions: BACOPP-D regimen appears as a feasible and safe treatment protocol in patients with advanced HL. The observed efficacy and lack of major toxicity, respectively, are encouraging. However, our results are preliminary and should be confirmed in a larger number of patients and with a longer follow-up.

\section{3}

Mitoxantrone/bendamustine/prednisolone (MBP) in indolent lymphomas - clinical results of a phase I/II study

Nickenig C. (1), Emmerich B. (2), Reiser M. (3), Abenhardt W., Hiddemann W. (1), Dreyling M. (1)

(1) Dept. of Medicine III, University Hospital Grosshadern/LMU, Munich, (2) Dept of Medicine, University Hospital Innenstadt/LMU, Munich,

(3) Dept. of Medicine, University Hospital Cologne

Introduction: The clinical course of indolent lymphomas is characterized by frequent relapses and a continously declining survival curve. Even in relapse, the disease is sensitive to conventional chemotherapy. Bendamustine is a well characterized alkylating drug which has been proven to be highly effective in indolent lymphomas.

Methods: A phase I/II study was initiated to define the maximally tolerated dose of Bendamustine in a combined chemotherapy regimen (MBP) based on the previous MCP scheme of the German Low Grade Lymphoma Study Group (Mitoxantrone $8 \mathrm{mg} / \mathrm{m}^{2} \mathrm{~d} 1+2$, Bendamustine $80-120 \mathrm{mg} / \mathrm{m}^{2} \mathrm{~d} 1+2$, Prednisone $\left.25 \mathrm{mg} / \mathrm{m}^{2} \mathrm{~d} 1-5\right)$. Bendamustine dose was started at $80 \mathrm{mg} / \mathrm{m}^{2}$ and increased in $20 \mathrm{mg} / \mathrm{m}^{2}$ steps if no dose limiting toxicity was observed in a 3 patient cohort.

Results: Currently, 12 patients with relapsed malignant lymphomas have been treated. Whereas generally, BMP was well tolerated, a subset of patients showed a significant hematotoxicity grade 4 after subsequent cycles of chemotherapy $\left(4 / 6\right.$ pts. at $80 \mathrm{mg} / \mathrm{m}^{2}, 1 / 3$ patients at $100 \mathrm{mg} / \mathrm{m}^{2}$ and $2 / 3$ pts. at $120 \mathrm{mg} / \mathrm{m}^{2}$ Bendamustine). Thus, the mitoxantrone dose was fixed at $10 \mathrm{mg} / \mathrm{m}^{2} \mathrm{~d} 1$ and the Bendamustine dose was reduced to $80 \mathrm{mg} / \mathrm{m}^{2}$. Interestingly, 7 of 9 patients $(78 \%)$ evaluable of this heavily pretreated patien population achieved a remission ( $2 \mathrm{CR}, 4 \mathrm{PR}, 1 \mathrm{MR}$ ). Especially, one ongoing complete remission was observed in a patient with relapsed mantle cell lymphoma.

Discussion: Our study results confirm a Bendamustin-containing regimen as a highly effective and generally well tolerated therapeutic regimen. However, because of cumulative hematotoxicity, the optimal Bendamustine dose has to be exactly defined. Based on the results of the current phase I/II study, a multicenter phase II study is planned to evaluate a combined immuno-chemotherapy (Rituximab-MBP) in relapsed indolent lymphomas.

\section{4}

\section{Bendamustine and rituximab act synergistically in vitro and are effective in the treatment of indolent and mantle-cell lymphomas pretreated with purine analogs}

Rummel M.J., Kim S.Z., Chow K.U., Weidmann E., Hoelzer D., Mitrou P.S.

Universitätsklinik Frankfurt am Main

The cytotoxic agent bendamustine combines a purine-like benzimidazol and bifunctionally alkylating nitrogen mustard group. The drug is active in a variety of lymphoproliferative disorders without crossresistance to cyclophosphamide. We investigated simultaneous combination of rituximab with bendamustine and found synergistic effects of apoptosis in vitro on lymphoma cell lines (DOHH-2, WSU-NHL) as well as on ex vivo cells of patients with CLL $(n=5)$. Apoptosis was determined by Annexin V and JC-1 (disruption of mitochondrial membrane potential). Rituximab was applied at a dose of $10 \mu \mathrm{g} / \mathrm{ml}$, where $80-95 \%$ of CD20-molecules per cell were sufficiently blocked. Noteworthy, the synergistic effects of rituximab in combination with bendamustine were evenly strong when rituximab was applied at a dose, at which approximately half of the CD20 molecules per cell were saturated. We furthermore demonstrated that the chemosensitizing effect of rituximab was complement-independent and that this effect critically depends on caspase-7. Based on our in vitro results we initiated a phase-II study to examine efficacy and toxicity of bendamustine combined with rituximab as therapy of 1 st, 2 nd or 3 rd relapse. All patients had previously received fludarabine or cladribine containing treatment. Treatment schedule: 6 cycles of Rituximab $375 \mathrm{mg} / \mathrm{qm}$ day 1 combined with 4 cycles of bendamustine $90 \mathrm{mg} / \mathrm{qm}$ day $2+3$, every four weeks. Included entities: immunocytomas, mantle cell, follicle center, and marginal zone lymphomas. 28 patients entered the study, 18 are evaluable for response. 4 pts had 3 prior therapies, 4 pts 2 prior tx, and 10 pts 1 prior tx.

\begin{tabular}{lrccc}
\hline Entity & $\mathrm{n}$ & $\mathrm{CR}(\%)$ & $\mathrm{PR}(\%)$ & $\mathrm{CR}+\mathrm{PR}(\%)$ \\
\hline Foll & 5 & $5(100)$ & & $5(100)$ \\
Mantle & 5 & $2(40)$ & $2(40)$ & $4(80)$ \\
IC & 6 & $2(33)$ & $4(67)$ & $6(100)$ \\
MZ & 2 & 2 & & $2(100)$ \\
Overal & 18 & $11(61)$ & $6(33)$ & $17(94)$ \\
\hline
\end{tabular}

Foll $=$ follicular; mantle $=$ mantle-cell $; \mathrm{IC}=$ immunocytoma $; \mathrm{MZ}=$ marginal zone.

Results out of 18 evaluable patients: CR 61\%, PR 33\%, overall response rate $94 \%$. The presented combination induced considerable low hematological toxicity with only $4 \%$ granulocytopenias grade 3 and 4 . One patien with a mantle cell lymphoma being refractory to 2 prior treatments died due to lymphoma. 1 bacterial pneumonia has been observed. So far, it appears in this ongoing study that the combination of bendamustine and rituximab seems to be very effective in the treatment of relapsed indolent and mantle cell lymphomas reaching an overall response rate of $94 \%$ and a CR rate of $61 \%$.

\section{5}

\section{Secondary malignancies}

Scheinpflug K., Jentsch-Ullrich K., Koenigsmann M., Franke A.

Department of Hematology / Oncology , „Otto-von-Guericke“-University of Magdeburg, Germany

Objectives: As the success of modern cancer therapy has increased the duration of survival and curability of many patients, recognition of long-term complications of therapy also has increased. The most severe and often lifethreatening late complication of both multiagent chemotherapy and radiation therapy is the development of secondary malignancies.

Material and Methods: We report on 84 patients ( median age 67.5 years ) suffering from secondary malignancies. Patients with solid tumors following hematologic neoplasms and patients with hematologic neoplasms following successful therapy of solid cancers as well as patients with two different hematologic malignancies were enrolled and included in analysis. Association with previous therapies was investigated. Our special attention was for patients with multiple secondary neoplasias among therapy-induced malignancies

Results: The most frequent primary cancers were high-grade $(13 / 84=$ $15,5 \%)$ and low-grade lymphoma $(8 / 84=9,5 \%)$, Hodgkin's disease $(7 / 84$ $=8,3 \%)$ and chronic lymphocytic leukemia $(12 / 84=14,3 \%)$ as hematologic neoplasms and solid tumors such as breast $(5 / 84=6 \%)$ and uterine carcinoma $(6 / 84=7,1 \%)$, colorectal cancer $(4 / 84=4,8 \%)$ and melanoma $(4 / 84=4,8 \%)$. Secondary malignancies were mostly hematologic neoplasms ( high-grade lymphoma 11/84 = 13,1\%; low-grade lymphoma 9/84 $=10,7 \%$; acute myelogenous leukemia $6 / 84=7,1 \%$; myelodysplastic syndrome $7 / 84=8,3 \%$; multiple myeloma $5 / 84=6 \%$ and Hodgkin's disease $4 / 84=4,8 \%$ ) followed by solid cancers: colorectal cancer $6 / 84=7,1 \%$; renal cell carcinoma $5 / 84=6 \%$ and prostate cancer $5 / 84=6 \%$. Latency between primary and secondary malignancies was 0-324 months, for instance 54 months ( median ) for secondary acute myelogenous leukemia and 59 months ( median ) for high-grade lymphoma. Patients with transformed low-grade lymphoma (into high-grade lymphoma ) and Richter's syndrome as well as patients with the occurrence of two different malignancies within 3 months were excluded from investigation for therapy-association.

Conclusions: Secondary therapy-induced malignancies are seen because of successful initial treatment. Attemps should be made to reduce this most severe long-term complication, especially for curable neoplasms, such as high-grade lymphoma or Hodgkin's disease. 


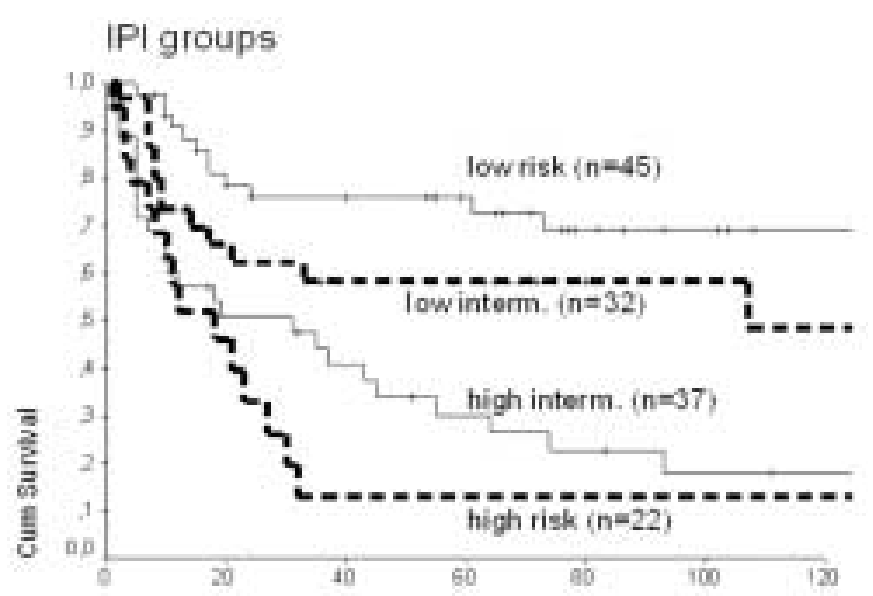

SUFWNAL (morite)

high dose + BM/stem cell transplantation

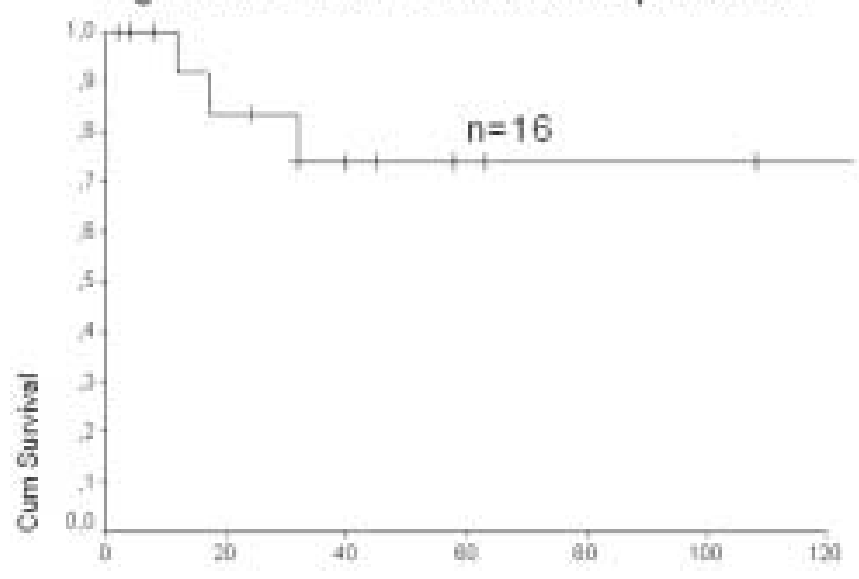

SURMVAL imerdm:

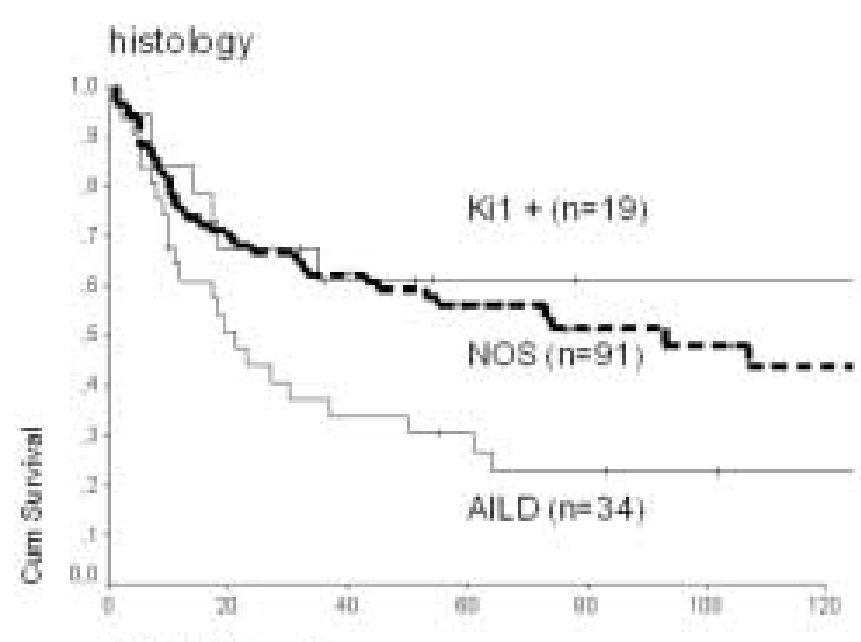

SURMVAL (morths)

218

Sequential high-dose chemotherapy with autologous stem cell support in combination with the anti-cd20 antibody rituximab (mabthera ${ }^{\circledR}$ ) in relapsed aggressive non-hodgkin's lymphoma preliminary safety and feasibility data

Staak J.O., Glossmann J.P., Diehl V., Engert A., Josting A.

UNI Köln, Klinik I für Innere Medizin

Objectives: Combination chemotherapy can cure patients (pts) with NonHodgkin's lymphoma (NHL), but those with relapse still have a poor prognosis. High-dose chemotherapy (HDCT) with autologous stem cell support (ASCT) can improve the outcome of these pts as shown in the precursor

(54\%). B-Symptoms were present in $43 \%$. Most frequent extranoda . garding the IPI, 5Y-OS was $85 \%$ for low risk, $58 \%$ for low intermediate, $32 \%$ for high-intermed. and $13 \%$ for high risk. Patients who received high dose therapy had a $5 \mathrm{Y}-\mathrm{OS}$ of $74 \%$ and a 5 year relapse free survival of $58 \%$. Conclusions: The IPI is a relevant prognostic score in T-cell NHL. Early intensified treatment seems to stabilize remission. Diagnostic safety of T-cel lymphoma might be improved using cytology combined with Facs Scan. 
study (ORR 48\%: 32\% CR, 16\% PR; FFTF/OS of 42\%/54\%; Data recently published). Chemosensitivity and achievement of minimal disease status prior to HDCT are important prognostic factors in NHL pts treated with HDCT. Rituximab demonstrated encouraging activity in aggressive NHL and showed low toxicity in the setting of combined immunochemotherapy. Material and Methods: We modified the intensified salvage program by addition of rituximab to the chemotherapy cycles. Eligibility criteria include pts with age 18-65 years and eligible for HDCT with histologically proven CD20+ relapsed NHL. Treatment program consists of two cycles DHAP (dexamethasone, cytarabine, cisplatin) plus rituximab $\left(375 \mathrm{mg} / \mathrm{m}^{2}\right)$; pts with PR or CR receive cyclophosphamide $\left(4 \mathrm{~g} / \mathrm{m}^{2}\right)$ followed by PBSC harvest methotrexate $8 \mathrm{~g} / \mathrm{m}^{2}$ and vincristine $1,4 \mathrm{mg} / \mathrm{m}^{2}$ plus rituximab; and etoposide $2 \mathrm{~g} / \mathrm{m}^{2}$ each plus rituximab. The final myeloablative course is BEAM plus rituximab followed by ASCT.

Results: So far, 10 pts (median age 51 years, range 22-64) with relapsed aggressive NHL have been enrolled (stage I/II: 4, stage III/IV: 6). All pts had CHOP-like regimens as first-line therapy. The median time to progression was 14 months. This chemoimmunotherapy combination regimen was well tolerated in all pts without side effects exceeding the toxicity expected from chemotherapy alone. 9/10 pts were available for restaging after 2 cycles DHAP with 2 CR, 6 PR, 1 PD. Treatment was discontinued in one pt due to development of severe heart failure. Stem cell harvest was successful in $7 / 7$ patients. However, no pt is yet available for final restaging evaluation.

Conclusions: Preliminary data suggest feasibility and safety of rapid sequential administration of DHAP and high doses of cyclophosphamide, methotrexate and etoposide, each in combination with rituximab withou affecting the tolerability of the final myeloablative BEAM. The combination regimen allows effective mobilization of stem cells and is so far effective in this small group of pts with relapsed aggressive NHL. A total of 20 pts is planned to document feasibility and safety followed by a prospective randomized multicenter trial combining HDSCT with rituximab for evaluation of efficacy.

\section{9}

Radioimmunotherapy (RAIT) with 131-I rituximab: comparable uptake of radionuclide into lymphoma sites and clinical efficacy with or without preinfusion of unlabeled rituximab except for patients with marked splenomegaly

von Schilling C. (1), Wolf I. (2), Schwarz K. (1), Schmidt (1), Sandherr M. (1), Schwaiger M. (2), Peschel C. (1), Scheidhauer K. (2)

(1) III. Medizinische Klinik, (2) Nuklearmedizinische Klinik, TU München Objectives: $\alpha$-CD20 RAIT using murine antibodies requires preinfusion of unlabeled antibody to clear circulating lymphoma burden and to minimise non-specific binding of the radioconjugate. This may be different in radioconjugates using chimeric antibodies. We sought to define the value of preinfusing cold rituximab $(\mathrm{R})$ prior to $131-\mathrm{I}$ rituximab RAIT.

Material and Methods: In ongoing phase I/II trials, 54 patients (pts) with CD20+ NHL have now been treated with 131-I R. Of these, 47 have been followed until progression or remission of at least 1 year duration: 24 treated with non-myeloablative, and 23 with myeloablative doses supported by autologous PBSCT. Detailed whole-body and organ dosimetry and biodistribution studies (planar scintigraphy, with calculation of biological terminal half-life, residence times, and lymphoma/non-involved organ uptakes by the MIRDOSE algorithm) \pm preinfusion of $2.5 \mathrm{mg} / \mathrm{kg}$ cold $\mathrm{R}$ prior to administration of the radioconjugate are available from 35 of these. Treatment was administered with or without preinfusion of cold $\mathrm{R}$, depending on whether the lymphoma vs. total body dose ratio seemed to improve after preinfusion in late (day 5 or 7 ) scans or not.

Results: Overall, terminal T1/2 without preinfusion of cold $\mathrm{R}$ varied around a median of $88 \mathrm{~h}$ (range $34-127 \mathrm{hrs}, 24 / 35 \mathrm{pts} 79-96 \mathrm{hrs}$ ). T1/2 was further extended for a median of 14 hrs by preinfusing cold R. Tumor vs. nontumor ratios improved in 11/35 pts, but markedly only in $1 \mathrm{pt}$ with extensive splenomegaly. In pts with improved tumor- vs. non-tumor ratios, uptake was effectively enhanced only beyond 5 days, where overall activity was already greatly reduced. It is questionable whether this low proportion of additional radiation adds significantly to the effect achieved by the radioconjugate infused without addition of cold rituximab. Clinical evaluation revealed that preinfusion of cold $\mathrm{R}$ did not predict for enhanced clinical activity. Clinical, and possibly methodological aspects (myeloablative vs. non-myeloablative $\mathrm{Tx}$ ) had a greater influence on outcome.

Conclusions: In our limited series, $\alpha$-CD20 RAIT with 131-I R, as opposed to treatment with murine radioconjugates, does not require preinfusion of cold antibody to enhance clinical activity, while very likely leading to equivalent remission rate and duration. This would mean that $131-\mathrm{I} \mathrm{R}$ is a cheap and equally effective alternative to both $4 \times 375 \mathrm{mg} / \mathrm{msq} \mathrm{R}$ as well as to RAIT with murine conjugates.
Bendamustine/Mitoxantrone/Rituximab (BMR): A new effective regimen for refractory or relapsed indolent lymphomas

Weide R., Heymanns J., Schneider A., Pandorf A., Köppler H.

Oncology group practice, Koblenz

Background: Bendamustine and mitoxantrone have been shown to be potent cytotoxic drugs for the treatment of relapsed or refractory indolent lymphomas. The anti-CD20 monoclonal antibody rituximab has produced an overall response rate of $50 \%$ as a single agent in relapsed or refractory indolent lymphomas. We evaluated a combination of bendamustine, mitoxantrone and rituximab (BMR) to improve these results.

Objective: Open label, single centre pilot study for patients (pts) with relapsed or refractory, CD20-positive indolent lymphoma or chronic lymphocytic leukemia. The therapy consisted of bendamustine (B) $\left(80 \mathrm{mg} / \mathrm{m}^{2}\right.$, day $1-3),\left(80 \mathrm{mg} / \mathrm{m}^{2}\right.$, day $1-2$ in B-CLL), mitoxantrone (M) $\left(10 \mathrm{mg} / \mathrm{m}^{2}\right.$, day 1$)$, rituximab (R) $\left(375 \mathrm{mg} / \mathrm{m}^{2}\right.$, week $\left.2,3,4,5\right)$. Reduction of B dose according to pts' clinical condition was permitted. The first 20 pts received the full B dose, thereafter B was reduced down to $90 \mathrm{mg} / \mathrm{m}^{2}$, day $1-2$. BM was repeated on day 36 or when hematological parameters had recovered. The maximum therapy consisted of 1 BMR-application, followed by 5 BM applications. Treatment was stopped when the disease responded with a partial remission (PR) or a complete remission (CR).

Results: Between 3/99 and 04/02 42 pts received the BMR-regimen (4 secondary high grade lymphoma, 25 indolent lymphoma, 12 B-CLL, 1 BPLL). The mean B dose for lymphoma pts was $191 \mathrm{mg} / \mathrm{m}^{2}$ per course, divided into 3 applications on day $1-3$, and $169 \mathrm{mg} / \mathrm{m}^{2}$ for B-CLL/B-PLL pts, divided into 2 applications on day 1-2. The median age was 67 years (range 36-82) and their performance score ranged from 0-3. Median number of previous treatment regimens was 2 (1-7). Of the lymphoma pts 26 had stage IV disease, 3 stage III. Of the B-CLL / B-PLL pts 11 were Rai stage IV (Binet C) and 2 Rai stage II (Binet B). Overall response rate was $98 \%$ (41/42) with 19 pts achieving a CR (45\%) and 22 pts achieving a PR (52 $\%)$. Median time to progression is 26 months (1-36) and response is still durable in $29 / 42$ pts $(69 \%)$ (1+ to $36+$ months after therapy). A symptomatic, reversible grade 3 or 4 hematotoxicity occurred in 6/42 pts $(14 \%)$. A non-symptomatic grade 3 or 4 hematotoxicity was seen in $18 / 42$ pts (43 $\%)$. No major non-hematological toxicity was observed.

Conclusion: BMR is a well tolerated, very effective outpatient treatment for relapsed or refractory $\mathrm{CD} 20$ positive indolent lymphoid malignancies. The suggested B dose for lymphoma pts is $90 \mathrm{mg} / \mathrm{m}^{2}$, day 1 and 2, and for pts with B-CLL $80 \mathrm{mg} / \mathrm{m}^{2}$, day 1 and 2 , respectively.

\section{1}

Monotherapy with the anti-CD20 antibody rituximab in patients with post-transplant lymphoproliferative disease (PTLD) results of a multicentre phase II study

Zeidler K. (1), Oertel S.H. (1), Grefer J. (1), Reinke P. (1), Jonas S. (1), Verschuuren E. (2), Jäger U. (3), Jerkeman M. (4), Niedermeyer J. (5), Hummel M. (6), Kapell S. (6), Anagnostopoulos I. (7), Dörken B. (1), Riess H. (1)

(1) Humboldt Universität Berlin, Charite- Campus Virchow Klinikum, (2) Academic Hospital Groningen, (3) Universität Wien, AKH, (4) Lund University Hospitaal, (5) MH- Hannover, (6) Deutsches Herzzentrum Berlin, (7) Freie Universität Berlin

Objectives: : PTLD is a life-threatening disease of B-cell origin after solid organ transplantation. It occurs with an incidence of $1-10 \%$. There are no generally accepted treatment guidelines for PTLD. In patients (pts.) with PTLD the use of cytostatic drugs is limited due to the risk of serious infectious complications. Because of the low toxicity profile, treatment with the anti-CD20 monoclonal antibody rituximab is a promising alternative approach.

Material and Methods: : We conducted a multicentre, open phase II trial investigating rituximab as monotherapy in 25 patients (pts.) with PTLD. 7 pts. were kidney-, 6 lung-, 6 heart-, 5 liver- and 1 kidney/pancreas transplant recipients. Rituximab was given as 1st line therapy in 15, 2nd line in 8 and 3rd line in 2 pts. The treatment consisted of four infusions rituximab $375 \mathrm{mg} / \mathrm{m}^{2}$ on days $1,8,15$ and 22 . CT- scans and sonography were used to evaluate the outcome 4-6 weeks after therapy.

Results: : The mean age was 49.9 years (23.0-73.1), and the mean follow up time is 15.6 months (0.6-53.6). There were 18 diffuse large B cell-, 2 marginal zone-, 1 Burkitt lymphoma and 4 polymorphic lymphoproliferations. The therapy was well tolerated and no severe adverse events were observed. Adverse events were limited to two infusion related reactions (pain/fever) $\mathrm{WHO}^{\circ} 1$. The mean overall survival is 41.2 months with 20 pts. still alive. 
One patient died from chronic rejection and one due to progressive PTLD. Three pts. had pre-existing, recurrent, severe infections, and died of sepsis months after rituximab treatment, without signs of PTLD at autopsy. 13 pts. $(52 \%)$ achieved a complete remission with a mean duration of 25.1 months. Partial remission was observed in 1 pt., minor remission in 2 pts., no change in 8 pts. and 1 pt. experienced progressive disease. Overall response rate is $64 \%$.

Conclusions: : Rituximab proved to be well tolerated and effective in the treatment of PTLD. Adverse events were rare and of low grade. Furthermore pts. benefit from the short duration of therapy. Monotherapy with rituximab must be considered as first line therapy in PTLD.

\section{Myelodysplastic Syndromes}

\section{2}

\section{Low-dose melphalan in elderly patients with high-risk myelodysplastic syndrome or secondary acute myeloid leukemia: criteria predicting response}

\author{
Benz D. (1), Bowen D. (2), Gelly K. (2), Kanz L. (1), Denzlinger C. (1)
}

(1) Abteilung II, Medizinische Klinik, Universität Tübingen, (2) Molecular and Cellular Pathology, University of Dundee, UK

There is no standard treatment for elderly patients with high-risk myelodysplastic syndrome (MDS) or secondary or relapsed acute myeloid leukemia (s/rAML), apart from best supportive care. Median survival of these patients is less than half a year. We treated 32 elderly patients (range 59-81 years) with one of these diseases with $2 \mathrm{mg}$ melphalan orally once a day. 5 patients had refractory anemia with excess of blasts- 1 (RAEB-1), 8 patients RAEB-2, 14 patients SAML (including 2 patients with RAEB in tranformation, RAEB-t), 3 patients myeloproliferative chronic myelomonocytic leukemia (CMML) and 2 patients rAML. We observed 7 complete peripheral responses (CRs) as defined by $\mathrm{Hb}>12 \mathrm{~g} / \mathrm{dl}$, platelets $>100 \mathrm{G} / \mathrm{l}$, granulocytes $>1.5 \mathrm{G} / 1$ and absence of peripheral blasts. In a patient entering with a deletion in q13 a normal karyotype was documented in remission. CR patients had RAEB-2, RAEB-t or sAML. In all responding patients, improvements in blood counts were noted before week 5 of treatment.Melphalan treatment was stopped when a CR was obtained. CRs lasted for $13+$ to 55 weeks. Relapse after CR was generally responsive to retreatment with melphalan. In addition to CRs, 3 patients had transient (less than 8 weeks) in creases in platelet counts, and 1 patient had a reduction in leukemic skin infiltrates. 4 patients had stable disease for at least 8 weeks. All but two of these favourable effects were observed in patients with normal karyotype and with normal or hypocellular bone marrow. None one of the 11 patients with hypercellular bone marrow or of the 5 patients with complex cytogenetic abnormalities had a response. This was in contrast to a $50 \% \mathrm{CR}$ rate in patients with normal or reduced bone marrow cellularity and normal cytogenetics or del 13q. Treatment was generally well tolerated apart from a mild and transient initial worsening of cytopenias which was observed in most patients and lasted for up to 4 weeks. None of the responders had infectious or bleeding complications requiring hospitalization during this period. We conclude that low-dose melphalan is a well tolerated out-patient regimen with promising activity in elderly patients with high-risk MDS or sAML. Low-dose melphalan appears to have little effect in patients with leukemic bone marrow hyperplasia. We are currently conducting a randomized placebo-controlled study in elderly patients with high-risk MDS patients presenting with normal or hypocellular bone marrow.

\section{How to identify very-low-risk patients with myelodysplastic syndromes (MDS)}

Germing U. (1), Strupp C. (1), Hildebrandt B. (2), Aivado M. (1), Giagounidis A. (3), Haas R. (1), Gattermann N. (1)

(1) Uniklinik Düsseldorf, Klinik für Hämatologie, Onkologie und Klinische Immunologie, (2) Universität Düsseldorf, Inst. für Humangenetik, (3) St. Johannes.Hospital Duisburg, Klinik für Hämatologie, Onkologie und Klinische Immunologie

Several tools for predicting survival and AML evolution in MDS have been established in the past. Scoring sys-tems, using medullary blast count, blood count and karyotype can identify groups of patients with a rela-tively good prognosis. The new WHO classification of MDS proposes low-risk entities which show dysplastic features within the erythroid lineage only. We combined the WHO proposals with our scoring system in order to identify pa- tients with a favourable prognosis. Based on the German MDS Registry in Düsseldorf, we applied the Düsseldorf Score to our patient population of 614 patients with RA or RARS according to the FAB-classification. $30 \%$ of the patients showed only dyserythropoiesis whereas $70 \%$ belonged to the category of multilineage dysplasia. The Düsseldorf-Score identified a lowrisk group with a median survival times of about 68 months. When we applied the score to patients belonging to the WHO categories that show signs of dyserythropoiesis only, a more exclusive low-risk group was identified Median survival time in such a very-low-risk group according to the Düsseldorf-Score was 99 months $(n=81)$. This group comprises $44 \%$ of the pure dyserythropoietic group and $13 \%$ of all RA and RARS patients. AML evolution occurred in 1 out of 81 very-low-risk patients identified by the Düsseldorf-Score. In summary, if a scoring system is applied to MDS categories characterized by dyserythropoiesis only, a very-low-risk-group can be identified, whose prognosis does not seem to be adversely affected by the diagnosis of MDS. These patients should be spared the imponderability of experi-mental treatment protocols

\section{AC133-positive stem cells carry the del $(5 q)$ in the clinical 5q-syndrome}

Giagounidis A.A.N. (1), Haase S. (1), Peceny R. (3), Germing U. (2), Aivado M. (2), Schroeder M. (1), Aul C. (1)

(1) St. Johannes Hospital Duisburg, (2) Heinrich-Heine-Universität Düsseldorf, (3) Universitätsklinikum Essen, Klinik für Knochenmarktransplantation

The 5q-syndrome is a subset of myelodysplastic syndromes characterized by macrocytic anemia, thrombocytosis, mild leukopenia and a low probability of transformation into acute myeloid leukemia.

Objectives: Recent investigations suggest that the deletion of the long arm of chromosome 5 occurs at the hematopoietic stem cell (HSC) level, involving CD34+CD38- HSCs, but also CD34+CD19+ pro-B-cells. Thus, a lympho-myeloid pluripotent stem cell seems to be the primary target in most patients. In an attempt to further elucidate the most primitive stem cell bearing the $\operatorname{del}(5 \mathrm{q})$ we isolated $\mathrm{CD} 34+\mathrm{AC} 133+$ cells in 8 patients with the 5q-syndrome.

Material and Methods: CD34+AC133+ cells are a subset of CD34+ hematopoietic progenitors with multilineage, long-term culture initiating capacities. Using magnetic bead technology, we isolated these cells from bone marrow samples of 5q-patients. With the aid of fluorescence-in-situhybridizytion we identified the $\operatorname{del}(5 \mathrm{q})$ in the isolated cells

Results: 40 to $73 \%$ of all CD34+AC133+ cells carried the 5q-deletion. $\mathrm{Pu}$ rity of the AC133-preparation was checked using flow cytometry. AC133+ cells made up 40 to $84 \%$ of all isolated cell material carried the 5q-deletion. Conclusions: These results indicate that the $\operatorname{del}(5 q)$ is present in uncommitted, multilineage progenitor cells of human bone marrow staining positive for CD34 and AC133.

\section{Low-dose cytarabine in the treatment of patients with the 5q-syndrome}

Giagounidis A.A.N. (1), Haase S. (1), Germing U. (2), Hildebrandt B. (2), Heinsch M. (1), Aivado M. (2), Schroeder M. (1), Aul C. (1)

(1) St. Johannes Hospital Duisburg, (2) Heinrich-Heine-Universität Düsseldorf

The 5q-syndrome is a myelodysplastic disorder with excellent prognosis translating into a projected median survival of 145 months and a very low cumulative risk for acute leukemias of about $5 \%$. It is therefore sensible to be reluctant about aggressive treatment strategies; a primary goal should be to try and avoid secondary organ damage caused by transfusion-induced hemosiderosis.

Objectives: Attempts to improve transfusion dependence in patients with the $5 \mathrm{q}$-syndrome have included various drugs such as pyridoxine, glucocorticoids, danazol, and others. A phase II study investigating the effect of all-trans-retinoic acid (ATRA) in these patients is under way, and eligible patients should be included into the protocol available at the author's corresponding address.

Material and Methods: We report on seven patients treated with low-dosecytarabine $\left(20 \mathrm{mg} / \mathrm{m}^{2}\right.$ for up to 21 days s.c. or i.v.)for the clinical $5 \mathrm{q}$-syndrome, who were either treated before activation of the ATRA trial, were not eligible for the protocol or failed to respond to ATRA. 
Results: Five patients had refractory anemia, one had refractory anemia with ring sideroblasts, and one had refractory anemia with excess of blasts with a blast count of less than $10 \%$. All patients had typical morphology of the bone marrow with mononuclear megakaryocytes. Two had additional trisomy 21 in the malignant bone marrow cells. Three patients achieved complete remission defined as normalization of hemoglobin $(>12 \mathrm{~g} / \mathrm{dl})$, thrombocytes $(>150.000 / \mu \mathrm{l}$ but less than $350.000 / \mu \mathrm{l})$ and leucocytes between 4000 and 10.000/ $\mu 1$. Four patients did not respond. None of the patients with an additional chromosomal aberration responded to therapy. Repetitive fluorescence-in-situ hybridization of one responding patien showed a sharp reduction in the number of metaphases lacking part of the chromosome 5 . This suggests a cytoreductive rather than a differentiation effect responsible for the hematologic improvement. Two responding patients relapsed after 10 and 12 months. One is in ongoing remission for six months. Low-dose cytarabine may be a useful treatment to achieve transfusion freedom for a limited time in 5q-syndrome patients. However, it seems not possible to induce long-term remission with this treatment strategy.

\section{The 5q-syndrome: An update on 76 cases}

Giagounidis A.A.N. (1), Germing U. (2), Haase S. (1), Willems H. (1), Hildebrandt B. (2), Schlegelberger B. (3), Schoch C. (4), Aivado M. (2), Aul C. (1)

(1) St. Johannes Hospital Duisburg, (2) Heinrich-Heine-Universität Düsseldorf, (3) Medizinische Hochschule Hannover, (4) Klinikum Großhadern der LMU München

The new WHO classification restricts the designation ,5q-syndrome“ to patients having $<5 \%$ medullary and peripheral blast count and an isolated cytogenetic deletion on the long arm of chromosome 5 .

Objectives: In order to evaluate this definition and to elaborate more characteristics of the disease, we report on 76 patients with typical bone marrow morphology of the 5q-syndrome studied during the last 26 years and classified according to FAB criteria. We compared these cases to 28 patients with multiple chromosomal aberrations including del $(5 q)$.

Results: Gross cytogenetic analysis revealed $28 x \operatorname{del}(5)(q 13 q 33)$, 9x $\operatorname{del}(5)(\mathrm{q} 13 \mathrm{q} 31), 9 x \operatorname{del}(5)(\mathrm{q} 22 \mathrm{q} 33), 3 x \operatorname{del}(5)(\mathrm{q} 12 \mathrm{q} 32), 2 \mathrm{x} \operatorname{del}(5)(\mathrm{q} 12 \mathrm{q} 33)$, and once $\operatorname{del}(5)(\mathrm{q} 13 \mathrm{q} 34), \quad \operatorname{del}(5)(\mathrm{q} 14 \mathrm{q} 31), \quad \operatorname{del}(5)(\mathrm{q} 14 \mathrm{q} 32), \quad$ and $\operatorname{del}(5)(\mathrm{q} 14 \mathrm{q} 33)$ each. In 10 patients it was impossible to delineate the exact breakpoints on 5q. 10 patients had one additional chromosomal anomaly in at least two metaphases, the most common being trisomy 21 ( 2 cases). 68\% of patients had refractory anemia (RA), $16 \%$ refractory anemia with ring sideroblasts (RARS), and 16\% refractory anemia with excess blasts (RAEB). 2 patients had more than $10 \%$ medullary blast cells. No patient was found having blasts in the peripheral blood and a normal medullary blast count. $10 \%$ of patients displayed only low amounts of mononuclear megakaryocytes. Median age of the entire study population was 67 years (range, 33 to 83), median hemoglobin $8.35 \mathrm{~g} / \mathrm{dl}$ (range, 3.4 to 12.2) at a median MCV of $92 \mathrm{fl}$ (range, 78 to 140). Median platelet counts were $253.000 / \mu 1$ (range, 34.000 to $1.540 .000 / \mu 1$ ). Erythropoetin levels were measured in 20 patients and grossly elevated in all of them (median 1000 U/l, range 122 to 2880). Projected median overall survival (OS) for patients not aggressively treated with an isolated $\operatorname{del}(5 \mathrm{q})$ is 145 months. With one additional chromosomal anomaly, median OS drops to 46 months. Patients with multiple chromosomal aberrations including del(5q) have a median OS of 6 months. We did not find any significant difference in OS according to the gross cytogenetic breakpoint, nor was the cut-off point of $5 \%$ medullary blasts significant in terms of OS

Conclusions: The 5q-syndrome definition should be extended to patients with 5 to $10 \%$ medullary blast cells and to patients with one additional chromosomal anomaly.

\section{7}

\section{Amifostine long-term application vs. supportive care in patients with low-risk MDS}

Haase D., Gassmann W., Stitz E., Vehmeyer K., Steffens R., Saake A., Hahnfeld S., Söling U., Griesinger F., Binder C., Kliche K.-O., Schulz T., Ludwig W.-D., Steidl C., Schanz J., Trümper L., Wörmann B.

Georg-August-Universität Göttingen, Abt. Hämatologie und Onkologie, Robert-Koch-Str. 40, 37075 Göttingen

It is the aim of our study, to examine the impact of amifostine on impaired hematopoiesis and disease progression in a randomized comparison with supportive care in patients with low-risk MDS. Stratification is performed according to cytogenetics. The therapy-schedule is biphasic with an inten- sive induction of $500 \mathrm{mg}$ amifostine $4 \mathrm{x} / \mathrm{week}$, followed by a maintenance of $500 \mathrm{mg}$ once a week for further 48 weeks. Sequential bone marrow biopsies with central morphology, cytogenetics, colony assays and FACS-analyses are performed before start of therapy and after 4, 25 and 51 weeks. Primary endpoints are hemoglobine, leucocytes, platelets and transfusions. As yet 38 pts. were randomized with the following FAB-classification: 18 RA, 15 RARS, 3 RAEB, 1 CMML, 1 no FAB. Median age was 62.5 yrs. (21-70). The median observation period amounts to 52 weeks. Preliminary findings are as follows: In the supportive care group $4 / 19$ pts. - and in the amifostine group $1 / 19$ pts. have died. Follow-up data are available from 28 pts. (14 in each therapy-arm). The courses of blood counts (according to international consensus criteria) are given in the table below ( $\mathrm{P}=$ platelets, $\mathrm{L}=$ leucocytes, $\mathrm{Hb}=$ hemoglobine):

Twenty pts. were dependent on transfusions of erythrocytes (11 pts. in the supportive care arm and 9 receiving amifostine). A significant reduction of erythrocyte transfusions was achieved in 2 pts. in the amifostine-group as compared to none in the supportive care arm.

\begin{tabular}{lllllll}
\hline Therapy-arm & $\begin{array}{l}\mathrm{P}, \\
\text { decrease }\end{array}$ & $\begin{array}{l}\mathrm{Hb}, \\
\text { decrease }\end{array}$ & $\begin{array}{l}\mathrm{L}, \\
\text { decrease }\end{array}$ & $\begin{array}{l}\mathrm{P}, \\
\text { increase }\end{array}$ & $\begin{array}{l}\mathrm{Hb}, \\
\text { increase }\end{array}$ & $\begin{array}{l}\mathrm{L}, \\
\text { increase }\end{array}$ \\
\hline Supportive & $57 \%$ & $29 \%$ & $43 \%$ & $29 \%$ & $14 \%$ & $21 \%$ \\
Amifostine & $36 \%$ & $35 \%$ & $14 \%$ & $50 \%$ & $29 \%$ & $64 \%$ \\
\hline
\end{tabular}

An increase of transfusion need was observed in 6/11 pts. with supportive care treatment as compared to $0 / 9$ receiving amifostine.

\section{8}

Response to combination therapy with valproic acid and alltrans retinoic acid in a patient with SAML/MDS

\author{
Kündgen A. (1), Aivado M. (1), Strupp C. (1), Hildebrandt B. (2), Haas R. \\ (1), Germing U. (1), Gattermann N. (1)
}

(1) Department of Hematology, Oncology, and Clinical Immunology; (2) Institute of Human Genetics; Heinrich-Heine-University, Düsseldorf, Germany

Impaired maturation of hematopoietic precursors is an important feature of advanced myelodysplastic syndromes. Differentiation therapy may therefore help to improve hematopoiesis and counteract leukemic transformation. In vitro studies with leukemic cells support a synergistic differentiating effect of all-trans retinoic acid (ATRA) and histone deacetylase (HDAC) inhibitors, which are a promising new class of anticancer agents. We examined the clinical efficacy of this drug combination in a patient with MDS For HDAC inhibition, we chose the antiepileptic drug valproic acid (VPA), the differentiating effect of which has only recently been discovered. In November, 2001, we diagnosed refractory anemia with excess of blasts $(10 \%$, RAEB I) in a 62-year-old man. Blast cells were not detectable in the peripheral blood at that time. Cytogenetic analysis revealed trisomy 8 and monosomy 5. Comorbidity precluded intensive chemotherapy, and the patient received a trial of thalidomide $(300 \mathrm{mg} / \mathrm{d})$, which was not successful. In April, 2002, the patient presented with increased transfusion dependency, thrombocytopenia, leukocytosis, and $29 \%$ peripheral blasts. Bone marrow cytology showed transformation into AML (FAB M1/M2, 30\% blasts). On cytogenetic analysis, only trisomy 8 was found in 4 out of 20 metaphases. After obtaining informed consent, we started valproic acid (administered orally, with monitored blood levels between 50 and $100 \mu \mathrm{g} / \mathrm{ml}$ ), and ATRA (at a dose of $80 \mathrm{mg} / \mathrm{m}^{2} / \mathrm{d}$ in two divided doses for 7 consecutive days, every other week).

The treatment was well tolerated. After six weeks, there was complete clearance of peripheral blasts, and the WBC dropped from $54,100 / \mu 1$ to $2,900 / \mu 1$. Bone marrow cytology showed a partial remission (10\% blasts). There was no cytogenetic response (trisomy 8 in three metaphases). Transfusion frequency was not significantly reduced. We conclude that ATRA plus VPA can achieve a drastic reduction of blast cells in sAML/MDS, probably by inducing differentiation and/or apoptosis of these cells. It remains to be seen whether this translates into a survival advantage for the patient. 
High frequency and heterogeneity of aberrant P15INK4B gene methylation in chronic myelomonocytic leukaemia (CMMoL)

Lehmann U., Tessema M., Länger F., Kreipe H.

Institute of Pathology, Medizinische Hochschule Hannover, D-30625 Hannover, Germany

Objectives: Chronic myelomonocytic leukaemia (CMMoL) is a stem cell disorder with a high incidence of blast transformation, which belongs to the category of myelodysplastic/myeloproliferative diseases according to the new WHO classification. For the myelodysplastic syndrome (MDS) an aberrant methylation of the tumour suppressor gene p15INK4B has been shown. Concerning CMMoL there are so far only very limited data available regarding the epigenetic inactivation of this important cell cycle regulator. Therefore, the methylation patterns and the frequency of epigenetic inactivation of the p15INK4B gene in CMMoL was investigated.

Methods: Different methylation specific PCR (MSP) and genomic sequencing protocols were employed for the analysis of $\mathrm{CpG}$ island methylation of p15INK4B to establish a reliable and quantitative methylation assay. The analysis of genomic DNA extracted from formalin-fixed paraffin-embedded bone marrow trephines from a series of CMMoL patients $(n=30)$ enabled a direct comparison with the morphological features of the respective biopsies and the clinical data. Also RNA for quantitative mRNA studies was extracted from formalin-fixed paraffin-embedded trephines.

Results: Hypermethylation of the CpG-island in the p15INK4B gene is a frequent event in CMMoL. But the exact frequency of detection depends very much on the methodology employed because the methylation patterns in different patients display strong inter- and intraindividual heterogeneity. Inactivation of the p15INK4B cell cycle regulator by hypermethylation was demonstrated by measuring mRNA transcript levels using quantitative realtime PCR technology.

Conclusions: Aberrant methylation of the p15INK4B gene can contribute to the discrimination of the disease from reactive hyperplastic status of the bone marrow. Its predictive power regarding the evolution to blast crisis has to be addressed in future studies.
Patients with myelodysplastic syndrome show a high frequency of $T$ cell response to wilms tumour 1 (WT1), proteinase 3 , mucin and telomerase

Mailaender V., Scheibenbogen C., Letsch A., Schmittel A., Thiel E., Keilholz $U$.

Universitätsklinikum Benjamin-Franklin, Medizinische Klinik III, Hämatologie, Onkologie und Transfusionsmedizin, Freie Universität Berlin, Hindenburgdamm 30, 12200 Berlin, Germany

Objectives: We have shown recently that $\mathrm{T}$ cell responses to the leukemia associated antigens (LAA) WT1 and proteinase 3 can be detected in approximately half of the patients with AML. Since overexpression of WT1 is detected in peripheral blood of a proportion of MDS patients and related to the progression of the disease we now studied T cell responses to WT1 in MDS patients and patients with secondary AML. In addition we analysed T cell reactivity to other LAA (proteinase 3, mucin, telomerase).

Material and Methods: PBMCs were stimulated with peptides derived from WT1 (AA position 126-134 and 187-195), proteinase 3 (169-177), mucin (12-20 and 950-958) and telomerase (540-548) for $2 \mathrm{~h}$ followed by a $16 \mathrm{~h}$ incubation with Brefeldin A to inhibit protein secretion and stained for CD3, CD8 and IFNg. Further phenotypic characterization of IFNg positive cells was done by staining for CD45RA and CXCR4. WT1 mRNA level in peripheral blood (PB) was measured by quantitative realtime RT-PCR using recombinant plasmid as control standard. Values were normalized to PBGD housekeeping gene expression level in $\mathrm{pg} / \mathrm{pg}$.

Results: 7 of 16 patients were HLA-A2 positiv (5 MDS and 2 secondary AML) and could be analysed. T cell responses specific for WT1 peptides were detected in 4 out of 7 patients (ranging from $0.20 \%$ to $1.59 \% \mathrm{IFNg}$ producing $\mathrm{CD} 3+\mathrm{CD} 8+\mathrm{T}$ cells), for proteinase 3 peptide in 2 out of 6 patients $(0.14 \%$ and $0.44 \%)$, for mucin peptides in 1 out of $3(1.96 \%)$, and for telomerase in 3 out of 3 (ranging from $0.40 \%$ to $2.03 \%$ ). Only one patient with secondary AML showed no reactivity against the tested peptides. Further phenotypic characterization of LAA specific IFNg secreting T cells revealed expression of CD45RA in 4 out of 4 patients consistent with an effector phenotype. In two patients analysed IFNg secreting $\mathrm{T}$ cells were pos- 
itive for CXCR4, a chemokine receptor mediating homing to bone marrow. In 3 of 3 patients with WT1-specific T cells WT1 expression could be detected in PB ranging from $1.82 * 10^{-3}$ to $4.02 * 10^{-1}$

Conclusions: In this study we detected a high frequency $\mathrm{T}$ cell response to LAA in patients with MDS and secondary AML. Phenotypic characterization suggested that these T cells belong to the cytotoxic effector cell population and have the ability to migrate to bone marrow. The presence of such $\mathrm{T}$ cell responses suggests that the myelodysplastic preleukemic or leukemic cells are highly immunogenic and have profound implications for immunotherapy in MDS.

\section{1}

Different expression of enzymes of the oxidative metabolism in MDS patients and healthy donors of different age

Pfeilstöcker M. (1,2), Karlic H. (2), Lohninger A. (3), Laschan C. (1), Lapin A. (4), Temml C. (4), Pittermann E. (2)

(1) 3rd Medical Department Hanusch Hospital, Vienna, (2) Ludwig Boltzmann Institute for Leukemia Research and Hematology, Vienna

(3) Department of Medical Chemistry, University of Vienna,

(4) Sozialmedizinisches Zentrum Sophienspital, Vienna

Objectives: Myelodysplastic syndromes (MDS) comprise a heterogeneous group of clonal stem cell disorders with a high median age at time of diagnosis which may evolve from different pathogenetic pathways. Changes in key enzymes of oxidative metabolism in blood are known to be associated with the ageing process and many diseases. Regulation of these enzymes takes place on the transcriptional level. Aim of this study was to quantify mRNA synthesis of different mitochondrial carnitine acyltransferases (MCAs), microsomal carnitine palmitoyltransferase GRP58 and OCTN2 (organic cation transporter) to detect possible differences in gene expression in MNC from MDS patients and healthy donors of different age groups

Methods: Using quantitative reverse transcription real time PCR (RTQPCR) mRNA synthesis of MCAs, GRP58, OCTN in MNC from peripheral blood of 23 healthy persons (mean age 45 years), from blood and bone marrow of 23 MDS patients (6 RA, 12 RARS, 4 RAEB, 1 RAEBt - mean age 79 years) and from blood of 11 age matched controls was compared.

Results: Concerning different MCAs a downregulation of 50\% in MDS patients and healthy elderly was found for carnitine palmitoyltransferases CPT1A and CPT1B, a reduction higher than $85 \%$ was detected for CPT2 and CRAT ( carnitin acetyltransferase) and OCTN. Different expression between normal age matched donors and MDS patients was only found in GRP58 which was reduced by $43 \%$ in elderly persons and by $96 \%$ in MDS No differences were found for expression between men and women, blood or bone marrow samples and MDS subtypes.

Conclusions: A similar downregulation of mitochondrial carnitine acyltransferases and OCTN2 transcription in MDS patients and healthy elderly persons indicates the importance of using age adjusted controls. Although a possible correlation between altered transcription rates of enzymes the oxidative metabolism in older persons and pathogenesis of MDS remains to be established, a possible role of these genes has to be considered for development of MDS.

\section{2}

\section{Gender-related differences in cytogenetic findings from 488} patients with myelodysplastic syndromes

\section{Schanz J., Schabla R., Haase D.}

Department of Haematology and Oncology, Georg-August-University of Göttingen, Robert-Koch-Straße 40, 37075 Göttingen

It is well known that there are gender-related differences in cytogenetic findings in patients with myelodysplastic syndromes (MDS), i.e. the incidence of the $5 q$-syndrome. It was our aim to examine these differences in our cytogenetic database of 488 patients with MDS. We examined 272 patients (pts.) of male and 216 pts. of female gender. Mean age was 57.6 $\pm 19,9$ years in male and $57.1 \pm 19.1$ years in female patients. There were no differences in the distribution of FAB-subgroups between male and female pts. We found gender-related differences in the occurrence of cytogenetic findings associated with poorer prognosis: Female patients showed significantly more abnormal karyotypes $(60.2 \%$ vs. $48.9 \%$ in male pts., $\mathrm{p}=0.0130)$, AA karyotypes (only abnormal metaphases) $(13.2 \%$ vs. $24.5 \%$ in male pts. $\mathrm{p}=0,0013)$ and cytogenetic evolution $(16.7 \%$ vs. $9.6 \%$ in male pts. $\mathrm{p}=0.0238$ ). The size of the abnormal clone at the time of first cytogenetic diagnosis was $80.9 \%$ (Mean value) in female and $71,6 \%$ in male pts $(\mathrm{p}=0.0175)$. On the other hand, male pts. showed a higher number of aberrations per case: Mean value was 3.15 in male and 2.54 in female patients $(\mathrm{p}=0.0284)$. We found no significant differences in the occurrence of complex abnormalities and non-clonal-instability. Differences in median survival between the two groups were not evident (19.5 months in female vs. 20.6 months in male pts., $p>0.05$ ).

We suppose that our findings can be explained by the high incidence of prognostically favourable $5 q$ - syndrome in female patients. $5 q$ - was found in $37.96 \%$ of female pts. and in $19.55 \%$ of male pts. with abnormal karyotype $(\mathrm{p}=0.0011)$. Other karyotype alterations (i.e. $-7,+8,-20 / 20 \mathrm{q}-,-5)$ showed no gender-related differences in the frequency of their occurence. The implications of these findings will be shown in detail.

\section{3}

Perioperative management of cardiac surgery in a patient with haemolytic paroxysmal nocturnal haemoglobinuria

Knobloch K. (1), Zardo P. (1), Gohrbandt B. (1), Fischer S. (1), Leyh R.G. (1), Tiede A. (2), Ganser A. (2), Schubert J. (3)

(1) Division of Cardiothoracic and Vascular Surgery, Hannover Medical School; (2) Department of Hematology/Oncology, Hannover Medical School; (3) Internal Medicine I, Saarland University Medical School, Homburg/Saar

Objectives: $\mathrm{PNH}$, is an acquired hemolytic anemia associated with thrombopenia and an abnormal susceptibility to venous thromboses. In cardiac surgery, PNH would lead to life threatening complications. Cytopenia would increase the risk of perioperative infection and bleeding. $\mathrm{PNH}$-associated complement activation is further exaggerated by extracorporal circulation leading to putative hemolytic crisis.

Case presentation: A patient who developed hemolytic PNH 2 years after treatment of SAA 8 years ago was still on CsA because of cytopenia. $\mathrm{He}$ had clinically manifest cardiac failure (NYHA III) and severe combined vitium of the aortic valve with leading incompetence (grade III to IV) by echocardiography and major stenosis of the LAD on coronary angiography. Therefore, aortic valve replacement and coronary revascularisation was decided. On admission, laboratory tests exhibited marked leucopenia, anemia, and thrombopenia, completely diminished haptoglobin, and an LDH level of 1215 U/l. Flow cytometric analysis revealed about $75 \%$ GPI-deficient neutrophils. Preoperatively, the patient received G-CSF $300 \mu \mathrm{g} \mathrm{3x} /$ week in order to increase the neutrophil counts. In addition, transfusions of RBC were given until a normal hemoglobin level was reached.

After initiation of anesthesia the procedure including cardiopulmonary bypass was started. The aortic valve was completely excised and a $23 \mathrm{~mm}$ porcine aortic valve prothesis was inserted. Furthermore, the LAD was revascularized with the left internal mammary artery. Extracorporeal circulation was discontinued after 108 minutes with an aortic cross-clamp time of 67 minutes. Intraoperatively, 3 units of RBC, 2 units of FFP, and 1 unit of platelets were transfused. Antibiotic prophylaxis was performed using ceftriaxon over 5 days. Perioperatively, the patient received mannitol twice prophylactically. On POD 5, no further hemolysis was detected with even normal haptoglobin values and no thrombosis was evident under intravenous heparin anticoagulation for 11 days. G-CSF was applied 3 times within one week postoperatively and stopped thereafter. CsA therapy was continued and the patient discharged from hospital on POD 15.

Conclusion: Cardiac surgery can be done in patients with PNH even with the use of extracorporeal circulation. Special emphasis should be given to optimal preoperative preparation including G-CSF and intensive perioperative RBC and platelet transfusion, fluid management and antibiotic prophylaxis.

\section{4}

Thalidomide for the treatment of myelodysplastic syndromes (MDS)

Strupp C. (1), Germing U. (1), Hildebrandt B. (2), Haas R. (1), Gatter$\operatorname{mann} N$. (1)

(1) Klinik für Hämatologie/Onkologie, Heinrich-Heine-Universität Düsseldorf, (2) Institut für Humangenetik, Heinrich-Heine-Universität Düsseldorf

Cytokines such as TNF-alpha may not only lead to increased apoptosis of hematopoietic cells in MDS but also to enhanced microvascularity of the bone marrow stroma. Thalidomide reduces bFGF-stimulated angiogenesis, inhibits TNF-alpha, modulates adhesion molecules and induces differentiation of the human leukemia cell line K562 in vitro. We examined the efficacy and tolerability of thalidomide in 53 patients ( $27 \mathrm{men} / 26$ women) with MDS :22 RA, 9 RARS, 6 RAEB, 12 RAEBT, 4 CMML. Median age was 66 years (range 52-81). Patients belonged to the following cytogenetic groups: 17 complex abnormal karyotypes, 24 normal karyotypes, 4 cases 
with 5q- and 8 single aberrations. Patients received thalidomide at an escalating dose from 100 to $500 \mathrm{mg} / \mathrm{d}$ (median $300 \mathrm{mg}$ ). Administration of the drug was discontinued in 22 out of 53 patients after a median of 3 months (1-14) because of skin rash (3), intolerable fatigue (18) and polyneuropathy (1) disregarding clinical response. 44 patients were evaluable for response. Treatment responses were classified according to the IWG-criteria. 26 patients showed hematological improvement with 10 partial remissions in cluding two cytogenetic major responses, 7 major response with transfusion independence, 9 patients showed minor response with decrease of transfusion frequency. Clinical response is now observed for a median duration of 12 months (2-25). 3 Patients (1 RA, 2 RAEBT) relapsed after a partial remission lasting 16 months (median). 7 patients showed progressive disease, 2 patients showed stable disease, 9 patients showed no response. In summary, a therapeutic benefit was achieved in 26 of 53 study patients (49\%).

\section{5}

\section{Outpatient treatment in patients with myelodysplastic syndrome}

Steegmanns R. (1), Köppler H. (1), Heymanns J. (1), Jacobs G. (2), Schimke J. (2), Steinmetz T. (3), Schmitz S. (3), Weide R. (1)

\section{(1) Oncology group practice Koblenz, (2) Saarbrücken, (3) Köln}

Objective: Evaluation of feasability and outcome in patients with myelodysplastic syndrome (MDS) who were diagnosed and treated completely on an outpatient basis.

Methods: Retrospective analysis of 165 consecutive, unselected MDS-patients who were diagnosed and treated between 06/95-08/01 in three community based oncology group practices.

Results: The median age of the patients was 72 years (37-90), 80 were male and 85 female. The distribution of patients in MDS-subgroups was as follows: 89 (54\%) RA, 30 (18\%) RARS, 14 (9\%) RAEB-1, 9 (5\%) RAEB-2, $16(10 \%)$ CMMOL and $7(4 \%)$ unclassified. In 97 patients cytogenetics were performed (59\%), therefore the Düsseldorf score was chosen for prognostic analysis of the whole cohort and IPSS was used for the group of patients with cytogenetic data. Treatment consisted in best supportive care only in 87 patients $(53 \%)$, palliative chemotherapy in 18 patients $(11 \%)$ and curative intended chemotherapy in 6 patients $(3 \%) .54$ patients $(33 \%)$ needed no treatment. During the treatment period 161 hospitalisations occurred 78 patients were hospitalised $(47 \%)$. The median frequency of hospitalisation was $2 /$ patient (1-7). The median duration of hospitalisation was 8 days (1-155). The reasons for hospitalisation were as follows: 115 disease related (71\%), 40 comorbidity $(25 \%)$ and $6(4 \%)$ unknown.

Overall survival analysis according to Düsseldorf score revealed the following survival:

Low risk: 71 months (1-85). Intermediate risk: 25 months (1-253). High risk: 15 months (4-91) with a median observation time of 18 months (1253). Overall survival according to IPSS was: Low risk: 71 months (1-253) Intermediate-1: 24 months (2-64). Intermediate-2: 21 months (4-91). High risk: 6 months (1 patient) with a median observation time of 18 months (1$253)$. During the observation period 63 patients have died $(38 \%) .15$ patients died at home $(24 \%), 41$ patients died in hospital $(65 \%)$, and 7 patients $(11 \%)$ died at an unknown location.

Conclusions: All necessary diagnostic procedures to establish a diagnosis of MDS can be performed on an ambulant basis. Palliative as well as supportive therapy is feasable in the outpatient setting. The median survival is comparable to results achieved in hospital based departments. A considerable percentage of patients can die at home.

\section{Multiple Myeloma - Basic Research}

\section{6}

\section{Enrichment of MUC1-reactive memory T cells in bone marrow of multiple myeloma patients}

\section{Beckhove P. (1), Witzens M. (2), Choi C. (1), Ho A.D. (2), Goldschmidt H.} (2), Schirrmacher V. (1)

(1) Deutsches Krebsforschungszentrum, Heidelberg, (2) Medizinische Klinik und Poliklinik V, Universität Heidelberg

Objective: Recently, the common tumor-associated antigen MUC1 has been shown to be expressed on about $90 \%$ of malignant plasma cells in multiple myeloma (MM). In breast cancer, MUC1 is known as a potential target for T cells. Previously, we could demonstrate that patients with breast cancer accumulate tumor reactive memory $\mathrm{T}$ cells in their bone marrow (BM), but not peripheral blood (PB). This study was performed to investigate the content and reactivity of tumor-specific memory $\mathrm{T}$ cells in BM and PB from MM patients with respect to possible use in immunotherapeutic strategies. Methods: Paired BM and PB samples from 42 HLA-A2 pos. MM patients and 11 HLA-A2 pos. normal donors were tested for frequency of TAA-specific CD8 T cells by HLA-A2 tetramer-analysis using MUC1 derived peptide LLLTVLTV (12-20) as TAA or for frequency of tumor-reactive CD8 memory $\mathrm{T}$ cells in $40 \mathrm{~h}$ short term IFN gamma ELISPOT assay using autologous monocyte-derived dendritic cells as antigen presenting cells after loading with LLLLTVLTV Muc1-derived peptide or HLA-A2 binding HLVEALYLV (34-42) peptide derived from insulin as control antigen. Results: The frequencies of MUC1-specific CD8 T cells in PB and BM of 30 tested patients varied between $0-6,4 \%$ (Median 0,6\%) of CD8 T cells. In contrast, PB and BM of 11 normal donors contained only $0-0,25 \%$ (Median $<0,1 \%$ ) tetramer binding CD8 T cells. Enrichment of MUC1 specific CD8 T cells (>0,3\% of CD8 T cells) was found in PB and BM from 16 out of 30 patients $(53 \%)$. Using short term IFN gamma ELISPOT functional-assay we detected enrichment of MUC1-reacticve CD8 memory T cells in BM from 6 out of 12 patients. In contrast, in corresponding PB, MUC1-reactive T cells were detected in only 1 out of 9 patients. The frequencies of MUC1reactive CD8 memory $\mathrm{T}$ cells varied between 1:390-1: 3350, (Median $1: 1052, \mathrm{BM})$, and 1:3340 (PB).

Conclusions: MUC1 specific T cells are highly enriched in PB and BM of about $50 \%$ of MM patients. We detected high amounts of MUC1-derived peptide specific CD8 memory T cells capable of IFN gamma secretion upon appropriate restimulation in BM but not $\mathrm{PB}$ of MM patients. Our data sug gest that $\mathrm{MM}$ induces tumor immune responses that are directed against MUC1 on tumor cells. Such responses result in generation of functional memory T cells that are localized within patients' BM. Thus, autologous BM-derived memory T cells reactivated in vitro with MUC1 pulsed dendritic cells might be useful for future immunotherapy of MM.

\section{7}

Tyrphostin AG 490 induces apoptosis in multiple myeloma cells in the absence and presence of bone marrow stromal cells but does not act via the STAT3, ERK, or Akt pathway

\section{Chatterjee M., Hönemann D., Herrmann P. , Bommert K., Dörken B.} Bargou R.C.

Department of Hematology, Oncology, and Tumorimmunology, RobertRössle Cancer Center at the Max Delbrück Center for Molecular Medicine, Charité, Campus Berlin-Buch, Humboldt University of Berlin, Germany

Recently it has been reported that constitutive activation of STAT3 signaling confers resistance to apoptosis in human myeloma cell lines. Accoddingly, it has been demonstrated that the Jak2 inhibitor tyrphostin AG 490 inhibits STAT3 phosphorylation and induces apoptosis in certain human myeloma cell lines. Therefore, tyrphostins are thought to be useful drugs for the treatment of multiple myeloma. However, data concerning the role of tyrphostins in human MM were obtained from experiments performed with cell lines and without considering the bone marrow microenvironment, which has been found to confer resistance to drug treatment in MM. Data on primary MM cells are missing and data describing the specificity and potential side effects of typhostins are limited. Here we show that treatment with AG 490 induces apoptosis of human MM cells in the absence or presence of bone marrow stromal cells to a similar extend. This effect could be observed with both cell lines and primary cells from bone marrow aspirates of MM patients. Of interest, other cell types such as B-CLL cells or stromal cells were unaffected by AG 490 treatment. Nevertheless, a strong in vitro growth inhibting effect of hematopoietic stem cells was observed. Signaling analysis revealed that AG 490 treatment led to significant STAT3 inhibition only in the absence of BMSCs and only at very high dosages. In the presence of BMSCs and at lower but already apoptosis inducing dosages no inhibition of STAT3 could be observed. Also no significant inhibition of MAPK-ERK1,2 or Akt activation could be observed upon AG 490 treatment neither in the presence nor in the absence of BMSCs. Although these data indicate that tyrphostins are of potential interest for the treatment of MM they do not act MM specific and one has to expect side effects in particular on the hematopoietic system. Furthermore, the apoptosis inducing effect of AG 490 is not mediated by inhibition of the STAT3 or other common pathways related to the malignant growth of MM. Thus, the precise mechanism of action of AG 490 in MM still has to be elucidated. 
In the presence of bone marrow stromal cells human multiple myeloma cells become independent of the IL-6/gp130/STAT3 pathway

Chatterjee M. (1), Hönemann D. (1), Lentzsch S. (1), Bommert K. (1), Sers C. (2), Herrmann P. (1), Mathas S. (1), Dörken B. (1), Bargou R.C. (1)

(1) Department of Hematology, Oncology, and Tumorimmunology, Robert-Rössle Cancer Center at the Max-Delbrück-Center for Molecular Medicine, Charité, Campus Berlin-Buch, Humboldt University of Berlin, Germany, (2) Institute of Pathology, University Hospital Charité, Humboldt University of Berlin, Germany

The IL-6/gp130/STAT3 pathway has been reported to play an important role in the pathogenesis of multiple myeloma (MM) and for survival of MM cells. However, most data concerning the role of IL-6 and IL-6-triggered signaling pathways were obtained from experiments performed with MM cell lines and without considering the bone marrow microenvironment. Thus, the precise role of IL- 6 and its intracellular signaling pathways for survival of human MM cells is still unclear. Here we show that treatment of human MM cells (IL-6-dependent MM cell line INA-6 and primary MM cells) with the IL-6 receptor-antagonist Sant7 or with an anti-gp130 mAb induced apoptosis if the cells were cultured in the absence of bone marrow stromal cells (BMSCs). In contrast, apoptosis could not be observed if the MM cells were cocultured with BMSCs. The analysis of intracellular pathways revealed that Sant7 and anti-gp130 mAb were effectively inhibiting the phosphorylation of gp130 and STAT3 in the absence and presence of BMSCs, whereas ERK1,2 phosphorylation was only slightly affected. In contrast, treatment with farnesyl transferase inhibitor FPT III induced of apoptosis in MM cells in the absence or presence of BMSCs, and led to a complete inhibition of the Ras/MAPK pathway. These observations indicate that the IL-6/gp130/STAT3 pathway is not essential for survival of human myeloma cells if they are grown in the presence of cells from the bone marrow microenvironment. Furthermore, we provide evidence that farnesyl transferase inhibitors might be useful for the development of novel therapeutic strategies for the treatment of MM.

\section{9}

\section{Establishment of a new xenograft tumour model for multiple myeloma in NOD/SCID mice}

\section{Dietz P. (1), Westhof A. (2), Merget-Millitzer H. (1)}

(1) MainGen Biotechnologie GmbH, Frankfurt/ Main, Germany; (2) Baxter Oncology GmbH, Frankfurt/Main, Germany

Multiple Myeloma is a B-cell malignancy. Current treatment protocols have improved overall survival of patients but many patients ultimately relapse. Therefore, multiple myeloma still remains an incurable disease and it is of topical interest to develop alternative treatment strategies. For preclinical testing of the efficacy of new drugs or treatment modalities, appropriate animal models are essential.

Objectives: As part of a research project to develop a new immunotherapy strategy for multiple myeloma we established a preclinical animal model in NOD/SCID mice using the human plasmacytoma cell line INA-6. Our aim was to obtain solid tumour formation in contrast to other known models.

Material and Methods: INA-6 cells were cultured in the presence of human IL-6 and 5 Mio cells were transplanted s.c. into NOD/SCID mice. To supplement for human IL-6 in vivo INA-6 cells were co-transplanted with IL-6 producing stroma cells in some experimental settings. Tumour growth was monitored over several weeks.

Results: Around $60-80 \%$ of mice developed INA-6 cell derived tumours in several independent experiments. A treatable tumour mass of around $0,2 \mathrm{~g}$ was usually (or consistently) reached after 2-3 weeks. It was observed that solid INA-6-tumours show a strong expansive growth pattern. Interestingly, tumour growth turned out to be IL-6 independent in vivo although INA-6 cell proliferation depends on exogenous human IL-6 in vitro. The additional transplantation of human IL-6 producing cells did not influence primary tumour growth or tumour progression. These results indicate that supplementation of human IL-6 in vivo is dispensable for tumour formation. The functionality of this animal model was proven by treatment of INA-6-tumour bearing mice with the glucocorticoid drug Dexamethasone alone or in combination with All-trans retenoic acid. As expected tumour growth was significantly decreased in all animals treated. The application of both drugs showed to be slightly more effective than treatment with Dexamethasone alone. These results confirm the feasibility of the xenograft model.
Conclusions: We established a reproducible tumour model for multiple myeloma based on solid tumour formation of human plasmacytoma cell xenografts in NOD/SCID mice. The animal model provides a useful tool to test and evaluate potential drugs or novel therapeutic strategies in multiple myeloma.

\section{0}

Number of clonotypic cells in peripheral blood of a patient with extramedullary plasmocytoma correlates with tumor growth

Fenk R., Schneider P., Kronenwett R., Kobbe G., Ak M., Steidl U., Strupp $K$., Emde T., Giagounidis A., Haas $R$.

Klinik für Hämatologie, Onkologie und klinische Immunologie, HeinrichHeine-Universität Düsseldorf

Background: We describe a patient with multiple myeloma who achieved complete remission with negative immunfixation after high-dose melphalan chemotherapy and autologous peripheral blood stem cell transplantation (PBSCT). Four months after tranplantation the patient suffered from extramedullary relapse with tumor masses emerging from the skull. He showed positive immunofixation and no evidence of infiltration of myeloma cells in the bone marrow. After treatment with local radiation and unrelated allogeneic PBSCT with non-myeloablative conditioning a 99\% tumor regression was observed for 7 days. After reoccurrence of extramedullary tumor manifestations immunosuppression was tapered and acute graft versus host disease (GvHD) evolved accompanied by a new regression of tumor masses. Following successfull treatment of GvHD tumor progression was observed. Although CED-chemotherapy reduced tumor manifestations again, the patient died because of pneumococcal septicemia with progressive disease.

Methods: We determined the number of clonotypic cells in the peripheral blood with a patient-specific quantitative real-time PCR using an allele specific oligonuceotides (ASO) primer and an ASO-TaqMan probe complementary to the complementerity determining region 3 of the rearranged immunoglobulin heavy chain $(\mathrm{IgH})$ gene.

Results: The number of clonotypic cells in the peripheral blood ranged from negativity (sensitivity $10 \mathrm{E}-5$ ) to $0,04 \mathrm{IgH}$ copies/beta-actin copies, thus spanning a range of 3,5 logs. The median number of clonotypic cells during tumor progression was $0,007 \mathrm{IgH}$ copies/beta-actin copies versus 0,0005 during tumor regression $(\mathrm{p}<0.05)$. Moreover the dynamic changes of residual tumor cells reflected the clinical course of this patient as increasing and decreasing copy numbers exactly matched time points of tumor progression and regression.

Conclusions: The number and kinetic of clonotypic cells in the peripheral blood of a patient with multiple myeloma correlated with extramedullary tumor growth. This emphasises the importance of the peripheral blood as the compartment where circulating B-cells of the myeloma clone not only promote spread of the disease but also can be targeted by graft-versusmyeloma effector cells. Furthermore, in the rare case of extramedullary manifestation of multiple myeloma real-time IgH-PCR provides a diagnostic tool.

\section{1}

Identification of genes associated with multiple myeloma and monoclonal gammopathy of undetermined significance using the myeloma microarray

Gaiger A. (1,4), Fonseca R. (2), Jelinek D. (3), Ordonez N. (1), Pyle R. (1), Molesh D. (1), Likes R. (1), Jaeger U. (4) Carter L. (1), Jeffery E. (1), Lechner K. (4), Persing D. (1), Greipp P.(2)

(1) Corixa Corporation, Seattle; Washington 98104; (2) Division of Hematology and Internal Medicine, Mayo Clinic, Rochester, MN 55905, USA; (3) Department of Immunology, Mayo Clinic, Rochester, MN 55905, USA; (4) 1st Dept. of Internal Medicine, Div. of Hematology, University of Vienna, Austria

Gene expression profiling using DNA microarrays has great potential to improve the understanding, diagnosis, and management of multiple myeloma. The near completion of the human genome sequencing project further increases the analytical power of this technology. A cDNA microarray uniquely suitable for the analysis of multiple myeloma has been developed, the Myeloma Microarray. To identify genes that are dysregulated in plasma cell disorders 6 subtracted libraries were constructed to enrich for myeloma or plasma cell specific cDNA sequences. cDNA pools from monoclonal gammopathy of undetermined significance (MGUS) (1 library), smouldering multiple myeloma (SMM) and multiple myeloma (MM) (3 libraries) and myeloma cell lines (2 libraries) were subtracted against cDNA pools of re- 
lated normal hematopoietic tissues (including CD138+ selected normal bone marrow derived plasma cells) or normal non-hematopoietic tissues. 6,000 cDNA fragments were then analyzed using DNA microarray technology. Genes dysregulated in plasma cell disorders were identified using pairs of fluorescence-labeled cDNA probes synthesized from MGUS, SMM, MM and myeloma cell lines $(n=35)$ and normal tissue poly $A+$ RNAs $(n=35)$. Over 420,000 hybridization signals were analyzed. Gene cluster analyse demonstrated significant similarities between MGUS and SMM, clearly distinguishing the gene expression profile of these 2 diseases from that of multiple myeloma. Expression patterns of 52 genes overexpressed in multiple myeloma were confirmed and characterized further by Real Time PCR using a panel of cDNAs comprising of multiple myeloma (including CD138+ sorted myeloma cells), normal tissues and MACS sorted hematopoietic subpopulations. In addition to genes known to be associated with MM or plasma cells (including CD138, VEGF receptor, IL6 receptor), we identified 3 novel genes Ly1728, Ly1732 and Ly1851 which are highly overexpressed in the majority of multiple myeloma patients. Ongoing studies evaluate the diagnostic and prognostic value of these genes.

\section{2}

\section{New options for multiple myeloma treatment: Inhibition of gp130 signaling}

Gramatzki M., Bakker F., Wohlfarth A., Burger, R., Guenther A.

Div. of Hematology/Oncology, Department of. Medicine III, University of Erlangen-Nuernberg, Erlangen, Germany

Interleukin-6 (IL-6) is one of the most important growth and survival factors in multiple myeloma (MM) and inhibition with specific monoclonal antibodies led to clinical responses in MM patients. However, human malignant plasma cells become rapidly resistant due to mechanisms not fully understood. Therefore, we focused on the investigation of IL-6 and other gp130 cytokines and the intracellular gp130 signaling network. With the human myeloma cell line INA-6, which is in vitro strictly IL-6 dependent, we could establish a SCID mouse xenograft model. Analyzing INA-6 tumors explanted from these animals, rescue mechanisms such as autocrine IL-6 production and cytokine replacement by Leukemia Inhibitory Factor or Oncostatin M could be demonstrated. gp130 Monoclonal antibodies successfully prevented myeloma cell growth in SCID mice. For INA-6 plasma cells, growth is due to constitutive Ras activation and gp130 mediated signaling to the JAK/STAT pathway. The inhibition of JAK2 by tyrphostin AG490 led to a strong dose-dependent inhibition of myeloma cell growth and induction of apoptosis. In addition, farnesyltransferase inhibitors (FTI), which were developed to block the activation of Ras, were tested. Treatment of the cell lines with FTI (FTI-277, L744.832 and FPT inhibitor III) resulted in a significant dose-dependent growth inhibition. Surprisingly, we found that FT response did not correlate with the $\mathrm{N}-/ \mathrm{K}$-Ras mutation status and that all three inhibitors strongly induced apoptosis without affecting cell cycle. Because K-Ras is known to be more resistant to FTI than N-Ras, we measured the membrane binding of N-/K-Ras by Western blotting and found no effect of FTI on K-Ras at effective dosages. Thus, FTI effectively block myeloma cell growth, however, the anti-myeloma activity is not caused by the inhibition of Ras. IL-6 can also activate the phosphatidylinositol 3-kinase (PI3K)/Akt pathway. Therefore, we tested the sensitivity of the five myeloma cell lines to specific inhibitors of either PI3K or its downstream target, mTOR, the activator of p70S6 kinase. Inhibition of PI3K with LY294002 blocked the growth of all cell lines in a dose dependent manner. Moreover, the p70S6K inhibitor rapamycin blocked myeloma cell growth at concentrations significantly lower than those needed to block the growth of other hematopoetic tumor cell lines $(72-98 \%$ at $2 \mathrm{nM})$. Therefore, inhibition of $\mathrm{mTOR} / \mathrm{p} 70 \mathrm{~S} 6 \mathrm{~K}$ can be considered as an interesting therapeutic option.

\section{3}

\section{The anti-myeloma activity of Farnesyltransferase inhibitors is not caused by an inhibition of Ras}

Guenther A., Wohlfarth A., Bakker F., Burger R., Gramatzki M.

Div. of Hematology/Oncology, Department of Medicine III, University of Erlangen-Nürnberg, Erlangen, Germany

Activation of the Ras/MAPK pathway, by IL- 6 or by mutations in the N- or $\mathrm{K}$-Ras gene, is essential for the growth of malignant plasma cells. Since proteins of the Ras family are active only in their membrane bound form, they have to be prenylated by farnesyltransferase, which enables them to anchor to the cell membrane. Notably, the K- Ras protein (and possibly also $\mathrm{N}$-Ras) can alternatively be prenylated by geranylgeranyltransferase if the farnesyltranseferase is blocked. Specific inhibitors of these enzymes have been used to block growth of various tumor cells and some of these compounds have entered clinical trials. However, it is still controversial whether the antitumor effect of farnesyltransferase inhibitors (FTIs) is due to inhibition of Ras or whether other signaling pathways are involved. We tested the effect of three farnesyltransferase inhibitors (FTI-277, L744.832 and FPT inhibitor III) on the growth and survival of five myeloma cell lines. In al five cell lines, the Ras/MAPK pathway is activated either by N-Ras (INA-6, L363) or K-Ras (JK-6L, RPMI8226) mutations or by an autocrine IL-6 loop (U266). Treatment of the cells with all three FTIs resulted in a significan and dose-dependent inhibition of cell growth as measured by $\left[{ }^{3} \mathrm{H}\right]-$ Thymidine uptake. However, the sensitivity to FTI treatment varied between the cell lines but surprisingly we observed no correlaton to the N-/K-Ras mutation status. Furthermore, all three inhibitors induced a marked increase in the rate of apoptosis without affecting cell cycle. This is surprising since Ras is thought to be mainly responsible for myeloma cell proliferation. In order to determine whether the anchorage of Ras to the cell membrane was blocked by FTIs at effective dosages, cell lysates were prepared upon FTI treatment divided in a membrane and a cytosolic fraction and analyzed by Western Blotting with antibodies specific for N- and K-Ras. As expected, FTI-277 was not able to block the anchorage of K-Ras to the cell membrane, indicating alternative prenylation by geranylgeranyltransferase. In contrast, the amount of N-Ras protein was elevated but N-Ras was still present in the membrane bound form. Thus, FTIs effectively block myeloma cell growth and may represent an interesting therapeutic option. Our results strongly suggest that anti-myeloma activity is not caused by the inhibition of Ras but rather involves other prenylated signaling proteins.

\section{4} Prognostic value of $13 q 14$ deletion and gain of chromosome 9
detected by Fluorescence in situ hybridization (FISH) in patients with multiple myeloma

Hahn C. (1), Urban T. (1), Mezger J. (2), Maintz C. (3), Schmidt-Wolf I.G.H. (1), Glasmacher A. (1), and the VRID study group

(1) UK Bonn, Medizinische Klinik und Poliklinik I, (2) St. VincentiusKrankenhäuser Karlsruhe, (3) Onkologische Praxis, Würselen

Objective: Partial or total deletion of chromosome 13 has been reported to be an independent adverse prognostic factor in patients with multiple myeloma (MM). However, conventional cytogenetics are successful in only 20 to $60 \%$ of patients. This rate can be improved by the use of FISH methodology. Using FISH, we studied the prognostic value of deletion of $13 q 14$ and in addition gain of chromosome 9 ( $\mathrm{chr} 9)$, which is one of the most frequent aberrations in MM.

Methods: FISH analysis was performed on bone marrow slides obtained from 71 patients (pts.) ( 36 females, 35 males) with MM at different stages of disease (stage I 13 pts, stage II 7 pts, stage III 48 pts). In $38 \%$ of cases the specimen was obtained at the time of initial diagnosis, $44 \%$ of patients received no prior therapy. Locus-specific probe for band 13q14 (Rb-1) and chromosome enumeration probe CEP 9 (Vysis, Bergisch Gladbach, Germany) were used according to standard protocols and analysis was successful in 58 pts and 69 pts, respectively.

Results: Deletion of $13 q 14$ (13q-) was detected in $18 / 58$ patients $(31 \%)$ Median survival from time of initial diagnosis in patients with $13 \mathrm{q}-\mathrm{vs}$. patients with normal $13 \mathrm{q}$ was significantly different: 887 days vs. 2838 days, $\mathrm{p}=0.032$. Gain of chromosome $9(+9)$ was found in $34 / 69$ patients $(49 \%)$. There was a trend towards a prolonged survival in patients with normal chr 9 vs. patients with +9 (median survival: 4738 vs. 1205 days, $\mathrm{p}=0.066$ ) Combining chr 9 and 13q14 results, the group with normal chr 9 and 13q14 has a significantly better overall survival than all other groups $(p=0.045)$. Median survival was not yet reached in group normal chr 9/normal 13q14 $(n=19)$ and was 495 days in group normal chr 9/13q- $(n=8), 2463$ days in group +9/normal 13q14 $(\mathrm{n}=22)$ and 887 days in group $+9 / 13 \mathrm{q}-(\mathrm{n}=9)$.

Conclusions: (1) Not only 13q- seems to be an adverse prognostic factor in MM.

(2) The importance of +9 has to be evaluated in a multivariate analysis and will require further follow-up. Supported by Leukämie-Initiative Bonn e.V. 
Cytostatic effects of aminopterin-albumin conjugate (AMPT-SA) in tumor cell lines

Hartung G. (1), Eschen N. (1), Bauder-Wüst U. (1), Schrenk H.-H., Sinn H. (2), Kremer P. (3) Frei E. (2)

(1) Division of Hematology/Oncology, Department of Internal Medicine, University of Rostock; (2) German Cancer Research Center, Heidelberg, (3) Neurosurgical Department, Kopfklinikum, University of Heidelberg, Germany

The antifolate aminopterin (AMPT) preceeded methotrexate (MTX) in clinical development but was not established to general use due its increased toxicity compared to MTX in the clinical setting. Recently we have reported on the increased metabolism of albumin-conjugates such as MethotrexateAlbumin (MTX-HSA) in malignant tumors and the feasibility to use albumin as carrier for drug targeting and decreasing systemic toxicity. Consequently, AMPT was covalently bound to serum albumin (AMPT-SA) at a 1:1 molar ratio. Biodistribution, tolerability and efficacy of this novel conjugate were studied in 3 tumor cell lines (multiple myeloma RPMI-8226, C6-glioma and A240286S-lung-adenocarcinoma). The cell lines (0.5 x 106 cells/ml) were cultured up to 72 hours in supplemented RPMI 1640 standard medium with various concentrations of AMPT-SA ranging from 0.1$5.0 \mu \mathrm{g} / \mathrm{ml}$ or with Lyso Tracker red and aminofluorescin-SA for means of counterstaining the lysosomes and evaluating the uptake of albumin into the lysosomes by confocal fluorescence microscopy. Within 24 hours efficient uptake of albumin into the lysosomes of all three cell lines could be demonstrated by colocalisation of up to $90 \%$ of aminofluorescin-SA with Lyso Tracker red in the lysosomes. Within 72 hours cell growth was inhibited $>50 \%$ in all cell lines at concentrations of $5.0 \mu \mathrm{g} / \mathrm{ml}$ AMPT-SA. In comparison with MTX-HSA the IC 50 could be achieved with AMPT-SA at half the dose. In accordance with animal studies, our results suggest that AMPT-SA might be a more effective albumin-bound antimetabolite than MTX-HSA, which is currently in clinical development.

\section{6}

\section{Expression of receptor activator of NF-kappa B ligand (RANKL)} by human myeloma cells

\section{Heider U., Jakob C., Zavrski I., Langelotz C., Eucker J., Schulz C.O.,} Possinger K., Sezer 0.

Department of Hematology and Oncology, Universitätsklinikum Charité, Humboldt-Universität, 10098 Berlin, Germany.

Objectives: Increased bone resorption is a hallmark of multiple myeloma (MM). It was proposed that myeloma cells produce osteoclast activating factors $(\mathrm{OAF})$, but these factors remained to be clarified. Recently, a system of cytokines was identified as key mediators in osteoclastogenesis, including the receptor activator of NF-kappa B ligand (RANKL) and osteoprotegerin (OPG). RANKL (synonym: TRANCE) is a member of the tumor necrosis factor (TNF) superfamily. It binds to its specific receptor, receptor activator of NF-kappa B (RANK), which is located on preosteoclasts RANKL induces differentiation, formation and prolonged survival of preosteoclasts. Osteoprotegerin acts as a decoy receptor of RANKL and inhibits its biological effects. It could be demonstrated that RANKL plays a crucial role in the pathogenesis of benign and malignant bone disease. Recent studies implicated that an increased RANKL/OPG ratio causes osteolytic activity in multiple myeloma. Therefore we analyzed the expression of RANKL on bone marrow plasma cells from patients with multiple myeloma and patients without cancer-induced bone disease.

Material and Methods: Bone marrow aspirates were derived from patients with multiple myeloma and individuals without malignant bone disease. Three colour flow cytometry was performed and myeloma cells were identified as CD38 strongly positive/ CD138 positive cells. RANKL expression was determined using a monoclonal antibody against human RANKL (R\&D systems). Additionally, immunocytologic staining of bone marrow from myeloma patients with anti-RANKL antibody was performed.

Results: By both methods, it could be shown that plasma cells from myeloma patients express RANKL. On plasma cells from patients without cancerinduced bone disease, no significant expression of RANKL could be detected.

Conclusions: These findings suggest that RANKL is produced by myeloma cells, leads to an imbalance in the RANKL/ OPG axis and promotes osteoclastogenesis. This is of clinical interest, since correcting the imbalance in the RANKL/ OPG system by osteoprotegerin could be an useful treatment of osteolytic bone disease in multiple myeloma.
Proteom mapping of myeloma cell lines and first comparison with highly enriched cd138+ malignant and normal plasma cells

Heumann K., Lannert H., Eckstein V., Lenze A., Ho A.D.

Department of Internal Medicine V, University of Heidelberg, Hospitalstr. 3, 69115 Heidelberg

Objectives: The term proteome was coined by Wilkins and Williams as meaning the entire protein complement of a given genome, that is, the proteins that are expresssed by the genome. Expressed proteins are typically modified in a variety of ways, such as phosphorylation and glycosylation. Proteomics attempts to catalog and characterize these proteins, compare variations in their expression level under different conditions, notably sickness versus health. This method allows to identify for example new epitopes for immunotherapeutic strategies. For that, we focus our proteom-investigations on two myeloma cell lines RPMI-8226 and IM9 to establish 2D-gelelectrophoresis and to evaluate optimal protein concentrations, followed by first comparisons between CD138+ highly enriched normal human and malignant myeloma cells.

Methods: A) Protein samples of human myeloma cell lines: RPMI-8226 and IM9 were at first analyzed. Sample preparation, determination of optimal protein concentrations, 2D-gel-electrophoresis with a 1D-separation isoelectric focusing (IEF) with Immobilized $\mathrm{pH}$ gradient (IPG) strips (17cm, pH 3-10), 2D-separation with SDS-PAGE were performed and described in Proteome Works System (BioRad) and via internet available. After silver staining, imaging and image analysis was performed by BIORAD's PDQuest-software 7.0.

B) Mononuclear cells were isolated by a standard Ficoll-Hypaque gradient separation method from aspirations of bone marrow from healthy donors and myeloma patients. A FACS Vantage SE cell sorter (BD) was used to highly enrich (>99\%) CD138+ (200kDa) cells.

Results: Using PDQuest a computer image analysis program for gel comparison, detection and quantitation of protein spots between the two myeloma cell lines and the fractions from BM of CD138+ cells. 1. Specific protein spots for metabolism of glycolysis (e.g. Glycerinalde 3-phosphate dehydrogenase, GAPDH, pI 8.9, MW $38.4 \mathrm{kDa}$ ) and Ig light chains by RPMI8226 and IM9 were detected. 1. The protein pattern of the both myeloma cell-lines are complex and differ slightly. The generation of a protein database of myeloma cells is running. 2. First pattern analyses of the CD138+ fractions from BM cells differ also between healthy donors and patients. Conclusion: We suggest proteomic analyses between normal and malignan CD138+ cells, myeloma cell lines respectively are an excellent tool for understanding the cell biology of this worse disease and to develop new therapeutic strategies.

\section{8}

A novel recombinant bispecific single-chain antibody, Wue-1xCD3, induces T-cell mediated cytotoxicity directed against autologous primary human myeloma cells

Hönemann D. (1,2), Kufer P. (3), Rimpler M. (1,2), Chatterjee M. (1,2), Bommert K. (1), Riechert F. (2), Wolf A. (4), Baeuerle P. (4), Riethmüller G. (3), Dörken B. (1,2), Greiner A. (5), Bargou R.C. (1,2)

(1) Max Delbrück Center for Molecular Medicine, Robert-Rössle-Str. 10 D-13122 Berlin-Buch, Germany, (2) Humboldt University of Berlin, University Medical Center Charité, Robert-Rössle-Klinik,, Department of Internal Medicine, Lindenberger Weg 80, D-13122 Berlin, Germany, (3) Institute of Immunology, University of Munich, Munich, Germany, (4) Micromet Inc., Munich, Germany, (5) Institute of Pathology, University of Würzburg, Würzburg, Germany

The development of antibody-based strategies for the treatment of malignant plasma cell disorders has been hampered by the fact that suitable plasmacell-specific surface antigens have been missing so far. Although normal and malignant plasmacells express a number of well characterized surface markers they all have turned out to be not plasmacell-specific. However, very recently a novel monoclonal antibody, designated Wue-1, has been generated, which specifically binds to the cell surface of normal and malignant human plasma cells. Therefore, Wue-1 represents an interesting and promising candidate to develop novel immunotherapeutic strategies for the treatment of multiple myeloma and immunocytoma. An interesting variant of an antibody-based strategy is the bispecific antibody approach. In particular, recombinant bispecific single-chain antibodies are interesting candidates that show exceptional biological properties discriminating these molecules from conventional antibodies. We generated a novel plasma cell-directed recombinant bispecific single-chain antibody, Wue-1xCD3 (bscWue- 
$1 x C D 3)$ and analyzed the biological properties of this antibody using the MM cell line NCI-H929 and primary cells from the bone marrow or peripheral blood of patients with multiple myeloma or immunocytoma and autologous or allogeneic T cells. We were able to show that bscWue-1xCD3 induces efficient T-cell mediated cell death of the NCI-H929 cells and primary myeloma or immunocytoma cells in 10/12 cases analyzed. Target cell lysis could be blocked by the parental monoclonal antibody, Wue-1, indicating the specificity of this effect. To our knowledge this is the first plasma cell-directed bispecific antibody described so far, showing promising result as new potential agent for the treatment of malignant plasma cell disorders.

\section{9}

\section{Deletion of chromosome $13 q$ is a frequent abnormality in multiple myeloma evolving from a preexisting monoclonal gammopathy of undetermined significance}

Kaufmann H. (1), Ackermann J. (1), Nösslinger T. (2), Gisslinger H. (1), Krömer E. (3), Ludwig H. (4), Drach J. (1)

(1) AKH Wien, Univ. Klinik für Innere Medizin I, (2) Hamuschkrankenhaus, III. Med. Abteilung, (3) Univ. Wien, Inst. für Medizinische Biologie, (4) Wilhelminenspital, 1. Med. Abt. mit Onkologie; Wien, Österreich

Objectives: Monoclonal gammopathy of undetermined significance (MGUS) remains a stable condition in the majority of cases, but it may progress to multiple myeloma (MM) or a related lymphoproliferative disorder with a probability of about $1 \%$ per year. Molecular features associated with this progression or indicating a high risk of transformation are still poorly characterized. We investigated at the cytogenetic level whether or not patients (pts) with MM evolving from a preexisting MGUS (MM postMGUS) would be different from those with ,de-novo“ MM.

Methods: Interphase fluorescence in situ hybridization (FISH) combined with the detection of cytoplasmic immunoglobulins was used to study chromosomal aneuploidy in the clonal plasma cell (PC) population. The staus of chromosome 13q14 was determined by probes specific for $\mathrm{rb}-1$ and/or D13S319.

Results: Between 1995 and 5/2002, 27 pts with MM post-MGUS were identified. Time from the first recognition of MGUS and progression to MM ranged between 2 and 17 years (median, 8 years). Among pts with MM post-MGUS, a deletion of chromosome 13q [del(13q)] was detected by FISH in 17 pts $(63 \%)$. This was significantly more common than frequencies of a del(13q) in MGUS ( 17 out of 50 pts $=34 \%$ ), asymptomatic MM (9 out of $23 \mathrm{pts}=39 \%$ ) and newly diagnosed $\mathrm{MM}$ at stage II/III wihout a previous history of MGUS $(102$ out of $245 \mathrm{pts}=42 \%)(\mathrm{P}<.01)$. In 9 of the 27 pts with MM post-MGUS, paired samples of bone marrow PC were available for a direct comparison of the chromosome 13q status between MGUS and MM post-MGUS. In 4 of these pts, chromosome $13 q$ was found to be normal in PC from both MGUS and MM. Five pts had a del(13q) in MM post-MGUS, and the abnormality was also found in the PC from the MGUS phase of the disease.

Conclusion: Our results indicate that $\operatorname{del}(13 \mathrm{q})$ is a freuent finding in MM post-MGUS. Del(13q) is an early event in monoclonal gammopathies and does not emerge at the time of progression from MGUS to MM.

\section{0}

\section{Macrophage inflammatory protein 1-alpha (MIP-1a) triggers migration and signaling cascades mediating survival and proliferation in multiple myeloma cells}

Lentzsch S., Gries M., Bargou R., Dörken B., Mapara M.Y.

Humboldt University of Berlin, Robert-Roessle-Klinik, 13125 Berlin, Germany

Introduction: Recently, several studies have suggested that MIP-1a contributes to the pathophysiology of multiple myeloma (MM). Thus, it has been demonstrated that MIP-1a is an osteoclast stimulatory factor and that MIP-1a neutralizing antibody ( $\mathrm{ab}$ ) or antisense inhibit bone destruction and reduce tumor load in a SCID-mice model of MM. Furthermore, MM patients have significantly higher bone marrow plasma levels of MIP-1a than healthy controls.

Results: The current study was designed to determine the direct effects of MIP-1a on MM cells. We found expression of MIP-1a and its receptor CCR5 in MM cell lines and patient-derived primary cells. Furthermore, we were able to demonstrate that MIP-1a acts as a potent growth, survival and chemotactic factor in MM cells. MIP-1a specific migration could be observed in a dose-dependent fashion up to 21 fold compared to control. MIP1a-induced signaling involved activation of the AKT/PKB and the MAPK pathway. In addition, inhibition of AKT activation by the PI3-Kinase (PI3-
K) inhibitors wortmannin or LY 294002 did not influence MAPK activation, suggesting that there is no crosstalk between MIP-1a-dependent activation of the PI3-K/AKT and ERK pathway.

Conclusion: Our data suggest that besides the role of development of osteolytic bone destruction, MIP-1a also directly affects cell signaling pathways mediating growth, survival and migration in MM cells and provide evidence that MIP-1a might play a pivotal role in the pathogenesis of MM.

\section{1}

Multiple bone marrow derived cytokines stimulate signaling cascades and mediate survival and proliferation in multiple myeloma (MM)

Lentzsch S., M. Chatterjee M., Mathas St., Mapara M.Y., Dörken B., Bargou R.

Humboldt University of Berlin, Robert-Roessle-Klinik, 13125 Berlin, Germany

Introduction: Survival and proliferation of MM cells are believed to be critically dependent on the specific conditions in the bone marrow microenvironment (BMM). Cell-cell interactions and soluble factors are crucial components in this process. Several studies have demonstrated IL-6-dependent activation of STAT-3 signaling pathways regulating proliferation and survival of MM cells. So far, IL-6 antibody therapies have failed to induce remissions in patients with MM questioning the singular role of IL-6 in MM. Results: In the present study we investigated the role of important cytokines and chemokines from the BMM and evaluated their effects on different signaling pathways (MAPK, PI-3K/Akt, STAT-3) relevant for MM. Using western blotting we investigated the ability of different cytokines (IL-6, LIF, VEGF, BFGF, SDF-1a, TNFa, MIP-1a, SCF, IL-1ß, IL-3) to activate these pathways. All of these cytokines activated MAPK pathway. Pretreatment with MEK1 inhibitor PD 98059 showed specific inhibition of cytokine-induced ERK1/2 phosphorylation. IL-6, LIF, VEGF, ßFGF, SDF-1a, MIP-1a and IL-1ß were able to activate PI-3K/Akt pathway. Cytokine in duced phosphorylation of Akt was completely abrogated by PI-3Kinase inhibitors. Phosphorylation of STAT-3 was only induced by IL-6 and LIF Furthermore we could show that cytokines (IL-6, LIF, VEGF, BFGF SDF1a, MIP-1a, IL-1ß), which activated both, MAPK and PI-3K/Akt pathway, were able to induce proliferation of MM cell lines and survival of primary MM cells.

Conclusions: In our study, we provide evidence that in addition to IL-6 a number of different BMM-derived factors might redundantly trigger important growth and survival-promoting pathways such as PI3-K/AKT and the MAPK in MM. Therefore, blocking of such pathways rather than blocking a single growth factor might be a promising approach to develop novel treatment strategies in MM.

\section{2}

Trisomies of chromosomes 1q, 9q, and 11q in multiple myeloma - non-random distribution of three highly frequent aberrations

Liebisch P. (1), Wellmann A. (1), Wendl C. (1), Roth K. (1), Benner A. (2), Stilgenbauer S. (1), Döhner H. (1)

(1) Medizinische Klinik III, Universitätsklinikum Ulm, (2) Zentrale Einheit Biostatistik, Deutsches Krebsforschungszentrum Heidelberg

Background: Deletion of chromosome 13q (13q-) is a powerful adverse prognostic parameter in multiple myeloma (MM). The true incidence and prognostic value of various other aberrations are still unknown. We developed a comprehensive DNA probe set for the systematic evaluation of chromosomal imbalances in the disease. Applying this probe set to a small series of pts., trisomies of chromosomes 1q, 9q, and 11q (+1q, +9q, +11q) were the most frequent chromosomal gains detectable.

Aims: 1 . Evaluation of the precise incidence of $+1 q,+9 q,+11 q$ by FISH 2 . Correlation of $+1 \mathrm{q},+9 \mathrm{q}$, and $+11 \mathrm{q}$ with $13 \mathrm{q}-$ as the most frequent and the only prognostically significant myeloma-associated aberration. Methods: PAC and BAC probes mapping to chromosome bands 1q21, 1q43, 9q34, 11q22.3-q23.1, 11q25, and 13q14 were used. FISH was combined with immunocytology for clear detection of plasma cells. Fisher's exact test, CART analysis and cluster analysis were used for statistical analysis.

Results: $+9 \mathrm{q},+11 \mathrm{q}$, and $+1 \mathrm{q}$ were detectable in 108/220 (49\%), 118/258 $(46 \%)$, and $85 / 213(40 \%)$ of tumors, respectively. Deletions of chromosomes $11 \mathrm{q} / 9 \mathrm{q} / 1 \mathrm{q}(11 \mathrm{q}-/ 9 \mathrm{q}-/ 1 \mathrm{q}-)$ were detectable in $3 \% / 0 \% / 1 \%$. 13q- was identified in 199/426 (47\%) of tumors. We did statistical analyses for 144 pts. from whom data on all four chromosomal loci were available. 13q- was significantly less frequent in pts. with +11 q (31\% vs. $71 \%$, p<.001, Fisher's 
exact test) and $+9 \mathrm{q}(40 \%$ vs. $66 \%, \mathrm{p}=.003)$. In contrast $13 \mathrm{q}$ - was significantly more frequent in pts. with $+1 \mathrm{q}(65 \%$ vs. $44 \%, \mathrm{p}=.02)$. CART analysis confirmed the non-random distribution of $+1 q,+9 q,+11 q$, and $13 q-$ in MM. Cluster analysis identified a normal $11 \mathrm{q}$ status and the combination of $+11 \mathrm{q}$ and $+1 q$ to be predictive for $13 q$ - in our series.

Summary: Together with $13 q-,+1 q,+9 q$, and $+11 q$ are by far the most frequent chromosomal aberrations in MM. The four aberrations exhibit a nonrandom distribution and therefore may point to novel pathways in MM biology. The high incidence of $+1 q$ in pts. with $13 q$ - might reflect $+1 q$ as a secondary aberration. The significantly lower incidence of $13 q-$ in pts. with $+11 \mathrm{q}$ might point to $+11 \mathrm{q}$ as an abnormality defining a subgroup of myeloma pts. with a more favorable prognosis. The prognostic significance of $+1 \mathrm{q},+9 \mathrm{q},+11 \mathrm{q}$ is currently evaluated in phase III multicenter treatment trials.

Supported by a grant from the Deutsche Krebshilfe to P.L. (70-2899-Li I)

\section{Treosulfan is an effective inducer of caspase-dependent} apoptosis in myeloma cells from bone marrow of patients

Meinhardt G. (1), Eppinger E. (1), Jahrsdoerfer B. (1), Oellerich M. (1), Baumgart J. (2), Schmidmaier R. (1)

(1) Klinikum der Universität München, Medizinische Klinik - Innenstadt, Department of Hematology/Oncology, Munich, Germany, (2) Medac $\mathrm{GmbH}$, Wedel, Germany

Multiple myeloma (MM) remains incurable despite the introduction of high-dose chemotherapy protocolls followed by autologous stem cell transplantation. Median survival after relapse is usually short with myeloma cells being largely resistant to conventional therapeutic strategies. We evaluated the efficacy of the alkylating agent treosulfan, that has been used in the treatment of ovarian cancer for over twenty years. Cell death was independently assayed by flow cytometry using unfixed cells (Annexin V/PI staining) or fixed cells (hypodiploid DNA content). Bone marrow or periphera blood cells from patients were stained with an antibody suitable for detection of the individual myeloma clone (anti-CD38, anti-CD56 or antiCD138) together with propidium iodide (PI).

In vitro assays using NCI-H929 and U-266 myeloma cells demonstrated a strong apoptosis-inducing effect of treosulfan. In NCI-H929 and U266 cells, a four day incubation resulted in more than $61 \%$ cells being apoptotic or necrotic with treosulfan concentrations of $10 \mathrm{uM}$ and $89 \%$ with $30 \mathrm{uM}$ (Annexin V/PI). Melphalan, used as reference compound, showed only a slightly stronger death-inducing effect at equimolar concentrations. Western blo analysis demonstrated cleavage of caspase- 3 and -9 , indicating activation of this apoptosis pathway. Interestingly, treosulfan dose-dependently downregulated the anti-apoptotic Bcl-2 homologue Mcl-1. Furthermore, treosulfan lead to upregulation of the cdk inhibitor p21 in NCI-H929 cells, but not in U-266 cells. To evaluate the efficacy of treosulfan on primary myeloma cells, mononuclear cells from bone marrow aspirates or from periphera blood of 7 multiple myeloma patients were incubated with $100 \mu \mathrm{M}$ treosulfan or melphalan for 48 hours. Melphalan induced cell death in 58\% (+/$28 \%$ ) of myeloma cells after 2 days, whereas treosulfan treatment resulted in $66 \%(+/-22 \%)$ of apoptotic/necrotic cells. Thus, at equimolar concentrations treosulfan is at least as potent as melphalan. Clinical trials have determined the MTD of treosulfan to be $47 \mathrm{~g} / \mathrm{m}^{2}$, whereas high-dose melphalan is commonly used at a maximal dose of $200 \mathrm{mg} / \mathrm{m}^{2}$. The toxic effect upon non-myeloma cells in the bone marrow samples was similar for both agents. Mature data including additional patient samples, statistical analysis and further studies with drug combinations will be presented at the meeting. Taken together, treosulfan has promising potential as a candidate drug for clinical studies in myeloma patients

\section{4}

Induction of myeloma specific cytotoxic T cells using dendritic cells transfected with tumor-derived RNA

Milazzo C., Reichardt V., Müller M.R., Grünebach F., Brossart P.

Medizinische Klinik II, Eberhard-Karls-Universität Tübingen

Objectives: We investigated myeloma RNA transfection of dendritic cells (DC) to induce myeloma specific cytotoxic T cell (CTL) responses in vitro. By this methodology we hope to bypass the need for the identification of shared MM associated antigens or specific antigens such as idiotype (Id). Material and Methods: Monocyte derived DC from buffy coats, which were matched in the HLA class I haplotype to the myeloma cell lines LP-1 and U266, were used for RNA transfection. DC were electroporated with total myeloma cell line RNA and were used as antigen presenting cells for the induction of myeloma specific CTL. After a single restimulation with RNA transfected DC, cytotoxic activity of induced T cells was ananlyzed in a standard 51-Cr release assay.

Results: We found that RNA transfected DC induce CTL that lyse the LP-1 and U266 myeloma cells. Cell line specificity was demonstrated by cold target inhibition assay and MHC class I restriction was revealed by antibody blocking studies. Interestingly, LP-1 specific CTL showed no specificity for the idiotype when Id pulsed autologous DC were used as CTL targets. Since MUC1 is described as a myeloma associated antigen, we investigated whether MUC1 specificity added to the lytic activity of U266 specific CTL. As corresponding epitopes we tested the described HLA-A2 restricted peptides M1.1 and M1.2 and found a striking fine specificity for M1.2, assuming a possible immunodominance of this peptide.

Conclusions: We report here on the induction of myeloma specific CTL by RNA transfection of DC. Since RNA encodes the whole antigenic repertoire of tumor cells, myeloma RNA transfected DC could serve as potential vaccine inducing a polyclonal CTL response against different immunogenic antigens.

\section{5}

\section{Targeting the RAS-to-MAPK signaling pathway in multiple myeloma}

Morgan M., Sebil T., Ganser A., Reuter C.W.M.

Dept. Hematology / Oncology, MH-Hannover, Carl-Neuberg-Str. 1, 30625 Hannover

Objectives: Disruption of RAS-to-MAP kinase (MAPK/ERK) signaling has been implicated in the molecular pathogenesis of multiple myeloma. Inhibitors of RAS posttranslational modification (FTase, REPase and PPMTase inhibitors), RAF and MEK are promising, novel types of nontoxic cancer therapeutics. Farnesyl transferase inhibitors (FTIs) are currently being evaluated in phase I-III clinical trials. In order to assess the effects of FTIs, geranylgeranyl transferase inhibitors (GGTIs) and MEK inhibitors in myeloma cells, six multiple myeloma cell lines were subjected to a panel of these inhibitors and assayed for viability, colony formation, cell cycle progression, MAP kinase kinase (MEK-1/2) activation and induction of apoptosis.

Material and Methods: Cell viability was quantified by trypan blue exclusion, MTT and colony formation assays. Activation of the RAS-to-MAPK pathway during cell cycle progression was quantified by a phospho-MEK specific FACS assay. Induction of apoptosis was monitored by Annexin VPE/7-amino-actinomycin D (7-AAD) and TUNEL FACS assays. RAS processing was monitored by Western blotting.

Results: Multiple myeloma cell lines which harbor K-RAS (OPM-2, RPMI8226) and N-RAS (L-363, NCI-H929) mutations were found to have higher amounts of cell-cycle-dependent phospho-MEK in G0/G1 and G2/M as compared to cell lines with wild-type RAS. Interestingly, highest amounts of phospho-MEK were observed in OPM-2 and NCI-H929 which also express high levels of mutationally activated $(\mathrm{K} 650 \mathrm{M})$ and wild-type FGFR3, respectively. Potent growth inhibition ( $>80 \%$ as compared to DMSO solvent control) was observed in cell lines treated with FTI-277 (4/6), FTI L744,832 (5/6), GGTI-286 (5/6), GGTI-298 (6/6), GGTI-2147 (6/6) and MEK inhibitor U0126 (6/6). Co-treatment of RPMI-8226 cells with FTI L744,832 and U0126 led to synergistic growth inhibition. Treatment of OPM-2 cells with the CAAX-based inhibitors FTI-277 and FTI L-744,832 $(20 \mu \mathrm{M}, 45 \mathrm{~h})$ lead to an increase of PP-MEK-1/2-positive cells in G2/M but a decrease in G0/G1, whereas FPP-based FTI FPT-3 induced apoptosis. In contrast to GGTIs, incubation of multiple myeloma cell lines with FTIs also caused inhibition of $\mathrm{H}$ - and N-RAS processing.

Conclusions: Our results demonstrate that disruption of the RAS-to-MAPK pathway on different levels inhibits multiple myeloma cell growth. These data support a potential therapeutic usefulness of these inhibitors in the treatment of multiple myelomas. 
Comparison of two flow cytometric methods for detection of aneuploidy in Multiple Myeloma: Implications for investigation of residual disease

Oelschlaegel U. (1), Nowak R. (2), Heider T. (1), Naumann R. (1), Ehninger G. (1)

\section{(1) Universitätsklinik Dresden, (2) Krankenhaus Chemnitz gGmbH}

Myeloma cells can be flow cytometrically characterized with a combination of aneuploidy detection and immunophenotyping. Two different cell preparation methods have been compared. With Cycloscope ${ }^{T M}$ measurements were performed after erythrocyte lysing, immunophenotyping with CD138/CD38 in one tube, and cell permeabilization with a detergent within $6 \mathrm{~h}$ after bone marrow aspiration. Our inhouse Ethanol method included immunophenotyping of mononuclear cells with CD138 or CD38 and a subsequent overnight permeabilization with ethanol. Propidium iodide was the DNA stain in both methods.

In 165 patients with Multiple Myeloma/MGUS 266 parallel investigations have been performed. The CV of G0/G1 peak, influencing the sensitivity of DNA quantification, was significantly higher with Cycloscope ${ }^{T M}$ (3.16 vs. $2.60 ; \mathrm{p}<0.001)$. Both methods revealed aneuploid plasma cells in $60 \%$ $(n=159)$ of all measurements. The percentage of aneuploid cells was higher in the Ethanol method because of enrichment after density gradient. Paired analysis resulted in a good correlation of the two methods $(r=0.85)$. Thus, DNA index was significantly higher with Cycloscope ${ }^{\mathrm{TM}}$ (1.27 vs. 1.19; $\mathrm{p}>0.001)$. Despite the different range of indices they correlated well in paired analysis $(\mathrm{r}=0.90)$. In $18 \%(\mathrm{n}=49)$ of investigations discrepant results were present. Additional aneuploid clones with Cycloscope ${ }^{\mathrm{TM}}$ were often rather false aneuploid $(n=29)$. On the one hand the aneuploid clone could not be detected again in further measurements of the same patient with active disease. On the other hand comparative multiparameter immunophenotyping showed only CD38++CD19+CD56- normal plasma cells which should not be aneuploid. In the remaining 11 investigations no explanation could be given for the additional aneuploid clone with Cycloscope ${ }^{\mathrm{TM}}$. In further 7 of 8 measurements with aneuploid cells, present only in the Ethanol method, this aneuploidy was confirmed with both methods in follow up investigations. In the latter patient group a low overall plasma cell conten was present or the aneuploid clone represented only a plasma cell subpopulation.

Summerizing data, both methods could be used for detection of aneuploidies in Multiple Myeloma at diagosis. Regarding residual disease detection the Ethanol method seemed to provide a higher specificity with lower false positive measurements on the one hand and higher sensitivity with less false negative results on the other hand

\section{7}

\section{Real-time PCR detection of tumor burden in patients with} multiple myeloma (MM) after allogeneic stem cell transplantation (allo-Tpx)

\section{Raab M.-S., Cremer F.W., Görner M., Ho A.D, Goldschmidt H., Moos M.}

\section{Department of Internal Medicine V, University of Heidelberg, Germany}

Introduction: Allo-Tpx after myeloablative conditioning regimen is a potentially curative approach in treatment of MM with high transplant associated morbidity and mortality. Non-myeloablative conditioning regimens are associated with less toxicity and are considered to achieve sustained complete clinical remissions (CCR) due to a graft-versus-myeloma effect. Objectives: The aim of this study is to investigate quantitatively tumor burden in bone marrow (BM) and peripheral blood (PB) samples and to look for molecular complete remissions (MCR) in patients (pts) with MM who underwent non-myeloablative allo-Tpx.

Methods: Real-time PCR assay (TaqMan) for quantitative analysis of minimal residual disease (MRD) was based on the rearranged IgH-genes. PCR primers and the TaqMan probe were developed using the CDR2- and CDR3- hypervariable regions of the IgH. DNA content of each sample was normalized to $10^{5}$ cells/reaction using a bcl-2 TaqMan assay. The detection limit amounted to $1 / 10^{5}$ cells.

Results: Reduced conditioning regimen contained TBI (2Gy) and fludarabine $\left(3 \times 30 \mathrm{mg} / \mathrm{m}^{2}\right)(4 \mathrm{pts}), 2 \mathrm{pts}$ received melphalan $140 \mathrm{mg} / \mathrm{m}^{2}$ and fludarabine $\left(3 \times 30 \mathrm{mg} / \mathrm{m}^{2}\right) .4$ pts had matched unrelated and 2 pts sibling donors. Prior to allo-Tpx 5 pts were in partial remission (PR), one in progressive disease (PD). $26 \mathrm{BM}$ and $46 \mathrm{~PB}$ follow-up samples (range, day +165 to +420 ) were analyzed. In $9 \mathrm{BM}$ and in $25 \mathrm{~PB}$ samples no tumor cells could be detected. In the remaining samples the tumor load ranged from $0.44 \%$ to $80.2 \%$ in $\mathrm{BM}$ and from $0.004 \%$ to $2.7 \%$ in PB. 2 pts achieved ongoing MCR from the first samples analyzed (follow-up day +215 and +388 re- spectively). One further pt attained MCR after donor lymphocyte infusion (DLI) due to PD followed by chron. GvHD. In two pts PD was accompanied by a rise of the tumor burden in BM seen 21 days (from MCR to $2,8 \%$ ) and 74 days (from $4 \%$ to $14,7 \%$ ) respectively earlier than the clinical diagnosis of PD. One pt with a steady tumor load in BM remained in PR (day $+165)$.

Conclusions: Feasibility of the real-time IgH-PCR assay was demonstrated by a high correlation with the clinical outcome. Precocious detection of PD could be shown with this assay providing the opportunity of earlier treatment intervention. MCR could be obtained by allo-Tpx with non-myeloablative regimen as well as after DLI followed by chron. GvHD. These findings have to be proven by longer follow-up investigations and larger numbers of pts.

\section{8}

Detection of clonal monosomy 13 or large monosomic deletions of $13 q$ in a selected sample of 32 multiple myeloma patients using interphase FISH

Wieland C. (1), Schneider P. (2), Hildebrandt B. (1), Redman A. (1), Royer-Pokora B. (1)

(1) Institute of Human Genetics and Anthropology, Heinrich Heine University of Duesseldorf, Germany, (2) Department of Haematology, Oncology and Clinical Immunology, Heinrich-Heine University of Duesseldorf, Germany

Objectives: The aim of our study was to screen a cohort of 32 multiple myeloma (MM) patients without cytogenetically visible chromosomal aberrations involving chromosome 13 for the presence of deletions in the chromosomal region $13 \mathrm{q} 14$ or monosomy 13 .

Material and Methods: BACs from the critical region shown to be deleted in CLL and in multiple myeloma, covering the markers D13S663E, D13S1168, D13S1220, D13S319 and D13S272 were used in interphase FISH. Recently, the BCMS gene was mapped in this region, and this gene is discussed as a candidate tumor suppressor gene for B-CLL. Further genes identified in this region are RFP2 and BCMSUN. The following 13q14 BACs were used: RPCI-11 236M15, RPCI-11 346I19, RPCI-11 34F20 and RPCI-11 480P3. In cases with a deletion, the FISH analysis was extended using the BACs RPCI-11 $203 \mathrm{I} 16$ (700 kb proximal to RPCI-11 236M15) and RPCI-11 40A8 (700 kb distal to RPCI-11 480P3) and further BACs from within or outside the interval, to characterise the extension of the deletion. FISH was performed on interphase nuclei of methanol/acetic acid fixed bone marrow samples of the patients using one 13q14 BAC indirectly labelled with digoxigenin together with a $13 \mathrm{q}$ telomeric probe (PAC clone GS 163 C9) indirectly labelled with biotin to analyse for monosomy 13 Hybridization of the probes was detected with anti-DIG-FITC and avidin$\mathrm{Cy} 3$ respectively. Only bone marrow samples of the first aspiration were analysed, containing $>20 \%$ plasma cells. We scored 300 nuclei for each probe set. Cut off values for all probes were determined on 4 control samples.

Results: We found monosomy 13 or large deletions in $13 q$ from $13 q 14$ up to and including the telomer in $40,6 \%$ (13) of the patients. Nine of these patients had a poor prognosis and eight of them showed monosomy 13. A deletion was found only in one patient, where the four 13q14 BACs used, were absent. Additionally the two BACs RPCI-11 203 I16 and RPCI-11 $40 \mathrm{~A} 8$ were found to be absent in interphase nuclei of the patient presenting with the deletion. The size of the deletion is at least $2.2 \mathrm{Mb}$.

Conclusions: The results indicate a correlation of monosomy 13 or of large monosomic deletions of $13 \mathrm{q}$ from $13 \mathrm{q} 14$ up to and including the telomer with poor prognosis in MM in our selected patient group. To prove this, statistical computation has to be done. Furthermore microdeletions in 13q14 are found very rarely ( 1 case out of $32(3,1 \%)$ ) in MM.

\section{9}

\section{The p70S6 Kinase is essential for the proliferation of human} malignant plasma cells

Wohlfarth A., Bakker F., Burger R., Guenther A., Gramatzki M.

Div. of Hematology/Oncology, Department of. Medicine III, University of Erlangen-Nürnberg, Erlangen, Germany

The phosphatidylinositol 3-kinase (PI3K)/Akt pathway can promote growth of different cell types by protecting them against apoptosis or by stimulating proliferation. In multiple myeloma (MM) PI3K can be activated by several cytokines (interleukin-6 [IL-6], insulin-like growth factor [IGF], hepatocyte growth factor $[\mathrm{HGF}]$ ) or by the loss of the tumor suppressor gene PTEN The activation of PI3K leads to phosphorylation and activation of the ser- 
ine/threonine kinase Akt. One of the downstream targets of Akt is mTOR, which activates p70S6 kinase (p70S6K), a promotor of cell cycle progression. We used five human malignant plasma cell lines (JK-6L, INA-6, L363, U266 and RPMI 8226) and specific inhibitors of either PI3K (LY294002) or mTOR (rapamycin) to study the role of the PI3K/Akt/p70S6K signaling pathway in MM. Incubation with LY294002 resulted in a dose dependen reduction of cell growth $(25-80 \%)$, as measured by $\left[{ }^{3} \mathrm{H}\right]$-Thymidine uptake Rapamycin also inhibited myeloma cell growth in a dose dependent manner $(72-98 \%$ at $2 \mathrm{nM})$ at concentrations significantly lower than those needed to block the growth of other hematopoetic tumor cell lines. Thus, activation of the PI3K/Akt/p70S6K pathway is essential for myeloma cell growth. We analyzed the DNA content of cells treated with inhibitors to study their effect on cell survival and proliferation. LY294002 treatment induced both a strong increase in the number of apoptotic cells as well as in the number of cells in the G1 phase. In contrast, treatment with rapamycin mainly resulted in a strong increase in the number of resting cells. Our results suggest that proliferation induced by PI3K is mediated by mTOR. Since myeloma cells are highly sensitive to rapamycin, inhibition of mTOR/p70S6K may provide an interesting therapeutic option for patients with multiple myeloma.

\section{0}

A comparison of the clinical applicability of three collagen I degradation products for the evaluation of myeloma-induced bone disease

Zavrski I., Jakob C., Heider U., Langelotz C., Schulz C.-O., Eucker J., Possinger K., Sezer $O$.

Department of Oncology and Hematology, Universitätsklinikum Charité, Campus Mitte, Humboldt-Universität Berlin, Germany

Objectives: Bone destruction is a hallmark of multiple myeloma. Unfortunately, myeloma-induced bone disease shows no correlation with classica biochemical parameters of the myeloma activity. In this study, we examined whether three bone resorption markers, N-terminal telopeptide (NTx) and desoxypyridinoline (DPD) in urine, and C-terminal pyridinoline cross linked telopeptide of type I collagen (ICTP) in serum reflect the extent of the bone destruction in patients with multiple myeloma. Recent investigations suggest that ICTP is a serum marker relatively insensitive to variations in normal bone turnover, while urinary NTx and Dpd appear to be more sensitive to variations of physiological bone turnover like age and menopausal status.

Design and Methods: Thirty seven patients with a newly diagnosed multiple myeloma, 20 females and 17 males, entered this study. None of them had received either chemotherapy or bisphosphonates prior to the study. NTx and DPD were measured in urine by competitive enzyme immunoassays and ICTP was measured from the serum samples using a radioimmunoassay. At the time of diagnosis, all patients underwent an x-ray examination of the skeleton.

Results: The levels for ICTP, NTx and DPD were significantly different between dichotomized groups of myeloma patients with pathologic fractures or osteolytic lesions versus no radiological signs of skeletal involvemen $(\mathrm{P}=0.005, \mathrm{P}=0.007, \mathrm{P}=0.001$, respectively). Furthermore, serum ICTP level differed significantly between the groups without osteolytic lesions versus only one osteolytic lesion versus two or more osteolytic lesions $(\mathrm{P}=0.02)$, whereas no significant difference could be found for NTx and DPD levels between these three groups. Patients with skeletal pain on percussion or compression had significantly higher ICTP levels than patients withou $(\mathrm{P}=0.018)$, while NTx and DPD were not significantly different between these groups.

Conclusions: The data suggest that serum ICTP is the best bone resorption marker for the evaluation of the severity of myeloma-induced bone disease. A possible reason for the differences between the three collagen I degradation products is the fact that the cleavage points of the ICTP molecule are mainly affected by proteases which are activated under pathological conditions. Thus ICTP could preferentially be used to monitor pathological type I collagen degradation in multiple myeloma.

\section{Minimal Residual Disease}

\section{1}

Detection and quantification of cytoceratin 20 positive cells from different human coloncarcinoma cell lines by RT-PCR as an alternative to immunocytochemistry

Suh Y.R., Ansorge N., Schmitz F., Felderbauer P., Ritter P.R., Ellrichmann M., Völker A., Hower M., Goetze O., Schmidt W.E., Baier J.E.

Klinikum der Ruhr-Universität Bochum, St. Josef-Hospital, Medizinische Klinik I

Objectives: Dissemination of carcinoma cells is a prerequisite for the spread of micrometastases and development of solid metastases. We compared the sensitivity of immunocytochemical detection for disseminated tumor cells with quantitative cytoceratin 20 (CK20)-RT-PCR.

Material and Methods: CK20 positive cells from human coloncarcinoma cell lines (colo320, HT29 and caco2) were diluted in phosphate buffered saline (PBS) or in a suspension of CK20-negative mononuclear cells (PBMCs), either aspirated from a peripheral vein or bone marrow. PBMCs were isolated by centrifugation through Ficoll-Paque ${ }^{\circledR}$ gradient, washed in PBS and adjusted to a density of 1.000 .000 cells $/ \mathrm{ml}$. Cytoceratin positive cell $(\mathrm{s}) / \mathrm{ml}$ from the different cell lines were added in serial dilution. Cellsuspensions were divided and CK20-positivity was determined by immunocytochemistry and CK20-RT-PCR: RT-PCR was performed with the Lightcycler $^{\circledR}$-system, quantity of CK20-specific mRNA was shown as normalized ratio. For immunocytochemistry cells were centrifuged onto a slide, stained with the pancytoceratin-antibody A45B/B3 and quantified by microscopy. Finally, the sensitivity of both detection methods were compared.

Results: Comparing the sensitivity of both methods, we found quantitative RT-PCR to be as sensitive as the detection by immunocytochemistry. mRNA could be detected down to $1 \mathrm{ng}$ mRNA of all cell lines. HT29-cells showed an up to 50-fold higher normalized ratio compared to caco2- and colo320-cells. Diluted in 100.000 PBMCs $/ \mathrm{ml} 100$ cells $/ \mathrm{ml}$ of HT29- and 1000 cells/ml of caco2- and colo320-cells could be detected by RT-PCR Identical results were found in HT29-cells diluted in bone marrow aspirate and could be reproduced by microscopy.

Conclusions: Comparing the sensitivity of the immunocytochemical detection with quantitative CK20-RT-PCR, we found RT-PCR to be a useful tool to detect and quantify disseminated cells of human colon-carcinoma celllines in an in vitro model. Interestingly, we could describe an up to 50-fold higher normalized ratio in HT29-cells compared to caco2- and colo320cells and a higher sensitivity for the detection of HT29-cells, showing that HT29-cells have a higher amount of specific mRNA encoding for CK20 and that quantification of disseminated tumor cells by RT-PCR must be carefully discussed concerning tumor-entity.

\section{2}

\section{Quantification of different MLL-fusion transcripts for MRD-research}

Böll I., Schoch C., Haferlach T., Hiddemann W., Schnittger S.

Laboratory for Leukemia Diagnostics, Department of Internal Medicine III, Klinikum der Universität München-Grosshadern, Munich, Germany

Detection of minimal residual disease (MRD) is of common interest for the evaluation of prognosis and for therapeutic stratification in acute lymphoblastic leukemia (ALL) and acute myeloid leukemia (AML). In both leukemias rearrangements of the MLL-gene are found: while MLL-AF4 and MLL-ENL-rearrangements prevail in ALL, MLL-AF6, -AF9, -AF10 and ENL-rearrangements predominate in AML. Furthermore, $6 \%$ of AML show partial tandem duplications (PTD) within the MLL-gene.

As shown in the AMLCG study, patiens with MLL-translocations achieve high $\mathrm{CR}$ rates but with respect to survival they are considered unfavourable, as they frequently relapse. For these AML subgroups, the MRD-situation is of great interest for the identification of risk profiles and for early detection of relapses.

In order to establish one method to quantify all heterogeneous MLL-fusion transcripts in one approach, we used primer and probes which were positioned in exon 6 of the MLL-gene, because exon 6 can be found in almost all of the fusion-transcripts. Reverse primers were specific for the partner gene. Thus, several heterogeneous rearrangements can be quantified by realtime PCR.

Detected sensitivities as determined by limited dilution assays were 1:1.000-10.000 for MLL-PTD, 1:1.000 for MLL-AF4 and 1:10.000 for MLL-AF6, -AF9 and -ENL. As shown for other fusion transcripts, sensitiv- 
ity of the MLL specific real-time PCR is significantly higher than cytogenetics and FISH-analysis and thus is the method of choice for MRD detec tion in this AML group. In total 42 samples with MLL-AF4 (primary diagnosis (PD): $n=7$; follow up (FU): $n=35$ ), 13 samples with MLL-AF6 (PD: $\mathrm{n}=7$; FU: $n=6$ ), 24 samples with MLL-AF9 (PD: n=16; FU: n=8), 19 samples with MLL-ENL (PD: n=3; FU: $n=16$ ) and 175 samples with MLLPTD (PD: $n=119$; FU: $n=56$ ) have been measured by now. Data suggest a high correlation with clinical outcome and offer the possibility to detect molecular relapses 3 months before clinical relapse.

\section{3}

\section{Design and evaluation of a real-time RT-PCR assay for the detection of minimal residual disease in MLL/AF9-positive acute myeloid leukemias}

\section{Breitinger H., Scholl C., Fröhling S., Schlenk R.F., Döhner H., Döhner K}

Department of Internal Medicine III, University Hospital of Ulm, Germany

Detection of minimal residual disease (MRD) in acute myeloid leukemias (AML) associated with specific gene fusions has been recognized as an important diagnostic tool for the assessment of response to treatment and the individual risk of relapse. Nevertheless, for most of the AML specific gene fusions the prognostic value of MRD still has to be determined. Real-time RT-PCR is a novel technique that allows the identification of MRD by quantification of the chimeric transcript. In this study, we designed a real-time RT-PCR assay for the quantification of the four common fusion types resulting from $\mathrm{t}(9 ; 11)(\mathrm{p} 22 ; \mathrm{q} 23)$ to evaluate the predictive value of MLL/AF9 fusion transcript quantification for disease relapse. All patients (pts) were treated homogeneously within the multicenter treatment trials AML HD93 and AML HD98-A. 114 samples [bone marrow (BM) $n=57$; peripheral blood (PB) $n=50$; leukapherese (LP) $n=7$ ] from 16 of 21 pts with $t(9 ; 11)$ positive AML were available for real-time analysis. For each different fusion type a specific probe set for real-time RT-PCR was established. The fusion transcript copy number in each sample was reported as the normalized value of MLL/AF9 per 104 transcript copies of a housekeeping gene, porphobilinogen deaminase, as control. No correlation was found between the percentage of $\mathrm{BM}$ or $\mathrm{PB}$ blasts and the copy number of the fusion product at diagnosis. Furthermore, the copy number at diagnosis was not predictive of subsequent clinical outcome. After double induction therapy the fusion transcript was not detected anymore in 7 pts. $5 / 7$ pts are still alive and in CR one died in CR because of GvHD and one relapsed 11 months after diagnosis. This pt received sequential high-dose cytarabine and mitoxantrone instead of autologous or allogeneic SCT. In contrast, 5 pts still had detectable levels of the specific fusion transcript after induction therapy. 4/5 relapsed within 8 months (range 3,6 to 8,6 months); and one died in CR three weeks after autologous BMT due to veno occlusive disease. In conclusion, our study demonstrates the feasibility of MRD quantification by real-time RTPCR in $\mathrm{t}(9 ; 11)$ positive AML. Furthermore, these data suggest that the assessment of MRD after double induction therapy may predict relapse probability. Larger prospective studies are necessary to establish the prognostic role of MLL/AF9 fusion transcript quantification in the clinical management of $\mathrm{t}(9 ; 11)$ positive AML.

\section{4}

Real-time PCR for immunoglobulin $\mathrm{H}(\mathrm{IgH})$ rearrangements using TaqMan chemistry together with LightCycler technology for quantification of minimal residual disease (MRD) in B cell malignancies

Fenk R., Ak M., Kobbe G., Schneider P., Steidl U., Hünerlitürkoglu A., Rohr U.P., Emde T., Bernhard A., Haas R., Kronenwett R.

Klinik für Hämatologie, Onkologie und klinische Immunologie, HeinrichHeine-Universität Düsseldorf

Background: Quantitative PCR approaches for the detection of residual tumor cells are used to assess treatment response. In B cell malignancies allele-specific (ASO) PCR for IgH provides a tool for detection of MRD. Methods: Real-time PCR with the LightCycler technology offers the opportunity to quantify IgH copies. The small size of the complementerity determining region 3 of the IgH gene often prohibits the use of a patient-specific ASO-primer together with ASO-hybridisation probes. Therefore, we developped an IgH-PCR with an ASO-TaqMan probe on the LightCycler system. Absolute clonotypic cell numbers were calculated using an external standard curve derived from dilution of plasmids carrying the clonotypic insert Values were normalized by parallel detection of beta-actin copy numbers in the corresponding sample.
Results: Standard curves were calculated for five patients by linear regression with correlation coefficients of $r>0.98$ and clonotypic cells could be quantifyed with high specificity and a sensitivity of $10 \mathrm{E}-4$ to $10 \mathrm{E}-5$. A significant pearson-correlation of $\mathrm{r}=0.93$ for inter- and $\mathrm{r}=0.98$ for intraassay analysis indicates the reproducibility of the assay. Accuracy was tested with dilutions of the U266 cell line in normal peripheral blood mononuclear cells showing similiar sensitivity and reproducibility. After validation of the assay the kinetics of residual tumor burden were analysed in 4 patients with multiple myeloma and immunocytoma who achieved at least partial remission after high-dose chemotherapy and autologous peripheral stem cell transplantation (PBSCT). Intraindividual analysis of IgH copies / beta-actin copies in samples from bone marrow and peripheral blood showed a significant $(\mathrm{p}<0.03)$ reduction of clonotypic cells from the time of active disease to best response and also a significant $(\mathrm{p}<0.03)$ increase from remission to progressive disease. In one patient a continuous increase of residual tumor cells in the peripheral blood was observed 3 month before relapse.

Conclusions: Quantification of MRD in B cell malignancies can be reliable performed with real-time IgH-PCR using ASO-TaqMan probes, which can be adapted to the LightCycler system. This method can be used to evaluate the prognostic significance of residual malignant cells after therapy. This could be the rationale for treatment stratefications on a molecular level.

\section{5}

Clonal expansion of $\mathrm{T}$ lymphocytes in aplastic anemia identified by single cell techniques

Franzke A., Piao W., Czwalinna A., Ganser A.

\section{MH-Hannover}

Background: Although aplastic anaemia (AA) is of unknown aetiology, in vitro data and clinical responsiveness to immunosuppressive agents sugges that AA represents a T-cell mediated disease in a high proportion of patients. However, neither the immune response nor the nature of recognised target antigen(s) have been well characterised. For a better understanding of the initiating immune process in AA several groups focussed on the analysis of T-cell repertoire and yielded controversial results. So far, T cell clonality was exclusively studied in $\mathrm{T}$ lymphocyte bulk population which may mask the pathological $\mathrm{T}$ cell clone.

Aims: Therefore, we applied a single cell technique for studying $\mathrm{T}$ cell clonality in order to determine whether an antigen-driven response is involved in the development of bone marrow failure.

Methods: Single CD3+ T cells were isolated form 3 severe aplastic anaemia patients both from the bone marrow and peripheral blood prior to immunosuppresive therapy. T cell receptor $\beta$ gene rearrangements were amplified by seminested two step PCR covering all V $\beta$ and J $\beta$ families. Direct DNA sequencing was performed for PCR products to verify functional TCR $\beta$ chain rearrangements.

Results: About $90 \%$ of the isolated CD3 $+\mathrm{T}$ cells gave rise to a specific PCR product. In healthy controls 24 different $\mathrm{V} \beta$ and $13 \mathrm{~J} \beta$ gene segments families could be amplified. More than 70,40 , and 30 rearrangements could be detected in bone marrow derived T cells $(87,34,11$ in T cells of peripheral blood, respectively) of aplastic anaemia patient A, B, and C. In bone marrow 34, 27, 25 (in peripheral blood 41, 19, 16, respectively) potentially functional rearrangements were obtained from patient $\mathrm{A}, \mathrm{B}$, and $\mathrm{C}$ identified by DNA sequencing. Compared to the $\mathrm{V} \beta$ gene rearrangement of the healthy controls, the analysed aplastic anaemia patients show a highly restricted $T$ cell repertoire. Interestingly, a predominance of TCR V $\beta 13$ was found in all of the analysed aplastic anaemia patients: 13/34 (A), 6/27 (B), and 10/25 (C) in bone marrow derived T cells. The deduced amino acid sequences of the CDR3 region demonstrates in 1 out of 3 patients studied a clear clonal expansion.

Conclusion: These results show a restricted $\mathrm{T}$ cell repertoire with common predominance of $\mathrm{V} \beta$ and $\mathrm{J} \beta$ gene segments in patients with aplastic anaemia suggesting a pathological T lymphocyte response to shared antigen(s).

\section{6}

\section{Quantitative RT-PCR for circulating melanoma cells}

Keilholz U., Goldin-Lang P., Letsch A., Max N., Thiel E.

\section{UKBF, FU-Berlin}

A number of specific genes encoding for melanosomal proteins are selectively expressed in melanocytes and melanomas. The expression of the tyrosinase gene is most widely used for detection of circulating melanoma cells. 
Utilizing internal plasmid standards, we now established true quantitative RT-PCR assays for tyrosinase, MelanA/MART1 and gp100 employing the LightCycler system, which is accurately quantitating the mRNA signal ove a range of 1 to 10.000 tumor cells per $\mathrm{ml}$ of blood. This quantitative RTPCR system further facilitates quality control by exact quantitation of housekeeping RNA for every sample.

Ten melanoma cell lines, 20 tumor tissue specimens and 186 periphera blood samples from patients with cutaneous $(n=122)$ or uveal $(n=64)$ melanoma were analysed and compared to 21 control blood samples. In melanoma cell lines and tissue, the expression of all three markers is in a similar order of magnitude and slightly higher as compared to PBGD housekeeping gene expression. In peripheral blood, there is low background expression of gp100 (ratio to PBGD housekeeping gene up to $6.6 \times 10 \mathrm{E}-5$ ) and MelanA/MART1 (up to $2.2 \times 10 \mathrm{E}-5$ ) in healthy control blood samples. Tyrosinase is undetectable in healthy controls. Therefore, positive tyrosinase expression in melanoma patients was judged as a positive result whereas positive findings for MelanA/MART1 and gp100 were only judged as positive, if they exceeded the healthy volunteer background by factor 2 . Under these provisions, out of 186 blood samples from melanoma patients 58 were positive for tyrosinase, 54 for MelanA/MART1, and 7 for gp 100 There was high correlation between tyrosinase and MelanA/MART1. Quantitatively, the interpatient variability for each marker was surprisingly limited.

In conclusion, real-time RT-PCR offers excellent quality control options by quantifying housekeeping gene expression. MelanA/MART1 expression may be a more sensitive marker for melanoma, whereas the advantage of tyrosinase is the absence of detectable background expression. The assay system is now being applied for monitoring of clinical multicenter trials.

\section{7}

BCR-ABL positive colony forming progenitors persist in chronic myeloid leukemia patients in complete cytogenetic remission after treatment with imatinib

Merx K. (1), Lohmann J. (1), Müller M.C. (1), Sellhorst A. (1), König H. (1), Kreil S. (1), Paschka P. (1), Lahaye T. (1), Schoch C. (2), Gschaidmeier H. (3), Hehlmann R. (1), Reiter A. (1), Hochhaus A. (1)

(1) III. Medizinische Klinik, Universitätsklinikum Mannheim, (2) Medizinische Klinik III, Klinikum Großhadern, München, (3) Novartis Pharma GmbH, Nürnberg, Germany

Objectives: A significant proportion of patients (pts.) with chronic myeloid leukemia (CML) achieve a complete cytogenetic remission (CCR) after therapy with the selective tyrosine kinase inhibitor imatinib. To determine the source of residual disease detected in CML pts. in CCR on imatinib therapy, we have tested CFU-GM colonies grown from bone marrow (BM) mononuclear cells for BCR-ABL mRNA using a nested multiplex RT-PCR. In parallel, quantitative assessment of residual disease was performed by conventional cytogenetics and hypermetaphase fluorescence-in-situ-hybridization from BM samples, and real time RT-PCR for BCR-ABL transcripts from peripheral blood specimen.

Patients and Methods: Twelve CML pts. in $\mathrm{CP}$ were investigated, results from six pts. (4 male, 2 female, median age 54.5, range 31.0-64.0 years) are available. All pts. were in CCR after 75-631 days (median 251) of imatinib therapy. The presence or absence of BCR-ABL mRNA in CFU-GM was determined by a nested multiplex RT-PCR that simultaneously amplifies $\mathrm{BCR}-\mathrm{ABL}$ and normal $\mathrm{BCR}$ transcripts as internal control for adequate RNA/cDNA quality.

Results: In four pts. hypermetaphase FISH was negative investigating $77-$ 304 metaphases. The median ratio BCR-ABL/ABL detected by real time RT-PCR in PB specimens was $0.18 \%$ (range $0.019-2.5 \%$ ). The median number of colonies analyzed from each patient was 55 (range 76-40), of which 93\% (range 18-100\%) were informative. BCR-ABL positive CFUGM colonies were detected in all six patients (median $2.3 \%$, range 1.8 $33.3 \%$ ).

Conclusions: We conclude that residual disease detected by real time RTPCR in patients with complete cytogenetic response after therapy with imatinib is derived at least in part from clonogenic myeloid cells. These clonogenic cells may have the potential to contribute to subsequent relapse.
Comparative analysis of the immunocytochemical detection of minimal residual disease (MRD) of solid tumors using the manual microscopy and an automated cellular imaging system

Obeid W., Henrich S., Seifart U., Loechelt C., Wachtel A., Jaques G.

Klinikum der Phlipps Universität, Klinik für Hämatologie, Onkologie, Immunologie

Objectives: The presence of occult tumour cells, especially in the mononuclear cell fraction of bone marrow has been reported to represent an important prognostic indicator for patients with solid tumours. The role of the manual microscopy for the detection of the occult tumour cells is a standard and traditional method in pathology by detecting single epithelial cells in stained slides. The accuracy and the sensitivity of this labour-intense method depends on well trained eyes of the pathological reviewers. The automated cellular image analysis system (ACIS, ChromaVision Medical Systems, Inc.) was developed to identify and enumerate tumour cells in different sample types (bone marrow, peripheral blood) to save time and effort. Material and Methods: : Slides used in this study were prepared from specimens of bone marrow and leucapheresis products. Specimens consisted of normal bone marrow $(n=289)$, normal leucapheresis products $(n=672)$ both as negative controls, bone marrow from patients with small cell lung cancer (SCLC) $(n=1392)$ and with other solid tumors $(n=40)$, leucapheresis products from patients with solid tumors $(n=520)$ and with non-solid tumors $(\mathrm{n}=800)$. The slides prepared by cytocentrifugation of mononuclear cells were stained immunocytochemically with the monoclonal antibody against Pan-Cytokeratin (Ck), Epithelspecific Antigen (Esa) and Human Epithelspecific Antigen (HEA) and detected with the chromogen New Fuchsin as substrate. The slides were analysed with the manual microscope by three different reviewers.

Results: By manual microscopy we found in 116/1392 slides positive cells in bone marrow of patients with SCLC. No positive cells were found in $(n=289)$ bone marrow samples of patients non-solid tumors. In leucapheresis products $(n=520)$ of patients with solid tumours no positive cells were detected, but in 13/800 leucapheresis products of patients with non-solid tumours the reviewers found positive cells. By ACIS we found in 145/1392 slides positive cells in bone marrow of patients with SCLC. Slides prepared from $9 / 289$ bone marrow samples in the group of patients with nonsolid tumours showed positive cells. In leucapheresis products from patients with solid tumors 14/520 were positive, while in 32/800 slides from leucapheresis products from non-solid tumours positive cells were found.

Conclusions: Our results demonstrate that ACIS was in relation to the manual microscopy more sensitive and detected more often positive cells.

\section{9}

Kinetics in circulating tumor cells and prognosis in lung cancer after surgery

Pachmann K., Günzel R., Rolle A., Paachmann U.

Friedrich Schiller Universität Jena Klinik für Innere Medizin II, Transfusionsmedizinisches Zentrum Bayreuth und Fachkrankenhaus Coswig $\mathrm{GmbH}$

Whereas in hematological malignancies in most cases the main part pf the tumor consists of circulating tumor cells, solid tumors were long regarded as being exclusively local. The formation of metastases was regarded as a randomly occurring rare event. There is, however, increasing evidence that circulating tumor cells in solid tumors can be observed more frequently than expected so far.

Tracing circulating epithelial cells, we were able to detect such cells in $90 \%$ of lung cancer patients before surgery. The manipulation of the tumor did mobilize additional epithelial cells in $77 \%$ of patients and led to a reduction of the circulating cell count only in $23 \%$ of patients.Five months later a further increase in the number ov these cells was observed in $55 \%$ of patients (3/7 with initial reduction and 14/23 with initial increase). 5 relapses have occurred after now up to $11 / 2$ years observation, all of them in the group with continuous post-surgery increase in circulating epithelial cell numbers. Thus, this is the first report to our knowledge, indicating that there is a correlation rather of cell kinetics than of only the number of circulating tumor cells with prognosis and that an increase in these cells is predictive for early relapse. 
Molecular remission predicts progression-free survival in mantle cell lymphoma after peripheral blood stem cell transplantation

Pott C. (1), Schrader C. (1), Derner N. (1), Siebert R. (2), Harder S. (2), Brüggemann M. (1), Ritgen M. (1), Dreger P. (3), Kneba M. (1)

(1) Second Department of Medicine, (2) Institute of Human Genetics, University of Kiel, (3) AK St. Georg, Hamburg, Germany

Mantle cell lymphoma (MCL) is characterized by a poor response to cytostatic therapy and a short survival with a median of less than 3 years. Highdose chemotherapy followed by autologous peripheral stem cell transplantation (PBSCT) provides a potential curative option for the treatment of younger patients. The prognostic relevance of MRD detection after intensive conventional treatment and high dose therapy has been proven for follicular lymphoma. Aim of this study was the quantitative evaluation of MRD status and the evaluation of its prognostic impact on disease free survival and long-term remission of patients with mantle cell lymphoma after peripheral blood stem cell transplantation (PBSCT).

Material and Methods: 17 patients with histologically and cytogenetically confirmed MCL stage IV received high-dose chemo-radiotherapy with endoxan and TBI after initial therapy with 3-6 cycles CHOP and subsequent stem cell mobilisation with DexaBEAM. Quantitative MRD assessment was performed by RQ-PCR 3,6 and 12 months after PBSCT with clonal IgHCDR3 allele specific oligonucleotides (ASO) and a consensus JH primer/probe combination. Only patients with a minimum sensitivity of $1 \times 10-4$ were included in survival analysis.

Results: All patients (6/17) with clinical and molecular complete remission after PBSCT (sensitivity $5 \times 10-5$ to $10-5$ ) are still in complete remission (median observation time 41,5 months (range 16-92 months). All 11 patients with positive MRD results have relapsed regardless of clinical remis sion status after PBSCT. Median progression free and overall survival (OS) after PBSCT were 21 (10-42) and 41 (13-65) months in the MRD+ group. PFS as well as OS were significantly higher for patients with molecular remission $(\mathrm{p}=0.0015$ and $\mathrm{p}=0,0184)$.

Conclusions: The data indicate that molecular remission is a very strong prognostic factor predicting progression-free and over all survival in MCL. MRD quantitation may be used for risk assessment and possible early allogeneic treatment protocols including early non-myeloablative SCT in the situation of low tumor burden.

\section{1}

Monitoring of residual PML/RARA fusion transcripts in acute promyelocytic leukemia (APL) by quantitative real-time RT-PCR

Saußele S. (1), Weißer A. (1), Walz Ch. (1), Reiter A. (1), Büchner T. (2), Hochhaus A. (1), Willer A. (1), Hehlmann R. (1), Lengfelder E. (1)

(1) III. Medizinische Klinik, Fakultät für Klinische Medizin Mannheim der Universität Heidelberg, (2) Medizinische Universitätsklinik, Münster

The $t(15 ; 17)$ translocation is the cytogenetic hallmark of APL. At the molecular level it results in two chimeric fusion genes PML/RARA and RARA/PML. RT-PCR methods have been developed for monitoring residual PML/RARA fusion transcripts after therapy. PCR positivity (RT-PCR sensitivity of 10E-4) in hematological remission is associated with relapse. PCR negativity is of uncertain prognostic value, as relapses may also occur in PCR negative patients. To improve the monitoring of APL patients, we established a rapid and reliable real-time RT-PCR approach for the detection and quantification of residual PML-RARA transcripts using the LightCycler technology.

We retrospectively and prospectively analysed 416 samples (87 at diagnosis, 329 under follow-up) of 102 patients with APL by quantitative real-time PCR and compared the results with qualitative nested PCR and with clinical outcome.

All samples tested negative by real-time PCR were also negative by nested PCR (234/416). Of the 145 samples tested positive by nested PCR 143 were positive by real-time PCR. Two follow-up samples were negative possibly due to a reduced cDNA quality. In the remaining 37 samples with negative nested PCR, the real-time PCR was positive on very low levels (PMLRARA/ABL ratios $0.00015 \%$ to $2.6 \%$ ). This is likely due to a higher sensitivity of real-time PCR. Although the expression levels varied extensively from $707 \%$ to $6.6 \%$ at diagnosis, the amount of transcript levels in the follow-up samples correlated well with a decrease of the PML-RARA/ABL ratios under chemotherapy in all patients. In 8 patients with hematological relapse or recurrence of nested PCR positivity, both methods showed positive results. In four of these patients follow-up samples were available. The re- sults of follow-up PCRs in these patients correlated well to the therapy steps with a decrease under chemotherapy and an increase of transcript levels in the intervals without therapy.

We conclude that i) the established real-time PCR for PML/RARA is a reliable, fast and sensitive method for detection and monitoring of minimal residual disease in APL patients; ii) follow-up investigations in prospective studies are needed to estimate the prognostic influence of initially differen expression levels and of low expression levels under therapy; iii) as shown in the relapsed patients, the results of quantitative real-time PCR can contribute to decision-making in therapy.

\section{2}

Prognostic value of immunological minimal residual disease in adult lymphoblastic leukemia

Schieder H., Pries A., Schmitz N., Sonnen R.

Abteilung f. Hämatologie, AK St. Georg Hamburg

Objectives: In contrast to childhood acute lymphoblastic leukemia the prognostic value of immunological minimal residual disease (MRD) in adult lymphoblastic leukemia ( ALL) is still point of discussion. Objective of this study was to compare results of immunological MRD with morphologic and molecular findings in order to determine their prognostic value.

Material and Methods: 24 patients with ALL were treated according to current studies ( BMFT-ALL 6/99, 5/93). Bone marrow cytology, molecular MRD and two colour flow cytometry were used in sequential monitoring of MRD. Primarily used antibody combinations were CD 22/10,CD34/19, TdT/CD79a for B-lineage ALL and TdT/cCD3 (sCD3), CD1a/CD2 forTlineage ALL. Immunological MRD was definded as existence of pathological antibody levels in more than $0,09 \%$ of absolute cell count. Per patient 4 8 samples were examined, in 4 cases molecular MRD results were available.

Results: Six patients who reached immunological CR $(<009 \%$ of cells) which was confirmed by molecular MRD in 3 cases, are still in CR after end of treatment. Maximum observation time of disease free survival is 12 month. Inspite of morphological CR 2 patients showed phenotypic MRD and are still under maintainance therapy. Four patients had high dose chemotherapy in first morphological CR because of high risk disease ( e.g. B-precursor-ALL). All of them showed immunological MRD. Six patients with immunological MRD relapsed, 4 of them still under treatment. Absolute ( $\geq 0,3-1 \%$ of absolute count ) and follow up values of of MRD levels differed from patients staying in stable CR. Thus stable MRD $\geq 0,3 \%$ or increasing levels are correlated with an instable remission which was confirmed by later relapses in this study.

Conclusions: Immunological CR correlates with stable CR. Molecular MRD confirms this findings in most cases. Therefore immunological MRD detection has a prognostic relevance and is quick and less expensive in contrast to molecular MRD. Further evaluations with own data are planned to confirm reported results.

High transcription rates of fusion genes at diagnosis, low reduction rates during therapy as well as increases of transcription rates after therapy identify prognostically unfavorable subgroups in PML-RARA-, AML1-ETO-, or CBFB-MYH11-positive acute myeloid leukemia

\section{Schnittger S. Weisser M., Schoch C., Kern W., Hiddemann W., Haferlach T.}

Laboratory for Leukemia Diagnosis, Department of Internal Medicine III, Ludwig-Maximilians University of Munich, University Hospital Grosshadern, Munich, Germany

PML-RARA-, AML1-ETO- and CBFB-MYH11-fusion mRNAs are detectable in $20 \%$ of acute myeloid leukemias (AML) at diagnosis and are associated with a favorable prognosis, however, $10-30 \%$ of these patients relapse. Quantitative real time PCR provides a tool to exactly quantify fusion mRNA expression rates over a wide dynamic range. Using this method we have analyzed 296 such patients (pts) at diagnosis and 246 pts during and after therapy with a median of 4 samples/pt and a median follow up of 10 months (total analyses: $n=654$ ). The level of fusion gene expression at diagnosis varied substantially up to 2000 fold. A lower level correlated with a better overall survival $(\mathrm{p}=0.017)$ as well as with a better relapse free survival $(\mathrm{p}<0.001)$. Only eight of $100 \mathrm{pts}$ analyzed during the first four months of therapy had a tumor reduction of less than $3 \log$ and all relapsed. In contrast, only five of the 92 pts with tumor reduction of more than $3 \log$ within four months of therapy relapsed. In addition, eight pts showed increasing levels of expression during follow up and all relapsed. In conclusion, pa- 
tients with a high risk of relapse can be identified by 1) high levels of fusion gene expression at diagnosis; 2) less than $3 \mathrm{logs}$ of tumor reduction during the first four months of therapy; 3 ) increase of at least one log of the expression level. For early detection of relapses follow up intervals should not exceed three months.

\section{4}

Therapy control by quantitative analysis of minimal residual disease in patients with mantle cell lymphoma and diffuse large cell lymphoma

\section{Schueler F., Doelken M., Kiefer T., Doelken L., Hirt C., Doelken G.}

Ernst-Moritz-Arndt-Universität Greifswald, Klinik für Innere Medizin C, Hämatologie/Onkologie, Sauerbruchstr., D-17487 Deutschland

We report the use of a real-time quantitative PCR to analyze minimal residual disease (MRD) by determining the number of the lymphoma specific clonal immunoglobulin heavy chain rearrangement in patients with mantle cell lymphoma (MCL) and diffuse large cell lymphoma (DLCL).

122 samples (peripheral blood mononuclear cells, bone marrow and stem cell preparations) from six patients with MCL and 125 samples from two patients with diffuse large cell lymphomas were tested using the $A B$ PRISM SDS 7700 device. The maximum sensitivity of this assay is one IgH copy in $10^{\wedge} 5$ to $10^{\wedge} 6$ normal cells based on a control gene (K-ras) amplification in real time. The follow up duration was between 6 to 36 months. The patients with MCL were treated either with conventional chemotherapy regimens or autologous or allogeneic stem cell transplantation. All these patients had circulating mantle cells in their peripheral blood at first diagnosis that could be reduced but not eliminated neither by conventional chemotherapy regimens nor by autologous stem cell transplantation. All stem cell preparations were contaminated by lymphoma cells. One patient received an allogeneic (matched unrelated donor) stem cell transplantation. This patient had a molecular relapse after allogeneic transplantation. He was successfully treated with the monoclonal antibody rituximab. 4-8 weeks in advance the time of all clinical relapses a significant increase of circulating mantle cells was detected by this sensitive method.

The two patients with DLCL had one or more relapses after autologous stem cell transplantations that could be monitored by quantitative MRD analyses. Both received an allogeneic stem cell transplantation. One patient had a molecular and consecutive clinical relapse after the allogeneic transplantation and died from relapse. The other patient had no detectable circulating lymphoma cells after transplantation but died soon from transplant related complications.

The quantitative detection of circulating lymphoma cells in mantle cell lymphoma and DLCL during follow up reflects the clinical course of these diseases. The amount of contaminating lymphoma cells in stem cell preparations can be evaluated. Relapses are associated with increasing numbers of circulating lymphoma cells before the clinical relapse becomes evidence. Therefore, the therapy can not only be controlled by MRD analyses but also initiated even earlier. This might improve disease free or overall survival.

\section{5}

\section{The use of housekeeping genes for quantification of minimal residual disease in acute myeloid leukemia by real time} RT-PCR

Weisser M., Haferlach T., Schoch C., Hiddemann, Schnittger S.

\section{Medizinische Klinik III, Klinikum Grosshadern, LMU München}

Quantification of minimal residual disease (MRD) is of major interest in modern hematology and oncology. The most sensitive method for detection and quantification of MRD is polymerase chain reaction (PCR). Most approaches to quantify MRD rely on relative quantification. This means that the gene expression of the target gene is normalized to the expression of an endogenous control, the a so called housekeeping gene. We tested the feasibility of four commonly used housekeeping genes - cABL, glucose-6-phosphate dehydrogenase $(\mathrm{G} 6 \mathrm{PDH}), \beta 2$-microglobulin $(\beta 2 \mathrm{M})$ and porphobilinogen deaminase (PBGD) - for the quantitative analysis of AML1-ETO fusion transcripts in $\mathrm{t}(8 ; 21)$ positive acute myeloid leukemia (AML). First we examined the expression of AML1-ETO relative to the four control genes in seven patients at primary diagnosis. The expression level of the tested genes were normalized against each other. All tested control genes showed significant correlation to one another. The expression of PBGD, $\beta 2 \mathrm{M}$ and $\mathrm{cABL}$ showed the highest correlation (correlation coefficient (CC) $0,96-0,99)$ while G6PDH demonstrated the least correlation to the other genes (CC 0,88-0,93). Second we analyzed the expression of AML1-ETO relative to the four different genes in seven follow up samples of two pa- tients with AML. In the follow up samples all house keeping genes showed highly significant correlation (CC 0,999$)$. Finally we analyzed the expression of the four genes in the peripheral blood of seven healthy individuals in parallel. The expression level in these samples was quantified absolutely using standard curve analysis. G6PDH, cABL and $\beta 2 \mathrm{M}$ were expressed at stable levels in all volunteers. In contrast PBGD revealed a rather inhomogeneous expression in peripheral blood and did not correlate with the other control genes. Based on these data we suggest to use CABL for quantification of fusion genes in AML. Because

1) In primary diagnosis cABL belongs to to three genes with the best correlation coefficients with G6PDH being the worst.

2) Based on the results of normal blood samples PBGD was not recommendable, because of its variable expression.

3) $\beta 2 \mathrm{M}$ was expressed at levels not in the same range as AML1-ETO.

4) $\mathrm{cABL}$ demonstrated the most stable expression and the level is in the range of AML1-ETO in diagnostic material.

\section{Solid Tumors}

\section{6}

Differences in the assessment of recurrences after radiotherapy and chemotherapy with positron emission tomography (PET) using 18-fluorodesoxyglucose (FDG) in an experimental tumour system

de Wit M. (1), Raabe A. (2), Seegers B. (1), Buchert R. (3), Hossfeld D.K. (1)

(1) Medizinische Klinik II, (2) Abteilung für Experimentelle Radioonkologie, Radiologische Klinik, (3) Abteilung für Nuklearmedizin, Radiologische Klinik, Universitätsklinik Eppendorf, Hamburg

Positron emission tomography (PET) using 18-fluorodesoxyglucose (FDG) in the assessment of recurrences after radiotherapy (RT) and chemotherapy (CT) in an experimental tumour system.

Purpose: The study was performed to determine the influence of time and tumour volume after RT and CT on the sensitivity and specificity of FDGPET, and the benefit of FDG-PET concerning early recognition of recurrences in an experimental tumour system.

Methods: Subcutaneously growing rhabdomyosarcoma R1H of the rat were treated either with radiotherapy (total doses of 80 or $85 \mathrm{~Gy} ; 30$ fractions in 6 weeks) or with chemotherapy for a median of 8 applications ( 3 per week, range 3-20) using vincristine or doxorubicine or ifosfamide monotherapy or in combination. Tumour volume was determined twice a week by measuring the tumour in two perpendicular dimensions by vernier calipers. PET was performed weekly before, during and for 6 months after therapy using a conventional full-ring whole body PET-Scanner (Siemens CTI Ecat/Exact). Animals received 4-11 MBq FDG s.c. $3 \mathrm{~h}$ prior to PET acquisition. The PET results were evaluated qualitatively using a 6 score scale. PET results and actual tumour volume were compared. Sensitivity and specificity were calculated and the time difference was determined for recurrences diagnosed by volumetry compared to FDG-PET.

Results: After irradiation 8/20 tumours (40\%) were controlled locally, whereas chemotherapy did not achieve local control. Recurrent tumours were detectable by volume measurements when they reached a volume of $0.1 \mathrm{ccm}$. The intra-observer concordance of tumour diagnosis by PET was reproducible with a kappa coefficient of 0.89 (95\% C.I.: 0.83...0.94). Sensitivity increased with time, reflecting the increase in volume of recurring tumours. For recurrences of $0.2 \mathrm{ccm}$ neither false negative nor false positive PET results were obtained, whereas for tumours of $0.1 \mathrm{ccm}$ the sensitivity was $91 \%$ and the specificity was $67 \%$. Following irradiation tumours were recognised by PET $31,62,74$, and 81 days (median) before approaching volumes of $0.2 \mathrm{ccm}, 0.5 \mathrm{ccm}, 0.8 \mathrm{ccm}$, or $1.0 \mathrm{ccm}$, respectively. Chemotherapy did not achieve local tumour control and recurrences developed earlier Consequently time benefit of FDG-PET was smaller with 3, 12, 13, and 20 days (median) before approaching volumes of $0.2 \mathrm{ccm}, 0.5 \mathrm{ccm}, 0.8 \mathrm{ccm}$, or $1.0 \mathrm{ccm}$, respectively.

Conclusion: The experimental system allows reproducible detection of R1H-tumours using FDG-PET. The quality of FDG-PET as a diagnostic test is very good for tumours of more than $0.1 \mathrm{ccm}$, or if FDG-PET is performed later than 6 weeks after the end of irradiation. 
Markov model: Second- and third-line chemotherapy options for advanced ovarian cancer

\section{Fedders M. (1), Hartmann M. (2), Schneider A. (1), Oelschläger H. (3)}

(1) Klinik für Frauenheilkunde, (2) Apotheke des Klinikums, (3) Institut für Pharmazie, Lehrstuhl für pharmazeutische Chemie, Friedrich-SchillerUniversität Jena

Markov models are useful when a decision problem involves risk that is continuous over time, when the timing of events is important, and when important events may happen more than once. The ability of a Markov model, shown as a Markov-cycle tree, to represent different strategies and the time dependence of survival probabilities, utilities and costs allows an accurate representation of ovarian cancer settings that involve these issues. Consider a 55 years old patient, after optimal debulking surgery, with advanced ovarian cancer FIGO stage II-IV and is receiving adjuvant chemotherapy. For this patient a specific example is shown for two different second- and thirdline chemotherapy options after first-line chemotherapy with Paclitaxel/Carboplatin.

\section{8}

Ifosfamide, carboplatin and etoposide (ICE) as second-line treatment is active in pretreated progressive soft tissue sarcoma (STS)

Fiegl M., Schlemmer M., Wendtner C.-M., Abdel-Rahman S., Hiddemann W., Issels R.D.

\section{Medizinische Klinik III, Universitätsklinikum Großhadern, München}

Objectives: To evaluate the toxicity and efficacy of ifosfamide, carboplatin and etoposide (ICE) as second-line chemotherapy in chemo-pretreated STS Material and Methods: 20 patients with either locally advanced $(n=9)$ or combined metastatic $(n=11)$ disease showing progressive disease (PD) to previous doxorubicin/ifosfamide-based first-line chemotherapy were treated with four cycles of ICE (ifosfamide $1,5 \mathrm{~g} / \mathrm{sqm}$, carboplatin $100 \mathrm{mg} / \mathrm{sqm}$, etoposide $150 \mathrm{mg} / \mathrm{sqm} \mathrm{d} 1-4)$ with G-CSF ( $5 \mu \mathrm{g} / \mathrm{kg} / \mathrm{bw})$ support repeated at day 28. In addition regional hyperthermia was applied at day 1 and 3 (60min, Tmax: $42,5^{\circ} \mathrm{C}$ ) in 13 patients with locally advanced tumor in combination with ICE.

Results: A median of 4 cycles of ICE was given. Hematological toxicity (grade III: $n=9$, grade IV: $n=5$ ) occured in $70 \%$ of patients. Overall objective response rate (PR: $n=4)$ was $20 \%$. The other patients showed mixed response $(n=2)$, stable disease $(n=5)$ or PD $(n=9)$. After a median follow-up time of 10 months, the median time to progression was 3 months. Probability of overall survival for all patients was 11.7 months with nine patients beeing alive at present.

Conclusions: With significant but acceptable risk of toxicity the ICE regimen in combination with G-CSF shows promising efficacy as second-line therapy in patiens with chemo-pretreatred STS.

\section{9}

Role of surgery in patients (pts) with liver metastases (LM) from testicular germ cell tumors (GCT)

Hartmann J.T. (1), Oechsle K. (1), Rick O. (2), Gauler T. (3), Schöffski P. (4), Schleicher J. (5), L. Kanz (1), C. Bokemeyer (1)

(1) Medizinische Universitätsklinik Tübingen, (2) Charite Campus Mitte Berlin, (3) Universität Essen, (4) MH Hannover, (5) KH Stuttgart, Germany

Objectives: The presence of LM represents an independent poor prognosticator for pts with GCT.

Material and Methods: Data of 43 pts with metastatic GCT who had undergone liver resection were reviewed.

Results: Median age was 29 yrs (range, 18-54) at the time of diagnosis of LM. 35 pts $(81 \%)$ were initially diagnosed with LM and advanced GCT. 8 pts had metachronous LM occurring after a median interval of 16 mos (range, 6-103). All pts received chemotherapy (CT) prior to the resection of LM, $81 \%$ as first-line and $19 \%$ as second-line CT. 15 pts had a single liver lesion $(35 \%)$ and 28 pts $(65 \%)$ a disseminated liver involvement. Extrahepatic metastases were present in 38 pts $(88 \%)$ including retroperitoneal LN (86\%), lungs (74\%), mediastinal LN (23\%), bone (5\%), CNS (5\%) or other (21\%). $56 \%, 72 \%$ and $63 \%$ of pts had elevated AFP, HCG and LDH marker concentrations, respectively. Remission status after CT prior to liver resec- tion revealed PR m- in 28 pts (65\%), PRm+ 10 pts (23\%) and PD in 5 pts (12\%). 5 pts (12\%) had isolated LM after CT. 38 pts $(88 \%)$ had residual tumor masses in the retroperitoneum $(48 \%)$, in the lungs $(42 \%)$, mediastinal LN (5\%) and other sites (5\%). Liver surgery included enucleation/single segment resection in 29 pts $(68 \%)$ and hemihepatectomy/resection of $>3$ segments in 10 pts $(23 \%)$ (n.e. $n=4 / 9 \%)$. None of the pts died of postoperative complications. The rate of relevant surgical complications was $9 \%$ One pt required re-operation because of hepatic bleeding. Resection of LM yielded necrosis in $67 \%$, teratoma in $12 \%$ and vital carcinoma in $21 \%$. In 32 pts further resections have been performed revealing necrosis in $63 \%$, teratoma $(28 \%)$ and vital cancer in $9 \%$. In 12 of 32 pts $(38 \%)$ histologic results differed among LM and resections at other locations. After a median follow up of 23 mos (range, 8-216) 34 pts are alive, 28 with NED and 6 with persistent disease. Calculated 5-yr OS was 76\% (CI95\%, 58-93\%). 9 pts died of disease, 3 pts due to PD of LM and 6 pts due to systemic PD. Univariate analysis identified mediastinal $\mathrm{LN}$ involvement and refractoriness to $\mathrm{CT}$ as negative prognostic factors.

Conclusions: Surgery either following induction or second-line CT is a feasible approach and morbidity is mainly dependent on the extent of surgery. The high rate of vital carcinoma in liver specimen, different histologic results at residual tumor locations and the observed survival rate support a multidisciplinary approach including resection of all masses at any location.

\section{0}

\section{Is TuM2-PK able to discriminate reactive and malignant mesothelial cells?}

Hastka J. (1), Metzgeroth G. (1), Rebel M. (2), Bohrer M. (2), Maywald O. (1), Hehlmann R. (1)

(1) Universität Heidelberg, (2) III. Medizinische Universitätsklinik Mannheim

Pyruvate kinase (PK) is one of the key enzymes of the anaerobe glycolysis, which is expressed in various isoforms in different tissues. The majority of tumors express a certain isoenzyme of PK, called tumor M2-PK (TuM2$\mathrm{PK})$. This isoenzyme exists in an active tetrameric and an inactive dimeric form. The dimeric form is strongly overexpressed in tumor cells and due to its release to body fluids, it has proven to be a sensitive tumor marker in different human carcinomas. The aim of our study was to evaluate the use of $\mathrm{Tu}$ M2-PK in the discrimination between reactive and malignant mesothelial cells in immunohistochemistry.

We examined the expression of TuM2-PK on formalin-fixed, paraffin-embedded tissue sections of 3 cases of a malignant mesothelioma and 2 cases of a reactive mesothelial proliferation by immunohistochemistry. We used a monoclonal antibody, which recognizes only the dimeric form of TuM2PK (clon DF4, dilution $1: 10$, ScheBo/Giessen) and the ABC-POX visualization method (Vectastain/Vector). To improve the staining, heat-induced antigen retrieval was performed prior to the immunohistochemistry.

All of the examined cases revealed unequivocal positive reaction of the mesothelial cells, indicating the expression of TuM2-PK in reactive mesothelial proliferation and malignant mesotheliomas as well. The most intensive staining was observed in one of the cases with reactive mesothelial proliferation.

Our results show, that TuM2-PK can not be used for discrimination between reactive mesothelial proliferation and malignant mesothelioma, beeing expressed by both reactive and malignant mesothelial cells.

\section{1}

\section{XPA protein and resistance to chemotherapy in germ cell tumours of the testis}

Honecker F.U. (1,2), Mayer F. (2), Oosterhuis J.W. (1), Bokemeyer C. (2), Looijenga L.H.J. (1)

(1) Pathology/Laboratory for Exp. Patho-Oncology, Erasmus Medical Center, Josephine Nefkens Institute, 3000 DR Rotterdam, The Netherlands, (2) Department of Oncology, Hematology, Immunology, and Rheumatology, University of Tuebingen Medical Center, 72076 Tuebingen, Germany

Introduction: Germ cell tumours (GCTs) of the testis are the most common tumours in male adults aged 20-45 years. Their exceptional sensitivity to chemotherapy - more than $80 \%$ of all patients with distant metastases can be cured with cisplatinum-based therapy - has been ascribed to low levels of XPA, leading to DNA repair deficiency in the nucleotide excision repair (NER) system. 
Objectives: To assess the role of XPA in clinical resistance of GCT to chemotherapy.

Material and Methods: Immunohistochemistry (IHC) was performed to as sess XPA expression in two different groups of patients with GCT with documented clinical course: relapse-free patients $(n=62)$ with a follow-up $\geq 3$ years, and patients with refractory tumours $(n=23)$ that died of disease. Copy numbers of the XPA-gene were assessed by FISH analysis in cases with high XPA levels. In addition, Western blot analysis was performed to investigate XPA protein levels after exposure to cisplatin in different GCTderived cell lines, both sensitive and resistant to cisplatin

Results: Differences in XPA protein levels were observed between differen GCT histologies with increasing frequency in more differentiated components: positivity of cases was $30 \%$ in embryonal carcinoma, $51 \%$ in seminoma, $70 \%$ in yolk sac, $75 \%$ in choriocarcinoma and $100 \%$ in teratoma. No amplification of the XPA gene was found by FISH analysis. No differences in XPA levels were found between the two groups of responding and nonresponding patients. XPA was detected in $65 \%$ of cases in continuous remission, and in $70 \%$ of patients with resistant disease. Tumours resected at relapse after primary chemotherapy ( $n=7,6$ of them yolk sac tumours) in the refactory group were all positive for XPA. In contrast, XPA was not induced in cell lines on repeated courses of sublethal doses of cisplatin in vitro. Compared to other cell lines (MCF7, HeLa) levels of XPA were low, as assessed by Western blot analysis.

Conclusions: Differences in the levels of XPA were found between differen histologies, suggesting that XPA expression is regulated during differentiation, but no differences could be shown between chemotherapy-sensitive and -resistant tumours. Overall, it seems unlikely that this protein of the NER system plays a clinically relevant role in treatment resistance of GCT.

\section{2}

Report on a patient with a granulosa/theca cell tumor of the ovary successfully treated with Imatinib (Glivec) - 15 months of follow up

Jakob A., Geiger R., Hirsch F.W.

Klinikum Offenburg, Medizinische Klinik II

Objectives: Imatinib is a new type of tyrosine kinase inhibitor that selectively inhibits various tyrosine kinases including ABL, BCR-ABL, KIT and PDGF receptors. Earlier studies have shown that Imatinib is highly effective in the treatment of chronic myeloid leukemia and in gastrointestinal stromal tumor. Granulosa cell tumors are composed of granulosa cells with or with out an admixture of theca cells. In our case, immunostaining revealed overexpression of c-KIT by the tumor cells. Fewer than $10 \%$ of all ovarian tumors are of stromal origin. These tumors tend to have a more indolent course. However, once granulosa cell tumors have disseminated, there is no effective systemic therapy available.

Material and Methods: Case report: A 87-y-old woman presented to another hospital with abdominal pain (01/00). There was a palpable mass in her abdomen and a CT-scan showed a tumor of the left ovary with suspected peritoneal spread. A laparatomy was performed and the tumor with the left adnexes and an infiltrated part of the small bowel were resected. Histologic workup revealed a granulosa/theca cell tumor infiltrating the small bowe wall. The patient received no additional treatment. 10 months later a sonographic examination of the abdomen showed multiple metastases in the liver. First the patient received a hormonal therapy (letrozole), thereafter a chemotherapy with idarubicin. Both therapies were ineffective and in April 2001 we started treatment with Imatinib (Glivec 100-200mg per day).

Results: The patient improved significantly. The pattern of response resembled that which was seen in patients with gastrointestinal stromal tumors The lesions within the liver melted down, the LDH level decreased from 603 to $397 \mathrm{U} / \mathrm{l}$ and the liver enzymes markedly improved.

Conclusions: This is - to our knowledge - the first case in literature of a granulosa cell tumor with a durable response to treatment with the novel tyrosin kinase inhibitor Imatinib. We recommend immunostaining for c-KIT expression in such tumors and a clinical trial should be initiated.
Hepatic Doppler perfusion index (DPI) for detection of liver metastasis (LM) in cancer patients (CP) - A prospective controlled trial using color Doppler ultrasound (DCDU)

Lueders C., Metzner D., Wiefel M., Karthaus M.

Med. Klinik II, Ev. Johannes-Krankenhaus Bielefeld, 33611 Bielefeld

Early detection of LM remains a challenge. LM can be sensitively detected by ultrasound (US). Previous studies have shown that subtle changes in liver perfusion may precede US detection of LM in colo-rectal cancer (CRC). An increase in A. hepatica (AH) blood flow $(\mathrm{BF})$ and thus a change in liver perfusion can be measured using DCDU. Data comparing DCDU in CRC to other tumors are rare. This prospective trial evaluated DCDU for the detection of liver metastasis in $\mathrm{CP}$ with $\mathrm{CRC}$ in comparison to other tumors and a healthy control $(\mathrm{HC} ; \mathrm{n}=34)$. BF profiles were obtained with DCDU from the main portal vein (VP) and AH. DPI, defined as ratio of AH to total liver blood flow, was calculated. Clinical records, biopsy results, CT- or MRIscans were reviewed to confirm diagnosis of LM. From Feb 2001 to May 2002 , a total of 130 consecutive CP $(68 \mathrm{~m} / 62 \mathrm{f})$ entered this prospective trial. Pts had CRC $(n=40)$ and other solid tumors $(n=90)$, with overt LM in 37 pts (14 CRC/23 other tumors).

Results: In LM, a 3-fold increase in AH blood flow was observed (0.39 $1 / \mathrm{min} \mathrm{LM} \mathrm{vs} 0.201 / \mathrm{min}$ CP vs $0.11 \mathrm{l} / \mathrm{min} \mathrm{HC)}$. Mean DPI was 0.26 in $\mathrm{LM}(\mathrm{SD} 0.17)$ vs $0.14 \mathrm{CP}(\mathrm{SD} 0.1)$ vs $0.09 \mathrm{HC}(\mathrm{SD} 0.06)$. There was no difference in DPI between CRC and other tumor pts with LM (0.27 vs 0.27). Furthermore, DPI did not differ between CRC and other cancer pts without LM (0.17 vs 0.16$)$.

Conclusion: Our results suggest that DCDU measurement of the DPI and $\mathrm{AH}$ may give helpful further information for detection of LM in CP that may be useful for CRC as other tumor pts.

Prognostic factors for survival and factors associated with long-term remission in patients with advanced melanoma receiving cytokine-based treatments: Second analysis of a randomized EORTC Melanoma Group trial comparing interferon-a2a (IFNa) and interleukin 2 (IL-2) with or without cisplatin

Keilholz U. (1), Martus P. (2), Punt C.J.A. (3), Kruit W. (4), Mooser G. (5), Schadendorf D. (6), Liénard D. (7), Dummer R. (8), Koller G. (9), Voit C. (10), Eggermont A.M.M. (4)

(1) Med Klinik III, UKBF, FU Berlin, (2) Biometrie, UKBF, FU Berlin, (3) AZL Nijmegen, (4) DDHK Rotterdam, (5) Dermatologie, Uni Ulm, (6) Dermatologie UKRV, Berlin, (7) CPO, Lausanne, (8) Dermatologie, Uni Zürich, (9) Dermatologie, Uni Salzburg, (10) Dermatologie, Charité, Berlin

Purpose: To define prognostic factors for survival, and especially for longterm survival in a mature data set of patients with stage IV melanoma treated within a randomised trial of cytokine based protocols.

Methods: Long term follow up data on patients enrolled into an EORTC trial comparing IFNa plus IL-2 with or without cisplatin were collected. Univariate and multivariate Cox regression analysis is performed to define prognostic factors for survival. Characteristics of patients alive at 2 and 5 years after randomisation are compared to the entire cohort using $\mathrm{c} 2$ test. Results: The minimum potential follow up of the 131 evaluable patients is five years. 18 patients (14\%) were alive 2 years after randomisation, and 11 $(8 \%) 5$ years after randomisation. Pretreatment performance status (PS), serum lactate dehydrogenase (LDH) and tumor mass are significant predictors for survival, whereas site of metastases and number of sites are not significant. PS and LDH are the only independant prognostic factors. All except one patient alive at 2 and 5 years had a pretreatment PS of $100 \%$, and only 3 long term survivors had elevated pretreatment LDH. There is no association of site of metastases with long term survival. Response to treatment is a major predictor for long term survival, whereas addition of cisplatin did neither impact on overall survival probability nor long term survival.

Conclusion: The probability of long-term survival in stage IV melanoma patients after IL-2 based treatments is governed by pretreatment PS, serum $\mathrm{LDH}$, and response to treatment. Site of metastases, the basis for the M-subcategories of the new AJCC staging system, is not informative in this study. 
Influence of hypoxia on the efficacy of chemotherapeutic agents in germ cell tumor derived cell lines

Koch S., Mayer F., Bokemeyer C.

Medizinische Uniklinik Tübingen, Klinik für Hämatologie, Onkologie, Immunologie und Rheumatologie, Eberhard Karls Universität Tübingen

Objectives: Germ cell tumors (GCTs) of the male are very sensitive to chemotherapy. Nevertheless, $10-15 \%$ of the patients fail to achieve a longterm remission with available treatment options. A reduced oxygen tension/hypoxia has been described to limit the efficacy of various cytotoxic drugs and promote formation of metastasis. For cisplatin, the most active drug for GCTs, and mitomycin C, improved efficacy in hypoxia has been found in various models. Tumor oxygenation depends on the hemoglobin content of the blood, correction of tumor-associated anemia improves the $\mathrm{pO}_{2}$ in tumor tissue. In order to evaluate the preclinical rationale of raising the hemoglobin-level in anemic GCT patients, the impact of hypoxia on the efficacy of cytotoxic drugs commonly used in the treatment of GCTs was studied in embryonal carcinoma cell lines.

Material and Methods: The cell lines NT2, 2102 Ep and NCCIT were tested for their sensitivity towards cisplatin, oxaliplatin, carboplatin, gemcitabine, bleomycin, etopophos, irinotecan, and mitomycin $\mathrm{C}$ under normoxic (20\% $\mathrm{O}_{2}$ ) and hypoxic (continuous flow of $94 \% \mathrm{~N}_{2}, 5 \% \mathrm{CO}_{2}$, and $1 \% \mathrm{O}_{2}$ ) conditions using the MTT-assay. IC50 and IC90 under both conditions were compared. The doubling time of the cells were analysed.

Results: All drugs tested were less effective under hypoxic conditions, including mitomycin C (e.g. increase in IC50 in normoxia vs. hypoxia for 2102 Ep: 9.3fold) and cisplatin (2102 Ep: 5.5fold). The effect depended on both the drug and the cell line (NT2/cisplatin: 2fold; NCCIT/cisplatin 3.4fold; NT2/gemcitabine: 1.3fold; 2102 Ep/gemcitabine: 1.85fold NT2/carboplatin: 5.3fold). Cells showed a reduced doubling time under hypoxic conditions $(\mathrm{NT} 2<\mathrm{NCCIT}<2102 \mathrm{Ep}$ ), that did not correlate with the relative resistance. In addition NT2 cells showed signs of neuronal differentiation in hypoxia.

Conclusions: These results show that all tested cytotoxic agents including cisplatin and mitomycin $\mathrm{C}$ are more efficient under normoxic than hypoxic conditions in GCT cell lines. The relative resistance for a given compound depends on characteristics of the cells and on the drug, it does not correlate strictly with proliferation. A reduced cell growth (e.g. $2102 \mathrm{Ep}$ ) and a somatic differentiation (NT2) under hypoxia are potential mechanisms to explain the relative resistance. These results support the rationale to improve hemoglobin concentrations in patients with GCTs during chemotherapy.

\section{6}

\section{Peg-liposomal doxorubicin, etoposide, ifosfamide and regional hyperthermia (EILA + RHT) in the treatment of high risk soft tissue sarcomas: A phase I/II-study}

Lindner L.H. (1), Dreyling M. (1), Wendtner C.M. (1,2), Sauer H. (1), Hiddemann W. (1), Issels R.D. $(1,2)$

(1) Department of Internal Medicine III, Klinikum Grosshadern Medical Center (KGMC), (2) GSF - National Research Center for Environment and Health, Munich, Germany

Feasibility and activity of PEG-liposomal doxorubicin $\left(\right.$ Caelyx $\left.{ }^{\circledR}\right)$, etoposide and ifosfamide combined with regional hyperthermia (RHT) in the treatment of adult patients with high-risk soft tissue sarcomas (STS) will be assessed. To our knowledge, this is the first clinical trail where PEG-liposomal doxorubicin is combined with RHT for the treatment of soft tissue sarcomas. Additionally, the influence of RHT on serumpharmacokinetics of PEG-liposomal doxorubicin will be analysed.

PEG-liposomal doxorubicin $\left(\mathrm{Caelyx}^{\circledR}\right)$ is a novel drug formulation with a prolonged circulation time and preferential extravasation at tumor sites. In the treatment of STS it demonstrated equivalent activity to free doxorubicin with an improved toxic profile (Judson et al. 1999). First shown in the treatment of metastatic breast cancer, PEG-liposomal doxorubicin combined with RHT could have synergistic potential (Park et al., Proc. ASCO 2001). Kouloulias et al. demonstrated a strong correlation between average temperature and clinical response in the treatment of recurrent breast cancer with Caelyx ${ }^{\circledR}$ and hyperthermia (Clin. Cancer Res. 2002). RHT is suggested to increase the delivery of liposomal doxorubicin into solid tumors by increasing blood flow. Additionally, RHT could increase leakage of doxorubicin from liposomes, either by corruption of tumor microcirculation and consecutive $\mathrm{pH}$-decrease resulting into physicochemical destabilisation of the liposomal envelope or by direct membrane destabilisation at transition temperature.
In Phase I Caelyx ${ }^{\circledR}$ was started at a dose of $25 \mathrm{mg} / \mathrm{m}^{2}$ (dose level 1). All patients received two sessions of hyperthermia treatment $\left(60 \mathrm{~min}, \mathrm{~T}>42^{\circ} \mathrm{C}\right)$. The first session within 1-2 $\mathrm{h}$ of Caelyx ${ }^{\circledR}$-Infusion to increase liposome extravasation into the tumor and the second session $48-72 \mathrm{~h}$ after infusion to increase intratumoral doxorubincin release. Etoposide $125 \mathrm{mg} / \mathrm{m}^{2}$ was given on days 1 and 4 , and ifosfamide $1500 \mathrm{mg} / \mathrm{m}^{2}$ on days 1 to 4 . Thermochemotherapy was repeated every 3 weeks, a total of four courses was given before assessment of tumor response. Dose escalation of Caelyx ${ }^{\circledR}$ will be performed until DLT is reached. Currently, dose level 2 (Caelyx ${ }^{\circledR} 35$ $\mathrm{mg} / \mathrm{m}^{2}$ ) is investigated.

\section{7}

Molecular determinants of treatment response in human germ cell tumors

Mayer F. (1), Oosterhuis J.W. (2), Looijenga L.H.J. (2), Bokemeyer C. (1)

(1) Medizinische Universitätsklinik Tübingen, (2) Josephine-NefkensInstitute, Erasmus Medical Center Rotterdam

Objectives: Germ cell tumors (GCTs) are highly sensitive to cisplatin-based chemotherapy. The molecular basis of this feature is unclear. It is also unknown, by which means the intrinsically chemotherapy resistant mature teratomas (MTs) and a minority of refractory GCTs achieve their resistant phenotype. Various cellular pathways may influence the efficacy of chemotherapy. Their impact has not been investigated in a comprehensive study of tumor samples from clinically defined subgroups of GCT patients. Material and Methods: We investigated proteins involved in regulation of apoptosis, cell cycle control, DNA-mismatch repair (MMR), drug export and inactivation immunohistochemically on tumor samples of three patient groups: unselected patients $(n=20)$, patients with metastatic disease in continuous complete remission $(\mathrm{n}=12)$, and chemotherapy refractory patients $(n=24)$. MT components $(n=10)$ within samples from all groups were analyzed separately. The apoptotic index was studied by TUNEL-assay.

Results: Invasive GCTs of all groups showed a correlation between the fraction of P53 positive cells and apoptotic index ( $\mathrm{rs}=0.66, \mathrm{p}<0.001)$. The levels of the anti-apoptotic proteins BCL-2 and BCL-XL were low. P21 was hardly detectable and not correlated with P53-expression ( $r s=0.29, p=0.07)$. Refractory tumors had lower levels of MMR factor MLH1 in 35\%, the remainig cases were uniformly high $(\mathrm{p}<0.001)$. No further differences between the three patient groups were identified regarding any of the investigated parameters (all p-values $>0.08$ ) in invasive components, even though only individual samples from resistant cases showed a strong staining for MRP2, GSTpi, and LRP. In contrast to other components, MT showed an intense P21- and RB staining, and were mostly positive for MRP2, LRP, and GSTpi.

Conclusions: Our results indicate a multi-factorial basis for the chemosensitivity of GCTs with lack of transporters for cisplatin, lack of anti-apoptotic BCL-2 family members, an intact apoptotic cascade downstream of P53, lack of P21-induction by P53 and lack of RB. These findings suggest a preference for apoptosis over cell cycle arrest in reponse to upregulation of P53. Reduced expression of the MMR-factor MLH1 is promising target to explain the resistant phenotype of some refractory tumors. The upregulation of various factors interfering with chemotherapy efficacy and the ability for a P21-induced cell cycle arrest can explain the intrinsic chemoresistance of mature teratoma components.

\section{8}

Urokinase inhibitor WX-UK1 as anti-metastatic cancer therapy. Results form the first dose in man phase la study

Muehlenweg B. (1), Ullrich S. (1), Schmalix W. (1), Knoeller J. (2), Wilhelm O.G. (1), Bartz R. (1)

(1) Wilex AG, Grillparzerstr. 10, 81675 München; (2) Focus Clinical Drug Development GmbH, Stresemannallee 6, 41460 Neuss

The plasminogen activation system with its key components urokinase-type plasminogen activator (uPA), the cell surface receptor uPAR and the inhibitor PAI-1, plays an important role in cancer cell invasion and metastasis. Inhibition of this system results in reduction of primary tumor growth and metastasis and is therefore an attractive target for therapeutic intervention. WX-UK1 is a synthetic inhibitor of uPA and the first representative of this substance class in clinical development.

In this first dose in man, double-blind, randomized, three-way cross-over, placebo-controlled, phase I study pharmacokinetics, pharmacodynamics and safety and tolerability was investigated. Six escalating i.v. doses of WXUK1 were administered in the range of $0.01-0.3 \mathrm{mg} / \mathrm{kg}$ and each dose was given to six healthy, male volunteers. 
Maximum plasma concentrations were in each case seen at the end of the 30 min. infusion: thereafter, concentrations declined rapidly up to 4-6 h postdose, and the mean terminal phase half-lifes were in the range of 10-12 h Good dose proportionality was observed both for Cmax and AUC across all doses. Renal elimination plays only a minor role in the overall clearance of the compound: mean recoveries of the parent compound up to $24 \mathrm{~h}$ postdose were in the range of $0.25 \%$ to $0.82 \%$ of the administered dose.

The systemic safety profile of WX-UK1 was highly acceptable at all dose levels. No changes in vital signs, ECG parameters, general safety laboratory parameters and adverse event profiles were observed which could be attributed to the administration of the study drug. For the coagulation parameters PT, aPTT and TT minor increases were observed at doses of $0.05 \mathrm{mg} / \mathrm{kg}$ and higher (mean increases of 6-13\% after WX-UK1 compared to 0-5\% with placebo) at the end of infusion. Except for aPTT and PT (2.6\% and $4.6 \%$ above the upper limit of normal) at Cmax all parameters remained within normal limits. All values returned to baseline within $15 \mathrm{~min}$. and were regarded not clinically relevant. Bleeding times remained unchanged and there was no indication of a drug-induced hemolysis.

The promising results of this phase I healthy volunteer study warrants further development of WX-UK1 as an anti-metastatic compound for the treatment of solid, malignant tumors. A phase I/II trial in gastric, pancreatic, ovarian and head \& neck cancer patients will be launched in Q3 2002.

\section{9}

\section{Efficacy of trofosfamide in patients with advanced soft tissue sarcomas progressing during or after anthracycline-based treatment}

Oechsle K., Mayer F., Kanz L., Bokemeyer C., Hartmann J.T.

Medizinische Universitätsklinik Tübingen, Germany

Objectives: The number of cytostatic agents effective in patients (pts) with advanced soft tissue sarcoma (STS) is limited. We have reviewed the efficacy and toxicity of continuous low-dose oral trofosfamide in pts with metastatic STS who have progressed during or after anthracycline-based chemotherapy.

Material and Methods: 19 pts with histologically profen STS and a median age of 57 years (range, 27-78) were treated with oral trofosfamide 300 $\mathrm{mg} / \mathrm{d}$ for $1 \mathrm{wk}$ followed by $150 \mathrm{mg} / \mathrm{d}$ given continuously. All pts have been advised to secure an oral fluid input of $>1.5 \mathrm{~L} /$ day. Response to treatment was evaluated every $4 \mathrm{wks}$ and trofosfamide application was stopped when progressive disease or inacceptable toxicity occurred. 18 pts had received at least one antracycline-based chemotherapeutic regimen prior to trofosfamide. In $67 \%$ of these pts $(n=12)$ pretreatment has included ifosfamide. 14 of 18 pts progressed during first-line chemotherapy, in 4 pts a partial response has occurred and theses pts were subsequently off treatment for at least 3 mos. One pt who refused i.v. therapy was also treated with trofosfamide as first-line chemotherapy for advanced disease.

Results: 9 pts (47\%) achieved stable disease lasting for a median of 5.5+ mos (range, $1-9+$ ). 10 pts $(53 \%)$ had progressive disease at first response evaluation. The median time to progression was $10+$ wks (range, 4-37+). In two pts treatment was stopped due to toxicity: anemia $\mathrm{III}^{\circ}$ and emesis $\mathrm{II}^{\circ} / \mathrm{fa}$ tigue $\mathrm{III}^{\circ}$. In general, toxicity was mild (maximum $\mathrm{II}^{\circ}$ according to WHOcriteria): emesis, thrombopenia and neutropenia. Grade $\mathrm{III}^{\circ}$ toxicity was seen in 5 pts (26\%): 3 pts with anemia and 2 with fatigue syndrome. No hemorrhagic cystitis was seen. The overall survival is 7+ mos (range, 2-13+). One pts is still on therapy.

Conclusions: Although no objective remission was observed with oral lowdose trofosfamide in heavily pretreated STS pts progressing during or after previous anthracycline-based chemotherapy, almost half of pts have achieved disease stabilisation for a median time interval of $5.5 \mathrm{mos}$. The moderate toxicity profile observed in this study allows the consideration of trofosfamide as a reasonable palliative treatment option in STS

\section{0}

\section{Treatment of renal cancer by a recombinant antibody-TNF fusion protein}

Adrian N., Bauer St., Fadle N., Pfreundschuh M., Renner C.

Med. Klinik I, Universität des Saarlandes

Treatment of advanced renal cancer is still a clinical challenge as standard procedures such as chemotherapy or radiotherapy have demonstrated only limited success in recent years. New experimental treatment modalities for advanced and/or metastatic renal cancer are being explored by many groups with a prime focus on antibody and vaccine based approaches. The almost exclusive expression of the G250 antigen by more than $95 \%$ of all clear cell renal cancers renders this antigen to be a good candidate for antibody directed therapy. Clinical trials with a chimeric anti-G250 antibody revealed excellent tumor targeting properties with tumor uptake reaching $0.1 \%$ of the injected total protein. However, neither the naked antibody by itself or radio-conjugated variants $(131 \mathrm{I}, 90 \mathrm{Y})$ have demonstrated meaningful clinical responses. As immune-surveillance is known to play a pivotal role in renal cancer we aimed at designing a fusion protein consisting of the chimeric anti-G250 antibody and human TNF-a replacing the IgG1 $\mathrm{CH} 2 / \mathrm{CH} 3 \mathrm{Fc}$-domain by the cytokine. This immunocytokine (anti-G250TNF) should target the renal caner cell and exert its TNF-a activity locally by inducing apoptosis, inflammation and hemorrhagic necrosis. The immunocytokine was generated by recombinant DNA technology and expressed in CHO cells in serum-free media under GMP conditions. Production was optimized to achieve antibody levels up to $20 \mathrm{mg} / \mathrm{l}$. Immunoreactivity of the final construct was analyzed by cell binding. Recognition of the G250 antigen was slightly reduced when compared to the parental antiG250 antibody(5-10 fold). TNF activity was determined using a variety of either TNF-a resistant or sensitive cell lines. Our immunocytokine revealed an equivalent activity when compared with recombinant human TNF-a TNF-a dependent killing was observed down to the low $\mathrm{ng} / \mathrm{ml}$ level with a LD50 activity between 10-20 ng/ml. Human granulocytes were activated specifically by the immunocytokine to release super-peroxide in a dose and time dependent manner. TNF activity could be enhanced by the addition of human IFN-g. In conclusion, the specific delivery of human TNF-a by an anti-G250 antibody should be possible to achieve local enrichment of this cytokine in renal cancer tissue. We are currently up-scaling production yields in a GMP facility to transfer this approach into the clinic in the near future.

Tyrosinase peptide vaccination in high-risk melanoma patients: role of immunological adjuvants and first clinical results

Scheibenbogen C. (1), Letsch A. (1), Mailänder V. (1), Schadendorf D. (2), Bechrakis N. (3), Schmittel A. (1), Nagorsen D. (1), Thiel E. (1), Keilholz U. (1)

(1) Med. Klinik III, Hämatologie, Onkologie u. Transfusionsmedizin, Universitätsklinikum Benjamin Franklin, Hindenburgdamm 30, 12200 Berlin, (2) Hautklinik, Klinikum Mannheim, (3) Augenklinik, UKBF, Berlin

Objectives: Immunological adjuvants are used to augment the immunogenicity of MHC class I-restricted peptide vaccines, but this effect has rarely been systematically evaluated in a clinical trial. We have investigated in a phase I study the potency of immunological adjuvants to enhance the T cell response to MHC class I peptide vaccines.

Material and Methods: Melanoma patients with an increased risk for relapse who were clinically free of disease received vaccinations with MHC class I-restricted tyrosinase peptides alone or either with GM-CSF, or the T helper antigen keyhole limpet hemocyanin $(\mathrm{KLH})$, or a combination of both adjuvants. The primary endpoint was induction of tyrosinase-specific $\mathrm{T}$ cells and serial $\mathrm{T}$ cell monitoring was performed in unstimulated peripheral blood samples before and after the $2 \mathrm{nd}$, 4th, and 6 th vaccination by ELISPOT assay.

Results: Tyrosinase-specific IFN $\gamma$-producing T cells were detected as early as 2 weeks after the 2 nd vaccination in 5 of 9 patients vaccinated with tyrosinase peptides in combination with GM-CSF and KLH, but not in any patient vaccinated with tyrosinase peptides without adjuvants or in combination with either adjuvant alone. After 6 vaccinations tyrosinase-specific T cells were also found in patients immunized with peptides either without adjuvants (3 of 9 patients) or in combination with the single adjuvant GMCSF (4 of 9 patients), but not with KLH (0 of 10 patients). In an ongoing phase I/II study we now vaccinate high-risk patients with 2 or more previous resectable relapses with tyrosinase peptides in combination with GM$\mathrm{CSF}$ and KLH. So far 11 patients (median 3 previous relapses, range $2-7$ relapses) are evaluable confirming the high immunogenicity of this vaccine combination ( $\mathrm{T}$ cell responses in 10 of 11 patients with early $\mathrm{T}$ cell responses in 4 patients). Furthermore in 3 patients with relapses early during vaccination subsequent regression of metastatic lesions upon continuation of vaccination could be documented.

Conclusions: Our results show that the combination of GM-CSF and KLH is able to augment immunogenicity of MHC class I peptide vaccines and provide first evidence for clinical efficacy of this vaccine combination. 
Prognostic factors for development of metastases in ocular melanoma: $\geq 6$ years follow-up of 271 patients

Schmittel A. (1), Bechrakis N.E. (2), Mutlu D. (1), Servetopoulou F. (2), Scheibenbogen C. (1), Förster M.H. (2), Thiel E. (1), Keilholz U. (1)

(1) Medizinische Klinik III (Hämatologie, Onkologie und Transfusionsmedizin), (2) Augenklinik, Universitätsklinikum Benjamin Franklin, Hindenburgdamm 30, 12200 Berlin

Objectives: $15-40 \%$ of patients with ocular melanoma develop metastases in the course of disease, which is associated with a very poor prognosis. Since modern vaccination strategies are promising in high-risk cutaneous melanoma patients, the determination of prognostic factors in ocular melanoma is urgently required to be able to perform adjuvant vaccination trials with innovative regimens.

Material and Methods: We analysed the course of patients with ocular melanoma, who were first diagnosed between 1994 and 1995 in our hospital. Data on the primary tumor, primary therapy and follow-up were collected.

Results: 271 patients with a mean age of 57 (8-86) years received primary therapy in the Department of Ophthalmology for ocular melanoma in 1994 and $1995.86 \%$ of patients were available for a potential follow up. $24 \%$ had an involvement of the ciliary body, in $35 \%$ the tumor was located anterior of the equator and in $41 \%$ posterior. 10 out 271 patients had an extraocular tumor growth (EOG). The mean of the largest tumor diameter (LTD) was $12,7 \mathrm{~mm}$ (standard deviation 3,5 mm) and the mean thickness was $6,13 \mathrm{~mm}$ (standard deviation $3,0 \mathrm{~mm}$ ). A total of $17 \%$ developed metastases during the follow-up period. $35 \%$ of patients with ciliary body involvement and $70 \%$ of patients with EOG developed metastatic disease during the follow-up period. Ciliary body involvement as well as EOG were associated with a decreased time to progression (log-rank test for both factors $\mathrm{p}<0.01)$.

Conclusions: This is the first study with a large number of patients and an almost complete follow-up for $\geq 6$ years, which defines prognostic factors as a basis for adjuvant therapy protocols.

\section{3}

\section{Characterization of CXCL12 signaling in renal cell cancer} based on studies in A498 cells

Schrader A.J. (1,2), Buer J. (2), Templin M. (2), Probst-Kepper M. (2), Ohlmann C.H. (1), Olbert P. (1), Hofmann R. (1)

\section{(1) Philipps-Universität Marburg, (2) GBF Braunschweig}

Objectives: CXCL12 (SDF-1), a CXC-chemokine, and its specific receptor, CXCR4, have recently been shown to be involved in tumourgenesis, proliferation and angiogenesis in several malignant diseases. We were able to demonstrate a distinct and divergent downregulation of the ligand and upregulation of the receptor in primary renal carcinoma (RCC) of the clear cell type in comparison with adjacent normal kidney tissue. This expression pattern was also observed in A498 RCC cells. In this study, we used A498 cells to prove, that the CXCR4 protein is functionally active and to disclose its potential biological influence in kidney cancer.

Material and Methods: To prove that the CXCR4 protein is functionally active, rhCXCL12 $\alpha$ was investigated for its ability to induce changes of intracellular calcium levels in A498 cells. Moreover, differential expression of 1176 genes in rhCXCL12 $\alpha$ stimulated versus unstimulated A498 cells was investigated by cDNA array analysis using the Human Cancer 1.2 Array (Clontech).

Results: Employing simultaneous confocal microscopy-analysis we were able to show that rhCXCL12 $\alpha$ caused a rapid and robust increase of intracellular calcium in a significant fraction of A498 RCC cells, demonstrating that the CXCR4 receptor was functionally active. Using cDNA expression arrays, $31 / 1176$ genes were found to be significantly influenced by rhCXCL12 $\alpha$, which could be grouped into five major categories: genes involved in the CXCR4 signal transduction pathway (paxillin, c-src, shb, LAT, CD82), transcription factors (FBP2, EGR1, IRF1, NF- $\kappa$ B p100, I- $\kappa \mathrm{B} \alpha$ ), regulators of translation (hnRNP1, 9G8) and the cell cycle (cyclin-D, cyclin-G1, AHR, RbAp48), pro-apoptotic (procaspase-8, -10 and -6 , CRADD), as well as miscellaneous genes such as PAI-1, interleukins, cathepsin L precursor, neuregulin and ECK. Taken together, the synchronous upregulation of metastasis inhibiting proteins, of proapoptotic molecules and of negative cell cycle regulators was paralleled by a downregulation of molecules involved in cell cycle progression and metastasis.
Conclusions: The genetic changes reported here should provide new insights into the developmental paths leading to tumour progression with special focus on the role of CXCL12 signaling and may also aid the design of new approaches to therapeutic intervention.

\section{4}

Combination chemotherapy with 5-FU/Cisplatinum is effective in patients with hormone-refractory prostate cancer (HRPC) with poor prognosis

Schroeder M., Anhuf J., Heinsch M., Giagounidis A., Aul C.

Medizinische Klinik 2, St. Johannes-Hospital, An der Abtei 7-11, D-47166 Duisburg

Prostate cancer is the most common cancer in males. Standard therapy is hormone treatment. Patients with progressive disease developing visceral metastases under antihormonal treatment usually have a poor prognosis. Platinum agents have modest but potentially additive benefits in HRPC. We investigated the combination of 5-FU and cisplatinum in $21 \mathrm{pts}$, median age 68 years (range $49-81$ years), performance score $0-3$, median 1. Platinum was given in a dosage of $100 \mathrm{mg} / \mathrm{m}^{2}$ every 4 weeks. In pts presenting with a WHO performance score $>1$ the total cisplatinum dosage was splitted into 5 equal doses of $20 \mathrm{mg} / \mathrm{m}^{2} / \mathrm{d} .3$ patients with impaired renal function received oxaliplat $\left(140 \mathrm{mg} / \mathrm{m}^{2} \mathrm{~d} 1\right)$ instead of cisplatinum. 5-FU was given as continous intravenous infusion $\left(1000 \mathrm{mg} / \mathrm{m}^{2} \mathrm{~d} 1-5\right.$, repeated $\left.\mathrm{d} 29\right)$. Pretreatment consisted of antihormonal therapy in all patients. In addition 11/21 patients had previously been treated with other cytotoxic drugs. At time of salvage treatment, all pts showed signs of symptomatic tumor progression with development of visceral metastases: liver $n=10$, lung $n=4$, bone marrow $n=$ 3 , meningeal involvement $\mathrm{n}=2$, brain $\mathrm{n}=2$. The number of chemotherapy courses ranged from 1-14 (median 4).

Results: 9 patients achieved a partial response with documented regression of metastases. 5 patients with previously progressive cancer achieved stable disease. In 5 patients progression of prostate cancer was documented, and 2 patients died rapidly from complications of their malignancy. Median response duration was 8 mos for patients achieving PR, and 4 mos for patients with stable disease. With conventional antiemetic support GIT-toxicity was mild (nausea I $40 \%$ ). There was no patient with grade III $^{\circ}$ hematotoxicity. From these date we conclude that combination chemotherapy with 5FU/DDP is effective and well tolerated in pts with HRPC. In this negatively selected patient population there was a remarkable clinical benefit with improvement of quality of live and prolongation of survival in about $50 \%$ of our patients.

\section{5}

Evaluation of the importance of elderly patients in a community based oncology group practice

Steinmetz H.T., Klausmann U., Schmitz S.

Practice for oncology, Sachsenring 69, 50677 Cologne

Due to the increasing life expectancy, the number of elderly patients is growing rapidly. Apart from this, more than $70 \%$ of cancer cases are detected in patients older than 60 . Therefore, elderly cancer patients are becoming increasingly important for health services. It was the aim to determine the frequency of geriatric cancer patients, their diagnosis and therapies in a community based oncology group practice and to get an idea of the specific aspects of geriatric patients. We analysed the clinical records of all patients who had visited the office for the first time between Jan. 1st and June 30th, 2001 (6 mo). 118 out of 577 patients $(20,5 \%)$ were 70 years or older. 84 pat. $(71 \%)$ had a malignant disease and 51 of them $(60,7 \%)$ received chemotherapy. Further characteristics are given in the table:

\begin{tabular}{lcll}
\hline & no & range of age & mean of age \\
\hline all patients & $118(100 \%)$ & $70-98$ & 77,3 \\
non malignant disease & $34(28,8 \%)$ & $70-98$ & 80,8 \\
malignant disease & $84(71,2 \%)$ & $70-90$ & 75,9 \\
without chemotherapy & $33(28,0 \%)$ & $70-90$ & 76,1 \\
with chemotherapy & $51(43,2 \%)$ & $70-88$ & 75,7 \\
\hline
\end{tabular}




\begin{tabular}{lrcr}
\hline the 6 most frequent diagnoses & no & $\begin{array}{l}\text { no without } \\
\text { chemotherapy }\end{array}$ & $\begin{array}{l}\text { no with } \\
\text { chemotherapy }\end{array}$ \\
\hline colon-ca & 11 & 1 & 10 \\
lung-ca & 10 & 0 & 10 \\
rectal-ca & 9 & 3 & 6 \\
lymphoma (high + low grade) & 9 & 3 & 6 \\
plasmocytoma & 7 & 2 & 5 \\
breast-ca & 7 & 3 & 4 \\
all others & 31 & 21 & 10 \\
\hline
\end{tabular}

Conclusions: A significant number of geriatric patients are diagnosed and treated in community based oncology group practices. A considerable number of patients is treated with chemotherapy. The patients with malignan disease will be further analysed.

296

The combination of liposomal daunorubicin and ifosfamide is a well tolerated and active chemotherapy regime in advanced soft tissue sarcoma

Szelényi H., Keilholz U., Siehl J., Hütter G., Thiel E.

Universitätsklinikum Benjamin Franklin, Freie Universität Berlin, Medizinische Klinik III, Hindenburgdamm 30, 12200 Berlin

The combination of an anthracycline and ifosfamide is the most effective chemotherapeutic treatment for soft tissue sarcoma. Usually high cumulative doses of the anthracycline are given limiting their use, especially in elderly patients or patients with cardiac disease. To improve tolerability of this combination we combined liposomal daunorubicin (L-Dauno, DaunoXome ${ }^{\circledR}$ ) with a continuous infusion of high dose ifosfamide.

The regimen was: L-Dauno $100 \mathrm{mg} / \mathrm{m}^{2}$ and ifosfamide $5 \mathrm{~g} / \mathrm{m}^{2}$ over $24 \mathrm{~h}$ Mesna $\left(5 \mathrm{~g} / \mathrm{m}^{2}\right.$ over $\left.24 \mathrm{~h}\right)$ and G-CSF starting day 5 was given concomitantly. Cycles wer repeated every 4 weeks. Between 1996 and 200227 patients with a median age of 55 years (20 to 78 years) were treated. Initially, 5 patients were treated as a second line therapy after failure of a doxorubicin/ifosfamide regimen. However, none of these responded. Subsequently, 22 patients were treated first line. A total of 83 cycles (range 1 to 7 ) were administered as of $6 / 2002$. Toxicity was tolerable with 5 episodes (6\% of cycles) of ifosfamide related CNS toxicity grade II (next cycles ifosfamide administered over $48 \mathrm{~h}$ ) and 4 episodes (5\% of cycles) of neutropenic fever. Treatment had to be terminated due to a pseudo-allergic reaction to L-Dauno in one patient. No other non-hematological toxicity exceeding grade II, especially no cardiac toxicity was observed. Eight $(38 \%)$ out of the 22 patients without anthracycline pretreatment achieved a partial remission (PR), all after 2 treatment cycles, 6 patients $(27 \%)$ retained a stable disease and 7 patients (32\%) progressed; one patient was not evaluable. In 4 progressive patients subsequent chemotherapy was given with no efficacy. Median TTP was 4 months, median response duration 5 months. With respect to histology partial response rates were: 2 out of 7 PNET, 1 out of 5 leiomyosarcoma, 2out of 3 liposarcoma and 1out of 3 synovial sarcoma. The other two responding patients had a pleomorphic and a fibroblastic sarcoma.

Our data shows that the combination of L-Dauno and ifosfamide is an active and well tolerated chemotherapy regimen for first line therapy in soft tissue sarcoma, especially in elderly patients.

\section{Stem Cell Biology I}

297

\section{Prompt diffferentiation of a rare subset of human hemato-} poietic cells (HSC) stimulated by multiple cytokines in vitro

\section{Engelhardt M., Wider D., Follo M., Lübbert M., Guо Y.}

Hematology/Oncology Department, University Medical Center, 79106 Freiburg, Germany

HSC can be selected based on the efflux of the DNA-binding dye Hoechst (HO) and are identified as SP cells. We and others have previously demonstrated that BM, peripheral blood (PB) or cord blood (CB) SP cells are negative for lineage markers, significantly increased for LTC-IC and reconstitute lethally irradiated mice. Of interest is that few SP cells divide in cytokine-supported methylcellulose and thus are not classifiable as direct colony-forming cells (CFU), as previously reported for CD34+CD38- cells. The latter have, however, shown to proliferate in liquid culture (lc), suggest- ing their quiescent nature. Previous reports have focused on CD34+/CD38cells. Here we sought to analyze the differentiation potential of human SP cells before and after lc. We used a combination of morphologic analysis, cell and CFU content to examine the functional properties of SP as compared to non-SP (NSP) and CD34+ cells using serum-containing medium (SCM), serum-free medium (SFM) and different cytokine combinations Median frequencies of SP cells of $0.02 \%$ and absolute numbers of $2.5 \times 10 \mathrm{e} 4 /$ sort were obtained which indicates the rarity of these cells. Cells were initially cultured in SCM plus IL-3, SCF and Flt3 (FK3) and for later experiments with the addition of Tpo and IL-6 (FKT36). In cytokine-supported lc, SP cells on day 5 and 7 increased 1- to 2-fold, giving rise to CD34+ cells. CFU numbers showed a more substantial expansion, with a 7.3- and 5.2-fold difference as compared to NSP cells on day 5 and 7, respectively. SP cells from CB vs. AP and BM, FKT36 vs. FK3, and SFM vs. SCM all led to more enhanced cell and CFU-increases. A higher proliferative capacity with respect to total cellularity and CFU numbers was observed after lc. The analysis of unstained vs. HO-stained NSP demonstrated insignificant cell and CFU decreases due to the HO-staining which suggests $\mathrm{HO}$ based selection of HSC to be feasible. The comparison of SP, CD34+ and NSP cells revealed highest CFU numbers from SP cells. Our data demonstrate the low CFU potential of SP cells before lc, reflecting their immaturity and their requirement of cytokine stimulation before proliferation is initiated. Our results suggest that HO staining can be used to isolate quiescent vs. replicating HSC and is an ideal target for purging, gene therapy and expansion protocols. These cells provide a novel method to enrich HSC, thus preventing the discard of CD34- subsets that could be critical for hematopoietic engraftment.

\section{8}

Effect of 5-aza-2'-deoxycytidine (DAC) on proliferating normal human CD34+ cells: a model to study demethylation during myeloid differentiation

Guo Y., Engelhardt M., Wider D., Claus R., Lübbert M.

Hematology / Oncology Department, University of Freiburg Medical Center, 79106 Freiburg, Germany

DNA methylation is a major epigenetic mechanism controlling tissue- and development-specific gene regulation. During normal hematopoietic cell maturation, methylation of myeloid-specific genes (such as myeloperoxidase [MPO], lysozyme [LZM]) is concomitantly decreased with cell differentiation. To establish an in vitro model of normal hematopoietic differentiation induced by demethylation, we used CD34+ cells from apheresis specimens (AP) and analyzed their proliferation, viability, colony-forming potential (CFU), LZM and MPO expression and cell cycle status with and without DAC treatment. Since DAC effects cells in S phase, CD34+ cells from AP specimens were cultured with IMDM and 10\% FCS (SCM) or serum-free medium (SFM) in the presence of Flt3, SCF, and IL-3. SFM as compared to SCM led to increased cell and CFU numbers. Treatment with DAC was initiated on day (d) 3 of culture, either as a single 24 hour (hr) pulse or three $24 \mathrm{hr}$ pulses with concentrations ranging from 10 to $5000 \mathrm{nM}$. Cells were harvested on $\mathrm{d} 7$ and $\mathrm{d} 10$ of culture. With a single pulse of DAC, a 50\% growth inhibition as compared control cells was observed at concentrations between 500 to $1000 \mathrm{nM}$. Cell viability started to decrease at $200 \mathrm{nM}$ DAC in a dose-dependent fashion, with significant decreases with 500 to $1000 \mathrm{nM}$, nevertheless below 500nM was similar to controls. With three $24 \mathrm{hr}$ DAC pulses, both $50 \%$ growth inhibition and decreasing cell viability were already observed at $10 \mathrm{nM}$. CFU showed a $50 \%$ decrease between 10 to $50 \mathrm{nM}$ for both 1- and 3-d DAC pulses. A dose-dependent increase of MPO+ cells was observed with 10 to $5000 \mathrm{nM}$ DAC on d7 as compared to control cells, demonstrating most significant MPO-increases with 200 to $5000 \mathrm{nM}$ DAC. In order to ask whether DAC induces an enhanced responsiveness to G-CSF, DAC treatment was followed by G-CSF stimulation. As compared to preculture levels, both LZM and MPO positive cells increased on $\mathrm{d} 7$ of culture and even more with the addition of G-CSF. These data suggest a differentiation-enhancing role of DAC. The cell cycle analysis revealed a substantial decrease of G0/G1 cell numbers between $\mathrm{d} 0$ and $\mathrm{d} 7 \mathrm{ex}$ panded cells, but not between DAC-treated and untreated cells. Our current results reveal that DAC induces a dose-dependent inhibition of cell growth and can enhance differentiation of normal hematopoietic precursors. This model should be suitable for the establishment of patterns of analyzing methylation and gene expression during lineage specific maturation. 
Endothelial activity in peripheral blood-derived adult stem cells showing improved angiogenesis in-vivo

Huss R. (1), Heil M. (2), Göttgens B. (3)

(1) Pathologisches Institut der LMU München, (2) Max-Planck-Institut für Experimentelle Kardiologie, Bad Nauheim, (3) Cambridge Institute for Medical Research, Cambridge, UK

Objectives: Hematopoietic stem cells (HSC) are routinely isolated from the bone marrow, peripheral blood and umbilical cord blood, either unselected or based on the expression of stem cell associated antigens, e.g. CD34. There is also evidence that it is also possible to achieve hematopoietic reconstitution with large numbers of CD34-negative stem cells, which are considered to be ,adult stem cells“. Due to their plasticity, adult stem cells have revealed a considerable degree of pluripotency, which allows the generation of a variety of tissue- and organ-specific progenitors in-vivo and adult stem cells are suppose to maintain a steady-state of organ function of injury or disease. Here we investigate the endothelial activity and angiogenic potential of a murine adult stem cell line in-vitro and in-vivo.

Methods: The CD34-negative murine cell line RM26 was generated from peripheral blood mononuclear cells and was already successfully used for hematopoietic reconstitution (Lange et al. 1999). The expression of different endothelial-associated surface antigens and transcription factors was investigated under different growth and differentiation conditions in-vitro and the adult stem cells were also used in a murine ischemia model to analyse the influence of transplanted adult stem cells on de-novo angiogensis invivo.

Results: The surface expression of VEGF and VEGF-R is increased in adult stem cells under stress-mediating growth conditions like serum depletion, while they form endothelial-like structures on angiogenesis-inducing collagen. Besides a variety of mesodermal and erythroid / myeloid transcription factors, the adult stem cells also express different GATA-factors and the active SCL stem cell core enhancer, binding at least two Ets proteins. When the stem cells are infused intravenously in a murine ischemia model (ligation of the femoral arteria and measurement of the relative blood flow), stem cell-treated mice showed an improved collateral vessel formation and neo-angiogenesis as compared to untreated control mice, although immunhistochemistry could never reveal the presence of transplanted cells integrated in the capillary vessel wall.

Conclusion: Adult stem cells from the peripheral blood show a distinct endothelial activity and a capability to support neo-angiogenesis under the appropriate environmental signals like cellular stress or ischemia. Therefore, adult stem cells can be effectively used for cell-, tissue- and organ repair invivo.

$$
300
$$

Coexpression of CD38, HLA-DR and CDw90 in CD133 positive and negative stem cells using Multiparametric Four-Colour Flow Cytometry (MFC)

\section{Kahl C., Kleine H.-D., Freund M.}

Division of Hematology and Oncology, Department of Internal Medicine, University of Rostock, Germany

Recent findings indicates that human hematopoietic stem cell properties can be found among cells lacking CD34 and lineage commitment markers. CD133 is a new stem cell antigen that may provide an alternative to CD34 for the selection of hematopoietic cells for transplantation. It was postulated that the CD133 positive stem cell subset should be more immature than the CD34 stem cell. This study is investigating the coexpression of CD38, HLA-DR, CDw90 in CD34/CD133 positive and negative cells as a marker of immaturity. Bone marrow (5) or apheresis samples (28) were collected for bone marrow or peripheral blood stem cell transplantation at our department. Seven of the apheresis samples were collected after G-CSF mobilisation without chemotherapy for allogenic transplantation, the remaining 21 apheresis samples were collected after chemotherapy for autologous transplantation. By using a live gate for analysis sorting, 10.000 cells of the distribution of the activation pattern among the CD133 and CD34 positive or negative cells of the 33 samples were measured as follows: HLADR+/CD133+/CD34- 4,7\%, HLA-DR+/CD13+/CD34+ 59,7\%, HLADR+/CD133-/CD34+ 13,3\% cells, CD38+/CD133+/CD34- 4,2\%, CD38+/ CD133+/CD34+ 64,6\%, CD38+/CD133-/CD34+ 12,8\%, CDw90+/ 
CD133+/CD34- 0,21\%, CDw90+/CD133+/CD34+ 15,8\%, CDw90+/ CD133-/CD34+ 0,78\%. In comparison of the bone marrow (5) and G-CSF mobilized (7) stem cell source the coexpression of the activation pattern in CD133/CD34 positive or negative stem cells was higher in bone marrow than in apheresis samples. Only the CDw90+/CD133-/CD34+ (17,6\% vs. $9,8 \%)$ and CD38+/CD133-/CD34+ (1,4\% vs. $0,58 \%)$ subset in apheresis samples showed a higher expression of these markers.

In conclusion the percentage of cells with coexpression of the activation pattern was highest in the CD133+/CD34+ cell subset, following by the CD133-/CD34+ cell subset. These markers were low in expression in the CD133+/CD34- cell subset. This observation was independent of the stem cell source (apheresis samples vs. bone marrow). There is no evidence for the immaturity of the CD133+/CD34- stem cell subset in comparison with the CD133-/CD34+ stem cells using the expression of activation pattern.

\section{1}

\section{Advantages of a new two-step PCR using degenerate primers for clonal analysis of gene-marked human colony-forming peripheral blood progenitor cells}

Nagy K.Z. (1), Laufs S. (1), Gentner B. (1), Zeller W.J. (1), Fruehauf S. (2)

(1) D0200, German Cancer Research Center, Heidelberg, (2) Department of Internal Medicine V, University of Heidelberg

Stem cells, the natural units of tissue regeneration, hold big promise as a treatment for a multitude of diseases. Their extensive ability to proliferate and self-renew make them an attractive target for gene therapy. Understanding the contribution of single cells to repopulation is important for basic cellular biology research, for clinical transplantation medicine and also for gene therapy. To tag single cells, their genome must be marked individually, and this marker must be propagated to their progeny. Retroviral transduction of cells is an excellent candidate method for this aim. Several methods to detect individual stem cell gene marking such as ligation-mediated PCR or inverse PCR have been described which all require extensive preamplification preparation steps of DNA.

In order to rapidly detect retroviral integration sites from small amounts of DNA we adapted a two-step PCR with partly degenerate, arbitrary primers that bind to the genomic DNA adjacent to the retroviral vector insertion site. We tested seven degenerate primers on two retrovirally transduced HT1080 cell line clones. Two of the degenerate primers detected a junction between the vector long-terminal repeat (LTR) sequence of the provirus and the flanking human sequence in both cell line clones. Using a mixture of these two primers we were able to detect even multiple integration sites in one reaction. These junction sequences were confirmed by ligation-mediated PCR.

Enriched human CD34+ peripheral blood progenitor cells from five donors were transduced with the SF1m retroviral vector. Transduced CD34+ cells were plated in methylcellulose medium and were grown for 14 days. To assess the effectivity of transduction PCRs for proviral sequences were performed. 72 single provirus-positive colonies were analyzed by two-step PCR with the tested primer mixture. The junction could be amplified in $35 \%$ of provirus-positive colonies, even from small colonies ( $<200$ cells), emphasizing the high sensitivity of this method.

Two-step PCR is a useful and rapid method to analyze retroviral integration sites in hematopoietic stem cells after transduction. It allows to analyze small provirus-positive colonies in a day and has therefore advantageous features over conventional techniques to study gene marking of stem cells.

\section{Analysis of adherent CD34+ cells on immobilized extracellular} matrix proteins

Oswald J. (1), Pompe T. (2), Salchert K. (2), Streller U. (2), Boxberger S.

(1), Bornhäuser M. (1), Werner C. (2)

(1) Med. Klinik und Poliklinik I, Universitätsklinikum Dresden,

(2) Institut für Polymerforschung Dresden

Objectives: Hematopoietic stem cells are localized in niches of the bone marrow where proliferation, commitment, and terminal differentiation of primary HSCs occur. Homing, proliferation and differentiation of HSCs is triggered by interaction with stromal cells, extracellular matrix (ECM) proteins, cytokines, and growth factors that are present on stromal cells or bound to ECM macromolecules.

Material and Methods: To mimic the bone marrow microenvironment, surface modification of solid carriers was performed utilizing reactive polymer coatings for the immobilization of ECM proteins. A set of strategies for the covalent immobilization of Collagen I on polymer materials was established utilizing reactive polymer films of poly(octadecene alt maleic anhydride) as a versatile substrate. We compared the effect of the immobilized basic collagen molecule (tropocollagen) with in vitro reconstituted collagen fibrils. Results: Time lapse studies revealed a decreased motility of HSCs on fibrillar collagen indicating a strong adhesive contact of the cells with the immobilized fibrils. Both forms of immobilized collagen were found to support the expansion of CD34+ enriched hematopoietic stem cells but after longer cultivation in presence of SCF, FLT-3L and IL-3 more CD34+ progenitor cells were found on the fibrillar collagen than on tropocollagen. For the detailed characterization of adherent hematopoietic progenitor cells on artificial substrates Laser Scanning Cytometry (LSC) and Laser Scanning Microscopy (LSM) were adapted. The LSC is a microscope linked instrument that measures fluorescence and forward scatter of cells on a standard microscope slide. In addition to multicolour cytometric analysis equivalent to flow cytometry, this configuration offers the advantage to permit accurate relocation and microscopic visualization of analysed cells.

Conclusions: Laser Scanning Microscopy can complement cytometrical data by high-resolution imaging of individual cells. Both techniques were found to be applicable to adherent cells. Using these analytical tools we could show that fibrillar collagen give a better support for the expansion of CD34+ progenitor cells indicating a fundamental role of adhesive interactions for the expansion of CD34+ cells.

\section{3}

The microenvironment of the murine fetal liver cell line, AFT024, maintains primitive human hematopoiesis by counteracting contact mediated proliferation inhibition

Punzel M. (1), Gupta P. (2), Verfaillie C.M. (3)

(1) Medizinische Poliklinik der Universität Heidelberg, (2) Veterans Affairs Medical Center, Minneapolis, MN, (3) Stem Cell Institute, University of Minnesota

We have previously shown that maintenance of primitive human hematopoietic stem cells is poor when cultured in contact with marrow stromal feeders. However, when separated from stromal contact human progenitors can be maintained because adhesion mediated proliferation inhibition does not occur. In this study we demonstrate how the murine fetal liver cell line, AFT024, supports primitive human hematopoiesis better in contact cultures compared to primary feeders. We evaluated if better progenitor maintenance in contact with AFT024 cells can be explained by decreased adhesion itself or decreased adhesion mediated inhibition of proliferation. We show that primitive human hematopoietic cells adhered equally well to AFT024 and primary feeders, such as M2-10B4. Further, contact with metabolically inactive AFT024 cells prevented cell cycle progression and decreased maintenance of primitive progenitors to the same extent as contact with M2-10B4 feeders. However, contact with viable AFT024 feeders did not inhibit proliferation, suggesting that AFT024-factors counteract contact mediated inhibition of proliferation. Cytokine production by M2-10B4 and AFT024 cells was similar. Large-size O-sulfated heparan sulfate glycosaminoglycans, known to be important for hematopoietic support, were found only in AFT024-matrix. Therefore, we hypothesize that these factors may explain in part our observations. Finally, we show that more than $100 \%$ of primitive myeloid progenitors could be maintained for at least 5 weeks when cultured in contact with AFT024 feeders in the presence of Interleukin-3 and Macrophage Inflammatory Protein-1alpha. In conclusion, AFT024 cells produce factor(s), that counteract contact induced growth inhibition of primitive human hematopoietic progenitors, leading to expansion of these cells in contact with the microenvironment.

\section{4}

The intrinsic controlled division program of individual human hematopoietic progenitors can be influenced by adhesive interactions with a stem cell supporting microenvironment

Punzel M., Zhang T., Eckstein V., Ho A.D.

Medizinische Poliklinik, Universität Heidelberg

We recently demonstrated that primitive human hematopoietic stem cells (HSC) proliferate controlled by an intrinsically determined division program. This allows us to distinguish between primitive HSC and committed progenitors within 8-10 days from culture initiation. In this study we investigated if adhesive as well as microenvironmental factors may change the initial cell division program at a single cell level.

CD34+/CD38- cells from human cordblood $(n=18 ; 88-176$ cells per experiment) were sorted individually in 96 well plates and cultured for 10 days adherent to either fibronectin (FN) or to the murine fetal liver cell line 
AFT024. BSA coated wells were used as controls. After 10 days of culture we assessed the subsequent functional fate of each individual cell. Stem cell equivalent function was measured by Myeloid-Lymphoid Initiating Cells (ML-IC), committed function was determined using the colony forming cell (CFC) assay.

Compared to BSA cultures adhesion to fibronectin (FN) did not change neither the quiescent fraction $(12.5 \pm 2.1 \%$ [FN]vs. $13.4 \pm 2.1 \%$ [BSA] $)$ nor the asymmetric division rate $(23.1 \pm 1,9 \%$ [FN] versus $22.0 \pm 1.4 \%$ [BSA] $)$. Similar frequencies of ML-IC $(4.6 \pm 1.0 \%$ [BSA] and $6.2 \pm 3.2 \%$ [FN]) and CFC $(27.7 \pm 3.7 \%$ in BSA and $23.7 \pm 2.6 \%$ in $\mathrm{FN})$ could be detected after 10 days of culture. This indicates that adhesive interactions via FN do not change the cell division or functional maintenance in both types of progenitors. However, all stem cell related ML-IC had been recruited exclusively from the slow dividing fraction (<2divisions), whereas the majority of cells with CFC capacity has divided $>2$ times initially $(92.1 \pm 3.5 \%$ [BSA] and $80.3 \pm 5.7 \%[\mathrm{FN}])$

Surprisingly, contact to the stem cell supporting microenvironment of AFT024 not only increased the number of proliferating cells $(3,1 \pm 0.8 \%$ quiescent cells) but also changed the asymmetric division rate of the entire CD34+/CD38- population $(31.0 \pm 2.0 \%)$. In addition, compared to BSA significantly more primitive $(10.5 \pm 0.7 \%$ ML-IC) as well as committed progenitors $(54.1 \pm 7.3 \%$ CFC) could be detected in the CD34+/CD38- population after culture on AFT024 feeders. This suggests that in contrast to adhesive factors itself only interactions with a stem cell supporting microenvironment, such as AFT024, may influence the intrinsically determined division program. This results in maintenance as well as recruitment of primitive and committed progenitors from quiescence into cell cycle.

\section{5}

The granulocytic inducer C/EBPalpha inactivates the myeloid master regulator PU.1: possible role in lineage commitment decisions

Reddy A.V. (1), Iwama (2), Iotzova G. (1), Schulz M. (1), Elsasser A. (1), Vangala R.K. (1), Tenen D.G. (3), Hiddemann W. (1), Behre G. (1)

(1) Department of Medicine III, Ludwig-Maximilians-University Munich and GSF-National Research Center for Environment and Health, Munich, 81377, Germany, (2) Department of Immunology, Institute of Basic

Medical Sciences, University of Tsukuba Ibaraki, 305-8575, Japan,

(3) Harvard Institutes of Medicine, Harvard Medical School, Boston MA-02115, USA

Several transcription factors have been implicated to play a role in myelopoiesis. PU.1, an ets-family transcription factor, is required for the development of both myeloid and lymphoid lineages while the transcription factor CCAAT/enhancer binding protein family member C/EBPalpha is essential for granulocytic development. We present here the first evidence that C/EBPalpha blocks the function of PU.1. PU.1 and C/EBPalpha interact physically and co-localize in myeloid cells. As a consequence of this interaction C/EBPalpha can inhibit the function of PU.1 to activate a minimal promoter containing only PU.1 DNA binding sites. We further demonstrate that the leucine zipper in the DNA binding domain of C/EBPalpha interacts with the b3/b4 region in the DNA binding domain of PU.1, and as a result displaces the PU.1 co-activator c-Jun. Finally, C/EBPalpha blocks PU.1 induced dendritic cell development from CD34+ human cord blood cells. The functional blocking of PU.1 by C/EBPalpha could be the mechanism by which C/EBPalpha inhibits cell fates specified by PU.1, and directs cell development to the granulocytic lineage.

\section{6}

\section{Simultaneous expansion of granulocytic and megakaryocytic post-progenitor cells from CD34 ${ }^{\text {pos }}$-selected peripheral blood progenitor cells (PBPC) in serum-free liquid culture}

Scheding S., Albrecht A., Oelze S., Kiesewetter H., Salama A.

Charité, Campus Virchow Klinik, Department of Transfusion Medicine, Stem Cell Laboratory, Berlin, Germany

Objectives: The additional transplantation of ex-vivo generated hematopoietic cells represents a promising approach for the amelioration of neutropenia and thrombocytopenia after high-dose therapy and stem cell transplantation. We have previously defined conditions to effectively generate granulocytic or megakaryocytic post-progenitors in serum-free medium (Scheding et al., Exp.Hematol. 2000; Kratz-Albers et al., Exp.Hematol. 2000). The current study aimed to investigate whether granulocytic and megakaryocytic cells could be generated simultaneously.
Material and Methods: CD34 ${ }^{\text {pos }}$-cells of G-CSF-mobilized healthy donors were selected by MACS (mean purity: $97.9 \pm 0.6 \%$ ) and seeded at $3 \times$ $10^{4} / \mathrm{ml}$ in serum-free X-VIVO10 [X10] or CellGrow [CG] medium in teflon culture bags. Cultures were stimulated with SCF, G-CSF, and MGDF (all at $100 \mathrm{ng} / \mathrm{ml}$ ). After 10 days, cells were analyzed for the generation of granulocytic and megakaryocytic cells by flow-cytometry and standard colony assays.

Results: Total cellular expansion was $24.8 \pm 7.8$-fold and $19.4 \pm 4.9$-fold in $\mathrm{X} 10-$ and CG-medium, respectively. The majority of cells cultured in CG were $\mathrm{CD}^{\mathrm{pos}}(63.4 \pm 10.4 \%)$ compared to $34.3 \pm 11.4 \%$ in X10. The fractions of $\mathrm{CD} 15^{\text {pos }} \mathrm{CD} 11 \mathrm{~b}^{\text {neg }}$ cells were $44.7 \pm 6.5 \%$ [CG] and $27.8 \pm 12.6 \%$ $[\mathrm{X} 10] . \mathrm{CD} 11 \mathrm{~b}^{\text {pos }} \mathrm{CD} 16^{\text {neg }}$ cells were $4.7 \pm 2.2 \%[\mathrm{CG}]$ and $16.1 \pm 5.7 \%$ [X10]. Cell production rates (i.e. number of cells of a given phenotype generated from every seeded CD34 $4^{\text {pos }}$-PBPC) in CG-medium were $12.0 \pm 2.4$ $\left(\mathrm{CD} 61^{\mathrm{pos}}\right), 9.2 \pm 3.2\left(\mathrm{CD} 15^{\mathrm{pos}} \mathrm{CD} 11 \mathrm{~b}^{\mathrm{neg}}\right)$, and $0.95 \pm 0.4\left(\mathrm{CD} 11^{\mathrm{pos}} \mathrm{CD} 16^{\mathrm{neg}}\right)$ Corresponding production rates in $\mathrm{X} 10$ were $7.2 \pm 1.2\left(\mathrm{CD}^{\mathrm{pos}}\right), 8.2 \pm 5.8$ $\left(\mathrm{CD} 15^{\mathrm{pos}} \mathrm{CD} 11 \mathrm{~b}^{\text {neg }}\right)$, and $4.8 \pm 2.4\left(\mathrm{CD} 11^{\mathrm{pos}} \mathrm{CD} 16^{\text {neg }}\right)$. In addition to the generation of post-progenitor cells, total CD34 ${ }^{\text {pos }}$-cells as well as clonogenic progenitor cells were also amplified in this culture system (fold expansion CD34 ${ }^{\text {pos }}$-cells: $5.1 \pm 0.4$ [CG], $2.8 \pm 0.8$ [X10]; CFU-GM: $2.8 \pm 0.8$ [CG], $3.6 \pm 1.2$ [X10]; CFU-Meg: $5.0 \pm 1.8$ [CG], $4.8 \pm 1.9$ [X10]).

Conclusions: The data indicated that granulocytic and megakaryocytic cells can be effectively generated simultaneously. Moreover, compared to X10, cells generated in CG showed a higher fraction of megakaryocytic cells and immature granulocytic post-progenitor cells as well as higher CD34 expansion rates.

\section{7}

Detection of epithelial microchimerism in paraffin-embedded colon biopsies after hematopoietic stem cell transplantation (HSCT)

Spyridonidis A. (1), Schmitt-Gräff A. (2), Calow T. (1), Follo M. (1), Behringer D. (1), Finke J. (1)

(1) Department of Hematology / Oncology and (2) Department of Pathology, University Medical Center, Freiburg, Germany

Objectives: The large number of reports of somatic cell plasticity after human HSCT has highlighted the need to standardize the criteria required to establish that a tissue chimerism has occurred. Because of the 3-dimensional structure of tissue specimens, it is essential that the occurrence of cells that overlay or wrap around one another not be mistaken as the same cell and taken as evidence for marker colocalization. The clinical consequences of the epithelial chimerism after HSCT are unknown; clarification of this biological phenomenon can only be reached by the examination of material from a great number of transplanted patients. Since tissue biopsies are usually archived in pathology banks as paraffin-embedded material it is crucia to establish a technically secure method for the detection of epithelial chimerism in such material.

Methods: We combined Y-chromosome FISH with cytokeratine (CK) immunofluoresence staining and TOTO-3 nuclear label or CD45 staining in formalin-fixed paraffin-embedded biopsies. Up to now, we have tested colonic biopsies taken 3-70 weeks post sex-mismatched transplantation from 5 female patients. We collected serial thin optical sections of the $4 \mu \mathrm{m}$ thick biopsies by confocal laser-scanning microscopy and used the stack of $2 \mathrm{D}$ images to generate $3 \mathrm{D}$ reconstructions which provided evidence of a 3D colocalization of the 3 markers. We never found Y signal in control biopsies from sex-matched transplanted female patients.

Results: Although 2D analysis indicated the presence of $\mathrm{Y}+/ \mathrm{CK}+$ cells, further 3D analysis and CD45 label showed that in some cases the origin of the intraepithelial found mismatch genotype was due to infiltrating CD45+ cells. Donor-derived epithelial cells were detected by 3D colocalization of $\mathrm{CK}$ and Y-chromosome, presence of the Y-signal within the TOTO-3 labeled nucleus, CK-staining up to the nuclear membrane and absence of CD45+ or CD68+ cells in the region studied by FISH. Y+/CK+/CD45- cells were identified as isolated single cells in $2-10 \%$ of the colonic crypts in $5 / 5$ patients. 3D video projections of individual $\mathrm{Y}+/ \mathrm{CK}+/ \mathrm{CD} 45-$ cells will be presented.

Conclusion: Our study suggests the presence of microchimerism in epithelial tissues after human HSCT. Detection of epithelial chimerism requires the use of triple staining techniques and the use of confocal or deconvolution microscopic methods. Our detection methodology give access to examination of great number of paraffin-embedded archival material. 
Transcriptomal characteristics of CD105+ mesenchymal stem and progenitor cells of human bone marrow

Steidl U., Fenk R., Rohr U.-P., Roes N., Martin S., Aivado M., Gattermann $N$., Kronenwett R., Haas $R$.

Heinrich-Heine-Universität Düsseldorf; Klinik für Hämatologie, Onkologie und klinische Immunologie

Recent studies showed that bone marrow cells have differentiation capabilities which go far beyond their hematopoietic or stromal determination and thus CD34+ hematopoietic and CD105+ mesenchymal stem cells come into consideration for cellular therapy of a variety of degenerative diseases of different organs. In this study, we used cDNA array technology to obtain a diversified molecular insight into the genetic program of CD105+ cells from bone marrow of healthy volunteers $(n=5)$. Furthermore, we intraindividually compared the transcriptomes of immunomagnetically enriched CD105+ stromal cells and CD34+ hematopoietic cells to identify characteristic molecular features of either cell population. In total, 95 genes were significantly differentially expressed. We observed distinct patterns of transcription factors apparently being crucial for either stromal or hematopoietic cells. In all examined samples, 8 transcription factors were expressed 2- to 6.4-fold higher in CD105+ cells (STAT1, STAT2, NFKB, RBP7, NFX1, PUR1, CATF1, E2F1). Another set of 14 transcription factors was consistently 2- to 32-fold higher expressed in CD34+ cells (ETS2, GATA2, FLI1, ERG1, ELK3, ETR101, TFAP4, EGR1, TFCP2, BRM, GNAS1, INI1, DB1, HPF7). Looking at genes promoting cell cycle we found that 9 genes were 3- to 12.6-fold higher expressed in CD105+ cells (CDC2, CDC20, CDC25A, CDC28, PLK, cyclins A2 and B2, TFDP2, FBP) when compared to CD34+ cells. In CD34+ cells 3 cell cycle inhibitors (CDK inhibitors $1 \mathrm{C}$, 2A, 2D) were higher expressed. Taken together these data suggest a higher proliferation activity of CD105+ in comparison to CD34+ cells. This assumption was further supported by the finding that CD105+ cells expressed 20 DNA synthesis genes 2- to 17-fold higher than CD34+ cells. 4 genes involved in stress defence (SOD1, GRX, HSP71, GPX2) were expressed 5- to 12 -fold higher in CD105+ cells than in CD34+ cells. In summary, our data provide gene expression profiles of CD105+ stromal stem and progenitor cells from human bone marrow. The intraindividual comparison with CD34+ cells allowed the identification of patterns of transcription factors characteristic for either stromal or hematopoietic stem and progenitor cells. Further, our findings suggest a greater proliferation activity and stress defence capacity of CD105+ stromal cells. Our data may serve as a molecular basis for further research on adult stem cells from human bone marrow.

\section{9}

\section{Receptors of hypothalamic peptides corticotropin-releasing hormone and orexin/hypocretin are expressed in human CD34+ hematopoietic stem and progenitor cells and regulate expres- sion of transcription factors, surface receptors and cytokines}

Steidl U. (1), Schroeder T. (1), Selbach O. (2), Seres J. (3), Beaujean D. (3), Maercker C. (4), Rohr U.-P. (1), Fenk R. (1), Schaub S. (1), Aivado M. (1), Martin S. (1), Roes N. (1), Kliszewski S. (1), Gattermann N. (1), Haas H.L. (2), Bornstein S.R. (3), Haas R. (1), Kronenwett R. (1)

(1) Heinrich-Heine-Universität Düsseldorf; Klinik für Hämatologie, Onkologie und klinische Immunologie; (2) Heinrich-Heine-Universität Düsseldorf; Institut für Neurophysiologie; (3) Heinrich-Heine-Universität Düsseldorf; Klinik für Endokrinologie; (4) Deutsches Ressourcenzentrum für Genomforschung, Berlin/Heidelberg

Recent findings of Terskikh et al. (PNAS 2001) and gene expression data of our group suggest a molecular interrelation of hemato- and neuropoietic cells and let assume that both cell types share molecular signaling pathways. In this study, we used real-time RT-PCR and flow cytometry to show for the first time that human CD34+ cells express the corticotropin-releasing hormone receptors 1 and 2 (CRH-R1, CRH-R2) as well as the orexin/hypocretin receptors 1 and 2 (ORX-R1, ORX-R2) on mRNA as well as on protein level $(n=8)$. CRH-Rs as well as their natural ligands CRH and urocortin are predominantly expressed in the brain and the pituitary gland but also in some peripheral sites such as cardiac and sceletal muscle, epididymis, and the gastrointestinal tract and mediate neuroendocrine stress responses. ORX-Rs and their ligands orexin A and B are almost exclusively expressed in the nervous system and have been shown to play a role in sleep-wake control, energy homeostasis and regulation of activity of sympathetic neurons. Applying two color immunofluorescence we found that $\mathrm{CRH}-\mathrm{Rs}$ as well as ORX-Rs are higher expressed in the CD38dim subset within the CD34+ cell population suggesting they might be particularly relevant for developmentally early CD34+ cells. Both, CRH-Rs as well as
ORX-Rs are G protein-coupled receptors. To figure out the functional effects of CRH-R or ORX-R stimulation we incubated immunomagnetically selected CD34+ cells with either urocortin $(100 \mathrm{nM})$ or orexin A $(300 \mathrm{nM})$ for $24 \mathrm{~h}$. Afterwards expression of 1,185 genes was measured by cDNA array technology (Clontech Atlas Human 1.2 I) and compared to untreated controls $(n=6)$. CRH receptor stimulation by urocortin caused up-regulation of IFN- $\gamma$, IL-13, and retinoic acid receptor $\beta$, and down-regulation of integrin $\alpha 4$ mRNA expression. Stimulation of orexin receptors by orexin A resulted in increased expression of IL- 8 and serine/threonine kinase 2 (STK2), and in diminished expression of transcription factor YY1 and opioid receptor $\mu 1$. In summary, we newly demonstrated CRH receptor 1 and 2 and orexin receptor 1 and 2 expression in human CD34+ hematopoietic stem and progenitor cells. Furthermore, we could show that those receptors regulate mRNA expression of transcription factors, receptors and cytokines in CD34+ cells. Our data support the model of a close ontogenetic and molecular propinquity of hemato- and neuropoietic cells and provide novel insight into stem cell signaling.

\section{0}

Osteopontin affects the hematopoietic stem cell pool in vivo

Stier S. (1), Ko Y. (1), Forkert R. (1), Lutz C. (1), Neuhaus T. (1), Grünewald E. (1), Cheng T. (2), Dombkowski D. (2), Rittling S. (3), Scadden D. (2)

(1) Medizinische Poliklinik, Universität Bonn, (2) AIDS-Research Center, MGH Cancer Center, Boston MA, USA, (3) Rutgers-University, Piscataway NJ, USA

Hematopoiesis involves highly regulated proliferation and differentiation during which a small number of multipotent stem cells give rise to a large number of more differentiated progeny. The molecular mechanisms governing the size and kinetics of stem cell pool are not well defined. Osteopontin (OPN) is a secreted, highly acidic, RGD-containing glycoprotein which is both produced by multiple mature hematopoietic cells types and can affect their function. To our knowledge, the role of OPN in earlier events in hematopoiesis has not been explored. We observed that CD34+ hematopoietic cells differentially expressed and produced OPN when stimulated by early acting (KL, FL or TPO) versus late acting (G-CSF, GMCSF, IL-6 or IL-3) cytokines. To evaluate the functional role of OPN in primitive hematopoietic cells we compared OPN deficient mice to their wild-type littermate controls. Immunophenotypic analysis demonstrated a significantly higher proportion of the stem cell enriched Sca1+c-kit+lincells in OPN null mice compared with controls $(P=0.03, \mathrm{n}=8)$. Total cellularity and the proportion of differentiated hematopoietic cells were not altered, suggesting that the increase in stem cells was not due to a complete block in the differentiation of primitive cells in the absence of OPN. To more accurately assess the stem cell frequency in the bone marrow of OPN null mice, we performed functional, limiting dilution, long term culture-initiating cell (LTC-IC) assays. Bone marrow cells from OPN-/- mice gave rise to a significantly higher number of LTC-ICs compared with wild-type controls $(\mathrm{P}=.01, \mathrm{n}=5)$. To assess whether the increased number of stem cells was due to enhanced stem cell self-renewal, we performed serial bone marrow transplantation and could identify no difference in outcome in up to 4 sequential transplants. In addition, we could detect no difference in the cell cycle profile of stem cells of OPN+/+ versus OPN-/- animals. In contrast, a trend toward reduced apoptosis in the OPN-/- Sca1+c-kit+lin- bone marrow stem cell population was observed $(P=0.06, n=4)$ suggesting that enhanced survival of hematopoietic stem cells participate in the different stem cell pool size. In sum, OPN plays a role in regulating the hematopoietic stem cell compartment, affecting the size of the stem cell pool through a mechanism that may include alteration in programmed cell death. OPN may offer a target for manipulation to achieve stem cell expansion.

\section{1}

Peripheral blood CD34+ progenitor cells from polycythemia vera patients demonstrate evidence for morphological and structural differences

Wickenhauser C., Siebolts U., Perez F., Varus E., Thiele J.

Institut für Pathologie, Universität Köln, 50924 Köln

Objectives: Polycythemia vera (PV) is a clonal myeloproliferative disease characterized by a trilineage marrow proliferation (so-called panmyelosis) with increased production of red blood cells and platelets. Proliferation and differentiation of erythroid and megakaryocytic progenitors is regulated by several cytokines. PV CD34+ progenitors are known to be hypersensitive to growth factors, although their receptor expression is reduced or absent. In 
this study CD34+ peripheral blood (PB) cells from PV patients, $\mathrm{PB}$ derived from healthy donors and patients with secondary polycythemia (SP) were investigated.

Material and Methods: CD34+ progenitors were enriched by an immunomagnetic isolation method. Cytomorphological examination was performed by histochemical and ultrastructural techniques. The cytokine receptor expression was evaluated by immunocytochemistry and FACS analysis and receptor transcription was studied by RT-PCR

Results: The highest amount of CD34+ cells was found in the SP group, while their frequency was slightly lower in PV. Native PV CD34+ cells demonstrated considerable differences in shape and form and according to electron microscopy exhibited intracytoplasmatic organelles like mitochondria and a more extended Golgi apparatus. In contrast, the control and SP patients constituted a more uniformand unmature phenotype. No premature transcripts for glycophorin A (GypA) and CD41b, both markers for advanced erythropoiesis and megakaryopoiesis, could be detected in PV patients. Also, coexpression for early acting hematopoietic cytokine receptors IL-3Ra and c-Kit and for initial erythropoiesis (GypC) or megakaryopoiesis (CD61) was similar in the different groups.

Conclusions: Our findings indicate that CD34+ cells from PV patients belong to a more activated state but are quiescent and not premature compared to the corresponding population of the control group. In conclusion, although a nonspecific increase in the release of progenitor cells from the bone marrow cannot be excluded an inhibition or at least prolongation of the cell cycle is discussed as a putative pathogenetic feature of PV.

\section{Stem Cell Transplantation I}

\section{2}

\section{Unrelated hematopoietic stem cell transplantation for acute myeloid leukemia: results on 133 patients}

Basara N., Kraut L., Guenzelmann S., Pohl H.-J., Linck D., Tran V., Fauser A.A., Kiehl M.G.

\section{Klinik für KMT und Hämatologie/Onkologie, Idar-Oberstein}

Objectives: Transplantation of hematopoietic stem cells (HSCT) from unrelated donor is increasingly used for the treatment of acute myeloid leukemia (AML). AML patients (pts) lacking a suitable sibling donor have a probability of approx. $80 \%$ to find an HLA-compatible unrelated donor.

Methods: Between 1994 and 2000, 68 pts with primary AML received an unrelated HSCT in our center. We analysed the outcome, i.e. overall and disease-free survival in this group of patients. In addition, the outcome of this group of pts was compared to the results of 65 patients with AML treated with HSCT from related donors. Both groups were matched with regard to age, FAB classification, stage of the disease and HLA-compatibility of the donor.

Results: Median age of pts was 36 (range 16 to 55; unrelated) and 40 (range 22 to 61 ; related) years, respectively. The male to female ratio was $33 / 32$ and 33/35, respectively. Conditioning for HSCT consisted either of a busulfan-based regimen in 65 pts (30 related, 35 unrelated) or total body irradiation in 68 pts (35 related, 33 unrelated). Graft-versus-host disease prophylaxis consisted of cyclosporine and prednisolone without (related transplants, $72 \%$ ) or with methotrexate (unrelated transplants, $84 \%$ ). Seventy nine $\%$ of pts with unrelated HSCT and $77 \%$ of pts with related HSCT have received an HLA-A, -B, and -DRB1 loci matched transplants. Median and average post-transplant follow-up was 8 and 18 months, respectively, with the longest overall survival being 6 years. Disease free survival (DFS) in the unrelated group of pts at 5 years was $54 \%$ for transplants transplanted in first complete remission (CR1) $(n=16), 47 \%$ in second CR (CR2) $(n=16)$ and $13 \%$ for advancedstages of the disease $(>C R 2)(n=36)$. Overall survival at 5 years was $47 \%(\mathrm{CR} 1), 36 \%(\mathrm{CR} 2)$ and $9 \%(>\mathrm{CR} 2)$. The cumulative incidences for relapse in the unrelated group were $13 \%, 19 \%$ and $44 \%$ for the three groups, respectively. DFS in pts treated with related transplants at 5 years was $42 \%(\mathrm{CR} 1)(\mathrm{n}=25), 31 \%(\mathrm{CR} 2)(\mathrm{n}=9)$ and $25 \%(\mathrm{CR} 2),(\mathrm{n}=31)$. The cumulative incidences for relapse in related group were $20 \%, 22 \%$ and $48 \%$ for the three groups, respectively.

Conclusion: The results of this comparative study confirm that unrelated HSCT is an important treatment option for patients with standard and highrisk AML, if sibling donors are not available.

\section{Allogeneic stem cell transplantation in high risk myelofibrosis} with myeloid metaplasia (MMM)

Baurmann H., Kreißig C., Bräuninger S., Prumbaum M., Judith D., Yaman A., Kaltenhäuser J., Schwerdtfeger R.

Zentrum für Knochenmark- und Blutstammzelltransplantation, Deutsche Klinik für Diagnostik, Wiesbaden, Germany

Objectives: MMM is a clonal myeloproliferative disorder of later life not generally considered for allogeneic stem cell transplantation (alloSCT). We present the results of seven consecutive patients (pts) with MMM submitted to alloSCT during le last two years in our institution.

Material and Methods: Five males and two females with primary $(n=6)$ and secondary MMM following essential thrombocythemia $(n=1)$ were grafted with unmanipulated bone marrow $(n=6)$ or peripheral stem cells ( $n$ $=1)$ from a related $(\mathrm{n}=2)$ or unrelated $(\mathrm{n}=5)$ donor. Median age was 56 (41-63) years, median time from diagnosis to transplant 15 (6-117) months Six pts had intermediate risk, one pt high risk disease according to Dupriez et al (Blood 1996). Five pts were transfusion dependent, two had portal hypertension following liver cirrhosis and portal vein thrombosis, respectively, and one insulin dependent diabetes mellitus with diabetic nephropathy Three pts had a history of solid tumor: hypernephroma, ovarian carcinoma and bronchial carcinoma, the last beeing detected and treated by lobectomy just prior to alloSCT. Four pts received standard conditioning by $\mathrm{Bu} / \mathrm{Cy}$ or $\mathrm{TBI} / \mathrm{Cy}$, three pts a protocol of intermediate intensity containing either $\mathrm{TBI}(8 \mathrm{~Gy}) / \mathrm{Flud} / \mathrm{Cy}$ or $\mathrm{Bu}(12) /$ Flud. In unrelated transplants ATG was added before grafting.

Results: All pts engrafted at a median of 21 (11-28) days. Two pts with unrelated donors died: One from cerebral/optical aspergillosis at day 333, the other from decompensated liver cirrhosis with hepatorenal syndrome and thrombocytopenia at day 266 post BMT. At a median follow up of 11 (1-27) months probability of disease free survival is $60 \%$

Conclusions: Although the number of pts is still very small and more follow up is needed, our results seem encouraging. In a pt population with advanced disease, advanced age and significant co-morbidity allogeneic stem cell transplantation is a curative treatment option for MMM.

\section{4}

Allogeneic transplantation in the elderly ( $>60$ years): outcome after unrelated and related donor transplantation in patients with poor risk hematological malignancies

\section{Bertz H., Potthoff K., Grüllich C. Mauerer K., Finke J.}

Dept. of Hematology-Oncology Albert Ludwigs University Medical Center, D-79106 Freiburg

Due to high treatment related morbidity and mortality the accepted upper age limit for allogeneic transplantation is 55 years for high dose busulphan or TBI containing conditioning regimens. Since 1998 we transplanted 33 pts. $>=60$ years median age of 63,6 years (range 60-73)) with haematological malignancies with our reduced intensity conditioning (RIC) regimen FBM (Fludarabine 5x $30 \mathrm{mg} / \mathrm{m}^{2}$, BCNU $150 \mathrm{mg} / \mathrm{m}^{2}$, Melphalan $110 \mathrm{mg} / \mathrm{m}^{2}$ ). Diagnosis were AML/RAEB-T(25), MDS(4) low-grade NHL/MM(3), MPS(1). Remission at transplantation: untreated (10), persistent primary induction failure (8), relapse untreated(7), CR2(4), RAI III/IV(2), PR1(1) and CP1(1), mainly with a high leukaemic burden. In 12/33 a matched sibling served as the donor and in 21/33 a matched unrelated donor (MUD). Grafts were PBSC in 29 pts. and BM in 4 pts.. GvHD-Prophylaxis consisted of cyclosporine + mycophenolat-mofetil (31) or + mini-MTX (2). ATG-S ${ }^{\circledR}$ (Fresenius) was added in 20 MUD-transplants. All evaluable pts. (27/33) achieved CR (82\%). 10 pts. died of: infection(6), relapse(2) and $\operatorname{aGvHD}(2)$ resulting in a TRM of $24 \% .12$ pts. had acute GvHD ${ }^{\circ} \mathrm{II}-\mathrm{IV}$. Two relapsing pts. achieved 2. CR after donor lymphocyte infusions(1) or 2. TX(1). At a median of 545 days (range 28-915) 23 pts. are alive and in CR with a DFS of $58 \%$ and OS of $67 \% ; 17 / 21(81 \%)$ MUD-patients are alive. Conclusion: Allogeneic transplantation with our FBM-protocol is possible in the elderly with an unrelated and related donor. Even in poor risk diseases it leads to good response rates with little TRM. Earlier transplantation may avoid infectious complications (e.g. aspergillosis). 
Secondary allogeneic transplantation with an uniform fludarabine/thiotepa containing regimen improves survival in patients with relapse after autologous or allogeneic transplantation

Bertz H., Potthoff K., Bachl C., Behringer D., Finke J.

Dept. of Hematology-Oncology Albert Ludwigs University Medical Center, D-79106 Freiburg

Objective: Patients with relapse after autologous transplantation have no chance to achieve long term remission with salvage chemotherapy; donor lymphocyte infusions cure only $10 \%$ of patients with relapse after allogeneic transplantation (alloTx). Because standard high dose busulphan or TBI-containing conditioning regimens for second alloTx lead to a high treatment related morbidity and mortality, we introduced a fludarabine containing regime in combination with Thiotepa for conditioning in secondary alloTx (Fludarabine $5 \times 30 \mathrm{mg} / \mathrm{m}^{2}$; Thiotepa $3 \times 5 \mathrm{mg} / \mathrm{kg}(\mathrm{n}=24)$ or $4 \times 5 \mathrm{mg} / \mathrm{kg}$ $(\mathrm{n}=8)) .32$ pts. (median age of 41 yy $(18-64)$ ), who relapsed after autoTX $(n=20)$ or alloTX $(n=12)$, were transplanted. Diagnosis were NHL/Hodgkin disease (14), AML/RAEB-T (7), CML (3), MPS (1), ALL (4) and Multiple Myeloma (3). Remission at transplantation: CR2/3 (4), relapse 1 (10), relapse 2/3 (5), PR (1), AP/BC (4), progress (3) and resistant primary induction failure (5). In 8/32 a sibling served as the donor and in $24 / 32$ a volunteer unrelated donor (VUD). Grafts were PBSC $(n=29)$ and BM $(n=3)$. Time intervals between second alloTX and 1st autoTx were in median 1103 days (131-2266) and between 1st and 2nd allo TX 373 days (145-1367), respectively. GvHD-prophylaxis consisted of cyclosporine A + miniMtx (26) or mycophenolat-mofetil (3) or MTX (2). ATG-S ${ }^{\circledR}$ (Fresenius) was added in VUD-transplants $(\mathrm{n}=22)$

Results: 22 pts. achieved CR (70\%), 12/20 pts. (60\%) after primary autoTx and $10 / 12$ pts. (80\%) after prim. alloTX, 5 pts. PR (15\%) SD (2) and 3 pts. before evaluation. 14 pts. died of relapse (6), infection (6) and organ failure (2) resulting in a TRM at day +100 of $25 \%$ (6/20 pts (30\%) after autologous and $2 / 12(17 \%)$ after previous alloTX). 8 pts. had acute GvHD ${ }^{\circ} \mathrm{II}-{ }^{\circ} \mathrm{IV}$ and 24 pts. ${ }^{\circ} 0$-I. At a median of 373 days (range 7-1148) 18 pts. are alive with an OS of $56 \%$ and a PFS of $43 \%$. There is no difference in OS $(60 \%$ vs $50 \%$ ) between prim. auto vs. alloTX.

Conclusion: Secondary alloTX is a chance of long term survival with tolerable TRM after our Flu/TT protocol. Adding ATG-S ${ }^{\circledast}$ in VUD transplantation no GvHD ${ }^{\circ} \mathrm{IV}$ occurred and no pt. died due to GvHD. Secondary alloTX should be considered early after relapse to reduce infectious complication.

316

CD34+ enriched peripheral blood progenitor cells from unrelated donors for allografting of adult patients: High risk of graft-failure, infection and relapse despite donor lymphocyte add-back

Bornhäuser M., Platzbecker U., Hölig K., Ehninger G.

\section{Med. Klinik und Poliklinik I, Universitätsklinikum Dresden}

Objectives: Fifty-one adults with hematological malignancies were transplanted with CD34+ selected peripheral blood progenitor cells (PBPC) from unrelated donors.

Methods: The conditioning protocol contained total body irradiation $(\mathrm{n}=17)$ or combinations of busulfan and other alkylating agents $(n=34)$. ATG was infused in all patients. The median number of $\mathrm{CD} 3+\mathrm{T}$ cells infused with the graft after purification with the Isolex $300^{\circledR}$ system in the first cohort of 18 patients was $2.1 \times 105 / \mathrm{kg}$. Prophylactic donor lymphocyte infusion (DLI) containing $1 \times 105 \mathrm{CD} 3+\mathrm{T}$ cells was performed on day 21 in the following 33 patients who had received PBPC purified by the CliniMACS ${ }^{\circledast}$ system. Results: Early graft-failure occurred in 8/51 patients (16\%). After a median follow-up of 31 months (range 8-60), the probability of disease-free survival was $36 \%$ for the whole group. Reasons for death were opportunistic infections $(n=15)$, graft-versus-host disease $(\mathrm{GvHD}, \mathrm{n}=7)$ and relapse $(\mathrm{n}=4)$ Pre-transplant factors with significant impact on disease-free survival were cytomegalovirus status and risk category of underlying disease. Occurrence of graft-failure or graft-versus-host disease were associated with poor outcome.

Conclusion: Recipients of CD34+ selected PBPC from unrelated donors are at high-risk for infectious complications, relapse and graft-failure which can not be prevented by early reinfusion of unmodified donor lymphocytes.
Fludarabine and targeted busulfan as conditioning for patients with high-risk chronic myelogenous leukemia and myelodysplastic syndrome transplanted with hematopoietic stem cells from HLA-compatible related or unrelated donors

Bornhäuser M. (1), Deeg H.J. (2), Thiede C. (1), Radich J. (2), Slattery J.T. (2), Hansen J. (2), Appelbaum F.R. (2), Jenke A. (1), Anasetti C. (2), Ehninger $G$. (1)

(1) Med. Klinik und Poliklinik I, Universitätsklinikum Dresden, (2) Fred Hutchinson Cancer Research Center, University of Washington, Seattle, USA

Objectives: The hypothesis of this study was that fludarabine (FLU) can replace cyclophosphamide (CY) in combination with busulfan (BU) in patients with advanced MDS and CML and facilitate donor engraftment with less toxicity.

Material and Methods: The conditioning regimen consisted of intravenous FLU $30 \mathrm{mg} / \mathrm{m}^{2}$ from day -9 to day -6 , and oral BU given at $1 \mathrm{mg} / \mathrm{kg}$ every 6 $\mathrm{h} \times 16$ from day -5 to day -2 , with doses adjusted to target plasma levels of $900 \pm 100 \mathrm{ng} / \mathrm{ml}$ at steady state. GvHD prophylaxis was with cyclosporine A (CSP) and short course methotrexate (MTX). Forty-two patients were enrolled for the treatment of CML blast phase $(n=2)$, CML blast phase/remission $(n=2)$, MDS with high-risk refractory anemia (RA, $n=6)$, RA with excess of blasts (RAEB, $n=13$ ), RAEB in transformation (RAEB-t, $n=3$ ), untreated $(n=3)$ or treated acute myelogeneous leukemia (AML) developing from MDS ( $\mathrm{n}=7,4$ in remission/3 resistant), and chronic myelomonocytic leukemia $(\mathrm{CMML}, \mathrm{n}=6$ ). The median patient age was 52 (range 12-65) years. Mobilized blood stem cells were infused from HLA-compatible siblings $(n=16)$ and unrelated donors $(n=26)$.

Results: Engraftment was achieved in all patients evaluable. The KaplanMeier estimates of relapse-free survival at 12 months are $46 \%$ for patients with MDS and $42 \%$ for all patients. The probability of non-relapse mortality at day 100 was $6 \%$.

Conclusions: These data suggest that FLU in combination with targeted BU can facilitate engraftment of blood stem cells from matched siblings and unrelated donors associated with limited early extramedullary toxicity.

\section{8}

Chronic GvHD after allogeneic peripheral stem cell transplantation - non-infectious lung complications indicate frequent pulmonary involvement

Buchholz S., Dammann E., Franzke A., Stadler M., Eder M., Ganser A., Hertenstein $B$.

Abteilung Hämatologie und Onkologie der Medizinischen Hochschule Hannover

Several studies have reported a comparable incidence of acute GvHD but a higher incidence of chronic GvHD after transplantation of unmanipulated allogeneic peripheral stem cells (PBSCT) compared to bone marrow transplantation (BMT). We observed a high incidence of non-infectious lung complications (NILC) probably resembling chronic GvHD of the lung in patients developing cGvHD after allogeneic PBPCT. Chronic GvHD occurred in 42 of 73 evaluable patients ( 45 male, 28 female; median age 39 , range 19-64) who received an unmanipulated peripheral stem cell graf from HLA-identical donors (unrelated donor 34, sibling 39 ). Underlaying disease AML (24), ALL (16), CML (15), MDS (9), NHL (6), multiple myeloma (2), CLL (1). Preparative regimens consisted of conventional conditioning (BU/CP 26, TBI/CP 15, TBI/VP16 7) or reduced intensity conditioning (Flu/Bu 13, Flu / Melph 7, other 5). Radioimmunotherapy was used additionally to pretreatment therapy in 7 cases. GvHD-prophylaxis consisted of CSA / MTX (50); CSA/MMF (13) or CSA/Steroids (8) Chronic GvHD was limited in $25(60 \%)$ and extensive in 17 patients $(40 \%) .19$ patients were transplanted from unrelated donors and 23 from sibling donors. Chronic GvHD was diagnosed in median 173 days after PBSCT, range $100-534 \mathrm{~d}$. Organ involvement included skin in $26(62 \%)$, liver in 30 $(71 \%)$, gastrointestinal tract in $10(24 \%)$, enoral mucositis in $27(64 \%)$, eyes with sicca-symptoms in $9(21 \%)$, myositis in $4(10 \%)$, arthralgia in 2 cases $(5 \%) .9 / 42(21 \%)$ patients (6 male, 3 female; age 29-60, median 43 years) developed obstructive lung changes (in 2 patients with additional NILC restriction) without a detectable infectious cause. 4 patients were transplanted from unrelated donors and 5 patients from sibling donors. NILC occurred at a median of 8 month after transplantation with a wide range from 4-18 month. 7/9 patients responded to immunosuppressive treatment, 1 patient died from progressive pulmonary. This data indicate that the incidence of 
chronic GvHD of lung after transplantation of allogeneic peripheral stem cells may be increased compared to that reported after bone marrow transplantation.

\section{9}

Intensification of the conditioning regimen for high-risk AML patients with $\mathrm{Re}-188$-labelled anti-CD66 monoclonal antibody: tumour load at transplant is the only factor affecting outcome

Bunjes D. (1), Wiesneth M. (2), Buchmann I. (3), Duncker C. (1), Von Harsdorf S. (1), Schlenk R. (1), Grimminger W. (1), Seitz U. (3), Kotzerke J. (3), Dohr D. (4), Buck A. (3), Glatting G. (3), Bergmann L. (5), Döhner H. (1), Reske S.N. (3)

(1) Abteilung Hämatologie / Onkologie, Universitätsklinik Ulm, (2) DRK Blutspendezentrale Ulm, (3) Abteilung Nuklearmedizin Universitätsklinik Ulm, (4) Abteilung Strahlentherapie Universitätsklinik Ulm, (5) Abteilung Hämatologie / Onkologie Universitätsklinik Frankfurt

Objective: To evaluate the factors influencing outcome after intensified conditioning with Re-labelled anti-CD 66 monoclonal antibody

Methods: The patient cohort consisted of 31 males anf 26 females with a median age of 45 ( range 17-63 y). The diagnoses were AML 1.CR 20 patients (pts.), AML 2.CR 11 pts., AML good PR ( 5-15\% blasts) 13 pts. and AML PR ( $15-25 \%$ blasts) 13 pts. Radioimmunotherapy was followed by standard full-dose conditioning with total body irradiation $(12 \mathrm{~Gy})(\mathrm{n}=30)$ or busulfan 12,8 mg / kg i.v. ( $=27$ ) and cyclophosphamide $120 \mathrm{mg} / \mathrm{kg} \pm$ thiotepa $10 \mathrm{mg} / \mathrm{kg}$. Patients subsequently received a $\mathrm{T}$ cell depleted allogeneic graft from a HLA-compatible family donor ( $n=24)$, a matched unrelated donor $(n=23)$ or a haploidentical family donor $(n=6)$. In 4 patients an unmanipulated autologous graft was used.

Results: Dosimetry was performed prior to therapy and a favourable dosimetry was observed in all cases. Radioimmunotherapy with the labelled antibody provided a mean of $15.5 \mathrm{~Gy}$ of additional radiation to the marrow, the kidney was the normal organ receiving the highest dose of supplemental radiation (mean 7.4 Gy). Infusion-related toxicity due to the labeled antibody was minimal and no increase in treatment-related mortality due to the radioimmunoconjugate was observed. Day +30 and day +100 mortalities were $3 \%$ and $7 \%$ respectively. Late renal toxicity was observed in $14 \%$ of patients. After a median follow-up of 26 months (range 3-50 months) the probability of disease-free survival for the whole group of patients was $47 \%$. Outcome was determined by the tumour load at transplant with a disease-free survival rate for 44 patients in 1 . or 2 . CR or in good PR $(<15 \%$ blasts in the marrow at transplant) of $64 \%$ with only $8 \%$ disease-free survival for those with $>15 \%$ blasts in the marrow at transplant. Outcome was similar whether patients were in 1 . CR, 2. CR or good PR, whether they received a graft from an HLA-compatible family donor or a matched unrelated donor and was independent of whether TBI or Bu was used. Treatment failure was due to relapse and TRM in equal proportions.

Conclusions: These data suggest that our approach may improve outcome in high-risk AML patients provided that induction chemotherapy can achieve a least a good partial remission.

\section{0}

Treosulfan and fludarabine as conditioning for allogeneic blood stem cell transplantation - final analysis of a phase $\mathrm{I} / \mathrm{II}$ study

Casper J. (1), Knauf W. (2), Dölken G. (3), Wolff D. (1), Steiner B. (1), Kleine D. (1), Hammer U. (1), Wegener R. (1), Freund M. (1)

(1) Universität Rostock, (2) Klinikum Benjamin Franklin, FU Berlin, (3) Ernst-Moritz-Arndt Universität Greifswald

Intravenous treosulfan and fludarabine were used as conditioning agents prior to allogeneic blood stem cell transplantation, within a phase I/II study. Thirty patients with CML, AML, MDS, multiple myeloma, NHL cbcc, tNHL and CLL otherwise non-eligible for allogeneic transplantation and a median age of 49 years (range 20-60) were transplanted. Fourteen matched related donors (MRD) and 16 matched unrelated donors (MUD) donated bone marrow or peripheral blood stem cells. The preparative regimen consisted of treosulfan $10 \mathrm{~g} / \mathrm{m}^{2}$ i.v. d- 6 to d- 4 and fludarabine $30 \mathrm{mg} / \mathrm{m}^{2}$ i.v. d-6 to d-2. Patients with unrelated donors received ATG (rabbit) $10 \mathrm{mg} / \mathrm{kg} \mathrm{d}-4$ to $\mathrm{d}-2$ in addition. Cyclosporine-A was given as GvHD prophylaxis only. Leukocyte recovery $>0.5 \mathrm{Gpt} / 1$ and $>1 \mathrm{Gpt} / 1$ occurred by median day +9 (range $0-18$ ), resp. day +10 (range $0-19)$. Platelet recovery $(>50 \mathrm{Gpt} / \mathrm{l})$ occurred by median day +16.5 (range $8-$ not reached). Extramedullary toxicity was mild, not exceeding CTC ${ }^{\circ} \mathrm{II}$ except for ALAT/ASAT elevation in $33 \%$ (10/30 $\left.\mathrm{CTC}^{\circ} \mathrm{III}\right)$, bilirubine in $\left.13 \%\left(4 / 30 \mathrm{CTC}{ }^{\circ} \mathrm{III}\right)\right)$ resp. $3 \%(1 / 30$ CTC $\left.{ }^{\circ} \mathrm{IV}\right)$, diarrhoea in 3 (10\% CTC $\left.{ }^{\circ} \mathrm{III}\right)$ and obstipation in $3 \%(1 / 30$
$\left.\mathrm{CTC}^{\circ} \mathrm{III}\right)$ of the pts.. However, ALAT/ASAT elevations were pre-existing in 7 of 10 of the pts. with $\mathrm{CTC}^{\circ} \mathrm{III}$. Additionally in one patient a reversible peripheral polyneuropathy occurred (CTC $\left.{ }^{\circ} \mathrm{III}\right)$ and the cause of this polyneuropathy remains unclear. Complete chimerism was reached in $81 \%$ of pts. by day +28 and in $90 \%(26 / 29)$ at any time after transplantation. One pt. (CML) had a primary graft failure and recovered with his own hematopoiesis and one pt. (CLL 75\% donor at $d+28$ ) had lost all signs of donor hematopoiesis at day 45. Retrospectively, an allo-immunization to donor histocompatibility antigens prior to transplantation could be demonstrated. Another pt. with CLL remained a mixed chimera and 1 pt. died before a chimerism analysis was performed. Relapses occurred in 3 CML (cytogenetic), 2 AML (transplanted in beginning relapse or PR) and 1 pt. with a high-grade NHL. One pt. with a therapy refractory CLL required further treatment. The treatment-related mortality after a median observation time of 15 (1-32) months is $20 \%(6 / 30)$ including the one pt. with multiple myeloma who died due to complications of his accompanying cardiac amyloidosis. With an estimated overall survival of $76 \%$, further evaluation of the treosulfan and fludarabine combination may lead to a promising toxicity-reduced conditioning regimen.

\section{1}

\section{Angiosarcomas of the heart - follow-up of 2 cases after} highdose-chemotherapy and autologous transplant

Dingeldein G., Hoffmann M., Weiss B., Henrich D., Uppenkamp M.

Medizinisch Klinik A, Klinikum Ludwigshafen, Bremserstr.63, Ludwigshafen

Objectives:Angiosarcomas of the heart are rare solid tumors with a worse prognosis. We present two cases, which received a high-dose chemotherapy with autologous stem cell transplantation with a follow up of 13 month. Methods: Two young men (age 37+47y.) were transferred in our clinic of heart surgery in our heartcenter. The patients were operated under the initial diagnosis of myxoma of the heart, but the intraoperative and histopathological findings revealed angiosarcomas of the right atrial and ventricle. In both cases a radical tumor operation was not possible because of size and infiltration. Both tumors were highly mitotic (G3). The staging revealed no further manifestations of the angiosarcomas. A typical therapy for sarcomas with adriamycin $75 \mathrm{mg} / \mathrm{m}^{2}$ (d1) and ifosfamide $1500 \mathrm{mg} / \mathrm{m}^{2}$ (d1-4) was applied. After the 4. cycles of therapy a stem cell-harvest of 3,3 and $6,8 \times 106$ /kg KG CD 34-pos- cells was performed. Both patients received an autologous stem cell-transplantation after ICE- high-dose chemotherapy (ifosfamide/ carboplatin/etoposid), in order to prolong the overall survival.

Results: Toxicities were a mucositis WHO grade III and in both cases a neutropenic fever WHO grade III-IV, which resolved with antibiotic therapy. The leukocyte-take was on day $10+11$, thrombocyte-take was on day $13+15$. Both patients are still alive and disease-free after a follow up of 13 month. One patient developed 5 months after highdose-chemotherapy and autologous transplant a carcinoma in situ of the bladder, which could be resected in toto. As an additional therapeutic option an allogene heart-transplant is planned in one patient.

Conclusion: The angiosarcomas of the heart are very rare and aggressive tumors with short survival-time. Allogene heart-transplantation alone is not sufficient to prolong survival, due to distant metastases, especially to the brain. High-dose chemotherapy with autologous stem cell transplantation followed by heart transplantation could be a therapeutic option for young patients to prolong their survival.

\section{Secondary cancer after haematopoietic stem cell transplanta-} tion

Ditschkowski M., Trenschel R., Steckel N., Lollert A., Ottinger H., Beelen D.W., Schaefer U.W., Elmaagacli A.H.

\section{Klinik für Knochenmarktransplantation, Uniklinikum Essen}

To evaluate the incidence and associated risk factors of secondary malignan neoplasms we analysed 1368 patients who had undergone haematopoietic stem cell transplantation at our centre between 1975 and 2001.

All secondary solid malignancies $(n=20)$, Hodgkin's diseases $(n=2)$ and non-Hodgkin lymphomas ( $\mathrm{n}=7$ including EBV associated lymphoproliferative disease) were included, secondary leukaemia and myelodysplastic syndromes were not considered. The patient collective was subdivided into three groups referring to the graft type: 17 pts. (patients) had syngeneic (median follow-up: 31 months), 147 pts. autologous (median follow-up: 25 months), and 1204 pts. allogeneic bone marrow or peripheral blood stem cell transplants, respectively (median follow- up: 196 months). 
A total number of 29 secondary malignant neoplasms was observed. In the syngeneic and autologous transplantion group, 1 patient each developed secondary solid cancer. Among the allogeneic transplanted pts., 27 malignant neoplasms occurred. Median age of all pts. with secondary cancer (18 male, 11 female) was 35 years at time of transplant and the median followup was 85 months. Secondary neoplasms were diagnosed on an average of 65 months (median) after transplantation. Eighteen of 29 pts. who developed secondary cancer suffered from acute, 19 pts. from chronic graft versus host disease (GvHD). Except 5 pts. who had chemotherapeutic conditioning all other pts. with secondary malignancies received total body irradiation during conditioning. Among the solid cancers, malignant neoplasms of skin and mucous membranes were most prevalent $(n=12)$. However, no statistical significant risk factors for the development of secondary cancer could be identified.

In conclusion, the risk of transplantation associated tumor development especially after allogeneic transplant increases with longer follow-up. This underlines the need for a close monitoring concerning secondary cancer.

\section{Is there a risk to transmit GB Virus-C by progenitor/stem cell transplantation?}

\section{Eder G., Pavlova B., Pittermann-Höcker E.}

L. Boltzmann Institut für Leukämieforschung und Hämatologie, Wien

The frequency of GB Virus-C infected blood donors varies between $1 \%$ and $4 \%$. The tissue tropism of the virus is not well understood. A suspected association between liver diseases and GBV-C infection could not be confirmed. Tucker et al recently reported the detection of a replicative strand of the virus in spleen and bone marrow, but not in tonsils, lymphnodes or other lymphatic tissues. We reported earlier a high frequency of GBV-C in hematological disorders $(62 \%)$ with a significant higher portion of malignant diseases (M. Hodgkin, NHL, AL, MM - 72\%) compared to clonal stem cell disorders with a long latent period (MDS and MPE - 28\%) not associated with blood transfusion, chemotherapy, elevated liver enzyme levels or viral co-infection.

Objectives: The aim of the study was to provide further in vivo evidence for infection and replication of GBV-C in hematopoetic cells. For this purpose CD34+ stem cells of a GBV-C infected chimpanzee were tested for GBV-C RNA.

Material and Methods: CD 34+ cells were mobilized by G-CSF (Filgrastim ${ }^{\circledR} 1$ million U/kg s.c. for five consecutive days), a method utilized since several years in baboons and macaques. A direct capture test with coated magnetic beads and nested PCR technique with primers of the 5'UTR and NS3 region was used.

Results: GBV-C RNA was detected in MNC, mature granulocytes and lymphocytes. The isolated CD34 + cells were found positive for GBV-C RNA with NS3 primers but negative with 5'UTR. The remaining CD34 negative cells were positive for both primer combinations. However, after separation of the lymphocytes with pan-T and pan-B coated paramagnetic beads this fraction remained GBV-C RNA negative.

Conclusions: The presence of GBV-C RNA in a CD34+ cell population proved to be a possible replication site of the virus. The strong positivity of the lymphocytic fraction confirmed the finding of others. However, the high frequency of GBV-C positivity in AML cases suggests that stem cells are the first choice of replication site. Further studies are necessary to confirm or to dismiss a possible role of the virus as an exogenous stimulus to cell deregulative processes.

\section{4}

\section{Influence of the donor CMV serostatus on the incidence of CMV infection in allogeneic stem cell transplant recipients}

Eibl M. (1), Quehenberger F. (2), Zinke-Cerwenka W. (1), Sormann S. (1), Linkesch W. (1)

(1) Division of Hematology - Department of Internal Medicine, (2) Institute of Medical Informatics, Statistics and Documentation; Graz, Austria

Objectives: The incidence of CMV infection can be minimized, if both donor and recipient are seronegative pre transplant. If the recipient is seropositive, the optimal donor serostatus has to be determined. We evaluated the donor CMV serostatus as a risk factor for CMV reactivation, CMV disease and survival in seropositive stem cell transplant recipients.

Patients and methods: From 1996 to 200273 consecutive patients, aged from 19 to 64 years (median: 42), were allografted with either bone marrow $(n=30)$ or peripheral blood progenitor cells $(n=42)$ or cord blood $(n=1)$ from their related $(\mathrm{n}=39)$ or unrelated $(\mathrm{n}=34)$ donors. Conventional dose conditioning was performed in 58 and reduced conditioning in 15 patients. Antithymocyte globuline as part of the conditioning was administered in 41 patients. The following CMV donor/recipient status could be found: negative/negative: $n=20$, positive/positive: $n=23$, positive/negative: $n=7$, and negative/positive: $n=23$. CMV monitoring with qualitative PCR was performed twice weekly. Premptive therapy using either gancyclovir, foscarnet or cidofovir was initiated after CMV reactivation (defined as two consecutive positive PCR results without any symptoms).

Results: Among the CMV seropositive recipients the probability of CMV reactivation at one year was $32 \%$ if the donor was seropositive, and $71 \%$ if the donor was seronegative $(\mathrm{p}=0.004)$. The probability of CMV disease at one year was $32 \%$ with positive donors and $64 \%$ with negative donors $(\mathrm{p}=$ $0.04)$. Overall survival at three years was $29 \%$ and $21 \%$, respectively $(p=$ 0.73). Eight patients died from CMV disease. They were all seropositive themselves, but six out of them had a negative donor.

Conclusion: Regarding the incidence of CMV reactivation, CMV disease and death from CMV disease in seropositive patients, donor seropositivity is associated with an improved outcome. This effect is most likely mediated by transfer of specific donor $\mathrm{T}$ cells.

\section{5}

Improved survival after experimental bone marrow transplantation by the induction of hemoxygenase I prior to conditioning

Gerbitz A., Wilke A., Ewing P., Eissner G., Andreesen R., Holler E.

University Clinic Regensburg, Dep. of Hematology and Oncology

Acute GvHD remains the main cause of high mortality after allogeneic bone marrow transplantation. The current pathophysiological concept of acute GvHD involves on one hand damage and activation of the host by the conditioning regimen, on the other hand release of cytotoxic cytokines from various cells of donor and host origin. Current strategies in GvHD treatment focus on the blockade of cytokine-receptors, on cytokines themselves or on $\mathrm{T}$ cell depletion. The emphasis to protect the host against irradiation induced damage has led to reduced conditioning regimens, but this approach may affect anti-tumor efficacy at least in some underlying diseases.

The enzyme hemeoxygenase 1 (HO-1) has been shown to protect a variety of cells from induction of programmed cell death by ionising irradiation. This enzyme is involved in the first step of heme degradation and is therefore constitutively expressed in the spleen. It is also inducible in a wide range of tissues such as liver, skin and gut. A potent inducer of HO-1 aside from heme is the Cobalt salt of Protoporphyrine IX (CoPP).

In an experimental murine MHC haploidentical transplantation model using highdose conditioning, we were able to show that the induction of HO-1 prior to irradiation is followed by improved survival and reduced acute GvHD: Approximately $50 \%$ of the control animals die due to acute GvHD within the first 2 weeks compared to $20 \%$ in the CoPP treated group. Longterm survival by day 70 is also improved from $15 \%$ to approximately $70 \%$. Administration of 125 ug CoPP on day -2 and -1 results in reduced serum TNF $\alpha$ levels at day +4 after transplant. Spontaneous TNF $\alpha$ secretion by peritoneal macrophages is reduced when animals are treated with CoPP prior to irradiation. In addition, $\mathrm{TNF} \alpha$ release by macrophages after stimulation with bacterial lipopolysaccharide (LPS) is markedly affected showing a loss of responsiveness to this potent stimulus and trigger of GvHD. Macrophages lacking TNF $\alpha$ production show cytoplasmatic HO-1 expression whereas PBS treated cells do not. In summary, these results demonstrate a survival benefit of animals treated with CoPP prior to conditioning which is associated and possibly explained by reduced TNF $\alpha$ release by macrophages in response to LPS. In further studies the direct effect of HO1 expression on tissue damage after conditioning will be explored.

\section{6}

Successfull related cord blood transplantation for high risk ALL in a 5-year old boy

Guenther C. (1), Führer M. (2), Brandl M.(1), Hahn U. (1), Bender-Goetze C. (2), Knabe H.(1)

(1) BSB Bayerische Stammzellbank gGmbH, Robert-Koch-Allee 7, 82131 Gauting, (2) Kinderpoliklinik der Universität München, Pettenkoferstr. 8a, 80336 München, Germany

We report on the first related cord blood transplantation performed in Bavaria (GER) in July 2000

Patient: The child, born 1995, suffered from ALL, diagnosed in Oct. 1999 with initial high leukocyte count $(>100.000 / \mu \mathrm{l})$, pre-T-ALL immunophenotype and a cytogenetic abnormality (del 9p13). The boy was treated in ac- 
cordance to the German ALL-BFM-99 protocol. He responded poorly to therapy (long persistance of leukemic cells, continuing cytogenetic deletion and minimal residual disease). The clinical course was complicated by cerebral seizures with the need for mechanical ventilation and episodes of fever. The search for an unrelated bone marrow donor initiated in March 2000 was not successfull.

Cord blood donor: After the birth of the healthy brother in May 2000 the cord blood was collected, processed and cryopreserved. HLA-typing showed a complete match.

Transplantation: In June 2000 an autologous back-up was gained. The patient was in morphologic remission but still MRD positive. The conditioning regimen consisted of TBI (12 Gy) and Vepesid (60 m/kg), GvHD prophylaxis of CyA. The cryopreserved cord blood was thawed and the DMSO washed out to improve the viability of transplanted cells. The final volume transplanted was $75 \mathrm{ml}$. The number of vital nuclear cells was low with $1,13 \times 10^{\wedge} 7 / \mathrm{kg}$. The time from thawing to transfusion did not exceed $3 \mathrm{~h}$ Hematopoetic reconstitution: The post-transplant period was uneventfull with granulocyte reconstitution $>500 / \mu \mathrm{l}$ on day 19 and the last thrombocyte transfusion 2 months after transplantation. The boy was discharged on day 43

GvHD, infections and chimerism: The patient experienced a short period of acute GvHD grade I, but no signs of chronic GvHD were noted until now (2 years after tlx). Except for an episode of Herpes Zoster no severe infectious complications were noted. He is a complete chimera (short tandem repeats) after transplantation. No further immunosuppresion had to be given.

Conclusion: The successfull transplantation of related HLA-identical cord blood in a young boy with high risk ALL shows the feasability of the alternative stem cell source even with a very low amount of vital nuclear cells (< $3 \times 10^{\wedge} 7 \mathrm{MNC} / \mathrm{kg}$ ). The hematopoetic reconstitution was rapid and the posttransplantion period without major complications. We suggest that cord blood asservation and transplantation should be considered as a routine procedure in siblings of children with malignant diseases.

\section{7}

Treatment of pure red cell aplasia after peripheral blood stem cell tranplantation (PBSCT) with plasma exchange and erythropoietin - a case report

Haack A. (1), Albrecht I. (1), Zimmer K. (1), Jenke A. (2), Ehninger G. (2), Bornhäuser M. (2), Hölig K. (1)

(1) Transfusionsmedizin, (2) Medizinische Klinik und Poliklinik I, Klinikum der Technischen Universität, Dresden, Germany

Objectives: Major ABO-incompatibility normally does not affect overall survival after allogeneic PBSCT but can give rise to delayed reticulocyte engraftment. We report on a case of long-lasting pure red cell aplasia (PRCA) Methods: A male 46 years old patient with multiple myeloma $(\operatorname{IgA})$ was treated with allo-PBSCT from a male HLA-identical unrelated donor. The patient was blood group $\mathrm{O}$ with high titres of isoagglutinins (anti-A1 1:4096; anti-A2 1: 2048), the blood group of donor was A2. Analysis of anti-A antibody titres was performed with indirect antihumanglobulin test in ID-Microtyping technique (Fa. Diamed; Bensheim, Germany).

Conditioning regimen consisted of Busulfan $(16 \mathrm{mg} / \mathrm{kg})$ and Fludarabin $\left(120 \mathrm{mg} / \mathrm{m}^{2}\right)$, Methotrexate and Cyclosporin were administrated for GvHDprophylaxis.

Engraftment of myelopoiesis and megakaryopoiesis was reached at day 14 and 16 respectively, but no donor erythropoiesis could be detected at day 219 after PBSCT. 5 doses of Rituximab were given without any success Therapeutic plasma Exchange (TPE) was introduced at day +219.14 treatments were performed over a period of 9 weeks using the Cobe Spectra (Gambro BCT, Lakewood, US) cell separator. During each TPE one patient plasma volume was removed and 5\% humanalbumin solution was used as replacement fluid. Additionally rHuEPO was administered at a dose of 1000 IE subcutaneously 3 times a week for 2 weeks and 3 x 3000 IE for another 4 weeks.

Results: The patient tolerated all TPE procedures without any side effects Anti- A1 and A2- titres declined continuously after initiation of TPA-treatment. At day +286 Anti-A2 disappeared and first donor red cells were detectable. The patient became independent from red cell transfusions at day +280 and complete erythropoiesis of donor type could be shown at day +357 . Actually he is well and in remission with complete donor chimerism. Anti-A1 antibody is still detectable at a titre of $1: 1$.

Conclusion: TPE in connection with rHu EPO is a safe and efficient treatment for PRCA after ABO-majorincompatible PBSCT.
Sirolimus in the treatment of acute and chronic graft-versushost-disease - a pilot study

Hahn J., Wittnebel S., Poetscher Ch., Andreesen R. , Holler E.

Klinikum der Universität Regensburg, Abteilung für Hämatologie und Internistische Onkologie

Background: Treatment of patients (pts) with steroid-refractory acute Graftversus-Host-Disease (sr aGvHD) or extensive chronic GvHD (ext cGvHD) is still unsatisfying, making new treatment options necessary. We therefore evaluated in a pilot study the toxicity and efficacy of sirolimus (rapamycin) in the treatment of pts with sr aGvHD or ext cGvHD as third- or fourth-line treatment.

Methods: We treated 17 pts with overall 19 treatment periods. Indications were $\mathrm{sr}$ aGvHD $(n=4)$ after second-line antibody treatment, ext cGvHD with $(n=5)$ and without $(n=10)$ lung involvement after second- or third-line treatment. In general sirolimus was added to the ongoing treatment regimen in an average dose of 1 to $2 \mathrm{mg}$ /day orally, aiming at serum trough levels of 10 to $15 \mathrm{ng} / \mathrm{ml}$.

Results: Treatment was stopped in 14 of 19 cases due to nephrotoxicity/microangiopathy $(n=5)$, infections/viral reactivations $(n=5)$, relapse $(n=1)$ or patient related reasons $(n=3)$. In 10 out of 14 cases stopped, treatment period was too short or compliance too insecure to evaluate these cases. Three of these 10 pts died early under therapy due to pneumonia $(n=2)$ or sepsis $(n=1)$. Median observation time was 204 days (range 121-422) in those 9 cases analyzed, compared to 18 days (range 5-183 days) in thoses 10 cases not evaluated. Six out of nine pts responded, defined as stable disease or reduction or cessation of steroid treatment. Responders were 1 of 4 pts treated with sr aGvHD, 3 of 5 pts with ext cGvHD with lung involvement and 2 of 10 pts with ext cGvHD without lung involvement. In pts treated with sr aGvHD we found a remarkably high rate of endothelial toxicity as 3 of 4 pts developed a HUS/TTP-like syndrome.

Conclusion: Sirolimus seems to be a promising new immunosuppressive agent especially in pts with ext cGvHD with lung involvement, but there are still a lot of open questions concerning dosing, timing, toxicity and safety of this drug in pts with acute or chronic GvHD to be adressed in further prospective clinical trials.

\section{9}

Lineage specific chimerism analysis of different hematopoietic cell lines after allogeneic hematopoietic cell transplantation

Hamann M. (1), Pihusch M. (1,3), Böck S. (1), Link T. (1), Heller T. (1), Rolf B. (2), Pihusch R. (1,3), Hiller E. (1), Kolb H.-J. (1,3)

(1) Department of Hematology and Oncology, (2) Institute of Legal Medicine, (3) José Carreras Transplantation Unit, University of Munich, Germany

Graft-versus-host disease (GvHD) is one of the major complications following allogeneic hematopoietic cell transplantation (AHCT). As evidence exists that not the chimerism of leucocytes, but the chimerism of dendritic cells (DC) and T-cells (TC) seems to be important for the initiation of GvHD, we analysed the chimerism of leucocytes, DC and TC.

At the time of engraftment, 6 and 12 months after AHCT blood samples were taken from 169 voluntary patients (108 male/61 female; 93 PBSCT/76 BMT; 93 related Tx/76 unrelated Tx) with different hematological disorders (51 AML, 42 CML, 25 ALL, 16 NHL, 14 MM, 7 CLL, 4 SAA, 4 OMF, 3 MDS, 2 Hodgkin's Disease, 1 SCA). DC were isolated from the peripheral blood by fluorescence activated cellsorting. The purity of isolated DC was $>95 \%$. TC were isolated by MACS (LS/MS-positive selection columns). The chimerism analyses were performed by using FISH- or STR-PCR-technique.

There were differences between the lineage specific chimerisms: At the time of engraftment $73,3 \%$ of the analysed patients showed a complete chimerism (38,5\% GvHD), 23,4\% a mixed chimerism (44,4\% GvHD) and $3,3 \%$ no chimerism (100\% GvHD) of peripheral blood DC1 (CD123+). A complete DC2 (CD11c+) chimerism showed $76,0 \%$ patients $(41,2 \%$ GvHD), a mixed chimerism $21,2 \%(57,1 \%$ GvHD) and no chimerism $2,8 \%$ $(33,3 \%$ GvHD). The leucocyte chimerism was complete in $74,2 \%(48,7 \%$ GvHD) and mixed in $25,8 \%(48,1 \%$ GvHD) of the patients. $76,6 \%$ of the analysed patients had a complete chimerism (51,7\% GvHD) and $23,4 \%$ a mixed chimerism $(33,3 \%$ GvHD) of TC. 6 months after the BMT the patients were analysed again: At this time the DC1 chimerism was complete in $94 \%$ and mixed in $6 \% .98 \%$ had a complete DC2 chimerism (20\% GvHD) and $2 \%$ a mixed chimerism. A complete leucocyte chimerism showed $85,7 \%$ ( $25 \%$ GvHD), $14,3 \%$ of chimerisms were mixed ( $0 \%$ GvHD). $81,8 \%$ of the TC chimerisms were complete and $18,2 \%$ mixed $(33,3 \% \mathrm{GvHD})$. 
After 12 months $92,9 \%$ had a complete DC1 chimerism, 7,1\% a mixed chimerism. $95,8 \%$ showed a complete chimerism of DC2, 4,2\% a mixed chimerism. Of the analysed leucocyte chimerisms were $94,9 \%$ complete and $5,1 \%$ mixed.

There are significant differences in the lineage specific chimerism analysis. Due to the clinical situation of the patients the leucocyte chimerism is correlated with hematopoietic reconstitution, whereas DC- and TC-chimerism may predict the induction of immune tolerance and GvHD.

\section{0}

Low regimen related toxicity of radioimmunotherapy with 188Rhenium-labelled anti-CD66abce antibody in addition to standard and reduced intensity conditioning regimens prior to allogeneic stem cell transplantation

Hertenstein B. (1), Hofmann M. (2), Dammann E. (1), Eder M. (1), Buchholz S. (1), Franzke A. (1), Stadler M. (1), Diedrich H. (1), Forstmeyer D. (1), Reuter C. (1), Wittwer S. (1), Ganser A. (1)

(1) Abteilung Hämatologie und Onkologie und (2) Abteilung

Nuklearmedizin der Medizinischen Hochschule Hannover

Radioimmunotherapy (RIT) with 188Rhenium labeled anti-CD66abce antibodies in the conditioning regimen for allogeneic stem cell transplantation has recently been reported to efficiently reduce the relapse rate in patients with high risk leukemias. There remain concerns, however, about the regimen related toxicity, in particular the incidence of gastrointestinal complications and GvHD with the use of unmanipulated stem cell transplants.

We report our experience with this method in 14 patients (median age 47ys. 23-63) receiving RIT as part of the conditioning regimen for high risk leukemia (12 AML, 1 CMML, 1 ALL). 6 patients were transplanted in relapse, 8 patients in $\mathrm{CR}$ ( $>1 . \mathrm{CR}$ or high risk due to cytogenetics or response to induction therapy). Donors were HLA-matched siblings in 3 and HLAmatched unrelated donors in 11 cases. Conditioning consisted of Busulfan $(16 \mathrm{mg} / \mathrm{kg}) /$ Cyclophosphamide $(120 \mathrm{mg} / \mathrm{kg})(\mathrm{n}=6)$, Fludarabin $150 \mathrm{mg} / \mathrm{m}^{2}$ and either Busulfan $(8 \mathrm{mg} / \mathrm{kg})(\mathrm{n}=5)$ or Melphalan $\left(180 \mathrm{mg} / \mathrm{m}^{2}\right)(\mathrm{n}=3)$. Reasons for reduced intensity conditioning were age (57-62 ys., $n=5)$ or previous toxicity $(\mathrm{n}=2)$. All patients received unmanipulated grafts (PBSC 13, BM 1). GvHD-prophylaxis consisted of CSA / MTX $(n=9)$ or MMF $(n=5)$ Patients grafted from an unrelated donor received ATG as part of the conditioning regimen. RIT resulted in a bone marrow irradiation of a median 11 Gy (range 6-15). The doses delivered to the kidneys were 9 Gy (3-12), to the liver 2,6 Gy (1-7), and to the lung 6 Gy $(4-8,5)$, respectively.

All patients engrafted. The transplant related mortality was low in this high risk population. Only one patient died of veno-occlusive disease of the liver. Furthermore, we observed a low incidence of acute and chronic GvHD in $3 / 14$ and 3/9 patients, respectively. With a limited follow up of 10 month $4 / 6$ patients transplanted not in CR relapsed again, and $6 / 8$ patients transplanted in CR remain in remission. Our data demonstrate that the addition of RIT to either standard or intensity reduced chemotherapy based conditioning regimens did not increase the regimen related toxicity or GvHD-incidence.

\section{1}

Follow up of patients with indolent lymphoma treated with CHOP/Rituximab induction treatment followed by high dose chemo/radiotherapy and autologous PBSC-transplantation as first line therapy

\section{Heß G., Flohr T., Huber C., Kolbe K., Derigs G., Fischer T.}

\section{Med. Klinik, Johannes Gutenberg-Universität, Mainz}

To evaluate safety and feasibility of a combined modality treatment with CHOP/Rituximab followed by high dose consolidation chemotherapy (HDT) in patients with untreated indolent lymphoma we performed a clinical phase I/II study. Fifteen patients without prior chemotherapy with advanced and progressive FCL/MCL have been enrolled into a treatment protocol combining CHOP/ Rituximab-CHOP therapy with subsequent consolidation high dose therapy and autologous stem cell support. A close follow up of immune reconstitution was included. Overall, 15 patients were enrolled and 13 patients completed the entire treatment protocol without major toxicities or increased infectious complications. One patient was excluded due to withdrawal of consent after achieving CR prior to HDT. One patient was taken off study with signs of disease progression after CHOP/Rituximab. All patients showed stable engraftment after high dose therapy. Response rates appear to be favourable, indicating additional effect of Rituximab and HDT. Overall, 12 of 13 patients achieved CR/CRu and one patient PR. Two patients have relapsed now. Follow up of immune reconstitution displayed transient severe combined immunodeficiency with slow normalization of the cellular and humoral compartments over 12 months post HDT. We conclude, that high dose chemotherapy can be safely applied following treatment with CHOP plus Rituximab. Despite delay of B-cell and CD 4 cell reconstitution, infectious complications during followup are rare. Efficacy in this small cohort of patients was high, suggesting an additional therapeutic effect of Rituximab combination chemotherapy followed by HDT. Actual follow up data of will be presented.

\section{2}

Follow up of a phase I/II Study for the evaluation of Rituximab in vivo purging in combination with CD34-positive selected autologous stem cell transplantation for salvage therapy in B-NHL

Heß G. (1), Flohr T. (2), Kolbe K. (1), Gamm H. (1), Huber C. (1), Derigs H.G. (2)

(1) III. Med. Klinik, Johannes Gutenberg Universität, Mainz;

(2) III. Medizinische Klinik, Krankenhaus Höchst, Frankfurt am Main

We have performed a clinical study to evaluate the safety, feasibility and efficacy of Rituximab included into a sequential salvage protocol for CD20+ B-NHL in relapse or induction failure. Overall, 37 patients with CD20+ BNHL in relapse or induction failure received Rituximab combined with DexaBEAM (R-DexaBEAM) for stem cell mobilization. Ex vivo selection of CD34 positive cells was performed using the CliniMacs ${ }^{\circledR}$ positive-selection method in patients who mobilized more than $5 \times 106 / \mathrm{kg}$ CD34+ cells ( $\mathrm{n}$ $=19$ ). Two doses of Rituximab were included into the high dose therapy regimen (HDT)

R-DexaBEAM was well tolerated and 36 of 37 patients mobilized sufficient numbers of CD34+ blood stem cells to receive HDT. Application of R-DexaBEAM resulted in significant depletion of peripheral B-cells. All patients showed stable engraftment of hematopoesis after HDT. One treatment related death occurred after HDT due to sepsis. During follow up a high rate of CMV-reactivation was seen, both in patients who received purged or unpurged stem cells. With preemptive ganciclovir medication no CMV disease occured A prolonged combined immunodeficiency was observed post HDT, with half of patients demonstrating a T4-cell count below $200 / \mu 1$ at day +180 and subnormal serum immunoglobuline levels for up to 3 years in a substantial proportion of patients.

Remission rate post HDT was 94\% (CR/CRu: 30/35; PR: 3/35). With a follow up of up to 42 months 14 patients have relapsed until now. Actual overall and progression-free survival will be presented. The treatment protocol has proven feasible with high purging efficiency and encouraging remission rates in this heavily pretreated patient population. Prolonged impairment of the immune system post transplant needs special attention. Efficacy of this treatment protocol is currently been investigated in an ongoing multicenter study.

\section{3}

\section{CMV infection in autologous stem cell transplantation}

Hoffmann M., Dingeldein G., Henrich D., Weiß B., Uppenkamp M.

Medizinische Klinik A, Klinikum Ludwigshafen

Objectives: High dose therapy (HDT) with autologous stem cell transplantation (ASCT) is now gold standard in the therapy of certain haematological malignancies. Infections are mainly during the neutropenic phase. Less often such disease occurs as „late infections“. There are few publications about CMV infections as complication of HDT followed by ASCT. Established risk factors in retrospective studies are CMV seropositivity and haematological malignancies. Aim of this retrospective study was the evaluation of the rate and severity of CMV infection and disease in patients with ASCT which were not screened routinely for CMV.

Material and Methods: From 6/96 until 9/01 63 patients were treated with HDT and ASCT. There were 79 transplantations ( 12 double/2 triple). The median age was 54 years (range 25-74 years). 49 patients had haematological neoplasms, 14 had a solid tumour. Laboratory examination for CMV was executed only when clinical symptoms or laboratory results were suspicious for CMV infection.

Results: CMV infection or disease were seen in 5 of 79 transplantations $(6 \%)$ or 5 of 63 patients $(8 \%)$. Clinical symptoms were fever (after exclusion of other causes), thrombocytopenia, mucositis and pneumonia. One woman died due to CMV pneumonia. Patients were treated with ganciclovir and some with CMV Hyperimmunoglobulin too. Two patients relapsed few weeks after successful first therapy. All patients had haematological malignancies. Risk factors for infection in the early transplantation period like 
several prior therapies, myeloablative conditioning regimens and severe mucositis had no negative effect. CD34-count of the transplant and age showed no influence.

Conclusions: Morbidity for CMV infection was in our patient group rather low. In our opinion therefore CMV screening is not indicated as routine procedure. It should follow clinical symptoms or laboratory results.

Platelet flow cytometric findings in patients undergoing conditioning therapy for allogeneic hematopoietic stem cell transplantation

Höhnberg B., Pihusch R., Pihusch M., Rank A., Penovici M., Murmann K., Reinhardt B., Kolb H.J., Hiller E.

Medizinische Klinik III Großhadern, Klinikum der Ludwig-MaximiliansUniversität München

Objectives: The conditioning regimen preceding hematopoietic stem cell transplantation (HSCT) causes a rapid decrease of platelet count and signs of disseminated intravascular coagulation, possibly indicating platelet activation. As impacts during the conditioning regimen may predict later transplantation-associated complications, we investigated changes in platelet membrane glycoproteins and the liberation of microparticles following dif ferent phases of the conditioning regimen therapy including total body irradiation (TBI), chemotherapy and ATG (antithymocyte globulin)-therapy. Material and Methods: Platelet receptors and granula of 49 patients undergoing HSCT were evaluated by fluorescence activated cell cytometry (FACS) before and after the different phases of conditioning regimen (chemotherapy, total body irradiation, therapy with antithymocyte globulin) and final transplantation. We used a panel of FITC-conjugated monoclonal antibodies (CD9, CD36, CD29, CD49b, CD41a, CD61, CD42a, CD42b, CD45, CD62P, CD63, CD154, PAC-1) to characterize the membrane glycoproteins using a double staining with CD41-PE to detect the platelet-population. To investigate the degranulation of platelet dense bodies, we used mepacrine staining with and without TRAP-stimulation.

Results: Following chemotherapy a high surface expression of CD62P, low mepacrine staining and a reduced surface expression of CD42b (part of the GP Ib/V/IX complex) were found, indicating an irreversible activation of platelets. In addition, elevated levels of circulating microparticles were observed, which may reinforce the thrombosis risk in these patients . Treatment with ATG leads to an elevated surface expression of PAC-1-epitopes, which are neoepitopes appearing after activation of GP IIb/IIIa. However, a significant degranulation was not detectable, which may be the consequence of inhibitory influences on platelets during ATG-induced cytokine released syndrome. TBI and transplantation itself had no influence on platelets. Conclusions: This study is able to demonstrate activating effects on platelets by certain phrases of the conditioning regimen in patients receiving HSCT. Chemotherapy, in particular, leads to strong and irreversible platelet activation and a generation of microparticles which may cause an increased thrombosis risk. Our findings underline the impact of platelets on the pathogenesis of hemostatic complications during HSCT.

\section{5}

Efficiency of peripheral blood progenitor cell (PBPC) mobilisation- influence of donor characteristics

Hölig K. (1), Haack A. (1), Albrecht I. (1), Zimmer K. (1), Bornhäuser M. (2), Ehninger G. (2)

(1) Transfusionsmedizin, (2) Medizinische Klinik und Poliklinik I,

Klinikum der Technischen Universität, Dresden, Germany

Objectives: Stem cell mobilisation in healthy allogenic donors varies to a wide scale. Defining parameters to predict the mobilisation efficacy would be of great interest to optimise protocols of stem cell collection.

Methods: 875 healthy donors (592 men, 293 women) underwent G-CSF application and PBPC collection at our department between 12/1996 and 7/2001

G-CSF administration was performed in 3 dosages: filgrastim $10 \mu \mathrm{g} / \mathrm{kg} / \mathrm{day}$ on 5 days $(11,4 \%)$; lenograstim $7.5 \mu \mathrm{g} / \mathrm{kg} /$ day on 5 days $(66,4 \%)$; lenograstim $7.5 \mu \mathrm{g} / \mathrm{kg} /$ day on day 1 and $2,12.5 \mu \mathrm{g} / \mathrm{kg} /$ day on the following 3 days $(21,7 \%)$. Leukaphereses was performed at day 5 (and 6, if necessary). CD34 -concentration in peripheral blood (x106/L) at day 5 before 1st leukapheresis was analysed for correlation with the following parameters: sex, age, body mass index (BMI), nicotine and alcohol consumption of the donors, G-CSF dose and interval of G-CSF application (single dose versus divided dose).
Results: No correlation of CD 34 concentration in peripheral blood was found with G-CSF-dose. Consumption of alcohol had also no influence on stem cell mobilisation. Significant positive correlation of CD 34 concentration was found with BMI $(\mathrm{p}<0,0001)$ and the intervals of G-CSF application (divided versus single dose; $\mathrm{p}<0,0001$ ). Male donors mobilised significantly better than female donors $(\mathrm{p}<0,0001)$. A significantly negative correlation was found with donor age $(\mathrm{p}<0,001)$ and smoking status $(\mathrm{p}<0,05)$.

Conclusion: In our study PBPC mobilisation was most effective in young, male donors with higher BMI. Lifestyle (smoking) seems to be of any influence, but to a lesser extent. To reach best results, it could possibly be more important to give G-CSF in divided dose than to give higher doses of GCSF

\section{6}

\section{Non-myeloablative transplantation - comparison of related versus unrelated donors}

\section{Höpfner S., Görner M., Benner A., Ho A.D.}

\section{Universitätsklinikum Heidelberg, Medizinische Klinik V}

Introduction: Allogeneic transplantation has become the treatment of choice for numerous malignancies, but the majority of patients do not have an HLA-identical sibling donor. Unrelated donor transplantation is associated with higher rates of GvHD and serious infections and despite lower relapse rates, overall survival is lower. These observations have been made with the use of myeloablative conditioning regimens, and it is yet unclear wether they are valid in the context of non-myeloablative transplantation, which has been employed increasingly to reduce transplant related morbidity and mortality. Therefore, we reviewed our experience with non-myeloablative conditioning regimens, comparing related and unrelated donors.

Patients and Methods: From 11/97 to 02/02, 51 patients with hematological malignancies were treated with non-myeloablative allogeneic transplantation. 31 had a related donor (RD), the remaining 20 had an unrelated donor (UD). For conditioning, most patients (20 RD, 15 UD) received fludarabin and 2 Gy TBI, the others were treated with fludarabin in combination either with melphalan or cyclophosphamide/idarubicin/etoposide in non-myeloablative doses. Both groups were well matched for age and gender.

Results: The incidence of graft failure was higher in the unrelated donor group with $10 \%(\mathrm{n}=2)$ compared to $3 \%(\mathrm{n}=1)$ for the related donor group. The rate of acute GvHD II-IV until day 100 was $30 \%$ in the related donor group versus $50 \%$ for unrelated donors. There were more serious infections in the unrelated donor group with $25 \%$ compared to $16 \%$. At 2 years after transplantation, the progession free survival was $40 \%$ and $25 \%$ in the related und unrelated donor group respectively. The Kaplan Meier estimate of overall survival was $50 \%$ in both groups at 24 months. TRM was $13 \%$ for patients with related dononrs and $30 \%$ for those with unrelated donors

Conclusion: We observed a higher rate of graft failure in patients with an unrelated donor. There was also a trend for an increased rate of aGvHD IIIV and serious infectious complications. There was no statistically significant difference regarding progression free and overall survival. This suggests that the use of unrelated donors in non-myeloablative transplantation is a feasible alternative if a related donor is lacking. Future emphasis should be laid on development of further stategies to reduce the rate of GvHD and serious infections.

\section{7}

\section{Feasibility of daily busulfan targeting as part of conditioning therapy}

Jenke A., Freiberg-Richter J., Schleyer E., Ehninger G., Bornhäuser M.

Med. Klinik und Poliklinik I, Universitätsklinikum Carl Gustav Carus, Fetscherstraße 74, 01307 Dresden

Busulfan (BU) administered in a total dosage of $16 \mathrm{mg} / \mathrm{kg}$ over 4 days is widely used as a component in preparative regimens for allogeneic blood stem cell transplantation (BSCT). The individual bioavailability after oral BU administration differs extremely among the patients and depends on factors like conditioning regimen, age, disease and coadministered drugs. Several authors found an influence of the overall exposure of BU on the outcome after BSCT. Considering this relationship, a therapeutic drug monitoring of BU seems to be advantageous. In these reports BU plasma concentrations at steady state (Css) of $900+-100 \mathrm{ng} / \mathrm{ml}$ seemed to reduce the incidence of relapse or regimen related toxicity. We evaluated a therapeutic drug monitoring in thirteen patients with myelodysplastic syndrome or relapsed acute myeloid leukemia with high risk of regimen related toxicity undergoing BSCT. 
BU dosages were adjusted to achieve Css $900+-100 \mathrm{ng} / \mathrm{ml}$. All patients received a dose reduced conditioning regimen consisting of targeted $\mathrm{BU}$ and fludarabin intravenously $\left(4 \times 30 \mathrm{mg} / \mathrm{m}^{2}\right)$. BU was administered orally in 16 doses every 6 hours. The first dose of BU was fitted using ideal body weight. BU-Css was determined after dose 1, 5, 9 and if necessary after dose 13. BU plasma levels were measured by a modified HPLC method with a time reduced sample treatment. Corresponding the measured Css levels BU doses 4, 8, 12 and 16 were adjusted.

The mean starting dose of BU was $0.89+-0.097 \mathrm{mg} / \mathrm{kg}(1 \mathrm{mg} / \mathrm{kg}$ ideal body weight). In ten patients a dose reduction undergoing the administration of BU was necessary to achieve the target levels. The totally administered dose of BU ranged from $9.9 \mathrm{mg} / \mathrm{kg}$ to $18 \mathrm{mg} / \mathrm{kg}$ with a median dose of $13.8 \mathrm{mg} / \mathrm{kg}$. The average Css after doses 5 and 9 ranged from $808 \mathrm{ng} / \mathrm{ml}$ to $1155 \mathrm{ng} / \mathrm{ml}$ with a median value of $934 \mathrm{ng} / \mathrm{ml}(\mathrm{n}=13)$. An average exposure over 4 days of treatment could be obtained in 7 patients and ranged from $806 \mathrm{ng} / \mathrm{ml}$ to $1128 \mathrm{ng} / \mathrm{ml}$ (median $973 \mathrm{ng} / \mathrm{ml}$ ). All patients receiving the described conditioning regimen had a stable engraftment of periphery blood stem cells. The 100-day-non-relapse mortality was $16 \%$.

Although time and labor intensive, the therapeutic drug monitoring of $\mathrm{BU}$ is a feasible procedure in patients receiving BSCT to minimize the risk of regimen related toxicity. Dose modifications lead to the expected changes in drug exposure. Prospective and randomized clinical studies are warranted to investigate the impact of BU dose adjustment on the outcome after BSCT.

\section{8}

Different human endogenous retrovirus HERV-K mRNA expression patterns in umbilical cord blood, maternal blood and placenta

Karlic H. (1), Kozireva S. (2), Böhles H. (3), Haslberger A. (4), Lohninger A. (5), Pfeilstöcker M. (1,6)

(1) Ludwig Boltzmann Institute for Leukemia Research and Hematology, Vienna, (2) August Kirchenstein Institute of Microbiology and Virology, University of Latvia, Riga, (3) Universitäts Kinderklinik, Frankfurt, (4) Institute of Microbiology, University of Vienna, (5) Department of Medical Chemistry, University of Vienna (6) 3rd Medical Department, Hanusch Hospital, Vienna

Objectives: Human cord blood is used as alternative source of blood stem cells for allogeneic stem cell transplantation, these cells may also be used for gene therapy. HERV-K derived retrovirus-like particles which are known to be present in human placenta could therefore lead to unforeseen consequences in the use of retroviral vectors or following stem cell transplantation. The objective of this pilot study was to analyse the distribution of a highly conserved HERV-K pol sequence motif between maternal blood, placenta and cord blood.

Methods: Samples from 9 cases including two twin births, were analyzed both at the level of genomic DNA and mRNA using quantitative real time PCR (RTQPCR)for expression of HERV-K.

Results: The results obtained by RTQPCR from genomic DNA and from mRNA showed that HERV-K was randomly distributed in genomic DNA but mRNA expression appeared to be up to 50 fold higher in cord blood as compared to mother's blood or placenta. The mRNA expression pattern of 5 housekeeping genes (glucose 6-phosphate-dehydrogenase, B-actin and carnitine palmitoyltransferases CPT1A, CPT1B and CPT 2) was similar in cord blood, maternal blood and placenta.

Conclusion: The relatively high expression of HERV-K in some cord blood samples may influence the success of gene therapy with cord blood derived blood stem cells. As an increased expression of endogenous retroviruses such as HERV-K has also been associated with increased immune activity, the results of this pilot study may also provide a possible explanation for the higher extent of graft-versus-leukemia/host effects which are known to be associated with allogeneic cord blood transplantation.
Rapid engraftment of long term culture initiating cells (LTC-IC) from donor origin and significant prognostic impact of donor $T$ cell chimerism on disease free survival in non myeloablative stem cell transplantation

Keil F., Prinz E., Moser K., Kalhs P., Mannhalter C., Loidolt H., Worel N. Höcker P., Greinix H.T.

(1) Department of internal Medicine I, Bone Marrow Transplantation Unit, University of Vienna, (2) Department of Transfusion Medizine, University of Vienna, Institiute of molecular medicine University of Vienna.

Objectives: Non myeloablative allogeneic stem cell transplantation (NST) allows establishment of donor hematopoiesis without eradication of recipient stem cells by chemoradiotherapy. In the case of mixed chimerism monitoring of T cells and hematopoietic precursor cells is of high importance. It allows to define origin of hematopoietic recovery and to manipulate favorably the graft by infusion of donor leukocytes or increasing or tapering of immunosuppressive therapy.

Methods: We performed dose reduced SCT (conditioning therapy: fludarabine $90 \mathrm{mg} / \mathrm{m}^{2}$ followed by TBI of 2 Gy rad and infusion of unmanipulated PBSC)in 38 patients 11 with AML, 3 with MDS, 11 with NHL, 2 with CML, 7 with MM and 4 with renal cell carcinoma were analysed: Cyclosporine and mycophenolate mofetil were given to prevent GVHD and rejection. Median age was 55 with a range from 18 to 70 years. Chimerism was evaluated in unseparated blood and marrow and various cell subsets in cluding myeloid (CD33+, CD34+)and lymphatic lineage (CD3+, CD4+ CD8+) Chimerisdm was quantified by FISH and VNTR-PCR. Additionally the kinetic of early and late stem cells before and after reduced conditioning therapy was investigated.

Results: Allthough the conditioning therapy was nonmyeloablative we saw a significant decrease of repopulating stem cells defined as long term culture initiating cells (LTC-IC) to $22 \%$ but a rapid recovery of LTC-IC to $150 \%$ if compared with pretransplant value was seen for weeks after transplantation. Interestingly, all LTC-IC were from donor origin after 2 weeks in 7 analysed patients. Similar results were obtained for committed stem cells defined as colony forming cells. (CFC)

All patients who had less than $40 \%$ of donor T cells four weeks after after SCT ( 8 of $38=$ Group A) relapsed ore experienced graft rejection with 3 cases of graft failure and persistent aplasia which made a second stem cell transplantatiopon necessary. All other patients (30 of $38=$ Group B) showed stable mixed or complete myeloid and lymphatic donor chimerism between one and 25 months after SCT. Progression free survival was significantly better in the patients in group B calculated with the product limit method, according to Kaplan and Meier $(\mathrm{p}<0,00005)$ if compared with group A.

Conclusions: These results emphasize the importance of sequencial analyse of lineage specific chimerism to predict outcome after allogeneic nonmyeloablative SCT with dose reduced conditioning.

\section{0}

Transabdominal sonography and colour doppler imaging to evaluating intestinal acute graft versus host disease

Klein S.A. (1), Martin H. (1), Trojan J. (2), Hermann S. (1), Wassmann D. (1), Hoelzer D. (1), Dietrich C.F. (3)

(1) Uniklinik Frankfurt, Med. Klinik III, (2) Uniklinik Frankfurt, Med. Klinik II, (3) Caritas Krankenhaus Bad Mergentheim

Objectives: Acute gastrointestinal graft versus host disease (aGvHD) is a severe complication after allogeneic bone marrow transplantation. In addition to the clinical grading an objective non-invasive method to assess extend, severity, course and prognosis of aGvHD of the bowel is needed. Recently, we were able to describe by high resolution ultrasonography (HRU) and colour Doppler imaging (CDI) some typical sonographic findings in acute GvHD. Here we want to update these findings after the examination of a larger cohort of patients with acute GvHD.

Material and Methods: High resolution ultrasonography and colour Doppler imaging were utilised to describe the sonographic findings in aGvHD of the bowel. 34 patients with suspected aGvHD of the bowel and/or severe cutaneous aGvHD were investigated by HRU and CDI. Structure and thickness of the bowel wall and the blood flow pattern in the superior mesenteric artery (SMA) and in the bowel wall were evaluated.

Results: All patients showed thickened bowel wall segments, particularly in the ileocecal region. Even in patients without any clinical signs of bowel involvement sonographic changes were observed. In all patients, in those with as well as in those without intestinal symptoms an aGvHD was confirmed histologically. In patients with severe diarrhea $\left(>\mathrm{II}^{\circ}\right)$ sonographic signs of secretoric diarrhea (changes of motility and reduction of Kerckring's plicae 
circulares) were detected. Patients without clinical symptoms and those with a low grade $\left(\mathrm{I}^{\circ}\right.$ and $\left.\mathrm{II}^{\circ}\right)$ aGvHD had no sonographic signs of secretoric diarrhea. In most of the patients an increased arterial blood flow was demonstrated as a typical sign of an inflammatory bowel process. In contrast, some patients showed the unique pattern of ischemic bowel wall lesions and high resistance flow pattern in the SMA in conjunction with a thickened bowel wall. These patients did not respond to immunosuppressive therapy and died. However, all patients with a normal or increased arterial blood flow responded at least to the second or third line immunosuppressive treatment.

Conclusions: HRU is a useful tool to detect acute bowel GvHD even prior to clinical symptoms. Furthermore, the pattern with a main manifestation in the ileocecal region might help to differentiate between an infection (e.g pseudomembranous colitis or CMV colitis) and aGvHD of the bowel. Taken together, HRU and CDI can be applied to define the severity of aGvHD and to identify patients with a poor prognosis.

\section{1}

Superior survival for patients with early disease stages following allogeneic blood stem cell transplantation after minimal intensive conditioning

Kobbe G. (1), Fenk R. (1), Emde T. (1), Bernhardt A. (1), Aivado M. (1), Zohren F. (1), Schubert D. (1), Hünerlitürkoglu A. (1), Kronenwett R. (1), Neumann F. (1), Gattermann N. (1), Germing U. (1), Pape H. (2), Haas R. (1), Schneider P. (1)

(1) Medizinische Klinik A, (2) Klinik für Radiologische Onkologie, Heinrich Heine Universität Düsseldorf

We treated 44 patients (median age 52 years, range 26-68) with hematologic malignancies with a conditioning regimen of fludarabine $\left(3 \times 30 \mathrm{mg} / \mathrm{m}^{2}\right)$ and 200cGy TBI followed by allogeneic blood stem cells from matched siblings (31) or matched unrelated donors (13). Diagnoses were AML (5), CML (6), MDS (7), MM (13), Lymphoma (13). Forty-two patients received unmodified mobilized PBPCs (median 7.8x10E6CD34+ cells $/ \mathrm{kg}$, range 3.523.5) and 2 patients received unmodified marrow. Cyclosporine or tacrolimus and mycophenolate mofetil were given for GvHD prophylaxis Thirteen patients were transplanted during early disease stages (Group A, 1st or 2nd CR or 1st PR in MM or 1st chronic phase in CML and low blast count without high risk karyotype in MDS) whereas 31 patients had advanced disease or unfavourable cytogenetics (Group B).

Forty-one patients (93\%) engrafted. Six (15\%) had decreasing donor chimerism in blood and marrow after initial engraftment. Eighteen of 31 evaluable patients $(58 \%)$ with measurable disease at transplantation had objective responses. Overall, 10 of 13 patients (77\%) from group A are alive in remission after a median of 549 days (range 36-999). In this group 2 patients $(15 \%)$ died from treatment related causes and 1 patient died of progressive disease. In group B, 12 of 31 patients (39\%) are alive (6 in CR, 6 with active disease) after a median follow up of 289 days (range 90-1009). Six patients $(19 \%)$ died from treatment related complications and 13 patients died from disease progression. Fatal complications were seen in both groups and were almost always associated with GvHD. Acute GvHD occurred in 25 patients $\left(57 \%, 34 \%{ }^{\circ} \mathrm{I}-\mathrm{II}, 23 \%{ }^{\circ} \mathrm{III}-\mathrm{IV}\right)$. Thirteen of 32 patients $(41 \%)$ who survived at least 100 days developed chronic GvHD. Among 18 evaluable surviving patients (median follow up 580 days, range 179-1009) 10 have chronic GVHD (56\%, 39\% limited, $17 \%$ extensive).

We conclude that allogeneic immunotherapy following minimal intensive conditioning is an effective treatment for patients with hematologic malignancies. Major complications are acute and chronic GvHD, which need to be reduced in future trials. Patients with early disease stages have the greatest chance to become long term survivors, whereas patients with advanced disease have an increased risk for treatment failure from disease progression. We therefore suggest that this new transplantation modality should be offered early in the course of hematologic malignancies with poor prognosis.

\section{Reduction of treatment related mortality after matched unrelated donor transplantation by graft engineering and modified myeloablative conditioning}

Kobbe G., Fenk R., Emde T., Bernhardt A., Aivado M., Hünerlitürkoglu A., Kronenwett R., Neumann F., Germing U., Haas R., Schneider P.

\section{Medizinische Klinik A, Heinrich Heine Universität Düsseldorf}

To reduce treatment related mortality of matched unrelated donor transplantation we developed a new triple aklylator conditioning regimen of melphalan $140 \mathrm{mg} / \mathrm{m}^{2}$, thiotepa $10 \mathrm{mg} / \mathrm{kg}$, cyclophosphamide $120 \mathrm{mg} / \mathrm{kg}$ and ATG $15 \mathrm{mg} / \mathrm{kg}$. Mobilized peripheral blood progenitor cells were depleted of Tcells by CD34-selection with the Isolex 300i. Cyclosporine was given for additional GvHD prophylaxis and sequential T-cell addbacks were scheduled during the first 3 months after transplantation. Mycophenolate mofetil and basiliximab $(2 \times 20 \mathrm{mg})$ was given for GvHD prophylaxis when unmodified marrow was provided by the harvest center.

Seventeen patients received selected mobilized CD34 positive cells (median $4.42 \times 10 \mathrm{E} 6 / \mathrm{kg}$, range $1.96-8.30$, median number of residual CD3 positive cells $0.49 \times 10 E 5 / \mathrm{kg}$, range $0.07-5.90$ ) and 2 patients received marrow. Diagnoses were AML 7, ALL 3, CML 6, MM 2 and NHL/PV 1. All patients (median age 42, range 24-56) had high-risk features. Hematopoietic reconstitution was rapid with WBC $>1000 /$ ul after a median of 11 days (range 967 ) and platelet count $>50000 / \mathrm{ul}$ after a median of 13 days (range 8-22) Two Patients developed acute GvHD before DLI (2x grade I-II) and 9 patients developed aGvHD after DLI (6x grade I-II, $3 x$ grade III-IV). Two of 10 evaluable patients $(20 \%)$ have limited chronic GvHD. After a median follow up of 355 days (range 22-1099) 12 patients (63\%) are alive in remission. Four patients $(21 \%)$ died of disease progression and 3 patients $(16 \%)$ died of treatment related complications (CLS and respiratory failure 1, sepsis 2). TRM was significantly reduced in comparison to a historical contro group of 29 patients who received conventional TBI or busulfan based regimens followed by unmodified unrelated marrow or PBSC transplantation. Among those patients TRM was $66 \%$, DRM was $14 \%$ and 6 patients $(21 \%)$ are alive after a median of 1517 days after transplantation (range 625-3844) We conclude that TRM of matched unrelated donor transplantation can be substantially reduced by using a modified myeloablative conditioning regimen, T-cell depletion and sequential T-cell add backs in high-risk patients with hematologic malignancies.

\section{3}

Induction of stable mixed chimerism in fully MHC-mismatched Bone Marrow Transplantation (BMT) following nonmyeloablative conditioning with low-dose TBI and fludarabine and/or cyclophosphamide

\section{Leng C. (1), Lusatis S. (1), Sykes M. (2), Dörken B. (1), Mapara M.Y. (1)}

(1) Medizinische Klinik m. S. Hämatologie und Onkologie, Universitätsklinikum Charité, Campus Virchow Klinikum und Campus Buch, Humboldt Universität zu Berlin, (2) Transplantation Biology Research Center, Massachusetts General Hospital, Harvard Medical School, Boston, USA

High treatment related mortality and morbidity is still a major problem in the setting of unrelated BMT due to conditioning toxicity and development of graft versus host disease (GVHD). Since the therapeutic benefit of allogeneic BMT is largely related to an immune-mediated graft-versusleukemia effect (GVL), research has focused on the development of less toxic, nonmyeloablative conditioning regimens to achieve mixed chimerism, which then could serve as a platform for the administration of an allogeneic adoptive immunotherapy in the form of donor lymphocyte infusions (DLI). Furthermore such non-toxic conditioning regimen could be applied in the context of solid organ transplantation for the establishment of donor-specific tolerance. Based on a previously established nonmyeloablative conditioning regimen containing in vivo $\mathrm{T}$ cell depletion, cyclophosphamide (CTX) and thymic irradiation (TI), we wanted to address the issue whether further reduction in toxicity would be feasible by substituting TI with fludarabine (FLU), a purine analogue used in the therapy of hematological malignancies that is known to cause immunosuppression. In this study, the effect of FLU containing nonmyeloablative conditioning regimens was evaluated in a fully MHC-mismatched Balb/c (H2d) to C57BL/6 (H2b) strain combination. Conditioning consisted of in vivo T cell depletion (d -5) using anti-CD4 (GK1.5) and anti-CD8 (2.43) monoclonal antibodies followed by the treatment with either FLU $(30 \mathrm{mg} / \mathrm{kg}$ on $\mathrm{d}-4$ to -2$)$ and CTX (200 mg/kg, on d -1), FLU, CTX and TBI (200 cGy) or CTX and TBI (300 cGy). The application of CTX and FLU alone did not provide sufficient immunosuppression to prevent rejection of the bone marrow graft. In 
contrast all mice that recieved low-dose TBI engrafted and showed stable longterm mixed chimerism without development of GVHD. Compared to the CTX/TBI group we could not observe an enhanced T cell lymphopenia in mice that recieved additional FLU. However, donor chimerism tended to be higher in the FLU treated group despite reduced TBI dose. Donor-specific in vitro tolerance could be observed in all mice with mixed chimerism. Our results show that these new nonmyeloablative conditioning regimens lead to stable engraftment of fully MHC-mismatched BMC and donor-specific tolerance without development of GVHD. Studies are currently ongoing to further reduce treatment-related toxicity by reducing TBI-dose and applying costimulation blockade.

\section{4}

\section{Complications after nonmyeloablative stem cell transplanta-} tion (NST) in acute leukemias

Massenkeil G., Genvresse I., Reich G., Le Coutre P., Rackwitz S., Dörken B., Arnold R.

Medizinische Klinik mit Schwerpunkt Hämatologie und Onkologie, Universitätsklinikum Charité, Campus Virchow-Klinikum, HumboldtUniversität Berlin, Deutschland

Introduction: NST has extended the indications for allogeneic SCT to patients (pts) considered ineligible for standard high-dose conditioning. The expectations to NST were high, hoping that the high morbidity of standard high-dose radio- and chemotherapy could be reduced.

Objectives: To analyse nonrelapse complications after NST in 25 patients with ALL and AML.

Patients: 25 pts (9 ALL, 16 AML), median age 43 years (19-67), received NST after conditioning with fludarabine $180 \mathrm{mg} / \mathrm{m}^{2}$, busulfan $8 \mathrm{mg} / \mathrm{kg}$ and ATG $40 \mathrm{mg} / \mathrm{kg}$. Contraindications against standard high-dose conditioning regimens were age above 50 years $(n=7)$, relapse after standard SCT $(n=6)$, infections $(n=9)$, intensive radiotherapy $(n=2)$, and subdural haemorrhage $(n=1) .16 / 25$ pts $(64 \%)$ had uncontrolled disease before NST. Median fol low-up is 8 months (1-39 months). $12 / 25$ pts (48\%) have died from relapse. 13 pts. are alive, $12 / 13$ pts are in CR.

Results: Conditioning related complications were seizure after busulfan despite anticonvulsive prophylaxis in $1 \mathrm{pt}$, serum sickness after ATG in $1 \mathrm{pt}$ and SIRS after ATG in 1 pt. $1 / 25$ pts had a non-fatal graft failure after unrelated NST. Infectious complications occurred in $23 / 25$ pts (1 infectious episode in median per pt, range 1-4): septicemia in 6 pts, meningitis in $1 \mathrm{pt}$, pneumonia in 4 pts, invasive candida infection in 2 pts, fever of unknown origin in 6 pts, acute bronchitis in 3 pts, central venous line infection in 4 pts, CMV-reactivation in 7 pts, herpes simplex/zoster infection in 6 pts, VRE or MRSA in 3 pts, stomatitis III-IV ${ }^{\circ}$ in 7 pts. Severe infections were observed in $9 / 25$ pts $(36 \%)$. 1/25 pts died from sepsis with concomitant acute GvHD grade III. Acute GvHD grade III-IV was observed in 10/25 pts and chronic extensive GvHD in 8/18 evaluable pts. Complications others than GvHD and infections occurred in 3/25 pts and comprised: renal insufficiency and humerus fracture in $1 \mathrm{pt}$, renal insufficiency and facial palsy in $1 \mathrm{pt}$, and secondary insulin-dependent diabetes mellitus in $1 \mathrm{pt}$.

Conclusions: NST can cure pts. with advanced acute leukemias, who do not qualify for standard SCT. However, transplant related toxicity is high. Infections and GvHD remain major transplant-related problems in the first year after allogeneic NST.

\section{5}

\section{Perforin/Granzyme is the predominant effector mechanism of CTL in Graft versus Leukemia reaction}

McGoldrick S., Finke J., Grüllich C.

Uni-Klinik Freiburg

Introduction: Graft versus Leukemia $(\mathrm{GvL})$ reaction plays a major role in remission induction and maintenance after allogenic bone marrow (BMT) or stem cell transplantation (SCT). Conversely the Graft versus Host disease (GvHD) is a severe complication in tranplantation. Since cytotoxic T-Lymphocytes (CTL) are involved in both Graft versus Host Disease (GVHD) and Graft versus leukemia (GVL) after bone-marrow transplantation it is of great interest to elucidate the molecular mechanisms of cellular cytotoxicity in the transplantation setting. Two pathways are used by CTLs: the perforin/granzyme pathway and the death receptor (i.e. Fas, TNFa,TRAIL) pathway. Currently it is poorly understood how these mechanisms are being utilized by CTL.

Methods: To approach this question we first established a cellular allogenic mismatch system to measure cytotoxicity of CTL against EBV-Lymphoblasts (LCL) and matched donor-recipient pairs of patients undergoing
BMT/SCT for leukemia. Specific inhibitors of the different pathways were used to obtain evidence for the relevance of each pathway. We established an cytotoxicity assay that measures apoptosis in target-cells by fluorescence. We generated CTL against the target cells. For the cytotoxic assay effector cells were preincubated with an inhibitor of perforin/granzyme exocytosis or target cells were incubated with caspase-inhibitors which are the dominant effectors of receptor mediated apoptosis.

Results: In all alloreactive CTLs $(n=10)$ cytotoxicity almost exclusively depended on the perforin/granzyme mechanism with rapid kinetics. However, when exocytosis was inhibited a caspase- 8 dependend mechanism of cytotoxicity with slower kinetics was active. We then generated HLA-matched donor CTLs against leukemic blasts of patients undergoing BMT/SCT $(n=6)$. The data obtained in this setting completely confirmed the data obtained with LCL specific CTLs.

Conclusion: Our assay allows rapid and non radioactive measurement of donor CTL cytotoxicity against leukemic blasts of recepients of BMT/SCT. Thus possibly predicting the extend of a GvL reaction. We show that the perforin/granzyme mechanism is predominantly active in the rapid elimination of target cells. However, in circumstances where perforin/granzyme might be inhibited either by primary or secondary resistance of targets, the receptor mediated pathway could function as a salvage mechanism. Futher studies are being conducted to clarify these mechanisms.

\section{6}

\section{Evaluation of haematopoetic recovery after autologous blood} stem cell transplantation by soluble transferrin receptor

Metzgeroth G., Bergmann J., Schlegel F., Emig M., Maywald O., Eichler H., Hehlmann R., Hastka J.

III. Medizinische Universitätsklinik, Fakultät für Klinische Medizin Mannheim der Universität Heidelberg

The measurement of serum transferrin receptor (sTfR) has proved to be a valuable parameter in the assessment of body iron status and the erythropoietic marrow activity. If iron deficiency is excluded, sTfR provides a quantitative measure of the erythroid marrow mass. The aim of our study was to investigate, whether sTfR can be used as an early predictive parameter of successful engraftment after autologous blood stem cell transplantation (SCT). In 12 patients (6 multiple myeloma, 5 NHL, 1 Morbus Hodgkin), who underwent autologous peripheral blood stem cell transplantation, the concentration of STfR and the values of hemoglobin, leucocytes and thrombocytes in peripheral blood samples were determined daily. The sTfRmeasurement was performed by a latex-particle-enhanced immunonephelometric assay (N Latex sTfR by Dade-Behring, Liederbach, Germany, normal range: $0,81-1,75 \mathrm{mg} / \mathrm{l})$. Iron deficiency was excluded in all patients by bone marrow examination. All patients showed rapid and sustained haematopoetic recovery. The leukocytes reached the nadir values in median 6 days (range: 3-9) after SCT. They begun to increase in median 9 days after SCT (range: $8-14$ ), levels above 1000/ $\mu 1$ were observed in median after 11 days (range 9-17). The pre-therapy sTfR-concentration was within the normal range in all patients (median: $1,0 \mathrm{mg} / \mathrm{l}$, range: $0,90-1,18$ ). After chemotherapy, sTfR declined to a minimum of $0,68 \mathrm{mg} / \mathrm{l}$ (range: $0,49-0,82$ ), the minimal values were measured in median 8,5 days (range: 7-11) after SCT. The increase of the sTfR-concentration was observed in median 9,5 days (range: 9-14) after SCT, the pre-therapy values were reached after 12,5 days (range: 12-20).

In 7 patients the increase of STfR and leucocyte count was observed on the same day after SCT. In 3 other patients the leucocyte count increased 1 to 2 days earlier than the sTfR-concentration.

Our results show, that the sTfR-determination is not useful as a predictive parameter of successful SCT, because sTfR values start to rise first when the increment of the leucocyte count has already occured.

\section{7}

Incidence of cytomegalovirus infection after allogenic stem cell transplantation: A comparison of dose reduced and myeloablative conditioning

Oswald O. (1), Schetelig J. (2), Steuer N. (1), Nitsche A. (3), Siegert W. (1)

(1) CCM, Berlin, (2) Charité - CVK, Berlin, (3) TIB-Molbiol, Berlin

Objectives: The final treatment for patients with severe hematologic malignancies is often an allogenic hematopoietic cell transplantation (HCT). However the indication for myeloablative conditioning is frequently limited due to the patient's individual risk factors. The use of reduced intensity conditioning allows applying the indication of HCT on most patients. After allogenic stem cell transplantation viral infections which are harmless in 
healthy persons, like the human cytomegalovirus (HCMV), can cause severe complications in patients of immunocompromised state. Earlier studies have demonstrated that the mortality due to early infection might be decreased by using a dose reduced conditioning regime.

Material and Methods: In total, 82 patients with hematologic malignancies received an allogenic transplantation. 37 of these patients were given a myeloablative treatment, mainly consisting of cyclophosphamid and busulfan, whereas 45 patients received a dose-reduced conditioning regime consisting of fludarabine, busulfan and ATG. The patients were prospectively monitored weekly for CMV infection by CMV-PCR, APAAP and clinical observation. All blood samples were tested by qualitative PCR, APAAP and quantitative real time PCR.

Results: No difference between the reduced-intensity and the myeloablative group was found regarding the rate of CMV infection defined by quantitative TaqMan PCR (46\% reduced-intensity and $38 \%$ myeloablative, $\mathrm{p}=0.466$; median viral load $861 \mathrm{ge} / \mathrm{ml}$ vs.1369 ge/ml, p=0.327) or APAAP (33\% vs $21 \%, \mathrm{p}=0.242$; median antigenemia positive cells $2 / 10,000$ for both groups, $\mathrm{p}=0.790$ ) nor by clinical criteria for CMV disease or the need of antiviral therapy. Also a multivariate model including age, CMV serostatus of patient and donor and acute GVHD did not show a significant difference between the two groups.

Conclusions: The incidence and severity of HCMV infections was not dif ferent in the two compared groups. The conditioning regime itself seems to have only a low effect on long term immunosuppression after HCT.

\section{8}

Survey on autologous hematopoietic stem cell transplantations performed in germany in the year 2001: A report from the German registry for stem cell transplantations (DRST)

\section{Ottinger H.D. (1), Müller C. (2), Kubanek B. (3) Schmitz N. (4), Schaefer} U.W. (1)

(1) KMT Klinik Universitätsklinikum Essen, (2) ZKRD Ulm, (3) DRK Blutspendedienst Universitätsklinikum Ulm, (4) Allgemeines Krankenhaus St. Georg, Hamburg

To get an overview on the transplant activities of autologous hematopoietic stem cells (auto-HSCT)in Germany in the year 2001 a retrospective evaluation was performed by the national registry for stem cell transplantations named DRST.

The local data managers of all German transplant units cooperating with the DRST $(n=95)$ were asked to fill an activity survey sheet on auto-HSCT covering the following items: number of patients who had received an autoHSCT, underlying diseases/disease stage and used stem cell sources. Results were compared to former activity surveys for the years 1998-2000. The total number of auto-HSCTs (first transplants only) performed in Germany was 2.142 in the year 2001. The corresponding figures for the years $1998-2000$ were $2.444,2.228$ and 2.105. Peripheral blood was preferred over marrow as stem cell source in $>99 \%$ of the cases in 2001 . The distribution of the main indications in year 2001 was as follows: multiple myeloma $32 \%$, non-Hodgkin lymphomas $32 \%$, acute myeloic leukemia in first remision $7 \%$, germinal tumors $6 \%$, Hodgkin's disease $6 \%$, and chronic lymphatic leukemia $3 \%$. Of interest is that the frequency of auto-HSCT for multiple myeloma showed a continous increase from $1998(\mathrm{n}=422)$ to $2001(n=691)$, whereas the number of auto-HSCTs for breast cancer dropped from $n=490$ in year 1998 to $n=29$ in year 2001. A decline in frequency of auto-HSCTs was also observed for lung cancer (1998: $n=48$, 2001: $n=4)$ and ovarial cancer $(1998: n=66,2001: n=11)$. The five largest units (number of first transplants) in the year 2001 were the University Hospitals of Heidelberg $(n=127)$, Essen $(n=84)$, Tübingen $(n=70)$, Düsseldorf $(n=63)$, and Kiel $(n=63)$. In contrast, at 30 of the 95 active German units the frequency of first auto-HSCTs was $<10$.

Thus, (1) the decrease in total number of auto-HSCT performed in Germany observed during 1998-2000 has been stopped in the year 2001 and (2) multiple myeloma has become the leading indication for auto-HSCT whereas breast cancer and other solid tumors have meanwhile become rare indications for auto-HSCT.
Survey on allogeneic hematopoietic stem cell transplantations performed in Germany in the year 2001: A report from the german registry for stem cell transplantations

Ottinger H.D. (1), Müller C. (2), Kubanek B. (3), Schmitz N. (4), Schaefer U.W. (1)

(1) Universitätsklinikum Essen, (2) ZKRD Ulm, (3) Universitätsklinikum Ulm, (4) Allgem. Krankenhaus St. Georg Hamburg

To get an overview on the transplant activities of allogeneic hematopoietic stem cells (allo-HSCT) in Germany in the year 2001 a retrospective evaluation was performed by the German Registry for Stem Cell Transplantations named DRST.

The local data managers of all active German allo-HSCT units $(n=46)$ were asked to fill a transplant activity survey sheet covering the following items: number of transplants performed, indications, type of donor as well as stem cell source used, and percentage of transplants with reduced conditioning intensity (mini, micro transplants). Results were compared to those of the activity surveys for the years 1998-2000.

A total of 1.436 first allo-HSCT ( 56 with preceeding auto-HSCT) were performed in Germany in the year 2001. The corresponding figures for the years $1998-2000$ were $1.118,1.417$ and 1.438 , respectively. The use of peripheral blood as stem cell source increased from $50 \%$ in 1998 to $68 \%$ in 2001. Cord blood transplantations were rarely performed (1998: $n=3,2001$ : $\mathrm{n}=7$ ). Whereas the proportion of related to unrelated transplants was still 1.5 in 1998, it decreased to 0.9 in the year 2001. The distribution of main indications for allo-HSCT in the year 2001 were as follows: AML $29 \%$, ALL $18 \%$, CML $17 \%$ and MDS $10 \%$. Of interest is that the frequency of alloHSCT for MDS showed a continious increase over the last four years (1998: $\mathrm{n}=76,2001: \mathrm{n}=136$ ), wheras the number of transplants for CML is clearly declining (1998: $n=333,2001: \mathrm{n}=239)$. The five largest allo-HSCT units in Germany (number of first allo or mixed auto- plus allo transplants, no retransplants) were the University Hospitals of Essen $(n=132)$, Hamburg ( $n=$ $84)$, Munich $(n=83)$, Dresden $(n=77)$, and Wiesbaden $(n=70)$. Despite financial restrictions a conditionining protocol with reduced intensity was used in $27 \%$ of the cases $(n=391)$ in 2001.

Thus,

(1) MDS becomes a more and more important indication for allo-HSCT,

(2) the frequency of HSCTs for CML is declining, and

(3) Mini/micro allo-HSCT have not lost their popularity in the year 2001 despite financial restrictions.

\section{High dose melphalan and autologous stem cell support after VAD chemotherapy for treatment of systemic light chain (AL) amyloidosis}

Perz J., Schönland S., Witzens M., Lannert H., Breitkreuz I., Raab M., Ho A.D., Goldschmidt H.

\section{Medizinische Klinik und Poliklinik V, Universität Heidelberg}

Aims: The median survival of patients (pts) with AL amyloidosis without therapy is 7 months. Autologous stem cell transplantation (ASCT) after high dose melphalan (HD-M) has been recently reported. We evaluated the efficacy and the toxicity of a new therapeutic regimen combining conventional VAD (vincristine/adriamycin/dexamethason) chemotherapy and HD$\mathrm{M}$ followed by ASCT.

Methods: 21 pts (13 male, 8 female) with main AL amyloidosis manifestation of heart $(14 \%)$, kidney $(58 \%)$, liver or intestinum $(14 \%)$ or muscles (14\%) were initially treated with $1-4$ cycles VAD. Stem cells were mobilised with G-CSF after cyclophosphamide (4 g/m², $\mathrm{n}=4)$, cyclophosphamide $\left(1 \mathrm{~g} / \mathrm{m}^{2}\right) /$ adriamycine/dexamethason $(\mathrm{n}=3)$ or ifosfamide $\left(12 \mathrm{~g} / \mathrm{m}^{2}\right.$, $\mathrm{n}=13) .13$ pts recieved $200 \mathrm{mg} / \mathrm{m}^{2}$ melphalan, 3 pts $140 \mathrm{mg} / \mathrm{m}^{2}$ melphalan and 4 pts with markely reduced renal function $100 \mathrm{mg} / \mathrm{m}^{2}$ melphalan.

Results: 1 patient died after the first VAD cycle from sepsis, 3 pts $(15 \%)$ succumbed during the fist 3 months after ASCT due to cerebral hemorrhagy, intestinal hemorrhagy or neutropenic sepsis. One patient died 10 months after ASCT due to progressive heart failure. The follow up of 16 surviving pts $(76 \%)$ is actually between 6 and 52 months (median 11 months). In 7 pts complete hematological remission could be achieved, in 2 pts minimal response, in 5 pts steable disease, in 2 pts evaluation not possible (no detectable gammopathy).

Conclusions: HD-M followed by ASCT after conventional VAD is feasible in pts with systemic AL amyloidosis. By initial reduction of the clonal plasma cell disorder hematological response can be achieved. Longer follow up will indicate whether conventional chemotherapy prior to HD-Mand and ASCT could produce long term benefit. 